Supporting Information

\title{
Cobalt-Catalyzed Enantioselective Hydroarylation of 1,6-Enynes
}

Andrew Whyte, ${ }^{\dagger}$ Alexa Torelli, ${ }^{\dagger}$ Bijan Mirabi, ${ }^{\dagger}$ Liher Prieto,${ }^{\dagger} \S$ Jose F. Rodriguez, ${ }^{\dagger}$ and Mark Lautens $^{\dagger *}$

<mark.lautens@utoronto.ca>

${ }^{\dagger}$ Davenport Research Laboratories, Department of Chemistry, University of Toronto, 80 St. George Street, Toronto, Ontario M5S 3H6, Canada

${ }^{\S}$ Department of Organic Chemistry II, University of the Basque Country (UPV/EHU), 48080 Bilbao, Spain

\section{Table of Contents}

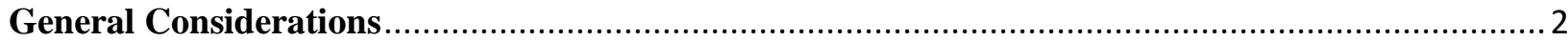

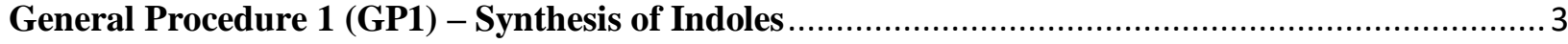

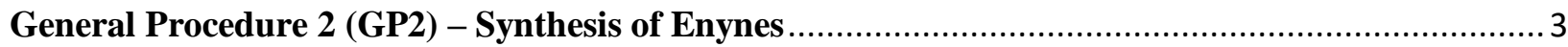

General Procedure 3 (GP3) - Cobalt-Catalyzed Hydroarylation .............................................. 4

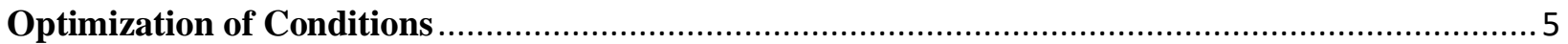

Optimization of NaBARF, temperature, and solvent ......................................................... 5

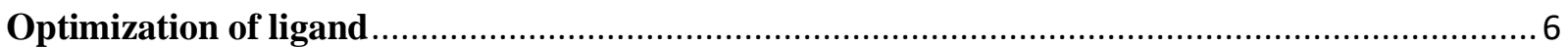

Optimization of other reaction parameters …................................................................. 7

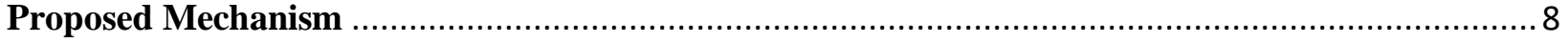

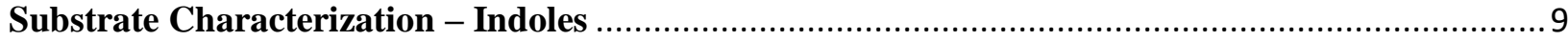

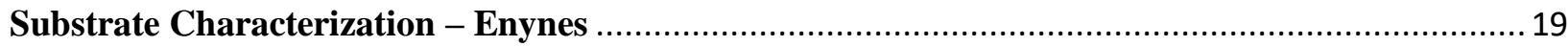

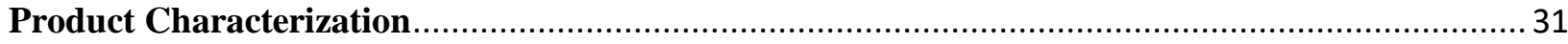

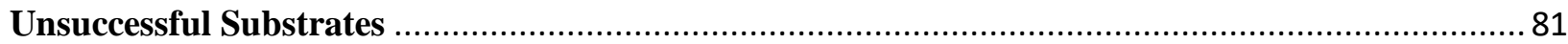

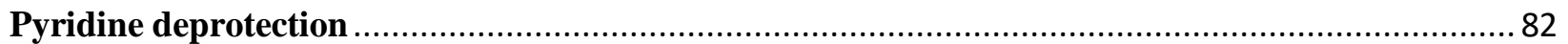

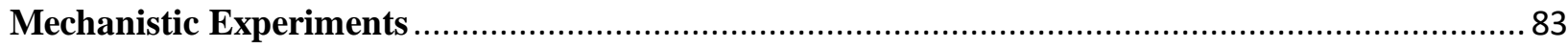

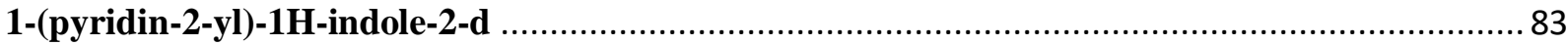

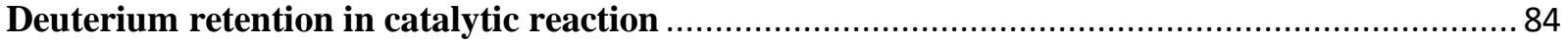

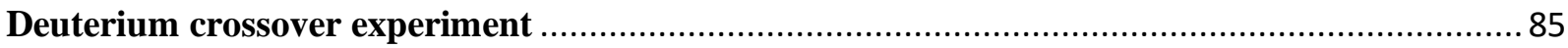

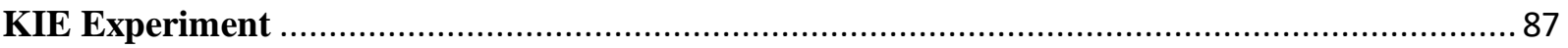

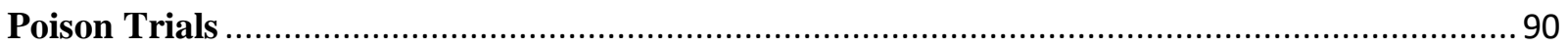

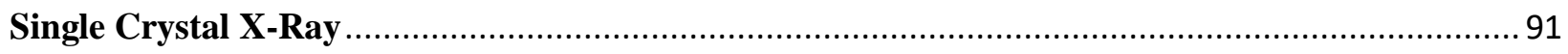

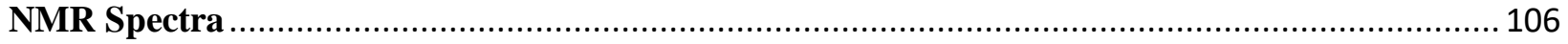




\section{General Considerations}

All reactions were performed under argon in a dry environment unless otherwise stated. Reaction progress was monitored by TLC using UV light or $\mathrm{KMnO}_{4}$ for visualization. THF was distilled over sodium/benzophenone. Dichloromethane was distilled over calcium hydride. Toluene was purchased from Fisher and used as received. Acetonitrile was purchased from Caledon and used as received. HPLC grade hexanes and isopropanol were purchased from Fisher. All other starting materials and reagents were purchased from Sigma, Alfa Aesar, Fisher, or Combi-Blocks and were used as received. $\mathrm{CoBr}_{2}$ was purchased from Sigma-Aldrich and was stored in a glovebox. $(R, R)$-QuinoxP was purchased from Strem. Zinc was purchased from Sigma and was acid washed prior to use and stored under an inert atmosphere. NaBARF was synthesized following literature procedure ${ }^{1}$. Catalytic reactions were performed in $2 \mathrm{dr}$ vials, equipped with a Teflon septa (ThermoScientific National B7995-15) and a stir bar (Fisher cat no. 14-513-57, 12 x 4.5 $\mathrm{mm})$.

${ }^{1} \mathrm{H},{ }^{13} \mathrm{C}$, and ${ }^{19} \mathrm{~F}$ NMR were obtained at $296 \mathrm{~K}$ on an Agilent DD2 500 equipped with a $5 \mathrm{~mm}$ Xses Cold Probe, or a Varian Mercury 300 or 400 or a Bruker Avance III 400. Measurements were referenced to the solvent. NMR data is referenced as chemical shift, multiplicity, coupling constant, integration. Carbons directly bound to boron were not observed due to quadrupolar relaxation. NMR yields were obtained using 1,3,5-trimethoxybenzene as an internal standard. HRMS were obtained on a JEOL AccuTOF-DART or ESI performed at the Advanced Instrumentation for Molecular Structure (AIMS) at the University of Toronto. IR spectra were acquired on a Perkin-Elmer Spectrum 100 instrument with a single-bounce diamond/ZeSe ATR accessory. Data is presented in wavenumbers (cm-1 ). Chiral HPLC was performed on Agilent 1100 or 1200 series operated by ChemStation LC 3D software.

${ }^{1}$ Park, J. G.; Jeon, I.-R.; Harris, T. D. Electronic Effects of Ligand Substitution on Spin Crossover in a Series of Diiminoquinonoid-Bridged Fe II 2 Complexes. Inorg. Chem. 2015, 54, 359-369. 
General Procedure 1 (GP1) - Synthesis of Indoles

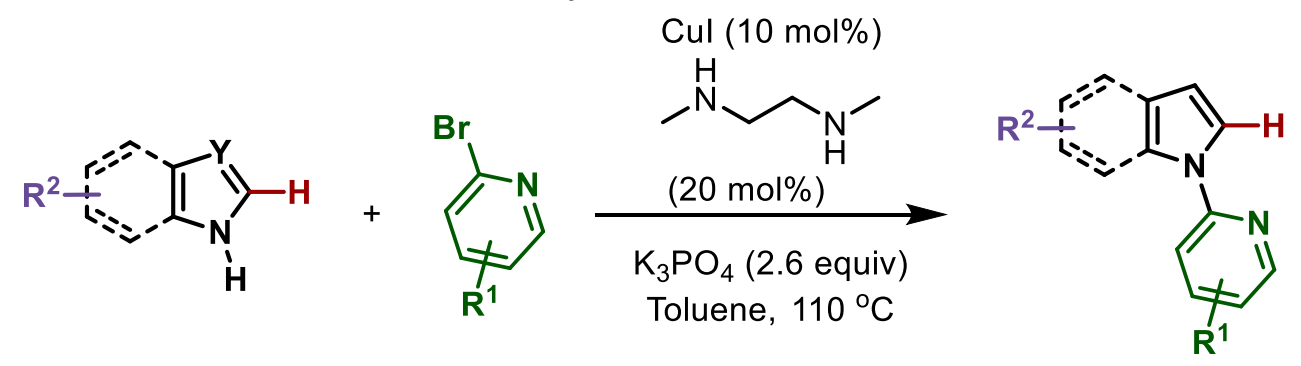

Procedure adapted from Ackermann (Org. Lett. 2011, 13, 3332-3335)

A dry $100 \mathrm{~mL}$ round bottom flask was purged with argon. The appropriate indole or $\mathrm{NH}$ heterocycle $(10 \mathrm{mmol})$ was added to the round bottom with $\mathrm{CuI}(190 \mathrm{mg}, 10 \mathrm{~mol} \%)$ and $\mathrm{K} 3 \mathrm{PO} 4$ (5.5 g, 2.6 equiv). Then 2-bromopyridine ( $1.8 \mathrm{~mL}, 1.5$ equiv) and DMEDA $(0.22 \mathrm{~mL}, 20 \mathrm{~mol} \%)$ was added in toluene $(12.5 \mathrm{~mL})$. The reaction was stirred at $110{ }^{\circ} \mathrm{C}$ for $24 \mathrm{~h}$. The reaction was cooled to room temperature and filtered over a silica pad. The product was isolated by flash column chromatography, typically $20 \%$-> 60\% DCM/pentane w/ $1 \%$ TEA. In instances where $\mathrm{N}$-pyridyl indole was difficult to separate from remaining bromopyridine the product was triturated or recrystallized from pentane or the reaction was run with 1.05-1.5 equivalents of indole relative to bromopyridine.

\section{General Procedure 2 (GP2) - Synthesis of Enynes}

Procedure following Yang's method. A. Lin, Z.-W. Zhang, J. Yang, Org. Lett. 2014, 16, 386389.

Allyl amine (1.1 equiv) was dissolved in DCM ( $2 \mathrm{M}$ ). Triethylamine (1 equiv) was added and the solution was cooled to $0{ }^{\circ} \mathrm{C}$. $p$-toluenesulfonyl chloride (1 equiv) was added portion wise at $0{ }^{\circ} \mathrm{C}$. Reaction was stirred at room temperature until completion was observed by TLC (typically X h). The reaction was quenched with $\mathrm{NaHCO} 3$ and diluted with DCM. The aqueous layer as extracted with DCM three times. The organic layer was washed twice with water, dried over magnesium sulfate, filtered, and concentrated. The $N$-allyl-p-toluenesulfonamides was subjected to the next step with no further purification.

$N$-allyl-p-toluenesulfonamides were dissolved in acetone ( $0.6 \mathrm{M})$. Potassium carbonate (1.5 equiv) was added. Propargyl bromide (1.5 equiv) was added dropwise. The solution was stirred at $60{ }^{\circ} \mathrm{C}$ until no starting material was observed on TLC. The reaction was filtered over a silica gel pad using ethyl acetate. The filtrate was concentrated in vacuo and columned in 5\% EtOAc/pentanes to yield the $N$-allyl-4-methyl- $N$-(propargyl)benzenesulfonamide as a white solid.

Reactions were typically performed on $4 \mathrm{mmol}$ scale. In a flame dried vial purged with argon aryl iodide was added, followed by the enyne (1.2 equiv), $\mathrm{Pd}(\mathrm{Cl})_{2}\left(\mathrm{PPh}_{3}\right)_{2}(4 \mathrm{~mol} \%)$, and copper iodide $(8 \mathrm{~mol} \%)$. The solids were dissolved in DMF or $\mathrm{MeCN}(1 \mathrm{M})$, then triethylamine (2.5 equiv) was charged. The reactions were stirred at $40{ }^{\circ} \mathrm{C}$ or $80^{\circ} \mathrm{C}$ until no starting material was observed on TLC, typically 4-6 hours. The reaction was quenched with sat. $\mathrm{NH}_{4} \mathrm{Cl}$, and diluted 
with EtOAc. The aqueous layer was extracted washed 5 times, dried over magnesium sulfate, filtered, and concentrated. The 1,6-enynes were purified by flash chromatography typically in 5$20 \%$ EtOAc/Pentanes.

\section{General Procedure 3 (GP3) - Cobalt-Catalyzed Hydroarylation}

An oven dried $2 \mathrm{dr}$ vial was cooled to room temperature and purged with argon. Then $(R, R)-$ QuinOxP (4.0 mg, $6 \mathrm{~mol} \%)$, Zn (2.6 mg, $20 \mathrm{~mol} \%)$, enyne ( $0.30 \mathrm{mmol}, 1.5$ equiv), C-H bond donor $(0.20 \mathrm{mmol})$, and $\mathrm{NaBARF}(13.3 \mathrm{mg}, 7.5 \mathrm{~mol} \%)$ were all added consecutively. In the case of liquid substrates, the substrates were weighed out separately and dissolved in DCM. Then $\mathrm{CoBr}_{2}(2.2 \mathrm{mg}, 5 \mathrm{~mol} \%$ ) was added to the reaction vial which was then sealed with a teflon septa. DCM $(1.0 \mathrm{~mL}, 0.2 \mathrm{M})$ was added and the reaction was stirred at $60{ }^{\circ} \mathrm{C}$ for $24 \mathrm{~h}$. Then reaction was filtered through a silica pad and purified with flash column chromatography in EtOAc/pentane mixtures. 


\section{Optimization of Conditions}

\section{Optimization of NaBARF, temperature, and solvent}

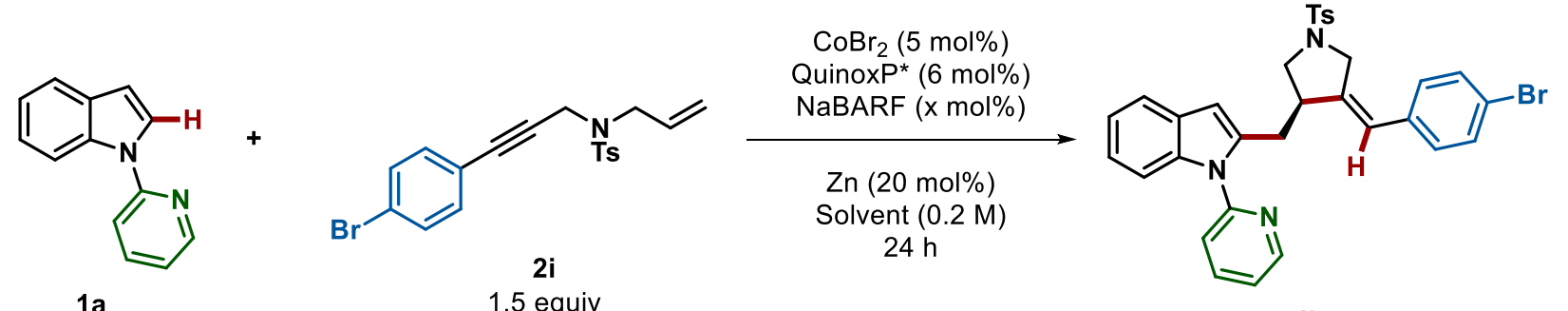

$1 \mathrm{a}$

1.5 equiv

$4 \mathbf{i}$

\begin{tabular}{|c|c|c|c|c|c|c|}
\hline $\begin{array}{c}\text { NaBARF } \\
(\mathrm{mol} \%)\end{array}$ & Temp. & Solvent & $\begin{array}{c}\text { Indole Left } \\
(\%)\end{array}$ & $\begin{array}{c}\text { Enyne Left } \\
\text { (equiv) }\end{array}$ & $\begin{array}{c}\text { NMR yield } \\
\text { (isolated) }\end{array}$ & er \\
\hline 10 & $40{ }^{\circ} \mathrm{C}$ & DCM & 87 & 1.45 & $5(7)$ & $90: 10$ \\
\hline 10 & $50{ }^{\circ} \mathrm{C}$ & DCM & 55 & 1.07 & $47(43)$ & $93: 7$ \\
\hline 10 & $60{ }^{\circ} \mathrm{C}$ & DCM & 35 & 0.93 & $60(58)$ & $95: 5$ \\
\hline 10 & $70{ }^{\circ} \mathrm{C}$ & DCM & 15 & 0.49 & $79(80)$ & $94: 6$ \\
\hline 10 & $80{ }^{\circ} \mathrm{C}$ & DCM & 13 & 0.57 & $85(83)$ & $93: 7$ \\
\hline 10 & $60{ }^{\circ} \mathrm{C}$ & DCE & 54 & 0.98 & $43(46)$ & $93: 7$ \\
\hline 10 & $80{ }^{\circ} \mathrm{C}$ & DCE & 93 & 1.40 & 7 & N.D. \\
\hline 5 & $60{ }^{\circ} \mathrm{C}$ & DCM & 75 & 1.30 & $25(25)$ & $95: 5$ \\
\hline 6 & $60^{\circ} \mathrm{C}$ & DCM & 2 & 0.49 & $98(91)$ & $95: 5$ \\
\hline 7.5 & $60{ }^{\circ} \mathrm{C}$ & DCM & 1 & 0.49 & $96(90)$ & 95.5:4.5 \\
\hline
\end{tabular}


Optimization of ligand

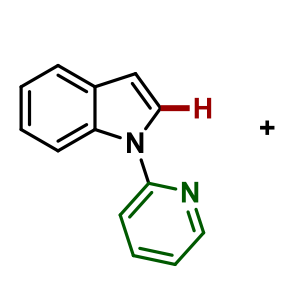

1a

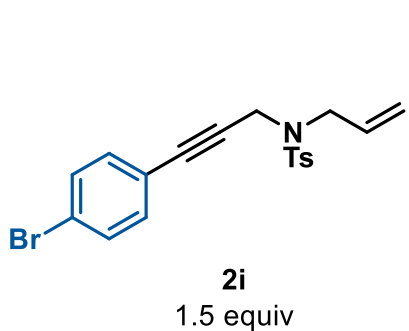

1.5 equiv
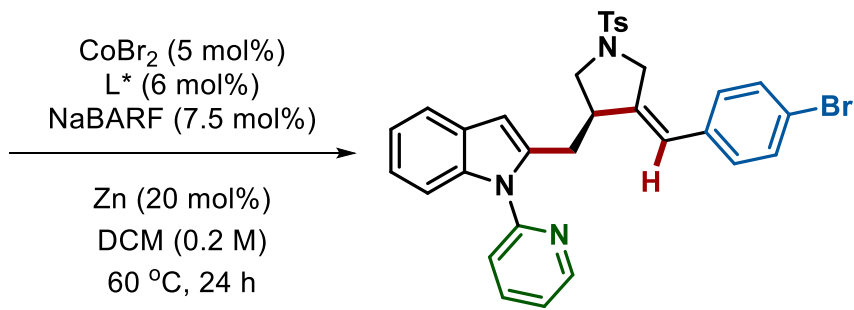

$4 i$

\begin{tabular}{|c|c|c|c|c|}
\hline L* & $\begin{array}{c}\text { Indole Left } \\
(\mathbf{\%})\end{array}$ & $\begin{array}{c}\text { Enyne Left } \\
\text { (equiv) }\end{array}$ & $\begin{array}{c}\text { NMR yield } \\
\text { (isolated) }\end{array}$ & er \\
\hline QuinoxP & $\mathbf{1}$ & $\mathbf{0 . 4 9}$ & $\mathbf{9 6}(\mathbf{9 0 )}$ & $\mathbf{9 5 . 5 : 4 . 5}$ \\
\hline L3 & 94 & 1.50 & 0 & n.d. \\
\hline L4 & 95 & 1.50 & 0 & n.d. \\
\hline L5 & 53 & 0.93 & 48 & $75: 25$ \\
\hline L6 & 96 & 1.50 & 0 & n.d. \\
\hline L7 & 93 & 1.48 & 0 & n.d. \\
\hline
\end{tabular}<smiles>C[C@H]1CC[C@@H](C)P1c1ccccc1P1[C@H](C)CC[C@H]1C</smiles>

L3<smiles>CC1CC[C@@H](C)P1c1ccccc1P1C(C)CC[C@H]1C</smiles>

L4<smiles>c1ccc(C2CC[C@@H](c3ccccc3)P2CCP2[C@H](c3ccccc3)CC[C@H]2c2ccccc2)cc1</smiles>

L5

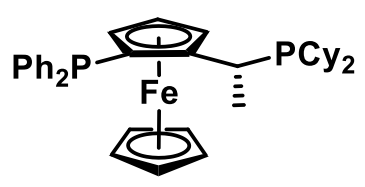

L6<smiles>c1ccc(-c2ccc3ccccc3c2-c2c(P(c3ccccc3)c3ccccc3)ccc3ccccc23)cc1</smiles>

L7 


\section{Optimization of other reaction parameters}

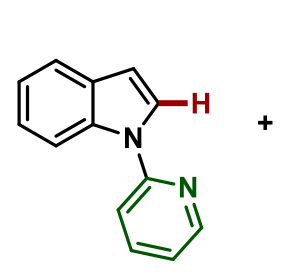

1 a

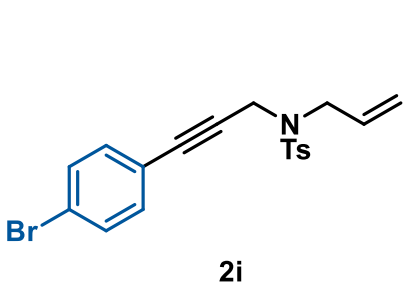

1.5 equiv

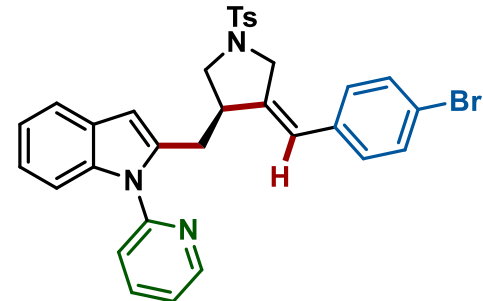

$4 i$

\begin{tabular}{|c|c|c|c|c|}
\hline Variation & $\begin{array}{c}\text { Indole Left } \\
(\mathbf{\%})\end{array}$ & $\begin{array}{c}\text { Enyne Left } \\
\text { (equiv) }\end{array}$ & $\begin{array}{c}\text { NMR yield } \\
\text { (isolated) }\end{array}$ & er \\
\hline None & $\mathbf{1}$ & $\mathbf{0 . 4 9}$ & $\mathbf{9 6}(\mathbf{9 0})$ & $\mathbf{9 5 . 5 : 4 . 5}$ \\
\hline THF instead of DCM & 95 & 0.17 & 0 & n.d. \\
\hline Toluene instead of DCM & 11 & 0.32 & 89 & $94: 6$ \\
\hline $\mathrm{MeCN}_{\text {instead of } \mathrm{DCM}}$ & 98 & 1.50 & 0 & n.d. \\
\hline $\mathrm{CoCl}_{2}$ instead of $\mathrm{CoBr}_{2}$ & 85 & 1.41 & 7 & $91: 9$ \\
\hline $\mathrm{CoI}_{2}$ instead of $\mathrm{CoBr}_{2}$ & 20 & 0.07 & 70 & $95.5: 4.5$ \\
\hline $\mathrm{Co}_{2}$ acac) ${ }_{2}$ instead of $\mathrm{CoBr}_{2}$ & 94 & 1.50 & 0 & n.d. \\
\hline 1.2 equiv enyne instead of 1.5 equiv & 3 & 0.16 & $93(86)$ & $95.5: 4.5$ \\
\hline 20 mol\% $\mathrm{ZnI}{ }_{2}$ instead of $\mathrm{NaBARF}$ & 61 & 0.85 & 36 & $94: 6$ \\
\hline $\mathrm{Mn}$ instead of $\mathrm{Zn}$ & 90 & 1.50 & 0 & n.d. \\
\hline
\end{tabular}




\section{Proposed Mechanism}

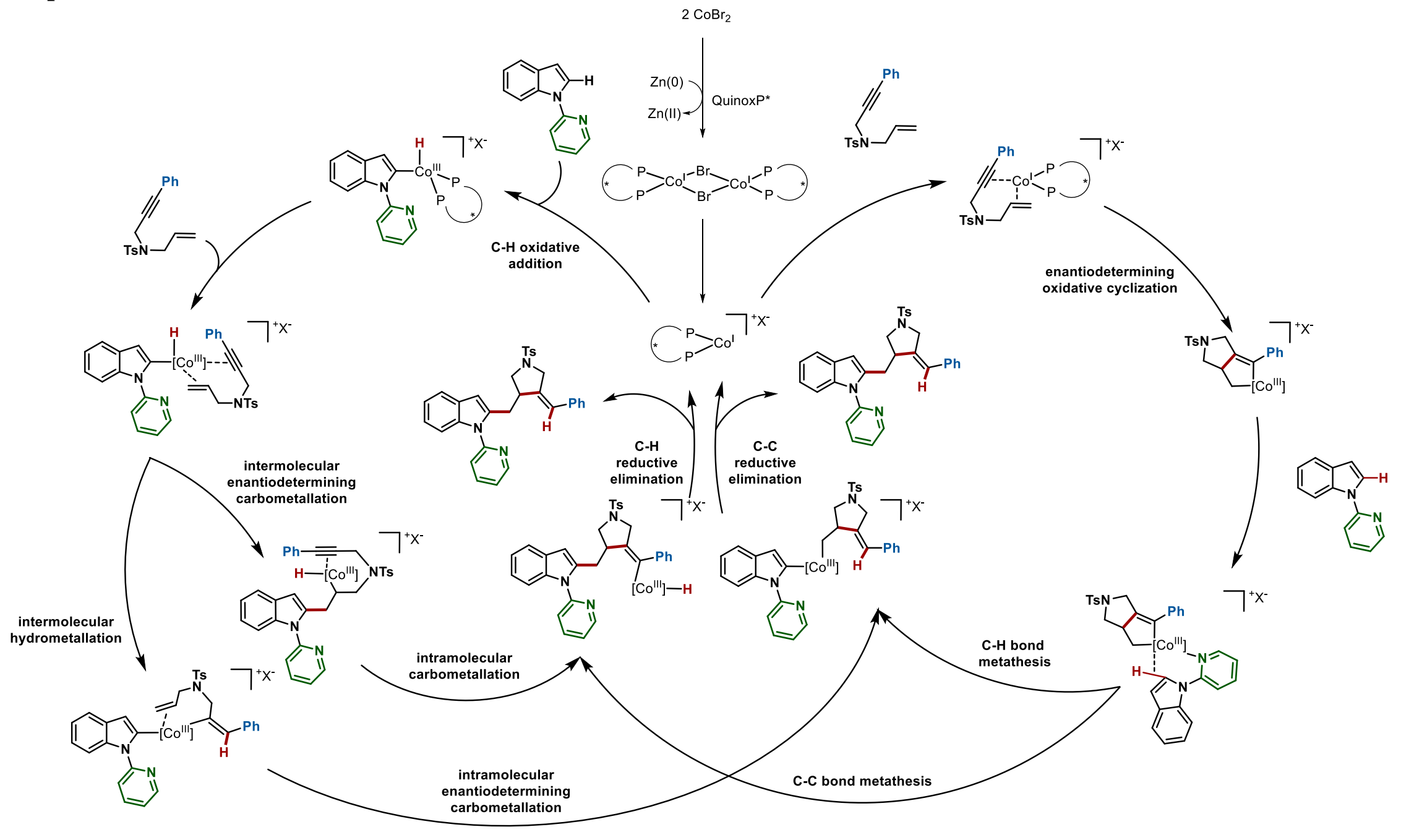




\section{Substrate Characterization - Indoles}

1-(pyridin-2-yl)-1H-indole (1a)<smiles>c1ccc(-n2ccc3ccccc32)nc1</smiles>

Synthesized following GP1. Characterization consistent with literature L. Ackermann, A. V. Lygin, Org. Lett. 2011, 13, 3332-3335.

${ }^{1}$ H NMR $\left(400 \mathrm{MHz}, \mathrm{CDCl}_{3}\right) \delta 8.44(\mathrm{ddd}, J=4.9,2.0,0.9 \mathrm{~Hz}, 1 \mathrm{H}), 8.11(\mathrm{dd}, J=8.3,0.9 \mathrm{~Hz}$, $1 \mathrm{H}), 7.64(\mathrm{ddd}, J=8.3,7.4,2.0 \mathrm{~Hz}, 1 \mathrm{H}), 7.60(\mathrm{~d}, J=3.5 \mathrm{~Hz}, 1 \mathrm{H}), 7.56(\mathrm{ddd}, J=7.7,1.3,0.8 \mathrm{~Hz}$, $1 \mathrm{H}), 7.34(\mathrm{dt}, J=8.2,1.0 \mathrm{~Hz}, 1 \mathrm{H}), 7.19$ (ddd, $J=8.2,7.1,1.3 \mathrm{~Hz}, 1 \mathrm{H}), 7.11(\mathrm{ddd}, J=8.2,7.1$, $1.0 \mathrm{~Hz}, 1 \mathrm{H}$ ), 7.01 (ddd, $J=7.4,4.9,1.0 \mathrm{~Hz}, 1 \mathrm{H}), 6.60$ (dd, $J=3.5,0.8 \mathrm{~Hz}, 1 \mathrm{H}$ ).

${ }^{13}$ C NMR $\left(101 \mathrm{MHz}, \mathrm{CDCl}_{3}\right) \delta$ 152.6, 149.0, 138.4, 135.2, 130.5, 126.0, 123.2, 121.3, 121.1, 120.1, 114.6, 113.1, 105.6.

\section{1-(pyrimidin-2-yl)-1H-indole (1b)}<smiles>c1cnc(-n2ccc3ccccc32)nc1</smiles>

Synthesized following and consistent with literature L. Ackermann, A. V. Lygin, Org. Lett. 2011, 13, 3332-3335.

${ }^{1} \mathbf{H}$ NMR $\left(400 \mathrm{MHz}, \mathrm{CDCl}_{3}\right) \delta 8.88(\mathrm{dd}, J=8.4,0.8 \mathrm{~Hz}, 1 \mathrm{H}), 8.71(\mathrm{~d}, J=4.8 \mathrm{~Hz}, 2 \mathrm{H}), 8.34(\mathrm{~d}, J$ $=3.7 \mathrm{~Hz}, 1 \mathrm{H}), 7.69(\mathrm{dt}, J=7.7,1.0 \mathrm{~Hz}, 1 \mathrm{H}), 7.41(\mathrm{ddd}, J=8.4,7.1,10 \mathrm{~Hz}, 1 \mathrm{H}), 7.35-7.27(\mathrm{~m}$, $1 \mathrm{H}), 7.03(\mathrm{t}, J=4.8 \mathrm{~Hz}, 1 \mathrm{H}), 6.77(\mathrm{dd}, J=3.7,0.8 \mathrm{~Hz}, 1 \mathrm{H})$.

${ }^{13} \mathrm{C}$ NMR $\left(101 \mathrm{MHz}, \mathrm{CDCl}_{3}\right) \delta 158.1,157.8,135.4,131.4,125.9,123.7,122.2,120.9,116.3$, 116.1, 107.0.

\section{1-(1H-indol-1-yl)ethan-1-one (1c)}<smiles>ClCn1ccc2ccccc21</smiles>

Synthesized and characterization consistent with literature following G. Özüduru, T. Schubach, M. M. K. Boysen, Org. Lett. 2012, 14, 4990-4993. 
${ }^{1}$ H NMR $\left(400 \mathrm{MHz}, \mathrm{CDCl}_{3}\right) \delta 8.45(\mathrm{~d}, J=8.3 \mathrm{~Hz}, 1 \mathrm{H}), 7.57(\mathrm{ddd}, J=7.7,1.4,0.7 \mathrm{~Hz}, 1 \mathrm{H}), 7.42$ (d, $J=3.8 \mathrm{~Hz}, 1 \mathrm{H}), 7.39-7.32(\mathrm{~m}, 1 \mathrm{H}), 7.31-7.26(\mathrm{~m}, 1 \mathrm{H}), 6.64(\mathrm{dd}, J=3.8,0.7 \mathrm{~Hz}, 1 \mathrm{H}), 2.64$ (s, 3H).

${ }^{13} \mathrm{C}$ NMR $\left(100 \mathrm{MHz}, \mathrm{CDCl}_{3}\right) \delta 168.6,135.6,130.4,125.2,125.1,123.6,120.8,116.5,109.2$, 24.0.

tert-butyl 1H-indole-1-carboxylate (1d)<smiles></smiles>

Synthesized and characterization consistent with literature following G. Özüduru, T. Schubach, M. M. K. Boysen, Org. Lett. 2012, 14, 4990-4993.

${ }^{1} \mathbf{H}$ NMR $\left(400 \mathrm{MHz}, \mathrm{CDCl}_{3}\right) \delta 8.19(\mathrm{~d}, J=8.3 \mathrm{~Hz}, 1 \mathrm{H}), 7.64(\mathrm{~d}, J=3.7 \mathrm{~Hz}, 1 \mathrm{H}), 7.62-7.56(\mathrm{~m}$, $1 \mathrm{H}), 7.35$ (ddd, $J=8.3,7.2,1.3 \mathrm{~Hz}, 1 \mathrm{H}), 7.29-7.23(\mathrm{~m}, 1 \mathrm{H}), 6.61(\mathrm{dd}, J=3.7,0.8 \mathrm{~Hz}, 1 \mathrm{H})$, $1.72(\mathrm{~s}, 9 \mathrm{H})$.

${ }^{13}$ C NMR $\left(100 \mathrm{MHz}, \mathrm{CDCl}_{3}\right) \delta 149.8,135.2,130.6,125.9,124.2,122.6,120.9,115.2,107.3$, 83.6, 28.2.

\section{1-tosyl-1H-indole (1e)}<smiles>[Y9]n1ccc2ccccc21</smiles>

Synthesized following PCT Int. Appl., 2011159067, characterization consistent with literature J. S. Alford, J. E. Spangler, H. M. L. Davies, J. Am. Chem. Soc. 2013, 135, 11712-11715.

${ }^{1} \mathbf{H}$ NMR $\left(400 \mathrm{MHz}, \mathrm{CDCl}_{3}\right) \delta 7.89(\mathrm{dd}, J=8.3,0.9 \mathrm{~Hz}, 1 \mathrm{H}), 7.64(\mathrm{~d}, J=8.3 \mathrm{~Hz}, 2 \mathrm{H}), 7.45(\mathrm{~d}, J$ $=3.7 \mathrm{~Hz}, 1 \mathrm{H}), 7.39(\mathrm{~d}, J=7.8 \mathrm{~Hz}, 1 \mathrm{H}), 7.19(\mathrm{ddd}, J=8.3,7.2,1.3 \mathrm{~Hz}, 1 \mathrm{H}), 7.13-7.07(\mathrm{~m}, 1 \mathrm{H})$, $7.07-7.01(\mathrm{~m}, 2 \mathrm{H}), 6.52$ (dd, $J=3.7,0.8 \mathrm{~Hz}, 1 \mathrm{H}), 2.16$ (s, 3H).

${ }^{13} \mathbf{C}$ NMR $\left(101 \mathrm{MHz}, \mathrm{CDCl}_{3}\right) \delta 145.0,135.3,134.9,130.8,129.9,126.8,126.4,124.6,123.3$, 121.5, 113.6, 109.1, 21.5.

\section{1-(4-methylpyridin-2-yl)-1H-indole (1g)}<smiles>Cc1ccnc(-n2ccc3ccccc32)c1</smiles>

Synthesized following GP1 on a $5 \mathrm{mmol}$ scale. Obtained $504 \mathrm{mg}$ of an orange oil (48\% yield). 
${ }^{1} \mathbf{H}$ NMR $\left(500 \mathrm{MHz}, \mathrm{CDCl}_{3}\right) \delta 8.43(\mathrm{dd}, J=5.0,0.8 \mathrm{~Hz}, 1 \mathrm{H}), 8.21(\mathrm{dq}, J=8.4,0.8 \mathrm{~Hz}, 1 \mathrm{H}), 7.72$ $(\mathrm{d}, J=3.5 \mathrm{~Hz}, 1 \mathrm{H}), 7.68(\mathrm{ddd}, J=7.8,1.3,0.8 \mathrm{~Hz}, 1 \mathrm{H}), 7.34-7.28(\mathrm{~m}, 2 \mathrm{H}), 7.24-7.20$ (m,

$1 \mathrm{H}), 7.00$ (ddd, $J=5.0,1.4,0.7 \mathrm{~Hz}, 1 \mathrm{H}), 6.72(\mathrm{dd}, J=3.5,0.8 \mathrm{~Hz}, 1 \mathrm{H}), 2.44$ (s, 4H).

${ }^{13} \mathbf{C}$ NMR $\left(126 \mathrm{MHz}, \mathrm{CDCl}_{3}\right) \delta 152.6,149.8,148.6,135.1,130.4,126.1,123.0,121.4,121.1$, 121.0, 115.3, 113.0, 105.3, 21.3.

IR (ATR) 3041, 1696, 1606, 1562, 1523, 1451, 1335, 1212, 1113, 1018, 883, 821, 767, 743, 713

HRMS (DART, M+H) Calculated for $\mathrm{C}_{14} \mathrm{H}_{13} \mathrm{~N}_{2} 209.1073$, found 209.1074

\section{1-(5-methylpyridin-2-yl)-1H-indole (1h)}<smiles>Cc1ccc(-n2ccc3ccccc32)nc1</smiles>

Synthesized following GP1 on a 5 mmol scale. Obtained $0.48 \mathrm{~g}$ of a crystalline solid (46\% yield) after recrystallization.

${ }^{1}$ H NMR $\left(500 \mathrm{MHz}, \mathrm{CDCl}_{3}\right) \delta 8.41(\mathrm{dp}, J=2.4,0.8 \mathrm{~Hz}, 1 \mathrm{H}), 8.19(\mathrm{dq}, J=8.4,0.9 \mathrm{~Hz}, 1 \mathrm{H}), 7.75$ $-7.69(\mathrm{~m}, 2 \mathrm{H}), 7.59(\mathrm{ddq}, J=8.3,2.4,0.8 \mathrm{~Hz}, 1 \mathrm{H}), 7.41-7.36(\mathrm{~m}, 1 \mathrm{H}), 7.34(\mathrm{ddd}, J=8.4,7.0$, $1.3 \mathrm{~Hz}, 1 \mathrm{H}), 7.25$ (ddd, $J=8.0,7.0,1.0 \mathrm{~Hz}, 1 \mathrm{H}), 6.74(\mathrm{dd}, J=3.5,0.9 \mathrm{~Hz}, 1 \mathrm{H}), 2.38(\mathrm{~s}, 2 \mathrm{H})$.

${ }^{13} \mathrm{C}$ NMR $\left(126 \mathrm{MHz}, \mathrm{CDCl}_{3}\right) \delta 150.4,149.0,139.0,135.1,130.3,129.7,126.1,123.0,121.1$, $121.0,114.3,112.7,105.0,17.8$.

IR (ATR) 3132, 3046, 1571, 1523, 1468, 1448, 1310, 1223, 1206, 816, 767, 738, 727

HRMS (DART, M+H) Calculated for $\mathrm{C}_{14} \mathrm{H}_{13} \mathrm{~N}_{2} 209.1073$, found 209.1074

MP $100-105^{\circ} \mathrm{C}$

\section{1-(5-bromopyridin-2-yl)-1H-indole (1i)}

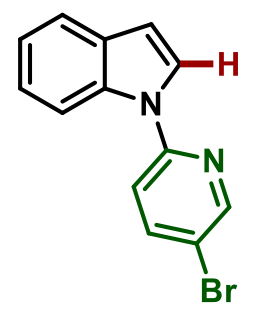

Synthesized following GP1 on a $5 \mathrm{mmol}$ scale. Obtained $0.95 \mathrm{~g}$ of a white solid (70\% yield).

${ }^{1} \mathbf{H}$ NMR $\left(500 \mathrm{MHz} \mathrm{CDCl}_{3}\right) \delta 8.61(\mathrm{dd}, J=2.5,0.7 \mathrm{~Hz}, 1 \mathrm{H}), 8.21(\mathrm{dq}, J=8.4,0.9 \mathrm{~Hz}, 1 \mathrm{H}), 7.87$ $(\mathrm{dd}, J=8.7,2.5 \mathrm{~Hz}, 1 \mathrm{H}), 7.68(\mathrm{ddd}, J=7.8,1.3,0.8 \mathrm{~Hz}, 1 \mathrm{H}), 7.66(\mathrm{dd}, J=3.4,0.4 \mathrm{~Hz}, 1 \mathrm{H}), 7.35$ $(\mathrm{dd}, J=8.7,0.7 \mathrm{~Hz}, 1 \mathrm{H}), 7.37-7.30(\mathrm{~m}, 1 \mathrm{H}), 7.27-7.22(\mathrm{~m}, 1 \mathrm{H}), 6.74(\mathrm{dd}, J=3.5,0.9 \mathrm{~Hz}$, $1 \mathrm{H})$. 
${ }^{13}$ C NMR $\left(126 \mathrm{MHz}, \mathrm{CDCl}_{3}\right) \delta 151.1,149.8,140.8,135.0,130.5,125.6,123.4,121.6,121.2$, $115.5,115.4,113.2,106.2$.

IR (ATR) 3142, 3046, 1571, 1523, 1468, 1448, 1310, 1224, 1203, 1023, 806, 741, 716

HRMS (DART, $\mathrm{M}+\mathrm{H}$ ) Calculated for $\mathrm{C}_{13} \mathrm{H}_{10} \mathrm{~N}_{2} \mathrm{Br} 273.0022$, found 273.0026

MP $46-48{ }^{\circ} \mathrm{C}$

\section{1-(5-chloropyridin-2-yl)-1H-indole (1j)}<smiles>Clc1ccc(-n2ccc3ccccc32)nc1</smiles>

Synthesized following GP1 on a $10 \mathrm{mmol}$ scale. Obtained $0.70 \mathrm{~g}$ of a white solid after recrystallization $(31 \%$ yield)

${ }^{1} \mathbf{H}$ NMR $\left(500 \mathrm{MHz}, \mathrm{CDCl}_{3}\right) \delta 8.52(\mathrm{dd}, J=2.6,0.7 \mathrm{~Hz}, 1 \mathrm{H}), 8.22(\mathrm{dq}, J=8.3,0.9 \mathrm{~Hz}, 1 \mathrm{H}), 7.74$ $-7.68(\mathrm{~m}, 2 \mathrm{H}), 7.66(\mathrm{~d}, J=3.5 \mathrm{~Hz}, 1 \mathrm{H}), 7.40-7.32(\mathrm{~m}, 2 \mathrm{H}), 7.28$ (ddd, $J=8.1,7.1,1.1 \mathrm{~Hz}$, $1 \mathrm{H}), 6.75(\mathrm{dd}, J=3.5,0.8 \mathrm{~Hz}, 1 \mathrm{H})$.

${ }^{13}$ C NMR $\left(126 \mathrm{MHz}, \mathrm{CDCl}_{3}\right) \delta$ 150.7, 147.5, 138.1, 135.0, 130.5, 127.4, 125.7, 123.4, 121.6, $121.2,114.8,113.2,106.1$.

IR (ATR) 3148, 3045, 1961, 1898, 1579, 1470, 1448, 1204, 814, 769, 742, 713

HRMS (DART, $\mathrm{M}+\mathrm{H}$ ) Calculated for $\mathrm{C}_{13} \mathrm{H}_{10} \mathrm{~N}_{2} \mathrm{Cl} 229.0527$, found 229.0525

MP $51-52{ }^{\circ} \mathrm{C}$

\section{3-methyl-1-(pyridin-2-yl)-1H-indole (1k)}<smiles>Cc1cc2ccccc2n1-c1ccccn1</smiles>

Synthesized following GP1. Characterization consistent with literature G. S. Kumar, M. Kapur, Org. Lett. 2016, 18, 1112-1115.

${ }^{1} \mathbf{H}$ NMR $\left(400 \mathrm{MHz}, \mathrm{CDCl}_{3}\right) \delta 8.55(\mathrm{ddd}, J=4.9,2.0,0.9 \mathrm{~Hz}, 1 \mathrm{H}), 8.25(\mathrm{dt}, J=8.3,0.9 \mathrm{~Hz}$, $1 \mathrm{H}), 7.78$ (ddd, $J=8.3,7.4,2.0 \mathrm{~Hz}, 1 \mathrm{H}), 7.61(\mathrm{ddd}, J=7.8,1.2,0.9 \mathrm{~Hz}, 1 \mathrm{H}), 7.53(\mathrm{q}, J=1.2 \mathrm{~Hz}$, $1 \mathrm{H}), 7.45$ (dt, $J=8.3,0.9 \mathrm{~Hz}, 1 \mathrm{H}), 7.32(\mathrm{ddd}, J=8.3,7.1,1.2 \mathrm{~Hz}, 1 \mathrm{H}), 7.26-7.21(\mathrm{~m}, 1 \mathrm{H}), 7.11$ (ddd, $J=7.4,4.9,0.9 \mathrm{~Hz}, 1 \mathrm{H}), 2.39$ (d, $J=1.2 \mathrm{~Hz}, 3 \mathrm{H})$. 
${ }^{13} \mathbf{C}$ NMR $\left(100 \mathrm{MHz}, \mathrm{CDCl}_{3}\right) \delta 152.6,148.9,138.2,135.3,131.1,123.2,123.2,120.8,119.4$, 119.0, 114.8, 114.0, 113.1, 9.7.

\section{4-methyl-1-(pyridin-2-yl)-1H-indole (11)}<smiles>Cc1cccc2c1ccn2-c1ccccn1</smiles>

Synthesized following GP1 on a $10 \mathrm{mmol}$ scale. Obtained $1.1 \mathrm{~g}$ of a thick oil (53\% yield).

${ }^{1} \mathbf{H}$ NMR $\left(500 \mathrm{MHz}, \mathrm{CDCl}_{3}\right) \delta 8.59$ (ddd, $\left.J=4.9,2.0,0.9 \mathrm{~Hz}, 1 \mathrm{H}\right), 8.06(\mathrm{dq}, J=8.3,0.8 \mathrm{~Hz}$, $1 \mathrm{H}), 7.80$ (ddd, $J=8.2,7.4,2.0 \mathrm{~Hz}, 1 \mathrm{H}), 7.76(\mathrm{~d}, J=3.5 \mathrm{~Hz}, 1 \mathrm{H}), 7.51(\mathrm{dt}, J=8.2,0.9 \mathrm{~Hz}, 1 \mathrm{H})$, $7.28-7.21(\mathrm{~m}, 1 \mathrm{H}), 7.16(\mathrm{ddd}, J=7.4,4.9,0.9 \mathrm{~Hz}, 1 \mathrm{H}), 7.06(\mathrm{dp}, J=7.1,0.9 \mathrm{~Hz}, 1 \mathrm{H}), 6.78(\mathrm{dd}$, $J=3.5,0.9 \mathrm{~Hz}, 1 \mathrm{H}), 2.63(\mathrm{~s}, 2 \mathrm{H})$.

${ }^{13} \mathbf{C}$ NMR $\left(126 \mathrm{MHz}, \mathrm{CDCl}_{3}\right) \delta 152.6,149.0,138.3,134.8,130.5,130.2,125.5,123.2,121.6$, 120.0, 114.7, 110.5, 103.9, 18.8 .

IR (ATR) 3049, 3016, 2916, 2859, 1694, 1584, 1522, 1470, 14365, 1344, 1306, 1263, 1213, 1149

HRMS (DART, M+H) Calculated for $\mathrm{C}_{14} \mathrm{H}_{13} \mathrm{~N}_{2} 209.1073$, found 209.1078

\section{4-(benzyloxy)-1-(pyridin-2-yl)-1H-indole (1m)}<smiles></smiles>

Synthesized following GP1 on a $10 \mathrm{mmol}$ scale. Obtained $1.95 \mathrm{~g}$ of a white solid (65\% yield).

${ }^{1} \mathbf{H}$ NMR $\left(500 \mathrm{MHz}, \mathrm{CDCl}_{3}\right) \delta 8.59(\mathrm{~d}, J=4.7 \mathrm{~Hz}, 1 \mathrm{H}), 7.85(\mathrm{dt}, J=8.7,2.7 \mathrm{~Hz}, 1 \mathrm{H}), 7.80(\mathrm{t}, J$ $=7.8 \mathrm{~Hz}, 1 \mathrm{H}), 7.68(\mathrm{~s}, 1 \mathrm{H}), 7.56(\mathrm{~d}, J=6.9 \mathrm{~Hz}, 2 \mathrm{H}), 7.50(\mathrm{~d}, J=8.7 \mathrm{~Hz}, 1 \mathrm{H}), 7.44(\mathrm{t}, J=6.9 \mathrm{~Hz}$, $2 \mathrm{H}), 7.37(\mathrm{t}, J=7.3 \mathrm{~Hz}, 1 \mathrm{H}), 7.24(\mathrm{ddd}, J=8.7,4.7,2.7 \mathrm{~Hz}, 1 \mathrm{H}), 7.16(\mathrm{dd}, J=7.3,4.7 \mathrm{~Hz}, 1 \mathrm{H})$, $6.95(\mathrm{~s}, 1 \mathrm{H}), 6.74(\mathrm{~d}, J=7.8 \mathrm{~Hz}, 1 \mathrm{H}), 5.28(\mathrm{~s}, 2 \mathrm{H})$.

${ }^{13}$ C NMR $\left(126 \mathrm{MHz}, \mathrm{CDCl}_{3}\right) \delta 152.6,152.4,149.0,138.4,137.5,136.5,128.5,127.8,127.4$, 124.6, 123.9, 121.3, 120.2, 114.8, 106.5, 102.9, 102.9, 70.0.

IR (ATR) 1583, 1493, 1472, 1439, 1391, 1335, 1263, 1221, 1060, 931, 788, 737, 719, 695, 641

HRMS (DART, $\mathrm{M}+\mathrm{H}$ ) Calculated for $\mathrm{C}_{20} \mathrm{H}_{17} \mathrm{~N}_{2} \mathrm{O} 301.1335$, found 301.1335

MP $81-82{ }^{\circ} \mathrm{C}$ 


\section{4-bromo-1-(pyridin-2-yl)-1H-indole (1n)}<smiles>Brc1cccc2c1ccn2-c1ccccn1</smiles>

Synthesized following GP1. Characterization consistent with literature G. S. Kumar, M. Kapur, Org. Lett. 2016, 18, 1112-1115.

${ }^{1} \mathbf{H}$ NMR $\left(500 \mathrm{MHz}^{\mathrm{CDCl}} 3\right) \delta 8.57(\mathrm{ddd}, J=4.9,1.9,0.9 \mathrm{~Hz}, 1 \mathrm{H}), 8.19(\mathrm{dt}, J=8.4,0.9 \mathrm{~Hz}$, $1 \mathrm{H}), 7.81$ (ddd, $J=8.2,7.4,1.9 \mathrm{~Hz}, 1 \mathrm{H}), 7.73$ (dd, $J=3.5,0.4 \mathrm{~Hz}, 1 \mathrm{H}), 7.43$ (dt, $J=8.4,0.9 \mathrm{~Hz}$, $1 \mathrm{H}), 7.38(\mathrm{dd}, J=7.6,0.9 \mathrm{~Hz}, 1 \mathrm{H}), 7.19(\mathrm{ddd}, J=7.4,4.9,0.9 \mathrm{~Hz}, 1 \mathrm{H}), 7.15$ (ddd, $J=8.2,7.6$, $0.4 \mathrm{~Hz}, 1 \mathrm{H}), 6.79(\mathrm{dd}, J=3.5,0.9 \mathrm{~Hz}, 1 \mathrm{H})$.

${ }^{13} \mathrm{C}$ NMR $\left(126 \mathrm{MHz}, \mathrm{CDCl}_{3}\right) \delta 152.2,149.0,138.6,135.4,130.9,126.5,124.2,124.0,120.6$, 114.8, 114.7, 112.3, 105.6.

\section{5-methyl-1-(pyridin-2-yl)-1H-indole (10)}<smiles></smiles>

Synthesized following GP1 on a $5 \mathrm{mmol}$ scale. Obtained $485 \mathrm{mg}$ of a white solid (47\% yield).

${ }^{1} \mathbf{H}$ NMR $\left(500 \mathrm{MHz}, \mathrm{CDCl}_{3}\right) \delta 8.58(\mathrm{ddd}, J=4.9,2.0,0.9 \mathrm{~Hz}, 1 \mathrm{H}), 8.18(\mathrm{dd}, J=8.5,0.8 \mathrm{~Hz}$, $1 \mathrm{H}), 7.77$ (ddd, $J=8.2,7.4,2.0 \mathrm{~Hz}, 1 \mathrm{H}), 7.74(\mathrm{~d}, J=3.5 \mathrm{~Hz}, 1 \mathrm{H}), 7.51(\mathrm{dt}, J=1.8,0.9 \mathrm{~Hz}, 1 \mathrm{H})$, $7.46(\mathrm{dt}, J=8.2,0.9 \mathrm{~Hz}, 1 \mathrm{H}), 7.19$ (ddt, $J=8.5,1.8,0.5 \mathrm{~Hz}, 1 \mathrm{H}), 7.13$ (ddd, $J=7.4,4.9,0.9 \mathrm{~Hz}$, $1 \mathrm{H}), 6.69(\mathrm{dd}, J=3.5,0.8 \mathrm{~Hz}, 1 \mathrm{H}), 2.54(\mathrm{~s}, 3 \mathrm{H})$.

${ }^{13} \mathrm{C}$ NMR $\left(126 \mathrm{MHz}, \mathrm{CDCl}_{3}\right) \delta 152.6,148.9,138.3,133.4,130.8,130.6,125.9,124.7,120.9$, $119.8,114.2,112.9,105.2,21.4$.

IR (ATR) 1589, 1524, 1457, 1305, 1224, 1149, 1042, 973, 892, 779, 740, 707, 611

HRMS (DART, M+H) Calculated for $\mathrm{C}_{14} \mathrm{H}_{13} \mathrm{~N}_{2} 209.1073$, found 209.1077

MP $58-59{ }^{\circ} \mathrm{C}$

\section{5-methoxy-1-(pyridin-2-yl)-1H-indole (1p)}


<smiles>COc1ccc2c(ccc3ncccc32)c1</smiles>

Synthesized following GP1. Characterization consistent with literature G. S. Kumar, M. Kapur, Org. Lett. 2016, 18, 1112-1115.

${ }^{1} \mathbf{H}$ NMR $\left(500 \mathrm{MHz}^{\mathrm{CDCl}} 3\right) \delta 8.55(\mathrm{ddd}, J=4.9,1.9,0.9 \mathrm{~Hz}, 1 \mathrm{H}), 8.18(\mathrm{dt}, J=9.0,0.8 \mathrm{~Hz}$, $1 \mathrm{H}), 7.78(\mathrm{ddd}, J=8.2,7.4,1.9 \mathrm{~Hz}, 1 \mathrm{H}), 7.70(\mathrm{dd}, J=3.5,0.5 \mathrm{~Hz}, 1 \mathrm{H}), 7.44$ (dt, $J=8.2,0.9 \mathrm{~Hz}$, $1 \mathrm{H}), 7.17-7.09(\mathrm{~m}, 2 \mathrm{H}), 6.95(\mathrm{ddd}, J=9.0,2.6,0.5 \mathrm{~Hz}, 1 \mathrm{H}), 6.65(\mathrm{dd}, J=3.5,0.8 \mathrm{~Hz}, 1 \mathrm{H})$, $3.88(\mathrm{~s}, 3 \mathrm{H})$.

${ }^{13} \mathrm{C}$ NMR $\left(126 \mathrm{MHz}, \mathrm{CDCl}_{3}\right) \delta$ 155.0, 152.5, 148.9, 138.3, 131.1, 130.2, 126.2, 119.7, 114.1, $113.9,112.7,105.3,102.9,55.7$.

\section{5-fluoro-1-(pyridin-2-yl)-1H-indole (1q)}<smiles>Fc1ccc2c(ccc3ncccc32)c1</smiles>

Synthesized following GP1. Characterization consistent with literature L. Liang, S. Fu, D. Lin, X.-Q. Zhang, Y. Deng, H. Jiang, W. Zeng, J. Org. Chem. 2014, 79, 9472-9480.

${ }^{1} \mathbf{H}$ NMR $\left(500 \mathrm{MHz}, \mathrm{CDCl}_{3}\right) \delta 8.55(\mathrm{ddd}, J=4.9,2.0,1.0 \mathrm{~Hz}, 1 \mathrm{H}), 8.28$ (ddt, $J=9.1,4.6,0.9$ $\mathrm{Hz}, 1 \mathrm{H}), 7.75(\mathrm{ddd}, J=8.2,7.4,2.0 \mathrm{~Hz}, 1 \mathrm{H}), 7.70(\mathrm{~d}, J=3.5 \mathrm{~Hz}, 1 \mathrm{H}), 7.37(\mathrm{dt}, J=8.2,1.0 \mathrm{~Hz}$, $1 \mathrm{H}), 7.33(\mathrm{dd}, J=9.1,2.6 \mathrm{~Hz}, 1 \mathrm{H}), 7.13(\mathrm{ddd}, J=7.4,4.9,0.9 \mathrm{~Hz}, 1 \mathrm{H}), 7.07$ (td, $J=9.1,2.6 \mathrm{~Hz}$, $1 \mathrm{H}), 6.67$ (dd, $J=3.5,0.9 \mathrm{~Hz}, 1 \mathrm{H})$.

${ }^{13}$ C NMR $\left(126 \mathrm{MHz}, \mathrm{CDCl}_{3}\right) \delta 158.6(\mathrm{~d}, J=236.7 \mathrm{~Hz}), 152.3,148.8,138.5,131.74,131.0(\mathrm{~d}, J$ $=10.0 \mathrm{~Hz}), 127.3,120.2,114.5(\mathrm{~d}, J=9.2 \mathrm{~Hz}), 114.0,111.2(\mathrm{~d}, J=25.4 \mathrm{~Hz}), 105.9(\mathrm{~d}, J=23.3$ $\mathrm{Hz}), 105.4(\mathrm{~d}, J=4.3 \mathrm{~Hz})$.

\section{5-bromo-1-(pyridin-2-yl)-1H-indole (1r)}<smiles></smiles>

Synthesized following GP1. Characterization consistent with literature G. S. Kumar, M. Kapur, Org. Lett. 2016, 18, 1112-1115. 
${ }^{1} \mathbf{H}$ NMR $\left(500 \mathrm{MHz}, \mathrm{CDCl}_{3}\right) \delta 8.56(\mathrm{ddd}, J=4.9,1.9,0.9 \mathrm{~Hz}, 1 \mathrm{H}), 8.15(\mathrm{dd}, J=8.8,0.8 \mathrm{~Hz}$, $1 \mathrm{H}), 7.82(\mathrm{ddd}, J=8.2,7.4,1.9 \mathrm{~Hz}, 1 \mathrm{H}), 7.78(\mathrm{dd}, J=2.0,0.5 \mathrm{~Hz}, 1 \mathrm{H}), 7.69$ (d, $J=3.5 \mathrm{~Hz}, 1 \mathrm{H})$, $7.43(\mathrm{dt}, J=8.2,0.9 \mathrm{~Hz}, 1 \mathrm{H}), 7.37(\mathrm{dd}, J=8.8,2.0 \mathrm{~Hz}, 1 \mathrm{H}), 7.18$ (ddd, $J=7.4,4.9,0.9 \mathrm{~Hz}, 1 \mathrm{H}$ ), $6.65(\mathrm{dd}, J=3.5,0.8 \mathrm{~Hz}, 1 \mathrm{H})$.

${ }^{13} \mathbf{C ~ N M R}\left(126 \mathrm{MHz}, \mathrm{CDCl}_{3}\right) \delta 152.2,149.0,138.5,133.8,132.0,126.9,125.9,123.5,120.4$, $114.8,114.4,114.3,104.9$.

\section{5-(((tert-butyldimethylsilyl)oxy)methyl)-1-(pyridin-2-yl)-1H-indole (1s)}

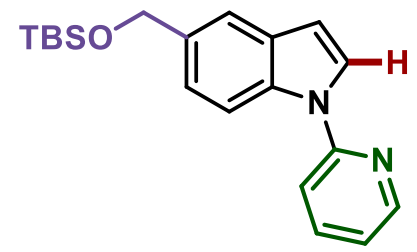

Synthesized following GP1 on a $2 \mathrm{mmol}$ scale. Obtained $360 \mathrm{mg}$ of a white solid (53\% yield).

${ }^{1} \mathbf{H}$ NMR $\left(500 \mathrm{MHz}, \mathrm{CDCl}_{3}\right) \delta 8.57(\mathrm{ddd}, J=4.9,2.0,0.9 \mathrm{~Hz}, 1 \mathrm{H}), 8.24(\mathrm{dd}, J=8.6,0.8 \mathrm{~Hz}$, $1 \mathrm{H}), 7.76(\mathrm{ddd}, J=8.2,7.4,2.0 \mathrm{~Hz}, 1 \mathrm{H}), 7.74(\mathrm{~d}, J=3.5 \mathrm{~Hz}, 1 \mathrm{H}), 7.67(\mathrm{dd}, J=1.7,0.8 \mathrm{~Hz}, 1 \mathrm{H})$, $7.46(\mathrm{dt}, J=8.2,0.9 \mathrm{~Hz}, 1 \mathrm{H}), 7.33(\mathrm{dd}, J=8.6,1.7 \mathrm{~Hz}, 1 \mathrm{H}), 7.13(\mathrm{ddd}, J=7.4,4.9,0.9 \mathrm{~Hz}, 1 \mathrm{H})$, $6.73(\mathrm{dd}, J=3.5,0.8 \mathrm{~Hz}, 1 \mathrm{H}), 4.92(\mathrm{~s}, 2 \mathrm{H}), 1.03(\mathrm{~s}, 9 \mathrm{H}), 0.19(\mathrm{~s}, 6 \mathrm{H})$.

${ }^{13} \mathrm{C}$ NMR $\left(126 \mathrm{MHz}, \mathrm{CDCl}_{3}\right) \delta 152.5,148.9,138.3,134.4,134.4,130.5,126.1,122.1,119.9$, $118.8,114.3,112.9,105.6,65.6,26.1,18.6,-5.0$.

IR (ATR) 2945, 2858, 1595, 1478, 1436, 1352, 1254, 1212, 1081, 845, 773, 728, 668, 617

HRMS (DART, H+M) Calculated for $\mathrm{C}_{20} \mathrm{H}_{27} \mathrm{~N}_{2} \mathrm{OSi} 339.1887$, found 339.1888

MP $31-32{ }^{\circ} \mathrm{C}$

\section{6-fluoro-1-(pyridin-2-yl)-1H-indole (1t)}<smiles>Fc1ccc2ccn(-c3ccccn3)c2c1</smiles>

Synthesized following GP1. Characterization consistent with literature L. Liang, S. Fu, D. Lin, X.-Q. Zhang, Y. Deng, H. Jiang, W. Zeng, J. Org. Chem. 2014, 79, 9472-9480.

${ }^{1} \mathbf{H}$ NMR $\left(400 \mathrm{MHz} \mathrm{CDCl}_{3}\right) \delta 8.57(\mathrm{ddd}, J=4.9,1.9,0.9 \mathrm{~Hz}, 1 \mathrm{H}), 8.09$ (dd, $J=10.8,2.4 \mathrm{~Hz}$, $1 \mathrm{H}), 7.80(\mathrm{ddd}, J=8.2,7.4,1.9 \mathrm{~Hz}, 1 \mathrm{H}), 7.66(\mathrm{~d}, J=3.5 \mathrm{~Hz}, 1 \mathrm{H}), 7.56(\mathrm{dd}, J=8.6,5.5 \mathrm{~Hz}, 1 \mathrm{H})$, 7.41 (dt, $J=8.2,1.0 \mathrm{~Hz}, 1 \mathrm{H}), 7.16$ (ddd, $J=7.4,4.9,1.0 \mathrm{~Hz}, 1 \mathrm{H}), 6.99$ (ddd, $J=9.3,8.6,2.4 \mathrm{~Hz}$, $1 \mathrm{H}), 6.69$ (dd, $J=3.5,0.9 \mathrm{~Hz}, 1 \mathrm{H})$.

${ }^{13}$ C NMR $\left(126 \mathrm{MHz}, \mathrm{CDCl}_{3}\right) \delta 160.4(\mathrm{~d}, J=238.0 \mathrm{~Hz}), 152.4,148.9,138.5(\mathrm{~d}, J=0.8 \mathrm{~Hz})$, $135.1(\mathrm{~d}, J=12.8 \mathrm{~Hz}), 126.7(\mathrm{~d}, J=1.2 \mathrm{~Hz}), 126.0(\mathrm{~d}, J=3.8 \mathrm{~Hz}), 121.5(\mathrm{~d}, J=10.2 \mathrm{~Hz}), 120.2$, $114.0,109.8(\mathrm{~d}, J=24.5 \mathrm{~Hz}), 105.6(\mathrm{~d}, J=0.9 \mathrm{~Hz}), 100.5(\mathrm{~d}, J=28.2 \mathrm{~Hz})$. 
${ }^{19}$ F NMR $\left(377 \mathrm{MHz}, \mathrm{CDCl}_{3}\right) \delta-119.17$.

6-chloro-1-(pyridin-2-yl)-1H-indole (1u)<smiles>Cc1cc2ccc(Cl)cc2n1-c1ccccn1</smiles>

Synthesized following GP1. Characterization consistent with literature L. Liang, S. Fu, D. Lin, X.-Q. Zhang, Y. Deng, H. Jiang, W. Zeng, J. Org. Chem. 2014, 79, 9472-9480.

${ }^{1} \mathbf{H}$ NMR $\left(400 \mathrm{MHz}, \mathrm{CDCl}_{3}\right) \delta 8.57(\mathrm{ddd}, J=4.9,1.9,0.9 \mathrm{~Hz}, 1 \mathrm{H}), 8.36(\mathrm{dt}, J=1.8,0.7 \mathrm{~Hz}$, $1 \mathrm{H}), 7.79$ (ddd, $J=8.4,7.4,1.9 \mathrm{~Hz}, 1 \mathrm{H}), 7.65(\mathrm{~d}, J=3.5 \mathrm{~Hz}, 1 \mathrm{H}), 7.56(\mathrm{dd}, J=8.4,0.5 \mathrm{~Hz}, 1 \mathrm{H})$, $7.40(\mathrm{dt}, J=8.2,0.9 \mathrm{~Hz}, 1 \mathrm{H}), 7.23-7.08(\mathrm{~m}, 2 \mathrm{H}), 6.68(\mathrm{dd}, J=3.5,0.9 \mathrm{~Hz}, 1 \mathrm{H})$.

${ }^{13}$ C NMR $\left(100 \mathrm{MHz}, \mathrm{CDCl}_{3}\right) \delta$ 152.2, 148.9, 138.5, 135.4, 129.1, 128.8, 126.3, 121.9, 121.7, 120.3, 114.2, 113.6, 105.5.

\section{6-bromo-1-(pyridin-2-yl)-1H-indole (1v)}<smiles></smiles>

Synthesized following GP1 on a $10 \mathrm{mmol}$ scale. Obtained $0.84 \mathrm{~g}$ of a white solid after recrystallization in DCM (31\% yield).

${ }^{1} \mathbf{H}$ NMR $\left(500 \mathrm{MHz}, \mathrm{CDCl}_{3}\right) \delta 8.55(\mathrm{ddd}, J=4.9,2.0,0.9 \mathrm{~Hz}, 1 \mathrm{H}), 8.53(\mathrm{dt}, J=1.6,0.7 \mathrm{~Hz}$, $1 \mathrm{H}), 7.76$ (ddd, $J=8.2,7.4,2.0 \mathrm{~Hz}, 1 \mathrm{H}), 7.62(\mathrm{~d}, J=3.5 \mathrm{~Hz}, 1 \mathrm{H}), 7.51$ (dd, $J=8.4,0.5 \mathrm{~Hz}, 1 \mathrm{H}$ ), $7.38-7.28$ (m, 2H), 7.14 (ddd, $J=7.4,4.9,0.9 \mathrm{~Hz}, 1 \mathrm{H}), 6.67$ (dd, $J=3.5,0.7 \mathrm{~Hz}, 1 \mathrm{H})$.

${ }^{13} \mathrm{C}$ NMR $\left(126 \mathrm{MHz}, \mathrm{CDCl}_{3}\right) \delta 152.1,148.9,138.6,135.8,129.2,126.3,124.5,122.1,120.4$, $116.9,116.6,114.2,105.6$.

IR (ATR) 1595, 1529, 1487, 1439, 1350, 1245, 1206, 1164, 1093, 877, 806, 773, 722, 662

HRMS (DART, M+H) Calculated for $\mathrm{C}_{13} \mathrm{H}_{10} \mathrm{~N}_{2} \mathrm{Br}_{1}$ 273.0022, found 273.0017

MP $85-90{ }^{\circ} \mathrm{C}$

\section{2-(1H-pyrrol-1-yl)pyridine (1w)}


<smiles>c1ccc(-n2cccc2)nc1</smiles>

Synthesized following GP1. Characterization consistent with literature H.-C. Ma, X.-Z. Jiang, J. Org. Chem. 2007, 72, 8943-8946.

${ }^{1} \mathbf{H}$ NMR $\left(400 \mathrm{MHz}, \mathrm{CDCl}_{3}\right) \delta 8.43(\mathrm{ddd}, J=4.9,1.9,0.9 \mathrm{~Hz}, 1 \mathrm{H}), 7.73(\mathrm{ddd}, J=8.2,7.4,1.9$ $\mathrm{Hz}, 1 \mathrm{H}), 7.55-7.50(\mathrm{~m}, 2 \mathrm{H}), 7.34-7.29(\mathrm{~m}, 1 \mathrm{H}), 7.10$ (ddd, $J=7.4,4.9,0.9 \mathrm{~Hz}, 1 \mathrm{H}), 6.39-$ $6.34(\mathrm{~m}, 2 \mathrm{H})$.

${ }^{13}$ C NMR (100 MHz, $\left.\mathrm{CDCl}_{3}\right) \delta 151.4,148.7,138.5,120.2,118.1,111.4,111.3$.

\section{2-(2-methyl-1H-pyrrol-1-yl)pyridine (1x)}<smiles></smiles>

Synthesized following GP1 on a $17.2 \mathrm{mmol}$ scale. Obtained $1.23 \mathrm{~g}$ of a yellow oil (45\% yield).

${ }^{1} \mathbf{H}$ NMR $\left(500 \mathrm{MHz}, \mathrm{CDCl}_{3}\right) \delta 8.52(\mathrm{ddd}, J=4.9,2.0,0.9 \mathrm{~Hz}, 1 \mathrm{H}), 7.77(\mathrm{ddd}, J=8.2,7.4,2.0$ $\mathrm{Hz}, 1 \mathrm{H}), 7.30(\mathrm{dt}, J=8.2,0.9 \mathrm{~Hz}, 1 \mathrm{H}), 7.19(\mathrm{ddd}, J=7.4,4.9,1.0 \mathrm{~Hz}, 1 \mathrm{H}), 7.09(\mathrm{dd}, J=3.1,1.8$ $\mathrm{Hz}, 1 \mathrm{H}), 6.23(\mathrm{t}, J=3.1 \mathrm{~Hz}, 1 \mathrm{H}), 6.07$ (ddq, $J=3.7,1.8,1.0 \mathrm{~Hz}, 1 \mathrm{H}), 2.45$ (d, $J=1.0 \mathrm{~Hz}, 3 \mathrm{H})$. ${ }^{13} \mathrm{C}$ NMR $\left(126 \mathrm{MHz}, \mathrm{CDCl}_{3}\right) \delta 152.8,148.8,138.1,129.2,120.9,120.2,117.0,110.2,109.1$, 14.2 .

IR (ATR) 3067, 3010, 2958, 2918, 1713, 1587, 1472, 1453, 1409, 1332, 1184, 1107, 882, 783, 741, 697, 660

HRMS (DART, M+H) Calculated for $\mathrm{C}_{10} \mathrm{H}_{11} \mathrm{~N}_{2}$ 159.0917, found 159.0915

\section{2-(furan-3-yl)pyridine (1aa)}<smiles>c1ccc(-c2ccoc2)nc1</smiles>

Synthesized following and consistent with literature Pospech, J., Spannenberg, A., Neumann, H., Beller, M. Chem. Eur. J. 2014, 20, 3135 - 3141

${ }^{1} \mathbf{H}$ NMR $\left(500 \mathrm{MHz}, \mathrm{CDCl}_{3}\right) \delta 8.42(\mathrm{ddd}, J=4.8,1.9,0.9 \mathrm{~Hz}, 1 \mathrm{H}), 7.91(\mathrm{dd}, J=1.9,0.9 \mathrm{~Hz}$, $1 \mathrm{H}), 7.37$ (td, $J=7.5,1.9 \mathrm{~Hz}, 1 \mathrm{H}), 7.34-7.30(\mathrm{~m}, 1 \mathrm{H}), 7.20(\mathrm{dt}, J=7.5,1.9 \mathrm{~Hz}, 1 \mathrm{H}), 6.88$ (ddd, $J=7.5,4.8,1.2 \mathrm{~Hz}, 1 \mathrm{H}), 6.76(\mathrm{dd}, J=1.9,0.9 \mathrm{~Hz}, 1 \mathrm{H})$. 
${ }^{13} \mathbf{C}$ NMR $\left(126 \mathrm{MHz}, \mathrm{CDCl}_{3}\right) \delta 151.4,149.3,143.5,140.9,136.1,126.9,121.3,119.7,108.4$.

Substrate Characterization - Enynes

N-allyl-4-methyl-N-(3-phenylprop-2-yn-1-yl)benzenesulfonamide (2a)

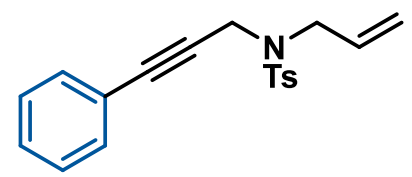

Synthesized following GP2. Characterization consistent with literature N. Cabrera-Lobera, P. Rodríguez-Salamanca, J. C. Nieto-Carmona, E. Buñuel, D. J. Cárdenas, Chem. Eur. J. 2018, 24, 784-788.

${ }^{1} \mathbf{H}$ NMR $\left(500 \mathrm{MHz}, \mathrm{CDCl}_{3}\right) \delta 7.77(\mathrm{~d}, J=8.3 \mathrm{~Hz}, 2 \mathrm{H}), 7.29-7.19(\mathrm{~m}, 5 \mathrm{H}), 7.09-7.03(\mathrm{~m}$, $2 \mathrm{H}), 5.79(\mathrm{ddt}, J=16.5,10.1,6.5 \mathrm{~Hz}, 1 \mathrm{H}), 5.33(\mathrm{dq}, J=16.5,1.3 \mathrm{~Hz}, 1 \mathrm{H}), 5.26(\mathrm{dq}, J=10.1$, $1.3 \mathrm{~Hz}, 1 \mathrm{H}), 4.30$ (s, $2 \mathrm{H}), 3.89$ (dt, $J=6.5,1.3 \mathrm{~Hz}, 2 \mathrm{H}), 2.31$ (s, $3 \mathrm{H})$.

${ }^{13} \mathbf{C ~ N M R}\left(126 \mathrm{MHz}, \mathrm{CDCl}_{3}\right) \delta 143.6,135.9,132.0,131.5,129.6,128.4,128.1,127.8,122.2$, $119.9,85.7,81.6,49.3,36.7,21.4$.

N-allyl-4-methyl-N-(3-(p-tolyl)prop-2-yn-1-yl)benzenesulfonamide (2b)

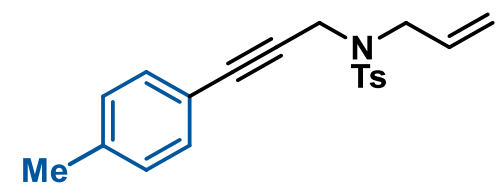

Synthesized following GP2. Characterization consistent with literature N. Cabrera-Lobera, P. Rodríguez-Salamanca, J. C. Nieto-Carmona, E. Buñuel, D. J. Cárdenas, Chem. Eur. J. 2018, 24, 784-788.

${ }^{1} \mathbf{H}$ NMR $\left(500 \mathrm{MHz}, \mathrm{CDCl}_{3}\right) \delta 7.77(\mathrm{~d}, J=8.3 \mathrm{~Hz}, 2 \mathrm{H}), 7.28-7.22(\mathrm{~m}, 2 \mathrm{H}), 7.04(\mathrm{dt}, J=7.8$, $0.7 \mathrm{~Hz}, 2 \mathrm{H}), 6.96(\mathrm{~d}, J=8.3 \mathrm{~Hz}, 2 \mathrm{H}), 5.80(\mathrm{ddt}, J=17.1,10.1,6.5 \mathrm{~Hz}, 1 \mathrm{H}), 5.33(\mathrm{dq}, J=17.1$, $1.3 \mathrm{~Hz}, 1 \mathrm{H}), 5.26(\mathrm{dq}, J=10.1,1.3 \mathrm{~Hz}, 1 \mathrm{H}), 4.30(\mathrm{~s}, 2 \mathrm{H}), 3.88(\mathrm{dt}, J=6.4,1.3 \mathrm{~Hz}, 2 \mathrm{H}), 2.35$ (s, $3 \mathrm{H}), 2.32(\mathrm{~s}, 3 \mathrm{H})$.

${ }^{13}$ C NMR $\left(126 \mathrm{MHz}, \mathrm{CDCl}_{3}\right) \delta 143.4,138.5,136.0,132.1,132.1,131.4,129.5,128.8,127.8$, $119.9,119.1,85.8,80.9,49.2,36.8,21.4$.

\section{$\mathrm{N}$-allyl-N-(3-(4-isopropylphenyl)prop-2-yn-1-yl)-4-methylbenzenesulfonamide (2c)}

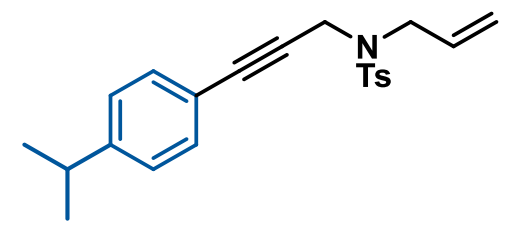

Synthesized following GP2 on a $5 \mathrm{mmol}$ scale. Obtained $1.45 \mathrm{~g}$ of a yellow oil (79\% yield) 
${ }^{1} \mathbf{H}$ NMR $\left(500 \mathrm{MHz}, \mathrm{CDCl}_{3}\right) \delta 7.77(\mathrm{~d}, J=8.2 \mathrm{~Hz}, 2 \mathrm{H}), 7.27-7.24(\mathrm{~m}, 2 \mathrm{H}), 7.10(\mathrm{~d}, J=8.0 \mathrm{~Hz}$, $2 \mathrm{H}), 7.00(\mathrm{~d}, J=8.2 \mathrm{~Hz}, 2 \mathrm{H}), 5.80(\mathrm{ddt}, J=17.1,10.1,6.5 \mathrm{~Hz}, 1 \mathrm{H}), 5.33(\mathrm{dq}, J=17.1,1.3 \mathrm{~Hz}$, $1 \mathrm{H}), 5.26(\mathrm{dq}, J=10.1,1.3 \mathrm{~Hz}, 1 \mathrm{H}), 4.30(\mathrm{~s}, 2 \mathrm{H}), 3.88(\mathrm{dt}, J=6.5,1.3 \mathrm{~Hz}, 2 \mathrm{H}), 2.87$ (hept, $J=$ $6.9 \mathrm{~Hz}, 1 \mathrm{H}), 2.34(\mathrm{~s}, 3 \mathrm{H}), 1.22(\mathrm{~d}, J=6.9 \mathrm{~Hz}, 6 \mathrm{H})$.

${ }^{13}$ C NMR $\left(126 \mathrm{MHz}, \mathrm{CDCl}_{3}\right) \delta 149.4,143.4,136.0,132.1,132.1,131.5,129.5,127.8,126.2$, $119.9,119.5,85.9,80.8,49.2,36.8,34.0,23.8,21.4$.

IR (ATR) 2963, 1597, 1430, 1347, 1161, 1087, 1057, 1006, 913, 824, 758, 665

HRMS (DART, M+H) Calculated for $\mathrm{C}_{22} \mathrm{H}_{26} \mathrm{~N}_{1} \mathrm{O}_{2} \mathrm{~S}_{1} 368.1679$, found 368.1675

N-(3-([1,1'-biphenyl]-4-yl)prop-2-yn-1-yl)-N-allyl-4-methylbenzenesulfonamide (2d)

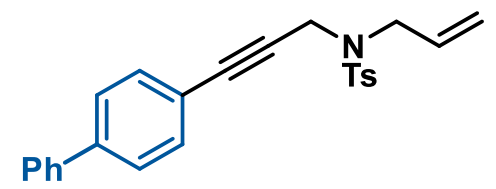

Synthesized following GP2. Characterization consistent with literature T. Xi, Z. Lu, ACS Catal. 2017, 7, 1181-1185.

${ }^{1} \mathbf{H}$ NMR $\left(500 \mathrm{MHz} \mathrm{CDCl}_{3}\right) \delta 7.80(\mathrm{~d}, J=8.3 \mathrm{~Hz}, 2 \mathrm{H}), 7.59-7.53(\mathrm{~m}, 2 \mathrm{H}), 7.51-7.42(\mathrm{~m}$, $4 \mathrm{H}), 7.41-7.33(\mathrm{~m}, 1 \mathrm{H}), 7.31-7.25(\mathrm{~m}, 3 \mathrm{H}), 7.14(\mathrm{~d}, J=8.3 \mathrm{~Hz}, 2 \mathrm{H}), 5.82$ (ddt, $J=17.2,10.1$, $6.5 \mathrm{~Hz}, 1 \mathrm{H}), 5.35$ (dq, $J=17.0,1.3 \mathrm{~Hz}, 1 \mathrm{H}), 5.29(\mathrm{dq}, J=10.1,1.3 \mathrm{~Hz}, 1 \mathrm{H}), 4.34(\mathrm{~s}, 2 \mathrm{H}), 3.92$ $(\mathrm{dt}, J=6.5,1.3 \mathrm{~Hz}, 2 \mathrm{H}), 2.36(\mathrm{~s}, 3 \mathrm{H})$.

${ }^{13} \mathrm{C}$ NMR $\left(126 \mathrm{MHz}, \mathrm{CDCl}_{3}\right) \delta 143.5,141.2,140.1,136.0,132.1,131.9,129.5,128.9,127.8$, $127.8,127.0,126.8,121.1,120.0,85.6,82.3,49.3,36.8,21.5$.

N-allyl-4-methyl-N-(3-(4-(thiophen-3-yl)phenyl)prop-2-yn-1-yl)benzenesulfonamide (2e)

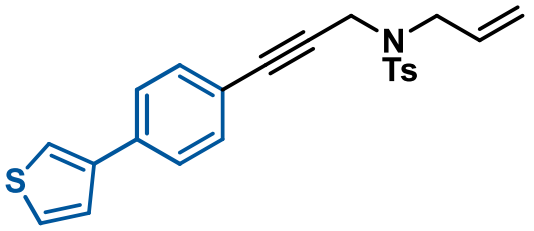

Synthesized following GP2 on a $5 \mathrm{mmol}$ scale. Obtained $0.95 \mathrm{~g}$ of a yellow solid (47\% yield).

${ }^{1} \mathbf{H}$ NMR $\left(500 \mathrm{MHz}, \mathrm{CDCl}_{3}\right) \delta 7.79(\mathrm{~d}, J=8.3 \mathrm{~Hz}, 2 \mathrm{H}), 7.51-7.44(\mathrm{~m}, 3 \mathrm{H}), 7.40(\mathrm{dd}, J=5.0$, $2.9 \mathrm{~Hz}, 1 \mathrm{H}), 7.37(\mathrm{dd}, J=5.0,1.4 \mathrm{~Hz}, 1 \mathrm{H}), 7.27(\mathrm{~d}, J=8.6 \mathrm{~Hz}, 2 \mathrm{H}), 7.10(\mathrm{~d}, J=8.5 \mathrm{~Hz}, 2 \mathrm{H})$, 5.81 (ddt, $J=16.5,10.1,6.5 \mathrm{~Hz}, 1 \mathrm{H}), 5.35(\mathrm{dq}, J=17.1,1.4 \mathrm{~Hz}, 1 \mathrm{H}), 5.28(\mathrm{dq}, J=10.0,1.2 \mathrm{~Hz}$, $1 \mathrm{H}), 4.33(\mathrm{~s}, 2 \mathrm{H}), 3.91(\mathrm{dt}, J=6.5,1.3 \mathrm{~Hz}, 2 \mathrm{H}), 2.35(\mathrm{~s}, 3 \mathrm{H})$.

${ }^{13} \mathrm{C}$ NMR $\left(126 \mathrm{MHz}, \mathrm{CDCl}_{3}\right) \delta 143.5,141.3,136.0,135.8,132.1,132.1,132.0,129.5,127.8$, $126.6,126.0,120.9,120.8,119.9,85.6,82.3,49.3,36.8,21.5$.

IR (ATR) 1668, 1595, 1499, 1447, 1345, 1323, 1161, 1119, 895, 779, 760

HRMS (DART, M+H) Calculated for $\mathrm{C}_{23} \mathrm{H}_{22} \mathrm{~N}_{1} \mathrm{O}_{2} \mathrm{~S}_{2} 408.1087$, found 408.1088

MP $75-77{ }^{\circ} \mathrm{C}$ 
N-allyl-N-(3-(4-methoxyphenyl)prop-2-yn-1-yl)-4-methylbenzenesulfonamide (2f)

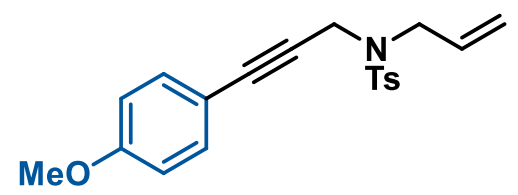

Synthesized following GP2. Characterization consistent with literature N. Cabrera-Lobera, P. Rodríguez-Salamanca, J. C. Nieto-Carmona, E. Buñuel, D. J. Cárdenas, Chem. Eur. J. 2018, 24, 784-788.

${ }^{1} \mathbf{H}$ NMR $\left(500 \mathrm{MHz}, \mathrm{CDCl}_{3}\right) \delta 7.77(\mathrm{~d}, J=8.3 \mathrm{~Hz}, 2 \mathrm{H}), 7.25(\mathrm{~d}, J=8.3 \mathrm{~Hz}, 2 \mathrm{H}), 7.01(\mathrm{~d}, J=8.9$ $\mathrm{Hz}, 2 \mathrm{H}), 6.76(\mathrm{~d}, J=8.9 \mathrm{~Hz}, 2 \mathrm{H}), 5.79(\mathrm{ddt}, J=17.1,10.0,6.4 \mathrm{~Hz}, 1 \mathrm{H}), 5.32(\mathrm{dq}, J=17.1,1.4$ $\mathrm{Hz}, 1 \mathrm{H}), 5.25(\mathrm{dq}, J=10.0,1.4 \mathrm{~Hz}, 1 \mathrm{H}), 4.28(\mathrm{~s}, 2 \mathrm{H}), 3.88(\mathrm{dt}, J=6.4,1.4 \mathrm{~Hz}, 2 \mathrm{H}), 3.78(\mathrm{~s}, 3 \mathrm{H})$, $2.35(\mathrm{~s}, 3 \mathrm{H})$.

${ }^{13}$ C NMR $\left(126 \mathrm{MHz}, \mathrm{CDCl}_{3}\right) \delta$ 159.6, 143.4, 136.0, 132.9, 132.1, 129.5, 127.8, 119.8, 114.3, $113.7,85.6,80.2,55.3,49.2,36.8,21.5$.

N-allyl-N-(3-(4-fluorophenyl)prop-2-yn-1-yl)-4-methylbenzenesulfonamide (2g)<smiles>C=CCN(S)CC#Cc1ccc(F)cc1</smiles>

Synthesized following GP2 on a $4 \mathrm{mmol}$ scale. Obtained $1.33 \mathrm{~g}$ of a white solid (95\% yield).

${ }^{1} \mathbf{H}$ NMR $\left(500 \mathrm{MHz}, \mathrm{CDCl}_{3}\right) \delta 7.77(\mathrm{~d}, J=8.3 \mathrm{~Hz}, 2 \mathrm{H}), 7.27-7.23(\mathrm{~m}, 2 \mathrm{H}), 7.07$ - $7.02(\mathrm{~m}$, $2 \mathrm{H}), 6.96-6.90(\mathrm{~m}, 2 \mathrm{H}), 5.79(\mathrm{ddt}, J=17.1,10.1,6.5 \mathrm{~Hz}, 1 \mathrm{H}), 5.32(\mathrm{dq}, J=17.1,1.4 \mathrm{~Hz}, 1 \mathrm{H})$, $5.26(\mathrm{dq}, J=10.0,1.2 \mathrm{~Hz}, 1 \mathrm{H}), 4.28(\mathrm{~s}, 2 \mathrm{H}), 3.88(\mathrm{dt}, J=6.5,1.3 \mathrm{~Hz}, 2 \mathrm{H}), 2.34(\mathrm{~s}, 3 \mathrm{H})$.

${ }^{13}$ C NMR $\left(126 \mathrm{MHz}, \mathrm{CDCl}_{3}\right) \delta 162.4(\mathrm{~d}, J=249.9 \mathrm{~Hz}), 143.5,136.0,133.4(\mathrm{~d}, J=8.2 \mathrm{~Hz})$, $132.0,129.5,127.8,119.9,118.3(\mathrm{~d}, J=3.3 \mathrm{~Hz}), 115.4(\mathrm{~d}, J=22.2 \mathrm{~Hz}), 84.6,81.4(\mathrm{~d}, J=1.6$ $\mathrm{Hz})$, 49.3, 36.6, 21.4.

${ }^{19}$ F NMR $\left(377 \mathrm{MHz}, \mathrm{CDCl}_{3}\right) \delta-110.5$.

IR (ATR) 1606, 1508, 1335, 1224, 1155, 1108, 1063, 940, 898, 842, 812, 764, 659, 629

HRMS (DART, $\mathrm{M}+\mathrm{H}$ ) Calculated for $\mathrm{C}_{19} \mathrm{H}_{19} \mathrm{~N}_{1} \mathrm{O}_{2} \mathrm{~F}_{1} \mathrm{~S}_{1} 344.1115$, found 344.1124

MP $43-45{ }^{\circ} \mathrm{C}$

N-allyl-N-(3-(4-chlorophenyl)prop-2-yn-1-yl)-4-methylbenzenesulfonamide (2h)<smiles>C=CCN(S)CC#Cc1ccc(Cl)cc1</smiles> 
Synthesized following GP2. Characterization consistent with literature N. Cabrera-Lobera, P. Rodríguez-Salamanca, J. C. Nieto-Carmona, E. Buñuel, D. J. Cárdenas, Chem. Eur. J. 2018, 24, 784-788.

${ }^{1} \mathbf{H}$ NMR $\left(500 \mathrm{MHz}, \mathrm{CDCl}_{3}\right) \delta 7.76(\mathrm{~d}, J=8.3 \mathrm{~Hz}, 2 \mathrm{H}), 7.27-7.23(\mathrm{~m}, 2 \mathrm{H}), 7.23-7.19(\mathrm{~m}$, $2 \mathrm{H}), 7.00-6.97(\mathrm{~m}, 2 \mathrm{H}), 5.79(\mathrm{ddt}, J=17.1,10.1,6.5 \mathrm{~Hz}, 1 \mathrm{H}), 5.31(\mathrm{dq}, J=17.1,1.4 \mathrm{~Hz}, 1 \mathrm{H})$, $5.26(\mathrm{dq}, J=10.1,1.3 \mathrm{~Hz}, 1 \mathrm{H}), 4.29(\mathrm{~s}, 2 \mathrm{H}), 3.88(\mathrm{dt}, J=6.5,1.3 \mathrm{~Hz}, 2 \mathrm{H}), 2.35(\mathrm{~s}, 3 \mathrm{H})$.

${ }^{13} \mathrm{C}$ NMR $\left(126 \mathrm{MHz}, \mathrm{CDCl}_{3}\right) \delta 143.5,136.0,134.4,132.7,132.0,129.5,128.4,127.8,120.7$, $120.0,84.5,82.8,49.3,36.6,21.4$.

N-allyl-N-(3-(4-bromophenyl)prop-2-yn-1-yl)-4-methylbenzenesulfonamide (2i)

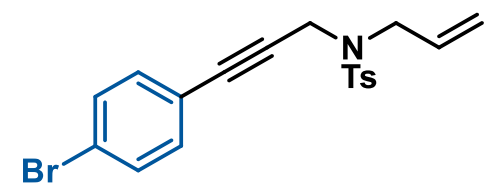

Synthesized following GP2. Characterization consistent with literature M. Ishida, Y. Shibata, K. Noguchi, K. Tanaka, Chem. Eur. J. 2011, 17, 12578-12581.

${ }^{1} \mathbf{H}$ NMR $\left(500 \mathrm{MHz}, \mathrm{CDCl}_{3}\right) \delta 7.79-7.73(\mathrm{~m}, 2 \mathrm{H}), 7.37(\mathrm{~d}, J=8.5 \mathrm{~Hz}, 2 \mathrm{H}), 7.27-7.23(\mathrm{~m}$, $2 \mathrm{H}), 6.92(\mathrm{~d}, J=8.6 \mathrm{~Hz}, 2 \mathrm{H}), 5.78(\mathrm{ddt}, J=16.6,10.1,6.5 \mathrm{~Hz}, 1 \mathrm{H}), 5.31(\mathrm{dq}, J=17.1,1.4 \mathrm{~Hz}$, $1 \mathrm{H}), 5.26(\mathrm{dq}, J=10.1,1.2 \mathrm{~Hz}, 1 \mathrm{H}), 4.28(\mathrm{~s}, 2 \mathrm{H}), 3.87(\mathrm{dt}, J=6.4,1.3 \mathrm{~Hz}, 2 \mathrm{H}), 2.37-2.33(\mathrm{~m}$, $3 \mathrm{H})$.

${ }^{13} \mathrm{C}$ NMR $\left(126 \mathrm{MHz}, \mathrm{CDCl}_{3}\right) \delta 143.5,136.0,132.9,132.0,131.4,129.5,127.8,122.6,121.1$, $120.0,84.6,83.0,49.3,36.7,21.5$.

N-allyl-4-methyl-N-(3-(4-(trifluoromethyl)phenyl)prop-2-yn-1-yl)benzenesulfonamide (2j)<smiles>C=CCN(C)CC#Cc1ccc(C(F)(F)F)cc1</smiles>

Synthesized following GP2. Characterization consistent with literature A. Lin, Z.-W. Zhang, J. Yang, Org. Lett. 2014, 16, 386-389.

${ }^{1} \mathbf{H}$ NMR $\left(500 \mathrm{MHz}, \mathrm{CDCl}_{3}\right) \delta 7.77(\mathrm{~d}, J=8.3 \mathrm{~Hz}, 2 \mathrm{H}), 7.50(\mathrm{dd}, J=8.7,0.7 \mathrm{~Hz}, 2 \mathrm{H}), 7.25(\mathrm{~d}, J$ $=8.3 \mathrm{~Hz}, 2 \mathrm{H}), 7.16(\mathrm{dd}, J=8.7,0.7 \mathrm{~Hz}, 2 \mathrm{H}), 5.79(\mathrm{ddt}, J=17.1,10.1,6.5 \mathrm{~Hz}, 1 \mathrm{H}), 5.32(\mathrm{dq}, J=$ $17.1,1.4 \mathrm{~Hz}, 1 \mathrm{H}), 5.27$ (dq, $J=10.1,1.4 \mathrm{~Hz}, 1 \mathrm{H}), 4.32(\mathrm{~s}, 2 \mathrm{H}), 3.89$ (dt, $J=6.5,1.4 \mathrm{~Hz}, 2 \mathrm{H})$, $2.33(\mathrm{~s}, 3 \mathrm{H})$.

${ }^{13} \mathrm{C}$ NMR (126 MHz, $\left.\mathrm{CDCl}_{3}\right) \delta 143.57,135.94,131.92,131.71,130.15(\mathrm{q}, J=32.7 \mathrm{~Hz}), 129.53$, $127.81,125.98(\mathrm{q}, J=1.4 \mathrm{~Hz}), 125.03(\mathrm{q}, J=3.8 \mathrm{~Hz}), 123.74(\mathrm{q}, J=272.2 \mathrm{~Hz}), 120.05,84.43$, 84.27, 49.43, 36.58, 21.39.

${ }^{19}$ F NMR $\left(377 \mathrm{MHz}, \mathrm{CDCl}_{3}\right) \delta-62.9$. 
ethyl 2-(4-(3-((N-allyl-4-methylphenyl)sulfonamido)prop-1-yn-1-yl)phenoxy)acetate (2k)<smiles>C=CCN(S)CC#Cc1ccc(OCC(=O)OCC)cc1</smiles>

Synthesized following GP2 on a $5 \mathrm{mmol}$ scale. Obtained $1.26 \mathrm{~g}$ of an orange oil (60\% yield).

${ }^{1} \mathbf{H}$ NMR $\left(500 \mathrm{MHz}, \mathrm{CDCl}_{3}\right) \delta 7.75(\mathrm{~d}, J=8.3 \mathrm{~Hz}, 2 \mathrm{H}), 7.24(\mathrm{~d}, J=8.3 \mathrm{~Hz}, 2 \mathrm{H}), 7.00(\mathrm{~d}, J=9.0$ $\mathrm{Hz}, 2 \mathrm{H}), 6.76(\mathrm{~d}, J=9.0 \mathrm{~Hz}, 2 \mathrm{H}), 5.77(\mathrm{ddt}, J=16.5,10.0,6.5 \mathrm{~Hz}, 1 \mathrm{H}), 5.30(\mathrm{dq}, J=16.5,1.4$ $\mathrm{Hz}, 1 \mathrm{H}), 5.24(\mathrm{dq}, J=10.0,1.4 \mathrm{~Hz}, 1 \mathrm{H}), 4.59(\mathrm{~s}, 2 \mathrm{H}), 4.28-4.22(\mathrm{~m}, 4 \mathrm{H}), 3.86(\mathrm{dt}, J=6.5,1.4$ $\mathrm{Hz}, 2 \mathrm{H}), 2.33$ (s, 3H), $1.28(\mathrm{t}, J=7.1 \mathrm{~Hz}, 3 \mathrm{H})$.

${ }^{13}$ C NMR $\left(126 \mathrm{MHz}, \mathrm{CDCl}_{3}\right) \delta 168.5,157.8,143.4,136.0,133.0,132.1,132.1,129.5,127.8$, $119.8,115.4,114.4,85.3,80.6,65.2,61.5,49.2,36.7,21.4,14.1$.

IR (ATR) 2982, 1755, 1604, 1507, 1442, 1346, 1297, 1197, 1150, 1084, 897, 732, 814, 754

HRMS (DART, $\mathrm{M}+\mathrm{H}$ ) Calculated for $\mathrm{C}_{23} \mathrm{H}_{26} \mathrm{~N}_{1} \mathrm{O}_{5} \mathrm{~S}_{1} 428.1526$, found 428.1527

\section{N-allyl-4-methyl-N-(3-(4-(((triisopropylsilyl)oxy)methyl)phenyl)prop-2-yn-1- yl)benzenesulfonamide (2l)}

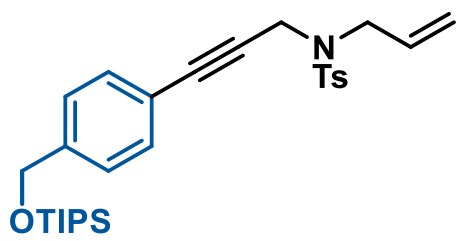

Synthesized following GP2 on a $5 \mathrm{mmol}$ scale. Obtained $2.00 \mathrm{~g}$ of a yellow oil (78\% yield).

${ }^{1}$ H NMR $\left(500 \mathrm{MHz}, \mathrm{CDCl}_{3}\right) \delta 7.77(\mathrm{~d}, J=8.3 \mathrm{~Hz}, 2 \mathrm{H}), 7.26-7.20(\mathrm{~m}, 4 \mathrm{H}), 7.03(\mathrm{~d}, J=8.2 \mathrm{~Hz}$, $2 \mathrm{H}), 5.80(\mathrm{ddt}, J=16.6,10.1,6.5 \mathrm{~Hz}, 1 \mathrm{H}), 5.33(\mathrm{dq}, J=16.6,1.2 \mathrm{~Hz}, 1 \mathrm{H}), 5.26(\mathrm{dq}, J=10.1$, $1.2 \mathrm{~Hz}, 1 \mathrm{H}), 4.80(\mathrm{~s}, 2 \mathrm{H}), 4.30(\mathrm{~s}, 2 \mathrm{H}), 3.89(\mathrm{dt}, J=6.5,1.2 \mathrm{~Hz}, 2 \mathrm{H}), 2.33(\mathrm{~s}, 3 \mathrm{H}), 1.20-1.13$ $(\mathrm{m}, 3 \mathrm{H}), 1.12-1.06(\mathrm{~m}, 18 \mathrm{H})$.

${ }^{13}$ C NMR $\left(126 \mathrm{MHz}, \mathrm{CDCl}_{3}\right) \delta 143.4,142.2,135.9,132.1,132.1,131.3,129.5,127.8,125.4$, $120.5,119.9,119.9,85.8,81.1,64.6,49.2,36.7,21.4,18.0,12.0$.

IR (ATR) 2942, 2864, 1462, 1349, 1329, 1161, 1116, 1091, 882, 812, 754, 678

HRMS (DART, M+H) Calculated for $\mathrm{C}_{29} \mathrm{H}_{42} \mathrm{~N}_{1} \mathrm{O}_{3} \mathrm{Si}_{1} \mathrm{~S}_{1} 512.2649$, found 512.2657

\section{N-allyl-4-methyl-N-(3-(m-tolyl)prop-2-yn-1-yl)benzenesulfonamide (2m)}




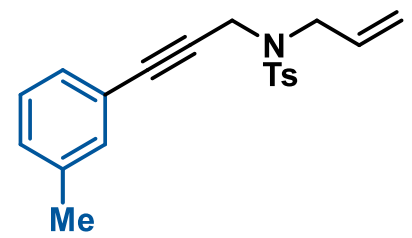

Synthesized following GP2 on a $4 \mathrm{mmol}$ scale. Obtained $0.70 \mathrm{~g}$ of a white solid (51\% yield).

${ }^{1} \mathbf{H}$ NMR $\left(500 \mathrm{MHz}, \mathrm{CDCl}_{3}\right) \delta 7.78(\mathrm{~d}, J=8.3 \mathrm{~Hz}, 2 \mathrm{H}), 7.29-7.24(\mathrm{~m}, 2 \mathrm{H}), 7.15-7.11(\mathrm{~m}$, $1 \mathrm{H}), 7.11-7.07(\mathrm{~m}, 1 \mathrm{H}), 6.90-6.86(\mathrm{~m}, 2 \mathrm{H}), 5.85-5.75(\mathrm{~m}, 1 \mathrm{H}), 5.33(\mathrm{dq}, J=17.0,1.3 \mathrm{~Hz}$, $1 \mathrm{H}), 5.27(\mathrm{dq}, J=10.0,1.3 \mathrm{~Hz}, 1 \mathrm{H}), 4.30(\mathrm{~s}, 2 \mathrm{H}), 3.89(\mathrm{dt}, J=6.5,1.3 \mathrm{~Hz}, 2 \mathrm{H}), 2.35(\mathrm{~s}, 3 \mathrm{H})$, $2.29(\mathrm{~s}, 3 \mathrm{H})$.

${ }^{13} \mathrm{C}$ NMR $\left(126 \mathrm{MHz}, \mathrm{CDCl}_{3}\right) \delta 143.4,137.8,136.0,132.0,132.0,129.5,129.3,128.6,128.0$, $127.8,122.0,119.9,85.9,81.2,49.2,36.7,21.4,21.2$.

IR (ATR) 1603, 1487, 1439, 1391, 1158, 1096, 994, 946, 907, 788, 758, 695, 665

HRMS (DART, M+H) Calculated for $\mathrm{C}_{20} \mathrm{H}_{22} \mathrm{~N}_{1} \mathrm{O}_{2} \mathrm{~S}_{1} 340.1366$, found 340.1370

MP $40-41{ }^{\circ} \mathrm{C}$

\section{N-allyl-N-(3-(3-methoxyphenyl)prop-2-yn-1-yl)-4-methylbenzenesulfonamide (2n)}

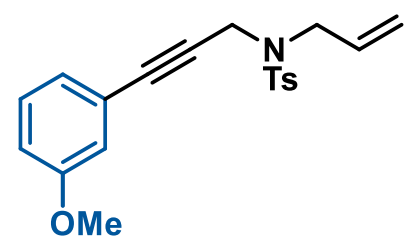

Synthesized following GP2. Characterization consistent with literature A. Lin, Z.-W. Zhang, J. Yang, Org. Lett. 2014, 16, 386-389.

${ }^{1} \mathbf{H}$ NMR $\left(500 \mathrm{MHz}, \mathrm{CDCl}_{3}\right) \delta 7.77(\mathrm{~d}, J=8.3 \mathrm{~Hz}, 2 \mathrm{H}), 7.28-7.24(\mathrm{~m}, 3 \mathrm{H}), 7.15(\mathrm{ddd}, J=8.3$, $7.6,0.5 \mathrm{~Hz}, 1 \mathrm{H}), 6.83(\mathrm{ddd}, J=8.3,2.6,1.0 \mathrm{~Hz}, 1 \mathrm{H}), 6.66(\mathrm{ddd}, J=7.6,1.4,1.0 \mathrm{~Hz}, 1 \mathrm{H}), 6.60$ $(\mathrm{dd}, J=2.6,1.4 \mathrm{~Hz}, 1 \mathrm{H}), 5.80(\mathrm{ddt}, J=17.1,10.0,6.5 \mathrm{~Hz}, 1 \mathrm{H}), 5.33(\mathrm{dq}, J=17.1,1.4 \mathrm{~Hz}, 1 \mathrm{H})$, $5.26(\mathrm{dq}, J=10.1,1.4 \mathrm{~Hz}, 1 \mathrm{H}), 4.30(\mathrm{~s}, 2 \mathrm{H}), 3.88(\mathrm{dt}, J=6.5,1.4 \mathrm{~Hz}, 2 \mathrm{H}), 3.77(\mathrm{~s}, 3 \mathrm{H}), 2.35$ (s, $3 \mathrm{H})$.

${ }^{13} \mathrm{C}$ NMR $\left(126 \mathrm{MHz}, \mathrm{CDCl}_{3}\right) \delta 159.1,143.6,135.9,132.0,129.5,129.2,127.8,124.0,123.2$, $119.9,117.0,114.3,85.6,81.5,55.2,49.3,36.7,21.4$.

\section{N-allyl-N-(3-(3-fluorophenyl)prop-2-yn-1-yl)-4-methylbenzenesulfonamide (2o)}

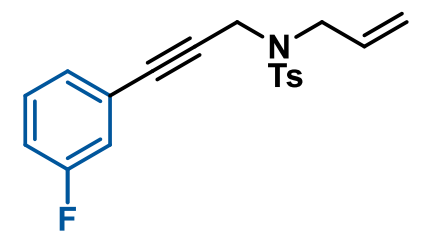

Synthesized following GP2. Characterization consistent with literature S.-C. Ren, F.-L. Zhang, J. Qi, Y.-S. Huang, A.-Q. Xu, H.-Y. Yan, Y.-F. Wang, J. Am. Chem. Soc. 2017, 139, 6050-6053. 
${ }^{1} \mathbf{H}$ NMR $\left(500 \mathrm{MHz}, \mathrm{CDCl}_{3}\right) \delta 7.77(\mathrm{~d}, J=8.3 \mathrm{~Hz}, 2 \mathrm{H}), 7.30-7.24(\mathrm{~m}, 2 \mathrm{H}), 7.20(\mathrm{td}, J=8.0$, $5.8 \mathrm{~Hz}, 1 \mathrm{H}), 6.99$ (tdd, $J=8.3,2.7,1.0 \mathrm{~Hz}, 1 \mathrm{H}), 6.87$ (dt, $J=7.7,1.3 \mathrm{~Hz}, 1 \mathrm{H}), 6.66$ (ddd, $J=9.4$, $2.7,1.5 \mathrm{~Hz}, 1 \mathrm{H}), 5.79(\mathrm{ddt}, J=17.1,10.1,6.5 \mathrm{~Hz}, 1 \mathrm{H}), 5.32(\mathrm{dq}, J=17.1,1.3 \mathrm{~Hz}, 1 \mathrm{H}), 5.27(\mathrm{dq}$, $J=10.1,1.3 \mathrm{~Hz}, 1 \mathrm{H}), 4.29$ (s, 2H), $3.88(\mathrm{dt}, J=6.5,1.3 \mathrm{~Hz}, 2 \mathrm{H}), 2.36$ (s, 3H).

${ }^{13}$ C NMR $\left(126 \mathrm{MHz}, \mathrm{CDCl}_{3}\right) \delta 162.1(\mathrm{~d}, J=246.6 \mathrm{~Hz}), 143.7,135.9,132.0,129.7(\mathrm{~d}, J=8.6$ $\mathrm{Hz}), 129.6,127.8,127.3(\mathrm{~d}, J=3.1 \mathrm{~Hz}), 124.0(\mathrm{~d}, J=9.5 \mathrm{~Hz}), 120.0,118.3(\mathrm{~d}, J=23.0 \mathrm{~Hz})$, $115.8(\mathrm{~d}, J=21.2 \mathrm{~Hz}), 84.4,82.7,49.4,36.6,21.4$.

${ }^{19}$ F NMR $\left(377 \mathrm{MHz}, \mathrm{CDCl}_{3}\right) \delta-112.9$.

N-allyl-4-methyl-N-(3-(3-(trifluoromethyl)phenyl)prop-2-yn-1-yl)benzenesulfonamide (2p)

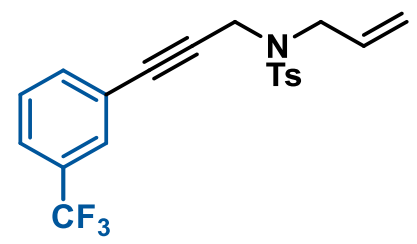

Synthesized following GP2 on a 4 mmol scale. Obtained $1.38 \mathrm{~g}$ of a yellow oil (88\% yield).

${ }^{1} \mathbf{H}$ NMR $\left(500 \mathrm{MHz}, \mathrm{CDCl}_{3}\right) \delta 7.78(\mathrm{~d}, J=8.3 \mathrm{~Hz}, 2 \mathrm{H}), 7.55-7.51(\mathrm{~m}, 1 \mathrm{H}), 7.40-7.35(\mathrm{~m}$, $1 \mathrm{H}), 7.30-7.24(\mathrm{~m}, 4 \mathrm{H}), 5.80$ (ddt, $J=17.1,10.1,6.5 \mathrm{~Hz}, 1 \mathrm{H}), 5.33$ (dq, $J=17.1,1.3 \mathrm{~Hz}, 1 \mathrm{H})$, $5.28(\mathrm{dq}, J=10.1,1.3 \mathrm{~Hz}, 1 \mathrm{H}), 4.32(\mathrm{~s}, 2 \mathrm{H}), 3.89(\mathrm{dt}, J=6.5,1.3 \mathrm{~Hz}, 2 \mathrm{H}), 2.32(\mathrm{~s}, 3 \mathrm{H})$.

${ }^{13}$ C NMR (126 MHz, $\left.\mathrm{CDCl}_{3}\right) \delta 143.7,135.9,134.6(\mathrm{q}, J=1.2 \mathrm{~Hz}), 132.0(\mathrm{~d}, J=1.7 \mathrm{~Hz}), 130.8$ $(\mathrm{q}, J=32.7 \mathrm{~Hz}), 129.5,128.7,128.1(\mathrm{q}, J=3.9 \mathrm{~Hz}), 127.9,124.95(\mathrm{q}, J=3.7 \mathrm{~Hz}), 123.6(\mathrm{q}, J=$ $272.5 \mathrm{~Hz}), 123.1,120.0,84.1,83.5,49.4,36.5,21.3$.

${ }^{19}$ F NMR $\left(377 \mathrm{MHz}, \mathrm{CDCl}_{3}\right) \delta-62.9$.

IR (ATR) 1436, 1324, 1234, 1157, 1161, 1093, 1078, 988, 892, 804, 792,752, 662

HRMS (DART, M+H) Calculated for $\mathrm{C}_{20} \mathrm{H}_{19} \mathrm{~N}_{1} \mathrm{O}_{2} \mathrm{~F}_{3} \mathrm{~S}_{1} 394.1083$, found 394.1088

ethyl 3-(3-((N-allyl-4-methylphenyl)sulfonamido)prop-1-yn-1-yl)benzoate (2q)

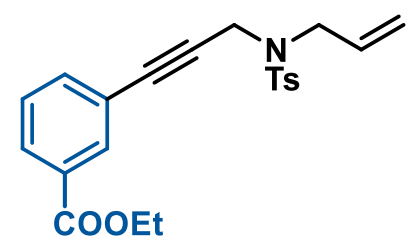

Synthesized following GP2 on a $5 \mathrm{mmol}$ scale. Obtained $0.44 \mathrm{~g}$ of a white solid (35\% yield).

${ }^{1} \mathbf{H}$ NMR $\left(500 \mathrm{MHz}, \mathrm{CDCl}_{3}\right) \delta 7.93(\mathrm{dt}, J=7.8,1.5 \mathrm{~Hz}, 1 \mathrm{H}), 7.79-7.73(\mathrm{~m}, 3 \mathrm{H}), 7.31(\mathrm{td}, J=$ $7.8,0.6 \mathrm{~Hz}, 1 \mathrm{H}), 7.27-7.21(\mathrm{~m}, 3 \mathrm{H}), 5.79(\mathrm{ddt}, J=16.5,10.0,6.4 \mathrm{~Hz}, 1 \mathrm{H}), 5.32(\mathrm{dq}, J=16.5$, $1.4 \mathrm{~Hz}, 1 \mathrm{H}), 5.26(\mathrm{dq}, J=10.0,1.4 \mathrm{~Hz}, 1 \mathrm{H}), 4.37(\mathrm{q}, J=7.1 \mathrm{~Hz}, 2 \mathrm{H}), 4.30(\mathrm{~s}, 2 \mathrm{H}), 3.88(\mathrm{dt}, J=$ 6.4, 1.4 Hz, 2H), 2.29 (s, 3H), 1.39 (t, $J=7.1 \mathrm{~Hz}, 3 \mathrm{H})$.

${ }^{13} \mathrm{C}$ NMR $\left(126 \mathrm{MHz}, \mathrm{CDCl}_{3}\right) \delta 165.7,143.7,135.8,135.5,132.5,132.0,130.6,129.5,129.3$, $128.2,127.8,122.6,120.0,84.7,82.6,61.2,49.3,36.6,21.4,14.3$. 
IR (ATR) 2987, 2923, 1711, 1595, 1483, 1344, 1326, 1227, 1160, 1116, 1083, 893, 747, 657

HRMS (DART, M+H) Calculated for $\mathrm{C}_{22} \mathrm{H}_{24} \mathrm{~N}_{1} \mathrm{O}_{4} \mathrm{~S}_{1} 398.1421$, found 398.1421

MP $61-62{ }^{\circ} \mathrm{C}$

N-allyl-N-(3-(3-bromophenyl)prop-2-yn-1-yl)-4-methylbenzenesulfonamide (2r)

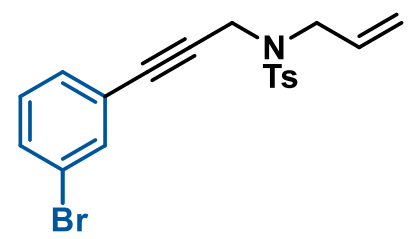

Synthesized following GP2 on a $4 \mathrm{mmol}$ scale. Obtained $1.11 \mathrm{~g}$ of a slightly yellow oil (68\% yield).

${ }^{1} \mathbf{H}$ NMR $\left(500 \mathrm{MHz}, \mathrm{CDCl}_{3}\right) \delta 7.76(\mathrm{~d}, J=8.3 \mathrm{~Hz}, 2 \mathrm{H}), 7.40(\mathrm{ddd}, J=8.1,2.0,1.1 \mathrm{~Hz}, 1 \mathrm{H}), 7.30$ $-7.26(\mathrm{~m}, 2 \mathrm{H}), 7.13-7.08(\mathrm{~m}, 2 \mathrm{H}), 7.04-7.00(\mathrm{~m}, 1 \mathrm{H}), 5.79$ (ddt, $J=17.1,10.0,6.4 \mathrm{~Hz}, 1 \mathrm{H})$, $5.31(\mathrm{dq}, J=17.1,1.4 \mathrm{~Hz}, 1 \mathrm{H}), 5.27(\mathrm{dq}, J=10.1,1.2 \mathrm{~Hz}, 1 \mathrm{H}), 4.29$ (s, $2 \mathrm{H}), 3.87(\mathrm{dt}, J=6.5$, $1.3 \mathrm{~Hz}, 2 \mathrm{H}), 2.38(\mathrm{~s}, 3 \mathrm{H})$.

${ }^{13} \mathrm{C}$ NMR $\left(126 \mathrm{MHz}, \mathrm{CDCl}_{3}\right) \delta 143.7,135.9,134.2,131.9,131.5,130.0,129.6,129.6,127.8$, 124.1, 121.9, 120.0, 84.1, 83.1, 49.4, 36.6, 21.5.

IR (ATR) 1595, 1559, 1478, 1347, 1155, 1093, 991, 931, 898, 815, 785, 752, 683, 665

HRMS (DART, $\mathrm{M}+\mathrm{H}$ ) Calculated for $\mathrm{C}_{19} \mathrm{H}_{19} \mathrm{~N}_{1} \mathrm{O}_{2} \mathrm{~S}_{1} \mathrm{Br}_{1} 404.0314$, found 404.3011

N-allyl-N-(3-(3,5-dimethoxyphenyl)prop-2-yn-1-yl)-4-methylbenzenesulfonamide (2s)

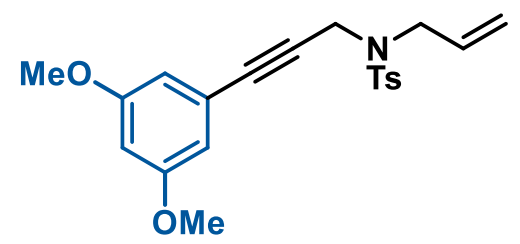

Synthesized following GP2 on a 4 mmol scale. Obtained $1.21 \mathrm{~g}$ of a yellow solid (79\%).

${ }^{1} \mathbf{H}$ NMR $\left(500 \mathrm{MHz}, \mathrm{CDCl}_{3}\right) \delta 7.77(\mathrm{~d}, J=8.3 \mathrm{~Hz}, 2 \mathrm{H}), 7.28-7.25(\mathrm{~m}, 2 \mathrm{H}), 6.40(\mathrm{t}, J=2.3 \mathrm{~Hz}$, $1 \mathrm{H}), 6.22(\mathrm{~d}, J=2.3 \mathrm{~Hz}, 2 \mathrm{H}), 5.79$ (ddt, $J=16.5,10.1,6.5 \mathrm{~Hz}, 1 \mathrm{H}), 5.32(\mathrm{dq}, J=17.1,1.3 \mathrm{~Hz}$, $1 \mathrm{H}), 5.26(\mathrm{dq}, J=10.1,1.3 \mathrm{~Hz}, 1 \mathrm{H}), 4.29(\mathrm{~s}, 2 \mathrm{H}), 3.88(\mathrm{dt}, J=6.5,1.3 \mathrm{~Hz}, 2 \mathrm{H}), 3.75(\mathrm{~s}, 6 \mathrm{H})$, $2.35(\mathrm{~s}, 3 \mathrm{H})$.

${ }^{13} \mathbf{C}$ NMR $\left(126 \mathrm{MHz}, \mathrm{CDCl}_{3}\right) \delta 160.4,143.6,135.9,132.0,129.5,127.8,123.5,119.9,109.6$, $101.2,85.6,81.3,55.3,49.3,36.7,21.4$.

IR (ATR) 1586, 1454, 1424, 1332, 1206, 1149, 1063, 951, 895, 827, 761, 656

HRMS (ESI, M+H) Calculated for $\mathrm{C}_{21} \mathrm{H}_{24} \mathrm{~N}_{1} \mathrm{O}_{4} \mathrm{~S}_{1} 386.1421$, found 386.1425

MP $94-95{ }^{\circ} \mathrm{C}$ 
N-allyl-N-(3-(2-methoxyphenyl)prop-2-yn-1-yl)-4-methylbenzenesulfonamide (2t)

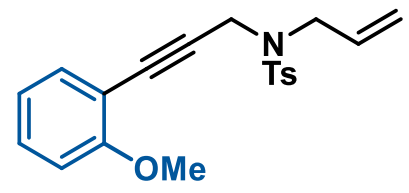

Synthesized following GP2. Characterization consistent with literature A. Lin, Z.-W. Zhang, J. Yang, Org. Lett. 2014, 16, 386-389.

${ }^{1} \mathbf{H}$ NMR $\left(400 \mathrm{MHz}, \mathrm{CDCl}_{3}\right) \delta 7.77(\mathrm{~d}, J=8.3 \mathrm{~Hz}, 2 \mathrm{H}), 7.24(\mathrm{ddd}, J=8.3,7.5,1.9 \mathrm{~Hz}, 1 \mathrm{H}), 7.22$ $-7.18(\mathrm{~m}, 2 \mathrm{H}), 6.93(\mathrm{dd}, J=7.8,1.9 \mathrm{~Hz}, 1 \mathrm{H}), 6.81(\mathrm{~m}, 2 \mathrm{H}), 5.80(\mathrm{ddt}, J=16.7,10.0,6.5 \mathrm{~Hz}$, $1 \mathrm{H}), 5.37(\mathrm{dq}, J=16.7,1.4 \mathrm{~Hz}, 1 \mathrm{H}), 5.25(\mathrm{dq}, J=10.0,1.4 \mathrm{~Hz}, 1 \mathrm{H}), 4.35(\mathrm{~s}, 2 \mathrm{H}), 3.91(\mathrm{dt}, J=$ $6.5,1.4 \mathrm{~Hz}, 2 \mathrm{H}), 3.79$ (s, 3H), 2.28 (s, 3H).

${ }^{13} \mathrm{C}$ NMR $\left(100 \mathrm{MHz}, \mathrm{CDCl}_{3}\right) \delta 160.0,143.3,136.0,133.3,132.1,129.8,129.4,127.8,120.1$, $120.0,111.5,110.5,85.7,82.3,55.6,49.1,37.1,21.4$.

N-allyl-N-(3-(2-fluorophenyl)prop-2-yn-1-yl)-4-methylbenzenesulfonamide (2u)<smiles>C=CCN(C)CC#Cc1ccccc1F</smiles>

Synthesized following GP2 on a $2.5 \mathrm{mmol}$ scale. Obtained $0.79 \mathrm{~g}$ of a yellow oil (90\% yield).

${ }^{1} \mathbf{H}$ NMR $\left(500 \mathrm{MHz}, \mathrm{CDCl}_{3}\right) \delta 7.76(\mathrm{~d}, J=8.3 \mathrm{~Hz}, 2 \mathrm{H}), 7.26(\mathrm{~m}, 1 \mathrm{H}), 7.22(\mathrm{~m}, 2 \mathrm{H}), 7.08-6.96$ $(\mathrm{m}, 3 \mathrm{H}), 5.79$ (ddt, $J=16.7,10.0,6.5 \mathrm{~Hz}, 1 \mathrm{H}), 5.35(\mathrm{dq}, J=16.7,1.4 \mathrm{~Hz}, 1 \mathrm{H}), 5.27(\mathrm{dq}, J=$ 10.0, 1.4 Hz, 1H), 4.33 (s, 2H), 3.89 (dt, $J=6.5,1.4 \mathrm{~Hz}, 2 \mathrm{H}), 2.27$ (s, 3H).

${ }^{13}$ C NMR $\left(126 \mathrm{MHz}, \mathrm{CDCl}_{3}\right) \delta 162.6(\mathrm{~d}, J=251.9 \mathrm{~Hz}), 143.5,135.7,133.3(\mathrm{~d}, J=1.3 \mathrm{~Hz})$, $131.9,130.2(\mathrm{~d}, J=8.0 \mathrm{~Hz}), 129.5,127.7,123.7(\mathrm{~d}, J=3.7 \mathrm{~Hz}), 120.2,115.3(\mathrm{~d}, J=20.8 \mathrm{~Hz})$, $110.8(\mathrm{~d}, J=15.5 \mathrm{~Hz}), 86.9(\mathrm{~d}, J=3.3 \mathrm{~Hz}), 79.2(\mathrm{~d}, J=0.9 \mathrm{~Hz}), 49.3,36.8,21.4$.

${ }^{19}$ F NMR $\left(377 \mathrm{MHz}, \mathrm{CDCl}_{3}\right) \delta-110.1$.

IR (ATR) 1490, 1454, 1347, 1260, 1218, 1161, 1099, 934, 898, 818, 755, 698, 662

HRMS (DART, $\mathrm{M}+\mathrm{H}$ ) Calculated for $\mathrm{C}_{19} \mathrm{H}_{19} \mathrm{~N}_{1} \mathrm{O}_{2} \mathrm{~F}_{1} \mathrm{~S}_{1} 344.1115$, found 344.1111

N-allyl-4-methyl-N-(3-(thiophen-2-yl)prop-2-yn-1-yl)benzenesulfonamide (2v)

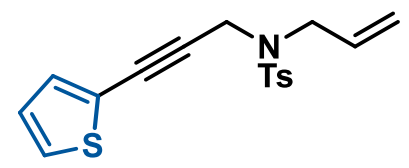

Synthesized following GP2. Characterization consistent with literature A. Lin, Z.-W. Zhang, J. Yang, Org. Lett. 2014, 16, 386-389. 
${ }^{1} \mathbf{H}$ NMR $\left(400 \mathrm{MHz}, \mathrm{CDCl}_{3}\right) \delta 7.75(\mathrm{~d}, J=8.3 \mathrm{~Hz}, 2 \mathrm{H}), 7.27(\mathrm{~d}, J=8.3 \mathrm{~Hz}, 2 \mathrm{H}), 7.19(\mathrm{dd}, J=$ $5.0,1.3 \mathrm{~Hz}, 1 \mathrm{H}), 6.92(\mathrm{dd}, J=3.6,1.3 \mathrm{~Hz}, 1 \mathrm{H}), 6.90(\mathrm{dd}, J=5.0,3.6 \mathrm{~Hz}, 1 \mathrm{H}), 5.78$ (ddt, $J=$ $17.1,10.1,6.4 \mathrm{~Hz}, 1 \mathrm{H}), 5.32(\mathrm{dq}, J=17.1,1.3 \mathrm{~Hz}, 1 \mathrm{H}), 5.26(\mathrm{dq}, J=10.1,1.3 \mathrm{~Hz}, 1 \mathrm{H}), 4.31(\mathrm{~s}$, $2 \mathrm{H}), 3.85(\mathrm{dt}, J=6.4,1.3 \mathrm{~Hz}, 2 \mathrm{H}), 2.36(\mathrm{~s}, 3 \mathrm{H})$.

${ }^{13}$ C NMR $\left(100 \mathrm{MHz}, \mathrm{CDCl}_{3}\right) \delta 143.6,135.8,132.2,132.0,129.6,127.7,127.2,126.8,122.1$, $120.0,85.7,78.9,49.3,36.9,21.5$.

\section{N-allyl-N-(hept-2-yn-1-yl)-4-methylbenzenesulfonamide (2w)}

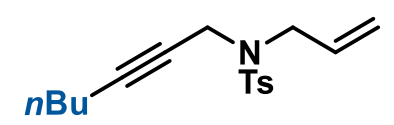

Synthesized following and consistent with literature T. Morimoto, K. Fuji, K. Tsutsumi, K.

Kakiuchi, J. Am. Chem. Soc. 2002, 124, 3806-3807.

${ }^{1} \mathbf{H}$ NMR $\left(500 \mathrm{MHz}, \mathrm{CDCl}_{3}\right) \delta 7.72(\mathrm{~d}, J=8.3 \mathrm{~Hz}, 1 \mathrm{H}), 7.27(\mathrm{~d}, J=8.3 \mathrm{~Hz}, 2 \mathrm{H}), 5.73$ (ddt, $J=$ $16.5,10.1,6.4 \mathrm{~Hz}, 1 \mathrm{H}), 5.26(\mathrm{dq}, J=17.1,1.3 \mathrm{~Hz}, 1 \mathrm{H}), 5.20(\mathrm{dq}, J=10.1,1.3 \mathrm{~Hz}, 1 \mathrm{H}), 4.05(\mathrm{t}, J$ $=2.3 \mathrm{~Hz}, 1 \mathrm{H}), 3.79(\mathrm{dt}, J=6.5,1.3 \mathrm{~Hz}, 1 \mathrm{H}), 2.40(\mathrm{~s}, 2 \mathrm{H}), 1.90(\mathrm{ddt}, J=6.8,4.6,2.3 \mathrm{~Hz}, 1 \mathrm{H})$, $1.26-1.07(\mathrm{~m}, 2 \mathrm{H}), 0.85-0.76(\mathrm{~m}, 1 \mathrm{H})$.

${ }^{13} \mathbf{C}$ NMR $\left(126 \mathrm{MHz}, \mathrm{CDCl}_{3}\right) \delta 143.2,136.2,132.2,129.3,127.8,119.5,86.2,72.2,48.9,36.3$, $30.4,21.8,21.5,18.1,13.5$.

\section{(3-(allyloxy)prop-1-yn-1-yl)benzene (2x)}

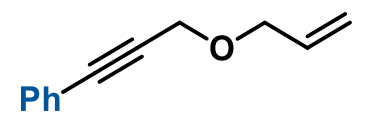

Synthesized following and consistent with literature T. Morimoto, K. Fuji, K. Tsutsumi, K. Kakiuchi, J. Am. Chem. Soc. 2002, 124, 3806-3807.

${ }^{1}$ H NMR $(500 \mathrm{MHz}, \mathrm{CDCl}) \delta 7.49-7.43(\mathrm{~m}, 2 \mathrm{H}), 7.35-7.28(\mathrm{~m}, 3 \mathrm{H}), 5.96$ (ddt, $J=17.2,10.4$, $5.8 \mathrm{~Hz}, 1 \mathrm{H}), 5.36(\mathrm{dq}, J=17.2,1.3 \mathrm{~Hz}, 1 \mathrm{H}), 5.25(\mathrm{dq}, J=10.4,1.3 \mathrm{~Hz}, 1 \mathrm{H}), 4.39(\mathrm{~s}, 2 \mathrm{H}), 4.15$ (dt, $J=5.8,1.3 \mathrm{~Hz}, 2 \mathrm{H})$.

${ }^{13} \mathbf{C}$ NMR $\left(126 \mathrm{MHz}, \mathrm{CDCl}_{3}\right) \delta 134.1,131.7,128.4,128.3,122.7,117.9,86.3,85.0,70.7,57.9$.

\section{diethyl 2-allyl-2-(3-phenylprop-2-yn-1-yl)malonate (2y)}

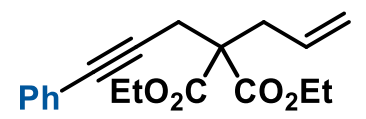

Synthesized following and consistent with literature T. Morimoto, K. Fuji, K. Tsutsumi, K. Kakiuchi, J. Am. Chem. Soc. 2002, 124, 3806-3807.

${ }^{1} \mathbf{H}$ NMR $\left(500 \mathrm{MHz}, \mathrm{CDCl}_{3}\right) \delta 7.39-7.33(\mathrm{~m}, 2 \mathrm{H}), 7.27-7.23(\mathrm{~m}, 3 \mathrm{H}), 5.69$ (ddt, $J=16.9$, $10.1,7.5 \mathrm{~Hz}, 1 \mathrm{H}), 5.21(\mathrm{ddt}, J=16.9,2.0,1.3 \mathrm{~Hz}, 1 \mathrm{H}), 5.14(\mathrm{ddt}, J=10.1,2.0,0.9 \mathrm{~Hz}, 1 \mathrm{H}), 4.21$ (qd, $J=7.5,2.0 \mathrm{~Hz}, 4 \mathrm{H}), 3.01(\mathrm{~s}, 2 \mathrm{H}), 2.87(\mathrm{dt}, J=7.5,1.3 \mathrm{~Hz}, 2 \mathrm{H}), 1.25(\mathrm{t}, J=7.5 \mathrm{~Hz}, 6 \mathrm{H})$. 
${ }^{13}$ C NMR $\left(126 \mathrm{MHz}, \mathrm{CDCl}_{3}\right) \delta 169.8,131.9,131.6,128.2,127.9,123.2,119.7,84.4,83.5,61.6$, 57.0, 36.6, 23.5, 14.1 .

N-allyl-N-(3-phenylprop-2-yn-1-yl)thiophene-2-sulfonamide (2z)<smiles>C=CCN(CC#Cc1ccccc1)S(=O)(=O)c1cccs1</smiles>

Synthesized following GP2 on a $10 \mathrm{mmol}$ scale. Obtained $2.80 \mathrm{~g}$ of a yellow solid (88\% yield).

${ }^{1} \mathbf{H}$ NMR $\left(500 \mathrm{MHz}, \mathrm{CDCl}_{3}\right) \delta 7.67(\mathrm{dd}, J=3.8,1.3 \mathrm{~Hz}, 1 \mathrm{H}), 7.56(\mathrm{dd}, J=5.0,1.3 \mathrm{~Hz}, 1 \mathrm{H}), 7.32$ $-7.23(\mathrm{~m}, 3 \mathrm{H}), 7.20-7.16(\mathrm{~m}, 2 \mathrm{H}), 7.07$ (dd, $J=5.0,3.8 \mathrm{~Hz}, 1 \mathrm{H}), 5.82$ (ddt, $J=17.1,10.0,6.4$ $\mathrm{Hz}, 1 \mathrm{H}), 5.37(\mathrm{dq}, J=17.1,1.4 \mathrm{~Hz}, 1 \mathrm{H}), 5.30(\mathrm{dq}, J=10.1,1.2 \mathrm{~Hz}, 1 \mathrm{H}), 4.34(\mathrm{~s}, 2 \mathrm{H}), 3.96(\mathrm{dt}, J$ $=6.4,1.3 \mathrm{~Hz}, 2 \mathrm{H})$.

${ }^{13}$ C NMR $\left(126 \mathrm{MHz}, \mathrm{CDCl}_{3}\right) \delta 139.4,132.5,132.1,131.7,131.6,128.5,128.2,127.5,122.1$, 120.1, 120.1, 85.9, 81.1, 49.4, 37.0.

IR (ATR) 3083, 2913, 1488, 1444, 1349, 1324, 1151, 1090, 1080, 949, 897, 760, 721, 649

HRMS (DART, $\mathrm{M}+\mathrm{H}$ ) Calculated for $\mathrm{C}_{16} \mathrm{H}_{16} \mathrm{~N}_{1} \mathrm{O}_{2} \mathrm{~S}_{2} 318.0617$, found 318.0612

MP $35-37^{\circ} \mathrm{C}$

\section{N,4-dimethyl-N-(3-phenylprop-2-yn-1-yl)benzenesulfonamide (2ab)}<smiles>CN(C)CC#Cc1ccccc1</smiles>

Synthesized following and consistent with literature M.-B. Li, X.-L. Tang, S.-K. Tian, Adv. Synth. Catal. 2011, 353, 1980-1984.

${ }^{1} \mathbf{H}$ NMR $\left(500 \mathrm{MHz}, \mathrm{CDCl}_{3}\right) \delta 7.75(\mathrm{~d}, J=8.3 \mathrm{~Hz}, 2 \mathrm{H}), 7.30-7.21(\mathrm{~m}, 5 \mathrm{H}), 7.12-7.07(\mathrm{~m}$, $2 \mathrm{H}), 4.25$ (s, 2H), 2.89 (s, 3H), 2.34 (s, 3H).

${ }^{13} \mathbf{C}$ NMR $\left(126 \mathrm{MHz}, \mathrm{CDCl}_{3}\right) \delta 143.6,134.2,131.5,129.5,128.1,128.0,122.1,85.9,81.4,40.7$, 34.6, 21.4.

\section{N-allyl-N,4-dimethylbenzenesulfonamide (2ac)}<smiles>C=CN(C)[13NH]</smiles>

Synthesized following and consistent with literature N. Sarkar, A. Banerjee, S. G. Nelson, J. Am. Chem. Soc. 2008, 130, 9222-9223. 
${ }^{1} \mathbf{H}$ NMR $\left(500 \mathrm{MHz}, \mathrm{CDCl}_{3}\right) \delta 7.66(\mathrm{~d}, J=8.3 \mathrm{~Hz}, 1 \mathrm{H}), 7.34-7.28(\mathrm{~m}, 1 \mathrm{H}), 5.75-5.63(\mathrm{~m}$, $1 \mathrm{H}), 5.18(\mathrm{dq}, J=7.0,1.4 \mathrm{~Hz}, 1 \mathrm{H}), 5.16(\mathrm{t}, J=1.4 \mathrm{~Hz}, 1 \mathrm{H}), 3.61(\mathrm{dt}, J=6.4,1.4 \mathrm{~Hz}, 1 \mathrm{H}), 2.65$ (s, 2H), 2.42 (s, 2H).

${ }^{13}$ C NMR $\left(126 \mathrm{MHz}, \mathrm{CDCl}_{3}\right) \delta 143.4,134.4,132.6,129.7,127.4,119.1,53.0,34.2,21.5$.

1-(4-phenylbut-3-yn-1-yl)pyridin-2(1H)-one (2ad)<smiles>O=c1ccccn1CCC#Cc1ccccc1</smiles>

Synthesized following Donets, P. A., and Cramer, N. Angew. Chem. Int. Ed. 2015, 54, 633 -637 on a $10 \mathrm{mmol}$ scale. Obtained $1.43 \mathrm{~g}$ of a white solid (64\% yield) after flash chromatography $5 \%$ -> 100\% EtOAc/pentane.

${ }^{1} \mathbf{H}$ NMR $\left(500 \mathrm{MHz}, \mathrm{CDCl}_{3}\right) \delta 7.39(\mathrm{ddd}, J=6.8,2.1,0.7 \mathrm{~Hz}, 1 \mathrm{H}), 7.36-7.30(\mathrm{~m}, 3 \mathrm{H}), 7.28-$ $7.24(\mathrm{~m}, 3 \mathrm{H}), 6.56(\mathrm{ddd}, J=9.1,1.4,0.7 \mathrm{~Hz}, 1 \mathrm{H}), 6.14(\mathrm{td}, J=6.7,1.4 \mathrm{~Hz}, 1 \mathrm{H}), 4.11(\mathrm{t}, J=6.4$ $\mathrm{Hz}, 2 \mathrm{H}), 2.89$ (t, $J=6.4 \mathrm{~Hz}, 2 \mathrm{H})$.

${ }^{13}$ C NMR $\left(126 \mathrm{MHz}, \mathrm{CDCl}_{3}\right) \delta 162.4,139.8,138.3,131.5,128.3,128.0,123.2,120.9,105.5$, 86.0, 83.1, 49.1, 19.6 .

IR (ATR) 2028, 2001, 1653, 1578, 1535, 1490, 1442, 1167, 1142, 8324, 755, 732, 693

HRMS (DART, M+H) Calculated for $\mathrm{C}_{15} \mathrm{H}_{14} \mathrm{NO} 224.10698$, found 224.10699

MP $67-68^{\circ} \mathrm{C}$ 
Product Characterization

(R,Z)-2-((4-benzylidene-1-tosylpyrrolidin-3-yl)methyl)-1-(pyridin-2-yl)-1H-indole (a)<smiles>[N][C]1CC(=Cc2ccccc2)C(Cc2cc3ccccc3n2-c2ccccn2)C1</smiles>

Synthesized following GP3. Isolated by flash column chromatography 15 -> 30\%

EtOAc/pentane. Obtained $105 \mathrm{mg}$ of a white solid (99\% yield).

${ }^{1} \mathbf{H}$ NMR $\left(500 \mathrm{MHz}, \mathrm{CDCl}_{3}\right) \delta 8.67(\mathrm{ddd}, J=4.9,2.0,0.9 \mathrm{~Hz}, 1 \mathrm{H}), 7.91(\mathrm{ddd}, J=8.1,7.5,2.0$ $\mathrm{Hz}, 1 \mathrm{H}), 7.69(\mathrm{~d}, J=8.3 \mathrm{~Hz}, 2 \mathrm{H}), 7.64-7.58(\mathrm{~m}, 1 \mathrm{H}), 7.48(\mathrm{dt}, J=8.1,0.9 \mathrm{~Hz}, 1 \mathrm{H}), 7.38-7.30$ (m, 4H), $7.30(\mathrm{~d}, J=8.3 \mathrm{~Hz}, 2 \mathrm{H}), 7.27-7.20(\mathrm{~m}, 1 \mathrm{H}), 7.20-7.14(\mathrm{~m}, 2 \mathrm{H}), 7.07(\mathrm{~d}, J=7.2 \mathrm{~Hz}$, $2 \mathrm{H}), 6.48(\mathrm{~d}, J=0.8 \mathrm{~Hz}, 1 \mathrm{H}), 6.13(\mathrm{q}, J=2.3 \mathrm{~Hz}, 1 \mathrm{H}), 4.17(\mathrm{dt}, J=14.8,2.3 \mathrm{~Hz}, 1 \mathrm{H}), 4.01$ (ddd, $J=14.8,2.3,0.8 \mathrm{~Hz}, 1 \mathrm{H}), 3.30$ (ddd, $J=14.8,4.8,0.8 \mathrm{~Hz}, 1 \mathrm{H}), 3.19(\mathrm{~m}, 2 \mathrm{H}), 3.11-3.01(\mathrm{~m}$, $1 \mathrm{H}$ ), 2.95 (ddd, $J=14.8,9.8,0.8 \mathrm{~Hz}, 1 \mathrm{H}), 2.41$ (s, 3H).

${ }^{13}$ C NMR $\left(126 \mathrm{MHz}, \mathrm{CDCl}_{3}\right) \delta 151.3,149.6,143.7,139.8,138.5,138.3,137.2,136.4,132.6$, 129.7, 128.6, 128.4, 128.1, 127.8, 127.1, 123.5, 122.2, 122.1, 121.0, 120.9, 120.3, 110.1, 104.1, $51.7,50.7,44.6,31.9,21.5$.

IR (ATR) 1581, 1470, 1454, 1436, 1347, 1167, 1151, 1095, 1036, 816, 781

HRMS (DART, M+H) Calculated for $\mathrm{C}_{32} \mathrm{H}_{30} \mathrm{~N}_{3} \mathrm{O}_{2} \mathrm{~S} 520.2045$, found 520.2048

MP $129-131{ }^{\circ} \mathrm{C}$

$\boldsymbol{\alpha}_{\boldsymbol{D}}^{\mathbf{2 0}}\left(\mathrm{c}=0.33, \mathrm{CH}_{2} \mathrm{Cl}_{2}\right) 13.30$

HPLC: IB, $50 \%$ IPA/Hex, $1.0 \mathrm{~mL} / \mathrm{min}$

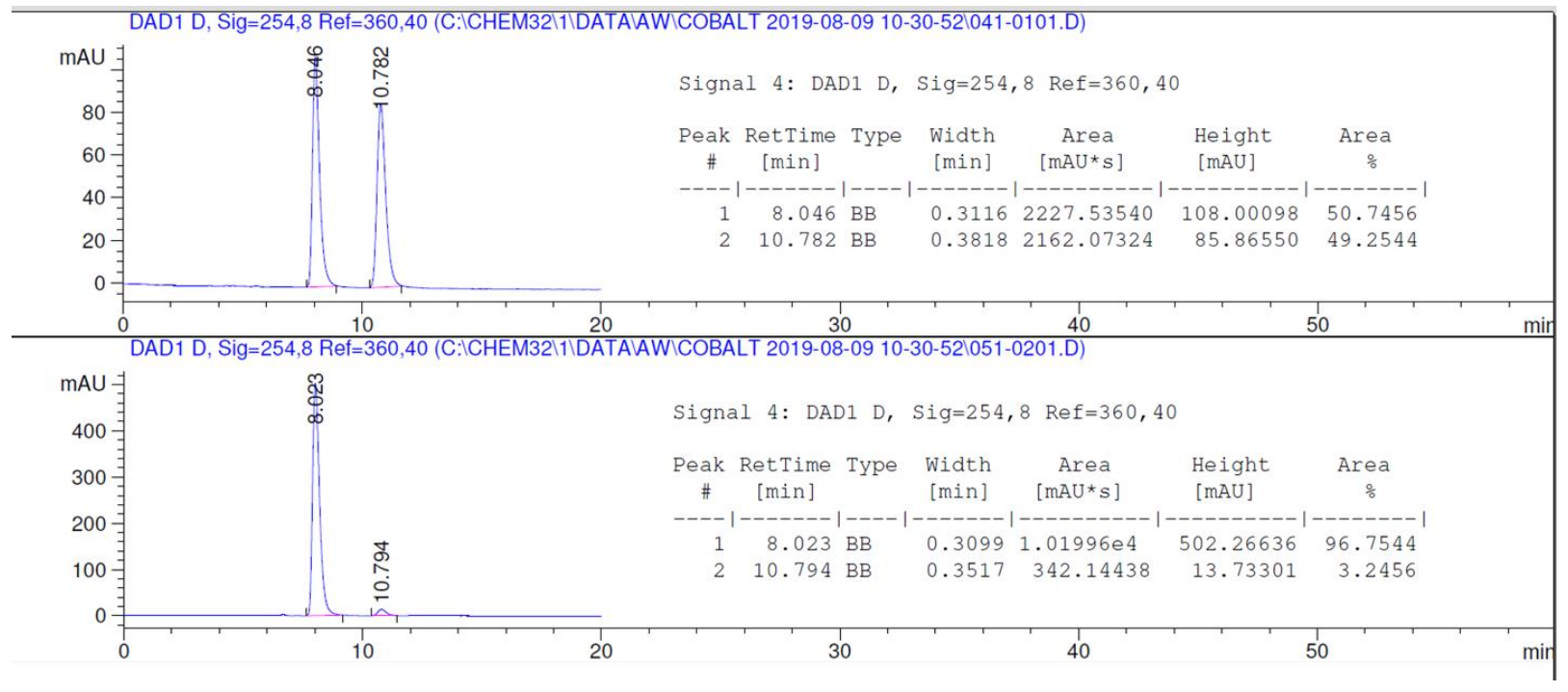


<smiles></smiles>

Synthesized following GP3. Isolated by flash column chromatography 10 -> 30\% EtOAc/pentane. Obtained $96 \mathrm{mg}$ of a white solid (92\% yield).

${ }^{1} \mathbf{H}$ NMR $\left(500 \mathrm{MHz}, \mathrm{CDCl}_{3}\right) \delta 8.79(\mathrm{~d}, J=4.7 \mathrm{~Hz}, 2 \mathrm{H}), 8.37(\mathrm{dq}, J=8.3,0.9 \mathrm{~Hz}, 1 \mathrm{H}), 7.71(\mathrm{~d}, J$ $=8.3 \mathrm{~Hz}, 2 \mathrm{H}), 7.56(\mathrm{ddd}, J=7.6,1.5,0.7 \mathrm{~Hz}, 1 \mathrm{H}), 7.36(\mathrm{t}, J=7.6 \mathrm{~Hz}, 2 \mathrm{H}), 7.29(\mathrm{~d}, J=8.3 \mathrm{~Hz}$, 2H), $7.28-7.19(\mathrm{~m}, 3 \mathrm{H}), 7.16(\mathrm{t}, J=4.8 \mathrm{~Hz}, 1 \mathrm{H}), 7.12(\mathrm{~d}, J=7.6 \mathrm{~Hz}, 2 \mathrm{H}), 6.48(\mathrm{~d}, J=0.9 \mathrm{~Hz}$, $1 \mathrm{H}), 6.31(\mathrm{q}, J=2.2 \mathrm{~Hz}, 1 \mathrm{H}), 4.27(\mathrm{ddd}, J=14.8,2.2,1.4 \mathrm{~Hz}, 1 \mathrm{H}), 4.07(\mathrm{dd}, J=14.8,2.2 \mathrm{~Hz}$, $1 \mathrm{H}), 3.68(\mathrm{dd}, J=13.8,3.1 \mathrm{~Hz}, 1 \mathrm{H}), 3.30-3.12(\mathrm{~m}, 4 \mathrm{H}), 2.40(\mathrm{~s}, 3 \mathrm{H})$.

${ }^{13} \mathbf{C}$ NMR $\left(126 \mathrm{MHz}, \mathrm{CDCl}_{3}\right) \delta 158.1,143.7,140.2,138.6,137.1,136.5,132.7,129.7,129.1$, 128.6, 128.1, 127.8, 127.1, 123.4, 123.0, 122.1, 120.0, 117.1, 114.5, 108.0, 51.6, 50.9, 44.6, 34.0, 21.5 .

IR (ATR) 1562, 1458, 1426, 1343, 1163, 1104, 906, 816, 726, 663

HRMS (DART, M+H) Calculated for $\mathrm{C}_{31} \mathrm{H}_{29} \mathrm{~N}_{4} \mathrm{O}_{2} \mathrm{~S} 521.2006$, found 521.2007

MP $84{ }^{\circ} \mathrm{C}$

$\boldsymbol{\alpha}_{\boldsymbol{D}}^{\mathbf{2 0}}\left(\mathrm{c}=0.19, \mathrm{CH}_{2} \mathrm{Cl}_{2}\right)-8.42$

HPLC: IB, 50\% IPA/Hex, $1.0 \mathrm{~mL} / \mathrm{min}$
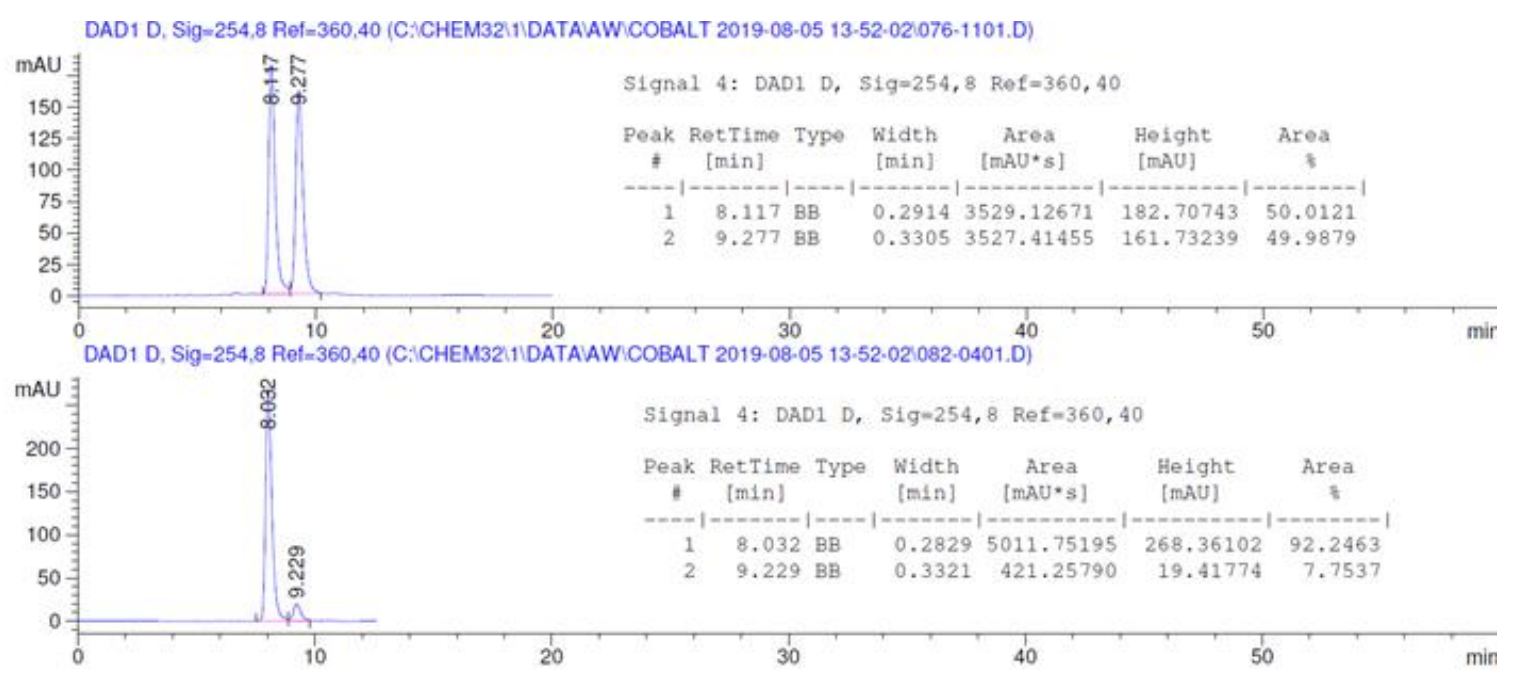
<smiles>Cn1c(C[C@@H]2C[C@@H]([AsH3])C/C2=C\c2ccccc2)cc2ccccc21</smiles>

Synthesized following GP3. Isolated by flash column chromatography 5 -> 30\% EtOAc/pentane. Obtained $83 \mathrm{mg}$ of a white solid (86\% yield).

${ }^{1} \mathbf{H}$ NMR $\left(500 \mathrm{MHz}, \mathrm{CDCl}_{3}\right) \delta 7.72(\mathrm{~d}, J=8.3 \mathrm{~Hz}, 2 \mathrm{H}), 7.68(\mathrm{dq}, J=8.4,0.9 \mathrm{~Hz}, 1 \mathrm{H}), 7.55-$ $7.52(\mathrm{~m}, 1 \mathrm{H}), 7.35(\mathrm{t}, J=7.6 \mathrm{~Hz}, 2 \mathrm{H}), 7.31(\mathrm{~d}, J=8.3 \mathrm{~Hz}, 2 \mathrm{H}), 7.29-7.25(\mathrm{~m}, 2 \mathrm{H}), 7.25-7.20$ $(\mathrm{m}, 1 \mathrm{H}), 7.15(\mathrm{~d}, J=7.1 \mathrm{~Hz}, 2 \mathrm{H}), 6.43(\mathrm{~d}, J=0.8 \mathrm{~Hz}, 1 \mathrm{H}), 6.42(\mathrm{q}, J=2.2 \mathrm{~Hz}, 1 \mathrm{H}), 4.34(\mathrm{ddd}, J$ $=14.8,2.2,1.6 \mathrm{~Hz}, 1 \mathrm{H}), 4.04(\mathrm{dd}, J=14.8,2.2 \mathrm{~Hz}, 1 \mathrm{H}), 3.46(\mathrm{ddd}, J=14.6,4.4,0.8 \mathrm{~Hz}, 1 \mathrm{H})$, $3.31-3.23(\mathrm{~m}, 2 \mathrm{H}), 3.16-3.11(\mathrm{~m}, 1 \mathrm{H}), 3.02(\mathrm{dd}, J=14.6,9.7 \mathrm{~Hz}, 1 \mathrm{H}), 2.81(\mathrm{~s}, 3 \mathrm{H}), 2.42(\mathrm{~s}$, $3 \mathrm{H})$.

${ }^{13} \mathrm{C}$ NMR $\left(126 \mathrm{MHz}, \mathrm{CDCl}_{3}\right) \delta 170.3,143.7,140.1,139.8,136.5,136.0,132.7,129.8,129.7$, $128.6,128.1,127.8,127.1,123.8,123.8,123.2,120.9$, 114.3, 110.7, 51.3, 50.8, 44.2, 35.1, 27.8, 21.5 .

IR (ATR) 1704, 1465, 1364, 1309, 1308, 1152, 1041, 1093, 910, 816, 736, 667

HRMS (DART, M+H) Calculated for $\mathrm{C}_{29} \mathrm{H}_{29} \mathrm{~N}_{2} \mathrm{O}_{3} \mathrm{~S} 485.1893$, found 485.1894

MP $149{ }^{\circ} \mathrm{C}$

$\boldsymbol{\alpha}_{D}^{\mathbf{2 0}}\left(\mathrm{c}=0.24, \mathrm{CH}_{2} \mathrm{Cl}_{2}\right) 17.50$

HPLC: IB, 50\% IPA/Hex, $1.0 \mathrm{~mL} / \mathrm{min}$

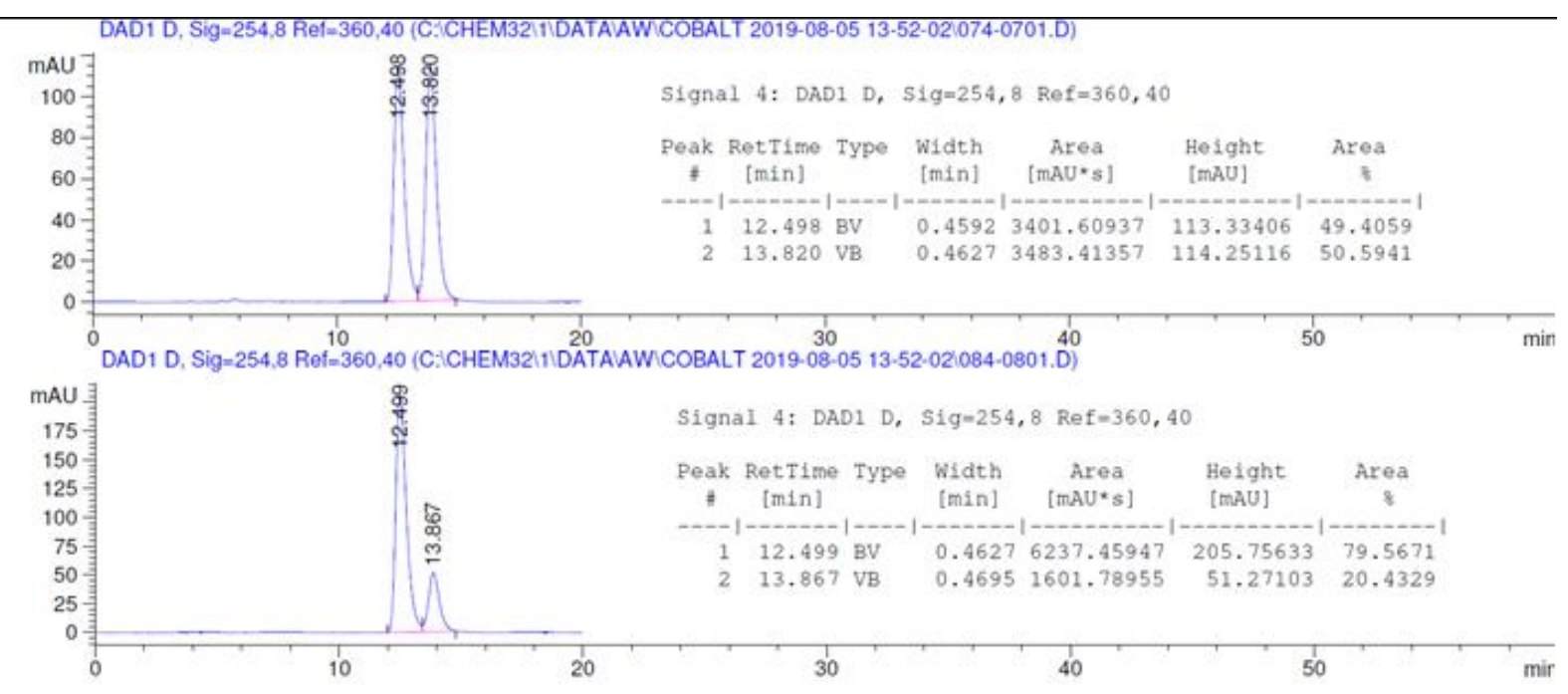




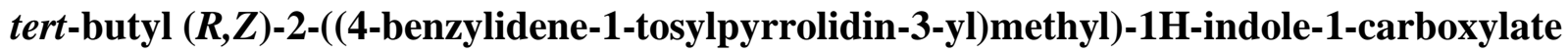
(3d)<smiles>C[As]C1CN(C(=O)c2ccccc2)C(=Cc2ccccc2)C1Cc1ccccc1</smiles>

Synthesized following GP3. Isolated by flash column chromatography 5 -> 30\% EtOAc/pentane. Obtained $86 \mathrm{mg}$ of a white solid (79\% yield).

${ }^{1} \mathbf{H}$ NMR $\left(500 \mathrm{MHz}, \mathrm{CDCl}_{3}\right) \delta 8.04(\mathrm{dt}, J=8.3,1.0 \mathrm{~Hz}, 1 \mathrm{H}), 7.71(\mathrm{~d}, J=8.3 \mathrm{~Hz}, 2 \mathrm{H}), 7.48(\mathrm{ddd}$, $J=7.5,1.5,0.7 \mathrm{~Hz}, 1 \mathrm{H}), 7.35(\mathrm{t}, J=7.2 \mathrm{~Hz}, 2 \mathrm{H}), 7.31(\mathrm{~d}, J=8.3 \mathrm{~Hz}, 2 \mathrm{H}), 7.29-7.19(\mathrm{~m}, 3 \mathrm{H})$, $7.13(\mathrm{~d}, J=7.2 \mathrm{~Hz}, 2 \mathrm{H}), 6.36(\mathrm{~d}, J=0.8 \mathrm{~Hz}, 1 \mathrm{H}), 6.34(\mathrm{q}, J=2.4 \mathrm{~Hz}, 1 \mathrm{H}), 4.28(\mathrm{dt}, J=14.7,2.4$ $\mathrm{Hz}, 1 \mathrm{H}), 4.11(\mathrm{dd}, J=14.7,2.4 \mathrm{~Hz}, 1 \mathrm{H}), 3.48$ (dd, $J=14.8,4.5 \mathrm{~Hz}, 1 \mathrm{H}), 3.31-3.26(\mathrm{~m}, 1 \mathrm{H})$, $3.26-3.21(\mathrm{~m}, 1 \mathrm{H}), 3.20-3.15(\mathrm{~m}, 1 \mathrm{H}), 3.03(\mathrm{ddd}, J=14.8,9.2,0.8 \mathrm{~Hz}, 1 \mathrm{H}), 2.42(\mathrm{~s}, 3 \mathrm{H}), 1.70$ $(\mathrm{s}, 9 \mathrm{H})$.

${ }^{13}$ C NMR $\left(126 \mathrm{MHz}, \mathrm{CDCl}_{3}\right) \delta 150.5,143.7,139.8,139.0,136.5,136.4,132.7,129.7,129.0$, 128.5, 128.1, 127.8, 127.1, 123.7, 123.7, 122.8, 120.1, 115.7, 109.3, 84.1, 51.7, 50.9, 44.0, 34.2, $28.3,21.5$.

IR (ATR) 1732, 1558, 1454, 1426, 1357, 918, 903, 877, 802, 726

HRMS (DART, M+H) Calculated for $\mathrm{C}_{32} \mathrm{H}_{35} \mathrm{~N}_{2} \mathrm{O}_{4} \mathrm{~S} 543.2312$, found 543.2324

MP $70-85{ }^{\circ} \mathrm{C}$

$\boldsymbol{\alpha}_{D}^{\mathbf{2 0}}\left(\mathrm{c}=0.25, \mathrm{CH}_{2} \mathrm{Cl}_{2}\right) 24.00$

HPLC: IB, 15\% IPA/Hex, $0.75 \mathrm{~mL} / \mathrm{min}$

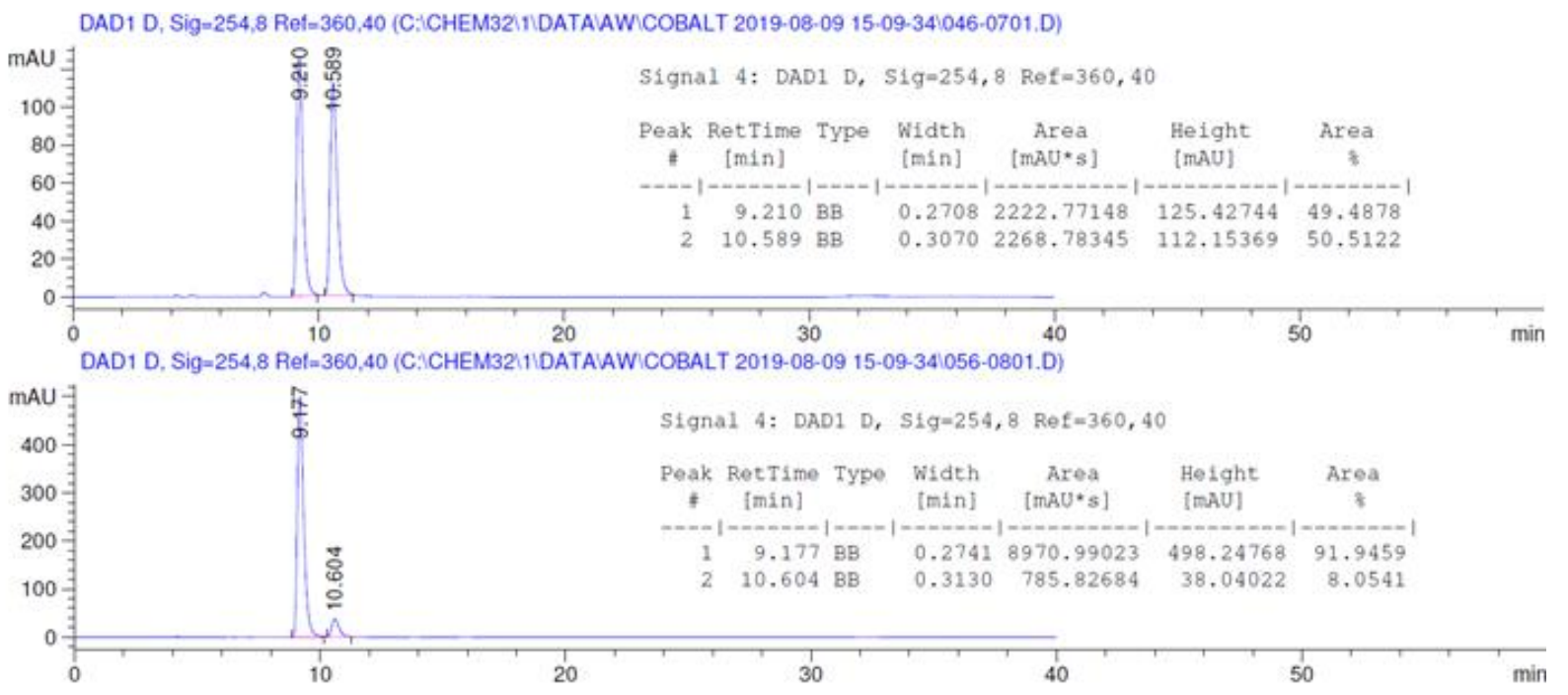




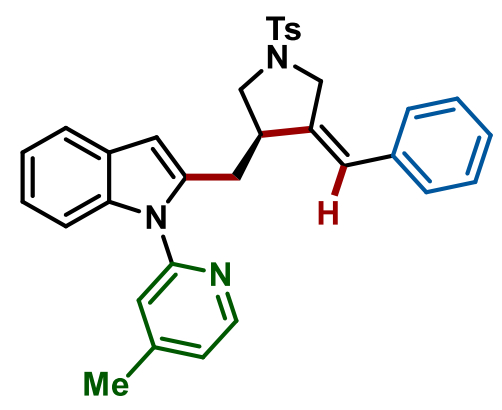

Synthesized following GP3. Isolated by flash column chromatography 10 -> 30\% EtOAc/pentane. Obtained $91 \mathrm{mg}$ of a white solid (85\% yield).

${ }^{1} \mathbf{H}$ NMR $\left(500 \mathrm{MHz}, \mathrm{CDCl}_{3}\right) \delta 8.51(\mathrm{dd}, J=5.0,0.7 \mathrm{~Hz}, 1 \mathrm{H}), 7.69(\mathrm{~d}, J=8.3 \mathrm{~Hz}, 2 \mathrm{H}), 7.62-$ $7.56(\mathrm{~m}, 1 \mathrm{H}), 7.37-7.26(\mathrm{~m}, 6 \mathrm{H}), 7.27-7.19(\mathrm{~m}, 1 \mathrm{H}), 7.20-7.12(\mathrm{~m}, 3 \mathrm{H}), 7.08-7.02(\mathrm{~m}$, $2 \mathrm{H}), 6.45(\mathrm{~d}, J=0.9 \mathrm{~Hz}, 1 \mathrm{H}), 6.11(\mathrm{q}, J=2.3 \mathrm{~Hz}, 1 \mathrm{H}), 4.14(\mathrm{dt}, J=14.8,2.3 \mathrm{~Hz}, 1 \mathrm{H}), 3.99$ (dd, $J=14.8,2.3 \mathrm{~Hz}, 1 \mathrm{H}), 3.30(\mathrm{ddd}, J=14.8,5.0,0.9 \mathrm{~Hz}, 1 \mathrm{H}), 3.23-3.12(\mathrm{~m}, 2 \mathrm{H}), 3.10-3.00$ (m, $1 \mathrm{H}), 2.94$ (ddd, $J=14.8,9.8,0.9 \mathrm{~Hz}, 1 \mathrm{H}), 2.46$ (s, 3H), 2.41 (s, 3H).

${ }^{13} \mathrm{C}$ NMR $\left(126 \mathrm{MHz}, \mathrm{CDCl}_{3}\right) \delta 151.3,150.1,149.2,143.7,139.8,138.3,137.2,136.4,132.6$, 129.7, 128.6, 128.4, 128.0, 127.8, 127.1, 123.5, 123.4, 122.0, 121.7, 120.8, 120.2, 110.2, 103.9, 51.7, 50.7, 44.7, 31.9, 21.5, 21.1 .

IR (ATR) 1607, 1458, 1343, 1163, 1047, 1041, 910, 823, 740, 667

HRMS (DART, M+H) Calculated for $\mathrm{C}_{33} \mathrm{H}_{32} \mathrm{~N}_{3} \mathrm{O}_{2} \mathrm{~S} 534.2210$, found 534.2205

MP $75-85{ }^{\circ} \mathrm{C}$

$\boldsymbol{\alpha}_{D}^{\mathbf{2 0}}\left(\mathrm{c}=0.23, \mathrm{CH}_{2} \mathrm{Cl}_{2}\right) 13.60$

HPLC: IA, 30\% IPA/Hex, $0.5 \mathrm{~mL} / \mathrm{min}$
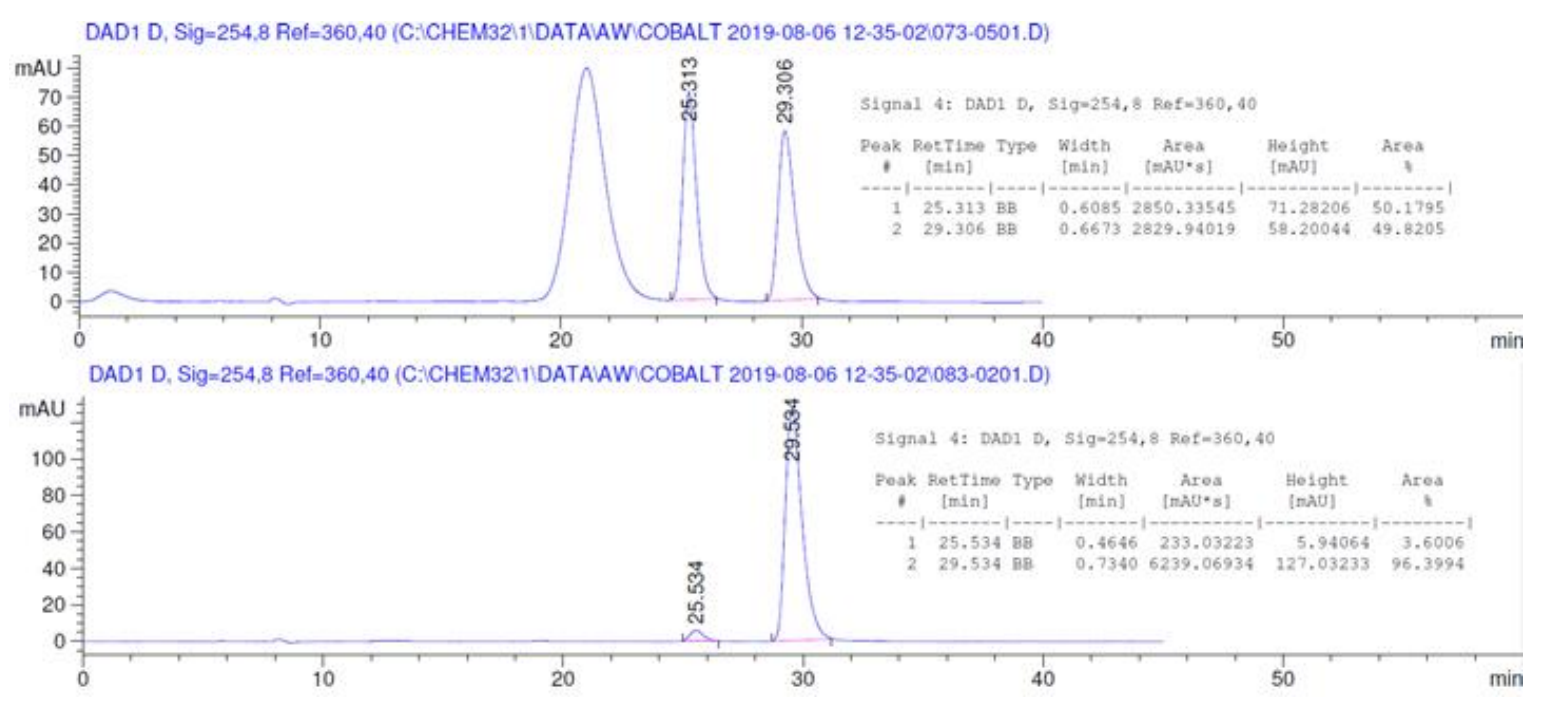


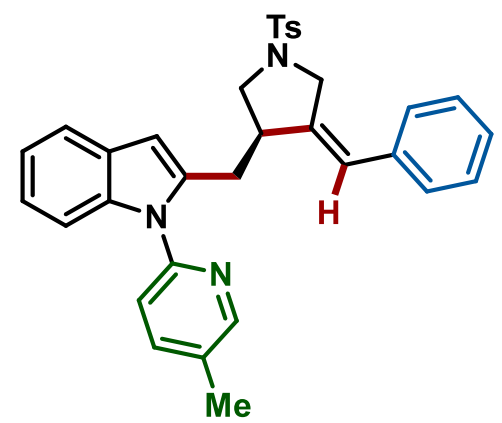

Synthesized following GP3. Isolated by flash column chromatography 10 -> 30\% EtOAc/pentane. Obtained $89 \mathrm{mg}$ of a white solid ( $83 \%$ yield).

${ }^{1} \mathbf{H}$ NMR $\left(500 \mathrm{MHz}, \mathrm{CDCl}_{3}\right) \delta 8.48(\mathrm{dt}, J=2.5,0.8 \mathrm{~Hz}, 1 \mathrm{H}), 7.74-7.66(\mathrm{~m}, 3 \mathrm{H}), 7.64-7.56$ $(\mathrm{m}, 1 \mathrm{H}), 7.39-7.28(\mathrm{~m}, 6 \mathrm{H}), 7.26-7.20(\mathrm{~m}, 1 \mathrm{H}), 7.20-7.12(\mathrm{~m}, 2 \mathrm{H}), 7.08(\mathrm{~d}, J=7.3 \mathrm{~Hz}, 2 \mathrm{H})$, $6.46(\mathrm{~d}, J=0.8 \mathrm{~Hz}, 1 \mathrm{H}), 6.13(\mathrm{q}, J=2.3 \mathrm{~Hz}, 1 \mathrm{H}), 4.17(\mathrm{dt}, J=14.8,2.3 \mathrm{~Hz}, 1 \mathrm{H}), 4.03(\mathrm{dd}, J=$ 14.8, $2.3 \mathrm{~Hz}, 1 \mathrm{H}), 3.26$ (dd, $J=14.9,4.8 \mathrm{~Hz}, 1 \mathrm{H}), 3.25-3.13(\mathrm{~m}, 2 \mathrm{H}), 3.11-3.02(\mathrm{~m}, 1 \mathrm{H}), 2.93$ (dd, $J=14.9,9.8 \mathrm{~Hz}, 1 \mathrm{H}), 2.45(\mathrm{~s}, 3 \mathrm{H}), 2.42$ (s, 3H).

${ }^{13}$ C NMR $\left(126 \mathrm{MHz}, \mathrm{CDCl}_{3}\right) \delta 149.9,148.9,143.7,139.8,139.1,138.3,137.4,136.4,132.6$, 132.1, 129.7, 128.6, 128.3, 128.1, 127.8, 127.1, 123.5, 122.0, 120.7, 120.6, 120.2, 110.1, 103.6, $51.7,50.8,44.5,31.8,21.5,18.1$.

IR (ATR) 1600, 1496, 1461, 1406, 1343, 1170, 1041, 906, 729, 663

HRMS (DART, M+H) Calculated for $\mathrm{C}_{33} \mathrm{H}_{32} \mathrm{~N}_{3} \mathrm{O}_{2} \mathrm{~S}_{1} 534.2210$, found 534.2210

MP $161-165^{\circ} \mathrm{C}$

$\boldsymbol{\alpha}_{D}^{\mathbf{2 0}}\left(\mathrm{c}=0.17, \mathrm{CH}_{2} \mathrm{Cl}_{2}\right) 9.41$

HPLC: IB, 50\% IPA/Hex, $1.0 \mathrm{~mL} / \mathrm{min}$

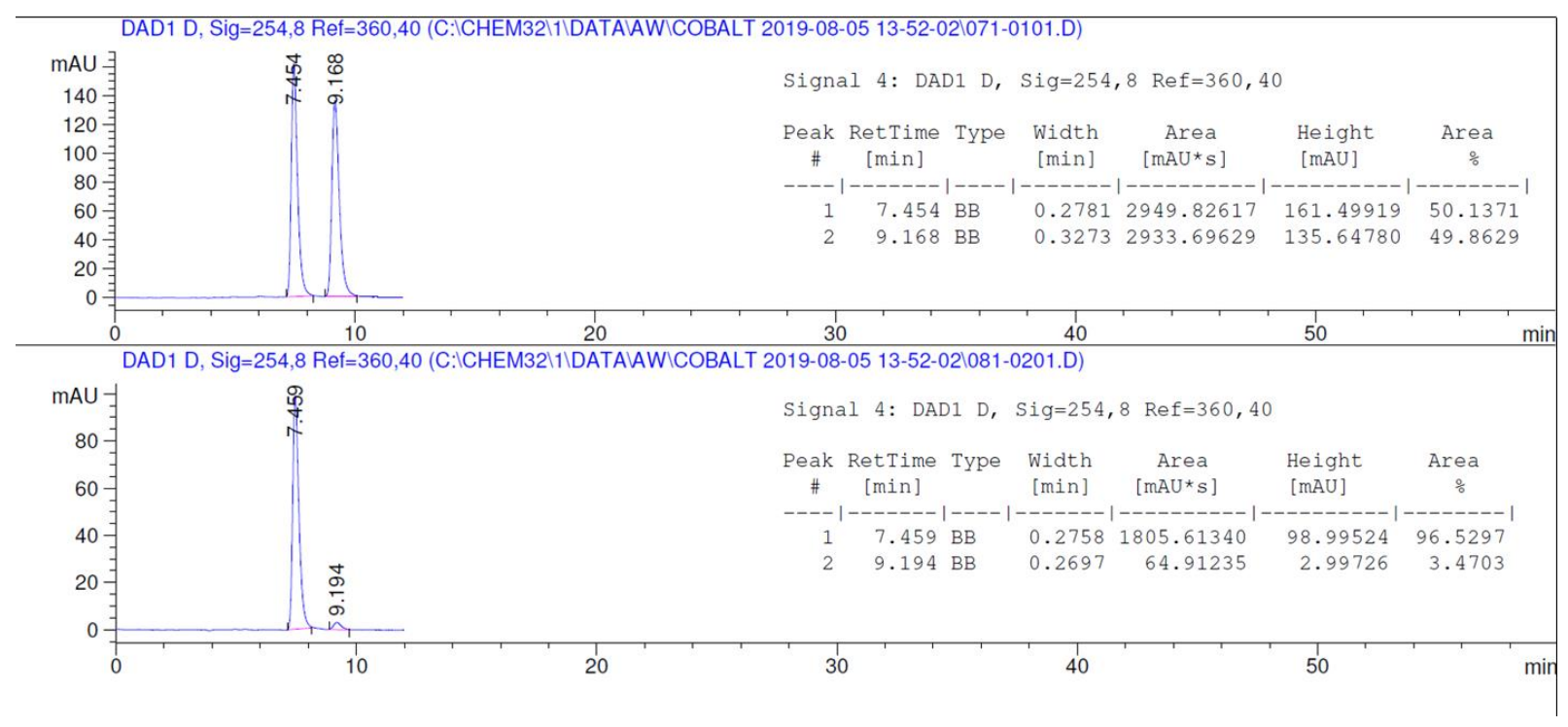




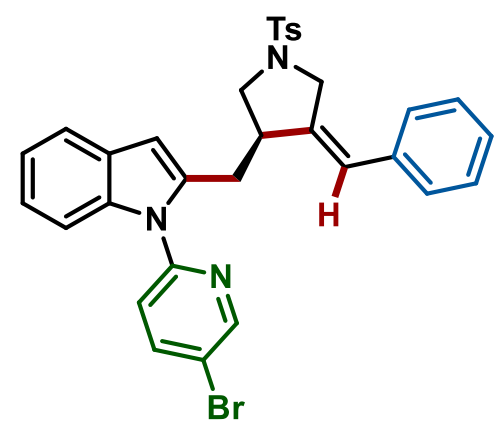

Synthesized following GP3. Isolated by flash column chromatography 10 -> 30\% EtOAc/pentane. Obtained $114 \mathrm{mg}$ of a white solid (95\% yield).

${ }^{1} \mathbf{H}$ NMR $\left(500 \mathrm{MHz}, \mathrm{CDCl}_{3}\right) \delta 8.69(\mathrm{dd}, J=2.5,0.7 \mathrm{~Hz}, 1 \mathrm{H}), 8.01(\mathrm{dd}, J=8.4,2.5 \mathrm{~Hz}, 1 \mathrm{H}), 7.68$ $(\mathrm{d}, J=8.2 \mathrm{~Hz}, 2 \mathrm{H}), 7.63-7.55(\mathrm{~m}, 1 \mathrm{H}), 7.40(\mathrm{dd}, J=8.4,0.7 \mathrm{~Hz}, 1 \mathrm{H}), 7.38-7.30(\mathrm{~m}, 3 \mathrm{H}), 7.30$ $(\mathrm{d}, J=8.2 \mathrm{~Hz}, 2 \mathrm{H}), 7.28-7.21(\mathrm{~m}, 1 \mathrm{H}), 7.22-7.14(\mathrm{~m}, 2 \mathrm{H}), 7.08(\mathrm{~d}, J=7.2 \mathrm{~Hz}, 2 \mathrm{H}), 6.48(\mathrm{~d}, J$ $=0.8 \mathrm{~Hz}, 1 \mathrm{H}), 6.15(\mathrm{q}, J=2.3 \mathrm{~Hz}, 1 \mathrm{H}), 4.17(\mathrm{dt}, J=14.8,2.3 \mathrm{~Hz}, 1 \mathrm{H}), 4.02(\mathrm{dd}, J=14.8,2.3$ $\mathrm{Hz}, 1 \mathrm{H}), 3.25$ (ddd, $J=15.0,5.0,0.8 \mathrm{~Hz}, 1 \mathrm{H}), 3.23-3.14(\mathrm{~m}, 2 \mathrm{H}), 3.14-3.04(\mathrm{~m}, 1 \mathrm{H}), 2.95$ (ddd, $J=15.0,9.7,0.8 \mathrm{~Hz}, 1 \mathrm{H}), 2.42(\mathrm{~s}, 3 \mathrm{H})$.

${ }^{13}$ C NMR $\left(126 \mathrm{MHz}, \mathrm{CDCl}_{3}\right) \delta 150.6,149.9,143.7,141.1,139.7,138.2,137.0,136.3,132.6$, 129.7, 128.6, 128.5, 128.1, 127.8, 127.2, 123.6, 122.4, 122.0, 121.3, 120.4, 118.5, 110.0, 104.6, 51.7, 50.7, 44.6, 31.8, 21.6, 21.5.

IR (ATR) 1556, 1455, 1396, 1307, 1209, 1167, 1113, 1056, 1025, 1017, 1005, 854, 796

HRMS (DART, M+H) Calculated for $\mathrm{C}_{33} \mathrm{H}_{32} \mathrm{~N}_{3} \mathrm{O}_{2} \mathrm{~S} 534.2210$, found 534.2209

MP $174{ }^{\circ} \mathrm{C}$

$\boldsymbol{\alpha}_{\boldsymbol{D}}^{\mathbf{2 0}}\left(\mathrm{c}=0.22, \mathrm{CH}_{2} \mathrm{Cl}_{2}\right) 6.36$

HPLC: IB, 50\% IPA/Hex, $1 \mathrm{~mL} / \mathrm{min}$

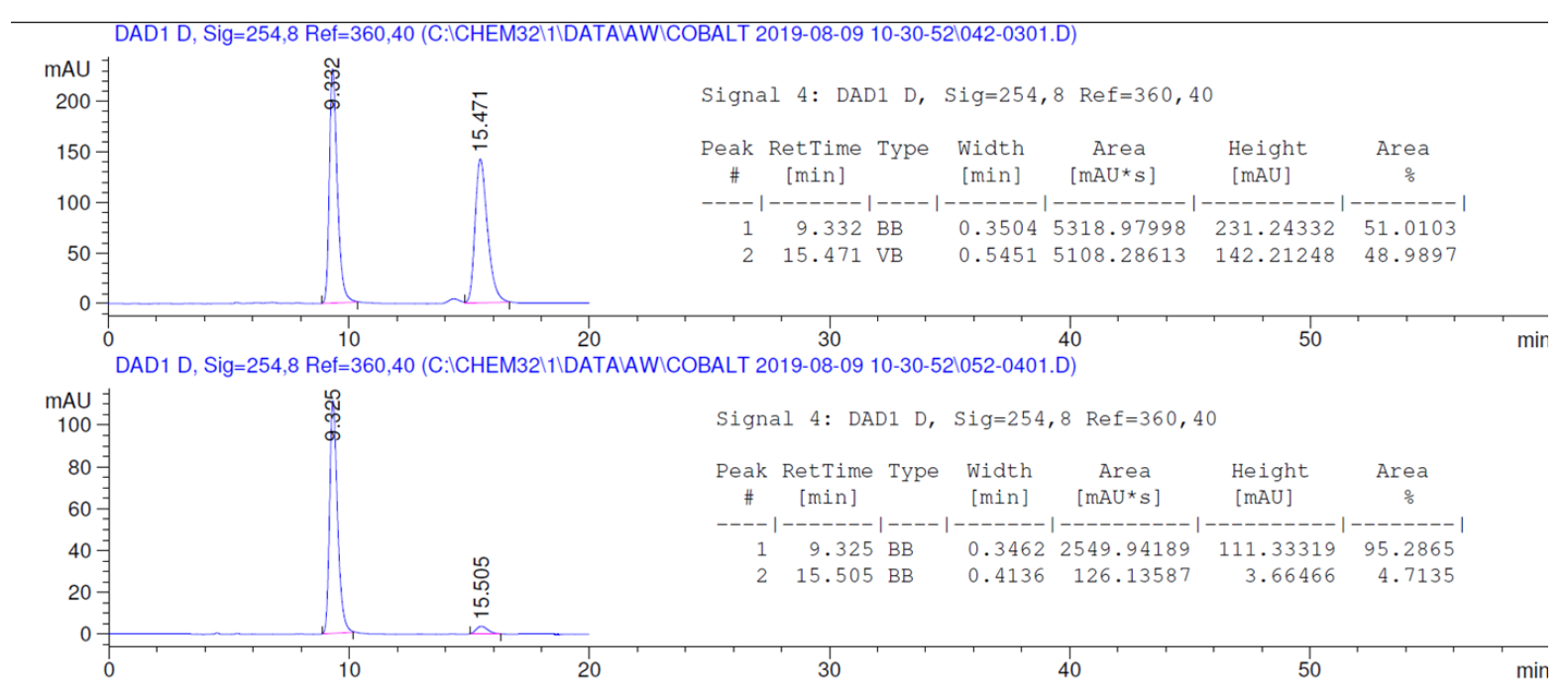




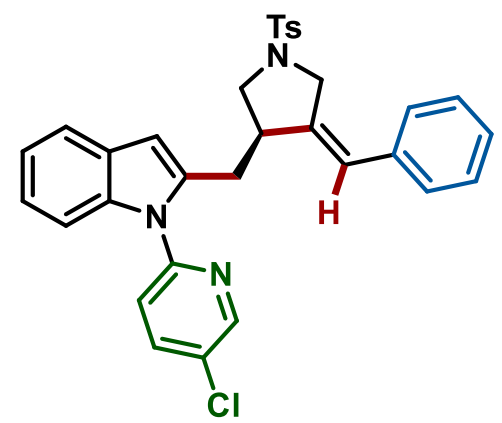

Synthesized following GP3. Isolated by flash column chromatography 10 -> 30\% EtOAc/pentane. Obtained $100 \mathrm{mg}$ of a white solid (90\% yield).

${ }^{1} \mathbf{H}$ NMR $\left(500 \mathrm{MHz}, \mathrm{CDCl}_{3}\right) \delta 8.60(\mathrm{dd}, J=2.7,0.7 \mathrm{~Hz}, 1 \mathrm{H}), 7.87(\mathrm{dd}, J=8.5,2.7 \mathrm{~Hz}, 1 \mathrm{H}), 7.69$ (d, $J=8.3 \mathrm{~Hz}, 2 \mathrm{H}), 7.64-7.57(\mathrm{~m}, 1 \mathrm{H}), 7.45(\mathrm{dd}, J=8.5,0.7 \mathrm{~Hz}, 1 \mathrm{H}), 7.38-7.27(\mathrm{~m}, 5 \mathrm{H}), 7.28$ $-7.21(\mathrm{~m}, 1 \mathrm{H}), 7.22-7.15(\mathrm{~m}, 2 \mathrm{H}), 7.08(\mathrm{~d}, J=7.3 \mathrm{~Hz}, 2 \mathrm{H}), 6.48(\mathrm{~d}, J=0.8 \mathrm{~Hz}, 1 \mathrm{H}), 6.15(\mathrm{q}, J$ $=2.3 \mathrm{~Hz}, 1 \mathrm{H}), 4.18(\mathrm{dt}, J=14.9,2.3 \mathrm{~Hz}, 1 \mathrm{H}), 4.02(\mathrm{dd}, J=14.9,2.3 \mathrm{~Hz}, 1 \mathrm{H}), 3.25$ (ddd, $J=$ $15.0,4.9,0.8 \mathrm{~Hz}, 1 \mathrm{H}), 3.23-3.14(\mathrm{~m}, 2 \mathrm{H}), 3.14-3.05(\mathrm{~m}, 1 \mathrm{H}), 2.95(\mathrm{dd}, J=15.0,9.7 \mathrm{~Hz}, 1 \mathrm{H})$, $2.42(\mathrm{~s}, 3 \mathrm{H})$.

${ }^{13}$ C NMR $\left(126 \mathrm{MHz}, \mathrm{CDCl}_{3}\right) \delta 149.5,148.4,143.7,139.7,138.3,138.2,137.0,136.3,132.6$, $130.2,129.7,128.6,128.5,128.1,127.8,127.2,123.6,122.4,121.5,121.2,120.4,110.0,51.7$, 50.7, 44.6, 31.8, 21.5.

IR (ATR) 1585, 1560, 1467, 1455, 1405, 1344, 1175, 1151, 1097, 1038, 814, 737, 665, 661

HRMS (DART, $\mathrm{M}+\mathrm{H}$ ) Calculated for $\mathrm{C}_{32} \mathrm{H}_{29} \mathrm{~N}_{3} \mathrm{O}_{2} \mathrm{~S}_{1} \mathrm{Cl}_{1} 554.1664$, found 554.1661

MP $176-180^{\circ} \mathrm{C}$

$\boldsymbol{\alpha}_{\boldsymbol{D}}^{\mathbf{2 0}}\left(\mathrm{c}=0.25, \mathrm{CH}_{2} \mathrm{Cl}_{2}\right) 7.18$

HPLC: IB, 50\% IPA/Hex, $1 \mathrm{~mL} / \mathrm{min}$
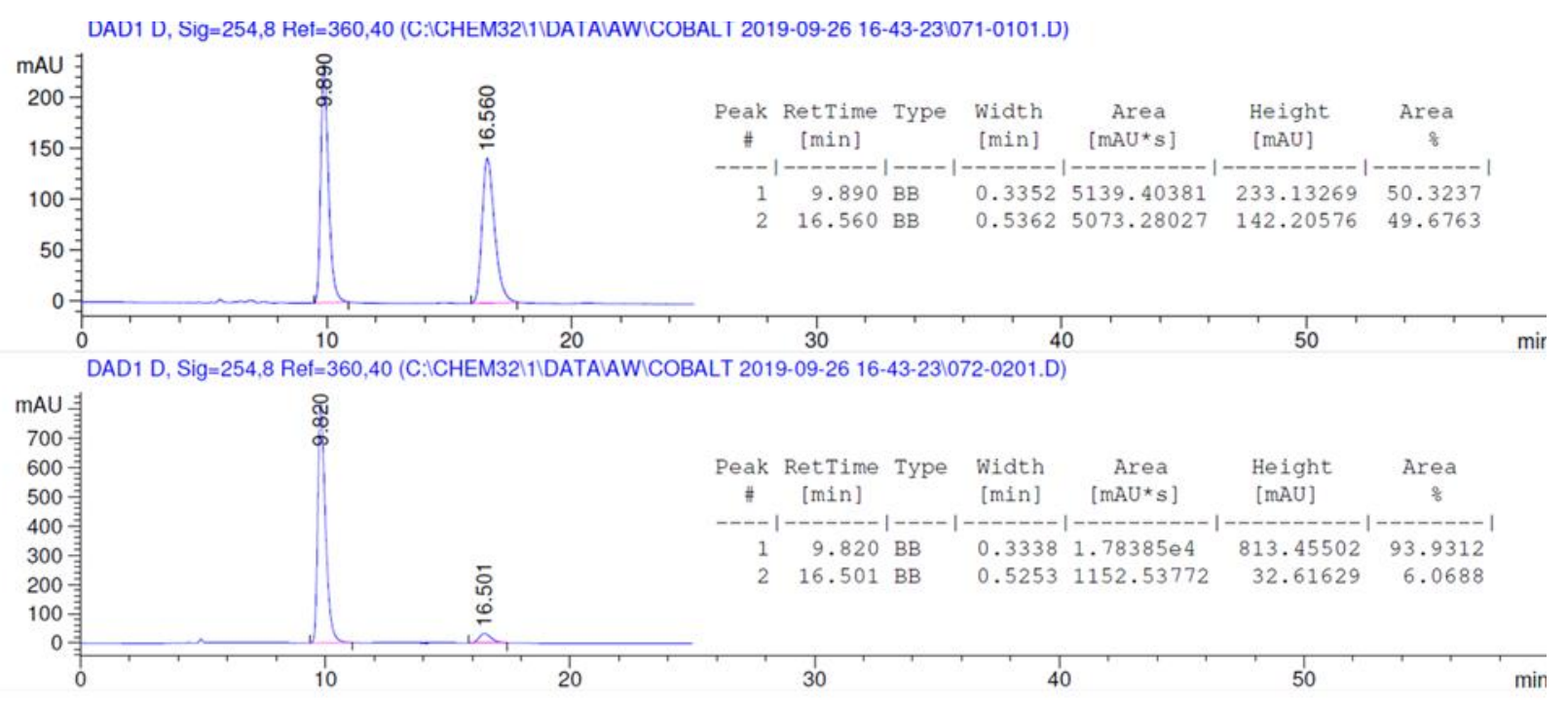

$(3 \mathbf{k})$<smiles>Cc1c(C[C@@H]2CN([As])CC2=Cc2ccccc2)n(-c2ccccn2)c2ccccc12</smiles>

Synthesized following GP3. Isolated by flash column chromatography 10 -> 30\% EtOAc/pentane. Obtained $100 \mathrm{mg}$ of a white solid (94\% yield).

${ }^{1} \mathbf{H}$ NMR $\left(500 \mathrm{MHz}, \mathrm{CDCl}_{3}\right) \delta 8.66(\mathrm{ddd}, J=4.9,2.0,0.8 \mathrm{~Hz}, 1 \mathrm{H}), 7.88(\mathrm{ddd}, J=8.0,7.2,2.0$ $\mathrm{Hz}, 1 \mathrm{H}), 7.66(\mathrm{~d}, J=8.3 \mathrm{~Hz}, 2 \mathrm{H}), 7.59-7.55(\mathrm{~m}, 1 \mathrm{H}), 7.45(\mathrm{dt}, J=8.0,0.8 \mathrm{~Hz}, 1 \mathrm{H}), 7.35-7.30$ $(\mathrm{m}, 4 \mathrm{H}), 7.28(\mathrm{~d}, J=8.3 \mathrm{~Hz}, 2 \mathrm{H}), 7.24-7.20(\mathrm{~m}, 1 \mathrm{H}), 7.20-7.16(\mathrm{~m}, 2 \mathrm{H}), 7.01(\mathrm{~d}, J=7.2 \mathrm{~Hz}$, $2 \mathrm{H}), 5.92(\mathrm{q}, J=2.3 \mathrm{~Hz}, 1 \mathrm{H}), 4.20(\mathrm{ddd}, J=14.9,2.3,1.7 \mathrm{~Hz}, 1 \mathrm{H}), 3.87(\mathrm{dd}, J=14.9,2.3 \mathrm{~Hz}$, $1 \mathrm{H}), 3.31$ (dd, $J=14.6,6.0 \mathrm{~Hz}, 1 \mathrm{H}), 3.16-3.07(\mathrm{~m}, 2 \mathrm{H}), 2.91(\mathrm{dd}, J=9.2,6.6 \mathrm{~Hz}, 1 \mathrm{H}), 2.88-$ $2.81(\mathrm{~m}, 1 \mathrm{H}), 2.40(\mathrm{~s}, 3 \mathrm{H}), 2.32(\mathrm{~s}, 3 \mathrm{H})$.

${ }^{13} \mathrm{C}$ NMR $\left(126 \mathrm{MHz}, \mathrm{CDCl}_{3}\right) \delta 151.8,149.5,143.6,139.6,138.4,136.6,136.4,134.0,132.3$, $129.7,129.3,128.5,128.0,127.8,127.0,123.5,122.3,121.8,120.9,120.4,118.7,112.0,109.8$, $51.5,50.6,45.4,29.5,21.5,9.2$.

IR (ATR) 1598, 1444, 1475, 1048, 1347, 1100, 1159, 819, 910, 670, 726

HRMS (DART, M+H) Calculated for $\mathrm{C}_{33} \mathrm{H}_{32} \mathrm{~N}_{3} \mathrm{O}_{2} \mathrm{~S} 534.2210$, found 534.2205

MP $115-120{ }^{\circ} \mathrm{C}$

$\boldsymbol{\alpha}_{\boldsymbol{D}}^{\mathbf{2 0}}\left(\mathrm{c}=0.21, \mathrm{CH}_{2} \mathrm{Cl}_{2}\right) 0.48$

HPLC: IA, 20\% IPA/Hex, $0.5 \mathrm{~mL} / \mathrm{min}$

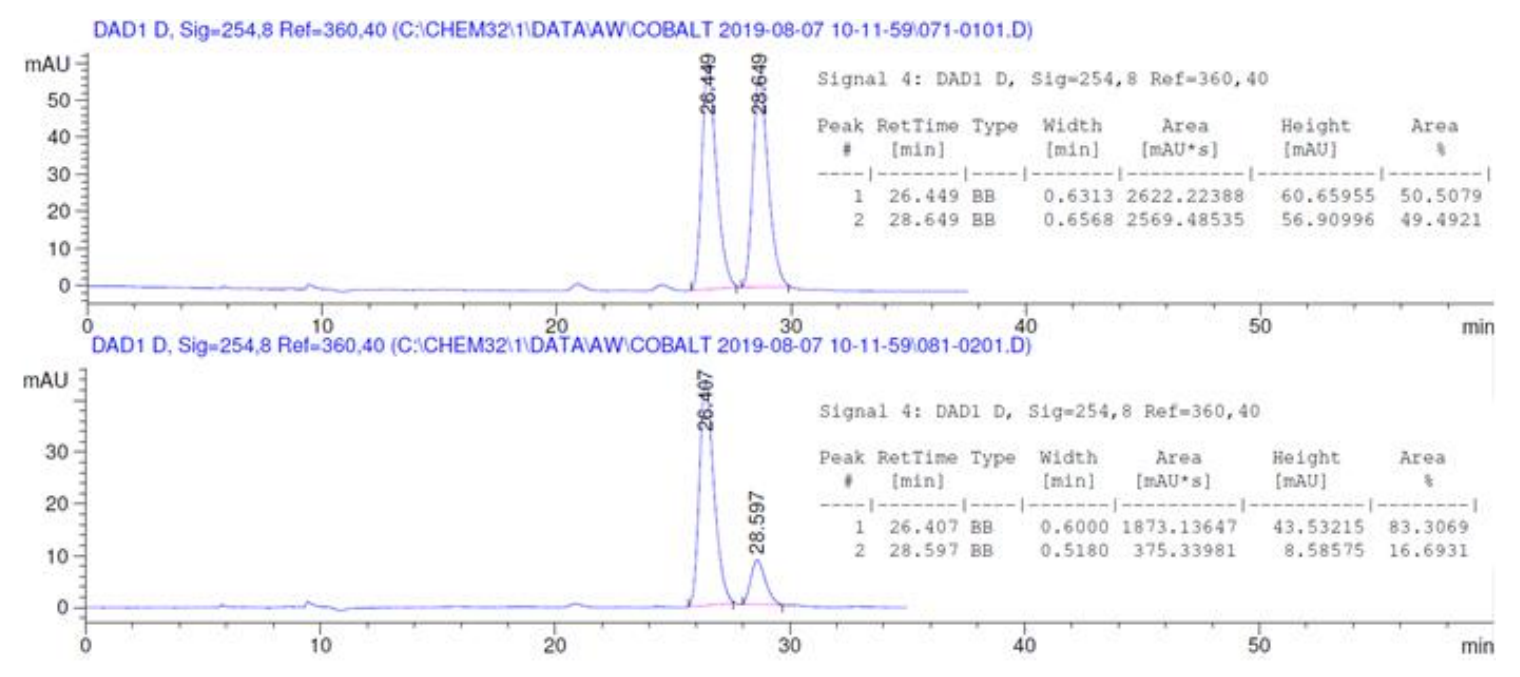



(31)<smiles>Cc1cccc2c1cc(C[C@@H]1CN([As])CC1=Cc1ccccc1)n2-c1ccccn1</smiles>

Synthesized following GP3. Isolated by flash column chromatography 10 -> 30\% EtOAc/pentane. Obtained $94 \mathrm{mg}$ of a white solid (88\% yield).

${ }^{1} \mathbf{H}$ NMR $\left(500 \mathrm{MHz}, \mathrm{CDCl}_{3}\right) \delta 8.66(\mathrm{ddd}, J=4.9,2.0,0.8 \mathrm{~Hz}, 1 \mathrm{H}), 7.90(\mathrm{ddd}, J=7.9,7.4,2.0$ $\mathrm{Hz}, 1 \mathrm{H}), 7.69(\mathrm{~d}, J=8.3 \mathrm{~Hz}, 2 \mathrm{H}), 7.47(\mathrm{dt}, J=7.9,0.8 \mathrm{~Hz}, 1 \mathrm{H}), 7.37-7.30(\mathrm{~m}, 3 \mathrm{H}), 7.30(\mathrm{~d}, J=$ $8.3 \mathrm{~Hz}, 2 \mathrm{H}), 7.27-7.19(\mathrm{~m}, 1 \mathrm{H}), 7.17(\mathrm{dq}, J=8.2,0.8 \mathrm{~Hz}, 1 \mathrm{H}), 7.11-7.03(\mathrm{~m}, 3 \mathrm{H}), 6.97(\mathrm{dt}, J$ $=7.1,0.8 \mathrm{~Hz}, 1 \mathrm{H}), 6.46(\mathrm{~d}, J=0.9 \mathrm{~Hz}, 1 \mathrm{H}), 6.11(\mathrm{q}, J=2.3 \mathrm{~Hz}, 1 \mathrm{H}), 4.17(\mathrm{dt}, J=14.7,2.3 \mathrm{~Hz}$, $1 \mathrm{H}), 4.01(\mathrm{dd}, J=14.7,2.3 \mathrm{~Hz}, 1 \mathrm{H}), 3.30(\mathrm{ddd}, J=14.8,4.7,0.9 \mathrm{~Hz}, 1 \mathrm{H}), 3.22-3.14(\mathrm{~m}, 2 \mathrm{H})$, $3.09-2.99(\mathrm{~m}, 1 \mathrm{H}), 2.94$ (ddd, $J=14.8,10.0,0.7 \mathrm{~Hz}, 1 \mathrm{H}), 2.58(\mathrm{~s}, 3 \mathrm{H}), 2.41$ (s, 3H).

${ }^{13} \mathrm{C}$ NMR $\left(126 \mathrm{MHz}, \mathrm{CDCl}_{3}\right) \delta 151.5,149.6,143.6,139.9,138.4,137.7,136.9,136.4,132.7$, $129.7,129.7,128.5,128.1,128.0,127.8,127.1,123.5,122.3,122.1,121.2,121.1,107.7,102.6$, 51.6, 50.7, 44.6, 32.1, 21.5, 18.7 .

IR (ATR) 1569, 1449, 1386, 1299, 1227, 1167, 1094, 1016, 987, 857, 787, 764, 710

HRMS (DART, M+H) Calculated for $\mathrm{C}_{33} \mathrm{H}_{32} \mathrm{~N}_{3} \mathrm{O}_{2} \mathrm{~S}_{1} 534.2210$, found 534.2211

MP $153-155^{\circ} \mathrm{C}$

$\boldsymbol{\alpha}_{\boldsymbol{D}}^{\mathbf{2 0}}\left(\mathrm{c}=0.19, \mathrm{CH}_{2} \mathrm{Cl}_{2}\right) 5.26$

HPLC: IB, 50\% IPA/Hex, $1 \mathrm{~mL} / \mathrm{min}$

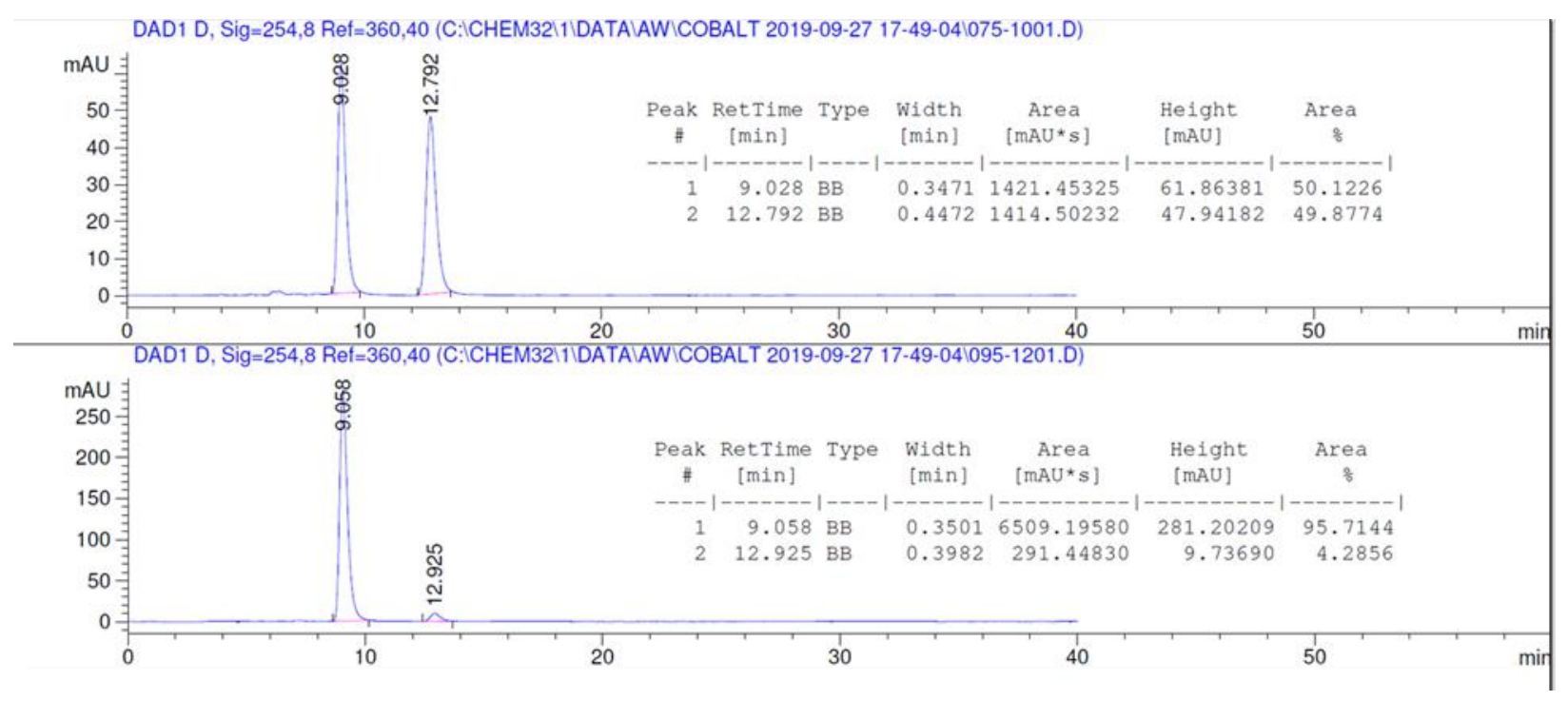


<smiles></smiles>

Synthesized following GP3. Isolated by flash column chromatography 15 -> 30\% EtOAc/pentane. Obtained $119 \mathrm{mg}$ of a white solid (95\% yield).

${ }^{1} \mathbf{H}$ NMR $\left(500 \mathrm{MHz}, \mathrm{CDCl}_{3}\right) \delta 8.67(\mathrm{ddd}, J=4.9,2.0,0.8 \mathrm{~Hz}, 1 \mathrm{H}), 7.90(\mathrm{td}, J=7.7,2.0 \mathrm{~Hz}$, $1 \mathrm{H}), 7.70(\mathrm{~d}, J=8.3 \mathrm{~Hz}, 2 \mathrm{H}), 7.56(\mathrm{~d}, J=7.6 \mathrm{~Hz}, 2 \mathrm{H}), 7.48(\mathrm{dt}, J=8.0,1.0 \mathrm{~Hz}, 1 \mathrm{H}), 7.44(\mathrm{t}, J=$ $7.5 \mathrm{~Hz}, 2 \mathrm{H}), 7.39-7.31(\mathrm{~m}, 4 \mathrm{H}), 7.28(\mathrm{~d}, J=8.3 \mathrm{~Hz}, 2 \mathrm{H}), 7.26-7.20(\mathrm{~m}, 1 \mathrm{H}), 7.13-7.03(\mathrm{~m}$, $3 \mathrm{H}), 6.99(\mathrm{dt}, J=8.2,0.7 \mathrm{~Hz}, 1 \mathrm{H}), 6.68(\mathrm{~d}, J=7.8 \mathrm{~Hz}, 1 \mathrm{H}), 6.62(\mathrm{~s}, 1 \mathrm{H}), 6.14(\mathrm{q}, J=2.3 \mathrm{~Hz}$, $1 \mathrm{H}), 5.27$ (s, 2H), 4.15 (dt, $J=14.8,2.3 \mathrm{~Hz}, 1 \mathrm{H}), 4.05(\mathrm{dd}, J=14.8,2.3 \mathrm{~Hz}, 1 \mathrm{H}), 3.30$ (dd, $J=$ 15.0, $4.7 \mathrm{~Hz}, 1 \mathrm{H}), 3.25(\mathrm{dd}, J=9.5,6.9 \mathrm{~Hz}, 1 \mathrm{H}), 3.17(\mathrm{dd}, J=9.5,4.8 \mathrm{~Hz}, 1 \mathrm{H}), 3.09-3.00$ (m, $1 \mathrm{H}), 2.90(\mathrm{dd}, J=15.0,10.0 \mathrm{~Hz}, 1 \mathrm{H}), 2.39(\mathrm{~s}, 3 \mathrm{H})$.

${ }^{13} \mathrm{C}$ NMR $\left(126 \mathrm{MHz}, \mathrm{CDCl}_{3}\right) \delta 152.0,151.4,149.6,143.7,139.9,138.7,138.5,137.5,136.8$, 136.4, 132.7, 129.8, 128.6, 128.5, 128.1, 127.8, 127.8, 127.5, 127.1, 123.4, 122.9, 122.3, 121.1, $119.2,103.9,102.5,101.2,70.1,51.8,50.8,44.5,31.9,21.5$.

IR (ATR) 1586, 1444, 1478, 1496, 1156, 1253, 1347, 1093, 736, 670

HRMS (DART, M+H) Calculated for $\mathrm{C}_{39} \mathrm{H}_{36} \mathrm{~N}_{3} \mathrm{O}_{3} \mathrm{~S} 626.2472$, found 626.2476

MP $85-87{ }^{\circ} \mathrm{C}$

$\boldsymbol{\alpha}_{D}^{\mathbf{2 0}}\left(\mathrm{c}=0.26, \mathrm{CH}_{2} \mathrm{Cl}_{2}\right) 6.15$

HPLC: IB, $60 \%$ IPA/Hex, $1.0 \mathrm{~mL} / \mathrm{min}$

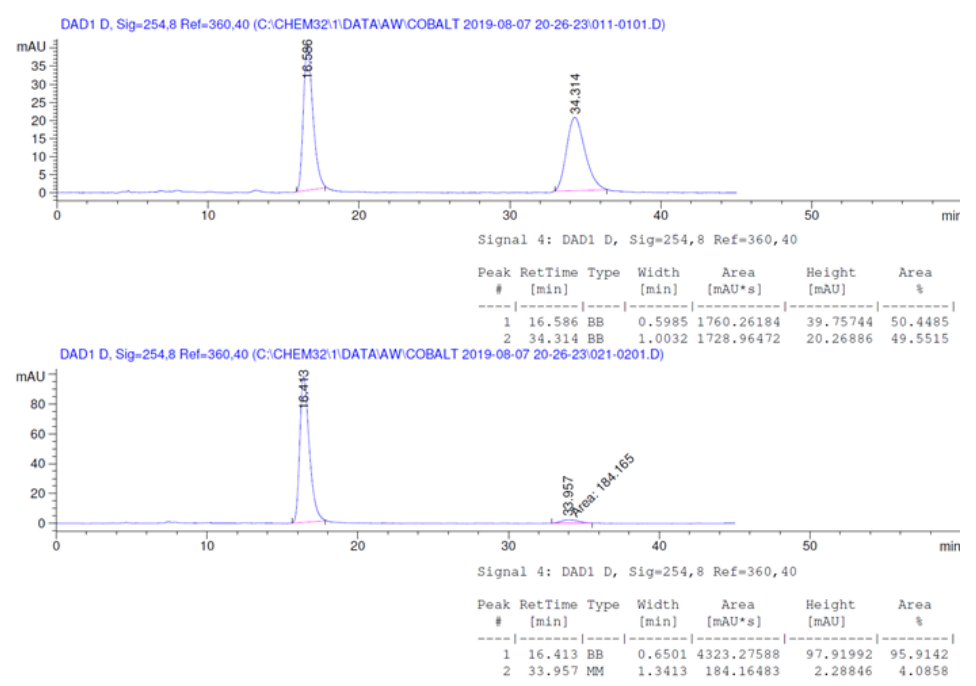



(3n)<smiles>[As]C1CC(=Cc2ccccc2)C(Cc2cc3c(Br)cccc3n2-c2ccccn2)C1</smiles>

Synthesized following GP3. Isolated by flash column chromatography 15 -> 30\% EtOAc/pentane. Obtained $119 \mathrm{mg}$ of a white solid (99\% yield).

${ }^{1} \mathbf{H}$ NMR $\left(500 \mathrm{MHz}, \mathrm{CDCl}_{3}\right) \delta 8.68(\mathrm{ddd}, J=4.9,2.0,0.9 \mathrm{~Hz}, 1 \mathrm{H}), 7.95-7.88(\mathrm{~m}, 1 \mathrm{H}), 7.70(\mathrm{~d}$, $J=8.3 \mathrm{~Hz}, 2 \mathrm{H}), 7.43(\mathrm{dt}, J=8.0,1.0 \mathrm{~Hz}, 1 \mathrm{H}), 7.38(\mathrm{ddd}, J=7.5,4.9,1.0 \mathrm{~Hz}, 1 \mathrm{H}), 7.35-7.30$ $(\mathrm{m}, 5 \mathrm{H}), 7.26-7.21(\mathrm{~m}, 2 \mathrm{H}), 7.06(\mathrm{~d}, J=6.9 \mathrm{~Hz}, 2 \mathrm{H}), 7.01(\mathrm{dd}, J=8.2,7.6 \mathrm{~Hz}, 1 \mathrm{H}), 6.49(\mathrm{~d}, J$ $=0.9 \mathrm{~Hz}, 1 \mathrm{H}), 6.12(\mathrm{q}, J=2.2 \mathrm{~Hz}, 1 \mathrm{H}), 4.15(\mathrm{dt}, J=15.0,2.2 \mathrm{~Hz}, 1 \mathrm{H}), 4.01(\mathrm{dd}, J=15.0,2.2$ $\mathrm{Hz}, 1 \mathrm{H}), 3.25(\mathrm{dd}, J=15.1,4.9 \mathrm{~Hz}, 1 \mathrm{H}), 3.22-3.15(\mathrm{~m}, 2 \mathrm{H}), 3.10-3.01(\mathrm{~m}, 1 \mathrm{H}), 2.92(\mathrm{ddd}, J=$ 15.1, 9.9, $0.7 \mathrm{~Hz}, 1 \mathrm{H}), 2.42$ (s, 3H).

${ }^{13} \mathrm{C}$ NMR $\left(126 \mathrm{MHz}, \mathrm{CDCl}_{3}\right) \delta 150.8,149.8,143.8,139.6,139.2,138.7,137.4,136.2,132.6$, 129.8, 129.0, 128.6, 128.1, 127.8, 127.2, 123.8, 123.6, 123.0, 122.8, 121.3, 114.1, 109.4, 103.7, 51.7, 50.6, 44.5, 31.9, 21.6.

IR (ATR) 1585, 1467, 1435, 1346, 1158, 1093, 1016, 815, 785, 763, 746

HRMS (DART, M+H) Calculated for $\mathrm{C}_{32} \mathrm{H}_{29} \mathrm{~N}_{3} \mathrm{O}_{2} \mathrm{~S}_{1} \mathrm{Br}_{1} 598.1158$, found 598.1151

MP $141-145^{\circ} \mathrm{C}$

$\boldsymbol{\alpha}_{D}^{\mathbf{2 0}}\left(\mathrm{c}=0.19, \mathrm{CH}_{2} \mathrm{Cl}_{2}\right) 17.89$

HPLC: IB, 50\% IPA/Hex, $1.0 \mathrm{~mL} / \mathrm{min}$

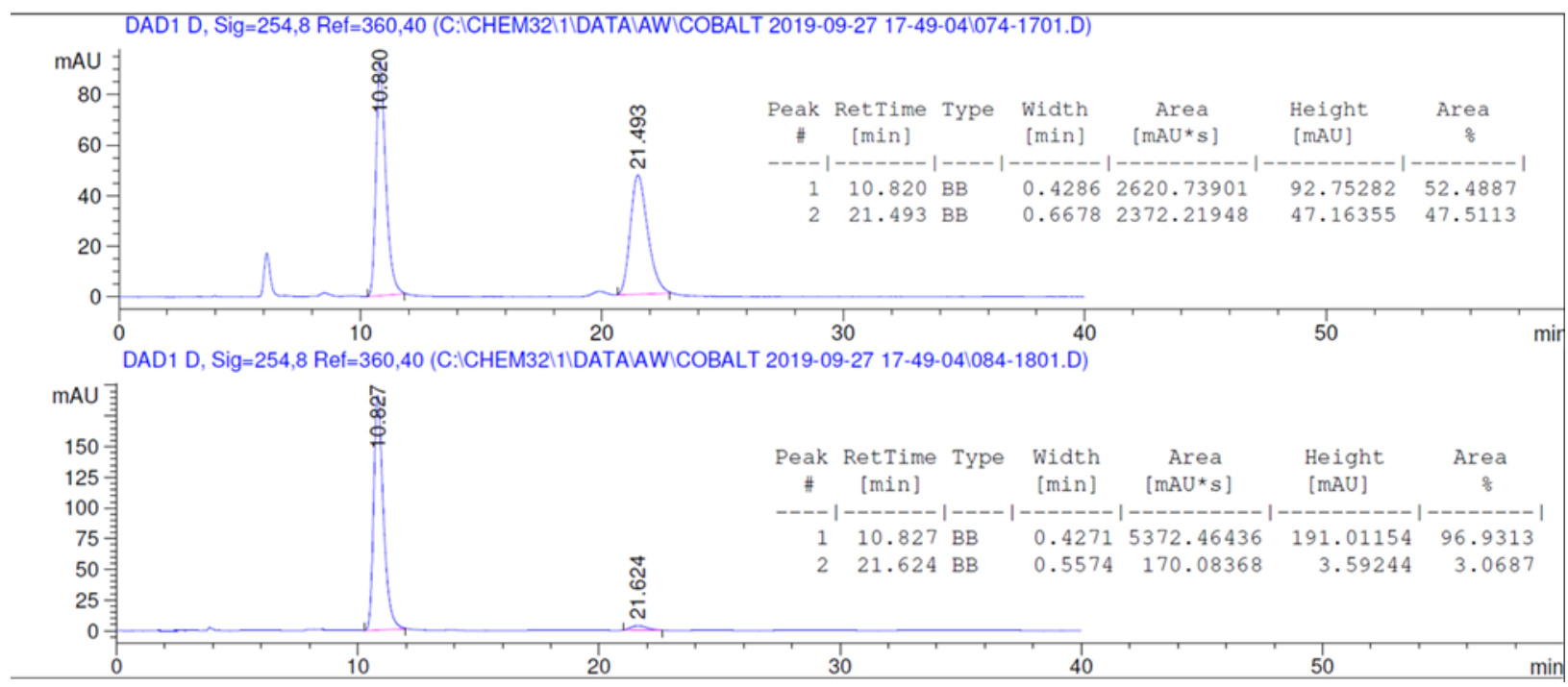



(3o)<smiles></smiles>

Synthesized following GP3. The product was isolated by flash column chromatography 15$>30 \%$ EtOAc/pentane. The product appeared as a white solid, $102 \mathrm{mg}$ (96\% yield).

${ }^{1} \mathbf{H}$ NMR $\left(500 \mathrm{MHz}, \mathrm{CDCl}_{3}\right) \delta 8.65(\mathrm{ddd}, J=4.9,2.0,0.8 \mathrm{~Hz}, 1 \mathrm{H}), 7.89(\mathrm{td}, \mathrm{J}=7.7,2.0 \mathrm{~Hz}, 1 \mathrm{H})$, $7.68(\mathrm{~d}, \mathrm{~J}=8.3 \mathrm{~Hz}, 2 \mathrm{H}), 7.46(\mathrm{dt}, \mathrm{J}=7.7,1.0 \mathrm{~Hz}, 1 \mathrm{H}), 7.39(\mathrm{dt}, \mathrm{J}=1.7,0.8 \mathrm{~Hz}, 1 \mathrm{H}), 7.37-7.27$ $(\mathrm{m}, 5 \mathrm{H}), 7.28-7.20(\mathrm{~m}, 2 \mathrm{H}), 7.07(\mathrm{~d}, \mathrm{~J}=7.3 \mathrm{~Hz}, 2 \mathrm{H}), 6.99(\mathrm{dd}, \mathrm{J}=8.5,1.7 \mathrm{~Hz}, 1 \mathrm{H}), 6.39(\mathrm{~s}$, $1 \mathrm{H}), 6.13(\mathrm{q}, \mathrm{J}=2.3 \mathrm{~Hz}, 1 \mathrm{H}), 4.16(\mathrm{dt}, \mathrm{J}=14.8,2.3 \mathrm{~Hz}, 1 \mathrm{H}), 4.01(\mathrm{dd}, \mathrm{J}=14.8,2.3 \mathrm{~Hz}, 1 \mathrm{H})$, 3.30 (ddd, $\mathrm{J}=14.9,4.8,0.9 \mathrm{~Hz}, 1 \mathrm{H}), 3.17(\mathrm{~d}, \mathrm{~J}=6.0 \mathrm{~Hz}, 2 \mathrm{H}), 3.05(\mathrm{dq}, \mathrm{J}=10.6,5.3 \mathrm{~Hz}, 1 \mathrm{H})$, 2.93 (dd, J = 14.9, $9.8 \mathrm{~Hz}, 1 \mathrm{H}), 2.46$ (s, 3H), 2.41 (s, 3H).

${ }^{13} \mathrm{C}$ NMR $\left(126 \mathrm{MHz}, \mathrm{CDCl}_{3}\right) \delta 151.5,149.5,143.6,139.9,138.5,138.3,136.4,135.5,132.6$, 130.2, 129.7, 128.7, 128.5, 128.1, 127.8, 127.1, 123.6, 123.4, 122.0, 120.8, 120.0, 109.8, 103.8, 51.7, 50.7, 44.6, 32.0, 21.5, 21.4 .

IR (ATR) 1592, 1444, 1478, 1345, 1156, 1100, 1045, 909, 820, 726, 670

HRMS (DART, M+H) Calculated for $\mathrm{C}_{33} \mathrm{H}_{32} \mathrm{~N}_{3} \mathrm{O}_{2} \mathrm{~S} 534.2210$, found 534.2216

MP $165-168{ }^{\circ} \mathrm{C}$

$\boldsymbol{\alpha}_{\boldsymbol{D}}^{\mathbf{2 0}}\left(\mathrm{c}=0.24, \mathrm{CH}_{2} \mathrm{Cl}_{2}\right)-0.83$

HPLC: IB, 50\% IPA/Hex, $1.0 \mathrm{~mL} / \mathrm{min}$

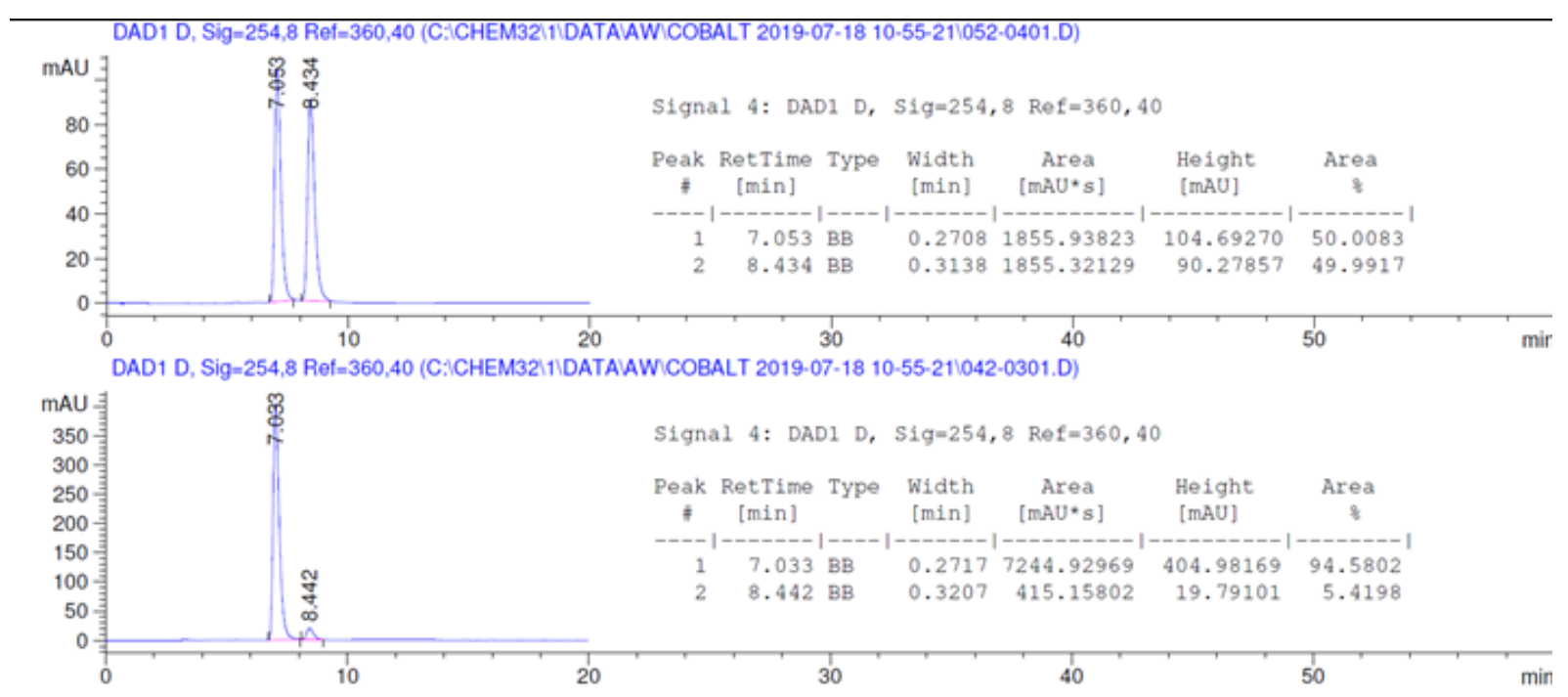


(R,Z)-2-((4-benzylidene-1-tosylpyrrolidin-3-yl)methyl)-5-methoxy-1-(pyridin-2-yl)-1Hindole (3p)<smiles>COc1ccc2c(c1)cc(C[C@@H]1CN([As])C/C1=C\c1ccccc1)n2-c1ccccn1</smiles>

Synthesized following GP3. The product was isolated by flash column chromatography 15$>30 \%$ EtOAc/pentane. The product appeared as a white solid, $107 \mathrm{mg}$ (97\% yield).

${ }^{1} \mathbf{H}$ NMR $\left(500 \mathrm{MHz}, \mathrm{CDCl}_{3}\right) \delta 8.65(\mathrm{ddd}, J=4.9,2.0,1.0 \mathrm{~Hz}, 1 \mathrm{H}), 7.89$ (ddd, $J=8.0,7.5,2.0$ $\mathrm{Hz}, 1 \mathrm{H}), 7.69(\mathrm{~d}, J=8.3 \mathrm{~Hz}, 2 \mathrm{H}), 7.45(\mathrm{dt}, J=8.0,1.0 \mathrm{~Hz}, 1 \mathrm{H}), 7.36-7.26(\mathrm{~m}, 6 \mathrm{H}), 7.25-7.21$ (m, 1H), $7.09-7.04(\mathrm{~m}, 3 \mathrm{H}), 6.82(\mathrm{dd}, J=8.9,2.5 \mathrm{~Hz}, 1 \mathrm{H}), 6.41(\mathrm{~d}, J=0.8 \mathrm{~Hz}, 1 \mathrm{H}), 6.13(\mathrm{q}, J$ $=2.3 \mathrm{~Hz}, 1 \mathrm{H}), 4.17(\mathrm{dt}, J=14.8,2.3 \mathrm{~Hz}, 1 \mathrm{H}), 4.00(\mathrm{dd}, J=14.8,2.3 \mathrm{~Hz}, 1 \mathrm{H}), 3.88(\mathrm{~s}, 3 \mathrm{H}), 3.29$ (dd, $J=14.8,4.7 \mathrm{~Hz}, 1 \mathrm{H}), 3.22-3.14(\mathrm{~m}, 2 \mathrm{H}), 3.10-3.01(\mathrm{~m}, 1 \mathrm{H}), 2.94(\mathrm{dd}, J=14.8,9.8 \mathrm{~Hz}$, $1 \mathrm{H}), 2.41$ (s, 3H).

${ }^{13}$ C NMR $\left(126 \mathrm{MHz}, \mathrm{CDCl}_{3}\right) \delta 154.9,151.4,149.6,143.7,139.8,138.8,138.5,136.4,132.6$, $132.3,129.7,129.0,128.6,128.1,127.8,127.1,123.5,122.0,120.7,111.8,111.0,104.0,102.3$, 55.8, 51.7, 50.7, 44.7, 32.1, 21.5.

IR (ATR) 1478, 1451, 1350, 1211, 1170, 1045, 903, 726, 670

HRMS (DART, $\mathrm{M}+\mathrm{H}$ ) Calculated for $\mathrm{C}_{33} \mathrm{H}_{32} \mathrm{~N}_{3} \mathrm{O}_{3} \mathrm{~S} 550.2159$, found 550.2154

MP $120-126^{\circ} \mathrm{C}$

$\boldsymbol{\alpha}_{\boldsymbol{D}}^{\mathbf{2 0}}\left(\mathrm{c}=0.55, \mathrm{CH}_{2} \mathrm{Cl}_{2}\right)-0.73$

HPLC: IB, $50 \%$ IPA/Hex, $1.0 \mathrm{~mL} / \mathrm{min}$

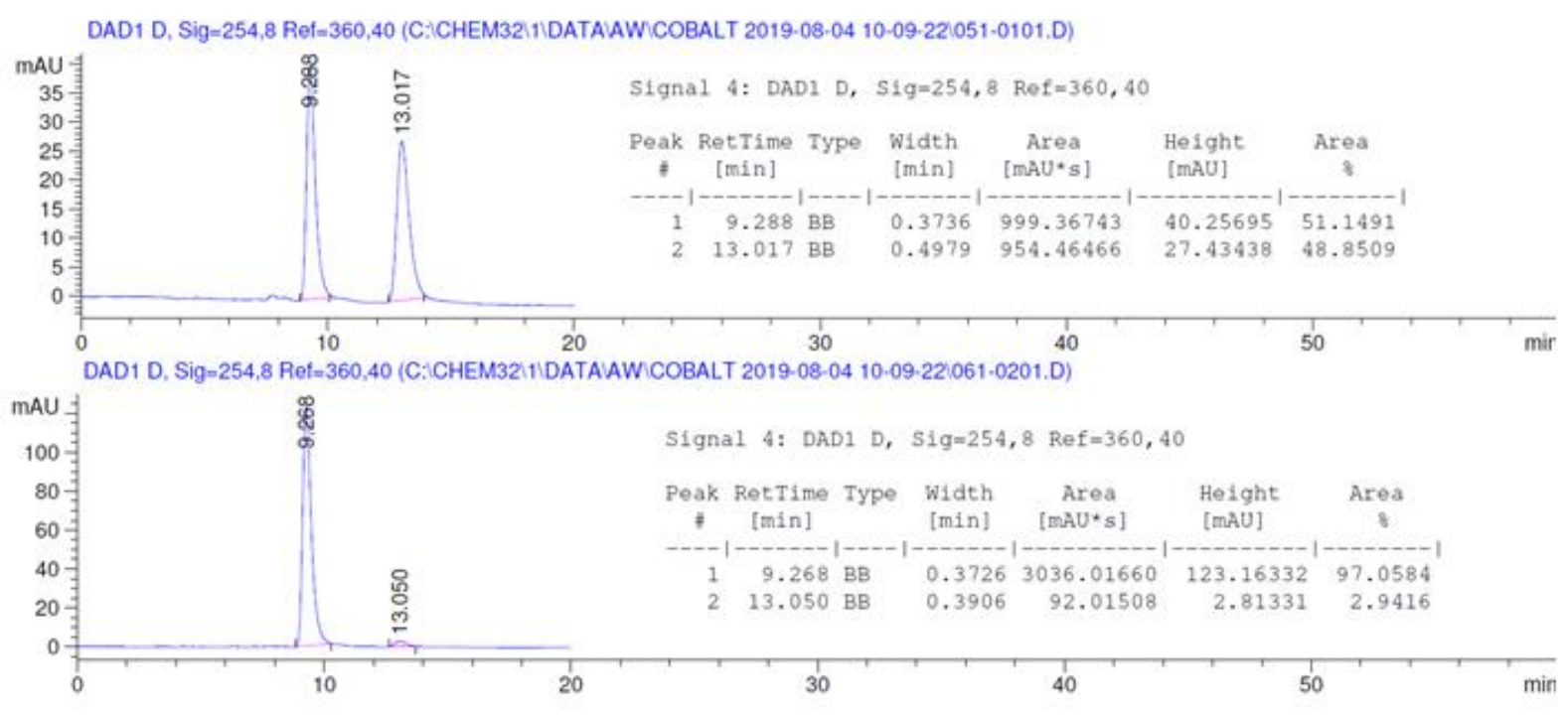


<smiles>Fc1ccc2c(c1)cc(C[C@@H]1CN([As])CC1=Cc1ccccc1)n2-c1ccccn1</smiles>

Synthesized following GP3. The product was isolated by flash column chromatography 10$>30 \% \mathrm{EtOAc/pentane.} \mathrm{The} \mathrm{product} \mathrm{appeared} \mathrm{as} \mathrm{a} \mathrm{white} \mathrm{solid,} 105 \mathrm{mg}$ (98\% yield).

${ }^{1} \mathbf{H}$ NMR $\left(500 \mathrm{MHz}, \mathrm{CDCl}_{3}\right) \delta 8.67(\mathrm{ddd}, J=4.9,2.0,0.9 \mathrm{~Hz}, 1 \mathrm{H}), 7.92(\mathrm{ddd}, J=8.1,7.5,2.0$ $\mathrm{Hz}, 1 \mathrm{H}), 7.68(\mathrm{~d}, J=8.3 \mathrm{~Hz}, 2 \mathrm{H}), 7.44(\mathrm{dt}, J=8.1,1.0 \mathrm{~Hz}, 1 \mathrm{H}), 7.36(\mathrm{ddd}, J=7.5,4.9,1.0 \mathrm{~Hz}$, $1 \mathrm{H}), 7.33(\mathrm{t}, J=7.6 \mathrm{~Hz}, 2 \mathrm{H}), 7.30(\mathrm{~d}, J=7.9 \mathrm{~Hz}, 2 \mathrm{H}), 7.28-7.19(\mathrm{~m}, 4 \mathrm{H}), 7.06(\mathrm{~d}, J=7.2 \mathrm{~Hz}$, $2 \mathrm{H}), 6.89(\mathrm{td}, J=9.1,2.5 \mathrm{~Hz}, 1 \mathrm{H}), 6.42(\mathrm{~d}, J=0.8 \mathrm{~Hz}, 1 \mathrm{H}), 6.11(\mathrm{q}, J=2.3 \mathrm{~Hz}, 1 \mathrm{H}), 4.16(\mathrm{dt}, J$ $=14.8,2.3 \mathrm{~Hz}, 1 \mathrm{H}), 3.98(\mathrm{dd}, J=14.8,2.3 \mathrm{~Hz}, 1 \mathrm{H}), 3.25(\mathrm{ddd}, J=14.8,4.9,0.8 \mathrm{~Hz}, 1 \mathrm{H}), 3.22-$ $3.13(\mathrm{~m}, 2 \mathrm{H}), 3.09-3.00(\mathrm{~m}, 1 \mathrm{H}), 2.95(\mathrm{dd}, J=14.8,9.7 \mathrm{~Hz}, 1 \mathrm{H}), 2.41(\mathrm{~s}, 3 \mathrm{H})$.

${ }^{13}$ C NMR $\left(126 \mathrm{MHz}, \mathrm{CDCl}_{3}\right) \delta 158.5(\mathrm{~d}, J=235.7 \mathrm{~Hz}), 151.1,149.7,143.7,139.9,139.6,138.7$, $136.3,133.8,132.5,129.7,128.8$ (d, $J=10.2 \mathrm{~Hz}), 128.6,128.1,127.8,127.2$, 123.62, 122.4, 121.0, $110.9(\mathrm{~d}, J=9.5 \mathrm{~Hz}), 110.1(\mathrm{~d}, J=25.9 \mathrm{~Hz}), 105.2(\mathrm{~d}, J=23.6 \mathrm{~Hz}), 103.9(\mathrm{~d}, J=4.2 \mathrm{~Hz})$, 51.6, 50.7, 44.6, 32.0, 21.5.

${ }^{19}$ F NMR $\left(377 \mathrm{MHz}, \mathrm{CDCl}_{3}\right) \delta-123.5$.

IR (ATR) 1596, 1350, 1454, 1478, 1437, 1163, 1100, 899, 667, 722

HRMS (DART, M+H) Calculated for $\mathrm{C}_{32} \mathrm{H}_{29} \mathrm{~N}_{3} \mathrm{O}_{2} \mathrm{FS} 538.1959$, found 538.1963

MP $87-95^{\circ} \mathrm{C}$

$\boldsymbol{\alpha}_{D}^{\mathbf{2 0}}\left(\mathrm{c}=0.28, \mathrm{CH}_{2} \mathrm{Cl}_{2}\right) 10.00$

HPLC: IB, 30\% IPA/Hex, $0.5 \mathrm{~mL} / \mathrm{min}$

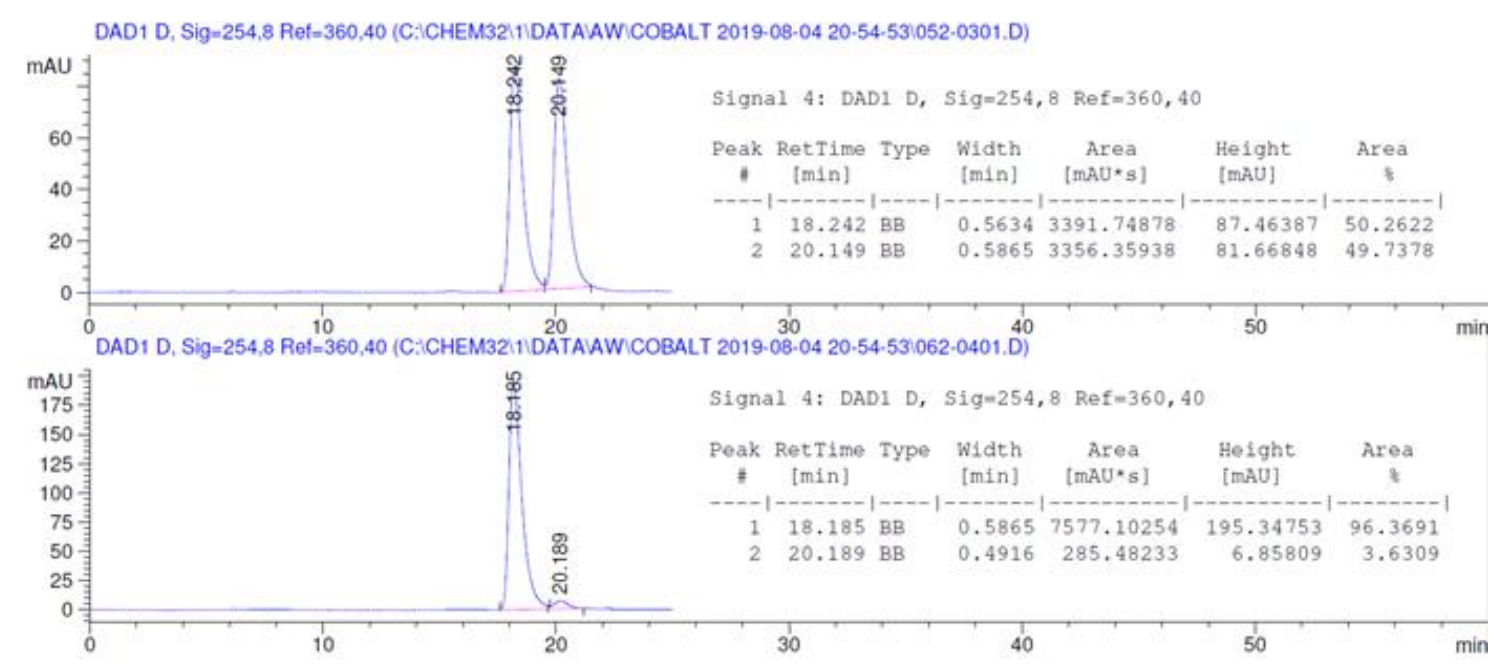



(3r)<smiles>[As]C1CC(=Cc2ccccc2)C(Cc2cc3cc(Br)ccc3n2-c2ccccn2)C1</smiles>

Synthesized following GP3. The product was isolated by flash column chromatography 10$>30 \%$ EtOAc/pentane. The product appeared as a white solid, $118 \mathrm{mg}$ (99\% yield).

${ }^{1} \mathbf{H}$ NMR $\left(500 \mathrm{MHz}, \mathrm{CDCl}_{3}\right) \delta 8.67(\mathrm{ddd}, J=4.9,2.0,0.8 \mathrm{~Hz}, 1 \mathrm{H}), 7.92(\mathrm{ddd}, J=8.0,7.5,2.0$ $\mathrm{Hz}, 1 \mathrm{H}), 7.70(\mathrm{dd}, J=1.9,0.6 \mathrm{~Hz}, 1 \mathrm{H}), 7.68(\mathrm{~d}, J=8.3 \mathrm{~Hz}, 2 \mathrm{H}), 7.42(\mathrm{dt}, J=8.0,1.0 \mathrm{~Hz}, 1 \mathrm{H})$, 7.37 (ddd, $J=7.5,4.9,1.0 \mathrm{~Hz}, 1 \mathrm{H}), 7.33(\mathrm{t}, J=7.6 \mathrm{~Hz}, 2 \mathrm{H}), 7.29(\mathrm{~d}, J=8.3 \mathrm{~Hz}, 2 \mathrm{H}), 7.25-7.21$ $(\mathrm{m}, 2 \mathrm{H}), 7.18(\mathrm{~d}, J=8.7 \mathrm{~Hz}, 1 \mathrm{H}), 7.05(\mathrm{~d}, J=7.6 \mathrm{~Hz}, 2 \mathrm{H}), 6.40(\mathrm{~d}, J=0.9 \mathrm{~Hz}, 1 \mathrm{H}), 6.09(\mathrm{q}, J=$ $2.3 \mathrm{~Hz}, 1 \mathrm{H}$ ), 4.15 (dt, $J=14.8,2.3 \mathrm{~Hz}, 1 \mathrm{H}), 3.97$ (dd, $J=14.8,2.3 \mathrm{~Hz}, 1 \mathrm{H}), 3.24$ (ddd, $J=14.8$, $4.9,0.9 \mathrm{~Hz}, 1 \mathrm{H}), 3.20-3.12(\mathrm{~m}, 2 \mathrm{H}), 3.07-3.00(\mathrm{~m}, 1 \mathrm{H}), 2.95(\mathrm{dd}, J=14.8,9.6 \mathrm{~Hz}, 1 \mathrm{H}), 2.41$ $(\mathrm{s}, 3 \mathrm{H})$.

${ }^{13} \mathrm{C}$ NMR $\left(126 \mathrm{MHz}, \mathrm{CDCl}_{3}\right) \delta 150.8,149.8,143.7,139.6,139.5,138.7,136.2,135.9,132.5$, 130.0, 129.7, 128.6, 128.0, 127.8, 127.2, 124.9, 123.7, 122.7, 122.6, 121.0, 114.0, 111.6, 103.3, 51.6, 50.6, 44.6, 31.8, 21.5.

IR (ATR) 1592, 1477, 1435, 1160, 1097, 1037, 913, 816, 733, 667

HRMS (DART, M+H) Calculated for $\mathrm{C}_{32} \mathrm{H}_{29} \mathrm{~N}_{3} \mathrm{O}_{2} \mathrm{SBr} 598.1158$, found 598.1152

MP $136-137{ }^{\circ} \mathrm{C}$

$\boldsymbol{\alpha}_{D}^{\mathbf{2 0}}\left(\mathrm{c}=0.27, \mathrm{CH}_{2} \mathrm{Cl}_{2}\right)-1.48$

HPLC: IB, 50\% IPA/Hex, $1.0 \mathrm{~mL} / \mathrm{min}$

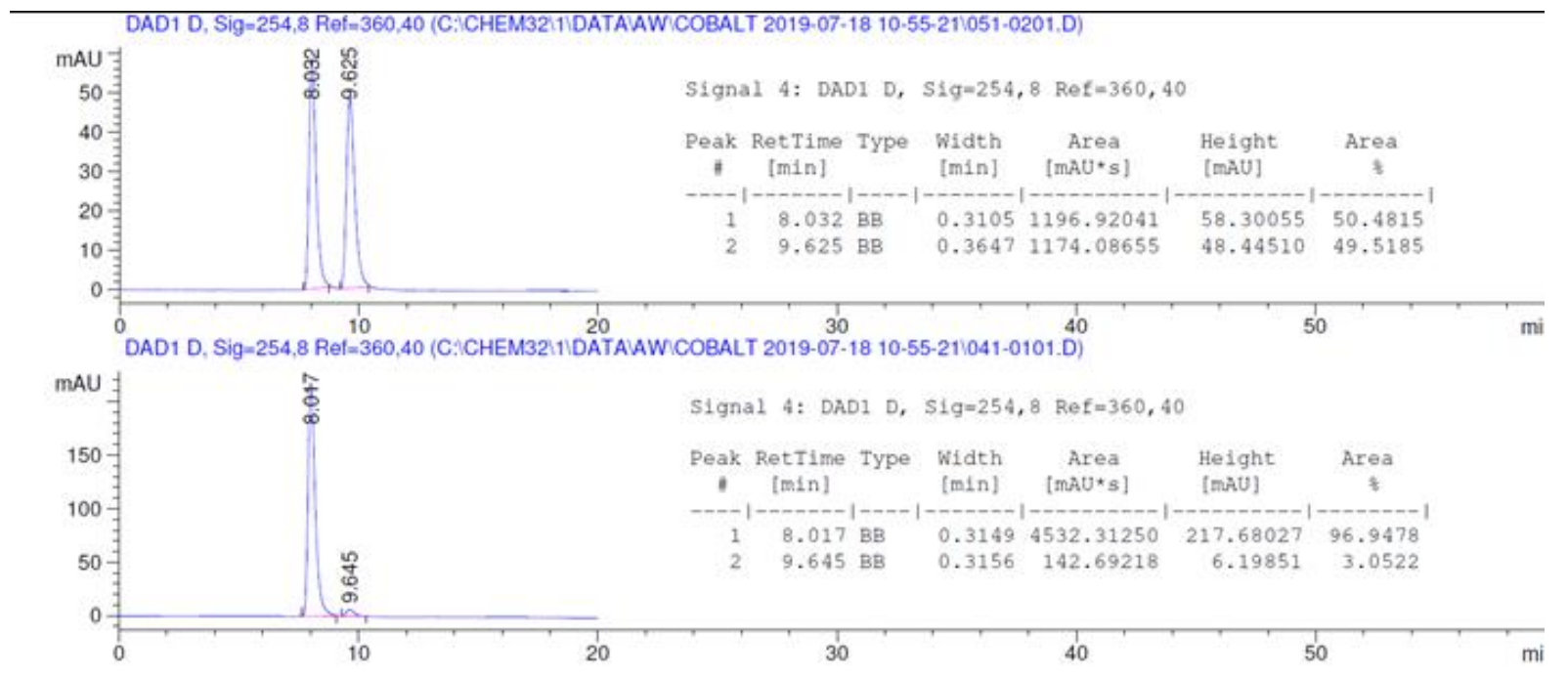


(R,Z)-2-((4-benzylidene-1-tosylpyrrolidin-3-yl)methyl)-5-(((tertbutyldimesthylsilyl)oxy)methyl)-1-(pyridin-2-yl)-1H-indole (3s)<smiles>CCCCOc1ccc2c(c1)cc(C[C@@H]1CN([As])CC1=Cc1ccccc1)n2-c1ccccn1</smiles>

Synthesized following GP3. The product was isolated by flash column chromatography 10$>30 \%$ EtOAc/pentane. The product appeared as a white solid, $52 \mathrm{mg}$ (39\% yield).

${ }^{1} \mathbf{H}$ NMR $\left(500 \mathrm{MHz}, \mathrm{CDCl}_{3}\right) \delta 8.66(\mathrm{ddd}, J=4.9,2.0,0.8 \mathrm{~Hz}, 1 \mathrm{H}), 7.90(\mathrm{ddd}, J=8.0,7.5,2.0$ $\mathrm{Hz}, 1 \mathrm{H}), 7.69(\mathrm{~d}, J=8.2 \mathrm{~Hz}, 2 \mathrm{H}), 7.55(\mathrm{dd}, J=1.7,0.8 \mathrm{~Hz}, 1 \mathrm{H}), 7.47(\mathrm{dt}, J=8.0,1.0 \mathrm{~Hz}, 1 \mathrm{H})$, $7.37-7.27(\mathrm{~m}, 6 \mathrm{H}), 7.27-7.19(\mathrm{~m}, 1 \mathrm{H}), 7.14(\mathrm{dd}, J=8.5,1.7 \mathrm{~Hz}, 1 \mathrm{H}), 7.06(\mathrm{~d}, J=7.2 \mathrm{~Hz}, 2 \mathrm{H})$, $6.44(\mathrm{~d}, J=0.9 \mathrm{~Hz}, 1 \mathrm{H}), 6.12(\mathrm{q}, J=2.3 \mathrm{~Hz}, 1 \mathrm{H}), 4.85(\mathrm{~s}, 2 \mathrm{H}), 4.16(\mathrm{dt}, J=14.8,2.3 \mathrm{~Hz}, 1 \mathrm{H})$, 4.00 (ddd, $J=14.8,2.3,0.8 \mathrm{~Hz}, 1 \mathrm{H}), 3.29$ (ddd, $J=14.8,4.9,0.9 \mathrm{~Hz}, 1 \mathrm{H}), 3.18(\mathrm{~m}, 2 \mathrm{H}), 3.05$ $(\mathrm{m}, 1 \mathrm{H}), 2.94(\mathrm{ddd}, J=14.8,9.8,0.8 \mathrm{~Hz}, 1 \mathrm{H}), 2.41(\mathrm{~s}, 3 \mathrm{H}), 0.97(\mathrm{~s}, 9 \mathrm{H}), 0.13(\mathrm{~s}, 6 \mathrm{H})$.

${ }^{13} \mathrm{C}$ NMR $\left(126 \mathrm{MHz}, \mathrm{CDCl}_{3}\right) \delta 151.4,149.6,143.7,139.8,138.5,136.5,136.4,134.1,132.6$, $129.7,128.5,128.3,128.1,127.8,127.1,123.5,122.1,121.1,120.9,118.0,109.9,104.2,65.6$, $51.7,50.7,44.6,32.0,26.1,21.5,18.5,-5.1$.

IR (ATR) 2950, 2927, 2852, 1587, 1470, 1436, 1343, 1304, 1251, 1162, 1092, 835, 775

HRMS (DART, M+H) Calculated for $\mathrm{C}_{39} \mathrm{H}_{46} \mathrm{~N}_{3} \mathrm{O}_{3} \mathrm{SiS} 664.3024$, found 664.3013

MP $70-75^{\circ} \mathrm{C}$

$\boldsymbol{\alpha}_{\boldsymbol{D}}^{\mathbf{2 0}}\left(\mathrm{c}=0.195, \mathrm{CH}_{2} \mathrm{Cl}_{2}\right)-5.13$

HPLC: IB, $15 \%$ IPA/Hex, $0.75 \mathrm{~mL} / \mathrm{min}$

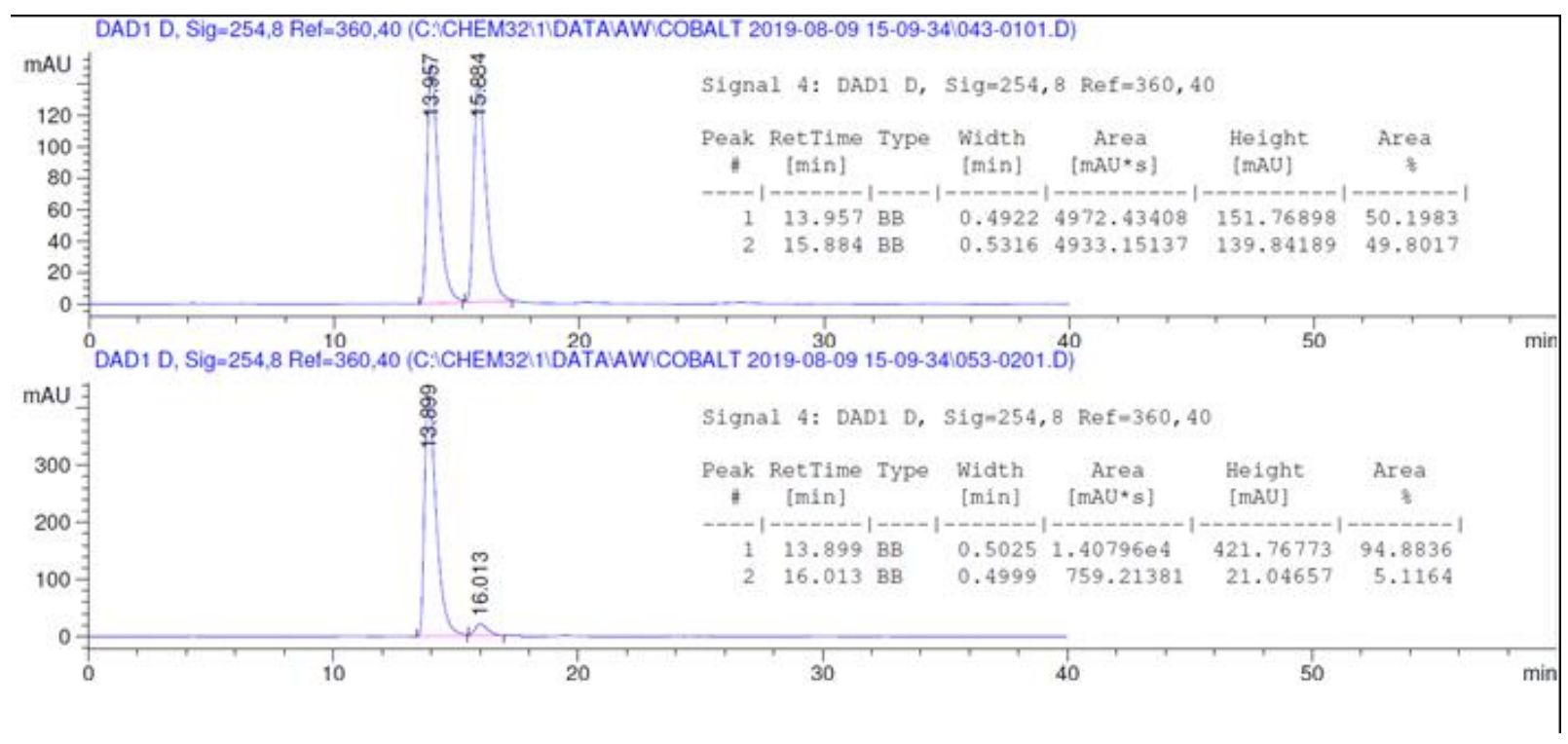




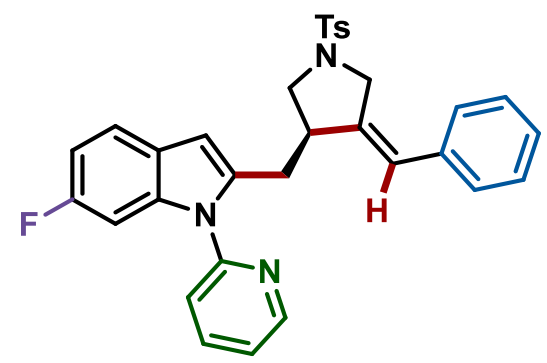

Synthesized following GP3. The product was isolated by flash column chromatography 10$>30 \%$ EtOAc/pentane. The product appeared as a white solid, $107 \mathrm{mg}$ (99\% yield).

${ }^{1} \mathbf{H}$ NMR $\left(500 \mathrm{MHz}, \mathrm{CDCl}_{3}\right) \delta 8.67$ (ddd, $\left.J=4.9,2.0,0.8 \mathrm{~Hz}, 1 \mathrm{H}\right), 7.92$ (ddd, $J=8.0,7.5,2.0$ $\mathrm{Hz}, 1 \mathrm{H}), 7.68(\mathrm{~d}, J=8.3 \mathrm{~Hz}, 2 \mathrm{H}), 7.49(\mathrm{dd}, J=8.6,5.4 \mathrm{~Hz}, 1 \mathrm{H}), 7.43(\mathrm{dt}, J=8.0,0.8 \mathrm{~Hz}, 1 \mathrm{H})$, 7.37 (ddd, $J=7.5,4.9,1.0 \mathrm{~Hz}, 2 \mathrm{H}), 7.33(\mathrm{t}, J=7.6 \mathrm{~Hz}, 4 \mathrm{H}), 7.29$ (d, $J=8.3 \mathrm{~Hz}, 3 \mathrm{H}), 7.27-7.19$ $(\mathrm{m}, 2 \mathrm{H}), 7.08-7.00(\mathrm{~m}, 3 \mathrm{H}), 6.93(\mathrm{ddd}, J=9.5,8.6,2.3 \mathrm{~Hz}, 1 \mathrm{H}), 6.44(\mathrm{~d}, J=0.8 \mathrm{~Hz}, 1 \mathrm{H}), 6.09$ $(\mathrm{q}, J=2.3 \mathrm{~Hz}, 1 \mathrm{H}), 4.16(\mathrm{dt}, J=14.7,2.3 \mathrm{~Hz}, 1 \mathrm{H}), 3.98(\mathrm{dd}, J=14.7,2.3 \mathrm{~Hz}, 1 \mathrm{H}), 3.24(\mathrm{dd}, J=$ 14.8, 4.7 Hz, 1H), $3.22-3.13(\mathrm{~m}, 2 \mathrm{H}), 3.07-2.98(\mathrm{~m}, 1 \mathrm{H}), 2.93(\mathrm{dd}, J=14.8,9.8 \mathrm{~Hz}, 1 \mathrm{H}), 2.41$ $(\mathrm{s}, 3 \mathrm{H})$.

${ }^{13} \mathrm{C}$ NMR $\left(126 \mathrm{MHz}, \mathrm{CDCl}_{3}\right) \delta 160.0(\mathrm{~d}, J=237.8 \mathrm{~Hz}), 151.0,149.8,143.7,139.7,138.7,138.7$ $(\mathrm{d}, J=3.8 \mathrm{~Hz}), 137.2$ (d, $J=12.0 \mathrm{~Hz}), 136.3,132.5,129.7,128.6,128.0,127.8,127.2,124.7$ (d, $J=1.0 \mathrm{~Hz}), 123.6,122.5,120.9(\mathrm{~d}, J=10.0 \mathrm{~Hz}), 120.8,109.4(\mathrm{~d}, J=24.2 \mathrm{~Hz}), 103.9,97.1(\mathrm{~d}, J$ $=27.2 \mathrm{~Hz}), 51.6,50.7,44.6,32.0,21.5$.

${ }^{19}$ F NMR $\left(377 \mathrm{MHz}, \mathrm{CDCl}_{3}\right) \delta-120.4$.

IR (ATR) 1582, 1482, 1470, 1435, 1349, 1168, 1150, 1094, 1016, 833, 815, 778, 738

HRMS (DART, M+H) Calculated for $\mathrm{C}_{32} \mathrm{H}_{29} \mathrm{~N}_{3} \mathrm{O}_{2} \mathrm{FS} 538.1959$, found 538.1960

MP $130-133{ }^{\circ} \mathrm{C}$

$\boldsymbol{\alpha}_{\boldsymbol{D}}^{\mathbf{2 0}}\left(\mathrm{c}=0.355, \mathrm{CH}_{2} \mathrm{Cl}_{2}\right) 11.83$

HPLC: IB, $15 \%$ IPA/Hex, $0.75 \mathrm{~mL} / \mathrm{min}$

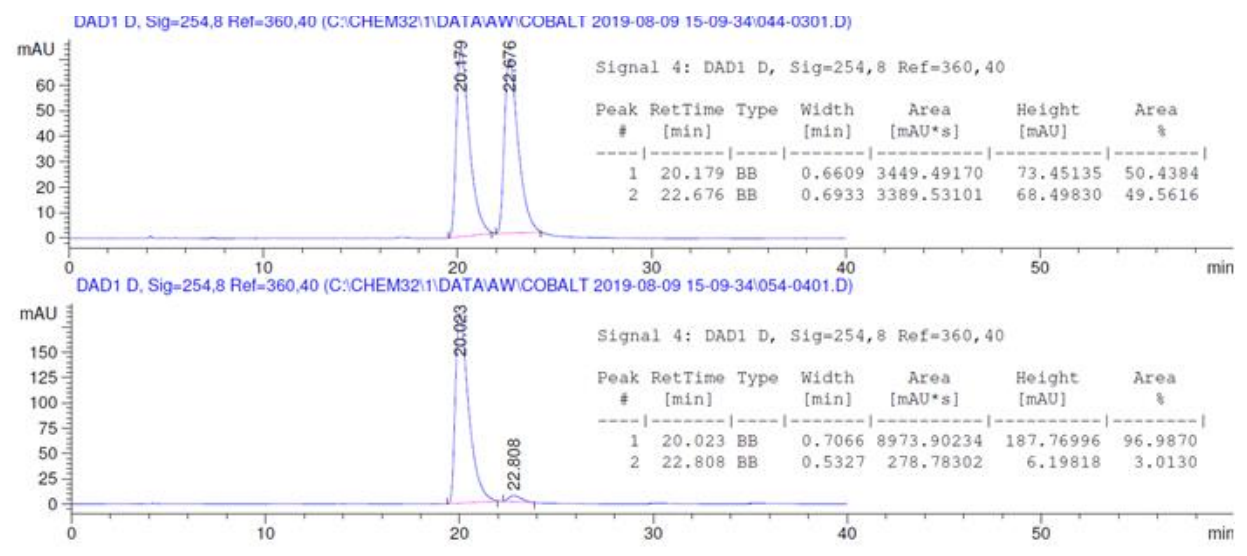




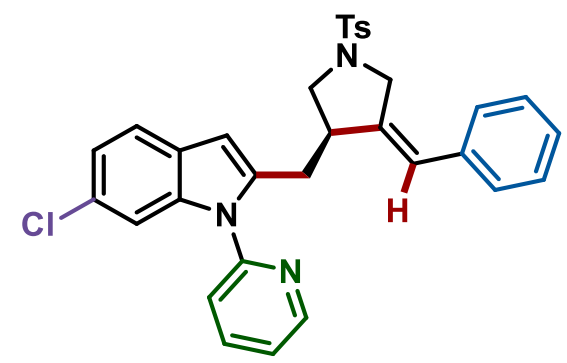

Synthesized following GP3. The product was isolated by flash column chromatography 10$>30 \%$ EtOAc/pentane. The product appeared as a white solid, $110 \mathrm{mg}$ (99\% yield).

${ }^{1} \mathbf{H}$ NMR $\left(500 \mathrm{MHz}, \mathrm{CDCl}_{3}\right) \delta 8.67(\mathrm{ddd}, J=4.8,2.0,0.8 \mathrm{~Hz}, 1 \mathrm{H}), 7.93(\mathrm{td}, J=7.7,2.0 \mathrm{~Hz}$, $1 \mathrm{H}), 7.68(\mathrm{~d}, J=8.3 \mathrm{~Hz}, 2 \mathrm{H}), 7.49(\mathrm{~d}, J=8.4 \mathrm{~Hz}, 1 \mathrm{H}), 7.43(\mathrm{dt}, J=7.7,1.0 \mathrm{~Hz}, 1 \mathrm{H}), 7.38$ (ddd, $J=7.7,4.8,1.0 \mathrm{~Hz}, 1 \mathrm{H}), 7.36-7.26(\mathrm{~m}, 5 \mathrm{H}), 7.27-7.19(\mathrm{~m}, 2 \mathrm{H}), 7.13(\mathrm{dd}, J=8.3,1.8 \mathrm{~Hz}$, $1 \mathrm{H}), 7.05(\mathrm{~d}, J=7.3 \mathrm{~Hz}, 2 \mathrm{H}), 6.44(\mathrm{~d}, J=0.8 \mathrm{~Hz}, 1 \mathrm{H}), 6.08(\mathrm{q}, J=2.3 \mathrm{~Hz}, 1 \mathrm{H}), 4.16(\mathrm{dt}, J=$ $14.9,2.3 \mathrm{~Hz}, 1 \mathrm{H}), 3.97$ (dd, $J=14.9,2.3 \mathrm{~Hz}, 1 \mathrm{H}$ ), 3.23 (ddd, $J=14.9,4.9,0.9 \mathrm{~Hz}, 1 \mathrm{H}$ ), $3.22-$ $3.12(\mathrm{~m}, 2 \mathrm{H}), 3.07-2.98(\mathrm{~m}, 1 \mathrm{H}), 2.94(\mathrm{dd}, J=14.9,9.7 \mathrm{~Hz}, 1 \mathrm{H}), 2.41(\mathrm{~s}, 3 \mathrm{H})$.

${ }^{13} \mathrm{C}$ NMR $\left(126 \mathrm{MHz}, \mathrm{CDCl}_{3}\right) \delta 150.8,149.8,143.7,139.6,139.1,138.8,137.6,136.2,132.5$, $129.7,128.6,128.1,128.0,127.8,127.2$, 126.9, 123.6, 122.6, 121.5, 121.0, 110.3, 103.9, 51.6, $50.7,44.5,31.9,21.5$.

IR (ATR) 1586, 1440, 1471, 1347, 1163, 1100, 1041, 906, 816, 722, 670

HRMS (DART, M+H) Calculated for $\mathrm{C}_{32} \mathrm{H}_{29} \mathrm{~N}_{3} \mathrm{O}_{2} \mathrm{SCl} 554.1664$, found 554.1671

MP $150-151{ }^{\circ} \mathrm{C}$

$\boldsymbol{\alpha}_{D}^{\mathbf{2 0}}\left(\mathrm{c}=0.11, \mathrm{CH}_{2} \mathrm{Cl}_{2}\right) 14.54$

HPLC: IB, 30\% IPA/Hex, $0.5 \mathrm{~mL} / \mathrm{min}$

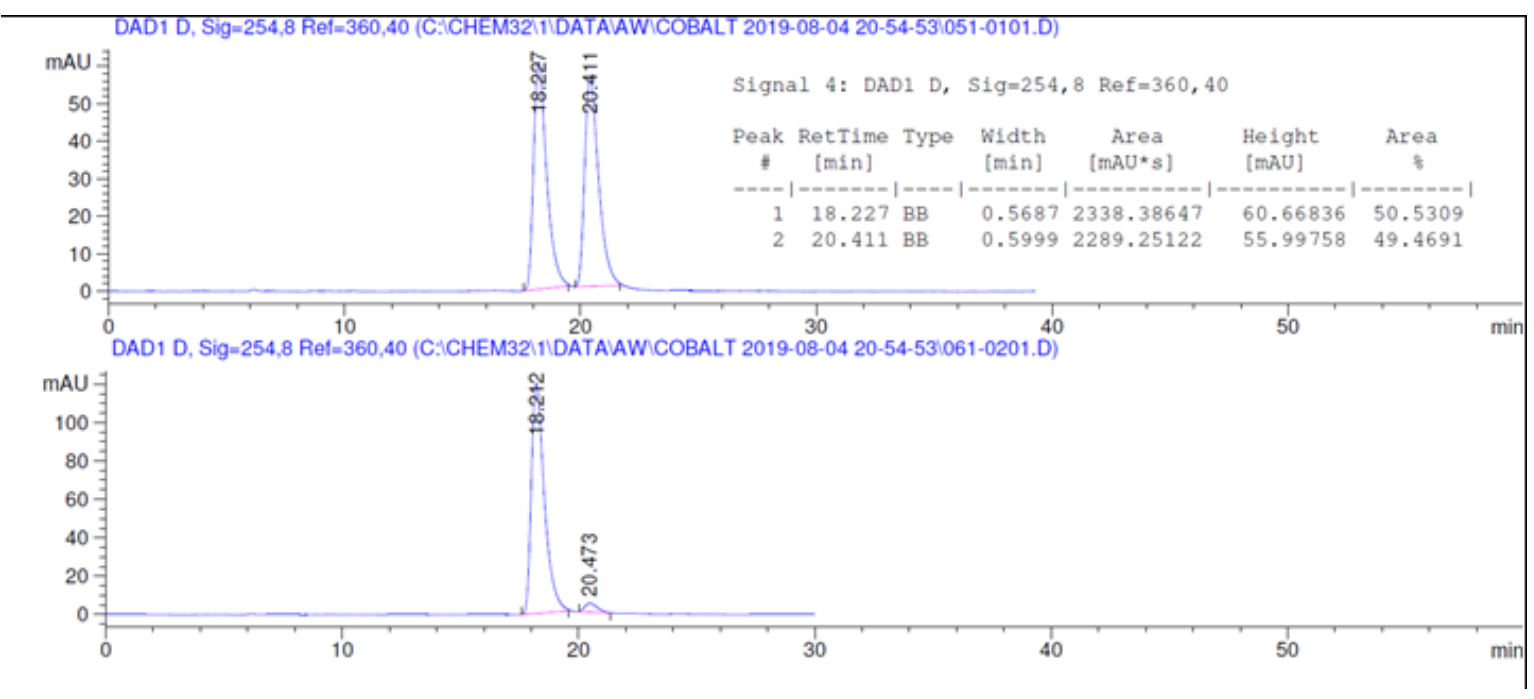




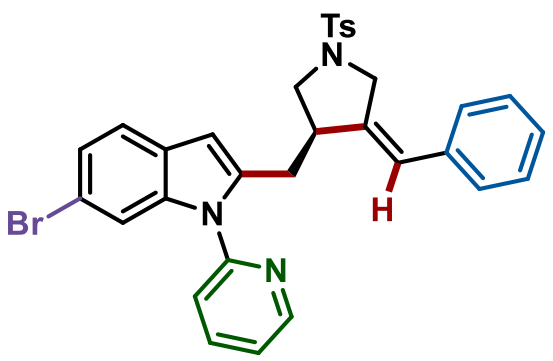

Synthesized following GP3. The product was isolated by flash column chromatography 10$>30 \%$ EtOAc/pentane. The product appeared as a white solid, $111 \mathrm{mg}$ (93\% yield).

${ }^{1} \mathbf{H}$ NMR $\left(500 \mathrm{MHz}, \mathrm{CDCl}_{3}\right) \delta 8.67(\mathrm{ddd}, J=4.9,2.0,0.8 \mathrm{~Hz}, 1 \mathrm{H}), 7.93(\mathrm{ddd}, J=8.0,7.5,2.0$ $\mathrm{Hz}, 1 \mathrm{H}), 7.68(\mathrm{~d}, J=8.3 \mathrm{~Hz}, 2 \mathrm{H}), 7.48-7.41(\mathrm{~m}, 3 \mathrm{H}), 7.38(\mathrm{ddd}, J=7.5,4.9,1.0 \mathrm{~Hz}, 1 \mathrm{H}), 7.33$ $(\mathrm{t}, J=7.6 \mathrm{~Hz}, 2 \mathrm{H}), 7.29(\mathrm{~d}, J=8.3 \mathrm{~Hz}, 2 \mathrm{H}), 7.26(\mathrm{dd}, J=8.3,1.7 \mathrm{~Hz}, 1 \mathrm{H}), 7.25-7.19(\mathrm{~m}, 1 \mathrm{H})$, $7.05(\mathrm{~d}, J=7.2 \mathrm{~Hz}, 2 \mathrm{H}), 6.44(\mathrm{~d}, J=0.8 \mathrm{~Hz}, 1 \mathrm{H}), 6.08(\mathrm{q}, J=2.3 \mathrm{~Hz}, 1 \mathrm{H}), 4.16(\mathrm{dt}, J=14.8,2.3$ $\mathrm{Hz}, 1 \mathrm{H}), 3.97$ (dd, $J=14.8,2.3 \mathrm{~Hz}, 1 \mathrm{H}), 3.26-3.12(\mathrm{~m}, 3 \mathrm{H}), 3.07-2.98(\mathrm{~m}, 1 \mathrm{H}), 2.93$ (ddd, $J=$ $14.8,9.7,0.7 \mathrm{~Hz}, 1 \mathrm{H}), 2.40$ (s, 3H).

${ }^{13} \mathrm{C}$ NMR $\left(126 \mathrm{MHz}, \mathrm{CDCl}_{3}\right) \delta 150.7,149.8,143.7,139.5,139.0,138.8,138.0,136.2,132.5$, $129.7,128.6,128.0,127.8,127.2,124.1,123.7,122.7,121.4,121.1,115.7,113.2,103.9,51.6$, $50.6,44.5,31.9,21.5$.

IR (ATR) 1589, 1437, 1471, 1347, 1163, 1097, 913, 1038, 816, 733, 667

HRMS (DART, M+H) Calculated for $\mathrm{C}_{32} \mathrm{H}_{29} \mathrm{~N}_{3} \mathrm{O}_{2} \mathrm{SBr} 598.1158$, found 598.1154

MP $144-145^{\circ} \mathrm{C}$

$\boldsymbol{\alpha}_{\boldsymbol{D}}^{\mathbf{2 0}}\left(\mathrm{c}=0.275, \mathrm{CH}_{2} \mathrm{Cl}_{2}\right) 8.00$

HPLC: IB, $15 \%$ IPA/Hex, $0.75 \mathrm{~mL} / \mathrm{min}$

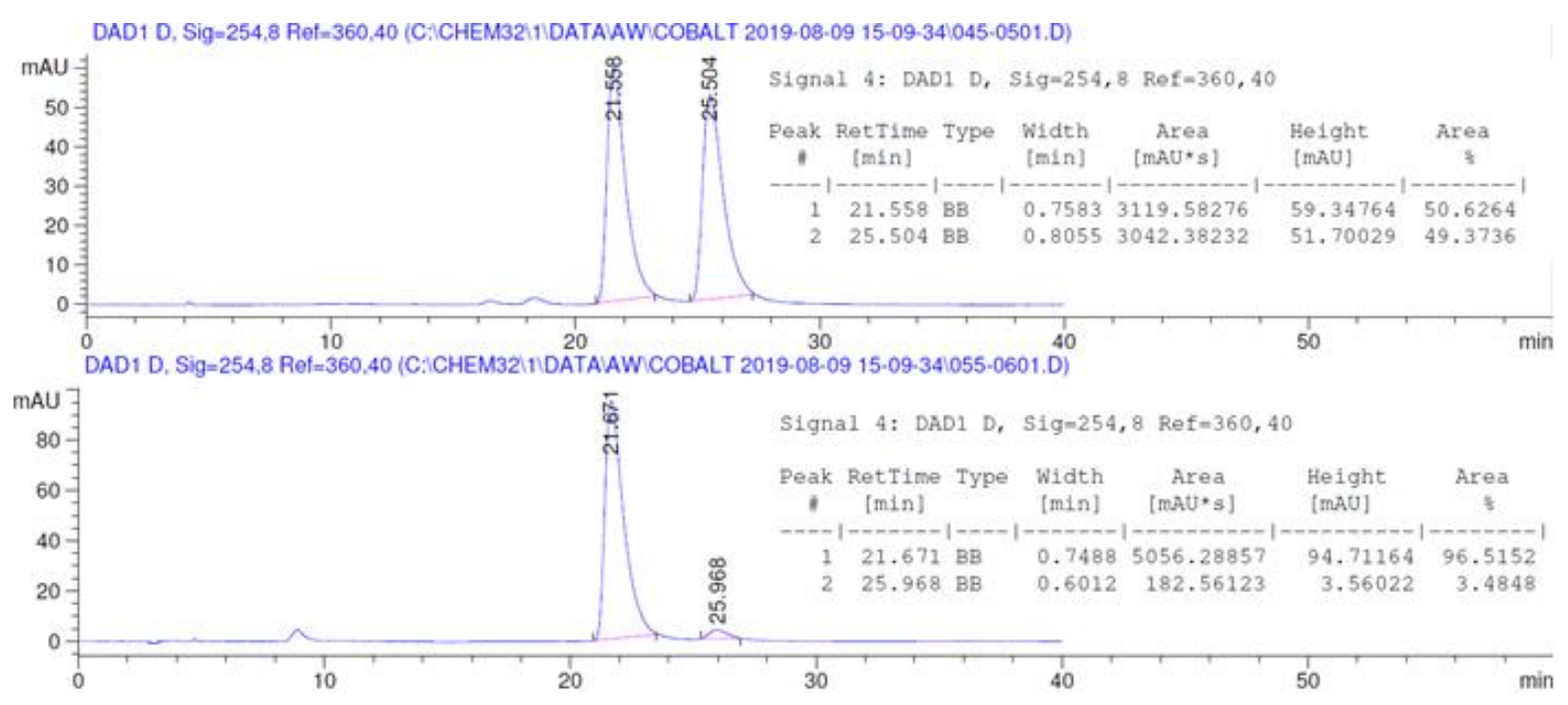


<smiles>[As]N1CC(=Cc2ccccc2)[C@H](Cc2cccn2-c2ccccn2)C1</smiles>

Synthesized following GP3 but $0.3 \mathrm{mmol}$ of the pyrrole and $0.2 \mathrm{mmol}$ of the enyne was employed. The product was isolated by flash column chromatography 10->30\% EtOAc/pentane. The product appeared as a white solid, $81 \mathrm{mg}$ (83\% yield).

${ }^{1} \mathbf{H}$ NMR $\left(500 \mathrm{MHz}, \mathrm{CDCl}_{3}\right) \delta 8.50(\mathrm{ddd}, J=4.9,2.0,0.9 \mathrm{~Hz}, 1 \mathrm{H}), 7.78(\mathrm{ddd}, J=8.2,7.4,2.0$ $\mathrm{Hz}, 1 \mathrm{H}), 7.69$ (d, $J=8.3 \mathrm{~Hz}, 2 \mathrm{H}), 7.35-7.27(\mathrm{~m}, 5 \mathrm{H}), 7.25-7.19(\mathrm{~m}, 2 \mathrm{H}), 7.08$ (d, $J=7.2 \mathrm{~Hz}$, $2 \mathrm{H}), 6.99(\mathrm{dd}, J=3.1,1.7 \mathrm{~Hz}, 1 \mathrm{H}), 6.24(\mathrm{t}, J=3.1 \mathrm{~Hz}, 1 \mathrm{H}), 6.17$ (q, $J=2.2 \mathrm{~Hz}, 1 \mathrm{H}), 6.09$ (dd, $J$ $=3.1,1.7 \mathrm{~Hz}, 1 \mathrm{H}), 4.14(\mathrm{dt}, J=14.8,2.2 \mathrm{~Hz}, 1 \mathrm{H}), 4.01(\mathrm{dd}, J=14.8,2.2 \mathrm{~Hz}, 1 \mathrm{H}), 3.33(\mathrm{dd}, J=$ 14.8, 5.2 Hz, 1H), 3.18 (m, 2H), 3.09 (m, 1H), 2.91 (dd, J = 14.8, $9.5 \mathrm{~Hz}, 1 \mathrm{H}), 2.40$ (s, 3H).

${ }^{13}$ C NMR $\left(126 \mathrm{MHz}, \mathrm{CDCl}_{3}\right) \delta 152.9,148.6,143.6,140.2,138.5,136.6,132.7,131.3,129.7$, 128.5, 128.1, 127.8, 127.0, 123.2, 121.2, 120.8, 117.1, 111.0, 109.4, 51.8, 50.8, 45.1, 32.1, 21.5. IR (ATR) 1585, 1477, 1439, 1333, 1148, 1093, 1065, 1050, 783, 722, 692, 667

HRMS (DART, M+H) Calculated for $\mathrm{C}_{28} \mathrm{H}_{28} \mathrm{~N}_{3} \mathrm{O}_{2} \mathrm{~S}_{1} 470.1897$, found 470.1895

MP $95-100{ }^{\circ} \mathrm{C}$

$\boldsymbol{\alpha}_{D}^{\mathbf{2 0}}\left(\mathrm{c}=0.15, \mathrm{CH}_{2} \mathrm{Cl}_{2}\right) 6.06$

HPLC: AS-H, 20\% IPA/Hex, $0.5 \mathrm{~mL} / \mathrm{min}$

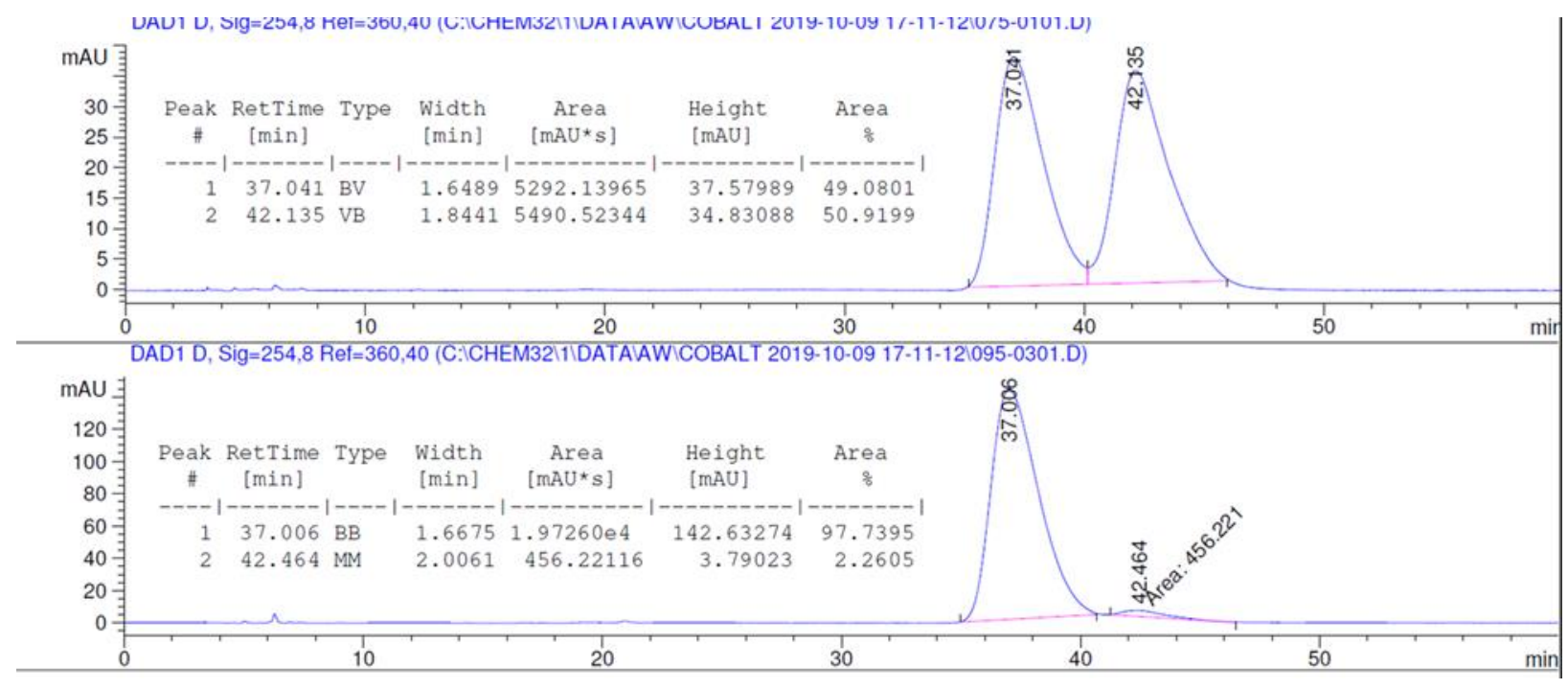


<smiles>Cc1ccc(C[C@@H]2CN([As])CC2=Cc2ccccc2)n1-c1ccccn1</smiles>

Synthesized following GP3. The product was isolated by flash column chromatography 10$>30 \%$ EtOAc/pentane. The product appeared as a white solid, $83 \mathrm{mg}$ ( $86 \%$ yield).

${ }^{1} \mathbf{H}$ NMR $\left(500 \mathrm{MHz}, \mathrm{CDCl}_{3}\right) \delta 8.62(\mathrm{ddd}, J=4.9,2.0,0.8 \mathrm{~Hz}, 1 \mathrm{H}), 7.85(\mathrm{td}, J=7.7,2.0 \mathrm{~Hz}$, $1 \mathrm{H}), 7.68(\mathrm{~d}, J=8.3 \mathrm{~Hz}, 2 \mathrm{H}), 7.36-7.27(\mathrm{~m}, 5 \mathrm{H}), 7.25-7.19(\mathrm{~m}, 2 \mathrm{H}), 7.04(\mathrm{~d}, J=7.4 \mathrm{~Hz}, 2 \mathrm{H})$, $5.98(\mathrm{q}, J=2.3 \mathrm{~Hz}, 1 \mathrm{H}), 5.94(\mathrm{~d}, J=3.8 \mathrm{~Hz}, 2 \mathrm{H}), 4.10(\mathrm{dt}, J=14.8,2.3 \mathrm{~Hz}, 1 \mathrm{H}), 3.96(\mathrm{dd}, J=$ $14.8,2.3 \mathrm{~Hz}, 1 \mathrm{H}), 3.21-3.08(\mathrm{~m}, 2 \mathrm{H}), 2.89-2.78(\mathrm{~m}, 2 \mathrm{H}), 2.60(\mathrm{dd}, J=16.2,11.1 \mathrm{~Hz}, 1 \mathrm{H})$, $2.41(\mathrm{~s}, 3 \mathrm{H}), 2.12(\mathrm{~s}, 3 \mathrm{H})$.

${ }^{13} \mathrm{C}$ NMR $\left(126 \mathrm{MHz}, \mathrm{CDCl}_{3}\right) \delta 151.9,149.5,143.6,140.1,138.2,136.5,132.6,130.3,129.7$, $129.1,128.5,128.0,127.8,127.0,123.2,122.7,122.3,107.8,107.2,51.7,50.7,45.0,31.6,21.5$, 13.1 .

IR (ATR) 1588, 1479, 1440, 1408, 1341, 1164, 1104, 1041, 794, 755, 702, 670

HRMS (DART, $\mathrm{M}+\mathrm{H}$ ) Calculated for $\mathrm{C}_{29} \mathrm{H}_{30} \mathrm{~N}_{3} \mathrm{O}_{2} \mathrm{~S}_{1} 484.2053$, found 484.2058

MP $56^{\circ} \mathrm{C}$

$\boldsymbol{\alpha}_{D}^{\mathbf{2 0}}\left(\mathrm{c}=0.195, \mathrm{CH}_{2} \mathrm{Cl}_{2}\right) 27.7$

HPLC: AD-H, 10\% IPA/Hexane, $0.5 \mathrm{~mL} / \mathrm{min}$

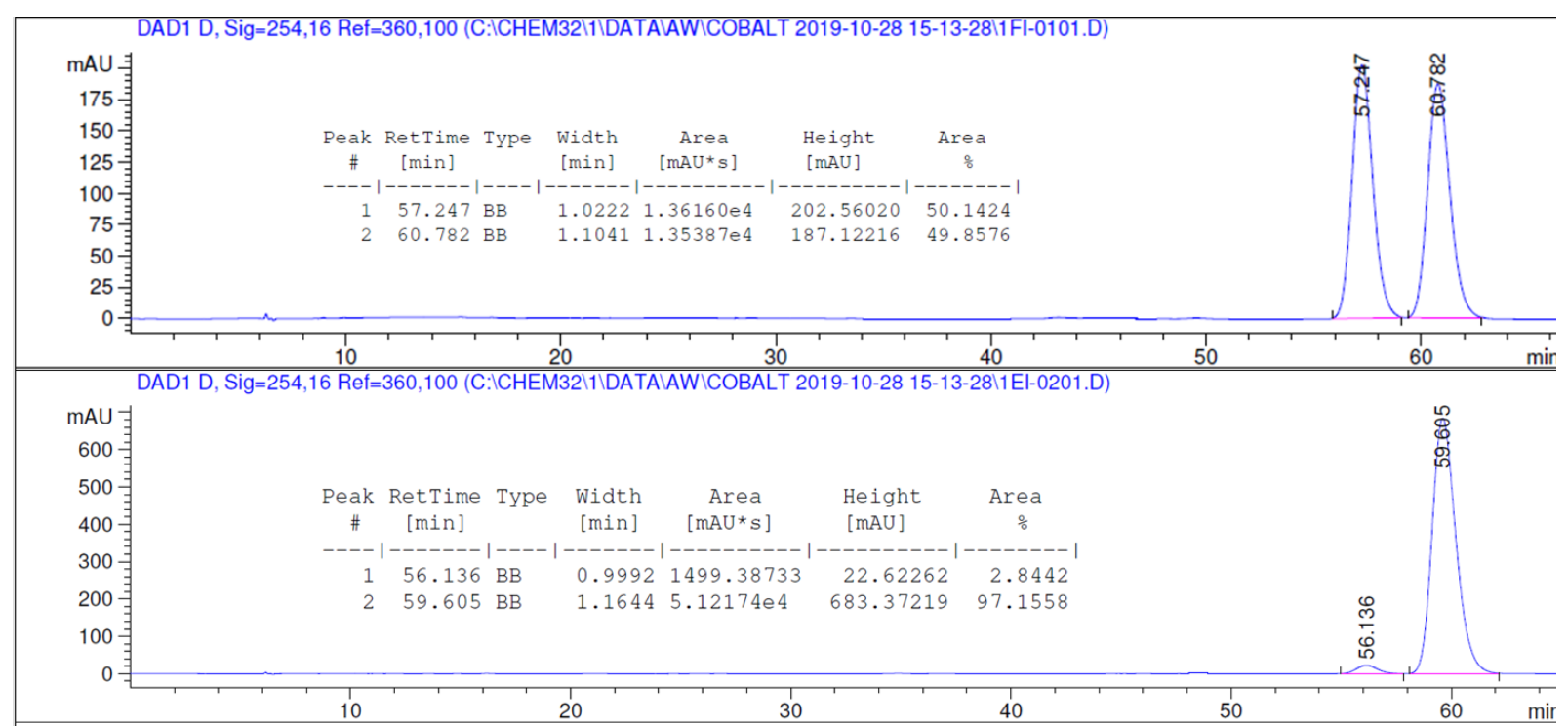




\section{(R,Z)-2-(2-((4-benzylidene-1-tosylpyrrolidin-3-yl)methyl)phenyl)pyridine (3y)}<smiles>[As]N1CC(=Cc2ccccc2)C(Cc2ccccc2-c2ccccn2)C1</smiles>

Synthesized following GP3 but $0.3 \mathrm{mmol}$ of the pyrrole and $0.2 \mathrm{mmol}$ of the enyne was employed. The product was isolated by flash column chromatography 10->20\% EtOAc/pentane. The product appeared as a white solid, $65 \mathrm{mg}$ (68\% yield).

${ }^{1} \mathbf{H}$ NMR $\left(500 \mathrm{MHz}, \mathrm{CDCl}_{3}\right) \delta 8.68(\mathrm{ddd}, J=4.9,1.9,1.0 \mathrm{~Hz}, 1 \mathrm{H}), 7.76(\mathrm{td}, J=7.7,1.9 \mathrm{~Hz}$, $1 \mathrm{H}), 7.65(\mathrm{~d}, J=8.3 \mathrm{~Hz}, 2 \mathrm{H}), 7.40-7.19(\mathrm{~m}, 13 \mathrm{H}), 7.02(\mathrm{~d}, J=7.2 \mathrm{~Hz}, 2 \mathrm{H}), 5.88(\mathrm{q}, J=2.3 \mathrm{~Hz}$, $1 \mathrm{H}), 4.10-4.02(\mathrm{~m}, 1 \mathrm{H}), 3.98-3.91(\mathrm{~m}, 1 \mathrm{H}), 3.29(\mathrm{dd}, J=13.3,5.5 \mathrm{~Hz}, 1 \mathrm{H}), 3.03(\mathrm{~d}, J=5.7$ $\mathrm{Hz}, 2 \mathrm{H}), 2.91-2.81(\mathrm{~m}, 1 \mathrm{H}), 2.77$ (dd, $J=13.3,9.5 \mathrm{~Hz}, 1 \mathrm{H}), 2.40$ (s, 3H).

${ }^{13}$ C NMR $\left(126 \mathrm{MHz}, \mathrm{CDCl}_{3}\right) \delta 160.1,149.0,143.5,140.5,139.9,137.5,136.6,136.5,132.7$, $130.8,130.1,129.7,128.5,128.0,127.8,126.9,126.7,124.2,123.1,121.9,51.5,50.6,46.3,37.0$, 21.5.

IR (ATR) 1587, 1471, 1445, 1341, 1158, 1092, 1033, 814, 751, 698, 663, 588

HRMS (DART, M+H) Calculated for $\mathrm{C}_{30} \mathrm{H}_{29} \mathrm{~N}_{2} \mathrm{O}_{2} \mathrm{~S}_{1} 481.1944$, found 481.1952

MP $65-70{ }^{\circ} \mathrm{C}$

$\boldsymbol{\alpha}_{\boldsymbol{D}}^{\mathbf{2 0}}\left(\mathrm{c}=0.375, \mathrm{CH}_{2} \mathrm{Cl}_{2}\right)-5.33$

HPLC: AS-H, 25\% IPA/Hex, $0.5 \mathrm{~mL} / \mathrm{min}$

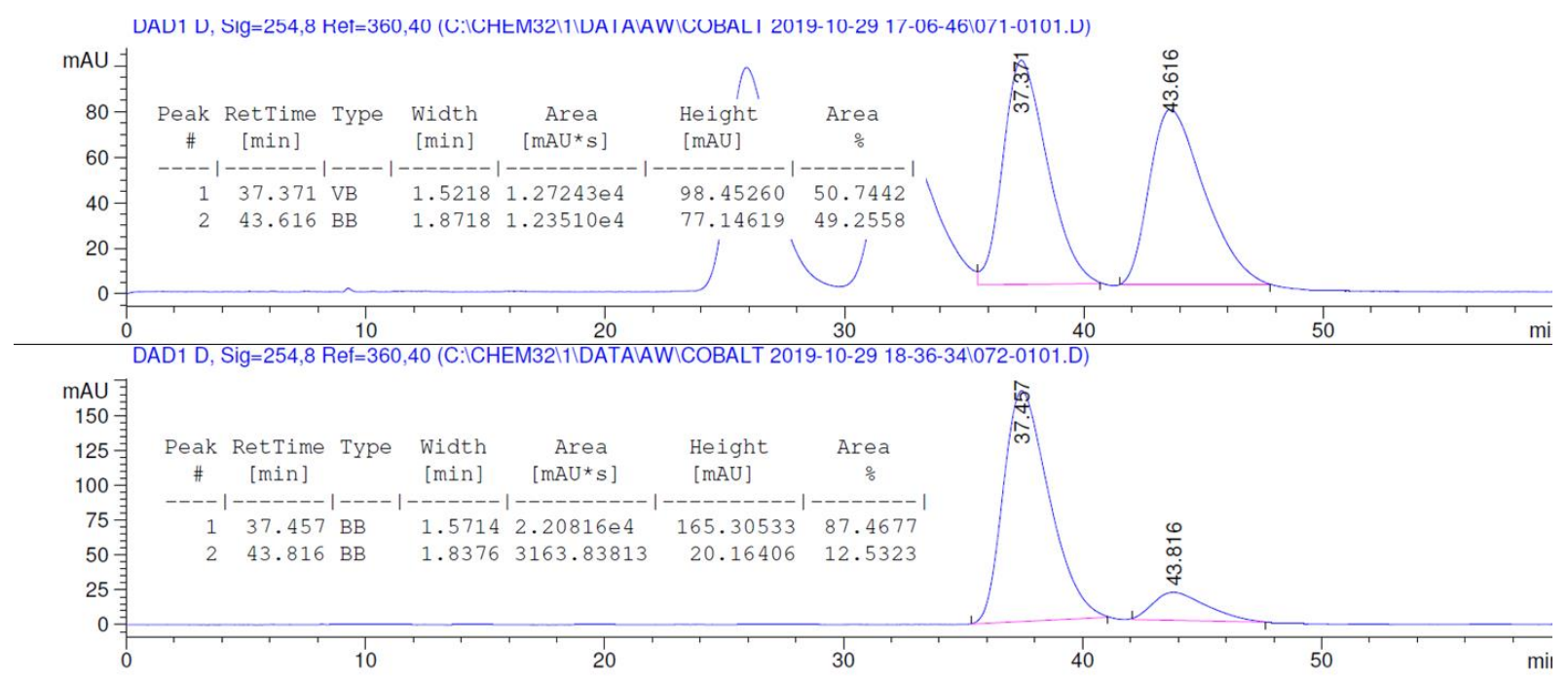




\section{(R,Z)-1-(2-((4-benzylidene-1-tosylpyrrolidin-3-yl)methyl)phenyl)ethan-1-one (3z)}<smiles>CC(=O)c1ccccc1C[C@@H]1CN([As])CC1=Cc1ccccc1</smiles>

Synthesized following GP3 but $0.3 \mathrm{mmol}$ of acetophenone and $0.2 \mathrm{mmol}$ of the enyne was employed. The product was isolated by flash column chromatography 10->20\% EtOAc/pentane. The product appeared as a white solid, $54 \mathrm{mg}$ (61\% yield).

${ }^{1} \mathbf{H}$ NMR $\left(500 \mathrm{MHz}, \mathrm{CDCl}_{3}\right) \delta 7.78(\mathrm{dd}, J=7.8,1.3 \mathrm{~Hz}, 1 \mathrm{H}), 7.70(\mathrm{~d}, J=8.2 \mathrm{~Hz}, 2 \mathrm{H}), 7.46-$ $7.41(\mathrm{~m}, 1 \mathrm{H}), 7.34$ (dddd, $J=7.8,6.7,3.8,1.5 \mathrm{~Hz}, 3 \mathrm{H}), 7.30(\mathrm{~d}, J=8.2 \mathrm{~Hz}, 2 \mathrm{H}), 7.24-7.18(\mathrm{~m}$, 2H), $7.14-7.09(\mathrm{~m}, 2 \mathrm{H}), 6.27(\mathrm{q}, J=2.3 \mathrm{~Hz}, 1 \mathrm{H}), 4.30(\mathrm{dt}, J=14.8,2.3 \mathrm{~Hz}, 1 \mathrm{H}), 4.02(\mathrm{dd}, J=$ $14.8,2.3 \mathrm{~Hz}, 1 \mathrm{H}), 3.38$ (dd, $J=12.8,5.7 \mathrm{~Hz}, 1 \mathrm{H}), 3.19(\mathrm{dd}, J=9.1,4.2 \mathrm{~Hz}, 1 \mathrm{H}), 3.13(\mathrm{~m}, 1 \mathrm{H})$, $3.03(\mathrm{dd}, J=9.1,6.4 \mathrm{~Hz}, 1 \mathrm{H}), 2.83(\mathrm{dd}, J=12.8,9.2 \mathrm{~Hz}, 1 \mathrm{H}), 2.59$ (s, 3H), $2.40(\mathrm{~s}, 3 \mathrm{H})$.

${ }^{13}$ C NMR $\left(126 \mathrm{MHz}, \mathrm{CDCl}_{3}\right) \delta 201.3,143.6,139.9,139.9,137.0,136.6,132.8,132.6,131.9$, $130.3,129.7,128.5,128.1,127.8,126.9,126.7,123.6,51.5,50.7,46.2,38.3,29.6,21.5$.

IR (ATR) 1673, 1600, 1457, 1341, 1245, 1150, 1091, 1048, 826, 807, 757, 692, 664

HRMS (DART, M+H) Calculated for $\mathrm{C}_{27} \mathrm{H}_{28} \mathrm{~N}_{1} \mathrm{O}_{3} \mathrm{~S}_{1} 446.1784$, found 446.1781

MP $117^{\circ} \mathrm{C}$

$\boldsymbol{\alpha}_{D}^{\mathbf{2 0}}\left(\mathrm{c}=0.10, \mathrm{CH}_{2} \mathrm{Cl}_{2}\right) 6.00$

HPLC: AD-H, 20\% IPA/Hex, $0.5 \mathrm{~mL} / \mathrm{min}$

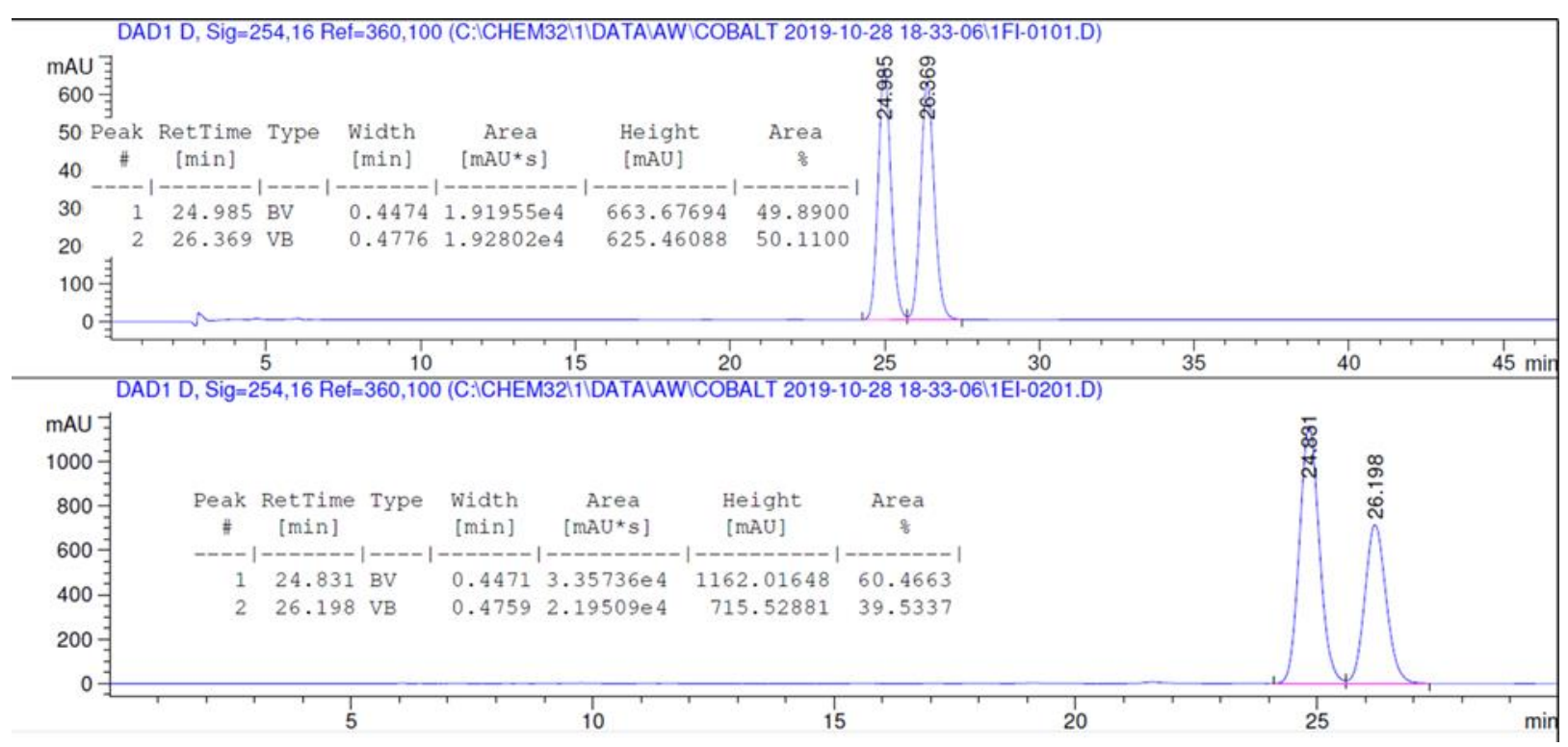




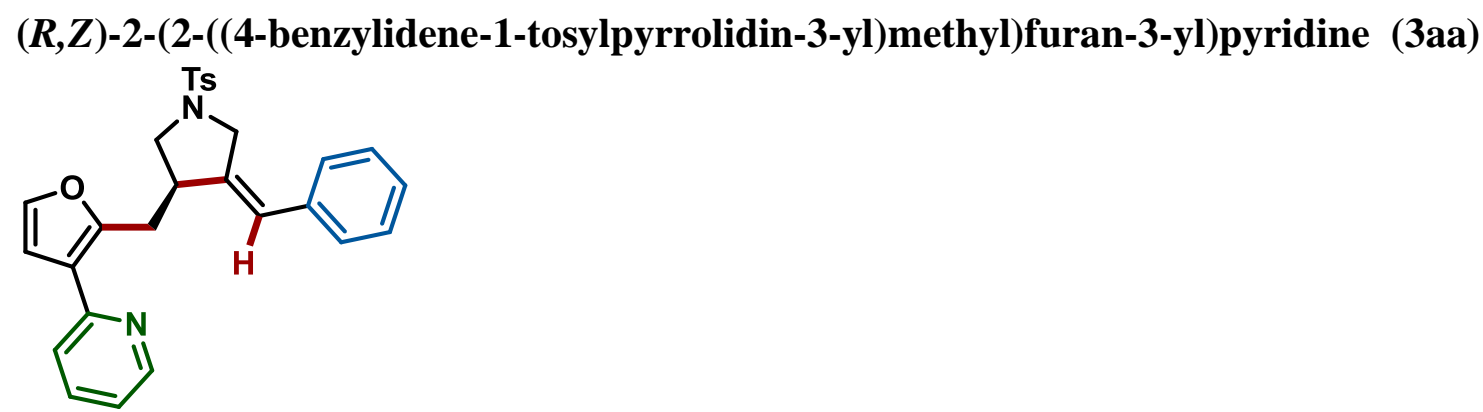

Synthesized following GP3. The product was isolated by flash column chromatography 15$>30 \% \mathrm{EtOAc/pentane.} \mathrm{The} \mathrm{product} \mathrm{appeared} \mathrm{as} \mathrm{a} \mathrm{white} \mathrm{solid,} 74 \mathrm{mg}$ (79\% yield).

${ }^{1} \mathbf{H}$ NMR $\left(500 \mathrm{MHz}, \mathrm{CDCl}_{3}\right) \delta 8.57(\mathrm{ddd}, J=4.8,1.9,1.0 \mathrm{~Hz}, 1 \mathrm{H}), 7.68(\mathrm{~d}, J=8.3 \mathrm{~Hz}, 2 \mathrm{H}), 7.65$ $(\mathrm{td}, J=7.7,1.9 \mathrm{~Hz}, 1 \mathrm{H}), 7.38(\mathrm{dt}, J=8.0,1.0 \mathrm{~Hz}, 1 \mathrm{H}), 7.36(\mathrm{~d}, J=1.9 \mathrm{~Hz}, 1 \mathrm{H}), 7.35-7.24(\mathrm{~m}$, 4H), $7.25-7.18(\mathrm{~m}, 1 \mathrm{H}), 7.11(\mathrm{ddd}, J=7.7,4.8,1.0 \mathrm{~Hz}, 1 \mathrm{H}), 7.08-7.02(\mathrm{~m}, 2 \mathrm{H}), 6.69(\mathrm{~d}, J=$ $1.9 \mathrm{~Hz}, 1 \mathrm{H}), 6.23(\mathrm{t}, J=2.2 \mathrm{~Hz}, 1 \mathrm{H}), 4.16(\mathrm{dt}, J=14.6,2.2 \mathrm{~Hz}, 1 \mathrm{H}), 4.08(\mathrm{dd}, J=14.7,2.2 \mathrm{~Hz}$, $1 \mathrm{H}), 3.52(\mathrm{dd}, J=14.2,5.6 \mathrm{~Hz}, 1 \mathrm{H}), 3.39-3.27(\mathrm{~m}, 2 \mathrm{H}), 3.27-3.15(\mathrm{~m}, 2 \mathrm{H}), 2.40(\mathrm{~s}, 3 \mathrm{H})$.

${ }^{13} \mathrm{C}$ NMR $\left(126 \mathrm{MHz}, \mathrm{CDCl}_{3}\right) \delta 153.0,152.5,149.4,143.5,141.1,139.7,136.6,136.4,132.8$, $129.7,128.5,128.1,127.8,127.0,123.3,121.5,121.0,110.0,52.0,50.7,44.2,31.5,21.5$.

IR (ATR) 2007, 1613, 1341, 1156, 1092, 1041, 1002, 814, 789, 743, 705, 695, 663

HRMS (DART, $\mathrm{M}+\mathrm{H}$ ) Calculated for $\mathrm{C}_{28} \mathrm{H}_{27} \mathrm{~N}_{2} \mathrm{O}_{3} \mathrm{~S} 471.17363$, found 171.17369

MP $47-50{ }^{\circ} \mathrm{C}$

$\boldsymbol{\alpha}_{D}^{\mathbf{2 0}}\left(\mathrm{c}=0.245, \mathrm{CH}_{2} \mathrm{Cl}_{2}\right)-11.4$

HPLC: AS-H 25\% IPA/Hex, $0.5 \mathrm{~mL} / \mathrm{min}$

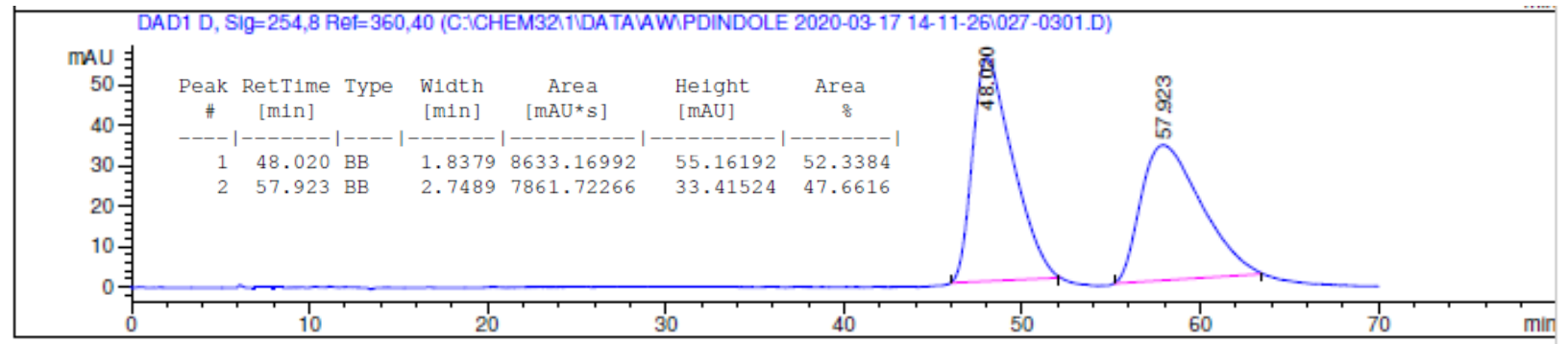

DAD1 D, Sig=254,8 Ref=360,40 (C.lCHEM32 1 \DATAMWMDINDOLE 2020-03-17 14-11-26l026-0201.D)

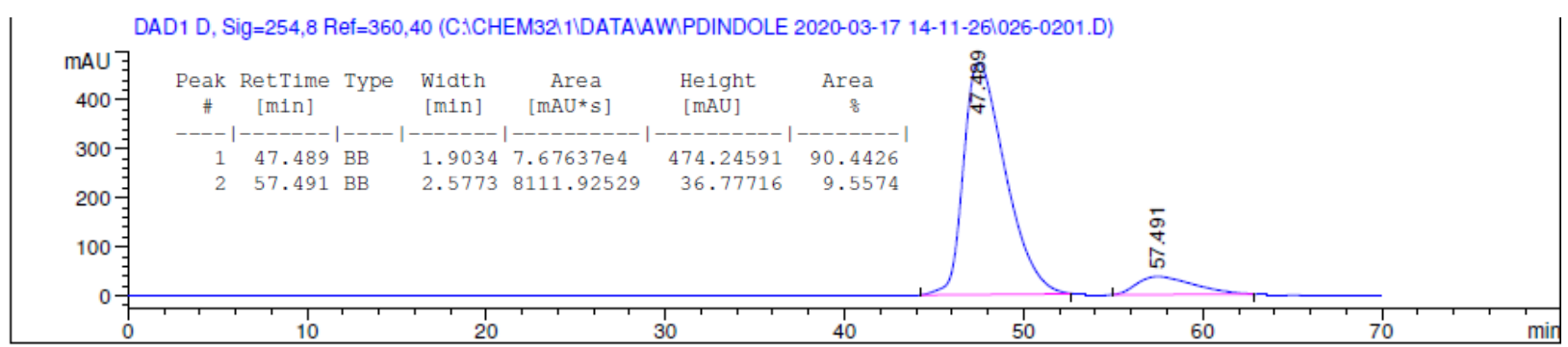




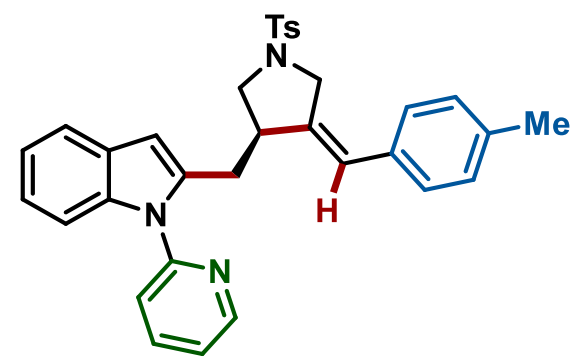

Synthesized following GP3. The product was isolated by flash column chromatography 15$>30 \%$ EtOAc/pentane. The product appeared as a white solid, $99 \mathrm{mg}$ (93\% yield).

${ }^{1} \mathbf{H}$ NMR $\left(500 \mathrm{MHz}, \mathrm{CDCl}_{3}\right) \delta 8.67$ (ddd, J = 4.9, 2.0, $\left.0.8 \mathrm{~Hz}, 1 \mathrm{H}\right), 7.94-7.84(\mathrm{~m}, 1 \mathrm{H}), 7.68(\mathrm{~d}$, $\mathrm{J}=8.3 \mathrm{~Hz}, 2 \mathrm{H}), 7.64-7.57(\mathrm{~m}, 1 \mathrm{H}), 7.48(\mathrm{dt}, \mathrm{J}=8.0,0.8 \mathrm{~Hz}, 1 \mathrm{H}), 7.36-7.33(\mathrm{~m}, 2 \mathrm{H}), 7.29(\mathrm{~d}, \mathrm{~J}$ $=8.3 \mathrm{~Hz}, 2 \mathrm{H}), 7.19-7.15(\mathrm{~m}, 2 \mathrm{H}), 7.14(\mathrm{~d}, \mathrm{~J}=7.9 \mathrm{~Hz}, 2 \mathrm{H}), 6.96(\mathrm{~d}, \mathrm{~J}=7.9 \mathrm{~Hz}, 2 \mathrm{H}), 6.47(\mathrm{~d}, \mathrm{~J}$ $=0.8 \mathrm{~Hz} 1 \mathrm{H}), 6.08(\mathrm{q}, \mathrm{J}=2.3 \mathrm{~Hz}, 1 \mathrm{H}), 4.15(\mathrm{dt}, \mathrm{J}=14.7,2.3 \mathrm{~Hz}, 1 \mathrm{H}), 3.99(\mathrm{dd}, \mathrm{J}=14.7,2.3 \mathrm{~Hz}$, $1 \mathrm{H}), 3.29$ (ddd, J = 14.8, 4.7, $0.8 \mathrm{~Hz}, 1 \mathrm{H}), 3.19-3.15(\mathrm{~m}, 2 \mathrm{H}), 3.03(\mathrm{~m}, 1 \mathrm{H}), 2.94$ (dd, J = 14.8, $9.8 \mathrm{~Hz}, 1 \mathrm{H}), 2.41(\mathrm{~s}, 3 \mathrm{H}), 2.35(\mathrm{~s}, 3 \mathrm{H})$.

${ }^{13} \mathrm{C}$ NMR $\left(126 \mathrm{MHz}, \mathrm{CDCl}_{3}\right) \delta 151.3,149.6,143.6,138.7,138.5,138.3,137.2,137.0,133.5$, 132.6, 129.7, 129.3, 128.4, 128.0, 127.8, 123.3, 122.2, 122.1, 121.0, 120.9, 120.2, 110.1, 104.1, 51.7, 50.7, 44.6, 31.9, 21.5, 21.2.

IR (ATR) 2259, 1510, 1589, 1448, 1475, 1350, 1163, 1097, 1035, 910, 788, 733, 670

HRMS (DART, M+H) Calculated for $\mathrm{C}_{33} \mathrm{H}_{32} \mathrm{~N}_{3} \mathrm{O}_{2} \mathrm{~S} 534.2208$, found 534.2207

MP $76-82{ }^{\circ} \mathrm{C}$

$\boldsymbol{\alpha}_{\boldsymbol{D}}^{\mathbf{2 0}}\left(\mathrm{c}=0.3, \mathrm{CH}_{2} \mathrm{Cl}_{2}\right) 5.33$

HPLC: IB, 50\% IPA/Hex, $1.0 \mathrm{~mL} / \mathrm{min}$

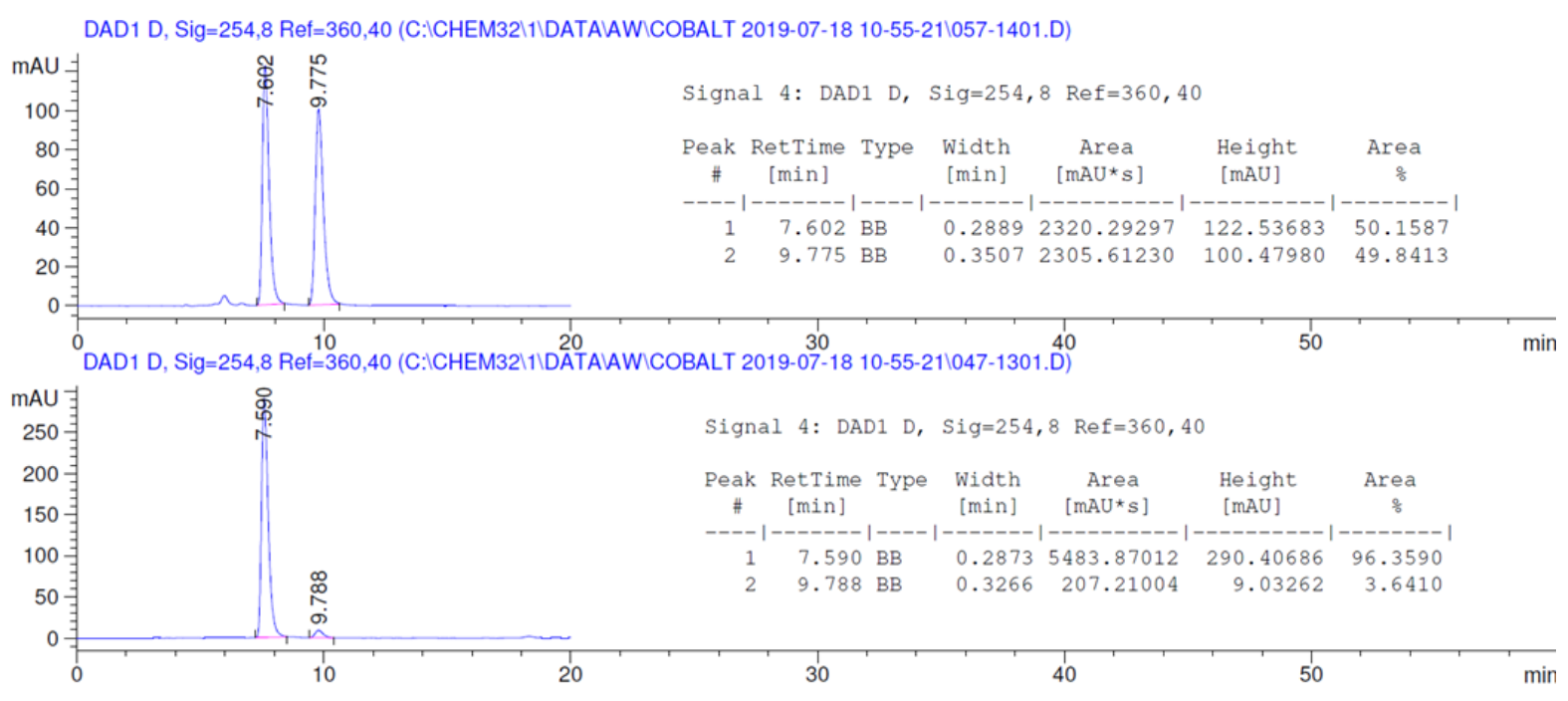


(R,Z)-2-((4-(4-isopropylbenzylidene)-1-tosylpyrrolidin-3-yl)methyl)-1-(pyridin-2-yl)-1Hindole $(4 c)$<smiles>CC(C)c1ccc(/C=C2\C[NH+]([NH3+])CC2Cc2cc3ccccc3n2-c2ccccn2)cc1</smiles>

Synthesized following $\mathbf{G P 3}$ at $80^{\circ} \mathrm{C}$. The product was isolated by flash column chromatography $15->30 \%$ EtOAc/pentane. The product appeared as a white solid, $87 \mathrm{mg}$ (77\% yield).

${ }^{1} \mathbf{H}$ NMR $\left(500 \mathrm{MHz}, \mathrm{CDCl}_{3}\right) \delta 8.67(\mathrm{ddd}, J=4.9,2.0,0.8 \mathrm{~Hz}, 1 \mathrm{H}$ ), 7.91 (ddd, $J=8.0,7.5,2.0$ $\mathrm{Hz}, 1 \mathrm{H}), 7.69(\mathrm{~d}, J=8.2 \mathrm{~Hz}, 2 \mathrm{H}), 7.62-7.56(\mathrm{~m}, 1 \mathrm{H}), 7.48(\mathrm{dt}, J=8.0,1.8 \mathrm{~Hz}, 1 \mathrm{H}), 7.37-7.31$ $(\mathrm{m}, 2 \mathrm{H}), 7.30(\mathrm{~d}, J=7.9 \mathrm{~Hz}, 2 \mathrm{H}), 7.20(\mathrm{~d}, J=7.9 \mathrm{~Hz}, 2 \mathrm{H}), 7.18-7.15(\mathrm{~m}, 2 \mathrm{H}), 7.01(\mathrm{~d}, J=8.2$ $\mathrm{Hz}, 2 \mathrm{H}), 6.47(\mathrm{~d}, J=0.8 \mathrm{~Hz}, 1 \mathrm{H}), 6.10(\mathrm{q}, J=2.3 \mathrm{~Hz}, 1 \mathrm{H}), 4.17(\mathrm{dt}, J=14.8,2.3 \mathrm{~Hz}, 1 \mathrm{H}), 4.01$ (ddd, $J=14.8,2.3,0.8 \mathrm{~Hz}, 1 \mathrm{H}$ ), 3.28 (ddd, $J=14.9,4.7,0.8 \mathrm{~Hz}, 1 \mathrm{H}), 3.20-3.14(\mathrm{~m}, 2 \mathrm{H}), 3.04$ (m, 1H), $2.97-2.86(\mathrm{~m}, 2 \mathrm{H}), 2.41(\mathrm{~s}, 3 \mathrm{H}), 1.26(\mathrm{~d}, J=6.9 \mathrm{~Hz}, 6 \mathrm{H})$.

${ }^{13}$ C NMR $\left(126 \mathrm{MHz}, \mathrm{CDCl}_{3}\right) \delta 151.3,149.6,147.9,143.6,138.8,138.5,138.4,137.2,133.9$, 132.6, 129.7, 128.4, 128.1, 127.8, 126.6, 123.3, 122.2, 122.1, 121.0, 120.9, 120.2, 110.1, 104.0, 51.7, 50.8, 44.6, 33.8, 32.0, 23.9, 21.5.

IR (ATR) 1586, 1440, 1468, 1347, 1163, 1100, 1055, 913, 816, 785, 722, 667

HRMS (DART, M+H) Calculated for $\mathrm{C}_{35} \mathrm{H}_{36} \mathrm{~N}_{3} \mathrm{O}_{2} \mathrm{~S} 562.2523$, found 562.2524

MP $76-82{ }^{\circ} \mathrm{C}$

$\boldsymbol{\alpha}_{\boldsymbol{D}}^{\mathbf{2 0}}\left(\mathrm{c}=0.195, \mathrm{CH}_{2} \mathrm{Cl}_{2}\right) 3.00$

HPLC: IB, 50\% IPA/Hex, $1.0 \mathrm{~mL} / \mathrm{min}$
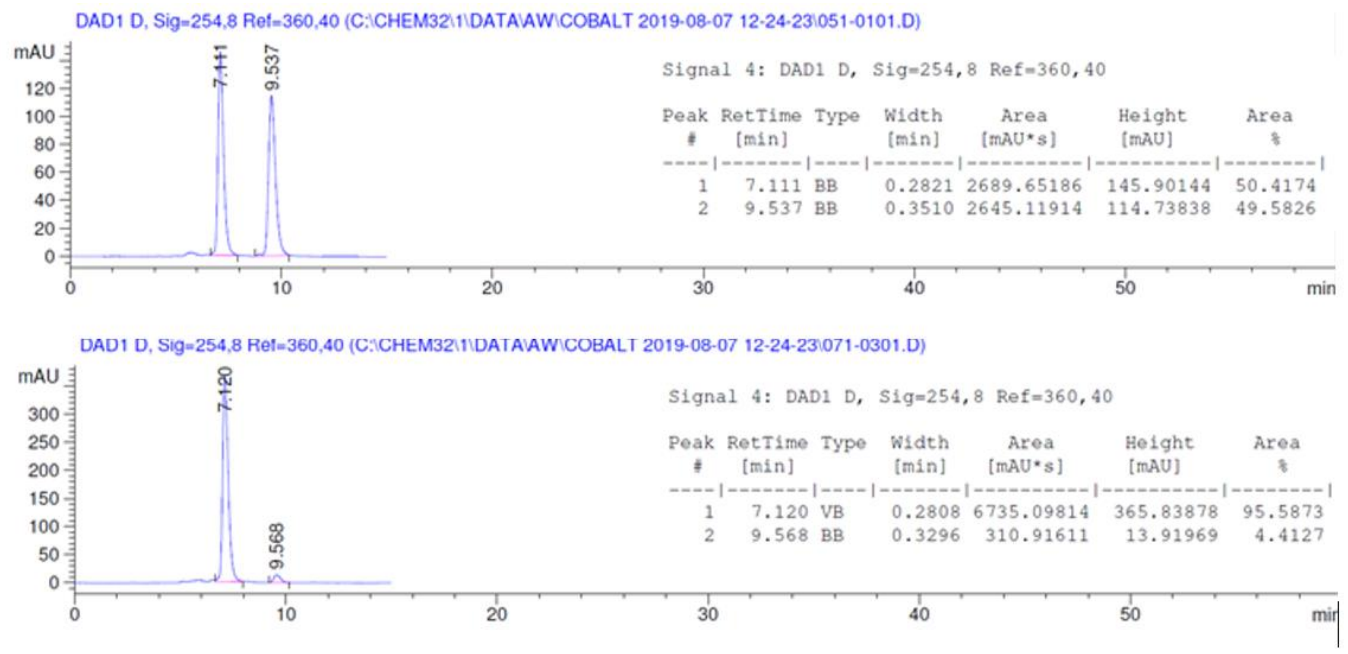
<smiles>[As]N1CC=C(c2ccc(-c3ccccc3)cc2)C1</smiles>

Synthesized following $\mathbf{G P 3}$ at $80^{\circ} \mathrm{C}$. The product was isolated by flash column chromatography $15->30 \%$ EtOAc/pentane. The product appeared as a white solid, $88 \mathrm{mg}$ (74\% yield).

${ }^{1} \mathbf{H}$ NMR $\left(500 \mathrm{MHz}, \mathrm{CDCl}_{3}\right) \delta 8.68(\mathrm{~d}, J=4.9 \mathrm{~Hz}, 1 \mathrm{H}), 7.92(\mathrm{td}, J=7.7,2.0 \mathrm{~Hz}, 1 \mathrm{H}), 7.70(\mathrm{~d}, J$ $=8.0 \mathrm{~Hz}, 2 \mathrm{H}), 7.63-7.54(\mathrm{~m}, 5 \mathrm{H}), 7.51-7.43(\mathrm{~m}, 4 \mathrm{H}), 7.39-7.34(\mathrm{~m}, 3 \mathrm{H}), 7.30(\mathrm{~d}, J=8.0$ $\mathrm{Hz}, 2 \mathrm{H}), 7.19-7.16(\mathrm{~m}, 2 \mathrm{H}), 7.14(\mathrm{~d}, J=8.0 \mathrm{~Hz}, 2 \mathrm{H}), 6.48(\mathrm{~s}, 1 \mathrm{H}), 6.15(\mathrm{~d}, J=2.3 \mathrm{~Hz}, 1 \mathrm{H})$, $4.21(\mathrm{~d}, J=14.9 \mathrm{~Hz}, 1 \mathrm{H}), 4.05(\mathrm{~d}, J=14.9 \mathrm{~Hz}, 1 \mathrm{H}), 3.31(\mathrm{dd}, J=14.9,4.9 \mathrm{~Hz}, 1 \mathrm{H}), 3.22-3.17$ $(\mathrm{m}, 2 \mathrm{H}), 3.08(\mathrm{~m}, 1 \mathrm{H}), 2.97(\mathrm{dd}, J=14.9,9.7 \mathrm{~Hz}, 1 \mathrm{H}), 2.41(\mathrm{~s}, 3 \mathrm{H})$.

${ }^{13} \mathrm{C}$ NMR $\left(126 \mathrm{MHz}, \mathrm{CDCl}_{3}\right) \delta 151.3,149.7,143.7,140.4,140.0,139.8,138.5,138.3,137.2$, $135.3,132.6,129.7,128.8,128.5,128.4,127.8,127.5,127.2,126.9,123.1,122.2$, 122.2, 121.0, $120.9,120.3,110.1,104.1,51.7,50.8,44.7,32.0,21.5$.

IR (ATR) 1590, 1469, 1455, 1436, 1341, 1157, 1092, 1036, 814, 783, 764, 742, 697, 663

HRMS (DART, M+H) Calculated for $\mathrm{C}_{38} \mathrm{H}_{34} \mathrm{~N}_{3} \mathrm{O}_{2} \mathrm{~S} 596.2366$, found 596.2373

MP $91-92{ }^{\circ} \mathrm{C}$

$\boldsymbol{\alpha}_{\boldsymbol{D}}^{\mathbf{2 0}}\left(\mathrm{c}=0.165, \mathrm{CH}_{2} \mathrm{Cl}_{2}\right) 2.34$

HPLC: IB, 50\% IPA/Hex, $1.0 \mathrm{~mL} / \mathrm{min}$
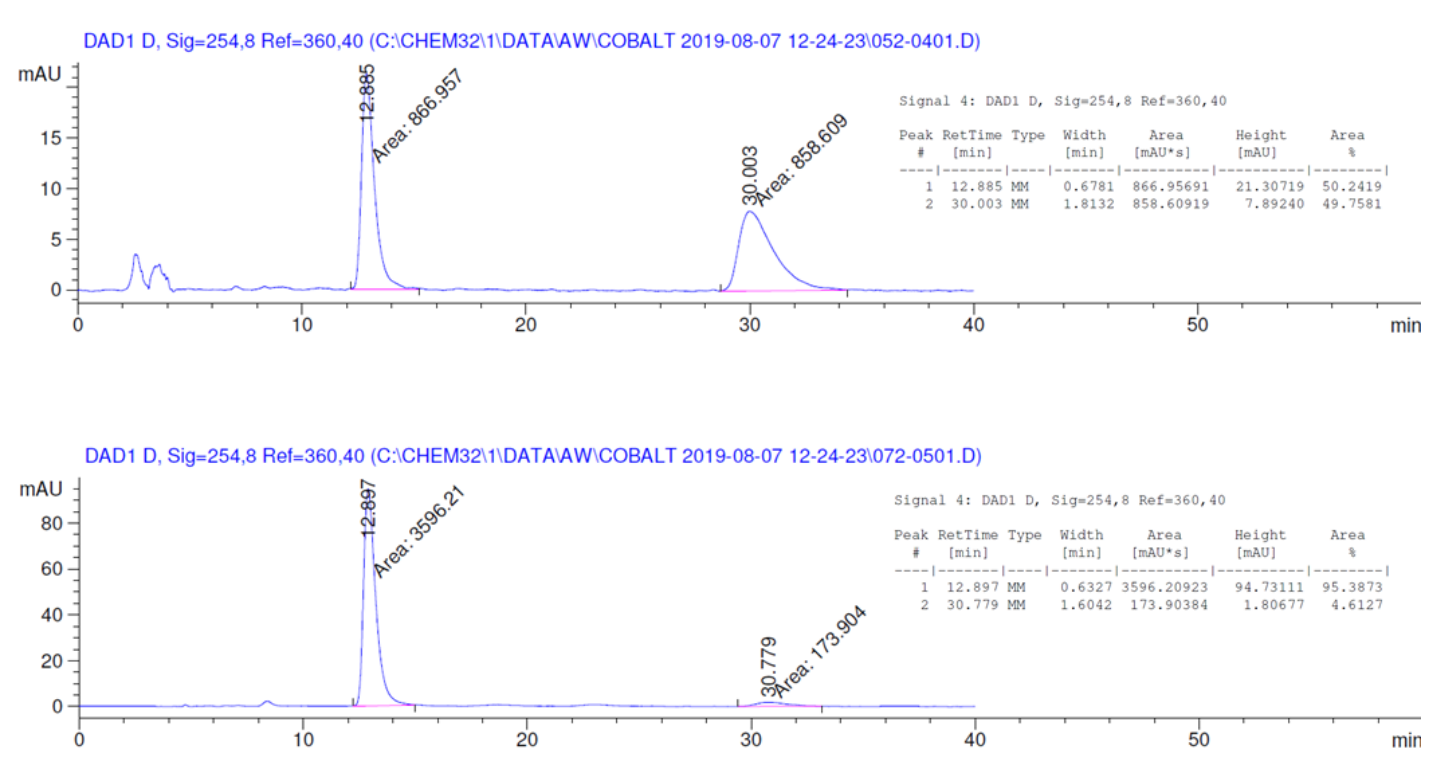
(R,Z)-1-(pyridin-2-yl)-2-((4-(4-(thiophen-3-yl)benzylidene)-1-tosylpyrrolidin-3-yl)methyl)1H-indole (4e)

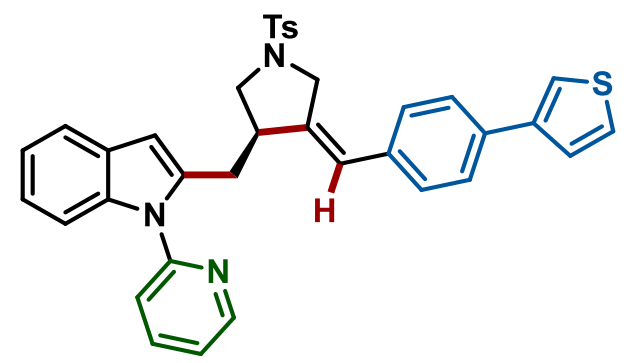

Synthesized following $\mathbf{G P 3}$ at $80^{\circ} \mathrm{C}$. The product was isolated by flash column chromatography $15->30 \%$ EtOAc/pentane. The product appeared as a white solid, $65 \mathrm{mg}$ (54\% yield).

${ }^{1} \mathbf{H}$ NMR $\left(500 \mathrm{MHz}, \mathrm{CDCl}_{3}\right) \delta 8.67(\mathrm{ddd}, J=4.9,2.0,0.8 \mathrm{~Hz}, 1 \mathrm{H}), 7.91$ (ddd, $J=8.0,7.4,2.0$ $\mathrm{Hz}, 1 \mathrm{H}), 7.69(\mathrm{~d}, J=8.2 \mathrm{~Hz}, 2 \mathrm{H}), 7.63-7.57(\mathrm{~m}, 2 \mathrm{H}), 7.56(\mathrm{~d}, J=8.3 \mathrm{~Hz}, 2 \mathrm{H}), 7.51-7.45(\mathrm{~m}$, $2 \mathrm{H}), 7.42-7.38(\mathrm{~m}, 2 \mathrm{H}), 7.38-7.31(\mathrm{~m}, 3 \mathrm{H}), 7.30(\mathrm{~d}, J=8.5 \mathrm{~Hz}, 2 \mathrm{H}), 7.20-7.14(\mathrm{~m}, 2 \mathrm{H})$, $7.09(\mathrm{~d}, J=8.3 \mathrm{~Hz}, 2 \mathrm{H}), 6.47(\mathrm{~d}, J=0.9 \mathrm{~Hz}, 1 \mathrm{H}), 6.12(\mathrm{q}, J=2.3 \mathrm{~Hz}, 1 \mathrm{H}), 4.18(\mathrm{dt}, J=14.8,2.3$ $\mathrm{Hz}, 1 \mathrm{H}), 4.03$ (dd, $J=14.8,2.3 \mathrm{~Hz}, 1 \mathrm{H}), 3.31$ (ddd, $J=14.9,4.9,0.9 \mathrm{~Hz}, 1 \mathrm{H}), 3.21-3.15(\mathrm{~m}$, 2H), $3.12-3.02(\mathrm{~m}, 1 \mathrm{H}), 2.96$ (ddd, $J=14.9,9.8,0.7 \mathrm{~Hz}, 1 \mathrm{H}), 2.41$ (s, 3H).

${ }^{13} \mathrm{C}$ NMR $\left(126 \mathrm{MHz}, \mathrm{CDCl}_{3}\right) \delta 151.3,149.6,143.7,141.6,139.8,138.5,138.3,137.2,135.1$, $134.5,132.6,129.7,128.5,128.4,127.8,126.5,126.4,126.1,123.1,122.2,122.1,121.0,120.9$, $120.4,120.3,110.1,104.1,51.7,50.8,44.7,31.9,21.5$.

IR (ATR) 1469, 1436, 1268, 1202, 1033, 1016, 865, 814, 707, 688

HRMS (DART, M+H) Calculated for $\mathrm{C}_{36} \mathrm{H}_{32} \mathrm{~N}_{3} \mathrm{O}_{2} \mathrm{~S}_{2} 602.1931$, found 602.1931

MP $103-107{ }^{\circ} \mathrm{C}$

$\boldsymbol{\alpha}_{\boldsymbol{D}}^{\mathbf{2 0}}\left(\mathrm{c}=0.2, \mathrm{CH}_{2} \mathrm{Cl}_{2}\right) 3.00$

HPLC: IB, 50\% IPA/Hex, $1.0 \mathrm{~mL} / \mathrm{min}$

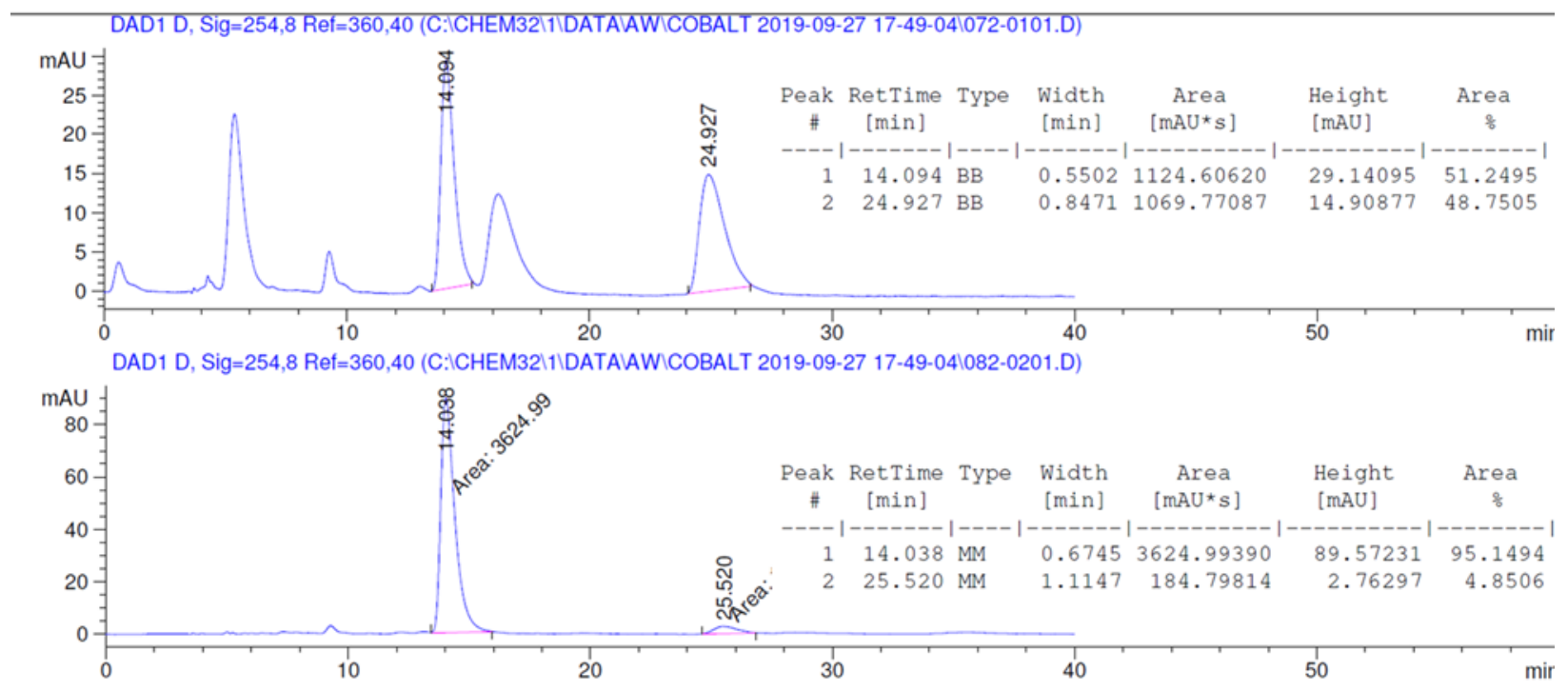




\section{(R,Z)-2-((4-(4-methoxybenzylidene)-1-tosylpyrrolidin-3-yl)methyl)-1-(pyridin-2-yl)-1H-}

indole (4f)

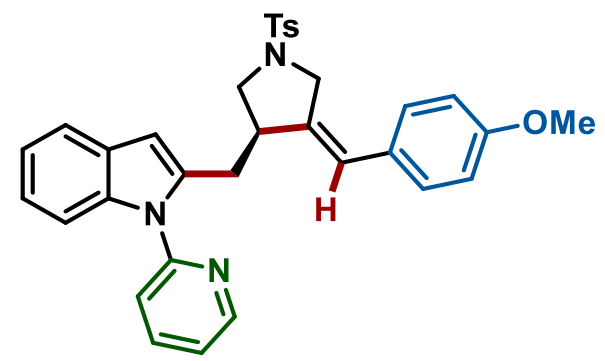

Synthesized following GP3. The product was isolated by flash column chromatography 15$>30 \% \mathrm{EtOAc/pentane.} \mathrm{The} \mathrm{product} \mathrm{appeared} \mathrm{as} \mathrm{a} \mathrm{white} \mathrm{solid,} 89 \mathrm{mg}$ (81\% yield).

${ }^{1} \mathbf{H}$ NMR $\left(500 \mathrm{MHz}, \mathrm{CDCl}_{3}\right) \delta 8.66(\mathrm{ddd}, \mathrm{J}=4.9,2.0,0.8 \mathrm{~Hz}, 1 \mathrm{H}), 7.90(\mathrm{ddd}, \mathrm{J}=8.0,7.4,2.0$ $\mathrm{Hz}, 1 \mathrm{H}), 7.68(\mathrm{~d}, \mathrm{~J}=8.3 \mathrm{~Hz}, 2 \mathrm{H}), 7.63-7.56(\mathrm{~m}, 1 \mathrm{H}), 7.47(\mathrm{dt}, \mathrm{J}=8.0,0.8 \mathrm{~Hz}, 1 \mathrm{H}), 7.38-7.31$ $(\mathrm{m}, 2 \mathrm{H}), 7.29(\mathrm{~d}, \mathrm{~J}=8.3 \mathrm{~Hz}, 2 \mathrm{H}), 7.20-7.12(\mathrm{~m}, 2 \mathrm{H}), 7.00(\mathrm{~d}, \mathrm{~J}=8.8 \mathrm{~Hz}, 2 \mathrm{H}), 6.86(\mathrm{~d}, \mathrm{~J}=8.8$ $\mathrm{Hz}, 2 \mathrm{H}), 6.46(\mathrm{~d}, \mathrm{~J}=0.8 \mathrm{~Hz}, 1 \mathrm{H}), 6.05(\mathrm{q}, \mathrm{J}=2.3 \mathrm{~Hz}, 1 \mathrm{H}), 4.13(\mathrm{dt}, \mathrm{J}=14.5,2.3 \mathrm{~Hz}, 1 \mathrm{H}), 3.97$ (dd, J = 14.5, $2.3 \mathrm{~Hz}, 1 \mathrm{H}), 3.81$ (s, 3H), 3.28 (ddd, J = 14.8, 4.7, $0.8 \mathrm{~Hz}, 1 \mathrm{H}), 3.19-3.14$ (m, 2H), 3.05-2.99 (m, 1H), $2.93(\mathrm{dd}, \mathrm{J}=14.8,9.8 \mathrm{~Hz}, 1 \mathrm{H}), 2.41(\mathrm{~s}, 3 \mathrm{H})$.

${ }^{13}$ C NMR $\left(126 \mathrm{MHz}, \mathrm{CDCl}_{3}\right) \delta 158.6,151.3,149.6,143.6,138.5,138.4,137.4,137.2,132.6$, 129.7, 129.3, 129.1, 128.4, 127.8, 122.9, 122.2, 122.1, 121.1, 120.9, 120.2, 114.0, 110.1, 104.0, $55.3,51.7,50.7,44.5,31.9,21.5$.

IR (ATR) 1586, 1477, 1437, 1343, 1163, 1091, 1037, 908, 816, 722, 667

HRMS (DART, M+H) Calculated for $\mathrm{C}_{33} \mathrm{H}_{32} \mathrm{~N}_{3} \mathrm{O}_{3} \mathrm{~S} 550.2159$, found 550.2158

MP $78-85{ }^{\circ} \mathrm{C}$

$\boldsymbol{\alpha}_{\boldsymbol{D}}^{\mathbf{2 0}}\left(\mathrm{c}=0.205, \mathrm{CH}_{2} \mathrm{Cl}_{2}\right) 6.83$

HPLC: IB, 50\% IPA/Hex, $1.0 \mathrm{~mL} / \mathrm{min}$

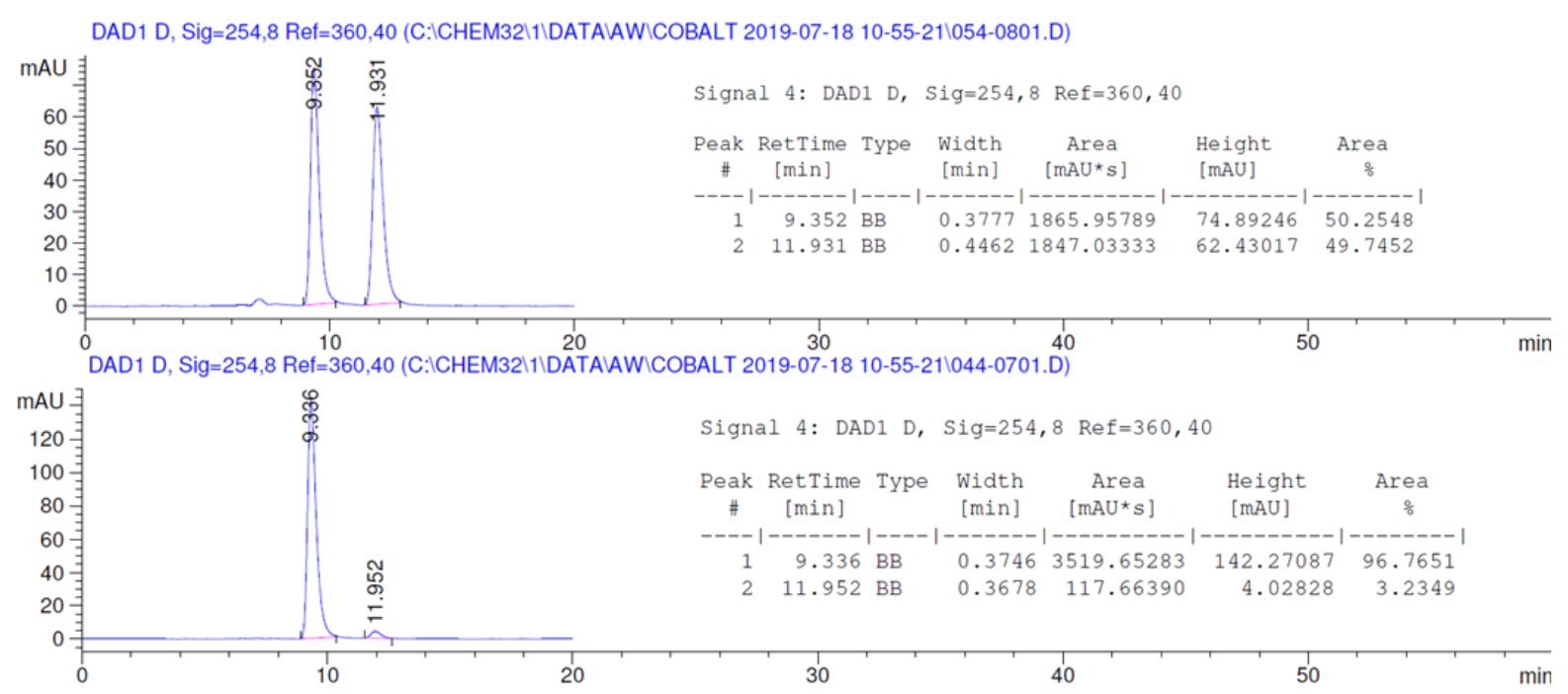




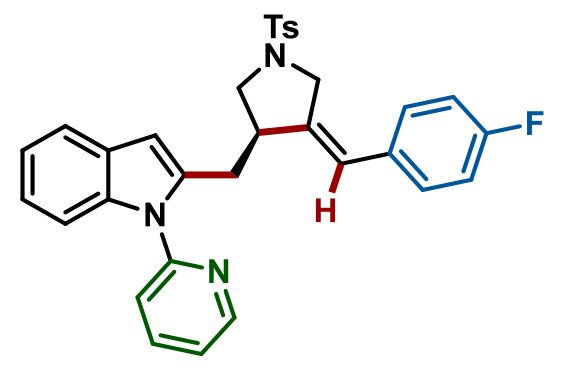

Synthesized following GP3. The product was isolated by flash column chromatography 15$>30 \%$ EtOAc/pentane. The product appeared as a white solid, $85 \mathrm{mg}$ (79\% yield).

${ }^{1} \mathbf{H}$ NMR $\left(500 \mathrm{MHz}, \mathrm{CDCl}_{3}\right) \delta 8.66(\mathrm{ddd}, J=4.9,2.0,0.8 \mathrm{~Hz}, 1 \mathrm{H}), 7.91(\mathrm{ddd}, \mathrm{J}=$ 8.0, 7.5, 2.0 $\mathrm{Hz}, 1 \mathrm{H}), 7.68(\mathrm{~d}, \mathrm{~J}=8.3 \mathrm{~Hz}, 2 \mathrm{H}), 7.63-7.56(\mathrm{~m}, 1 \mathrm{H}), 7.48(\mathrm{dt}, \mathrm{J}=8.0,0.8 \mathrm{~Hz}, 1 \mathrm{H}), 7.36-7.33$ $(\mathrm{m}, 2 \mathrm{H}), 7.30(\mathrm{~d}, \mathrm{~J}=8.3 \mathrm{~Hz}, 2 \mathrm{H}), 7.20-7.13(\mathrm{~m}, 2 \mathrm{H}), 7.04-6.99(\mathrm{~m}, 4 \mathrm{H}), 6.46(\mathrm{~d}, \mathrm{~J}=0.8 \mathrm{~Hz}$, $1 \mathrm{H}), 6.08(\mathrm{q}, \mathrm{J}=2.4 \mathrm{~Hz}, 1 \mathrm{H}), 4.11(\mathrm{dt}, \mathrm{J}=15.0,2.4 \mathrm{~Hz}, 1 \mathrm{H}), 3.95(\mathrm{dd}, \mathrm{J}=15.0,2.4 \mathrm{~Hz}, 1 \mathrm{H})$, 3.27 (ddd, J = 14.8, 4.9, $0.8 \mathrm{~Hz}, 1 \mathrm{H}), 3.18(\mathrm{~d}, \mathrm{~J}=5.8 \mathrm{~Hz}, 2 \mathrm{H}), 3.09-3.00$ (m, 1H), 2.94 (ddd, J = $14.8,9.7,0.8 \mathrm{~Hz}, 1 \mathrm{H}), 2.41(\mathrm{~s}, 3 \mathrm{H})$.

${ }^{13} \mathrm{C} \mathrm{NMR}\left(126 \mathrm{MHz}, \mathrm{CDCl}_{3}\right) \delta 161.66(\mathrm{~d}, J=247.7 \mathrm{~Hz}), 151.29,149.63,143.72,139.48(\mathrm{~d}, J=$ $2.1 \mathrm{~Hz}), 138.51,138.18,137.17,132.58,132.53(\mathrm{~d}, J=3.3 \mathrm{~Hz}), 129.73,129.64(\mathrm{~d}, J=8.0 \mathrm{~Hz})$, $128.37,127.80,122.39,122.20,122.17,121.01,120.94,120.25,115.50$ (d, $J=21.5 \mathrm{~Hz}), 110.12$, $104.12,51.70,50.58,44.50,31.89,21.53$.

${ }^{19}$ F NMR $\left(377 \mathrm{MHz}, \mathrm{CDCl}_{3}\right) \delta-114.3$.

IR (ATR) 1542, 1463, 1437, 1347, 1163, 1097, 906, 816, 722, 663

HRMS (DART, M+H) Calculated for $\mathrm{C}_{32} \mathrm{H}_{29} \mathrm{~N}_{3} \mathrm{O}_{2} \mathrm{FS} 538.1959$, found 538.1961

MP $154-155^{\circ} \mathrm{C}$

$\boldsymbol{\alpha}_{D}^{\mathbf{2 0}}\left(\mathrm{c}=0.155, \mathrm{CH}_{2} \mathrm{Cl}_{2}\right) 9.03$

HPLC: IB, 50\% IPA/Hex, $1.0 \mathrm{~mL} / \mathrm{min}$

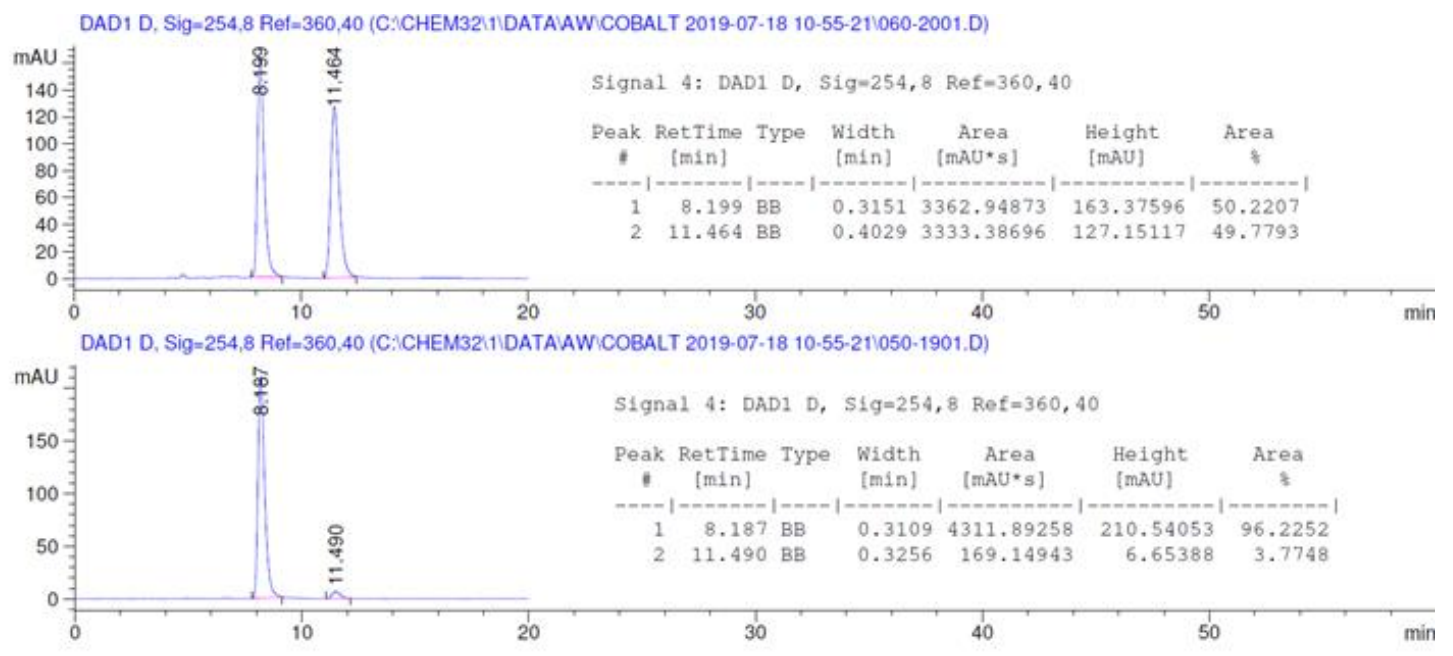




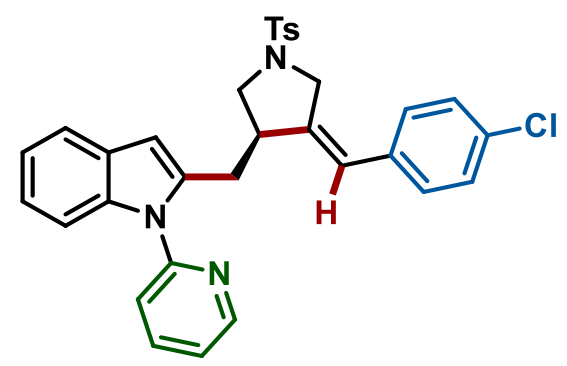

Synthesized following GP3. The product was isolated by flash column chromatography 15$>30 \%$ EtOAc/pentane. The product appeared as a white solid, $110 \mathrm{mg}$ (99\% yield).

${ }^{1} \mathbf{H}$ NMR $\left(500 \mathrm{MHz}, \mathrm{CDCl}_{3}\right) \delta 8.66(\mathrm{ddd}, J=4.9,2.0,0.9 \mathrm{~Hz}, 1 \mathrm{H}), 7.91(\mathrm{ddd}, J=8.0,7.5,2.0$ $\mathrm{Hz}, 1 \mathrm{H}), 7.68(\mathrm{~d}, J=8.3 \mathrm{~Hz}, 2 \mathrm{H}), 7.63-7.57(\mathrm{~m}, 1 \mathrm{H}), 7.48(\mathrm{dt}, J=8.0,0.9 \mathrm{~Hz}, 1 \mathrm{H}), 7.37-7.33$ $(\mathrm{m}, 2 \mathrm{H}), 7.32-7.27(\mathrm{~m}, 4 \mathrm{H}), 7.19-7.15(\mathrm{~m}, 2 \mathrm{H}), 6.98(\mathrm{~d}, J=8.5 \mathrm{~Hz}, 2 \mathrm{H}), 6.46(\mathrm{~d}, J=0.9 \mathrm{~Hz}$, $1 \mathrm{H}), 6.06(\mathrm{q}, J=2.3 \mathrm{~Hz}, 1 \mathrm{H}), 4.11(\mathrm{dt}, J=14.7,2.3 \mathrm{~Hz}, 1 \mathrm{H}), 3.95(\mathrm{ddd}, J=14.7,2.3,0.9 \mathrm{~Hz}$, $1 \mathrm{H}), 3.28$ (ddd, $J=14.8,5.0,0.9 \mathrm{~Hz}, 1 \mathrm{H}), 3.18(\mathrm{~d}, J=5.7 \mathrm{~Hz}, 2 \mathrm{H}), 3.11-3.02(\mathrm{~m}, 1 \mathrm{H}), 2.95$ (ddd, $J=14.8,9.7,0.7 \mathrm{~Hz}, 1 \mathrm{H}), 2.41(\mathrm{~s}, 3 \mathrm{H})$.

${ }^{13} \mathrm{C}$ NMR $\left(126 \mathrm{MHz}, \mathrm{CDCl}_{3}\right) \delta 151.3,149.6,143.8,140.6,138.5,138.1,137.2,134.8,132.9$, $132.5,129.7,129.3,128.7,128.4,127.8,122.4,122.2,122.2,121.0,121.0,120.3,110.1,104.2$, 51.7, 50.6, 44.6, 31.8, 21.5.

IR (ATR) 1586, 1437. 1468, 1340, 1170, 1090, 806, 906, 733, 663

HRMS (DART, M+H) Calculated for $\mathrm{C}_{32} \mathrm{H}_{29} \mathrm{~N}_{3} \mathrm{O}_{2} \mathrm{SCl} 554.1664$, found 554.1662

MP $126-128{ }^{\circ} \mathrm{C}$

$\boldsymbol{\alpha}_{\boldsymbol{D}}^{\mathbf{2 0}}\left(\mathrm{c}=0.135, \mathrm{CH}_{2} \mathrm{Cl}_{2}\right) 8.89$

HPLC: IB, 50\% IPA/Hex, $1.0 \mathrm{~mL} / \mathrm{min}$

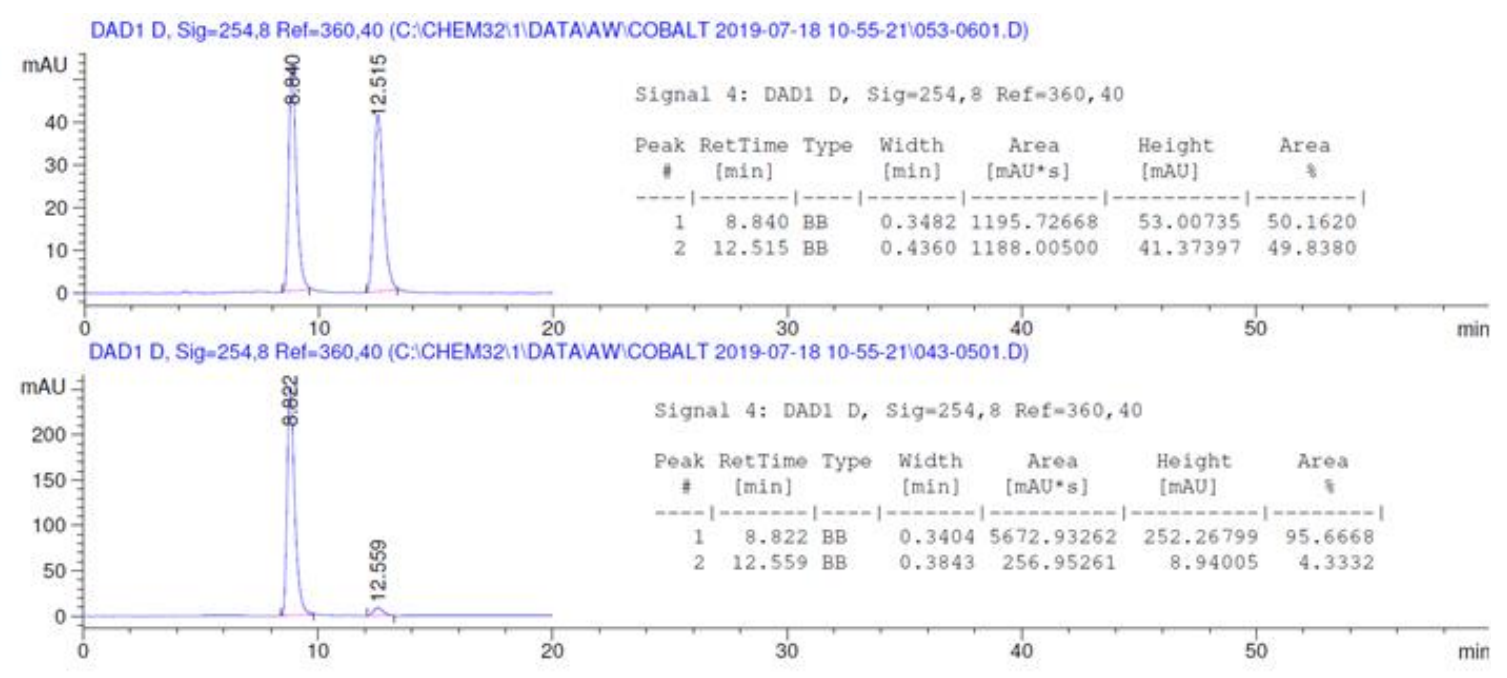




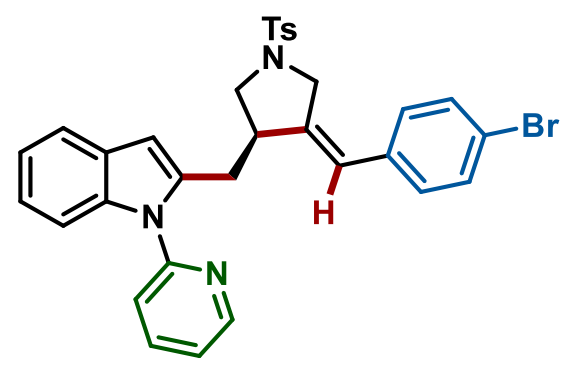

Synthesized following GP3. The product was isolated by flash column chromatography 15$>30 \%$ EtOAc/pentane. The product appeared as a white solid, $108 \mathrm{mg}$ (90\% yield).

${ }^{1} \mathbf{H}$ NMR $\left(500 \mathrm{MHz}, \mathrm{CDCl}_{3}\right) \delta 8.71-8.60(\mathrm{~m}, 1 \mathrm{H}), 7.91(\mathrm{td}, \mathrm{J}=7.7,2.0 \mathrm{~Hz}, 1 \mathrm{H}), 7.68(\mathrm{~d}, J=$ $8.3 \mathrm{~Hz}, 2 \mathrm{H}), 7.63-7.56(\mathrm{~m}, 1 \mathrm{H}), 7.47(\mathrm{~d}, J=7.7 \mathrm{~Hz}, 2 \mathrm{H}), 7.44(\mathrm{~d}, J=8.4 \mathrm{~Hz}, 2 \mathrm{H}), 7.38-7.31$ $(\mathrm{m}, 2 \mathrm{H}), 7.30(\mathrm{~d}, J=8.3 \mathrm{~Hz}, 2 \mathrm{H}), 7.20-7.13(\mathrm{~m}, 2 \mathrm{H}), 6.91(\mathrm{~d}, J=8.4 \mathrm{~Hz}, 2 \mathrm{H}), 6.46(\mathrm{~s}, 1 \mathrm{H})$, $6.04(\mathrm{q}, J=2.3 \mathrm{~Hz}, 1 \mathrm{H}), 4.10(\mathrm{dt}, J=14.8,2.3 \mathrm{~Hz}, 1 \mathrm{H}), 3.94(\mathrm{dd}, J=14.8,2.3 \mathrm{~Hz}, 1 \mathrm{H}), 3.27$ $(\mathrm{dd}, J=14.8,4.9 \mathrm{~Hz}, 1 \mathrm{H}), 3.18(\mathrm{~d}, J=5.7 \mathrm{~Hz}, 2 \mathrm{H}), 3.05(\mathrm{~m}, 1 \mathrm{H}), 2.95$ (dd, $J=14.8,9.6 \mathrm{~Hz}$, $1 \mathrm{H}), 2.41(\mathrm{~s}, 3 \mathrm{H})$.

${ }^{13} \mathrm{C}$ NMR $\left(126 \mathrm{MHz}, \mathrm{CDCl}_{3}\right) \delta 151.3,149.6,143.8,140.8,138.5,138.1,137.2,135.2,132.5$, $131.7,129.8,129.6,128.4,127.8,122.4,122.2$, 122.2, 121.0, 121.0, 121.0, 120.3, 110.1, 104.2, 51.7, 50.6, 44.6, 31.8, 21.5.

IR (ATR) 1593, 1477, 1437, 1347, 1163, 1090, 916, 816, 722, 660

HRMS (DART, $\mathrm{M}+\mathrm{H}$ ) Calculated for $\mathrm{C}_{32} \mathrm{H}_{29} \mathrm{~N}_{3} \mathrm{O}_{2} \mathrm{SBr} 598.1158$, found 598.1159

MP $117-118{ }^{\circ} \mathrm{C}$

$\boldsymbol{\alpha}_{D}^{\mathbf{2 0}}\left(\mathrm{c}=0.120, \mathrm{CH}_{2} \mathrm{Cl}_{2}\right) 6.67$

HPLC: IB, 50\% IPA/Hex, $1.0 \mathrm{~mL} / \mathrm{min}$

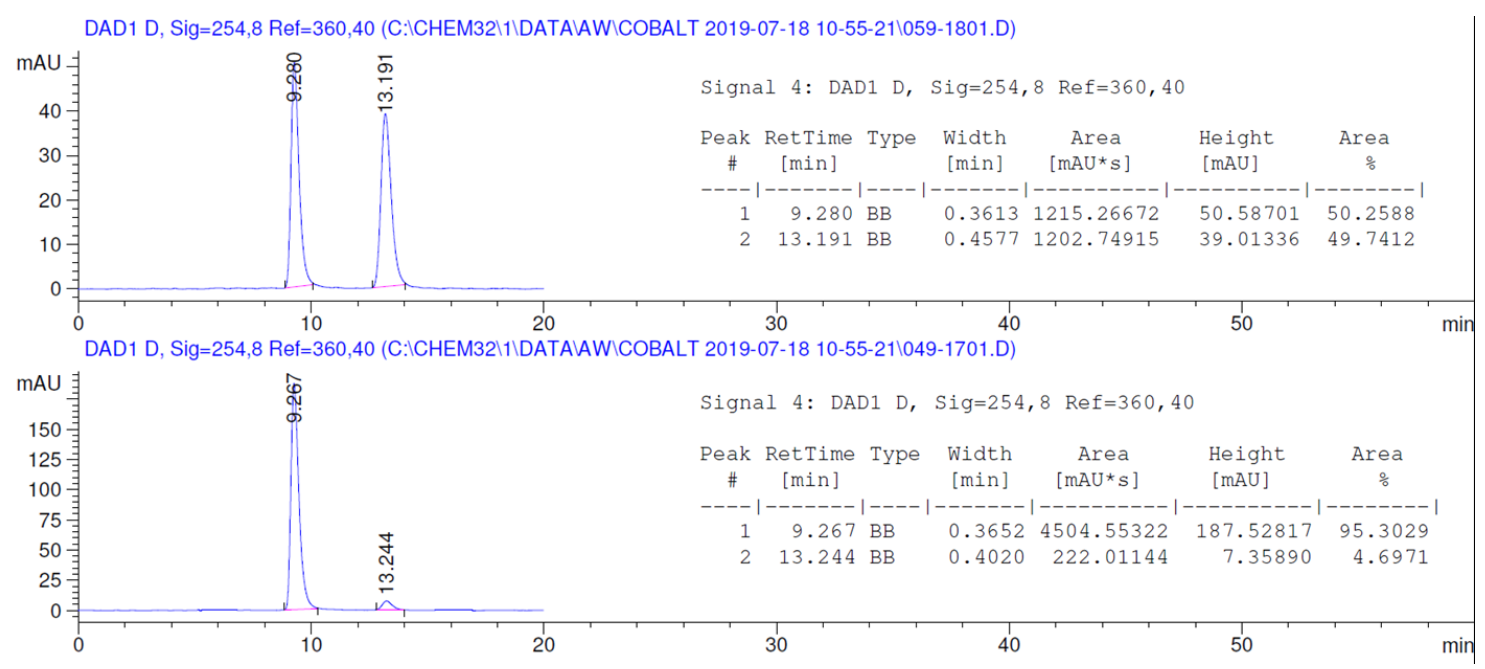


(R,Z)-1-(pyridin-2-yl)-2-((1-tosyl-4-(4-(trifluoromethyl)benzylidene)pyrrolidin-3yl)methyl)-1H-indole (4j)

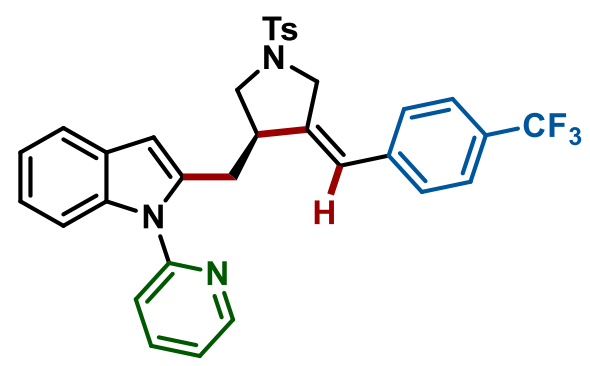

Synthesized following GP3. The product was isolated by flash column chromatography 15$>30 \%$ EtOAc/pentane. The product appeared as a white solid, $82 \mathrm{mg}$ (70\% yield).

${ }^{1} \mathbf{H}$ NMR $\left(500 \mathrm{MHz}, \mathrm{CDCl}_{3}\right) \delta 8.66(\mathrm{ddd}, J=4.9,2.0,1.0 \mathrm{~Hz}, 1 \mathrm{H}), 7.92(\mathrm{ddd}, J=8.1,7.5,2.0$ $\mathrm{Hz}, 1 \mathrm{H}), 7.69(\mathrm{~d}, J=8.3 \mathrm{~Hz}, 2 \mathrm{H}), 7.60(\mathrm{ddd}, J=5.9,3.1,0.7 \mathrm{~Hz}, 1 \mathrm{H}), 7.57(\mathrm{~d}, J=8.1 \mathrm{~Hz}, 2 \mathrm{H})$, $7.49(\mathrm{dt}, J=8.1,1.0 \mathrm{~Hz}, 1 \mathrm{H}), 7.37-7.33(\mathrm{~m}, 2 \mathrm{H}), 7.31(\mathrm{~d}, J=8.3 \mathrm{~Hz}, 2 \mathrm{H}), 7.19-7.16(\mathrm{~m}, 2 \mathrm{H})$, $7.15(\mathrm{~d}, J=8.1 \mathrm{~Hz}, 2 \mathrm{H}), 6.46(\mathrm{~d}, J=0.8 \mathrm{~Hz}, 1 \mathrm{H}), 6.14(\mathrm{q}, J=2.3 \mathrm{~Hz}, 1 \mathrm{H}), 4.15$ (dt, $J=14.9,2.3$ Hz, 1H), 3.98 (dd, $J=14.9,2.3 \mathrm{~Hz}, 1 \mathrm{H}), 3.29$ (ddd, $J=14.8,5.2,0.8 \mathrm{~Hz}, 1 \mathrm{H}), 3.20$ (dm, 2H), $3.15-3.06(\mathrm{~m}, 1 \mathrm{H}), 2.98$ (ddd, $J=14.8,9.6,0.7 \mathrm{~Hz}, 1 \mathrm{H}), 2.41(\mathrm{~s}, 3 \mathrm{H})$.

${ }^{13} \mathrm{C}$ NMR $\left(126 \mathrm{MHz}, \mathrm{CDCl}_{3}\right) \delta 151.3,149.6,143.8,142.7,139.8$ (q, $\left.J=1.2 \mathrm{~Hz}\right), 138.6,138.0$, $137.2,132.5,129.77,128.9(\mathrm{q}, J=32.5 \mathrm{~Hz}), 128.3,128.2,127.8,125.5(\mathrm{q}, J=3.8 \mathrm{~Hz}), 124.0(\mathrm{q}$, $J=271.9 \mathrm{~Hz}), 122.4,122.3,122.23,121.0,121.0,120.3,110.1,104.3,51.7,50.7,44.7,31.9$, 21.5.

${ }^{19}$ F NMR $\left(377 \mathrm{MHz}, \mathrm{CDCl}_{3}\right) \delta-62.9$.

IR (ATR) 1613, 1554, 1470, 1436, 1416, 1385, 1250, 1108, 1093, 1055, 1015, 814, 785

HRMS (DART, $\mathrm{M}+\mathrm{H}$ ) Calculated for $\mathrm{C}_{33} \mathrm{H}_{29} \mathrm{~N}_{3} \mathrm{O}_{2} \mathrm{~F}_{3} \mathrm{~S} 588.1927$, found 588.1928

MP $160-163{ }^{\circ} \mathrm{C}$

$\boldsymbol{\alpha}_{\boldsymbol{D}}^{\mathbf{2 0}}\left(\mathrm{c}=0.28, \mathrm{CH}_{2} \mathrm{Cl}_{2}\right) 3.57$

HPLC: IB, 50\% IPA/Hex, $1.0 \mathrm{~mL} / \mathrm{min}$

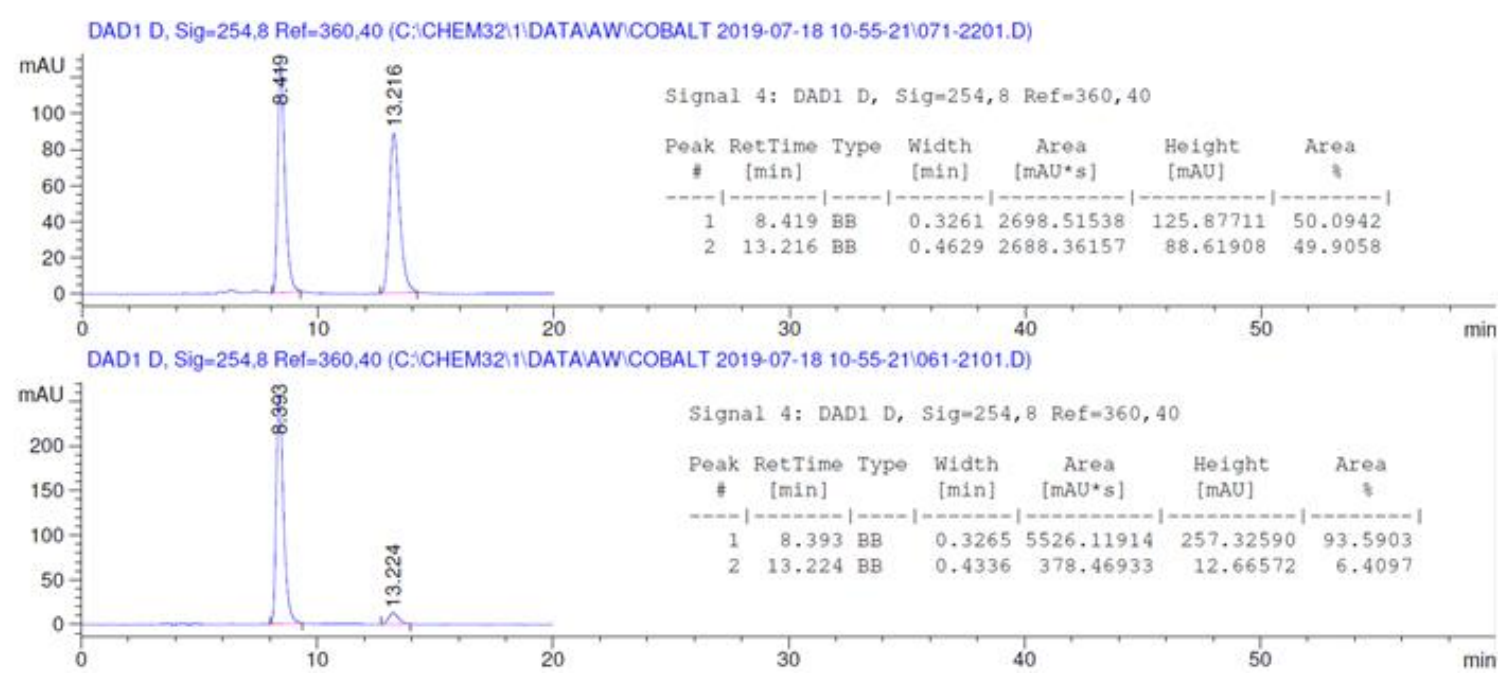


(R,Z)-1-(pyridin-2-yl)-2-((1-tosyl-4-(4-

(((triisopropylsilyl)oxy)methyl)benzylidene)pyrrolidin-3-yl)methyl)-1H-indole (4l)

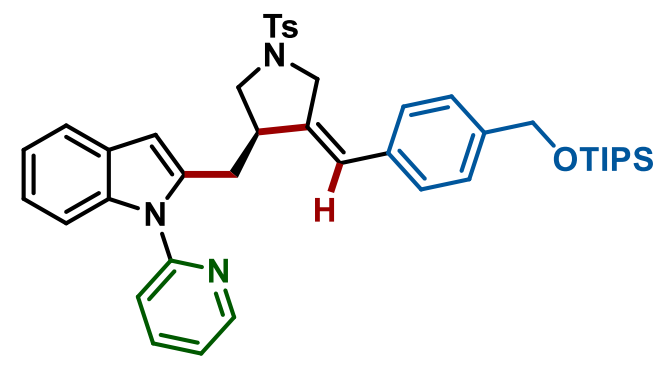

Synthesized following $\mathbf{G P 3}$ at $80{ }^{\circ} \mathrm{C}$. The product was isolated by flash column chromatography $15->30 \%$ EtOAc/pentane. The product appeared as a white solid, $103 \mathrm{mg}$ (73\% yield).

${ }^{1} \mathbf{H}$ NMR $\left(500 \mathrm{MHz}, \mathrm{CDCl}_{3}\right) \delta 8.66(\mathrm{ddd}, J=4.9,2.0,0.8 \mathrm{~Hz}, 1 \mathrm{H}), 7.94-7.86(\mathrm{~m}, 1 \mathrm{H}), 7.68(\mathrm{~d}$, $J=8.3 \mathrm{~Hz}, 2 \mathrm{H}), 7.63-7.56(\mathrm{~m}, 1 \mathrm{H}), 7.47(\mathrm{dt}, J=8.0,1.0 \mathrm{~Hz}, 1 \mathrm{H}), 7.38-7.26(\mathrm{~m}, 6 \mathrm{H}), 7.20-$ $7.12(\mathrm{~m}, 2 \mathrm{H}), 7.03(\mathrm{~d}, J=8.2 \mathrm{~Hz}, 2 \mathrm{H}), 6.46(\mathrm{~d}, J=0.8 \mathrm{~Hz}, 1 \mathrm{H}), 6.10(\mathrm{q}, J=2.3 \mathrm{~Hz}, 1 \mathrm{H}), 4.83(\mathrm{~s}$, $2 \mathrm{H}$ ), $4.16(\mathrm{dt}, J=14.8,2.3 \mathrm{~Hz}, 1 \mathrm{H}), 4.00(\mathrm{dd}, J=14.8,2.3 \mathrm{~Hz}, 1 \mathrm{H}), 3.29$ (ddd, $J=14.8,4.7,0.8$ $\mathrm{Hz}, 1 \mathrm{H}), 3.17(\mathrm{~m}, 2 \mathrm{H}), 3.04(\mathrm{~m}, 1 \mathrm{H}), 2.93(\mathrm{dd}, J=14.8,9.8 \mathrm{~Hz}, 1 \mathrm{H}), 2.41(\mathrm{~s}, 3 \mathrm{H}), 1.23-1.15$ $(\mathrm{m}, 3 \mathrm{H}), 1.11(\mathrm{~d}, J=6.7 \mathrm{~Hz}, 18 \mathrm{H})$.

${ }^{13}$ C NMR $\left(126 \mathrm{MHz}, \mathrm{CDCl}_{3}\right) \delta 151.3,149.6,143.6,140.7,139.2,138.5,138.3,137.2,134.9$, 132.6, 129.7, 128.4, 127.9, 127.8, 125.9, 123.3, 122.2, 122.1, 121.0, 120.9, 120.2, 110.1, 104.1, 64.7, 51.7, 50.8, 44.6, 31.9, 21.5, 18.1, 12.0 .

IR (ATR) 2897, 2257, 1550, 1512, 1491, 1172, 1098, 1051, 990, 854, 826, 740

HRMS (DART, $\mathrm{M}+\mathrm{H}$ ) Calculated for $\mathrm{C}_{42} \mathrm{H}_{52} \mathrm{~N}_{3} \mathrm{O}_{3} \mathrm{Si}_{1} \mathrm{~S}_{1} 706.3493$, found 706.3490

MP $120-122^{\circ} \mathrm{C}$

$\boldsymbol{\alpha}_{\boldsymbol{D}}^{\mathbf{2 0}}\left(\mathrm{c}=0.31, \mathrm{CH}_{2} \mathrm{Cl}_{2}\right) 3.87$

HPLC: IB, 50\% IPA/Hex, $1.0 \mathrm{~mL} / \mathrm{min}$
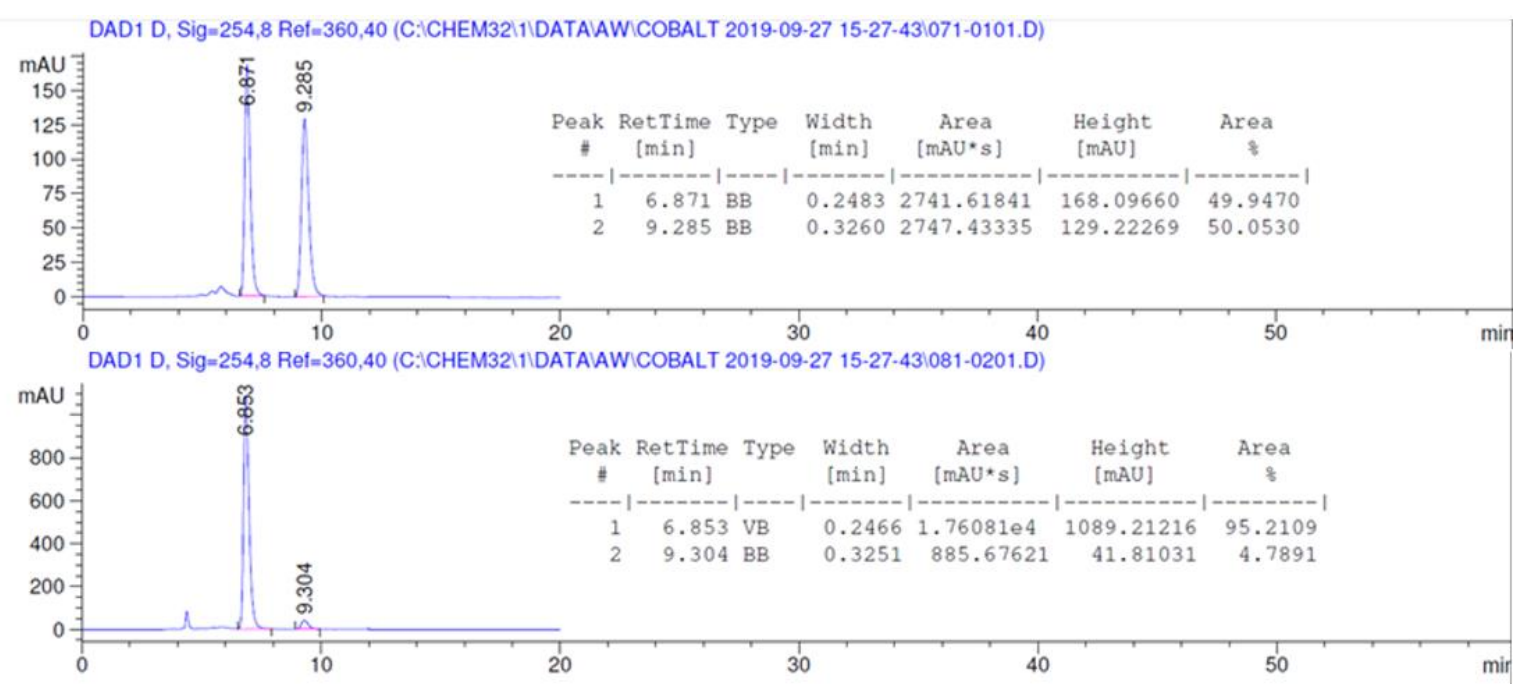


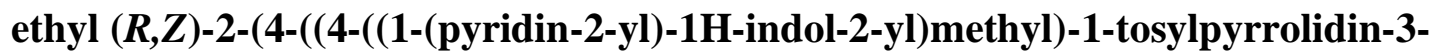
ylidene)methyl)phenoxy)acetate $(4 \mathrm{k})$

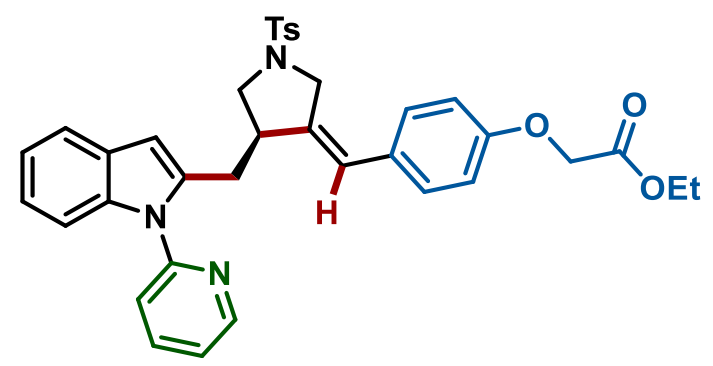

Synthesized following GP3 at $80{ }^{\circ} \mathrm{C}$. The product was isolated by flash column chromatography $15->30 \%$ EtOAc/pentane. The product appeared as a white solid, $63 \mathrm{mg}$ (51\% yield).

${ }^{1}$ H NMR $(500 \mathrm{MHz}) \delta 8.65(\mathrm{ddd}, J=4.9,2.0,0.8 \mathrm{~Hz}, 1 \mathrm{H}), 7.90(\mathrm{ddd}, J=8.0,7.5,2.0 \mathrm{~Hz}, 1 \mathrm{H})$, $7.67(\mathrm{~d}, J=8.3 \mathrm{~Hz}, 2 \mathrm{H}), 7.61-7.56(\mathrm{~m}, 1 \mathrm{H}), 7.46(\mathrm{dt}, J=8.0,0.8 \mathrm{~Hz}, 1 \mathrm{H}), 7.35-7.32(\mathrm{~m}, 2 \mathrm{H})$, $7.29(\mathrm{~d}, J=8.3 \mathrm{~Hz}, 2 \mathrm{H}), 7.18-7.12(\mathrm{~m}, 2 \mathrm{H}), 6.99(\mathrm{~d}, J=8.7 \mathrm{~Hz}, 2 \mathrm{H}), 6.86(\mathrm{~d}, J=8.8 \mathrm{~Hz}, 2 \mathrm{H})$, $6.45(\mathrm{~d}, J=0.8 \mathrm{~Hz}, 1 \mathrm{H}), 6.04(\mathrm{q}, J=2.3 \mathrm{~Hz}, 1 \mathrm{H}), 4.62(\mathrm{~s}, 2 \mathrm{H}), 4.28(\mathrm{q}, J=7.1 \mathrm{~Hz}, 2 \mathrm{H}), 4.11$ (dt, $J=14.6,2.3 \mathrm{~Hz}, 1 \mathrm{H}), 3.95(\mathrm{ddd}, J=14.6,2.3,0.8 \mathrm{~Hz}, 1 \mathrm{H}), 3.26(\mathrm{ddd}, J=14.8,4.8,0.8 \mathrm{~Hz}, 1 \mathrm{H})$, $3.18-3.12(\mathrm{~m}, 2 \mathrm{H}), 3.02(\mathrm{dq}, J=10.8,5.5 \mathrm{~Hz}, 1 \mathrm{H}), 2.92(\mathrm{ddd}, J=14.8,9.8,0.7 \mathrm{~Hz}, 1 \mathrm{H}), 2.41$ $(\mathrm{s}, 3 \mathrm{H}), 1.31(\mathrm{t}, J=7.1 \mathrm{~Hz}, 3 \mathrm{H})$.

${ }^{13} \mathrm{C}$ NMR $\left(126 \mathrm{MHz}, \mathrm{CDCl}_{3}\right) \delta 168.7,156.8,151.3,149.6,143.6,138.5,138.3,138.1,137.2$, 132.6, 130.2, 129.7, 129.4, 128.4, 127.8, 122.7, 122.2, 122.1, 121.0, 120.9, 120.2, 114.7, 110.1, $104.0,65.4,61.5,51.7,50.7,44.5,31.9,21.5,14.2$.

IR (ATR) 1586, 1469, 1455, 1306, 1199, 1180, 1112, 1034, 814, 782, 708, 668

HRMS (DART, M+H) Calculated for $\mathrm{C}_{36} \mathrm{H}_{36} \mathrm{~N}_{3} \mathrm{O}_{5} \mathrm{~S}_{1} 622.2370$, found 622.2374

MP $65-69^{\circ} \mathrm{C}$

$\boldsymbol{\alpha}_{\boldsymbol{D}}^{\mathbf{2 0}}\left(\mathrm{c}=0.29, \mathrm{CH}_{2} \mathrm{Cl}_{2}\right) 4.83$

HPLC: IB, 50\% IPA/Hex, $1.0 \mathrm{~mL} / \mathrm{min}$

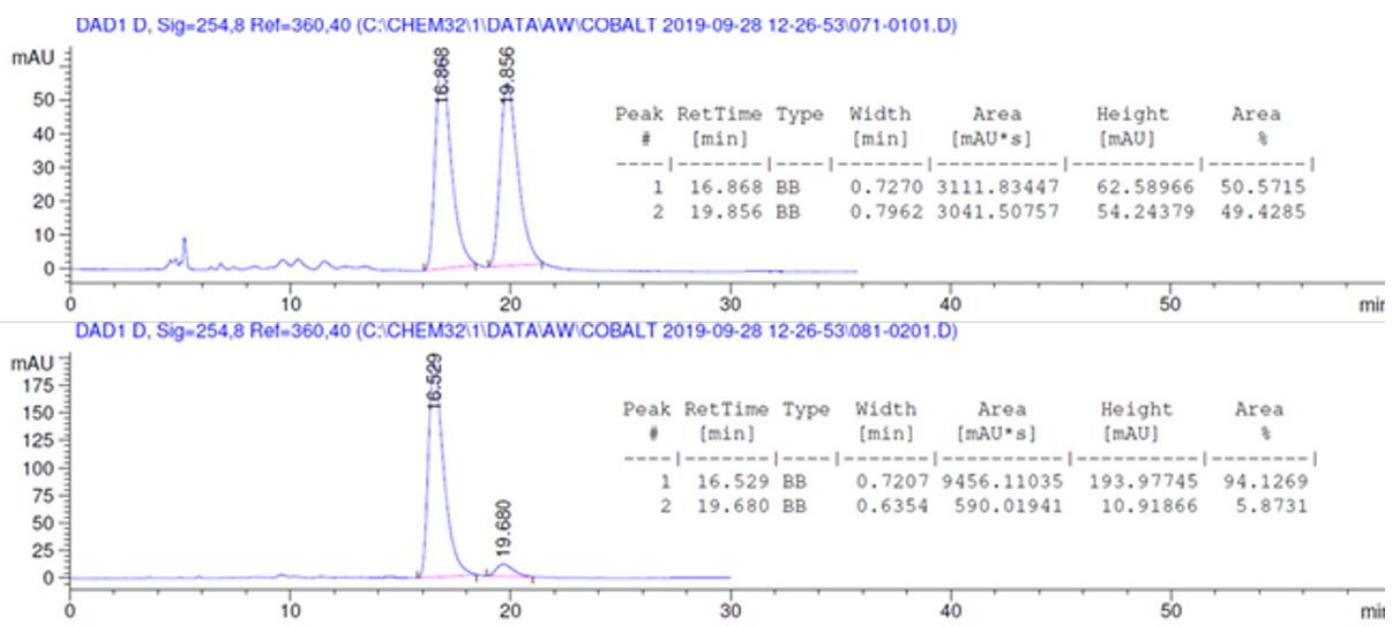


(R,Z)-2-((4-(3-methylbenzylidene)-1-tosylpyrrolidin-3-yl)methyl)-1-(pyridin-2-yl)-1H-indole $(4 \mathrm{~m})$

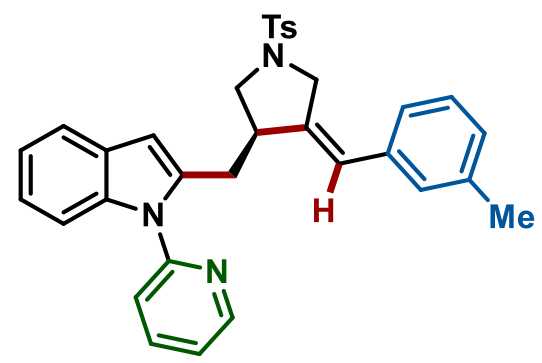

Synthesized following $\mathbf{G P 3}$ at $80{ }^{\circ} \mathrm{C}$. The product was isolated by flash column chromatography 15-> 30\% EtOAc/pentane. The product appeared as a white solid, $98 \mathrm{mg}$ (92\% yield).

${ }^{1}$ H NMR $\left(500 \mathrm{MHz}, \mathrm{CDCl}_{3}\right) \delta 8.67(\mathrm{ddd}, J=4.9,2.0,0.8 \mathrm{~Hz}, 1 \mathrm{H}), 7.91$ (ddd, $J=8.0,7.5,2.0$ $\mathrm{Hz}, 1 \mathrm{H}), 7.68(\mathrm{~d}, J=8.2 \mathrm{~Hz}, 2 \mathrm{H}), 7.63-7.57(\mathrm{~m}, 1 \mathrm{H}), 7.48(\mathrm{dt}, J=8.0,0.8 \mathrm{~Hz}, 1 \mathrm{H}), 7.38-7.31$ $(\mathrm{m}, 2 \mathrm{H}), 7.29(\mathrm{~d}, J=8.2 \mathrm{~Hz}, 2 \mathrm{H}), 7.22(\mathrm{t}, J=7.9 \mathrm{~Hz}, 1 \mathrm{H}), 7.20-7.13(\mathrm{~m}, 2 \mathrm{H}), 7.05(\mathrm{~d}, J=7.8$ $\mathrm{Hz}, 1 \mathrm{H}), 6.90-6.84(\mathrm{~m}, 2 \mathrm{H}), 6.46(\mathrm{~d}, J=0.8 \mathrm{~Hz}, 1 \mathrm{H}), 6.09(\mathrm{q}, J=2.3 \mathrm{~Hz}, 1 \mathrm{H}), 4.16(\mathrm{dt}, J=$ $14.8,2.3 \mathrm{~Hz}, 1 \mathrm{H}), 4.00$ (ddd, $J=14.8,2.3,0.8 \mathrm{~Hz}, 1 \mathrm{H}), 3.28$ (ddd, $J=14.8,4.8,0.9 \mathrm{~Hz}, 1 \mathrm{H}$ ), $3.17(\mathrm{~m}, 2 \mathrm{H}), 3.08-2.99(\mathrm{~m}, 1 \mathrm{H}), 2.93(\mathrm{ddd}, J=14.8,9.8,0.8 \mathrm{~Hz}, 1 \mathrm{H}), 2.41(\mathrm{~s}, 3 \mathrm{H}), 2.34(\mathrm{~s}$, $3 \mathrm{H})$.

${ }^{13}$ C NMR $\left(126 \mathrm{MHz}, \mathrm{CDCl}_{3}\right) \delta 151.3,149.6,143.6,139.5,138.5,138.3,138.1,137.2,136.3$, $132.7,129.7,128.9,128.4,128.4,127.9,127.8,125.0,123.6,122.2,122.1,121.0,120.9,120.3$, $110.1,104.1,51.7,50.7,44.6,31.9,21.5,21.5$.

IR (ATR) 1586, 1508, 1471, 1440, 1347, 1163, 1047, 1100, 910, 816, 794, 722, 670

HRMS (DART, M+H) Calculated for $\mathrm{C}_{33} \mathrm{H}_{32} \mathrm{~N}_{3} \mathrm{O}_{2} \mathrm{~S} 534.2210$, found 534.2207

MP $80-81{ }^{\circ} \mathrm{C}$

$\boldsymbol{\alpha}_{D}^{\mathbf{2 0}}\left(\mathrm{c}=0.16, \mathrm{CH}_{2} \mathrm{Cl}_{2}\right) 10.00$

HPLC: IB, 50\% IPA/Hex, $1.0 \mathrm{~mL} / \mathrm{min}$

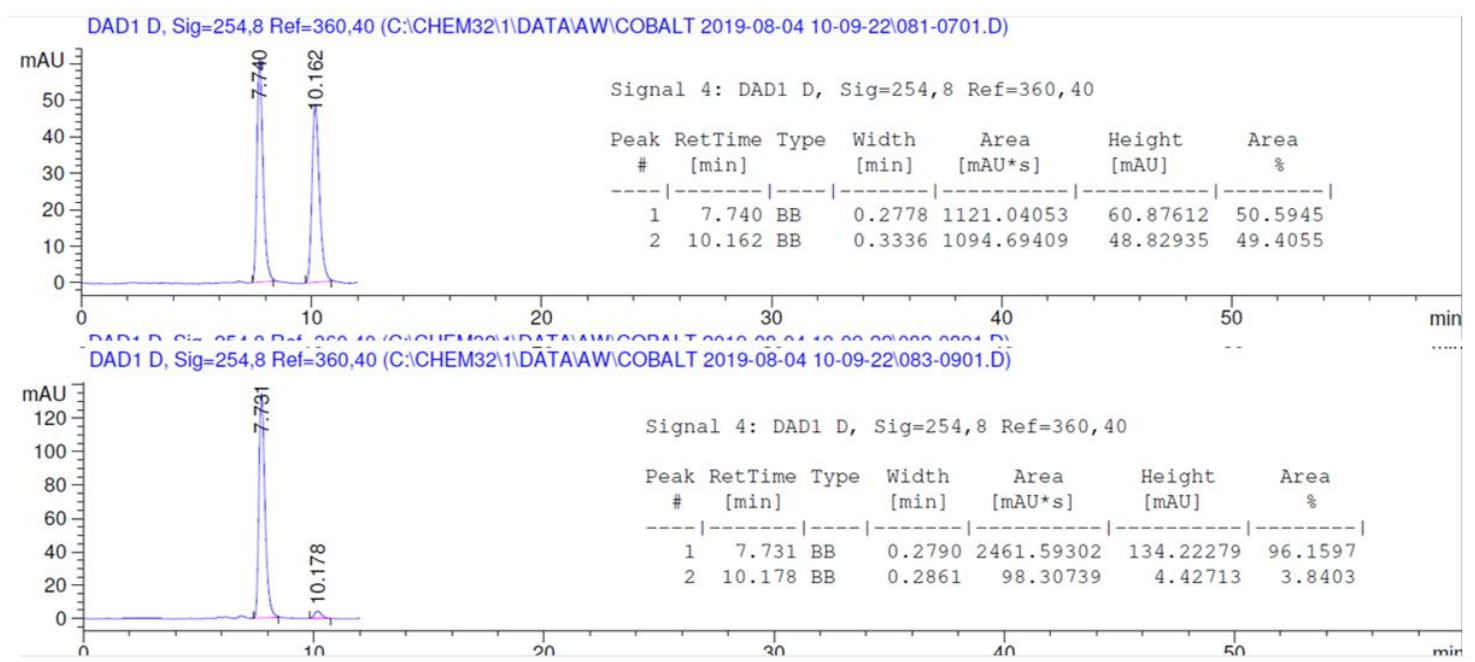


(R,Z)-2-((4-(3-methoxybenzylidene)-1-tosylpyrrolidin-3-yl)methyl)-1-(pyridin-2-yl)-1Hindole $(4 n)$

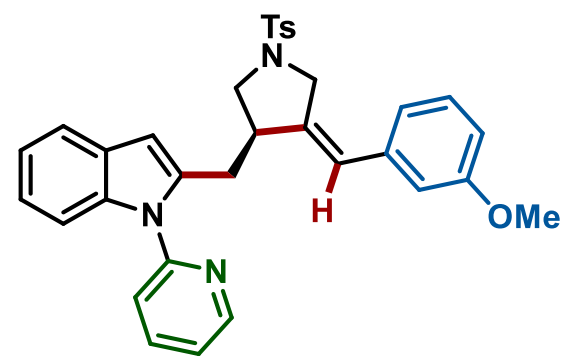

Synthesized following GP3 at $80{ }^{\circ} \mathrm{C}$. The product was isolated by flash column chromatography $15->30 \%$ EtOAc/pentane. The product appeared as a white solid, $99 \mathrm{mg}$ (90\% yield).

${ }^{1} \mathbf{H}$ NMR $\left(500 \mathrm{MHz}, \mathrm{CDCl}_{3}\right) \delta 8.66(\mathrm{ddd}, J=4.9,2.0,0.8 \mathrm{~Hz}, 1 \mathrm{H}), 7.91(\mathrm{ddd}, \mathrm{J}=8.0,7.5,2.0$ $\mathrm{Hz}, 1 \mathrm{H}), 7.68(\mathrm{~d}, \mathrm{~J}=8.3 \mathrm{~Hz}, 2 \mathrm{H}), 7.63-7.56(\mathrm{~m}, 1 \mathrm{H}), 7.48(\mathrm{dt}, \mathrm{J}=8.0,0.8 \mathrm{~Hz}, 1 \mathrm{H}), 7.38-7.31$ $(\mathrm{m}, 2 \mathrm{H}), 7.29(\mathrm{~d}, \mathrm{~J}=8.3 \mathrm{~Hz}, 2 \mathrm{H}), 7.25(\mathrm{t}, \mathrm{J}=7.9 \mathrm{~Hz}, 2 \mathrm{H}), 7.20-7.13(\mathrm{~m}, 2 \mathrm{H}), 6.78(\mathrm{ddd}, \mathrm{J}=$ 8.3, 2.0, 0.9 Hz, 1H), $6.66(\mathrm{dt}, \mathrm{J}=8.1,2.0 \mathrm{~Hz}, 1 \mathrm{H}), 6.57(\mathrm{t}, \mathrm{J}=2.0 \mathrm{~Hz}, 1 \mathrm{H}), 6.46$ (d, J = 0.9 Hz, $1 \mathrm{H}), 6.08$ (q, J = 2.3 Hz, 1H), 4.15 (dt, J = 14.8, $2.3 \mathrm{~Hz}, 1 \mathrm{H}), 3.98$ (dd, J = 14.9, 2.3 Hz, 1H), $3.80(\mathrm{~s}, 3 \mathrm{H}), 3.28(\mathrm{ddd}, \mathrm{J}=14.9,4.8,0.9 \mathrm{~Hz}, 1 \mathrm{H}), 3.17(\mathrm{~m}, 2 \mathrm{H}), 3.04(\mathrm{~m}, 1 \mathrm{H}), 2.94(\mathrm{dd}, \mathrm{J}=14.9$, $9.7 \mathrm{~Hz}, 1 \mathrm{H}), 2.41(\mathrm{~s}, 3 \mathrm{H})$.

${ }^{13} \mathrm{C}$ NMR $\left(126 \mathrm{MHz}, \mathrm{CDCl}_{3}\right) \delta 159.6,151.3,149.6,143.7,140.2,138.5,138.2,137.7,137.2$, 132.6, 129.7, 129.5, 128.4, 127.8, 123.4, 122.2, 122.1, 121.0, 120.9, 120.4, 120.3, 114.1, 112.3, $110.1,104.1,55.2,51.7,50.7,44.5,31.9,21.5$.

IR (ATR) 1586, 1474, 1437, 1341, 1163, 1090, 1037, 916, 816, 722, 667

HRMS (DART, M+H) Calculated for $\mathrm{C}_{33} \mathrm{H}_{32} \mathrm{~N}_{3} \mathrm{O}_{3} \mathrm{~S} 550.2159$, found 550.2161

MP $74-75^{\circ} \mathrm{C}$

$\boldsymbol{\alpha}_{\boldsymbol{D}}^{\mathbf{2 0}}\left(\mathrm{c}=0.150, \mathrm{CH}_{2} \mathrm{Cl}_{2}\right) 13.33$

HPLC: IB, 50\% IPA/Hex, $1.0 \mathrm{~mL} / \mathrm{min}$

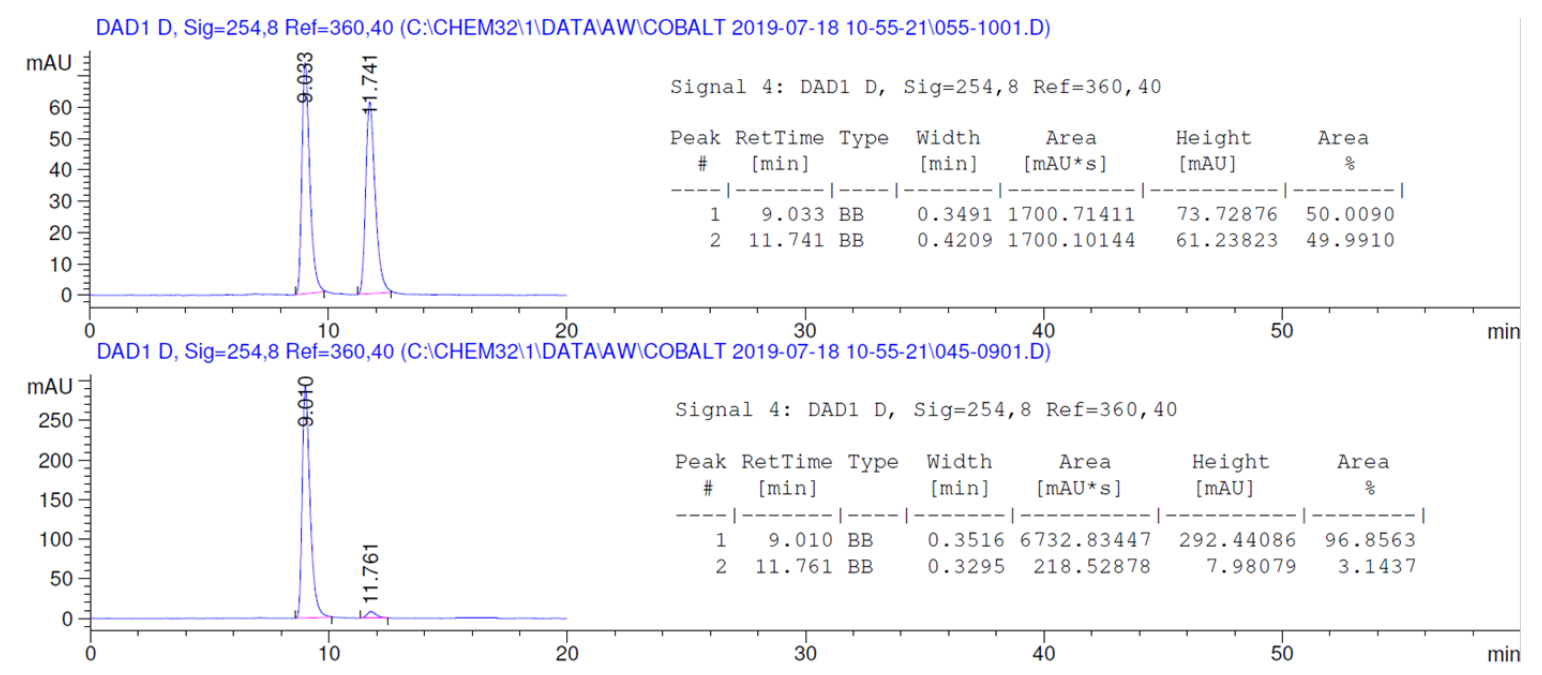




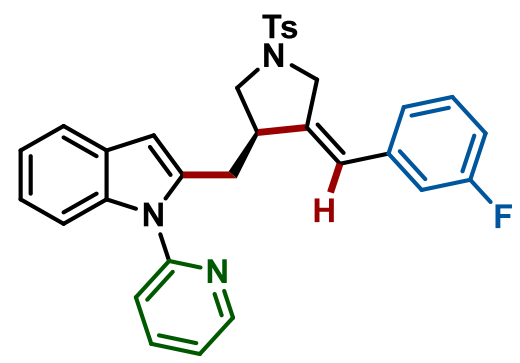

Synthesized following $\mathbf{G P 3}$ at $80{ }^{\circ} \mathrm{C}$. The product was isolated by flash column chromatography $15->30 \%$ EtOAc/pentane. The product appeared as a white solid, $66 \mathrm{mg}$ (61\% yield).

${ }^{1} \mathbf{H}$ NMR $\left(500 \mathrm{MHz}, \mathrm{CDCl}_{3}\right) \delta 8.66(\mathrm{ddd}, J=4.9,2.0,0.8 \mathrm{~Hz}, 1 \mathrm{H}), 7.91(\mathrm{ddd}, J=8.0,7.4,2.0$ $\mathrm{Hz}, 1 \mathrm{H}), 7.69$ (d, $J=8.3 \mathrm{~Hz}, 2 \mathrm{H}), 7.63-7.57(\mathrm{~m}, 1 \mathrm{H}), 7.48(\mathrm{dt}, J=8.0,0.8 \mathrm{~Hz}, 1 \mathrm{H}), 7.39-7.32$ $(\mathrm{m}, 2 \mathrm{H}), 7.34-7.26(\mathrm{~m}, 3 \mathrm{H}), 7.21-7.13(\mathrm{~m}, 2 \mathrm{H}), 6.93(\mathrm{tdd}, J=8.4,2.6,0.9 \mathrm{~Hz}, 1 \mathrm{H}), 6.83(\mathrm{~d}, J$ $=7.5 \mathrm{~Hz}, 1 \mathrm{H}), 6.74(\mathrm{dt}, J=10.0,2.1 \mathrm{~Hz}, 1 \mathrm{H}), 6.46(\mathrm{~d}, J=0.9 \mathrm{~Hz}, 1 \mathrm{H}), 6.08(\mathrm{q}, J=2.3 \mathrm{~Hz}, 1 \mathrm{H})$, 4.14 (dt, $J=14.9,2.3 \mathrm{~Hz}, 1 \mathrm{H}), 3.97$ (dd, $J=14.9,2.3 \mathrm{~Hz}, 1 \mathrm{H}), 3.28$ (ddd, $J=14.9,5.0,0.9 \mathrm{~Hz}$, $1 \mathrm{H}), 3.22-3.16(\mathrm{~m}, 2 \mathrm{H}), 3.12-3.02(\mathrm{~m}, 1 \mathrm{H}), 2.95(\mathrm{ddd}, J=14.9,9.8,0.7 \mathrm{~Hz}, 1 \mathrm{H}), 2.42(\mathrm{~s}, 3 \mathrm{H})$.

${ }^{13}$ C NMR $\left(126 \mathrm{MHz}, \mathrm{CDCl}_{3}\right) \delta 162.8(\mathrm{~d}, J=245.9 \mathrm{~Hz}), 151.3,149.6,143.8,141.4,138.5,138.5$ $(\mathrm{d}, J=7.7 \mathrm{~Hz}), 138.1,137.2,132.5,130.0(\mathrm{~d}, J=8.5 \mathrm{~Hz}), 129.8,128.4,127.8,123.8(\mathrm{~d}, J=2.8$ $\mathrm{Hz}), 122.5$ (d, $J=2.6 \mathrm{~Hz}), 122.2,122.2,121.0,121.0,120.3,114.7$ (d, $J=21.8 \mathrm{~Hz}), 114.0$ (d, $J$ $=21.2 \mathrm{~Hz}), 110.1,104.2,51.7,50.7,44.6,31.9,21.5$.

${ }^{19}$ F NMR $\left(377 \mathrm{MHz}, \mathrm{CDCl}_{3}\right) \delta-112.8$.

IR (ATR) 1532, 1475, 1447, 1343, 1173, 1104, 906, 785, 736, 678, 670

HRMS (DART, M+H) Calculated for $\mathrm{C}_{32} \mathrm{H}_{29} \mathrm{~N}_{3} \mathrm{O}_{2} \mathrm{FS} 538.1959$, found 538.1961

MP $119-123^{\circ} \mathrm{C}$

$\boldsymbol{\alpha}_{\boldsymbol{D}}^{\mathbf{2 0}}\left(\mathrm{c}=0.165, \mathrm{CH}_{2} \mathrm{Cl}_{2}\right) 6.06$

HPLC: IB, 50\% IPA/Hex, $1.0 \mathrm{~mL} / \mathrm{min}$
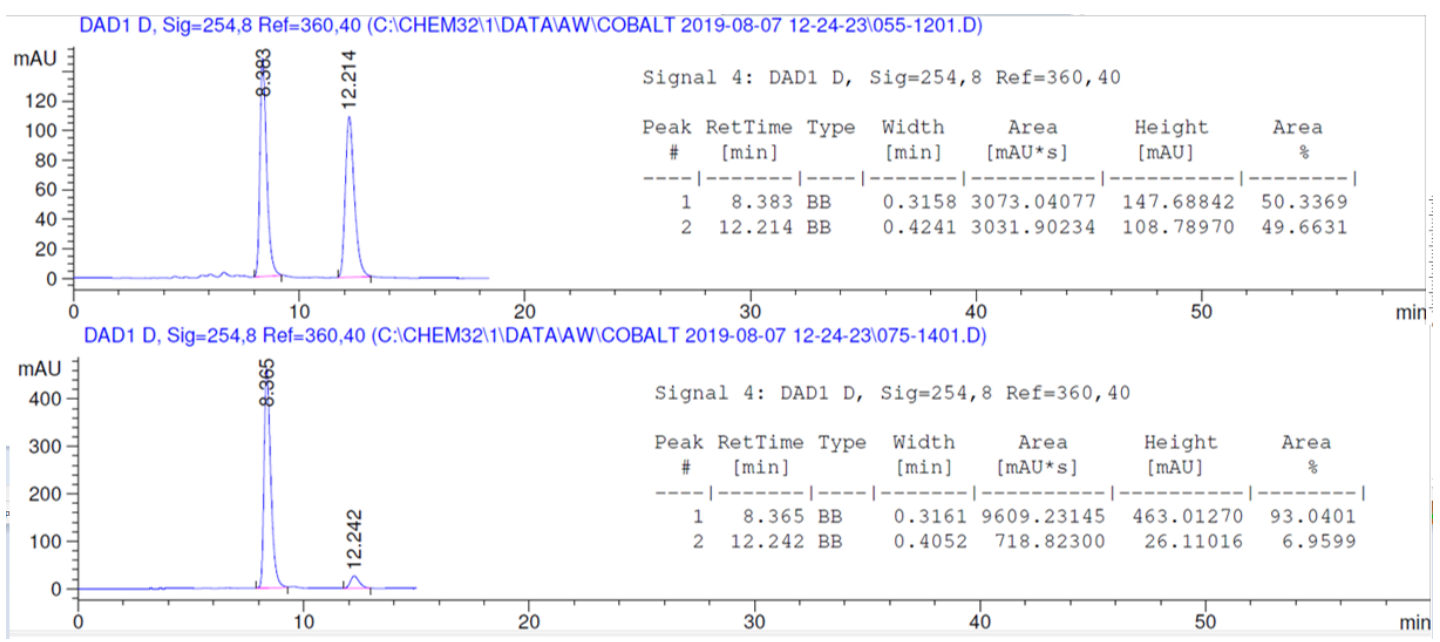
(R,Z)-1-(pyridin-2-yl)-2-((1-tosyl-4-(3-(trifluoromethyl)benzylidene)pyrrolidin-3yl)methyl)-1H-indole (4p)

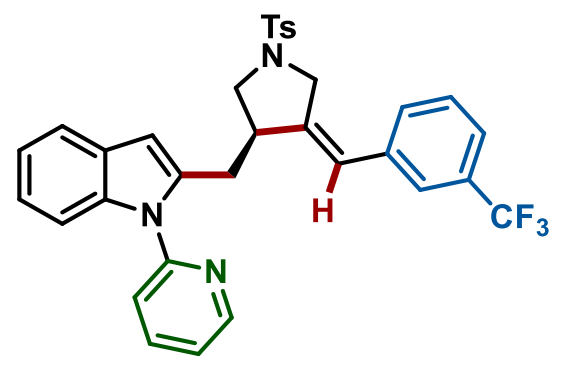

Synthesized following $\mathbf{G P 3}$ at $80{ }^{\circ} \mathrm{C}$. The product was isolated by flash column chromatography $15->30 \%$ EtOAc/pentane. The product appeared as a white solid, $75 \mathrm{mg}$ ( $64 \%$ yield).

${ }^{1} \mathbf{H}$ NMR $\left(500 \mathrm{MHz}, \mathrm{CDCl}_{3}\right) \delta 8.67(\mathrm{ddd}, J=4.9,2.0,0.8 \mathrm{~Hz}, 1 \mathrm{H}), 7.92(\mathrm{ddd}, J=8.0,7.4,2.0$ $\mathrm{Hz}, 1 \mathrm{H}), 7.68(\mathrm{~d}, J=8.3 \mathrm{~Hz}, 2 \mathrm{H}), 7.60(\mathrm{ddd}, J=6.1,3.1,0.8 \mathrm{~Hz}, 1 \mathrm{H}), 7.49(\mathrm{~m}, 2 \mathrm{H}), 7.45(\mathrm{t}, J=$ $7.7 \mathrm{~Hz}, 1 \mathrm{H}), 7.36(\mathrm{ddd}, J=7.6,4.9,1.1 \mathrm{~Hz}, 2 \mathrm{H}), 7.31(\mathrm{~d}, J=8.3 \mathrm{~Hz}, 2 \mathrm{H}), 7.27(\mathrm{~s}, 1 \mathrm{H}), 7.23(\mathrm{~d}, J$ $=7.4 \mathrm{~Hz}, 1 \mathrm{H}), 7.20-7.15(\mathrm{~m}, 2 \mathrm{H}), 6.47(\mathrm{~d}, J=0.9 \mathrm{~Hz}, 1 \mathrm{H}), 6.14(\mathrm{q}, J=2.3 \mathrm{~Hz}, 1 \mathrm{H}), 4.13(\mathrm{dt}, J$ $=15.0,2.3 \mathrm{~Hz}, 1 \mathrm{H}), 3.97(\mathrm{dd}, J=15.0,2.3 \mathrm{~Hz}, 1 \mathrm{H}), 3.29$ (ddd, $J=14.8,5.1,0.9 \mathrm{~Hz}, 1 \mathrm{H}), 3.23-$ $3.18(\mathrm{~m}, 2 \mathrm{H}), 3.13-3.06(\mathrm{~m}, 1 \mathrm{H}), 2.97(\mathrm{ddd}, J=14.8,9.6,0.7 \mathrm{~Hz}, 1 \mathrm{H}), 2.42(\mathrm{~s}, 3 \mathrm{H})$.

${ }^{13} \mathrm{C}$ NMR $\left(126 \mathrm{MHz}, \mathrm{CDCl}_{3}\right) \delta 151.3,149.6,143.8,142.1,138.6,138.0,137.2,137.1,132.5$, $130.9(\mathrm{~d}, J=32.2 \mathrm{~Hz}), 130.8(\mathrm{~d}, J=1.4 \mathrm{~Hz}), 129.8,129.0,128.3,127.8,125.0(\mathrm{q}, J=3.9 \mathrm{~Hz})$, $124.0(\mathrm{q}, J=272.5 \mathrm{~Hz}), 123.7(\mathrm{q}, J=3.8 \mathrm{~Hz}), 122.3,122.3,122.2,121.0,121.0,120.3,110.2$, 104.2, 51.7, 50.5, 44.6, 31.8, 21.5.

${ }^{19}$ F NMR $\left(377 \mathrm{MHz}, \mathrm{CDCl}_{3}\right) \delta-62.7$.

IR (ATR) 1589, 1485, 1437, 1333, 1166, 1125, 913, 785, 736, 663

HRMS (DART, M+H) Calculated for $\mathrm{C}_{33} \mathrm{H}_{29} \mathrm{~N}_{3} \mathrm{O}_{2} \mathrm{~F}_{3} \mathrm{~S} 588.1927$, found 588.1926

MP $70-71{ }^{\circ} \mathrm{C}$

$\boldsymbol{\alpha}_{D}^{\mathbf{2 0}}\left(\mathrm{c}=0.135, \mathrm{CH}_{2} \mathrm{Cl}_{2}\right) 7.41$

HPLC: IB, $40 \%$ IPA/Hex, $0.75 \mathrm{~mL} / \mathrm{min}$

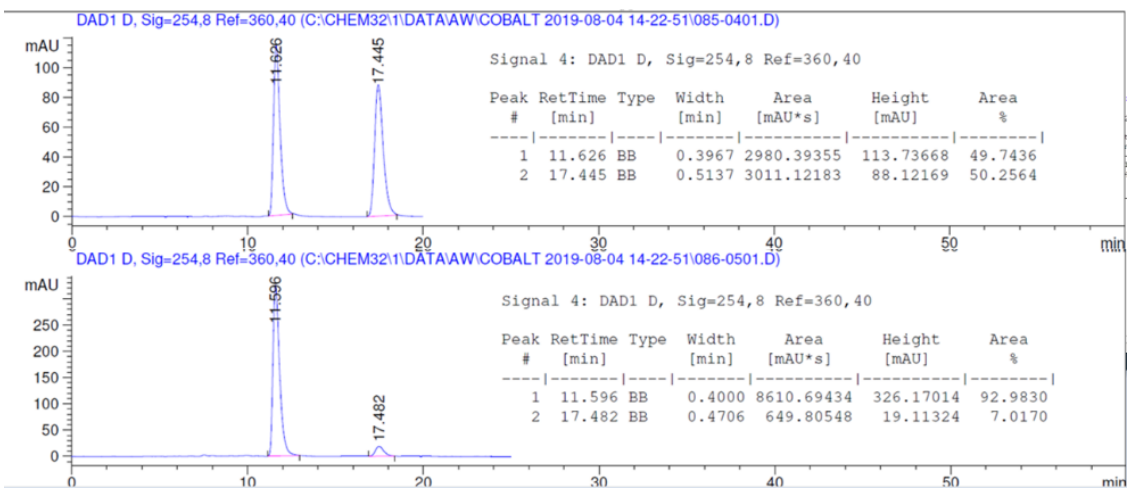


ethyl (R,Z)-3-((4-((1-(pyridin-2-yl)-1H-indol-2-yl)methyl)-1-tosylpyrrolidin-3ylidene)methyl)benzoate (4q)

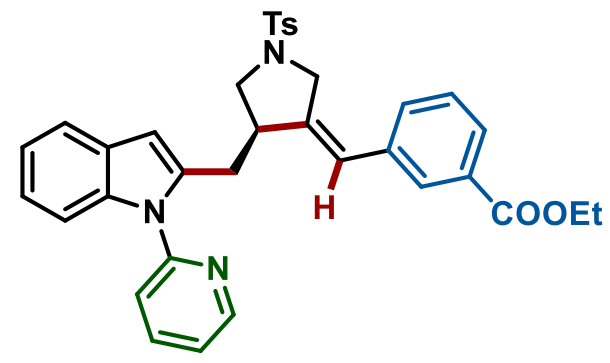

Synthesized following $\mathbf{G P 3}$ at $80{ }^{\circ} \mathrm{C}$. The product was isolated by flash column chromatography 15->30\% EtOAc/pentane. The product appeared as a white solid, $97 \mathrm{mg}$ (82\% yield).

${ }^{1} \mathbf{H}$ NMR $\left(500 \mathrm{MHz}, \mathrm{CDCl}_{3}\right) \delta 8.67$ (ddd, $\left.J=4.9,2.0,0.8 \mathrm{~Hz}, 1 \mathrm{H}\right), 7.94-7.87(\mathrm{~m}, 2 \mathrm{H}), 7.73(\mathrm{t}$, $J=1.8 \mathrm{~Hz}, 1 \mathrm{H}), 7.68(\mathrm{~d}, J=8.3 \mathrm{~Hz}, 2 \mathrm{H}), 7.62-7.57(\mathrm{~m}, 1 \mathrm{H}), 7.48(\mathrm{dt}, J=8.0,1.0 \mathrm{~Hz}, 1 \mathrm{H})$, $7.40(\mathrm{t}, J=7.7 \mathrm{~Hz}, 1 \mathrm{H}), 7.37-7.32(\mathrm{~m}, 2 \mathrm{H}), 7.29(\mathrm{~d}, J=8.3 \mathrm{~Hz}, 2 \mathrm{H}), 7.23(\mathrm{dt}, J=7.8,1.5 \mathrm{~Hz}$, $1 \mathrm{H}), 7.19-7.14(\mathrm{~m}, 2 \mathrm{H}), 6.47(\mathrm{~d}, J=0.9 \mathrm{~Hz}, 1 \mathrm{H}), 6.16(\mathrm{q}, J=2.3 \mathrm{~Hz}, 1 \mathrm{H}), 4.40(\mathrm{q}, J=7.2 \mathrm{~Hz}$, 2H), $4.14(\mathrm{dt}, J=14.9,2.3 \mathrm{~Hz}, 1 \mathrm{H}), 3.99(\mathrm{dd}, J=14.9,2.3 \mathrm{~Hz}, 1 \mathrm{H}), 3.29(\mathrm{ddd}, J=14.8,5.0,0.9$ $\mathrm{Hz}, 1 \mathrm{H}), 3.22-3.16(\mathrm{~m}, 2 \mathrm{H}), 3.08(\mathrm{~m}, 1 \mathrm{H}), 2.95(\mathrm{ddd}, J=14.8,9.8,0.7 \mathrm{~Hz}, 1 \mathrm{H}), 2.41(\mathrm{~s}, 3 \mathrm{H})$, $1.42(\mathrm{t}, J=7.1 \mathrm{~Hz}, 3 \mathrm{H})$.

${ }^{13} \mathrm{C}$ NMR $\left(126 \mathrm{MHz}, \mathrm{CDCl}_{3}\right) \delta 166.3,151.3,149.7,143.7,141.3,138.5,138.1,137.2,136.6$, $132.5,131.8,130.8,129.7,129.3,128.6,128.4,128.0,127.8,122.7,122.2,122.2,121.0,120.9$, $120.3,110.1,104.2,61.1,51.7,50.6,44.5,31.9,21.5,14.3$.

IR (ATR) 2961, 2853, 1714, 1581, 1468, 1435, 1343, 1268, 1201, 1154, 1091, 1017, 813, 782, 746, 707, 662

HRMS (DART, M+H) Calculated for $\mathrm{C}_{35} \mathrm{H}_{34} \mathrm{~N}_{3} \mathrm{O}_{4} \mathrm{~S}_{1} 592.2265$, found 592.2257

MP $69-70{ }^{\circ} \mathrm{C}$

$\boldsymbol{\alpha}_{D}^{\mathbf{2 0}}\left(\mathrm{c}=0.275, \mathrm{CH}_{2} \mathrm{Cl}_{2}\right) 5.09$

HPLC: IB, 50\% IPA/Hex, $1.0 \mathrm{~mL} / \mathrm{min}$

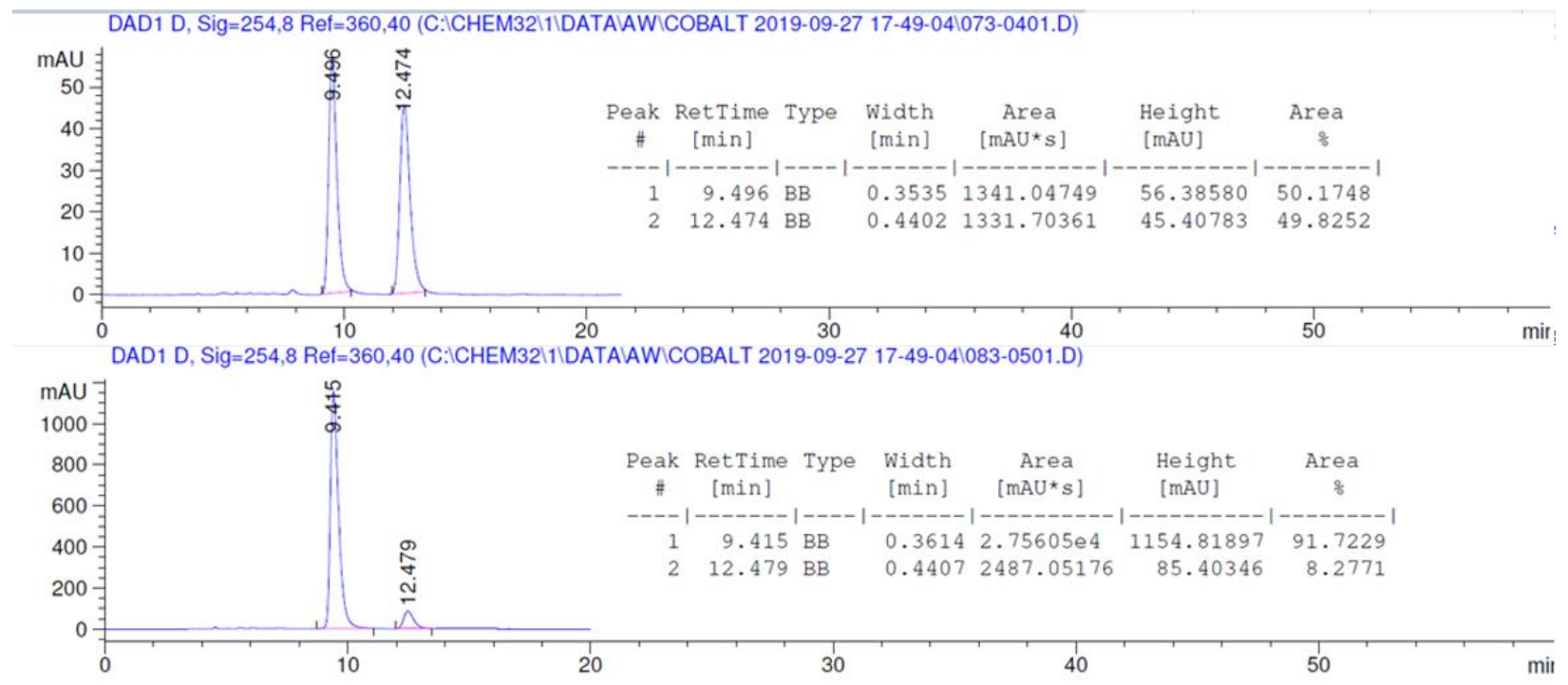


(R,Z)-2-((4-(3-bromobenzylidene)-1-tosylpyrrolidin-3-yl)methyl)-1-(pyridin-2-yl)-1Hindole $(4 r)$<smiles>[As]C1C/C(=C\c2cccc(Br)c2)[C@H](Cc2cc3ccccc3n2-c2ccccn2)C1</smiles>

Synthesized following GP3. The product was isolated by flash column chromatography 15$>30 \%$ EtOAc/pentane. The product appeared as a white solid, $103 \mathrm{mg}$ ( $86 \%$ yield).

${ }^{1} \mathbf{H}$ NMR $\left(500 \mathrm{MHz}_{\mathrm{CDCl}}\right) \delta 8.66(\mathrm{ddd}, \mathrm{J}=4.9,2.0,0.9 \mathrm{~Hz}, 1 \mathrm{H}), 7.92(\mathrm{ddd}, \mathrm{J}=7.9,7.5,2.0$ $\mathrm{Hz}, 1 \mathrm{H}), 7.68(\mathrm{~d}, \mathrm{~J}=8.3 \mathrm{~Hz}, 2 \mathrm{H}), 7.64-7.56(\mathrm{~m}, 1 \mathrm{H}), 7.48(\mathrm{dt}, \mathrm{J}=7.9,0.9 \mathrm{~Hz}, 1 \mathrm{H}), 7.37-7.33$ $(\mathrm{m}, 3 \mathrm{H}), 7.31(\mathrm{~d}, \mathrm{~J}=8.3 \mathrm{~Hz}, 2 \mathrm{H}), 7.23-7.13(\mathrm{~m}, 4 \mathrm{H}), 6.98(\mathrm{dt}, \mathrm{J}=7.8,1.3 \mathrm{~Hz}, 1 \mathrm{H}), 6.46(\mathrm{~d}, \mathrm{~J}=$ $0.9 \mathrm{~Hz}, 1 \mathrm{H}), 6.04(\mathrm{q}, \mathrm{J}=2.3 \mathrm{~Hz}, 1 \mathrm{H}), 4.12(\mathrm{dt}, \mathrm{J}=14.9,2.3 \mathrm{~Hz}, 1 \mathrm{H}), 3.96(\mathrm{dd}, \mathrm{J}=14.9,2.3 \mathrm{~Hz}$, $1 \mathrm{H}), 3.26$ (ddd, J = 14.8, 5.1, $0.9 \mathrm{~Hz}, 1 \mathrm{H}), 3.21-3.16(\mathrm{~m}, 2 \mathrm{H}), 3.11-3.01(\mathrm{~m}, 1 \mathrm{H}), 2.94(\mathrm{dd}, \mathrm{J}=$ $14.8,9.6 \mathrm{~Hz}, 1 \mathrm{H}), 2.42(\mathrm{~s}, 3 \mathrm{H})$.

${ }^{13} \mathrm{C}$ NMR $\left(126 \mathrm{MHz}, \mathrm{CDCl}_{3}\right) \delta 151.3,149.6,143.8,141.6,138.5,138.4,138.0,137.2,132.5$, $131.1,130.0,130.0,129.8,128.4,127.8,126.3,122.6,122.2,122.2,122.2$, 121.0, 120.3, 110.1, 104.2, 51.7, 50.5, 44.6, 31.9, 21.5.

IR (ATR) 1586, 1474, 1437, 1340, 1163, 1097, 916, 816, 733, 667

HRMS (DART, M+H) Calculated for $\mathrm{C}_{32} \mathrm{H}_{29} \mathrm{~N}_{3} \mathrm{O}_{2} \mathrm{SBr} 598.1158$, found 598.1151

MP $65-72{ }^{\circ} \mathrm{C}$

$\boldsymbol{\alpha}_{\boldsymbol{D}}^{\mathbf{2 0}}\left(\mathrm{c}=0.455, \mathrm{CH}_{2} \mathrm{Cl}_{2}\right) 3.52$

HPLC: IB, 50\% IPA/Hex, $1.0 \mathrm{~mL} / \mathrm{min}$

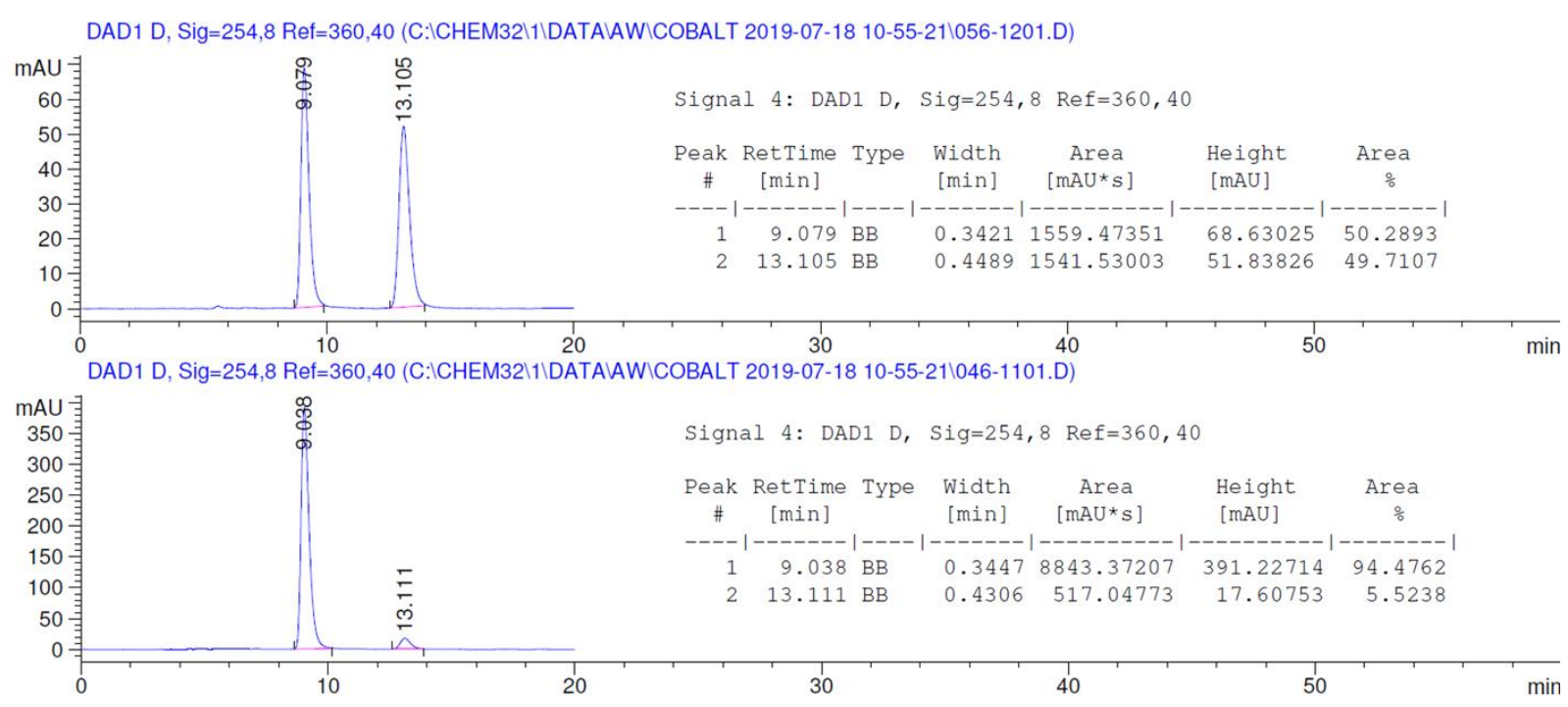


(R,Z)-2-((4-(3,5-dimethoxybenzylidene)-1-tosylpyrrolidin-3-yl)methyl)-1-(pyridin-2-yl)-1Hindole $(4 s)$<smiles>[CH2+][NH+]1C/C(=C\c2cc(OC)cc(OC)c2)C(Cc2cc3ccccc3n2-c2ccccn2)C1</smiles>

Synthesized following $\mathbf{G P 3}$ at $80{ }^{\circ} \mathrm{C}$. The product was isolated by flash column chromatography $15->30 \%$ EtOAc/pentane. The product appeared as a white solid, $109 \mathrm{mg}$ ( $94 \%$ yield).

${ }^{1} \mathbf{H}$ NMR $\left(500 \mathrm{MHz}, \mathrm{CDCl}_{3}\right) \delta 8.66(\mathrm{ddd}, J=4.9,2.0,0.8 \mathrm{~Hz}, 1 \mathrm{H}$ ), 7.91 (ddd, $J=8.0,7.5,2.0$ $\mathrm{Hz}, 1 \mathrm{H}), 7.67(\mathrm{~d}, J=8.2 \mathrm{~Hz}, 2 \mathrm{H}), 7.59$ (ddd, $J=6.1,3.1,0.8 \mathrm{~Hz}, 1 \mathrm{H}), 7.47(\mathrm{~d}, J=8.0 \mathrm{~Hz}, 1 \mathrm{H})$, $7.38-7.31(\mathrm{~m}, 2 \mathrm{H}), 7.29$ (d, $J=8.2 \mathrm{~Hz}, 2 \mathrm{H}), 7.16(\mathrm{dd}, J=6.0,3.2 \mathrm{~Hz}, 2 \mathrm{H}), 6.46(\mathrm{~d}, J=0.8 \mathrm{~Hz}$, $1 \mathrm{H}), 6.35(\mathrm{t}, J=2.2 \mathrm{~Hz}, 1 \mathrm{H}), 6.18(\mathrm{~d}, J=2.2 \mathrm{~Hz}, 2 \mathrm{H}), 6.02(\mathrm{q}, J=2.3 \mathrm{~Hz}, 1 \mathrm{H}), 4.12(\mathrm{dt}, J=$ $14.9,23 \mathrm{~Hz}, 1 \mathrm{H}), 3.97(\mathrm{dd}, J=14.9,2.3 \mathrm{~Hz}, 1 \mathrm{H}), 3.27(\mathrm{ddd}, J=14.5,4.8,0.8 \mathrm{~Hz}, 1 \mathrm{H}), 3.17(\mathrm{~m}$, 2H), $3.02(\mathrm{~m}, 1 \mathrm{H}), 2.94$ (ddd, $J=14.5,9.7,0.7 \mathrm{~Hz}, 1 \mathrm{H}), 2.41(\mathrm{~s}, 3 \mathrm{H})$.

${ }^{13}$ C NMR (126 MHz, $\left.\mathrm{CDCl}_{3}\right) \delta 160.7,151.3,149.6,143.7,140.4,138.5,138.3,138.2,137.2$, 132.6, 129.7, 128.4, 127.8, 123.5, 122.2, 122.1, 121.0, 120.9, 120.2, 110.1, 106.4, 104.1, 98.9, 55.3, 51.7, 50.7, 44.5, 31.9, 21.5.

IR (ATR) 1593, 1458, 1437, 1347, 1208, 1156, 1066, 906, 802, 726, 663

HRMS (DART, M+H) Calculated for $\mathrm{C}_{34} \mathrm{H}_{34} \mathrm{~N}_{3} \mathrm{O}_{4} \mathrm{~S} 580.2265$, found 580.2267

MP $69-70{ }^{\circ} \mathrm{C}$

$\boldsymbol{\alpha}_{\boldsymbol{D}}^{\mathbf{2 0}}\left(\mathrm{c}=0.35, \mathrm{CH}_{2} \mathrm{Cl}_{2}\right) 9.71$

HPLC: IB, 50\% IPA/Hex, $1.0 \mathrm{~mL} / \mathrm{min}$

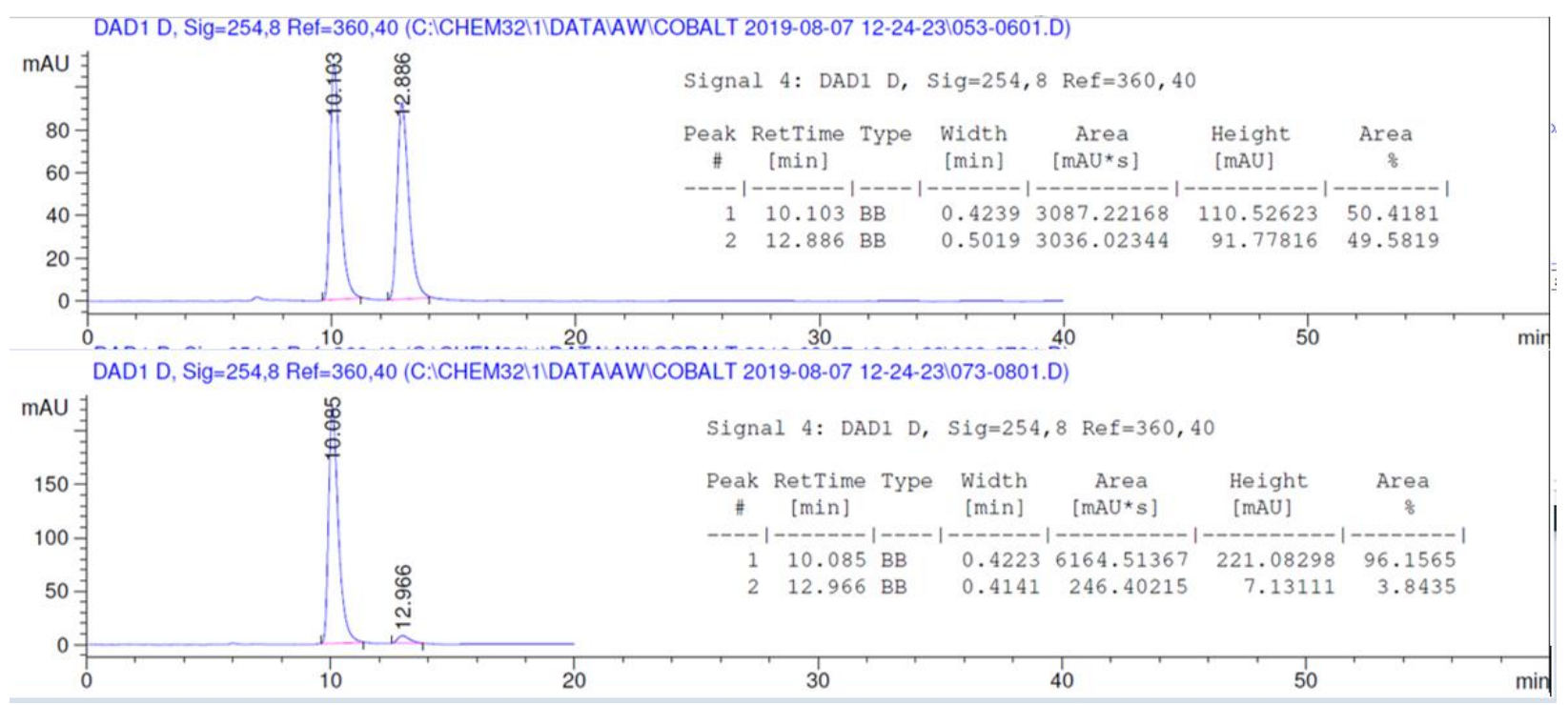




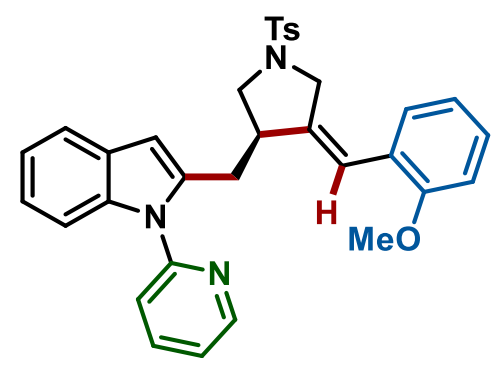

Synthesized following $\mathbf{G P 3}$ at $80{ }^{\circ} \mathrm{C}$. The product was isolated by flash column chromatography $15->30 \%$ EtOAc/pentane. The product appeared as a white solid, $79 \mathrm{mg}$ (72\% yield).

${ }^{1} \mathbf{H}$ NMR $\left(500 \mathrm{MHz}, \mathrm{CDCl}_{3}\right) \delta 8.67(\mathrm{ddd}, J=4.9,2.0,0.8 \mathrm{~Hz}, 1 \mathrm{H}), 7.90$ (ddd, $J=8.0,7.5,2.0$ $\mathrm{Hz}, 1 \mathrm{H}), 7.65(\mathrm{~d}, J=8.3 \mathrm{~Hz}, 2 \mathrm{H}), 7.62-7.56(\mathrm{~m}, 1 \mathrm{H}), 7.48(\mathrm{dt}, J=8.0,0.8 \mathrm{~Hz}, 1 \mathrm{H}), 7.36-7.31$ $(\mathrm{m}, 2 \mathrm{H}), 7.28(\mathrm{~d}, J=8.3 \mathrm{~Hz}, 2 \mathrm{H}), 7.22(\mathrm{ddd}, J=8.3,7.3,1.7 \mathrm{~Hz}, 1 \mathrm{H}), 7.18-7.13(\mathrm{~m}, 2 \mathrm{H}), 7.01$ $(\mathrm{dd}, J=7.6,1.7 \mathrm{~Hz}, 1 \mathrm{H}), 6.93(\mathrm{td}, J=7.6,1.1 \mathrm{~Hz}, 1 \mathrm{H}), 6.84(\mathrm{dd}, J=8.3,1.1 \mathrm{~Hz}, 1 \mathrm{H}), 6.47(\mathrm{~d}, J$ $=0.8 \mathrm{~Hz}, 1 \mathrm{H}), 6.42(\mathrm{q}, J=2.4 \mathrm{~Hz}, 1 \mathrm{H}), 4.10(\mathrm{ddd}, J=14.7,2.4,1.7 \mathrm{~Hz}, 1 \mathrm{H}), 3.92(\mathrm{ddd}, J=14.7$, $2.4,0.8 \mathrm{~Hz}, 1 \mathrm{H}), 3.76(\mathrm{~s}, 3 \mathrm{H}), 3.31$ (ddd, $J=14.8,4.8,0.8 \mathrm{~Hz}, 1 \mathrm{H}), 3.18(\mathrm{~m}, 2 \mathrm{H}), 3.12-3.01$ (m, 1H), $2.95(\mathrm{ddd}, J=14.8,9.9,0.7 \mathrm{~Hz}, 1 \mathrm{H}), 2.40(\mathrm{~s}, 3 \mathrm{H})$.

${ }^{13} \mathrm{C}$ NMR $\left(126 \mathrm{MHz}, \mathrm{CDCl}_{3}\right) \delta 156.5,151.3,149.7,143.5,139.7,138.5,138.4,137.2,132.7$, $129.6,128.5,128.5,128.4,127.8,125.3,122.1,122.0,121.0,120.8,120.4,120.2,118.1,110.5$, $110.1,104.0,55.4,51.8,50.6,44.5,32.0,21.5$.

IR (ATR) 1586, 1477, 1437, 1343, 1167, 1037, 1092, 908, 816, 722, 660

HRMS (DART, M+H) Calculated for $\mathrm{C}_{33} \mathrm{H}_{32} \mathrm{~N}_{3} \mathrm{O}_{3} \mathrm{~S} 550.2159$, found 550.2159

MP $78-80{ }^{\circ} \mathrm{C}$

$\boldsymbol{\alpha}_{\boldsymbol{D}}^{\mathbf{2 0}}\left(\mathrm{c}=0.18, \mathrm{CH}_{2} \mathrm{Cl}_{2}\right) 3.33$

HPLC: IB, 50\% IPA/Hex, $1.0 \mathrm{~mL} / \mathrm{min}$

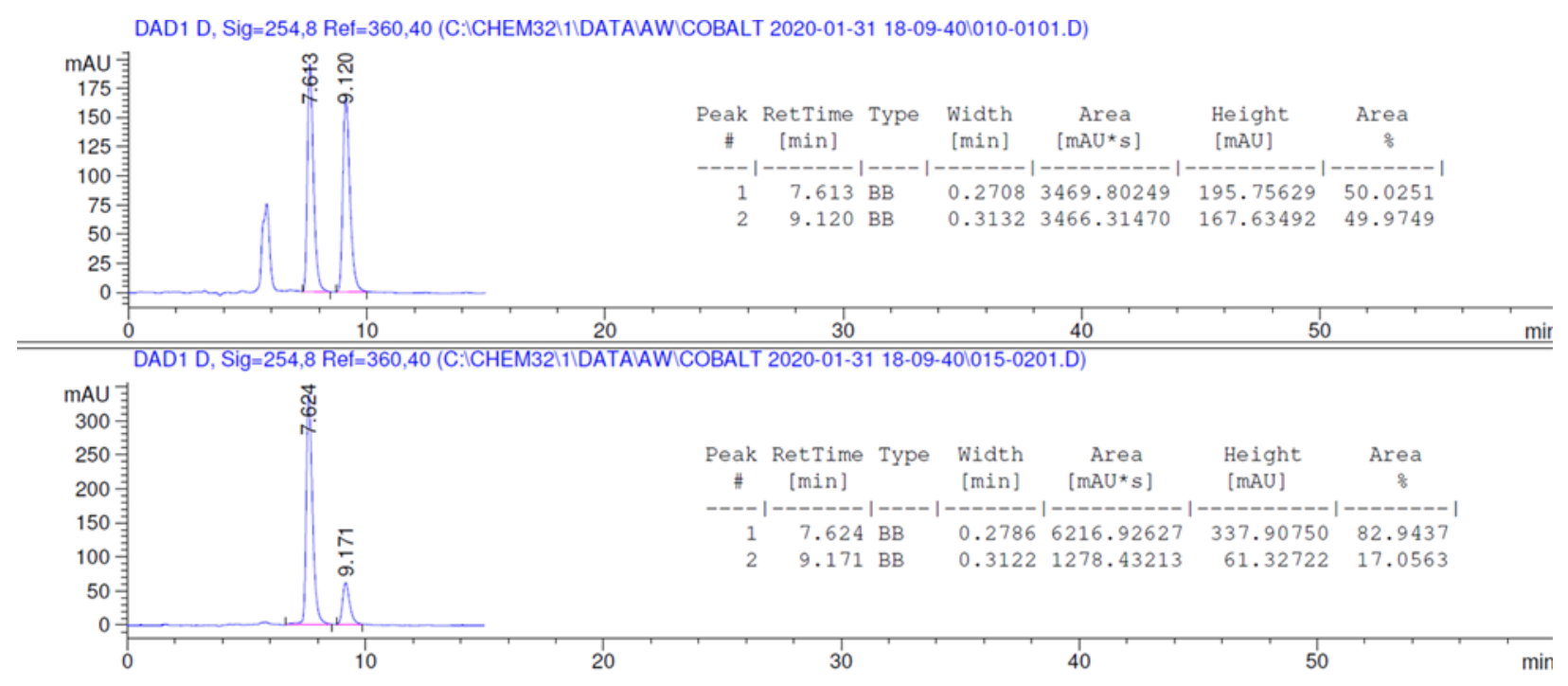


<smiles>Fc1ccccc1/C=C1\CN([As])C[C@H]1Cc1cc2ccccc2n1-c1ccccn1</smiles>

Synthesized following GP3. The product was isolated by flash column chromatography 15$>30 \%$ EtOAc/pentane. The product appeared as a white solid, $50 \mathrm{mg}$ (46\% yield).

${ }^{1} \mathbf{H}$ NMR $\left(500 \mathrm{MHz}, \mathrm{CDCl}_{3}\right) \delta 8.67(\mathrm{ddd}, J=4.9,2.0,0.8 \mathrm{~Hz}, 1 \mathrm{H}), 7.91(\mathrm{ddd}, J=8.0,7.5,2.0$ $\mathrm{Hz}, 1 \mathrm{H}), 7.67(\mathrm{~d}, J=8.3 \mathrm{~Hz}, 2 \mathrm{H}), 7.62-7.57(\mathrm{~m}, 1 \mathrm{H}), 7.49(\mathrm{dt}, J=8.0,0.8 \mathrm{~Hz}, 1 \mathrm{H}), 7.37-7.32$ (m, 2H), 7.29 (d, $J=8.3 \mathrm{~Hz}, 2 \mathrm{H}), 7.22$ (dddd, $J=8.1,7.2,5.2,1.8 \mathrm{~Hz}, 1 \mathrm{H}), 7.18-7.14(\mathrm{~m}, 2 \mathrm{H})$, $7.11(\mathrm{td}, J=7.5,1.2 \mathrm{~Hz}, 1 \mathrm{H}), 7.08-6.99(\mathrm{~m}, 2 \mathrm{H}), 6.46(\mathrm{~d}, J=0.8 \mathrm{~Hz}, 1 \mathrm{H}), 6.27(\mathrm{~s}, 1 \mathrm{H}), 4.10$ (dt, $J=14.9,2.2 \mathrm{~Hz}, 1 \mathrm{H}), 3.92(\mathrm{dd}, J=14.9,2.2 \mathrm{~Hz}, 1 \mathrm{H}), 3.29$ (ddd, $J=14.8,4.5,0.9 \mathrm{~Hz}, 1 \mathrm{H})$, $3.22-3.17$ (m, 2H), $3.11-3.03(\mathrm{~m}, 1 \mathrm{H}), 2.94$ (ddd, $J=14.8,10.0,0.7 \mathrm{~Hz}, 1 \mathrm{H}), 2.41$ (s, 3H).

${ }^{13} \mathrm{C}$ NMR $\left(126 \mathrm{MHz}, \mathrm{CDCl}_{3}\right) \delta 159.7(\mathrm{~d}, J=248.4 \mathrm{~Hz}), 151.3,149.7,143.7,142.2(\mathrm{~d}, J=1.4$ $\mathrm{Hz}), 138.5,138.1,137.2,132.6,129.7,128.9,128.8,128.8,128.4,127.8,124.2(\mathrm{~d}, J=13.1 \mathrm{~Hz})$, $124.0(\mathrm{~d}, J=3.6 \mathrm{~Hz}), 122.2,122.2,120.9,120.3,115.5(\mathrm{~d}, J=5.3 \mathrm{~Hz}), 115.5(\mathrm{~d}, J=22.2 \mathrm{~Hz})$, $110.1,104.1,51.8,50.7(\mathrm{~d}, J=2.6 \mathrm{~Hz}), 44.5,32.0,21.5$.

${ }^{19}$ F NMR $\left(377 \mathrm{MHz}, \mathrm{CDCl}_{3}\right) \delta-116.1$.

IR (ATR) 1612, 1502, 1485, 1471, 1436, 1349, 1173, 1055, 1018, 815, 770

HRMS (DART, M+H) Calculated for $\mathrm{C}_{32} \mathrm{H}_{29} \mathrm{~N}_{3} \mathrm{O}_{2} \mathrm{~F}_{1} \mathrm{~S}_{1} 538.1959$, found 538.1967

MP $123-128{ }^{\circ} \mathrm{C}$

$\boldsymbol{\alpha}_{\boldsymbol{D}}^{\mathbf{2 0}}\left(\mathrm{c}=0.20, \mathrm{CH}_{2} \mathrm{Cl}_{2}\right) 2.00$

HPLC: IB, 50\% IPA/Hex, $1.0 \mathrm{~mL} / \mathrm{min}$
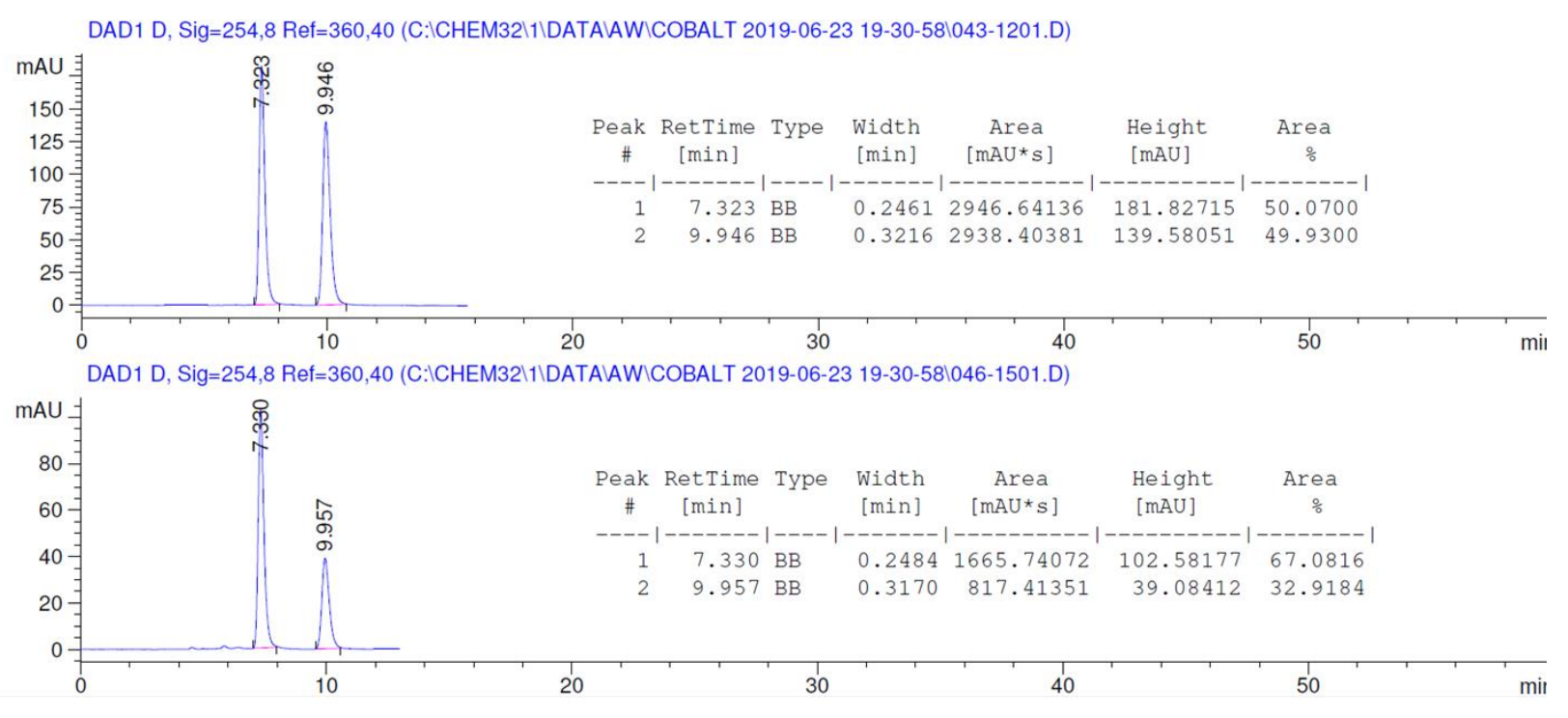
(R,Z)-1-(pyridin-2-yl)-2-((4-(thiophen-2-ylmethylene)-1-tosylpyrrolidin-3-yl)methyl)-1Hindole $(4 \mathrm{v})$<smiles>[As]C1CC(=Cc2cccs2)C(Cc2cc3ccccc3n2-c2ccccn2)C1</smiles>

Synthesized following GP3. The product was isolated by flash column chromatography 15$>30 \%$ EtOAc/pentane. The product appeared as a yellow solid, $42 \mathrm{mg}$ (40\% yield).

${ }^{1} \mathbf{H}$ NMR $\left(500 \mathrm{MHz}, \mathrm{CDCl}_{3}\right) \delta 8.65(\mathrm{ddd}, J=4.9,2.0,0.9 \mathrm{~Hz}, 1 \mathrm{H}), 7.90(\mathrm{ddd}, J=8.0,7.4,2.0$ $\mathrm{Hz}, 1 \mathrm{H}), 7.70(\mathrm{~d}, J=8.2 \mathrm{~Hz}, 2 \mathrm{H}), 7.62-7.56(\mathrm{~m}, 1 \mathrm{H}), 7.47(\mathrm{dt}, J=8.0,1.0 \mathrm{~Hz}, 1 \mathrm{H}), 7.37-7.32$ $(\mathrm{m}, 2 \mathrm{H}), 7.30(\mathrm{~d}, J=7.9 \mathrm{~Hz}, 2 \mathrm{H}), 7.28(\mathrm{~d}, J=5.0 \mathrm{~Hz}, 1 \mathrm{H}), 7.19-7.13(\mathrm{~m}, 2 \mathrm{H}), 7.00(\mathrm{dd}, J=$ $5.1,3.6 \mathrm{~Hz}, 1 \mathrm{H}), 6.80(\mathrm{~d}, J=3.5 \mathrm{~Hz}, 1 \mathrm{H}), 6.48-6.44(\mathrm{~m}, 1 \mathrm{H}), 6.30(\mathrm{q}, J=2.4 \mathrm{~Hz}, 1 \mathrm{H}), 4.08(\mathrm{dt}$, $J=15.3,2.3 \mathrm{~Hz}, 1 \mathrm{H}), 3.95(\mathrm{dd}, J=15.3,2.1 \mathrm{~Hz}, 1 \mathrm{H}), 3.27(\mathrm{ddd}, J=14.9,5.0,1.0 \mathrm{~Hz}, 1 \mathrm{H}), 3.23$ - $3.14(\mathrm{~m}, 2 \mathrm{H}), 3.11-3.02(\mathrm{~m}, 1 \mathrm{H}), 2.93(\mathrm{dd}, J=15.1,9.7 \mathrm{~Hz}, 1 \mathrm{H}), 2.41(\mathrm{~d}, J=0.7 \mathrm{~Hz}, 3 \mathrm{H})$.

${ }^{13} \mathrm{C}$ NMR $\left(126 \mathrm{MHz}, \mathrm{CDCl}_{3}\right) \delta 151.3,149.6,143.7,140.1,138.5,138.2,138.1,137.2,132.2$, 129.7, 128.4, 127.9, 127.4, 126.5, 125.7, 122.2, 122.1, 121.0, 120.9, 120.3, 116.3, 110.1, 104.1, $52.3,51.2,44.1,31.7,21.5$.

IR (ATR) 1586, 1469, 1455, 1436, 1342, 1211, 1159, 1092, 1016, 989, 813, 782, 706, 663

HRMS (DART, M+H) Calculated for $\mathrm{C}_{30} \mathrm{H}_{28} \mathrm{~N}_{3} \mathrm{O}_{2} \mathrm{~S}_{2} 526.1617$, found 526.1621

MP $80-90{ }^{\circ} \mathrm{C}$

$\boldsymbol{\alpha}_{\boldsymbol{D}}^{\mathbf{2 0}}\left(\mathrm{c}=0.225, \mathrm{CH}_{2} \mathrm{Cl}_{2}\right) 1.78$

HPLC: IB, 50\% IPA/Hex, $1 \mathrm{~mL} / \mathrm{min}$

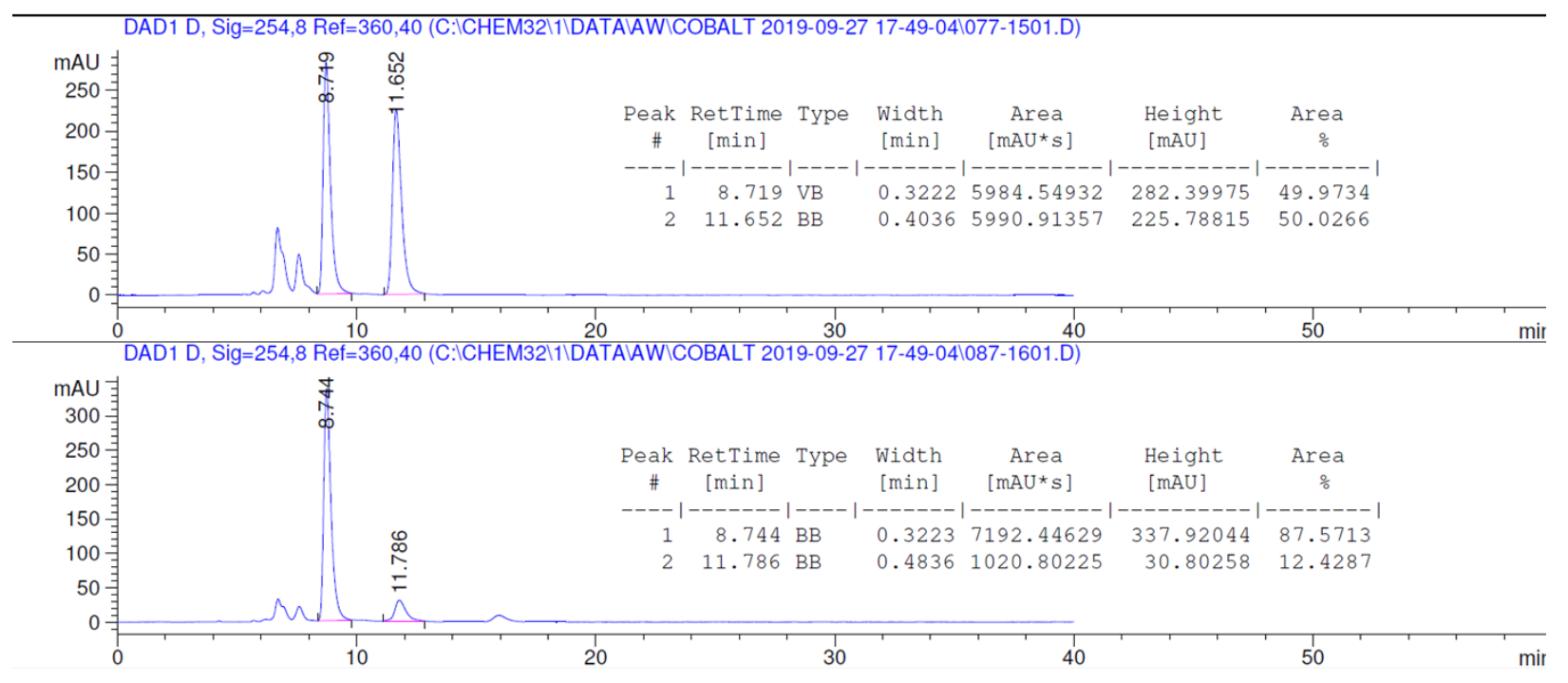




\section{(R,Z)-2-((4-pentylidene-1-tosylpyrrolidin-3-yl)methyl)-1-(pyridin-2-yl)-1H-indole (4w)}<smiles>CCCC=C1C[NH+]([As])C[C@H]1Cc1cc2ccccc2n1-c1ccccn1</smiles>

Synthesized following GP3. The product was isolated by flash column chromatography 15$>30 \%$ EtOAc/pentane. The product appeared as a white solid, $50 \mathrm{mg}$ (50\% yield).

${ }^{1} \mathbf{H}$ NMR $\left(500 \mathrm{MHz}, \mathrm{CDCl}_{3}\right) \delta 8.63(\mathrm{ddd}, J=4.9,2.0,0.8 \mathrm{~Hz}, 1 \mathrm{H}), 7.90(\mathrm{ddd}, J=8.0,7.5,2.0$ $\mathrm{Hz}, 1 \mathrm{H}), 7.67(\mathrm{~d}, J=8.2 \mathrm{~Hz}, 2 \mathrm{H}), 7.60-7.55(\mathrm{~m}, 1 \mathrm{H}), 7.46(\mathrm{dt}, J=8.0,0.8 \mathrm{~Hz}, 1 \mathrm{H}), 7.35-7.28$ $(\mathrm{m}, 4 \mathrm{H}), 7.17-7.12(\mathrm{~m}, 2 \mathrm{H}), 6.40(\mathrm{~d}, J=0.8 \mathrm{~Hz}, 1 \mathrm{H}), 5.10-5.01(\mathrm{~m}, 1 \mathrm{H}), 3.80$ (ddd, $J=14.0$, 2.6, $1.3 \mathrm{~Hz}, 1 \mathrm{H}), 3.68(\mathrm{dq}, J=14.0,1.0 \mathrm{~Hz}, 1 \mathrm{H}), 3.19-3.07(\mathrm{~m}, 2 \mathrm{H}), 3.04(\mathrm{dd}, J=9.4,4.7 \mathrm{~Hz}$, $1 \mathrm{H}), 2.85-2.74(\mathrm{~m}, 2 \mathrm{H}), 2.42(\mathrm{~s}, 3 \mathrm{H}), 1.86-1.76(\mathrm{~m}, 2 \mathrm{H}), 1.24-1.18(\mathrm{~m}, 2 \mathrm{H}), 0.87-0.82(\mathrm{~m}$, $3 \mathrm{H})$.

${ }^{13}$ C NMR $\left(126 \mathrm{MHz}, \mathrm{CDCl}_{3}\right) \delta 151.3,149.6,143.5,138.6,138.4,137.5,137.2,132.5,129.6$, $128.4,127.9,123.5,122.1,122.0,121.0,120.8,120.2,110.1,103.9,52.8,49.6,42.3,31.8,31.3$, 29.0, 22.2, 21.5, 13.9.

IR (ATR) 2428, 1586, 1447, 1471, 1350,1156, 10389, 917, 795, 663, 736

HRMS (DART, M+H) Calculated for $\mathrm{C}_{30} \mathrm{H}_{34} \mathrm{~N}_{3} \mathrm{O}_{2} \mathrm{~S}_{1} 500.2366$, found 500.2371

MP $56{ }^{\circ} \mathrm{C}$

$\boldsymbol{\alpha}_{D}^{\mathbf{2 0}}\left(\mathrm{c}=0.245, \mathrm{CH}_{2} \mathrm{Cl}_{2}\right) 18.7$

HPLC: IB, 50\% IPA/Hex, $1.0 \mathrm{~mL} / \mathrm{min}$
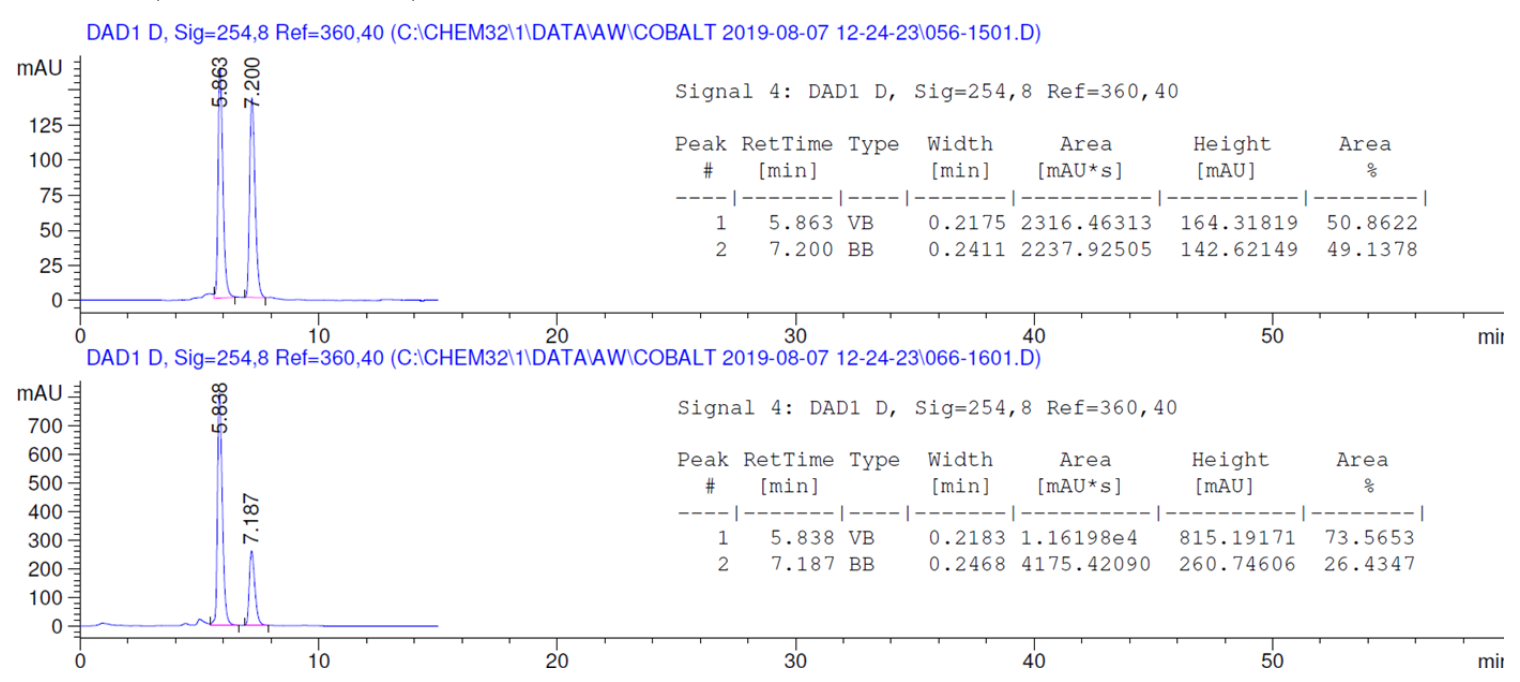
<smiles>C(=C1/COCC1Cc1cc2ccccc2n1-c1ccccn1)\c1ccccc1</smiles>

Synthesized following GP3. The product was isolated by flash column chromatography 15$>30 \% \mathrm{EtOAc/pentane.} \mathrm{The} \mathrm{product} \mathrm{appeared} \mathrm{as} \mathrm{an} \mathrm{oil,} 64 \mathrm{mg}$ (87\% yield).

${ }^{1} \mathbf{H}$ NMR $\left(500 \mathrm{MHz}, \mathrm{CDCl}_{3}\right) \delta 8.71(\mathrm{ddd}, J=4.9,2.0,0.9 \mathrm{~Hz}, 1 \mathrm{H}), 7.91(\mathrm{ddd}, J=8.0,7.4,2.0$ $\mathrm{Hz}, 1 \mathrm{H}), 7.61(\mathrm{ddd}, J=6.0,3.1,0.7 \mathrm{~Hz}, 1 \mathrm{H}), 7.49(\mathrm{dt}, J=8.0,1.0 \mathrm{~Hz}, 1 \mathrm{H}), 7.39-7.30(\mathrm{~m}, 5 \mathrm{H})$, $7.25-7.18(\mathrm{~m}, 2 \mathrm{H}), 7.17(\mathrm{dd}, J=6.0,3.2 \mathrm{~Hz}, 3 \mathrm{H}), 7.07$ (d, $J=7.2 \mathrm{~Hz}, 2 \mathrm{H}), 6.52$ (q, $J=0.8 \mathrm{~Hz}$, $1 \mathrm{H}), 6.21(\mathrm{q}, J=2.3 \mathrm{~Hz}, 1 \mathrm{H}), 4.64(\mathrm{dt}, J=14.1,2.3 \mathrm{~Hz}, 1 \mathrm{H}), 4.57(\mathrm{dd}, J=14.1,2.3 \mathrm{~Hz}, 1 \mathrm{H})$, $3.88(\mathrm{dd}, J=8.7,6.2 \mathrm{~Hz}, 1 \mathrm{H}), 3.72(\mathrm{dd}, J=8.7,4.6 \mathrm{~Hz}, 1 \mathrm{H}), 3.34(\mathrm{dd}, J=14.6,4.5 \mathrm{~Hz}, 1 \mathrm{H})$, $3.13-3.03(\mathrm{~m}, 1 \mathrm{H}), 3.01(\mathrm{dd}, J=14.6,10.0 \mathrm{~Hz}, 1 \mathrm{H})$.

${ }^{13}$ C NMR (126 MHz, $\left.\mathrm{CDCl}_{3}\right) \delta 151.4,149.7,144.5,139.1,138.4,137.3,137.2,128.5,128.5$, 127.9, 126.7, 122.2, 122.0, 121.3, 121.1, 120.8, 120.2, 110.1, 103.7, 72.3, 70.2, 45.1, 31.8.

IR (ATR) 1552, 1470, 1436, 1389, 1209, 1167, 927, 887, 866, 739

HRMS (DART, M+H) Calculated for $\mathrm{C}_{25} \mathrm{H}_{23} \mathrm{~N}_{2} \mathrm{O}_{1} 367.1805$, found 367.1808 $\boldsymbol{\alpha}_{D}^{\mathbf{2 0}}\left(\mathrm{c}=0.17, \mathrm{CH}_{2} \mathrm{Cl}_{2}\right)-45.8$

HPLC: AS-H, 20\% IPA/Hex, $0.5 \mathrm{~mL} / \mathrm{min}$

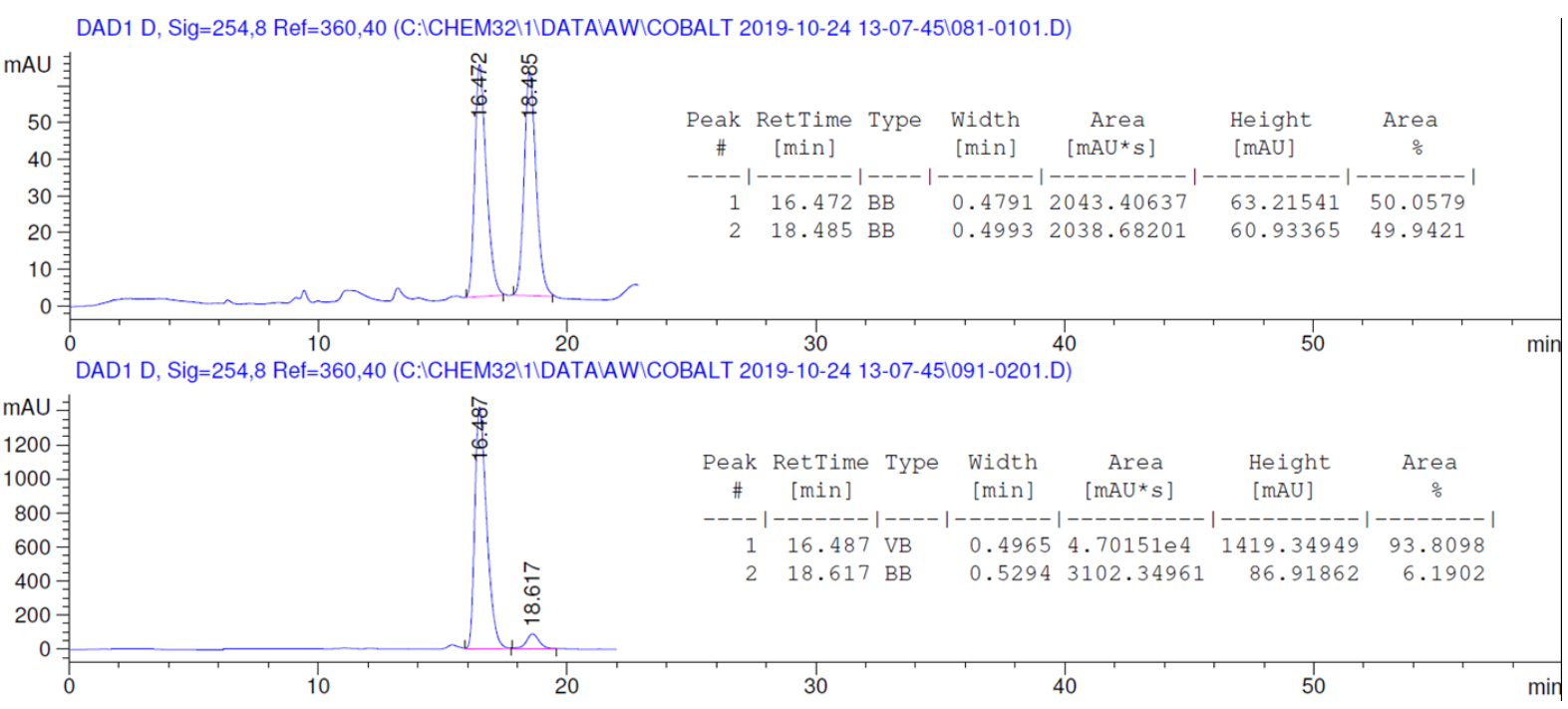


diethyl (R,E)-3-benzylidene-4-((1-(pyridin-2-yl)-1H-indol-2-yl)methyl)cyclopentane-1,1dicarboxylate $(4 y)$<smiles>CCOC(=O)C1(C(=O)OCC)CC(=Cc2ccccc2)C(Cc2cc3ccccc3n2-c2ccccn2)C1</smiles>

Synthesized following GP3. The product was isolated by flash column chromatography 15$>30 \% \mathrm{EtOAc/pentane.} \mathrm{The} \mathrm{product} \mathrm{appeared} \mathrm{as} \mathrm{an} \mathrm{oil,} 72 \mathrm{mg}$ (71\% yield).

${ }^{1}$ H NMR $\left(500 \mathrm{MHz}, \mathrm{CDCl}_{3}\right) \delta 8.69(\mathrm{ddd}, J=4.9,2.0,0.9 \mathrm{~Hz}, 1 \mathrm{H}), 7.90(\mathrm{td}, \mathrm{J}=7.7,2.0 \mathrm{~Hz}, 1 \mathrm{H})$, $7.64-7.56(\mathrm{~m}, 1 \mathrm{H}), 7.49(\mathrm{dt}, \mathrm{J}=7.7,0.9 \mathrm{~Hz}, 1 \mathrm{H}), 7.38-7.28(\mathrm{~m}, 4 \mathrm{H}), 7.24(\mathrm{~d}, \mathrm{~J}=7.2 \mathrm{~Hz}, 2 \mathrm{H})$, 7.19 (tt, J = 6.9, $1.4 \mathrm{~Hz}, 2 \mathrm{H}), 7.18-7.12(\mathrm{~m}, 2 \mathrm{H}), 6.56(\mathrm{~s}, 1 \mathrm{H}), 6.22(\mathrm{q}, \mathrm{J}=2.5 \mathrm{~Hz}, 1 \mathrm{H}), 4.21-$ $4.06(\mathrm{~m}, 4 \mathrm{H}), 3.47(\mathrm{ddd}, \mathrm{J}=15.1,4.7,1.0 \mathrm{~Hz}, 1 \mathrm{H}), 3.29(\mathrm{~d}, \mathrm{~J}=17.4 \mathrm{~Hz}, 1 \mathrm{H}), 3.21(\mathrm{dt}, \mathrm{J}=17.4$, $2.5 \mathrm{~Hz}, 1 \mathrm{H}), 2.99(\mathrm{~m}, 1 \mathrm{H}), 2.88(\mathrm{dd}, \mathrm{J}=15.1,9.9 \mathrm{~Hz}, 1 \mathrm{H}), 2.53(\mathrm{ddd}, \mathrm{J}=13.2,7.5,1.4 \mathrm{~Hz}, 1 \mathrm{H})$, $1.89(\mathrm{dd}, \mathrm{J}=13.2,9.7 \mathrm{~Hz}, 1 \mathrm{H}), 1.22(\mathrm{t}, \mathrm{J}=7.1 \mathrm{~Hz}, 4 \mathrm{H}), 1.18(\mathrm{t}, \mathrm{J}=7.1 \mathrm{~Hz}, 4 \mathrm{H})$.

${ }^{13} \mathrm{C}$ NMR $\left(126 \mathrm{MHz}, \mathrm{CDCl}_{3}\right) \delta 171.7,171.5,151.4,149.7,144.5,139.3,138.4,137.7,137.2$, 128.6, 128.3, 128.2 126.3, 122.6, 122.1, 121.8, 121.1, 120.7, 120.1, 110.0, 103.7, 61.6, 61.5, $59.2,43.5,39.1,39.0,32.9,14.0,14.0$.

IR (ATR) 1725, 1589, 1471, 1447, 1250, 1177, 1041, 913, 733

HRMS (DART, M+H) Calculated for $\mathrm{C}_{32} \mathrm{H}_{33} \mathrm{~N}_{2} \mathrm{O}_{4} 509.2435$, found 509.2434

$\boldsymbol{\alpha}_{\boldsymbol{D}}^{\mathbf{2 0}}\left(\mathrm{c}=0.1, \mathrm{CH}_{2} \mathrm{Cl}_{2}\right) 15.0$

HPLC: AD-H, 15\% IPA/Hex, $0.5 \mathrm{~mL} / \mathrm{min}$

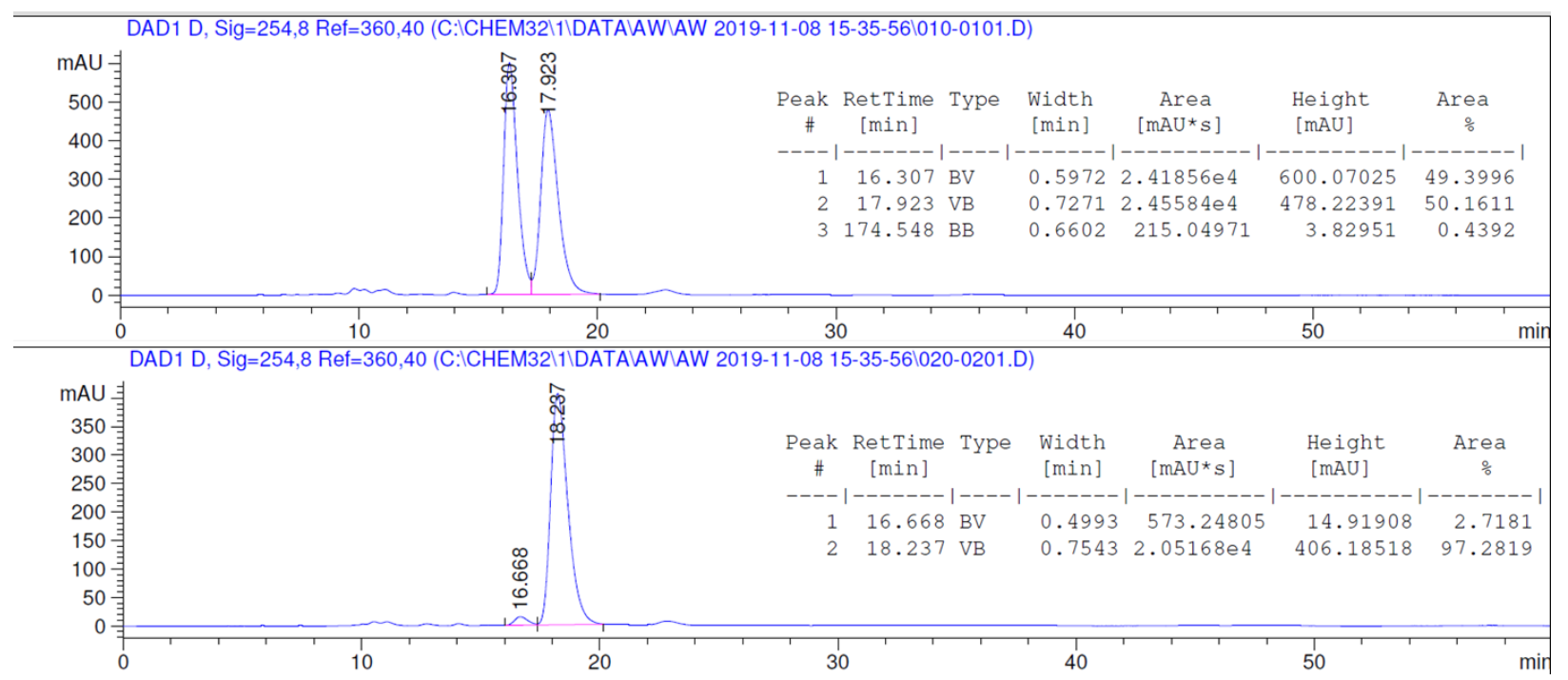




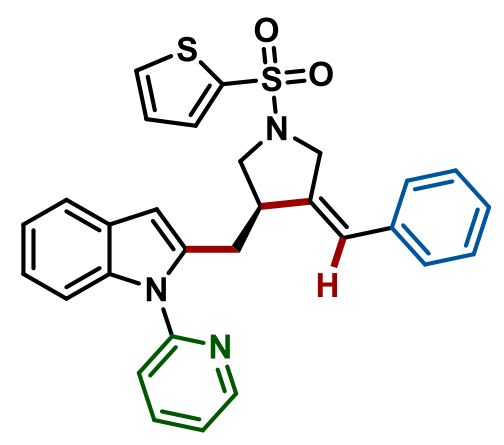

Synthesized following GP3. The product was isolated by flash column chromatography 15$>30 \% \mathrm{EtOAc/pentane.} \mathrm{The} \mathrm{product} \mathrm{appeared} \mathrm{as} \mathrm{a} \mathrm{white} \mathrm{solid,} 99 \mathrm{mg}$ (97\% yield).

${ }^{1} \mathbf{H}$ NMR $\left(500 \mathrm{MHz}, \mathrm{CDCl}_{3}\right) \delta 8.67$ (ddd, $\left.J=4.9,2.0,0.8 \mathrm{~Hz}, 1 \mathrm{H}\right), 7.91$ (ddd, $J=8.0,7.5,2.0$ $\mathrm{Hz}, 1 \mathrm{H}), 7.60$ (ddd, $J=6.1,3.1,0.7 \mathrm{~Hz}, 1 \mathrm{H}), 7.56(\mathrm{dq}, J=3.9,1.3 \mathrm{~Hz}, 2 \mathrm{H}), 7.48(\mathrm{~d}, J=7.9 \mathrm{~Hz}$, 1H), $7.37-7.30(\mathrm{~m}, 4 \mathrm{H}), 7.25-7.22(\mathrm{~m}, 1 \mathrm{H}), 7.19-7.14(\mathrm{~m}, 2 \mathrm{H}), 7.11(\mathrm{dd}, J=4.9,3.9 \mathrm{~Hz}$, 1H), $7.08(\mathrm{~d}, J=7.1 \mathrm{~Hz}, 2 \mathrm{H}), 6.49(\mathrm{~d}, J=0.8 \mathrm{~Hz}, 1 \mathrm{H}), 6.15(\mathrm{~d}, J=2.1 \mathrm{~Hz}, 1 \mathrm{H}), 4.22(\mathrm{dt}, J=$ 14.9, $2.3 \mathrm{~Hz}, 1 \mathrm{H}), 4.08$ (dd, $J=14.9,2.3 \mathrm{~Hz}, 1 \mathrm{H}), 3.30(\mathrm{ddd}, J=15.0,5.0,0.9 \mathrm{~Hz}, 1 \mathrm{H}), 3.25$ (m, $2 \mathrm{H}), 3.11(\mathrm{~m}, 1 \mathrm{H}), 2.96(\mathrm{dd}, J=15.0,9.7 \mathrm{~Hz}, 1 \mathrm{H})$.

${ }^{13} \mathrm{C}$ NMR $\left(126 \mathrm{MHz}, \mathrm{CDCl}_{3}\right) \delta 151.3,149.7,139.4,138.5,138.1,137.2,136.2,135.7,132.6$, 132.1, 128.6, 128.4, 128.1, 127.6, 127.2, 123.7, 122.2, 122.2, 121.0, 120.9, 120.3, 110.1, 104.1, 51.8, 50.9, 44.7, 32.0.

IR (ATR) 1556, 1476, 1454, 1382, 1278, 1210, 1166, 1116, 810, 778, 739, 689

HRMS (DART, M+H) Calculated for $\mathrm{C}_{29} \mathrm{H}_{25} \mathrm{~N}_{3} \mathrm{O}_{2} \mathrm{~S}_{2} 512.1461$, found 512.1454

MP $125-126{ }^{\circ} \mathrm{C}$

$\boldsymbol{\alpha}_{D}^{\mathbf{2 0}}\left(\mathrm{c}=0.365, \mathrm{CH}_{2} \mathrm{Cl}_{2}\right) 3.29$

HPLC: IB, 50\% IPA/Hex, $1 \mathrm{~mL} / \mathrm{min}$

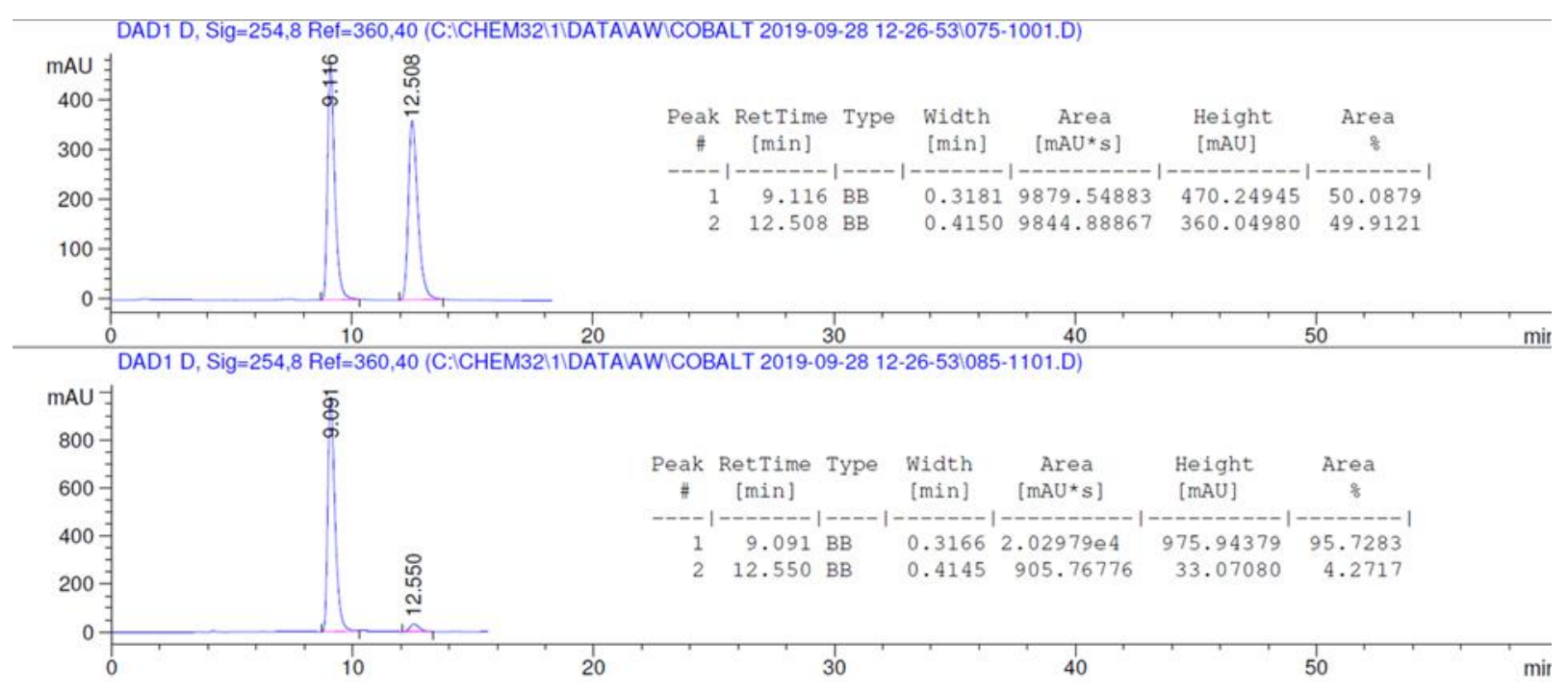




\section{Unsuccessful Substrates}

\section{C-H Bond donors}

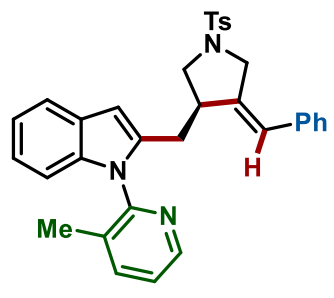

$7 \%$ yield e.r. N.D.

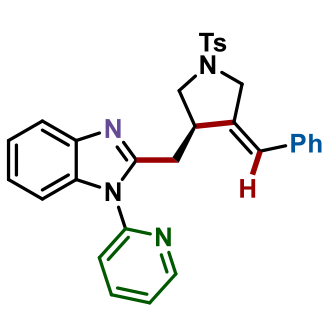

$21 \%$ conversion $0 \%$ yield

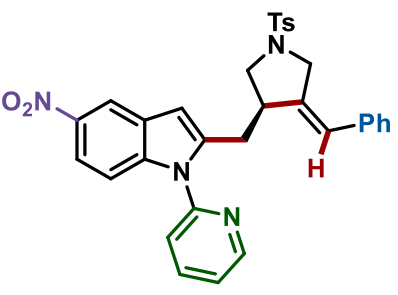

$0 \%$ conversion

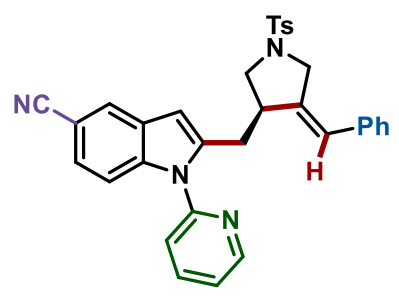

$0 \%$ conversion

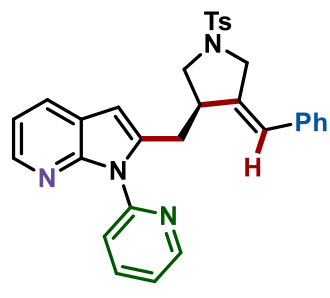

$0 \%$ conversion

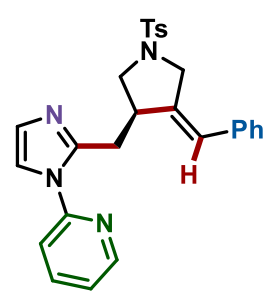

$75 \%$ conversion $0 \%$ yield

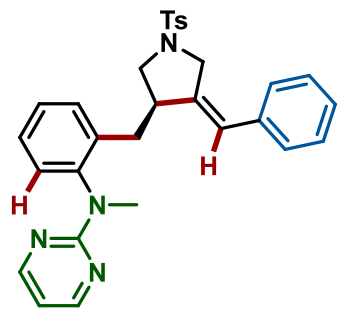

$0 \%$ conversion $0 \%$ yield<smiles>CC(=O)N(C)c1ccccc1C[C@@H]1CN([As])C/C1=C\c1ccccc1</smiles>

full conversion $0 \%$ yield

\section{Enynes}

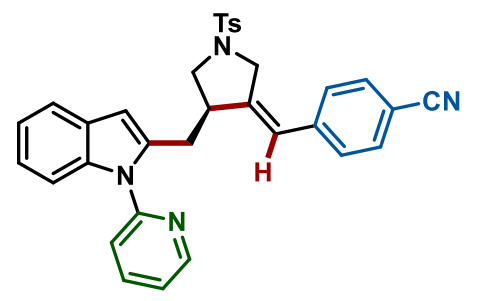

$0 \%$ conversion

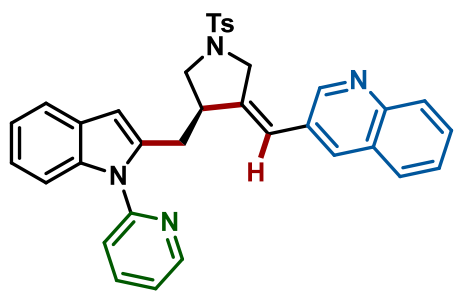

$6 \%$ conversion<smiles>O=C(c1ccccc1)N1C/C(=C\c2ccccc2)[C@H](Cc2cc3ccccc3n2-c2ccccn2)C1</smiles>

$9 \%$ conversion

$0 \%$ yield (enyne decomposed)<smiles>O=[N+]([O-])c1ccc(S(=O)(=O)N2CC(/C=C/c3ccccc3)C(Cc3cc4ccccc4n3-c3ccccn3)C2)cc1</smiles>

$8 \%$ conversion $7 \%$ NMR yield

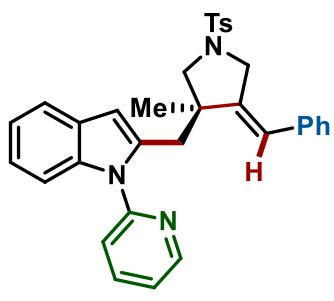

$0 \%$ conversion $0 \%$ NMR yield

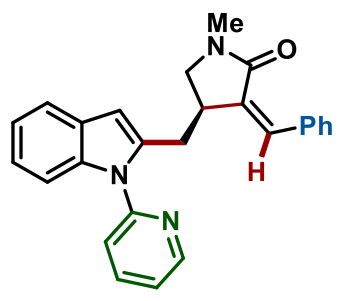

9\% conversion $0 \%$ yield<smiles>O=C1C=CC(c2cc3ccccc3n2-c2ccccn2)C2/C(=C\c3ccccc3)CCN12</smiles>

$7 \%$ conversion $6 \%$ yield 


\section{Pyridine deprotection}

((R,Z)-2-((4-benzylidene-1-tosylpyrrolidin-3-yl)methyl)-1H-indole)<smiles>[Y5]N1CC(=Cc2ccccc2)[C@H](Cc2cc3ccccc3n2[Pb])C1</smiles>

3a

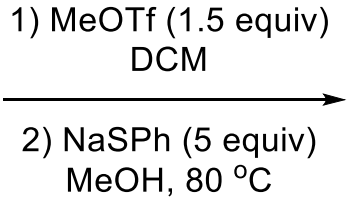

$\mathrm{MeOH}, 80^{\circ} \mathrm{C}$

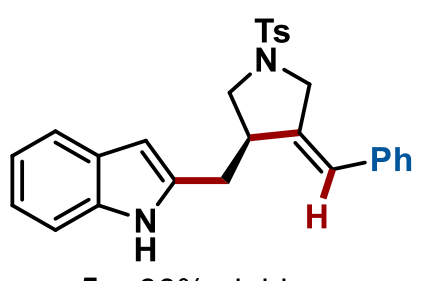

$5 a, 66 \%$ yield

Synthesized following Y.-F. Liang, L. Massignan, L. Ackermann, ChemCatChem 2018, 10, 2768-2772. Substrate 3a (103 mg, $0.2 \mathrm{mmol})$ was dissolved in DCM $(0.5 \mathrm{~mL})$. Methyl triflate ( $33 \mu \mathrm{L}, 0.3 \mathrm{mmol}, 1.5$ equiv) was added dropwise at $0{ }^{\circ} \mathrm{C}$. Methylation was slowly warmed to room temperature and stirred for $24 \mathrm{~h}$. The crude was rotovapped and dried. Then sodium thiophenolate (132 mg, 5.0 equiv) and $2 \mathrm{~mL}$ of $\mathrm{MeOH}$ were added and the reaction was stirred at $80{ }^{\circ} \mathrm{C}$ for $12 \mathrm{~h}$. Then $1 \mathrm{M} \mathrm{HCl}(10 \mathrm{~mL})$ was added and the reaction was extracted with DCM $(3 \mathrm{x}$ $10 \mathrm{~mL}$ ). The organic layers were dried under reduced pressure. The product was purified by flash column chromatography $10 \%$-> 20\% EtOAc/pentane. The product was obtained as a white solid (58 mg, 66\% yield).

${ }^{1} \mathbf{H}$ NMR $\left(500 \mathrm{MHz}, \mathrm{CDCl}_{3}\right) \delta 8.04(\mathrm{~s}, 1 \mathrm{H}), 7.69(\mathrm{~d}, J=8.2 \mathrm{~Hz}, 2 \mathrm{H}), 7.54$ (ddt, $J=7.7,1.4,0.8$ $\mathrm{Hz}, 1 \mathrm{H}), 7.36(\mathrm{t}, J=7.5 \mathrm{~Hz}, 2 \mathrm{H}), 7.33-7.24(\mathrm{~m}, 4 \mathrm{H}), 7.17-7.08(\mathrm{~m}, 4 \mathrm{H}), 6.35(\mathrm{q}, J=2.2 \mathrm{~Hz}$, $1 \mathrm{H}), 6.26(\mathrm{dt}, J=1.6,0.8 \mathrm{~Hz}, 1 \mathrm{H}), 4.27(\mathrm{dt}, J=14.8,1.6 \mathrm{~Hz}, 1 \mathrm{H}), 4.10(\mathrm{dd}, J=14.8,2.2 \mathrm{~Hz}$, $1 \mathrm{H}), 3.28-3.22(\mathrm{~m}, 1 \mathrm{H}), 3.20-3.11(\mathrm{~m}, 2 \mathrm{H}), 3.05(\mathrm{dd}, J=15.0,4.8 \mathrm{~Hz}, 1 \mathrm{H}), 2.87(\mathrm{dd}, J=$ 15.0, $9.1 \mathrm{~Hz}, 1 \mathrm{H}), 2.41(\mathrm{~s}, 3 \mathrm{H})$.

${ }^{13}$ C NMR $\left(126 \mathrm{MHz}, \mathrm{CDCl}_{3}\right) \delta 143.8,139.5,136.2,136.2,136.0,132.7,129.8,128.6,128.6$, 128.1, 127.7, 127.3, 123.8, 121.5, 120.0, 119.8, 110.6, 101.0, 51.6, 50.7, 45.2, 32.5, 21.5.

IR (ATR) 3338, 2974, 1590, 1475, 1454, 1433, 1380, 1321, 1166, 1135, 956, 820, 739, 700

HRMS (DART, $\mathrm{M}+\mathrm{H}$ ) Calculated for $\mathrm{C}_{27} \mathrm{H}_{27} \mathrm{~N}_{2} \mathrm{O}_{2} \mathrm{~S}_{1} 443.1788$, found 443.1780

MP $72{ }^{\circ} \mathrm{C}$

$\boldsymbol{\alpha}_{\boldsymbol{D}}^{\mathbf{2 0}}\left(\mathrm{c}=0.15, \mathrm{CH}_{2} \mathrm{Cl}_{2}\right) 7.33$

HPLC: IB, 50\% IPA/Hex, 1 mL/min

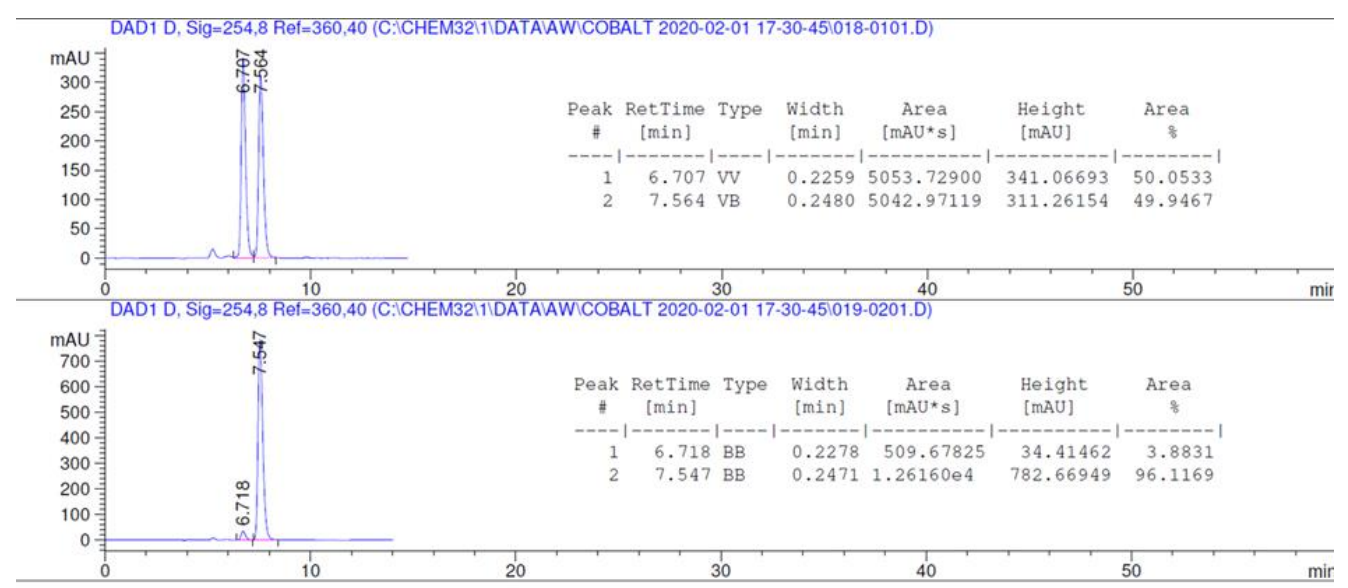


Mechanistic Experiments

Synthesis of deuterated substrate

1-(pyridin-2-yl)-1H-indole-2-d<smiles>[2H]c1cc2ccccc2n1-c1ccccn1</smiles>

$d-1 \mathrm{a}, 80 \% \mathrm{D}$

Synthesized following GP1 using deuterated indole (synthesized following X. Zhou, Y. Luo, L. Kong, Y. Xu, G. Zheng, Y. Lan, X. Li, ACS Catal. 2017, 7, 7296-7304.)

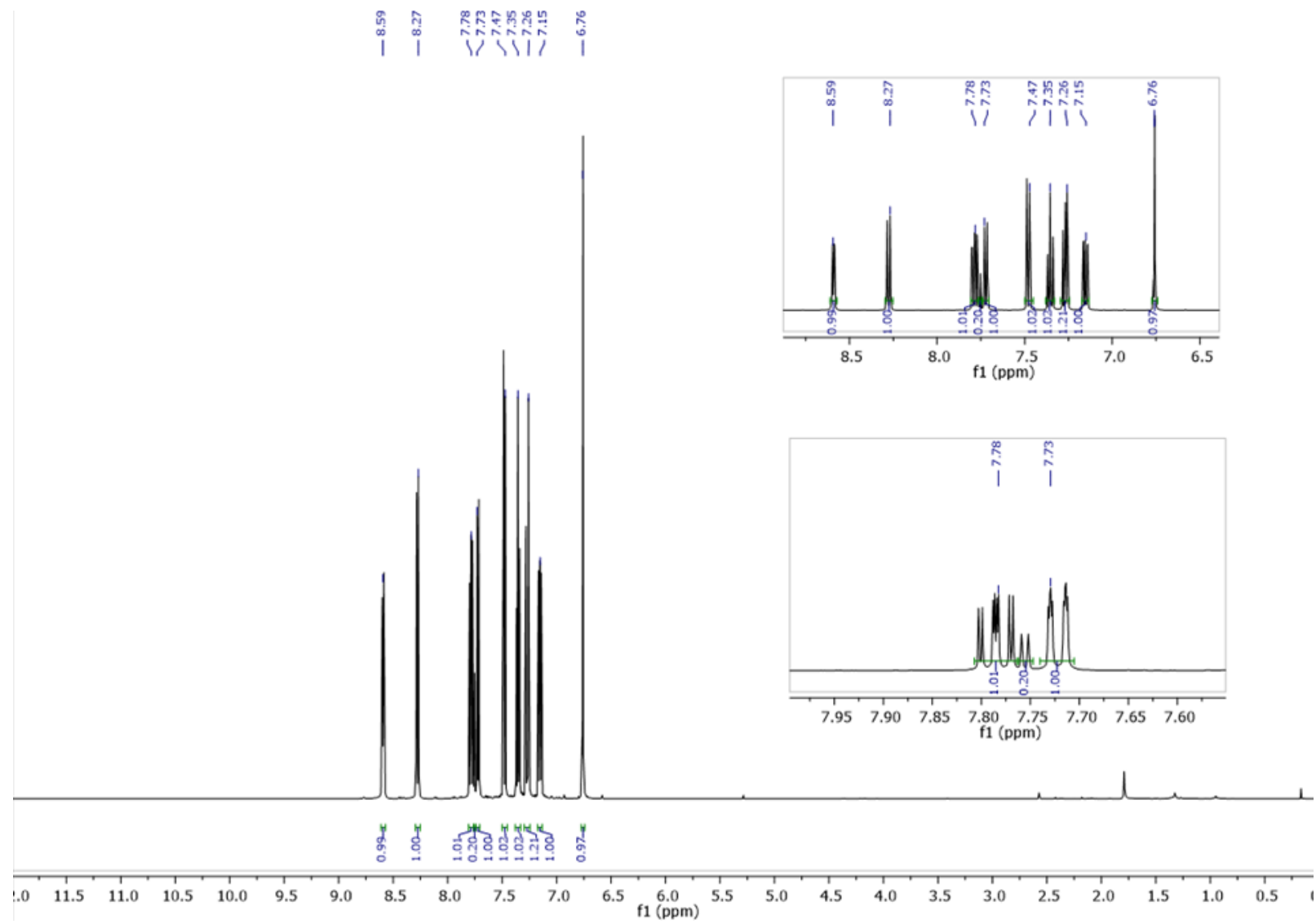




\section{Deuterium retention in catalytic reaction}

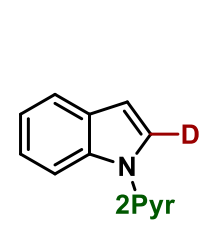

$d-1 a, 80 \%$ D
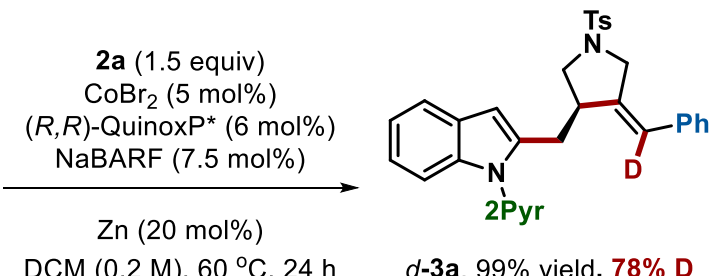

Synthesized following GP3 using deuterated indole. Obtained $105 \mathrm{mg}$ of a white solid.

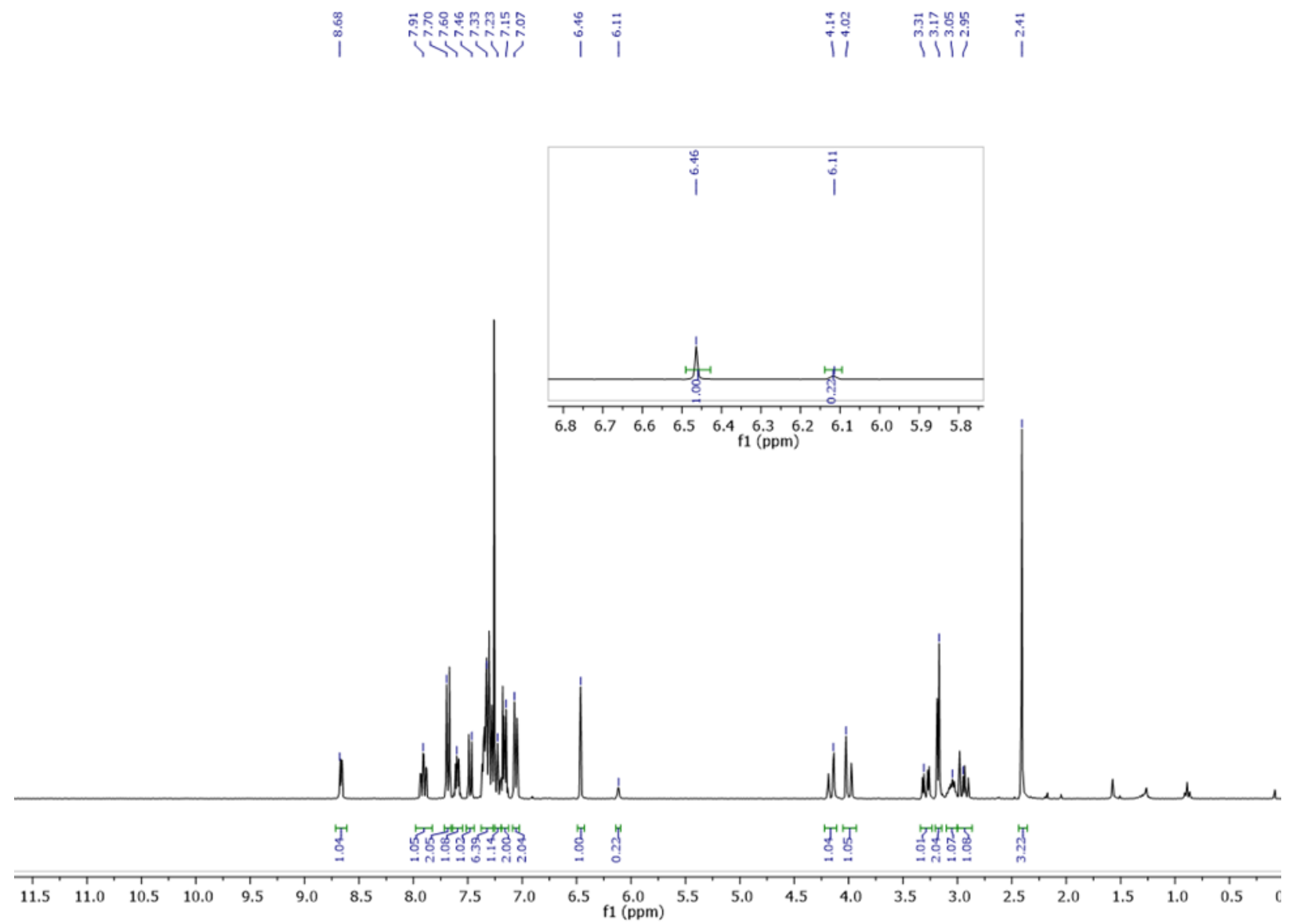




\section{Deuterium crossover experiment}

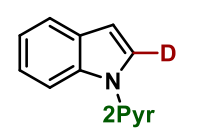

$d-1 \mathrm{a}, 80 \% \mathrm{D}$

(0.1 mmol)

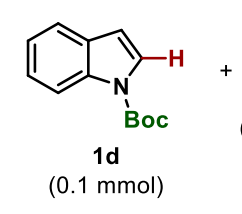

$2 a$

$(0.3 \mathrm{mmol})$

$(0.1 \mathrm{mmol})$

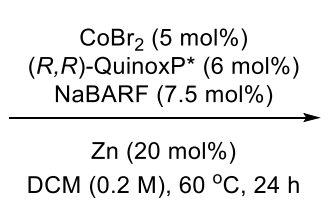

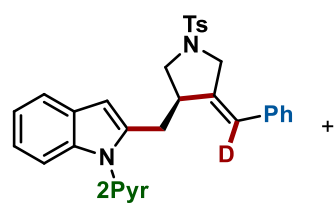

$d-3 a, 92 \%$ yield, $76 \%$ D

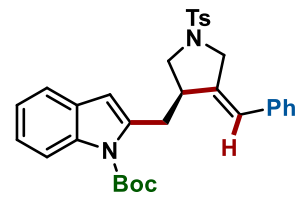

3 d, $38 \%$ yield, $0 \%$ D

Performed following GP3. Obtained $48 \mathrm{mg}$ of $d \mathbf{- 3 a}$ (92\% yield) and $21 \mathrm{mg}$ of $\mathbf{3 d}$ (38\% yield).<smiles></smiles>

d-3a, $92 \%$ yield, $76 \%$ D
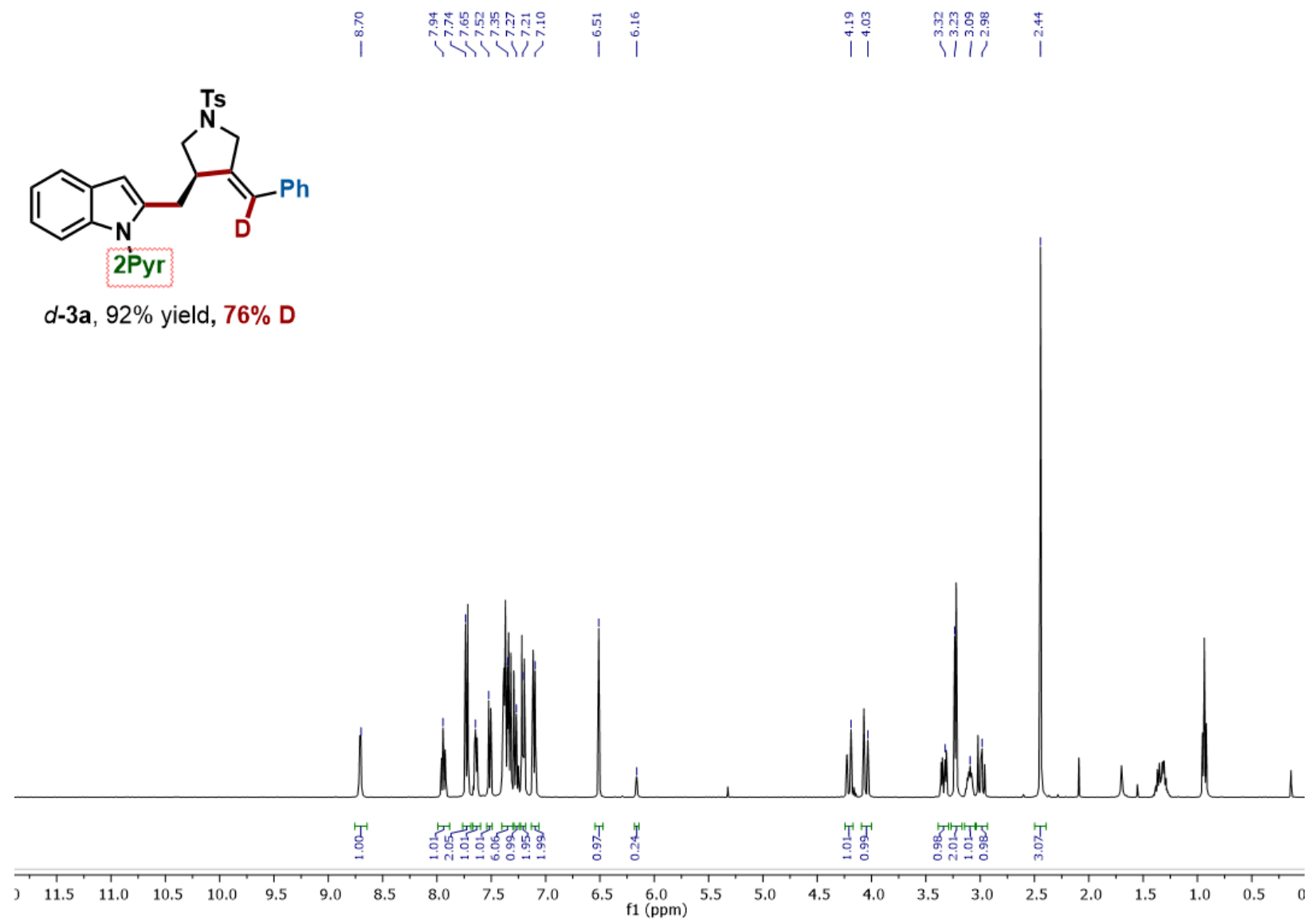


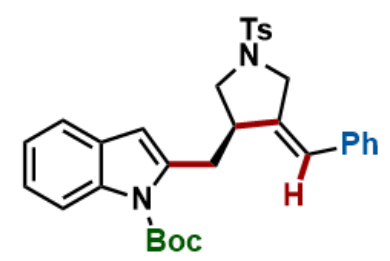

i

3d, $38 \%$ yield, $0 \% \mathrm{D}$

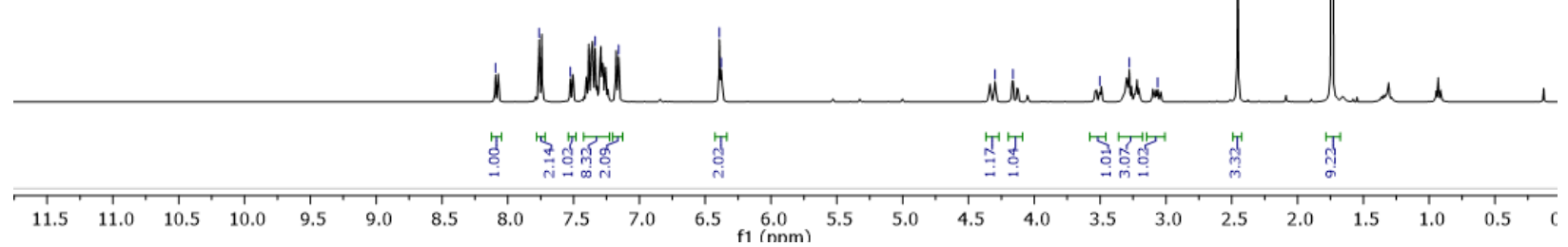




\section{KIE Experiment}

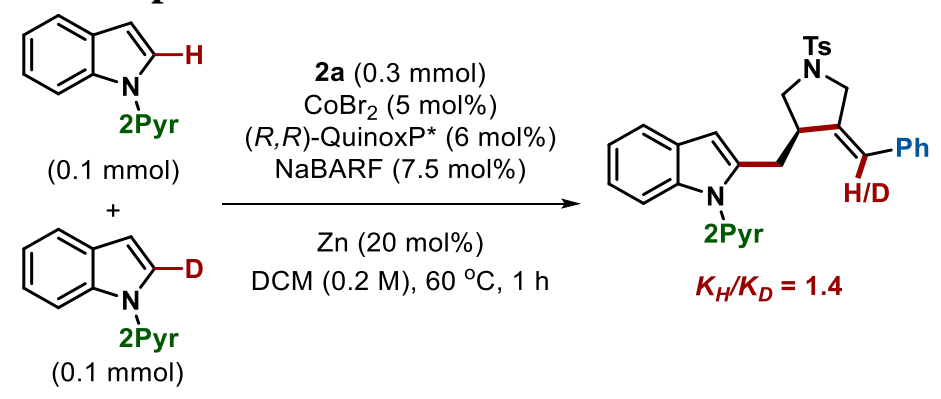

Performed following GP3 using a 1:1 ratio of deuterated and proto substrates. Reaction was stopped after $1 \mathrm{~h}$. KIE was determined by product distribution after isolation. Reactions were run to $4 \%$ completion. Repeated in duplicate. $0.58 / 0.42=1.38$ 
Trial 1 - Crude NMR
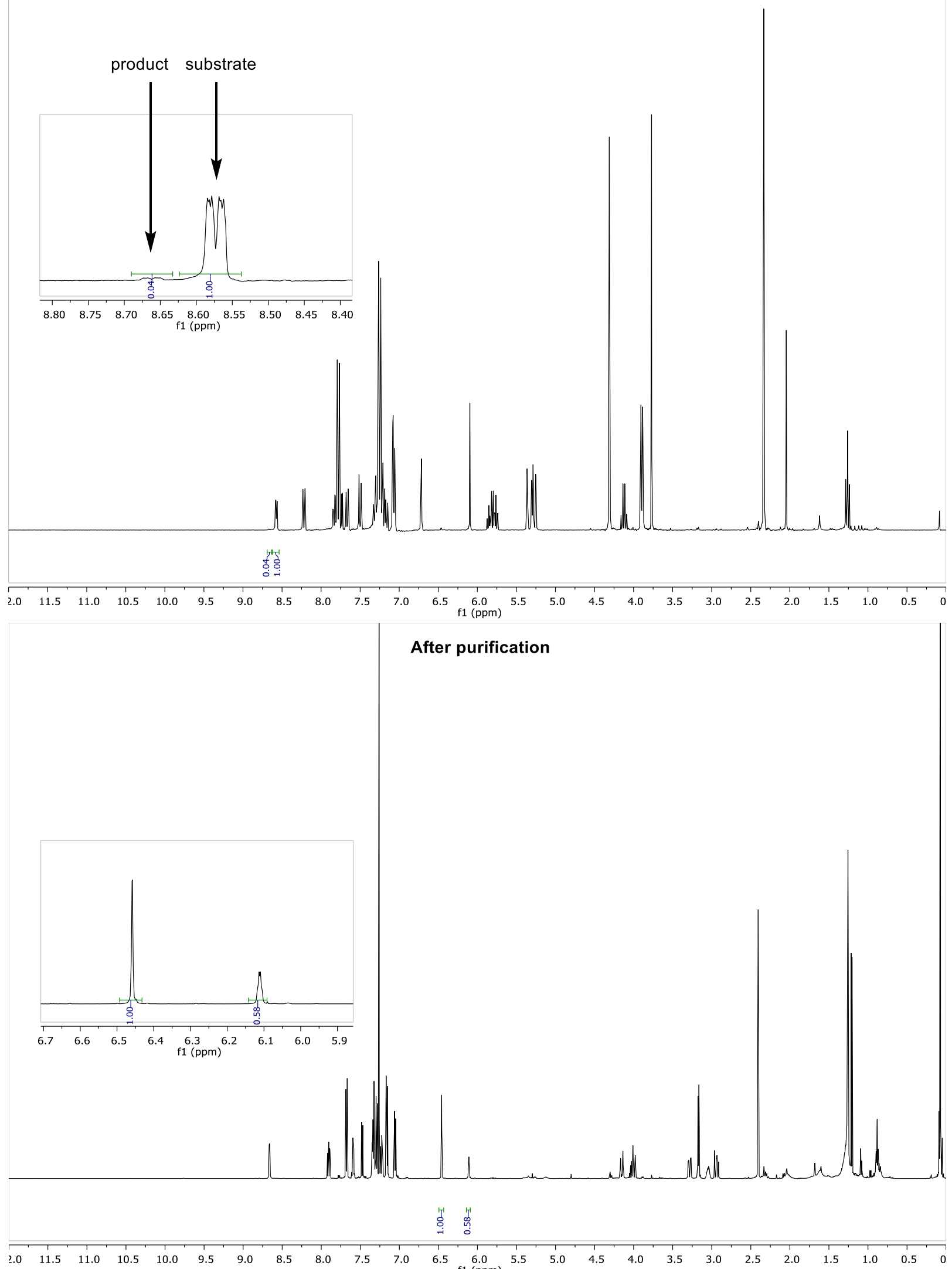


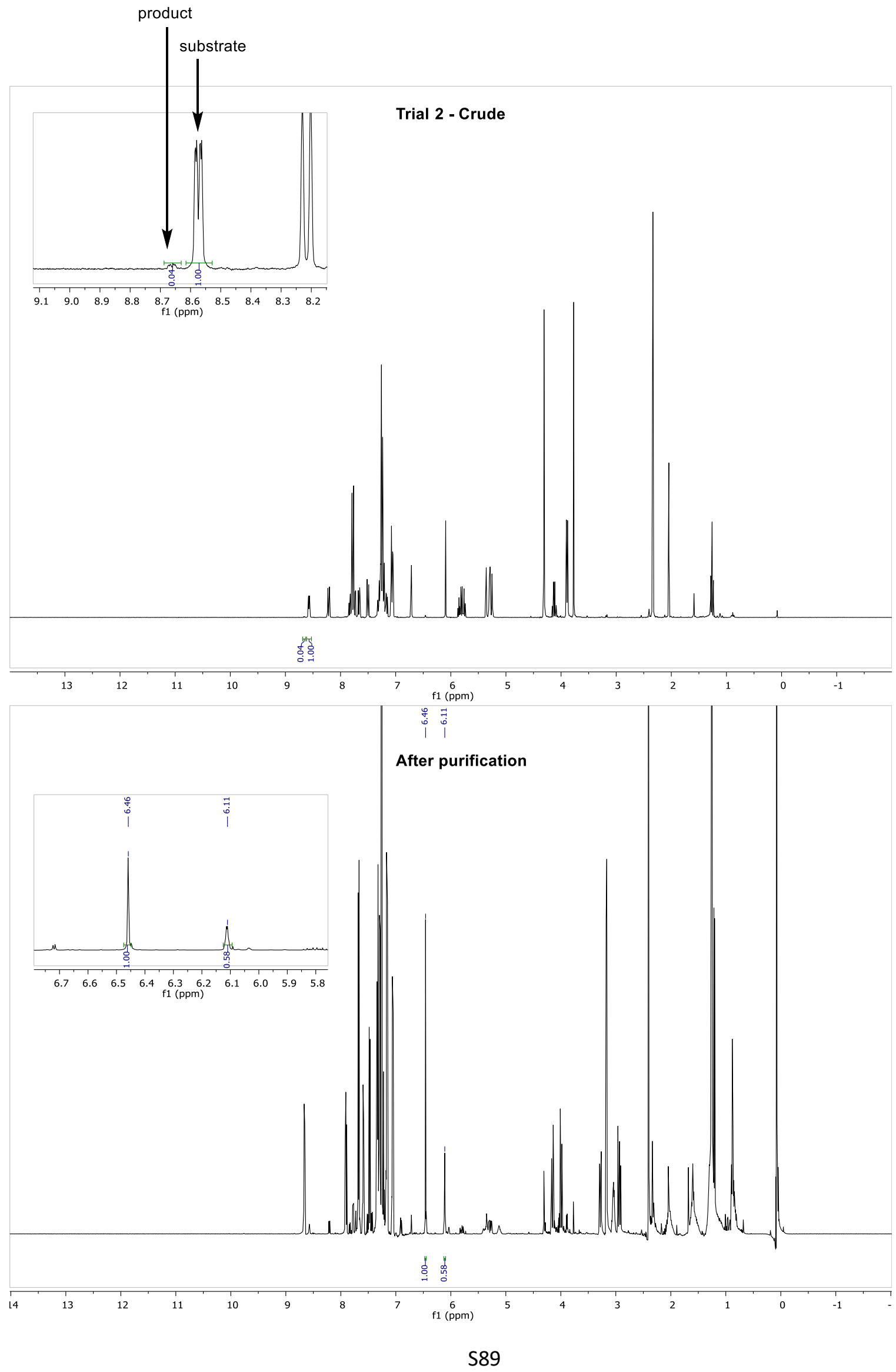




\section{Poison Trials}
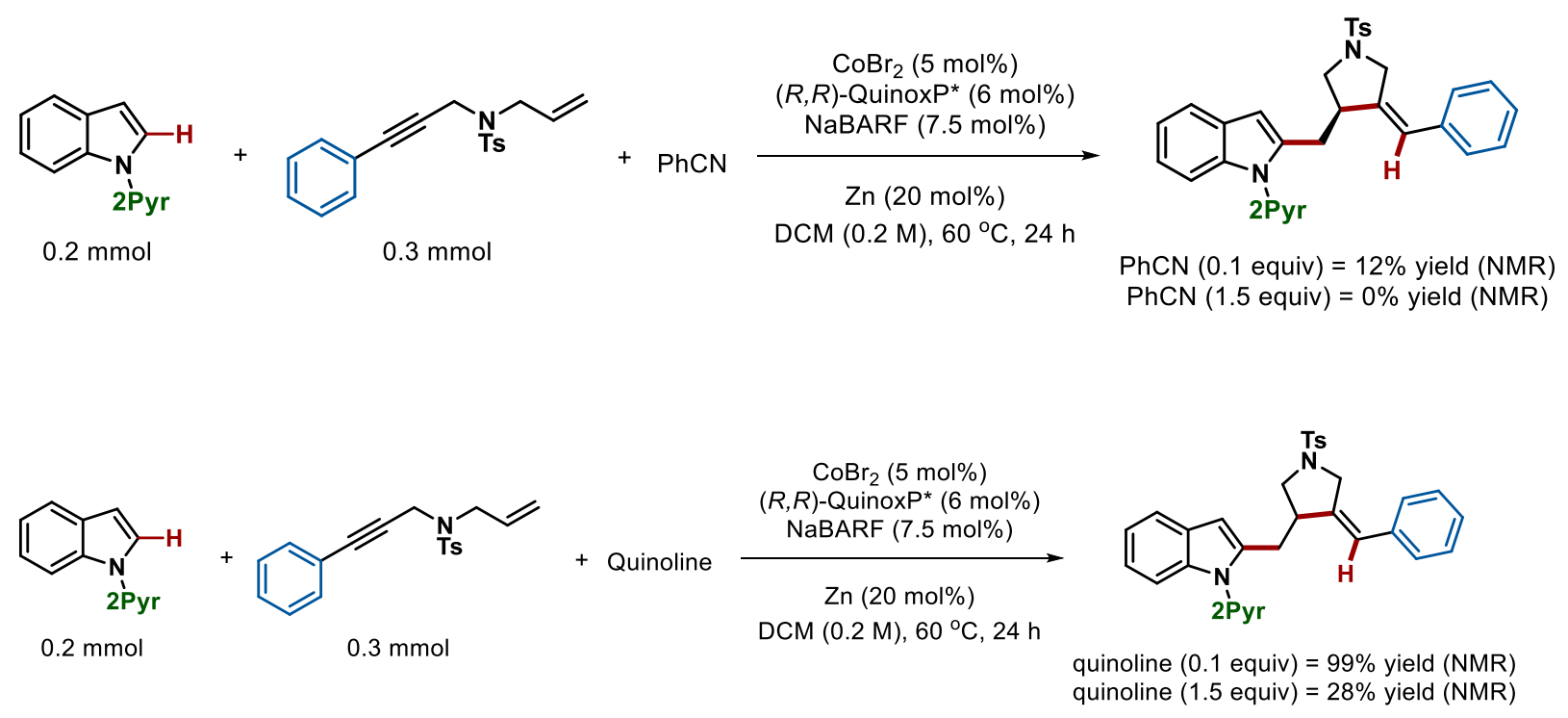


\section{Single Crystal X-Ray}

Table 1. Crystal data and structure refinement for d19195_a.

Identification code

Empirical formula

Formula weight

Temperature

Wavelength

Crystal system

Space group

Unit cell dimensions

Volume

$\mathrm{Z}$

Density (calculated)

Absorption coefficient

$\mathrm{F}(000)$

Crystal size

Theta range for data collection

Index ranges

Reflections collected

Independent reflections

Completeness to theta $=25.242^{\circ}$

Absorption correction

Max. and min. transmission

Refinement method

Data / restraints / parameters

Goodness-of-fit on $\mathrm{F}^{2}$

Final R indices [I $>2 \operatorname{sigma}(\mathrm{I})]$

$\mathrm{R}$ indices (all data)

Absolute structure parameter

Extinction coefficient

Largest diff. peak and hole d19195_a

$\mathrm{C} 32 \mathrm{H} 28 \mathrm{Cl} \mathrm{N} 3 \mathrm{O} 2 \mathrm{~S}$

554.08

150(2) K

$0.71073 \AA$

Orthorhombic

$\mathrm{P} 2{ }_{12} 2_{1}$

$\mathrm{a}=8.9830(5) \AA \quad \mathrm{a}=90^{\circ}$.

$\mathrm{b}=12.8241(7) \AA \quad \mathrm{b}=90^{\circ}$.

$\mathrm{c}=24.4677(16) \AA \quad \mathrm{g}=90^{\circ}$.

2818.7(3) $\AA^{3}$

4

$1.306 \mathrm{Mg} / \mathrm{m}^{3}$

$0.244 \mathrm{~mm}^{-1}$

1160

$0.250 \times 0.240 \times 0.030 \mathrm{~mm}^{3}$

1.665 to $27.498^{\circ}$.

$-9<=\mathrm{h}<=11,-16<=\mathrm{k}<=16,-31<=\mathrm{l}<=31$

44676

$6488[\mathrm{R}(\mathrm{int})=0.0435]$

$100.0 \%$

Semi-empirical from equivalents

0.7456 and 0.7034

Full-matrix least-squares on $\mathrm{F}^{2}$

6488 / 0 / 353

1.023

$\mathrm{R} 1=0.0359, \mathrm{wR} 2=0.0758$

$\mathrm{R} 1=0.0578, \mathrm{wR} 2=0.0849$

0.006(19)

$\mathrm{n} / \mathrm{a}$

0.174 and -0.300 e. $\AA^{-3}$ 
Table 2. Atomic coordinates $\left(\mathrm{x} 10^{4}\right)$ and equivalent isotropic displacement parameters $\left(\AA^{2} \mathrm{x}\right.$ $10^{3}$ )

for d19195_a. U(eq) is defined as one third of the trace of the orthogonalized $U^{i j}$ tensor.

\begin{tabular}{|c|c|c|c|c|}
\hline & $\mathrm{x}$ & $\mathrm{y}$ & $\mathrm{z}$ & $\mathrm{U}(\mathrm{eq})$ \\
\hline \multicolumn{5}{|l|}{ - } \\
\hline $\mathrm{Cl}(1)$ & $12206(1)$ & 3301(1) & $2507(1)$ & $60(1)$ \\
\hline$S(1)$ & $5577(1)$ & $3298(1)$ & $5470(1)$ & $35(1)$ \\
\hline $\mathrm{O}(1)$ & $5925(2)$ & $2259(1)$ & $5306(1)$ & $42(1)$ \\
\hline $\mathrm{O}(2)$ & $4265(2)$ & $3498(2)$ & $5785(1)$ & $50(1)$ \\
\hline $\mathrm{N}(1)$ & $5430(2)$ & $3983(2)$ & $4915(1)$ & $31(1)$ \\
\hline $\mathrm{N}(2)$ & $1528(2)$ & $6364(2)$ & $3889(1)$ & $29(1)$ \\
\hline $\mathrm{N}(3)$ & $3646(3)$ & 6913(2) & $3420(1)$ & $46(1)$ \\
\hline $\mathrm{C}(1)$ & $6684(3)$ & $3982(2)$ & $4522(1)$ & $29(1)$ \\
\hline$C(2)$ & $6227(3)$ & $4819(2)$ & $4119(1)$ & $28(1)$ \\
\hline $\mathrm{C}(3)$ & $4844(3)$ & $5359(2)$ & $4341(1)$ & $30(1)$ \\
\hline$C(4)$ & $4861(3)$ & $5067(2)$ & 4943(1) & $34(1)$ \\
\hline$C(5)$ & $3421(3)$ & $4940(2)$ & $4057(1)$ & $30(1)$ \\
\hline$C(6)$ & $2055(3)$ & $5530(2)$ & $4208(1)$ & $29(1)$ \\
\hline$C(7)$ & $1123(3)$ & $5389(2)$ & $4636(1)$ & $31(1)$ \\
\hline $\mathrm{C}(8)$ & $-48(3)$ & $6152(2)$ & $4605(1)$ & $31(1)$ \\
\hline $\mathrm{C}(9)$ & $-1255(3)$ & $6407(2)$ & $4938(1)$ & $36(1)$ \\
\hline$C(10)$ & $-2163(3)$ & $7222(2)$ & 4791(1) & $42(1)$ \\
\hline$C(11)$ & $-1892(3)$ & $7797(2)$ & $4315(1)$ & $44(1)$ \\
\hline $\mathrm{C}(12)$ & $-699(3)$ & $7570(2)$ & $3979(1)$ & $36(1)$ \\
\hline$C(13)$ & $220(3)$ & $6742(2)$ & $4127(1)$ & $29(1)$ \\
\hline$C(14)$ & $2183(3)$ & $6724(2)$ & $3398(1)$ & $33(1)$ \\
\hline$C(15)$ & $1351(4)$ & $6828(2)$ & $2927(1)$ & $48(1)$ \\
\hline$C(16)$ & $2073(5)$ & $7137(3)$ & $2453(2)$ & $67(1)$ \\
\hline$C(17)$ & $3582(5)$ & $7324(3)$ & $2471(2)$ & $72(1)$ \\
\hline $\mathrm{C}(18)$ & 4304(4) & 7198(3) & $2956(2)$ & $63(1)$ \\
\hline$C(19)$ & $6871(3)$ & $5074(2)$ & $3648(1)$ & $31(1)$ \\
\hline $\mathrm{C}(20)$ & $8195(3)$ & $4634(2)$ & $3383(1)$ & $29(1)$ \\
\hline $\mathrm{C}(21)$ & $8475(4)$ & $4862(2)$ & $2835(1)$ & $41(1)$ \\
\hline
\end{tabular}




$\begin{array}{lrlll}\mathrm{C}(22) & 9702(4) & 4470(2) & 2562(1) & 45(1) \\ \mathrm{C}(23) & 10685(3) & 3843(2) & 2844(1) & 37(1) \\ \mathrm{C}(24) & 10474(3) & 3625(2) & 3386(1) & 35(1) \\ \mathrm{C}(25) & 9240(3) & 4016(2) & 3653(1) & 32(1) \\ \mathrm{C}(26) & 7129(3) & 3786(2) & 5825(1) & 33(1) \\ \mathrm{C}(27) & 6969(4) & 4637(2) & 6172(1) & 46(1) \\ \mathrm{C}(28) & 8217(4) & 5052(2) & 6420(1) & 54(1) \\ \mathrm{C}(29) & 9626(4) & 4639(2) & 6325(1) & 52(1) \\ \mathrm{C}(30) & 9766(4) & 3796(2) & 5973(1) & 46(1) \\ \mathrm{C}(31) & 8530(3) & 3369(2) & 5724(1) & 38(1) \\ \mathrm{C}(32) & 10983(5) & 5098(3) & 6600(2) & 80(1)\end{array}$


Table 3. Bond lengths $[\AA]$ and angles $\left[{ }^{\circ}\right]$ for d19195_a.

\begin{tabular}{|c|c|}
\hline $\mathrm{Cl}(1)-\mathrm{C}(23)$ & $1.741(3)$ \\
\hline $\mathrm{S}(1)-\mathrm{O}(1)$ & $1.426(2)$ \\
\hline $\mathrm{S}(1)-\mathrm{O}(2)$ & $1.432(2)$ \\
\hline $\mathrm{S}(1)-\mathrm{N}(1)$ & $1.622(2)$ \\
\hline$S(1)-C(26)$ & $1.759(3)$ \\
\hline $\mathrm{N}(1)-\mathrm{C}(1)$ & $1.482(3)$ \\
\hline $\mathrm{N}(1)-\mathrm{C}(4)$ & $1.483(3)$ \\
\hline $\mathrm{N}(2)-\mathrm{C}(13)$ & $1.398(3)$ \\
\hline $\mathrm{N}(2)-\mathrm{C}(6)$ & $1.406(3)$ \\
\hline $\mathrm{N}(2)-\mathrm{C}(14)$ & $1.415(3)$ \\
\hline $\mathrm{N}(3)-\mathrm{C}(18)$ & $1.330(4)$ \\
\hline $\mathrm{N}(3)-\mathrm{C}(14)$ & $1.337(4)$ \\
\hline$C(1)-C(2)$ & $1.514(3)$ \\
\hline $\mathrm{C}(1)-\mathrm{H}(1 \mathrm{~A})$ & 0.9900 \\
\hline $\mathrm{C}(1)-\mathrm{H}(1 \mathrm{~B})$ & 0.9900 \\
\hline $\mathrm{C}(2)-\mathrm{C}(19)$ & $1.331(4)$ \\
\hline $\mathrm{C}(2)-\mathrm{C}(3)$ & $1.522(3)$ \\
\hline$C(3)-C(4)$ & $1.520(4)$ \\
\hline$C(3)-C(5)$ & $1.551(3)$ \\
\hline $\mathrm{C}(3)-\mathrm{H}(3 \mathrm{~A})$ & 1.0000 \\
\hline $\mathrm{C}(4)-\mathrm{H}(4 \mathrm{~A})$ & 0.9900 \\
\hline $\mathrm{C}(4)-\mathrm{H}(4 \mathrm{~B})$ & 0.9900 \\
\hline$C(5)-C(6)$ & $1.487(3)$ \\
\hline $\mathrm{C}(5)-\mathrm{H}(5 \mathrm{~A})$ & 0.9900 \\
\hline $\mathrm{C}(5)-\mathrm{H}(5 \mathrm{~B})$ & 0.9900 \\
\hline$C(6)-C(7)$ & $1.353(4)$ \\
\hline$C(7)-C(8)$ & $1.439(4)$ \\
\hline $\mathrm{C}(7)-\mathrm{H}(7 \mathrm{~A})$ & 0.9500 \\
\hline $\mathrm{C}(8)-\mathrm{C}(9)$ & $1.395(4)$ \\
\hline $\mathrm{C}(8)-\mathrm{C}(13)$ & $1.412(4)$ \\
\hline $\mathrm{C}(9)-\mathrm{C}(10)$ & $1.374(4)$ \\
\hline $\mathrm{C}(9)-\mathrm{H}(9 \mathrm{~A})$ & 0.9500 \\
\hline $\mathrm{C}(10)-\mathrm{C}(11)$ & $1.400(4)$ \\
\hline $\mathrm{C}(10)-\mathrm{H}(10 \mathrm{~A})$ & 0.9500 \\
\hline
\end{tabular}




\begin{tabular}{|c|c|}
\hline $\mathrm{C}(11)-\mathrm{C}(12)$ & $1.382(4)$ \\
\hline $\mathrm{C}(11)-\mathrm{H}(11 \mathrm{~A})$ & 0.9500 \\
\hline $\mathrm{C}(12)-\mathrm{C}(13)$ & $1.394(4)$ \\
\hline $\mathrm{C}(12)-\mathrm{H}(12 \mathrm{~A})$ & 0.9500 \\
\hline$C(14)-C(15)$ & $1.379(4)$ \\
\hline$C(15)-C(16)$ & $1.388(5)$ \\
\hline $\mathrm{C}(15)-\mathrm{H}(15 \mathrm{~A})$ & 0.9500 \\
\hline$C(16)-C(17)$ & $1.377(6)$ \\
\hline $\mathrm{C}(16)-\mathrm{H}(16 \mathrm{~A})$ & 0.9500 \\
\hline $\mathrm{C}(17)-\mathrm{C}(18)$ & $1.363(6)$ \\
\hline $\mathrm{C}(17)-\mathrm{H}(17 \mathrm{~A})$ & 0.9500 \\
\hline $\mathrm{C}(18)-\mathrm{H}(18 \mathrm{~A})$ & 0.9500 \\
\hline $\mathrm{C}(19)-\mathrm{C}(20)$ & $1.467(3)$ \\
\hline $\mathrm{C}(19)-\mathrm{H}(19 \mathrm{~A})$ & 0.9500 \\
\hline$C(20)-C(25)$ & $1.395(4)$ \\
\hline$C(20)-C(21)$ & $1.396(4)$ \\
\hline $\mathrm{C}(21)-\mathrm{C}(22)$ & $1.383(4)$ \\
\hline $\mathrm{C}(21)-\mathrm{H}(21 \mathrm{~A})$ & 0.9500 \\
\hline $\mathrm{C}(22)-\mathrm{C}(23)$ & $1.380(4)$ \\
\hline $\mathrm{C}(22)-\mathrm{H}(22 \mathrm{~A})$ & 0.9500 \\
\hline $\mathrm{C}(23)-\mathrm{C}(24)$ & $1.369(4)$ \\
\hline $\mathrm{C}(24)-\mathrm{C}(25)$ & $1.379(4)$ \\
\hline $\mathrm{C}(24)-\mathrm{H}(24 \mathrm{~A})$ & 0.9500 \\
\hline $\mathrm{C}(25)-\mathrm{H}(25 \mathrm{~A})$ & 0.9500 \\
\hline$C(26)-C(31)$ & $1.389(4)$ \\
\hline$C(26)-C(27)$ & $1.390(4)$ \\
\hline $\mathrm{C}(27)-\mathrm{C}(28)$ & $1.381(5)$ \\
\hline $\mathrm{C}(27)-\mathrm{H}(27 \mathrm{~A})$ & 0.9500 \\
\hline $\mathrm{C}(28)-\mathrm{C}(29)$ & $1.392(5)$ \\
\hline $\mathrm{C}(28)-\mathrm{H}(28 \mathrm{~A})$ & 0.9500 \\
\hline $\mathrm{C}(29)-\mathrm{C}(30)$ & $1.387(4)$ \\
\hline $\mathrm{C}(29)-\mathrm{C}(32)$ & $1.512(5)$ \\
\hline $\mathrm{C}(30)-\mathrm{C}(31)$ & $1.381(4)$ \\
\hline $\mathrm{C}(30)-\mathrm{H}(30 \mathrm{~A})$ & 0.9500 \\
\hline $\mathrm{C}(31)-\mathrm{H}(31 \mathrm{~A})$ & 0.9500 \\
\hline $\mathrm{C}(32)-\mathrm{H}(32 \mathrm{~A})$ & 0.9800 \\
\hline
\end{tabular}




\begin{tabular}{|c|c|}
\hline $\mathrm{C}(32)-\mathrm{H}(32 \mathrm{~B})$ & 0.9800 \\
\hline $\mathrm{C}(32)-\mathrm{H}(32 \mathrm{C})$ & 0.9800 \\
\hline $\mathrm{O}(1)-\mathrm{S}(1)-\mathrm{O}(2)$ & 119.99(13) \\
\hline $\mathrm{O}(1)-\mathrm{S}(1)-\mathrm{N}(1)$ & $106.76(11)$ \\
\hline $\mathrm{O}(2)-\mathrm{S}(1)-\mathrm{N}(1)$ & $106.69(12)$ \\
\hline $\mathrm{O}(1)-\mathrm{S}(1)-\mathrm{C}(26)$ & $107.33(13)$ \\
\hline $\mathrm{O}(2)-\mathrm{S}(1)-\mathrm{C}(26)$ & 108.79(13) \\
\hline $\mathrm{N}(1)-\mathrm{S}(1)-\mathrm{C}(26)$ & $106.55(12)$ \\
\hline $\mathrm{C}(1)-\mathrm{N}(1)-\mathrm{C}(4)$ & 107.07(19) \\
\hline $\mathrm{C}(1)-\mathrm{N}(1)-\mathrm{S}(1)$ & $118.75(17)$ \\
\hline $\mathrm{C}(4)-\mathrm{N}(1)-\mathrm{S}(1)$ & $119.83(17)$ \\
\hline $\mathrm{C}(13)-\mathrm{N}(2)-\mathrm{C}(6)$ & $108.4(2)$ \\
\hline $\mathrm{C}(13)-\mathrm{N}(2)-\mathrm{C}(14)$ & $126.2(2)$ \\
\hline $\mathrm{C}(6)-\mathrm{N}(2)-\mathrm{C}(14)$ & $125.4(2)$ \\
\hline $\mathrm{C}(18)-\mathrm{N}(3)-\mathrm{C}(14)$ & $116.9(3)$ \\
\hline $\mathrm{N}(1)-\mathrm{C}(1)-\mathrm{C}(2)$ & $102.45(19)$ \\
\hline $\mathrm{N}(1)-\mathrm{C}(1)-\mathrm{H}(1 \mathrm{~A})$ & 111.3 \\
\hline $\mathrm{C}(2)-\mathrm{C}(1)-\mathrm{H}(1 \mathrm{~A})$ & 111.3 \\
\hline $\mathrm{N}(1)-\mathrm{C}(1)-\mathrm{H}(1 \mathrm{~B})$ & 111.3 \\
\hline $\mathrm{C}(2)-\mathrm{C}(1)-\mathrm{H}(1 \mathrm{~B})$ & 111.3 \\
\hline $\mathrm{H}(1 \mathrm{~A})-\mathrm{C}(1)-\mathrm{H}(1 \mathrm{~B})$ & 109.2 \\
\hline$C(19)-C(2)-C(1)$ & $128.4(2)$ \\
\hline $\mathrm{C}(19)-\mathrm{C}(2)-\mathrm{C}(3)$ & $123.5(2)$ \\
\hline $\mathrm{C}(1)-\mathrm{C}(2)-\mathrm{C}(3)$ & $108.1(2)$ \\
\hline $\mathrm{C}(4)-\mathrm{C}(3)-\mathrm{C}(2)$ & $103.1(2)$ \\
\hline$C(4)-C(3)-C(5)$ & $110.9(2)$ \\
\hline $\mathrm{C}(2)-\mathrm{C}(3)-\mathrm{C}(5)$ & $110.8(2)$ \\
\hline $\mathrm{C}(4)-\mathrm{C}(3)-\mathrm{H}(3 \mathrm{~A})$ & 110.6 \\
\hline $\mathrm{C}(2)-\mathrm{C}(3)-\mathrm{H}(3 \mathrm{~A})$ & 110.6 \\
\hline $\mathrm{C}(5)-\mathrm{C}(3)-\mathrm{H}(3 \mathrm{~A})$ & 110.6 \\
\hline $\mathrm{N}(1)-\mathrm{C}(4)-\mathrm{C}(3)$ & $100.9(2)$ \\
\hline $\mathrm{N}(1)-\mathrm{C}(4)-\mathrm{H}(4 \mathrm{~A})$ & 111.6 \\
\hline $\mathrm{C}(3)-\mathrm{C}(4)-\mathrm{H}(4 \mathrm{~A})$ & 111.6 \\
\hline $\mathrm{N}(1)-\mathrm{C}(4)-\mathrm{H}(4 \mathrm{~B})$ & 111.6 \\
\hline $\mathrm{C}(3)-\mathrm{C}(4)-\mathrm{H}(4 \mathrm{~B})$ & 111.6 \\
\hline
\end{tabular}




$\begin{array}{ll}\mathrm{H}(4 \mathrm{~A})-\mathrm{C}(4)-\mathrm{H}(4 \mathrm{~B}) & 109.4 \\ \mathrm{C}(6)-\mathrm{C}(5)-\mathrm{C}(3) & 113.2(2) \\ \mathrm{C}(6)-\mathrm{C}(5)-\mathrm{H}(5 \mathrm{~A}) & 108.9 \\ \mathrm{C}(3)-\mathrm{C}(5)-\mathrm{H}(5 \mathrm{~A}) & 108.9 \\ \mathrm{C}(6)-\mathrm{C}(5)-\mathrm{H}(5 \mathrm{~B}) & 108.9 \\ \mathrm{C}(3)-\mathrm{C}(5)-\mathrm{H}(5 \mathrm{~B}) & 108.9 \\ \mathrm{H}(5 \mathrm{~A})-\mathrm{C}(5)-\mathrm{H}(5 \mathrm{~B}) & 107.8 \\ \mathrm{C}(7)-\mathrm{C}(6)-\mathrm{N}(2) & 108.8(2) \\ \mathrm{C}(7)-\mathrm{C}(6)-\mathrm{C}(5) & 129.3(2) \\ \mathrm{N}(2)-\mathrm{C}(6)-\mathrm{C}(5) & 121.9(2) \\ \mathrm{C}(6)-\mathrm{C}(7)-\mathrm{C}(8) & 108.7(2) \\ \mathrm{C}(6)-\mathrm{C}(7)-\mathrm{H}(7 \mathrm{~A}) & 125.6 \\ \mathrm{C}(8)-\mathrm{C}(7)-\mathrm{H}(7 \mathrm{~A}) & 125.6 \\ \mathrm{C}(9)-\mathrm{C}(8)-\mathrm{C}(13) & 119.4(2) \\ \mathrm{C}(9)-\mathrm{C}(8)-\mathrm{C}(7) & 134.1(3) \\ \mathrm{C}(13)-\mathrm{C}(8)-\mathrm{C}(7) & 106.4(2) \\ \mathrm{C}(10)-\mathrm{C}(9)-\mathrm{C}(8) & 119.1(3) \\ \mathrm{C}(10)-\mathrm{C}(9)-\mathrm{H}(9 \mathrm{~A}) & 120.4 \\ \mathrm{C}(8)-\mathrm{C}(9)-\mathrm{H}(9 \mathrm{~A}) & 120.4 \\ \mathrm{C}(9)-\mathrm{C}(10)-\mathrm{C}(11) & 121.0(3) \\ \mathrm{C}(9)-\mathrm{C}(10)-\mathrm{H}(10 \mathrm{~A}) & 119.5 \\ \mathrm{C}(11)-\mathrm{C}(10)-\mathrm{H}(10 \mathrm{~A}) & 119.5 \\ \mathrm{C}(12)-\mathrm{C}(11)-\mathrm{C}(10) & 121.3(3) \\ \mathrm{C}(12)-\mathrm{C}(11)-\mathrm{H}(11 \mathrm{~A}) & 119.3 \\ \mathrm{C}(10)-\mathrm{C}(11)-\mathrm{H}(11 \mathrm{~A}) & 119.3 \\ \mathrm{C}(11)-\mathrm{C}(12)-\mathrm{C}(13) & 117.7(3) \\ \mathrm{C}(11)-\mathrm{C}(12)-\mathrm{H}(12 \mathrm{~A}) & 121.2 \\ \mathrm{C}(13)-\mathrm{C}(12)-\mathrm{H}(12 \mathrm{~A}) & 121.2 \\ \mathrm{C}(12)-\mathrm{C}(13)-\mathrm{N}(2) & 130.8(2) \\ \mathrm{C}(12)-\mathrm{C}(13)-\mathrm{C}(8) & 121.5(2) \\ \mathrm{N}(2)-\mathrm{C}(13)-\mathrm{C}(8) & 107.6(2) \\ \mathrm{N}(3)-\mathrm{C}(14)-\mathrm{C}(15) & 123.3(3) \\ \mathrm{N}(3)-\mathrm{C}(14)-\mathrm{N}(2) & 115.7(3) \\ \mathrm{C}(14)-\mathrm{C}(15)-\mathrm{H}(15 \mathrm{~A}) & 120.9 \\ & \end{array}$




$\begin{array}{ll}\mathrm{C}(16)-\mathrm{C}(15)-\mathrm{H}(15 \mathrm{~A}) & 120.9 \\ \mathrm{C}(17)-\mathrm{C}(16)-\mathrm{C}(15) & 118.8(4) \\ \mathrm{C}(17)-\mathrm{C}(16)-\mathrm{H}(16 \mathrm{~A}) & 120.6 \\ \mathrm{C}(15)-\mathrm{C}(16)-\mathrm{H}(16 \mathrm{~A}) & 120.6 \\ \mathrm{C}(18)-\mathrm{C}(17)-\mathrm{C}(16) & 118.4(3) \\ \mathrm{C}(18)-\mathrm{C}(17)-\mathrm{H}(17 \mathrm{~A}) & 120.8 \\ \mathrm{C}(16)-\mathrm{C}(17)-\mathrm{H}(17 \mathrm{~A}) & 120.8 \\ \mathrm{~N}(3)-\mathrm{C}(18)-\mathrm{C}(17) & 124.3(4) \\ \mathrm{N}(3)-\mathrm{C}(18)-\mathrm{H}(18 \mathrm{~A}) & 117.8 \\ \mathrm{C}(17)-\mathrm{C}(18)-\mathrm{H}(18 \mathrm{~A}) & 117.8 \\ \mathrm{C}(2)-\mathrm{C}(19)-\mathrm{C}(20) & 129.8(2) \\ \mathrm{C}(2)-\mathrm{C}(19)-\mathrm{H}(19 \mathrm{~A}) & 115.1 \\ \mathrm{C}(20)-\mathrm{C}(19)-\mathrm{H}(19 \mathrm{~A}) & 115.1 \\ \mathrm{C}(25)-\mathrm{C}(20)-\mathrm{C}(21) & 116.9(2) \\ \mathrm{C}(25)-\mathrm{C}(20)-\mathrm{C}(19) & 123.8(2) \\ \mathrm{C}(21)-\mathrm{C}(20)-\mathrm{C}(19) & 119.3(2) \\ \mathrm{C}(22)-\mathrm{C}(21)-\mathrm{C}(20) & 122.1(3) \\ \mathrm{C}(22)-\mathrm{C}(21)-\mathrm{H}(21 \mathrm{~A}) & 118.9 \\ \mathrm{C}(20)-\mathrm{C}(21)-\mathrm{H}(21 \mathrm{~A}) & 118.9 \\ \mathrm{C}(23)-\mathrm{C}(22)-\mathrm{C}(21) & 118.6(3) \\ \mathrm{C}(23)-\mathrm{C}(22)-\mathrm{H}(22 \mathrm{~A}) & 120.7 \\ \mathrm{C}(21)-\mathrm{C}(22)-\mathrm{H}(22 \mathrm{~A}) & 120.7 \\ \mathrm{C}(24)-\mathrm{C}(23)-\mathrm{C}(22) & 121.1(3) \\ \mathrm{C}(24)-\mathrm{C}(23)-\mathrm{Cl}(1) & 119.1(2) \\ \mathrm{C}(22)-\mathrm{C}(23)-\mathrm{Cl}(1) & 119.9(2) \\ \mathrm{C}(23)-\mathrm{C}(24)-\mathrm{C}(25) & 119.6(3) \\ \mathrm{C}(23)-\mathrm{C}(24)-\mathrm{H}(24 \mathrm{~A}) & 120.2 \\ \mathrm{C}(25)-\mathrm{C}(24)-\mathrm{H}(24 \mathrm{~A}) & 120.2 \\ \mathrm{C}(24)-\mathrm{C}(25)-\mathrm{C}(20) & 121.6(2) \\ \mathrm{C}(24)-\mathrm{C}(25)-\mathrm{H}(25 \mathrm{~A}) & 119.2 \\ \mathrm{C}(20)-\mathrm{C}(25)-\mathrm{H}(25 \mathrm{~A}) & 119.2 \\ \mathrm{C}(31)-\mathrm{C}(26)-\mathrm{C}(27) & 120.3(3) \\ \mathrm{C}(31)-\mathrm{C}(26)-\mathrm{S}(1) & 119.5(2) \\ \mathrm{C}(27)-\mathrm{H}(27 \mathrm{~A}) & 120.4 \\ \end{array}$




$\begin{array}{ll}\mathrm{C}(26)-\mathrm{C}(27)-\mathrm{H}(27 \mathrm{~A}) & 120.4 \\ \mathrm{C}(27)-\mathrm{C}(28)-\mathrm{C}(29) & 121.2(3) \\ \mathrm{C}(27)-\mathrm{C}(28)-\mathrm{H}(28 \mathrm{~A}) & 119.4 \\ \mathrm{C}(29)-\mathrm{C}(28)-\mathrm{H}(28 \mathrm{~A}) & 119.4 \\ \mathrm{C}(30)-\mathrm{C}(29)-\mathrm{C}(28) & 118.9(3) \\ \mathrm{C}(30)-\mathrm{C}(29)-\mathrm{C}(32) & 120.4(4) \\ \mathrm{C}(28)-\mathrm{C}(29)-\mathrm{C}(32) & 120.7(3) \\ \mathrm{C}(31)-\mathrm{C}(30)-\mathrm{C}(29) & 120.7(3) \\ \mathrm{C}(31)-\mathrm{C}(30)-\mathrm{H}(30 \mathrm{~A}) & 119.7 \\ \mathrm{C}(29)-\mathrm{C}(30)-\mathrm{H}(30 \mathrm{~A}) & 119.7 \\ \mathrm{C}(30)-\mathrm{C}(31)-\mathrm{C}(26) & 119.8(3) \\ \mathrm{C}(30)-\mathrm{C}(31)-\mathrm{H}(31 \mathrm{~A}) & 120.1 \\ \mathrm{C}(26)-\mathrm{C}(31)-\mathrm{H}(31 \mathrm{~A}) & 120.1 \\ \mathrm{C}(29)-\mathrm{C}(32)-\mathrm{H}(32 \mathrm{~A}) & 109.5 \\ \mathrm{C}(29)-\mathrm{C}(32)-\mathrm{H}(32 \mathrm{~B}) & 109.5 \\ \mathrm{H}(32 \mathrm{~A})-\mathrm{C}(32)-\mathrm{H}(32 \mathrm{~B}) & 109.5 \\ \mathrm{C}(29)-\mathrm{C}(32)-\mathrm{H}(32 \mathrm{C}) & 109.5 \\ \mathrm{H}(32 \mathrm{~A})-\mathrm{C}(32)-\mathrm{H}(32 \mathrm{C}) & 109.5 \\ \mathrm{H}(32 \mathrm{~B})-\mathrm{C}(32)-\mathrm{H}(32 \mathrm{C}) & 109.5 \\ & \end{array}$

Symmetry transformations used to generate equivalent atoms: 
Table 4. Anisotropic displacement parameters $\left(\AA^{2} \times 10^{3}\right)$ for d19195_a. The anisotropic displacement factor exponent takes the form: $-2 p^{2}\left[h^{2} a^{* 2} U^{11}+\ldots+2 h k a^{*} b^{*} U^{12}\right]$

\begin{tabular}{|c|c|c|c|c|c|c|}
\hline & $\mathrm{U}^{11}$ & $\mathrm{U}^{22}$ & $\mathrm{U}^{33}$ & $\mathrm{U}^{23}$ & $\mathrm{U}^{13}$ & $\mathrm{U}^{12}$ \\
\hline $\mathrm{Cl}(1)$ & $48(1)$ & $84(1)$ & $49(1)$ & $3(1)$ & $25(1)$ & $10(1)$ \\
\hline$S(1)$ & $37(1)$ & $37(1)$ & $32(1)$ & $2(1)$ & $5(1)$ & $-4(1)$ \\
\hline $\mathrm{O}(1)$ & $54(1)$ & $32(1)$ & $40(1)$ & $1(1)$ & $-3(1)$ & $-7(1)$ \\
\hline $\mathrm{O}(2)$ & $40(1)$ & $63(1)$ & $48(1)$ & $6(1)$ & $16(1)$ & $-3(1)$ \\
\hline $\mathrm{N}(1)$ & $29(1)$ & $34(1)$ & $31(1)$ & $-1(1)$ & $5(1)$ & $2(1)$ \\
\hline $\mathrm{N}(2)$ & $27(1)$ & $25(1)$ & $35(1)$ & $4(1)$ & $1(1)$ & $0(1)$ \\
\hline $\mathrm{N}(3)$ & $36(1)$ & $40(1)$ & $63(2)$ & $12(1)$ & $9(1)$ & $-7(1)$ \\
\hline $\mathrm{C}(1)$ & $28(1)$ & $32(1)$ & $27(1)$ & $-1(1)$ & $2(1)$ & $3(1)$ \\
\hline $\mathrm{C}(2)$ & $25(1)$ & $28(1)$ & $29(1)$ & $-4(1)$ & $-4(1)$ & $1(1)$ \\
\hline $\mathrm{C}(3)$ & $26(1)$ & $27(1)$ & $37(1)$ & $-1(1)$ & $0(1)$ & $2(1)$ \\
\hline $\mathrm{C}(4)$ & $28(1)$ & $36(1)$ & $38(2)$ & $-4(1)$ & 2(1) & $6(1)$ \\
\hline $\mathrm{C}(5)$ & $29(1)$ & $26(1)$ & $36(1)$ & $1(1)$ & $-2(1)$ & $2(1)$ \\
\hline $\mathrm{C}(6)$ & $26(1)$ & $22(1)$ & $37(2)$ & $0(1)$ & $-3(1)$ & $-1(1)$ \\
\hline $\mathrm{C}(7)$ & $29(1)$ & $28(1)$ & $38(2)$ & $4(1)$ & $-2(1)$ & $-2(1)$ \\
\hline $\mathrm{C}(8)$ & $27(1)$ & $28(1)$ & $37(2)$ & $-3(1)$ & $-2(1)$ & $-5(1)$ \\
\hline $\mathrm{C}(9)$ & $31(2)$ & $37(1)$ & $40(2)$ & $-4(1)$ & $3(1)$ & $-5(1)$ \\
\hline $\mathrm{C}(10)$ & $33(2)$ & $44(2)$ & $48(2)$ & $-12(1)$ & $7(1)$ & $4(1)$ \\
\hline $\mathrm{C}(11)$ & $39(2)$ & $38(2)$ & $54(2)$ & $-7(1)$ & $-3(1)$ & $14(1)$ \\
\hline $\mathrm{C}(12)$ & $38(2)$ & $30(1)$ & $41(2)$ & $-1(1)$ & $-3(1)$ & $4(1)$ \\
\hline $\mathrm{C}(13)$ & $25(1)$ & $27(1)$ & $34(1)$ & $-5(1)$ & $-1(1)$ & $-1(1)$ \\
\hline $\mathrm{C}(14)$ & $38(2)$ & $21(1)$ & $41(2)$ & $5(1)$ & $5(1)$ & $3(1)$ \\
\hline $\mathrm{C}(15)$ & $50(2)$ & $52(2)$ & $42(2)$ & $2(1)$ & $0(2)$ & $16(2)$ \\
\hline $\mathrm{C}(16)$ & $86(3)$ & $72(2)$ & $43(2)$ & $13(2)$ & $8(2)$ & $41(2)$ \\
\hline $\mathrm{C}(17)$ & $83(3)$ & $57(2)$ & $77(3)$ & $31(2)$ & $43(2)$ & $31(2)$ \\
\hline $\mathrm{C}(18)$ & $50(2)$ & $47(2)$ & $90(3)$ & $24(2)$ & $28(2)$ & $3(2)$ \\
\hline $\mathrm{C}(19)$ & $30(1)$ & $31(1)$ & $31(1)$ & $3(1)$ & $-3(1)$ & $5(1)$ \\
\hline $\mathrm{C}(20)$ & $31(2)$ & $30(1)$ & $27(1)$ & $-1(1)$ & $0(1)$ & $-2(1)$ \\
\hline $\mathrm{C}(21)$ & $49(2)$ & $42(2)$ & $32(2)$ & $9(1)$ & $1(1)$ & $9(1)$ \\
\hline $\mathrm{C}(22)$ & $55(2)$ & $52(2)$ & $29(2)$ & $10(1)$ & $14(2)$ & $6(2)$ \\
\hline $\mathrm{C}(23)$ & $32(2)$ & $46(2)$ & $32(1)$ & $-1(1)$ & $10(1)$ & $0(1)$ \\
\hline $\mathrm{C}(24)$ & $28(1)$ & $48(2)$ & $29(1)$ & $2(1)$ & $0(1)$ & $1(1)$ \\
\hline & & & & S100 & & \\
\hline
\end{tabular}




$\begin{array}{lllllll}\mathrm{C}(25) & 28(1) & 47(2) & 22(1) & 0(1) & 0(1) & 1(1) \\ \mathrm{C}(26) & 46(2) & 32(1) & 21(1) & 1(1) & 3(1) & -3(1) \\ \mathrm{C}(27) & 61(2) & 47(2) & 28(2) & -6(1) & 9(1) & 0(2) \\ \mathrm{C}(28) & 85(3) & 45(2) & 31(2) & -9(1) & -2(2) & -9(2) \\ \mathrm{C}(29) & 71(2) & 49(2) & 38(2) & 8(1) & -15(2) & -16(2) \\ \mathrm{C}(30) & 47(2) & 47(2) & 44(2) & 7(1) & -9(2) & -2(1) \\ \mathrm{C}(31) & 46(2) & 34(1) & 32(1) & 3(1) & -1(1) & 2(1) \\ \mathrm{C}(32) & 94(3) & 79(3) & 68(3) & 10(2) & -34(2) & -34(2)\end{array}$


Table 5. Hydrogen coordinates ( $\left.\times 10^{4}\right)$ and isotropic displacement parameters $\left(\AA^{2} \times 10^{3}\right)$ for d19195_a.

\begin{tabular}{|c|c|c|c|c|}
\hline- & $\mathrm{x}$ & $\mathrm{y}$ & $\mathrm{z}$ & $\mathrm{U}(\mathrm{eq})$ \\
\hline \multicolumn{5}{|l|}{-} \\
\hline $\mathrm{H}(1 \mathrm{~A})$ & 6785 & 3295 & 4340 & 35 \\
\hline $\mathrm{H}(1 \mathrm{~B})$ & 7634 & 4160 & 4704 & 35 \\
\hline $\mathrm{H}(3 \mathrm{~A})$ & 4924 & 6131 & 4295 & 36 \\
\hline $\mathrm{H}(4 \mathrm{~A})$ & 5536 & 5524 & 5155 & 41 \\
\hline $\mathrm{H}(4 \mathrm{~B})$ & 3850 & 5095 & 5104 & 41 \\
\hline $\mathrm{H}(5 \mathrm{~A})$ & 3558 & 4975 & 3656 & 36 \\
\hline $\mathrm{H}(5 \mathrm{~B})$ & 3283 & 4198 & 4158 & 36 \\
\hline $\mathrm{H}(7 \mathrm{~A})$ & 1225 & 4872 & 4911 & 38 \\
\hline $\mathrm{H}(9 \mathrm{~A})$ & -1445 & 6022 & 5262 & 43 \\
\hline $\mathrm{H}(10 \mathrm{~A})$ & -2988 & 7398 & 5016 & 50 \\
\hline $\mathrm{H}(11 \mathrm{~A})$ & -2541 & 8354 & 4221 & 52 \\
\hline $\mathrm{H}(12 \mathrm{~A})$ & -512 & 7965 & 3658 & 44 \\
\hline $\mathrm{H}(15 \mathrm{~A})$ & 312 & 6691 & 2928 & 58 \\
\hline $\mathrm{H}(16 \mathrm{~A})$ & 1536 & 7219 & 2121 & 80 \\
\hline $\mathrm{H}(17 \mathrm{~A})$ & 4107 & 7536 & 2153 & 87 \\
\hline $\mathrm{H}(18 \mathrm{~A})$ & 5347 & 7321 & 2964 & 75 \\
\hline $\mathrm{H}(19 \mathrm{~A})$ & 6402 & 5625 & 3453 & 37 \\
\hline $\mathrm{H}(21 \mathrm{~A})$ & 7801 & 5300 & 2643 & 49 \\
\hline $\mathrm{H}(22 \mathrm{~A})$ & 9864 & 4630 & 2187 & 55 \\
\hline $\mathrm{H}(24 \mathrm{~A})$ & 11173 & 3207 & 3578 & 42 \\
\hline $\mathrm{H}(25 \mathrm{~A})$ & 9100 & 3861 & 4029 & 39 \\
\hline $\mathrm{H}(27 \mathrm{~A})$ & 6014 & 4930 & 6237 & 55 \\
\hline $\mathrm{H}(28 \mathrm{~A})$ & 8111 & 5630 & 6660 & 64 \\
\hline $\mathrm{H}(30 \mathrm{~A})$ & 10723 & 3510 & 5904 & 55 \\
\hline $\mathrm{H}(31 \mathrm{~A})$ & 8636 & 2792 & 5483 & 45 \\
\hline $\mathrm{H}(32 \mathrm{~A})$ & 11018 & 5850 & 6532 & 120 \\
\hline $\mathrm{H}(32 \mathrm{~B})$ & 11882 & 4770 & 6452 & 120 \\
\hline $\mathrm{H}(32 \mathrm{C})$ & 10928 & 4971 & 6995 & 120 \\
\hline
\end{tabular}


Table 6. Torsion angles $\left[{ }^{\circ}\right]$ for d19195_a.

\begin{tabular}{lc}
\hline $\mathrm{O}(1)-\mathrm{S}(1)-\mathrm{N}(1)-\mathrm{C}(1)$ & $-54.3(2)$ \\
$\mathrm{O}(2)-\mathrm{S}(1)-\mathrm{N}(1)-\mathrm{C}(1)$ & $176.25(18)$ \\
$\mathrm{C}(26)-\mathrm{S}(1)-\mathrm{N}(1)-\mathrm{C}(1)$ & $60.2(2)$ \\
$\mathrm{O}(1)-\mathrm{S}(1)-\mathrm{N}(1)-\mathrm{C}(4)$ & $171.2(2)$ \\
$\mathrm{O}(2)-\mathrm{S}(1)-\mathrm{N}(1)-\mathrm{C}(4)$ & $41.8(2)$ \\
$\mathrm{C}(26)-\mathrm{S}(1)-\mathrm{N}(1)-\mathrm{C}(4)$ & $-74.3(2)$ \\
$\mathrm{C}(4)-\mathrm{N}(1)-\mathrm{C}(1)-\mathrm{C}(2)$ & $-32.0(2)$ \\
$\mathrm{S}(1)-\mathrm{N}(1)-\mathrm{C}(1)-\mathrm{C}(2)$ & $-171.64(16)$ \\
$\mathrm{N}(1)-\mathrm{C}(1)-\mathrm{C}(2)-\mathrm{C}(19)$ & $-172.4(3)$ \\
$\mathrm{N}(1)-\mathrm{C}(1)-\mathrm{C}(2)-\mathrm{C}(3)$ & $7.5(2)$ \\
$\mathrm{C}(19)-\mathrm{C}(2)-\mathrm{C}(3)-\mathrm{C}(4)$ & $-161.7(2)$ \\
$\mathrm{C}(1)-\mathrm{C}(2)-\mathrm{C}(3)-\mathrm{C}(4)$ & $18.4(3)$ \\
$\mathrm{C}(19)-\mathrm{C}(2)-\mathrm{C}(3)-\mathrm{C}(5)$ & $79.7(3)$ \\
$\mathrm{C}(1)-\mathrm{C}(2)-\mathrm{C}(3)-\mathrm{C}(5)$ & $-100.3(2)$ \\
$\mathrm{C}(1)-\mathrm{N}(1)-\mathrm{C}(4)-\mathrm{C}(3)$ & $43.8(2)$ \\
$\mathrm{S}(1)-\mathrm{N}(1)-\mathrm{C}(4)-\mathrm{C}(3)$ & $-177.09(17)$ \\
$\mathrm{C}(2)-\mathrm{C}(3)-\mathrm{C}(4)-\mathrm{N}(1)$ & $-36.7(2)$ \\
$\mathrm{C}(5)-\mathrm{C}(3)-\mathrm{C}(4)-\mathrm{N}(1)$ & $82.0(2)$ \\
$\mathrm{C}(4)-\mathrm{C}(3)-\mathrm{C}(5)-\mathrm{C}(6)$ & $73.3(3)$ \\
$\mathrm{C}(2)-\mathrm{C}(3)-\mathrm{C}(5)-\mathrm{C}(6)$ & $-172.8(2)$ \\
$\mathrm{C}(13)-\mathrm{N}(2)-\mathrm{C}(6)-\mathrm{C}(7)$ & $-0.5(3)$ \\
$\mathrm{C}(14)-\mathrm{N}(2)-\mathrm{C}(6)-\mathrm{C}(7)$ & $-178.1(2)$ \\
$\mathrm{C}(13)-\mathrm{N}(2)-\mathrm{C}(6)-\mathrm{C}(5)$ & $178.8(2)$ \\
$\mathrm{C}(14)-\mathrm{N}(2)-\mathrm{C}(6)-\mathrm{C}(5)$ & $1.2(4)$ \\
$\mathrm{C}(3)-\mathrm{C}(5)-\mathrm{C}(6)-\mathrm{C}(7)$ & $-86.8(3)$ \\
$\mathrm{C}(3)-\mathrm{C}(5)-\mathrm{C}(6)-\mathrm{N}(2)$ & $94.0(3)$ \\
$\mathrm{N}(2)-\mathrm{C}(6)-\mathrm{C}(7)-\mathrm{C}(8)$ & $-0.5(3)$ \\
$\mathrm{C}(5)-\mathrm{C}(6)-\mathrm{C}(7)-\mathrm{C}(8)$ & $-179.7(2)$ \\
$\mathrm{C}(6)-\mathrm{C}(7)-\mathrm{C}(8)-\mathrm{C}(9)$ & $-176.7(3)$ \\
$\mathrm{C}(6)-\mathrm{C}(7)-\mathrm{C}(8)-\mathrm{C}(13)$ & $1.2(3)$ \\
$\mathrm{C}(13)-\mathrm{C}(8)-\mathrm{C}(9)-\mathrm{C}(10)$ & $0.5(4)$ \\
$\mathrm{C}(7)-\mathrm{C}(8)-\mathrm{C}(9)-\mathrm{C}(10)$ & $-0.4(4)$ \\
$\mathrm{C}(8)-\mathrm{C}(9)-\mathrm{C}(10)-\mathrm{C}(11)$ & \\
$\mathrm{C}(9)-\mathrm{C}(10)-\mathrm{C}(11)-\mathrm{C}(12)$ & \\
& \\
& \\
& \\
&
\end{tabular}




\begin{tabular}{|c|c|}
\hline$C(10)-C(11)-C(12)-C(13)$ & $0.5(4)$ \\
\hline $\mathrm{C}(11)-\mathrm{C}(12)-\mathrm{C}(13)-\mathrm{N}(2)$ & $-176.5(3)$ \\
\hline$C(11)-C(12)-C(13)-C(8)$ & $-0.2(4)$ \\
\hline $\mathrm{C}(6)-\mathrm{N}(2)-\mathrm{C}(13)-\mathrm{C}(12)$ & $178.1(3)$ \\
\hline $\mathrm{C}(14)-\mathrm{N}(2)-\mathrm{C}(13)-\mathrm{C}(12)$ & $-4.4(4)$ \\
\hline $\mathrm{C}(6)-\mathrm{N}(2)-\mathrm{C}(13)-\mathrm{C}(8)$ & $1.3(3)$ \\
\hline $\mathrm{C}(14)-\mathrm{N}(2)-\mathrm{C}(13)-\mathrm{C}(8)$ & $178.8(2)$ \\
\hline$C(9)-C(8)-C(13)-C(12)$ & $-0.4(4)$ \\
\hline$C(7)-C(8)-C(13)-C(12)$ & $-178.7(2)$ \\
\hline $\mathrm{C}(9)-\mathrm{C}(8)-\mathrm{C}(13)-\mathrm{N}(2)$ & $176.7(2)$ \\
\hline $\mathrm{C}(7)-\mathrm{C}(8)-\mathrm{C}(13)-\mathrm{N}(2)$ & $-1.5(3)$ \\
\hline $\mathrm{C}(18)-\mathrm{N}(3)-\mathrm{C}(14)-\mathrm{C}(15)$ & $-1.0(4)$ \\
\hline $\mathrm{C}(18)-\mathrm{N}(3)-\mathrm{C}(14)-\mathrm{N}(2)$ & $176.4(2)$ \\
\hline $\mathrm{C}(13)-\mathrm{N}(2)-\mathrm{C}(14)-\mathrm{N}(3)$ & $132.4(3)$ \\
\hline $\mathrm{C}(6)-\mathrm{N}(2)-\mathrm{C}(14)-\mathrm{N}(3)$ & $-50.5(3)$ \\
\hline $\mathrm{C}(13)-\mathrm{N}(2)-\mathrm{C}(14)-\mathrm{C}(15)$ & $-50.1(4)$ \\
\hline $\mathrm{C}(6)-\mathrm{N}(2)-\mathrm{C}(14)-\mathrm{C}(15)$ & $127.0(3)$ \\
\hline $\mathrm{N}(3)-\mathrm{C}(14)-\mathrm{C}(15)-\mathrm{C}(16)$ & $0.4(5)$ \\
\hline $\mathrm{N}(2)-\mathrm{C}(14)-\mathrm{C}(15)-\mathrm{C}(16)$ & $-176.9(3)$ \\
\hline$C(14)-C(15)-C(16)-C(17)$ & $0.2(5)$ \\
\hline$C(15)-C(16)-C(17)-C(18)$ & $-0.1(6)$ \\
\hline $\mathrm{C}(14)-\mathrm{N}(3)-\mathrm{C}(18)-\mathrm{C}(17)$ & $1.2(5)$ \\
\hline $\mathrm{C}(16)-\mathrm{C}(17)-\mathrm{C}(18)-\mathrm{N}(3)$ & $-0.6(6)$ \\
\hline$C(1)-C(2)-C(19)-C(20)$ & $-0.3(5)$ \\
\hline$C(3)-C(2)-C(19)-C(20)$ & $179.8(2)$ \\
\hline$C(2)-C(19)-C(20)-C(25)$ & $-16.5(4)$ \\
\hline$C(2)-C(19)-C(20)-C(21)$ & $165.6(3)$ \\
\hline$C(25)-C(20)-C(21)-C(22)$ & $2.1(4)$ \\
\hline$C(19)-C(20)-C(21)-C(22)$ & $-179.9(3)$ \\
\hline$C(20)-C(21)-C(22)-C(23)$ & $-0.7(5)$ \\
\hline $\mathrm{C}(21)-\mathrm{C}(22)-\mathrm{C}(23)-\mathrm{C}(24)$ & $-1.2(5)$ \\
\hline $\mathrm{C}(21)-\mathrm{C}(22)-\mathrm{C}(23)-\mathrm{Cl}(1)$ & $177.8(2)$ \\
\hline $\mathrm{C}(22)-\mathrm{C}(23)-\mathrm{C}(24)-\mathrm{C}(25)$ & $1.6(4)$ \\
\hline $\mathrm{Cl}(1)-\mathrm{C}(23)-\mathrm{C}(24)-\mathrm{C}(25)$ & $-177.4(2)$ \\
\hline$C(23)-C(24)-C(25)-C(20)$ & $-0.1(4)$ \\
\hline$C(21)-C(20)-C(25)-C(24)$ & $-1.7(4)$ \\
\hline
\end{tabular}




$\begin{array}{lc}\mathrm{C}(19)-\mathrm{C}(20)-\mathrm{C}(25)-\mathrm{C}(24) & -179.6(2) \\ \mathrm{O}(1)-\mathrm{S}(1)-\mathrm{C}(26)-\mathrm{C}(31) & 24.6(3) \\ \mathrm{O}(2)-\mathrm{S}(1)-\mathrm{C}(26)-\mathrm{C}(31) & 155.8(2) \\ \mathrm{N}(1)-\mathrm{S}(1)-\mathrm{C}(26)-\mathrm{C}(31) & -89.5(2) \\ \mathrm{O}(1)-\mathrm{S}(1)-\mathrm{C}(26)-\mathrm{C}(27) & -160.4(2) \\ \mathrm{O}(2)-\mathrm{S}(1)-\mathrm{C}(26)-\mathrm{C}(27) & -29.2(3) \\ \mathrm{N}(1)-\mathrm{S}(1)-\mathrm{C}(26)-\mathrm{C}(27) & 85.5(2) \\ \mathrm{C}(31)-\mathrm{C}(26)-\mathrm{C}(27)-\mathrm{C}(28) & -1.0(4) \\ \mathrm{S}(1)-\mathrm{C}(26)-\mathrm{C}(27)-\mathrm{C}(28) & -175.9(2) \\ \mathrm{C}(26)-\mathrm{C}(27)-\mathrm{C}(28)-\mathrm{C}(29) & 0.6(5) \\ \mathrm{C}(27)-\mathrm{C}(28)-\mathrm{C}(29)-\mathrm{C}(30) & 0.1(5) \\ \mathrm{C}(27)-\mathrm{C}(28)-\mathrm{C}(29)-\mathrm{C}(32) & -179.9(3) \\ \mathrm{C}(28)-\mathrm{C}(29)-\mathrm{C}(30)-\mathrm{C}(31) & -0.4(4) \\ \mathrm{C}(32)-\mathrm{C}(29)-\mathrm{C}(30)-\mathrm{C}(31) & 179.6(3) \\ \mathrm{C}(29)-\mathrm{C}(30)-\mathrm{C}(31)-\mathrm{C}(26) & 0.0(4) \\ \mathrm{C}(27)-\mathrm{C}(26)-\mathrm{C}(31)-\mathrm{C}(30) & 0.7(4) \\ \mathrm{S}(1)-\mathrm{C}(26)-\mathrm{C}(31)-\mathrm{C}(30) & 175.6(2)\end{array}$

Symmetry transformations used to generate equivalent atoms: 
NMR Spectra

S106 

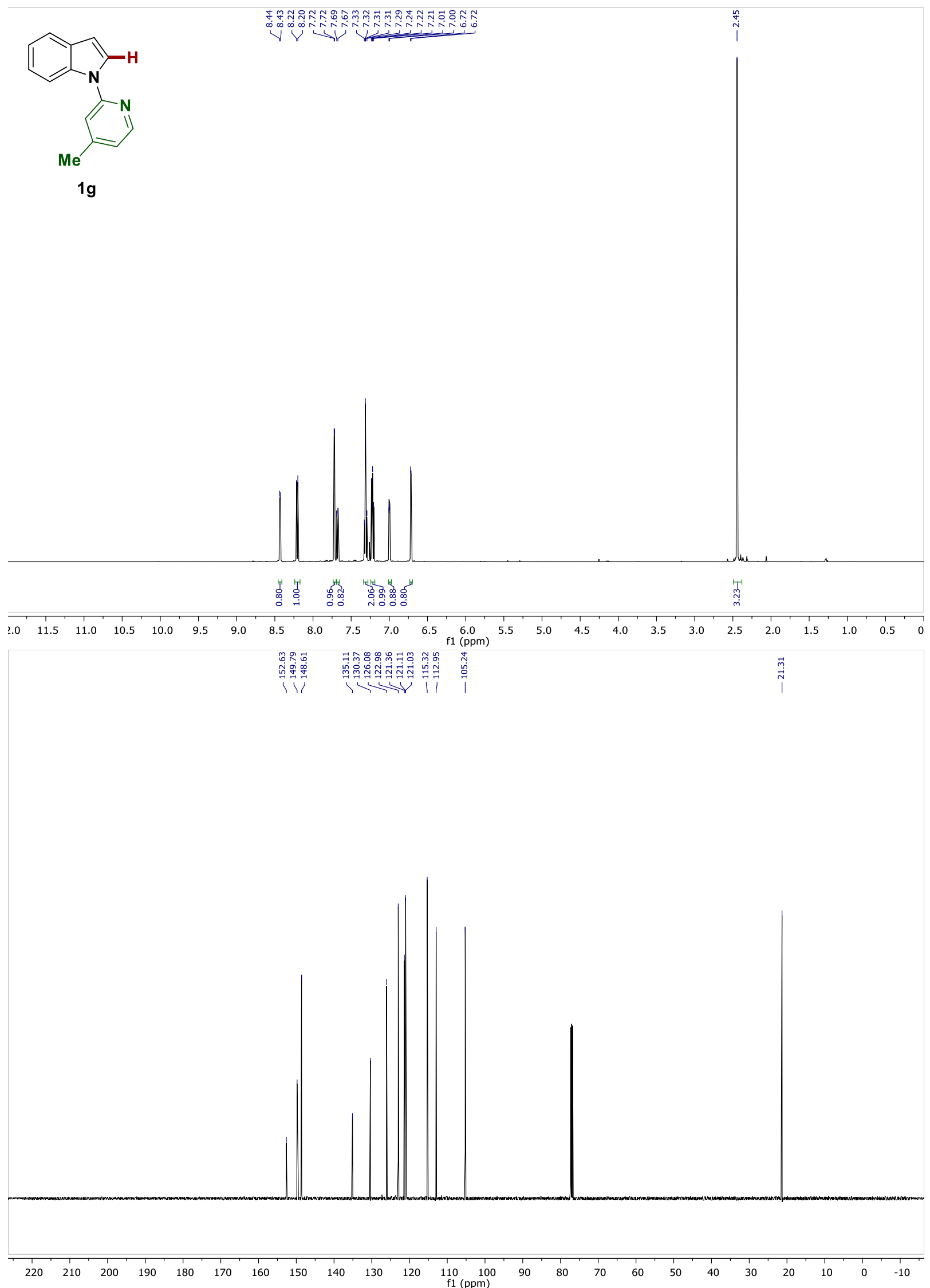

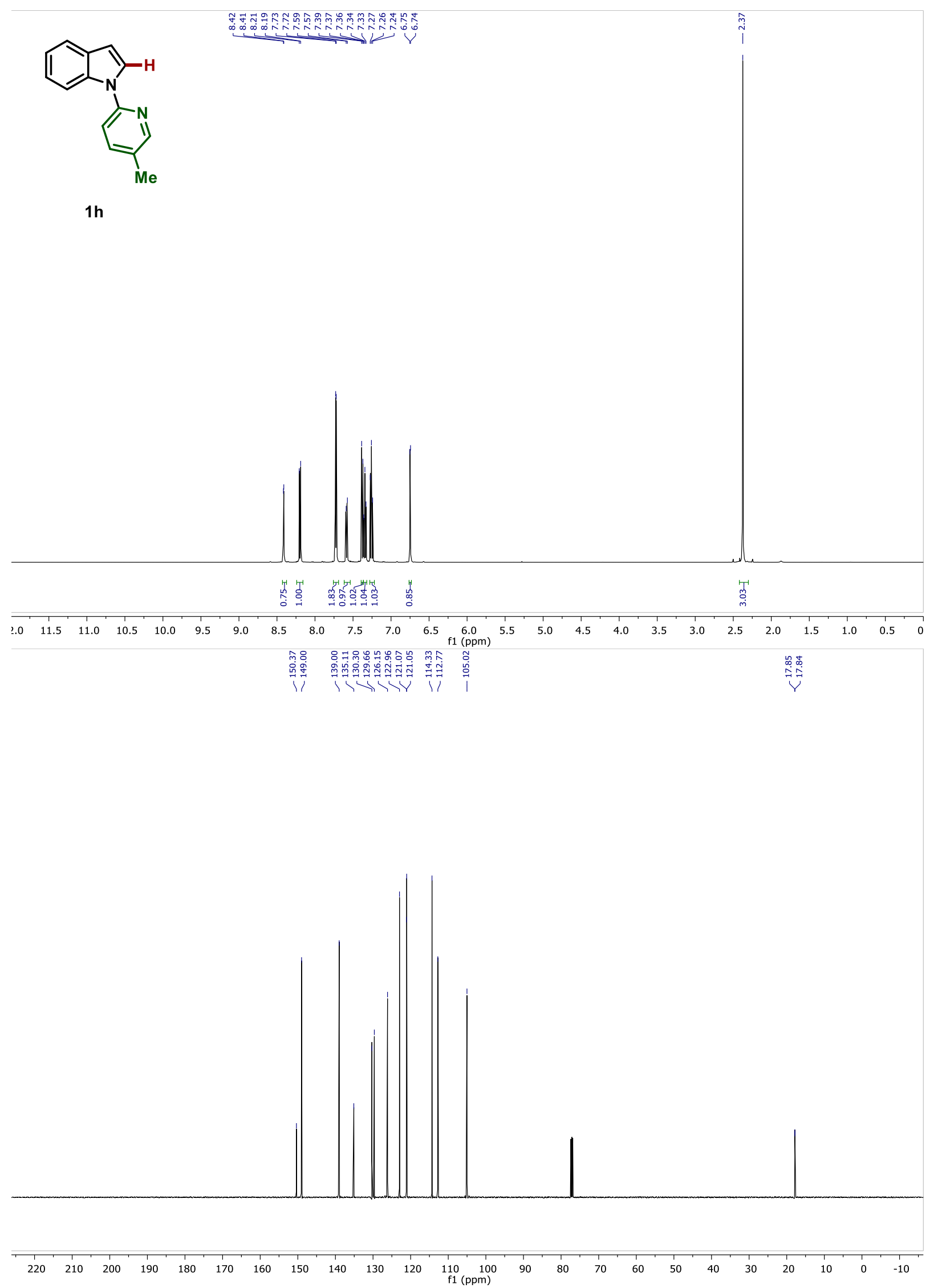


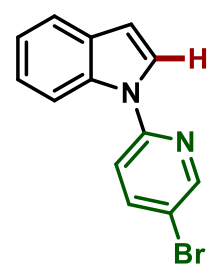

$1 i$

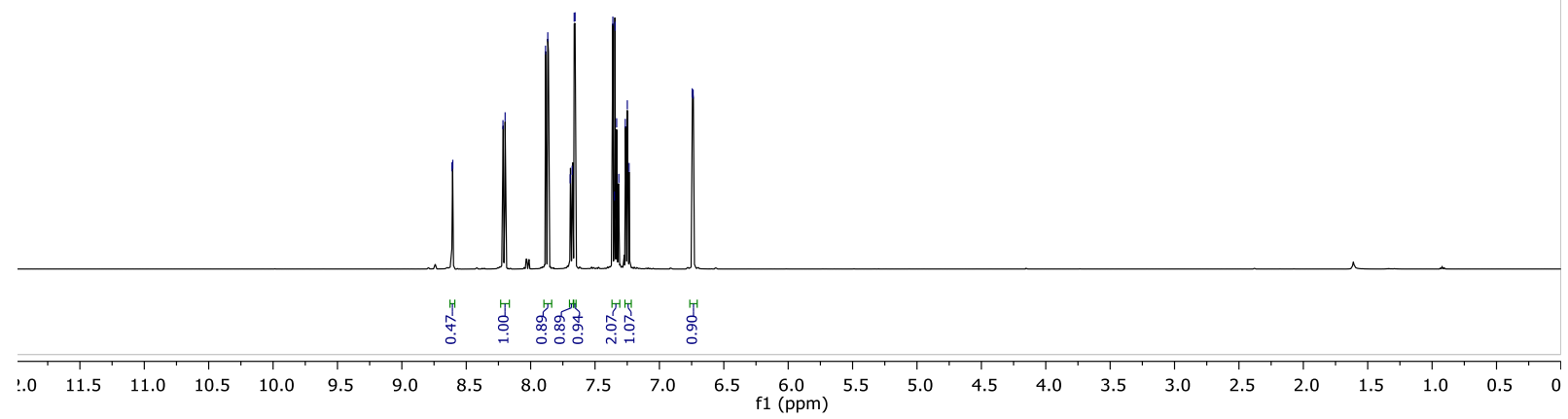

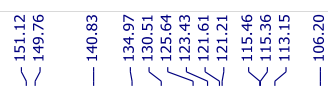

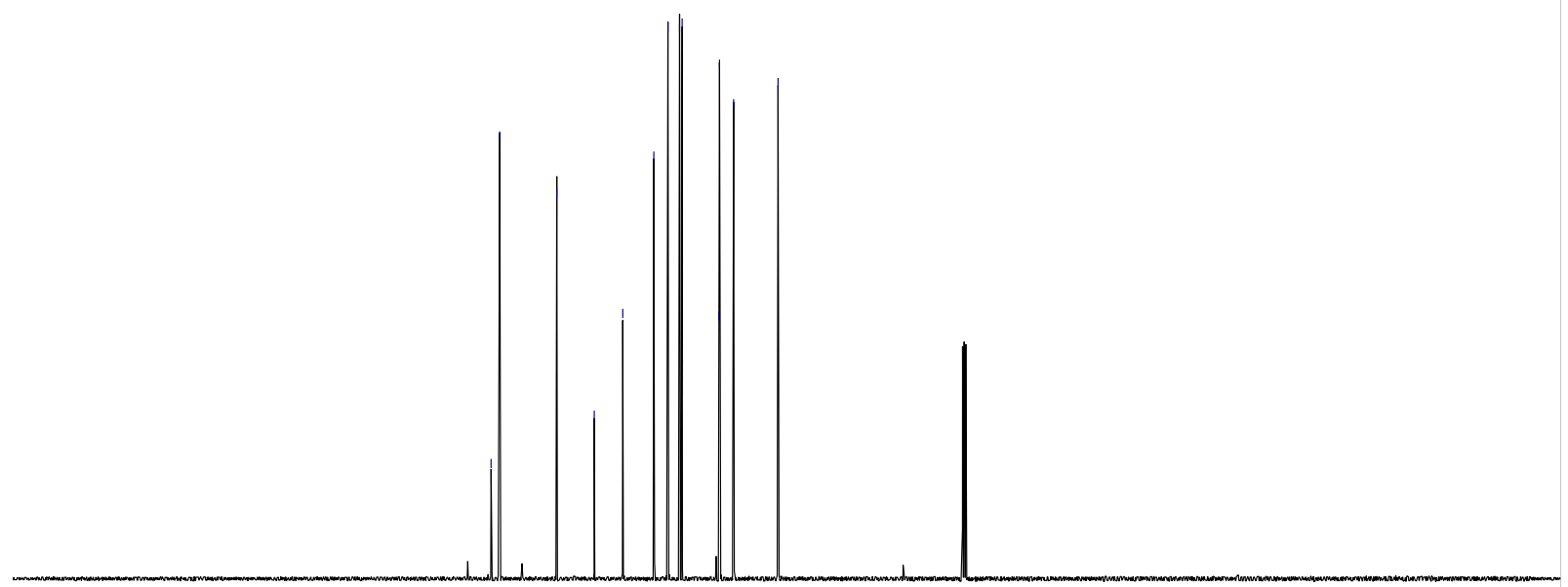

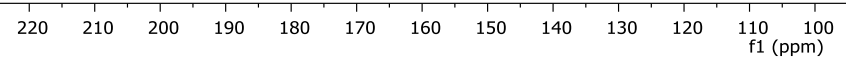



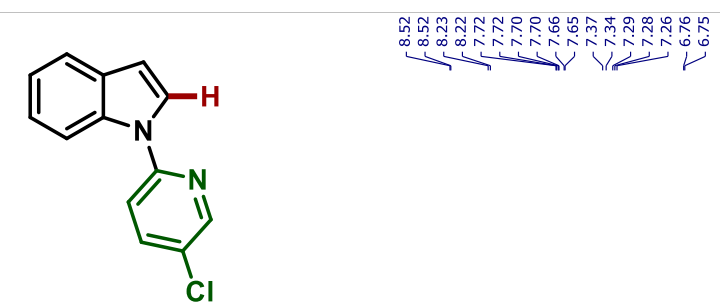

1j
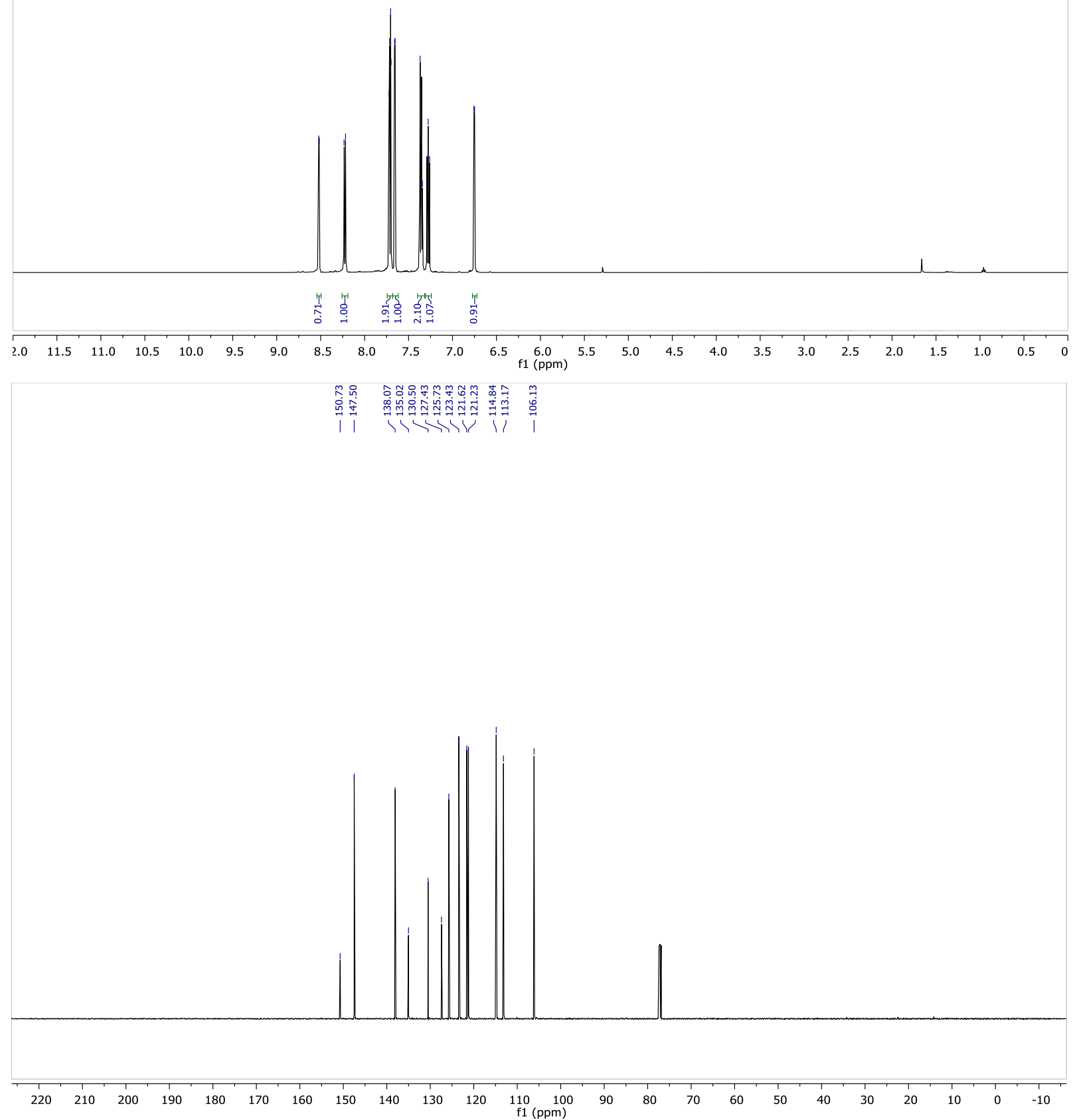

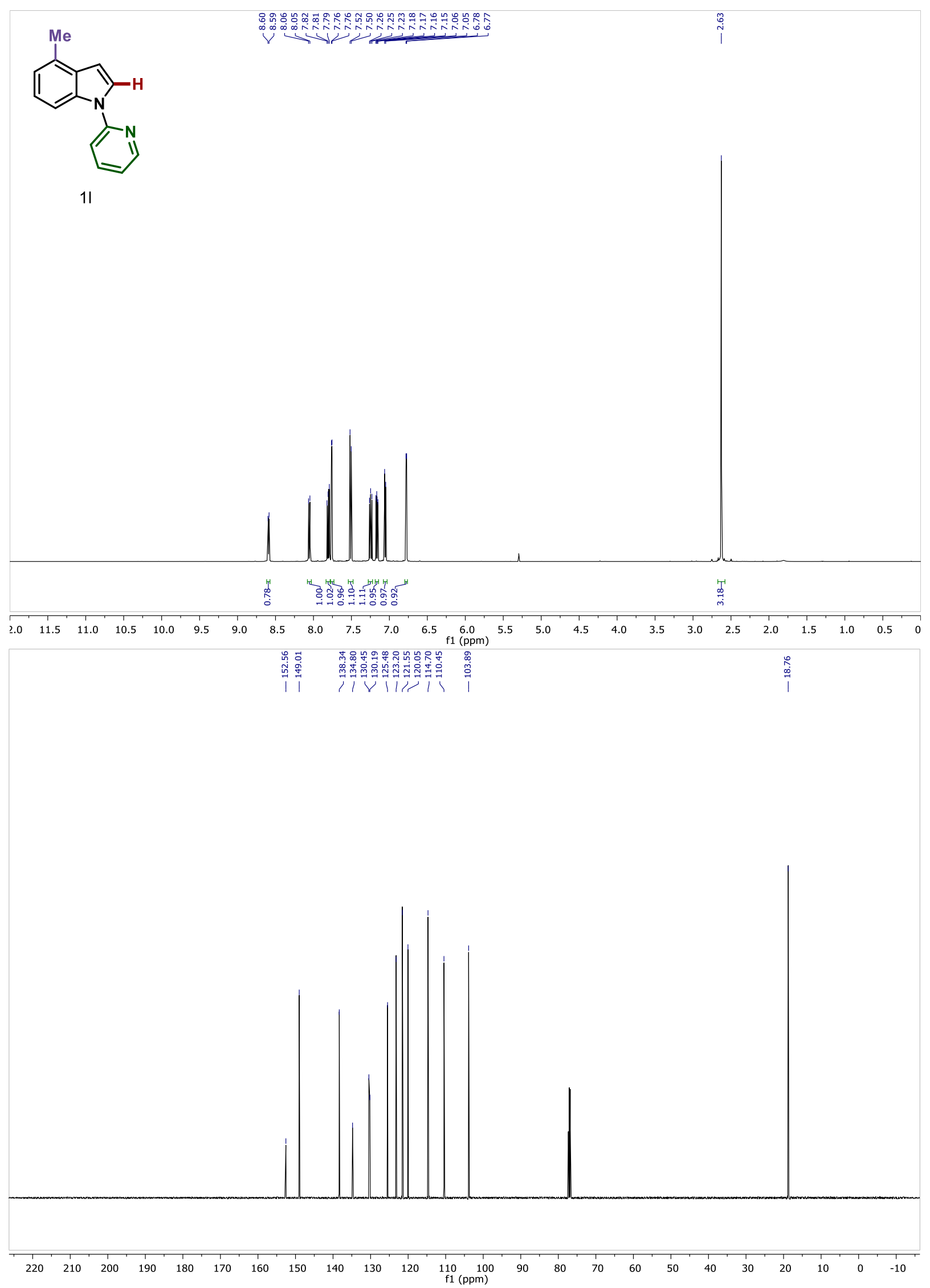

S111 

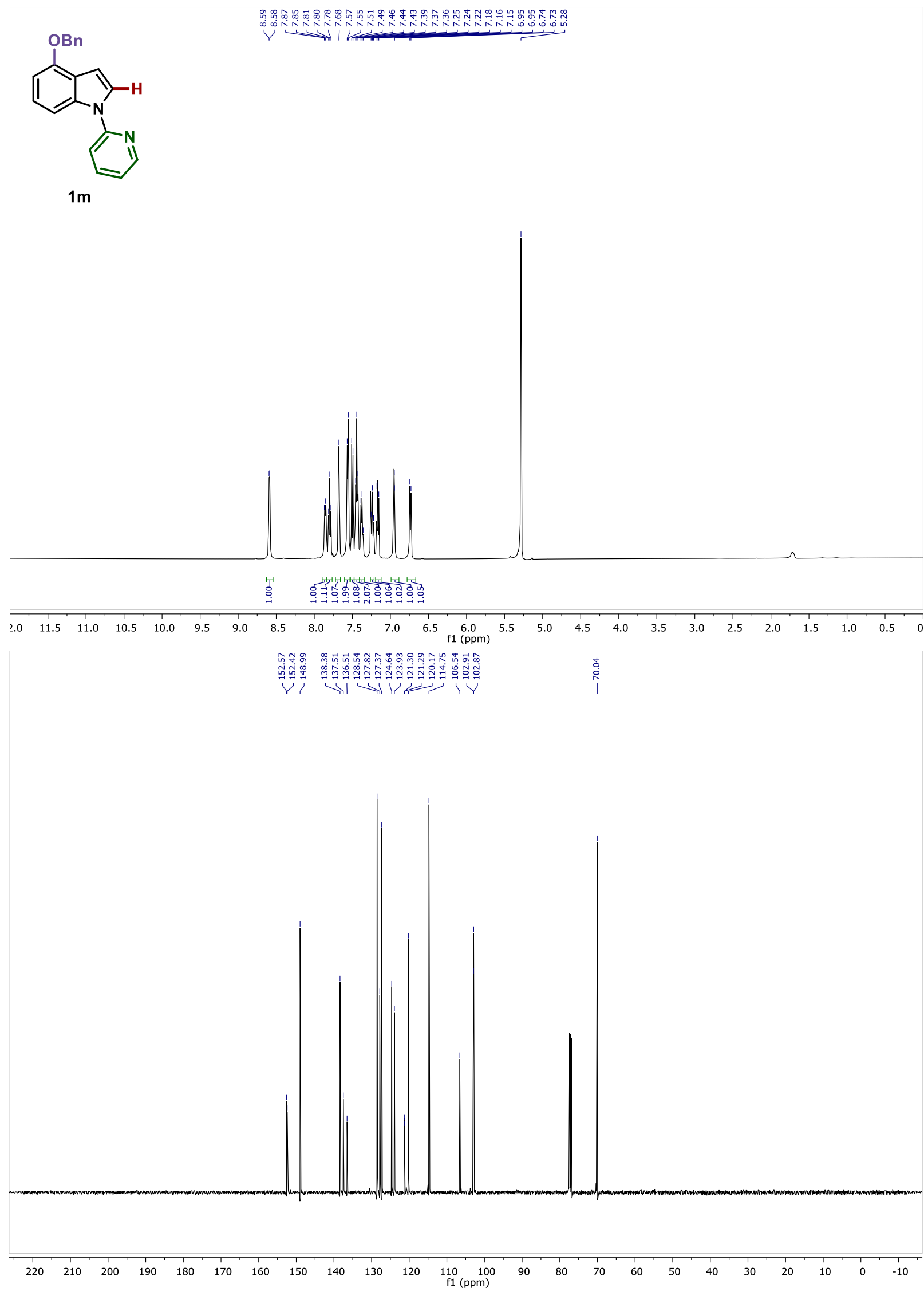


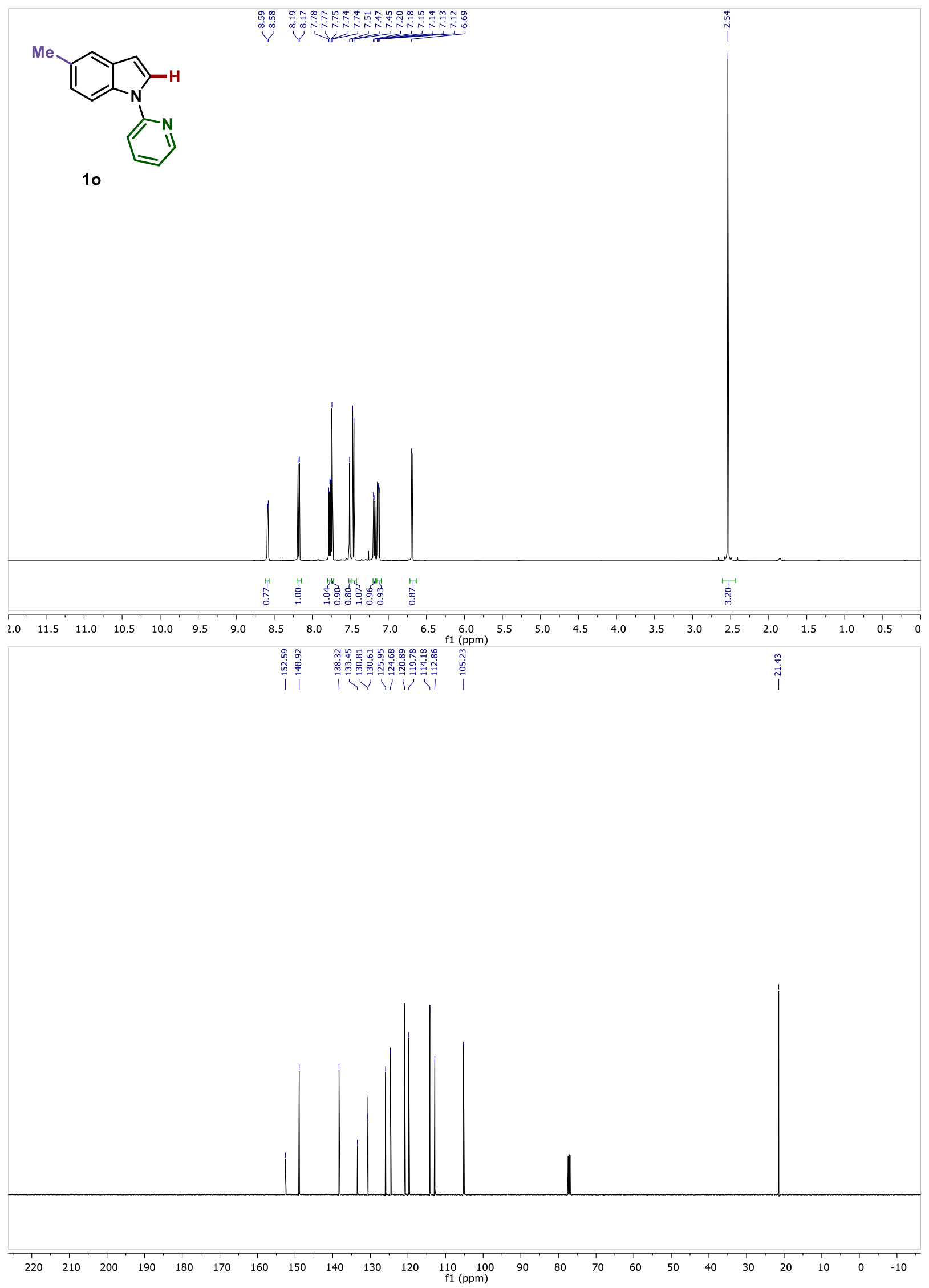

S113 

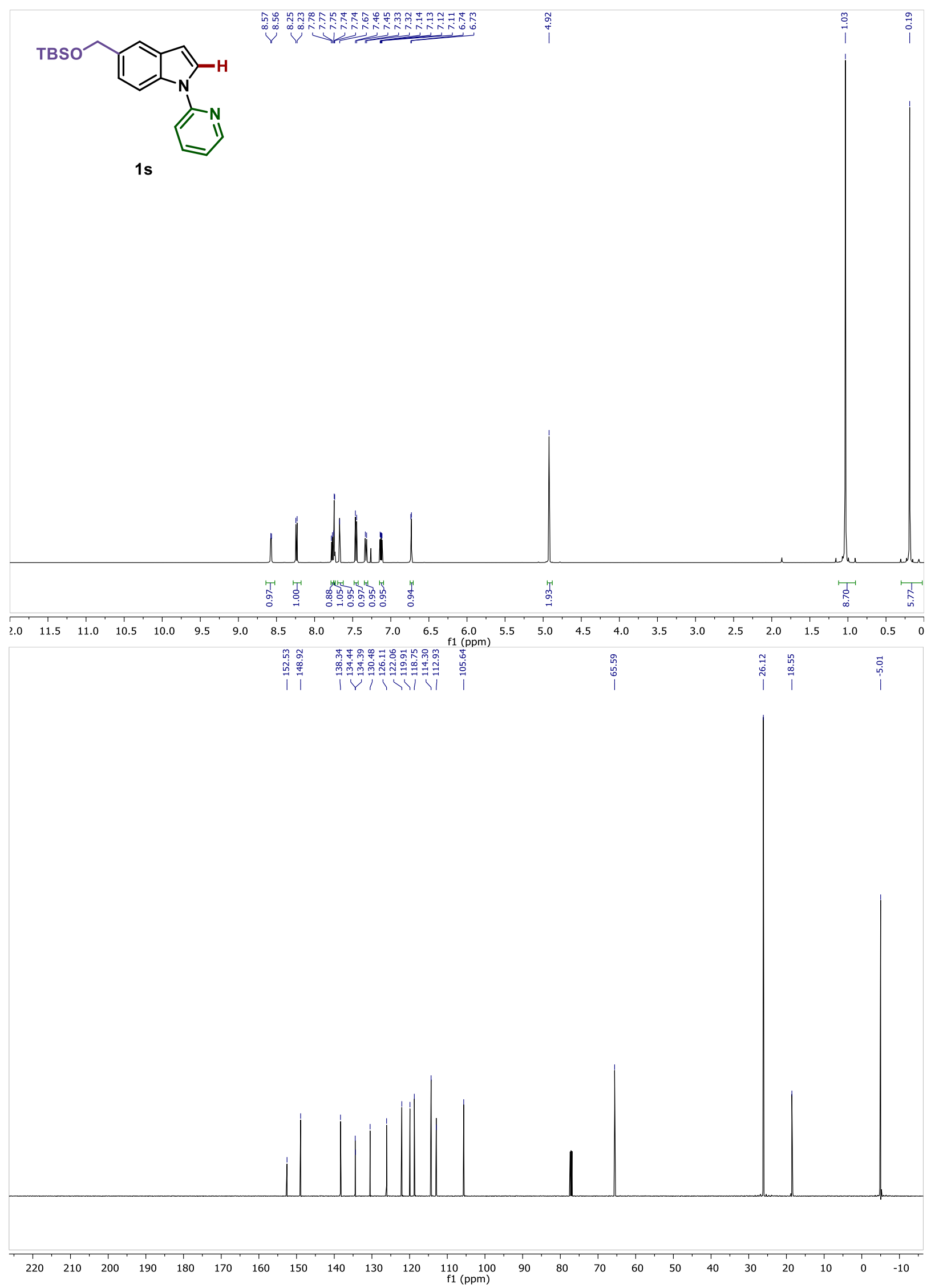


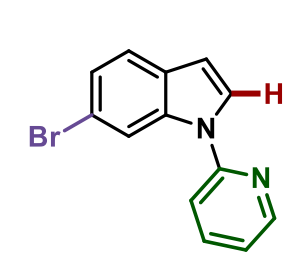

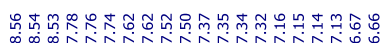

1v
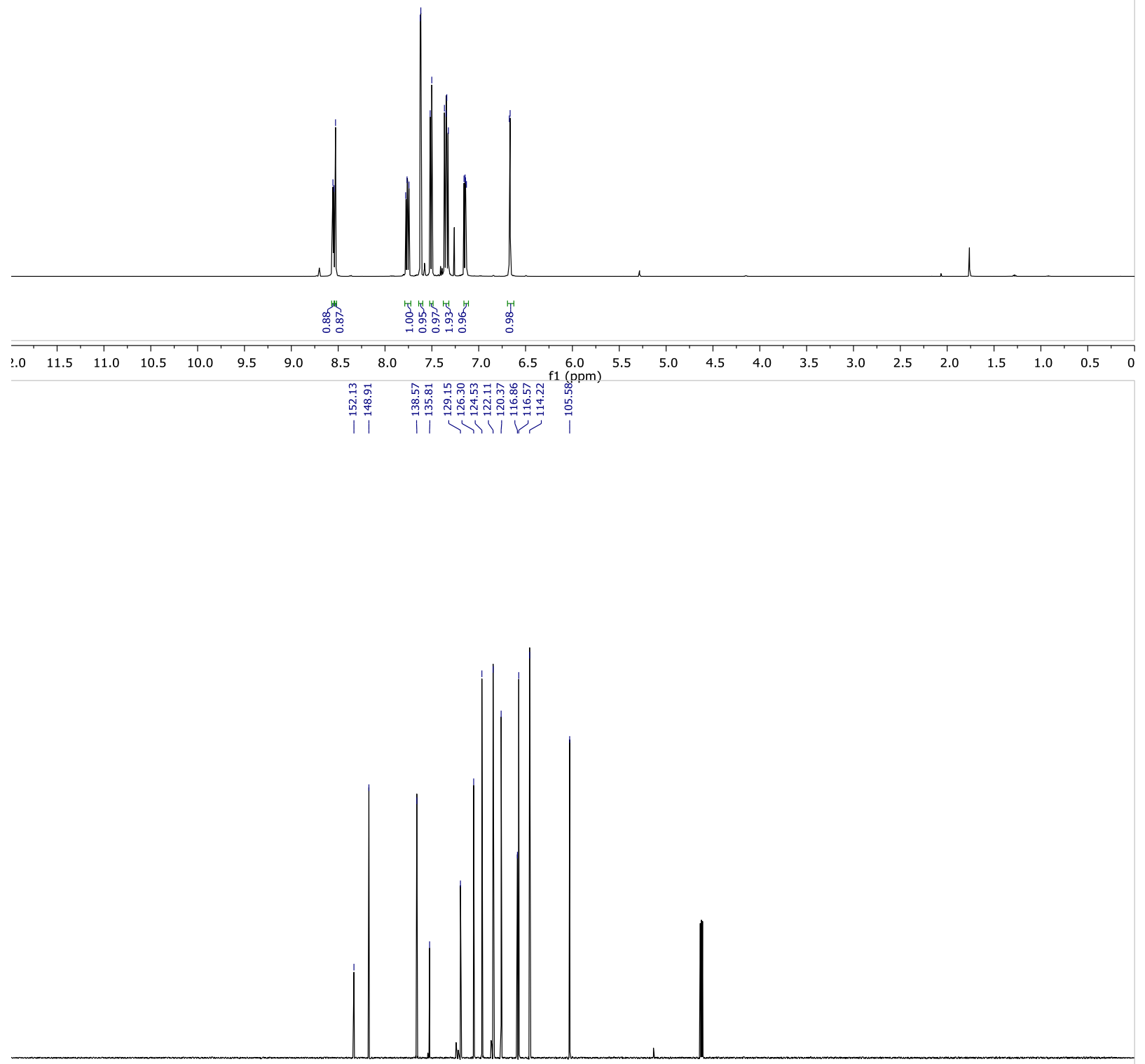

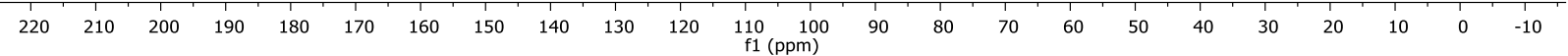



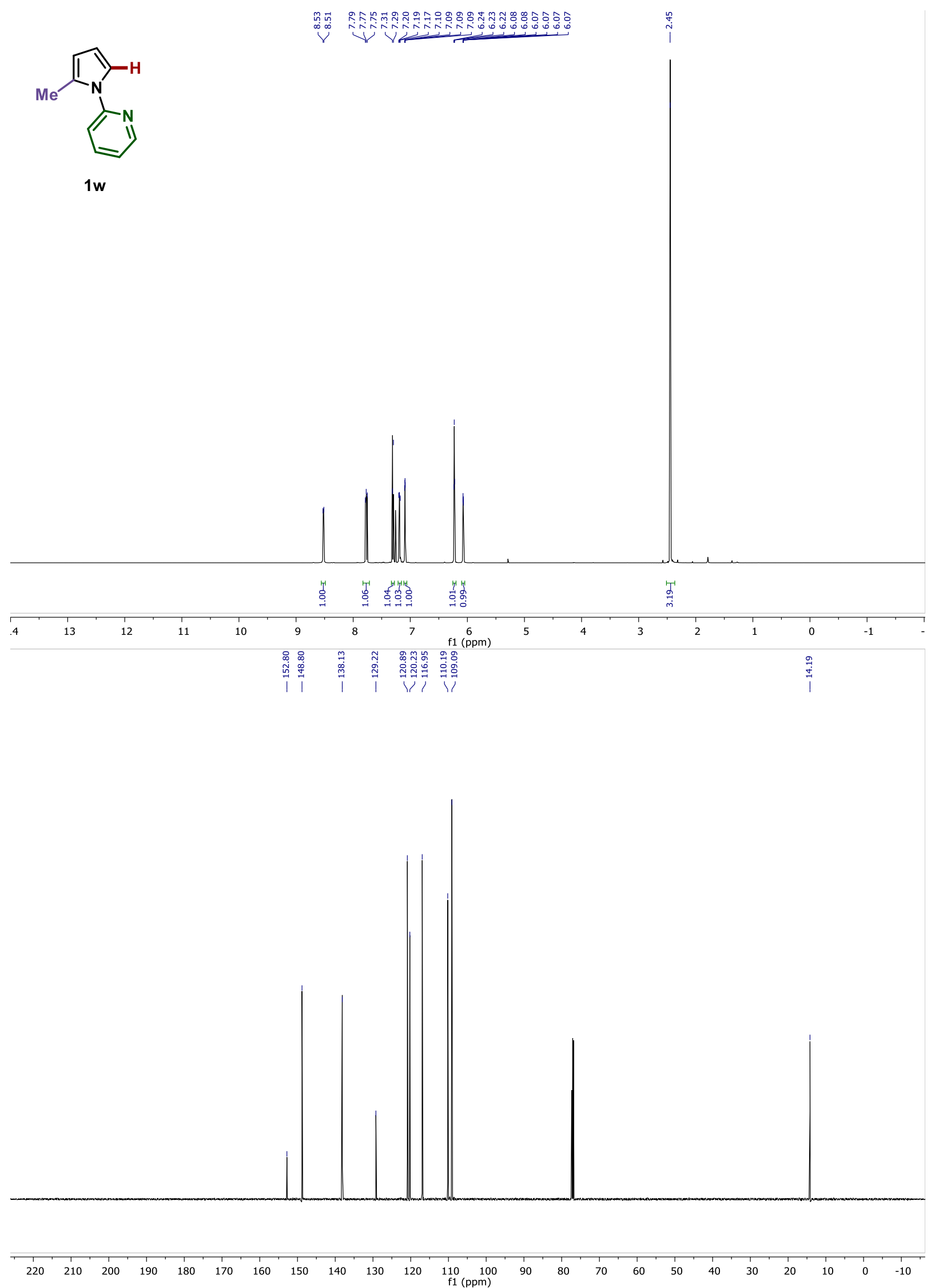

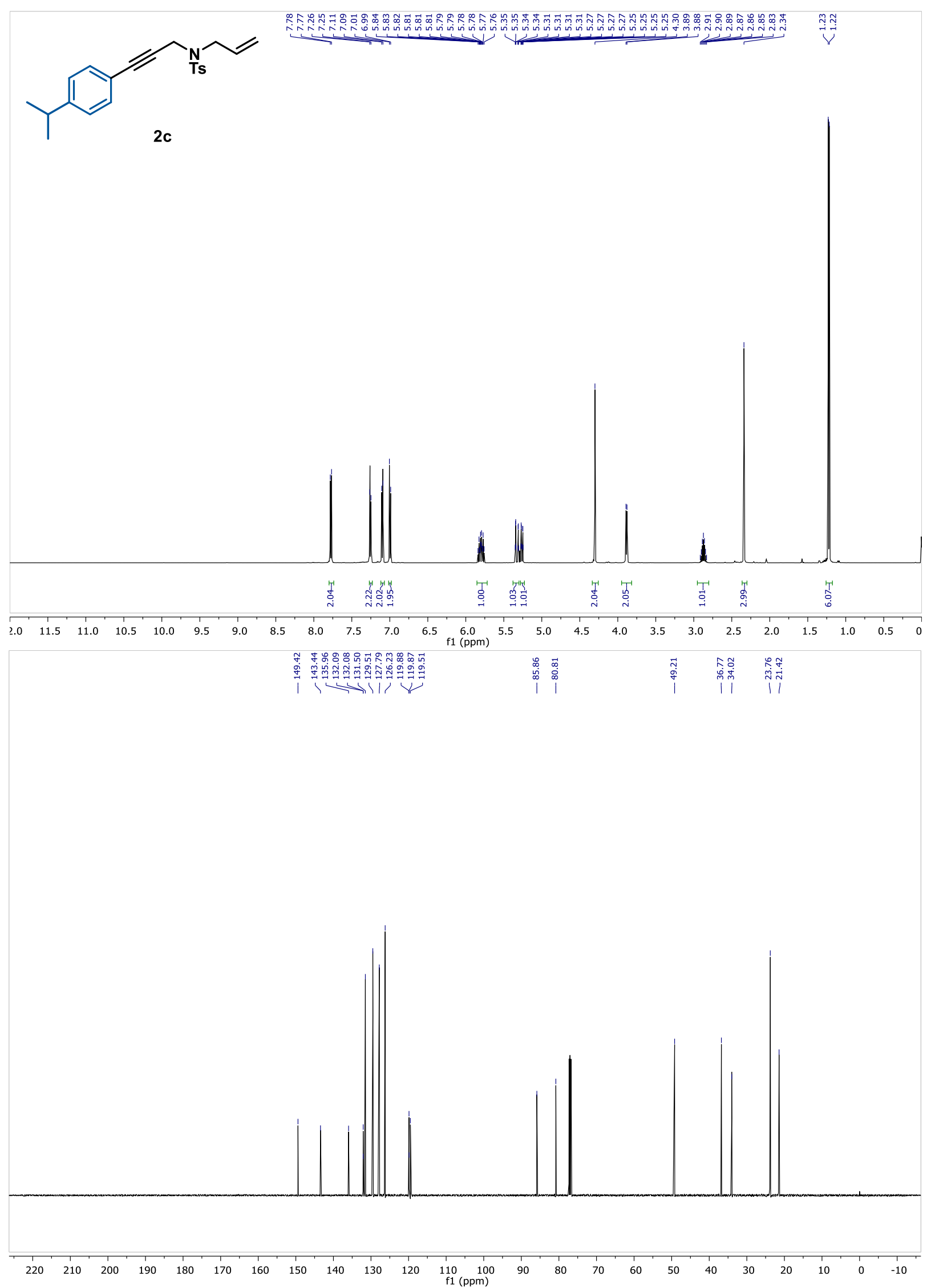

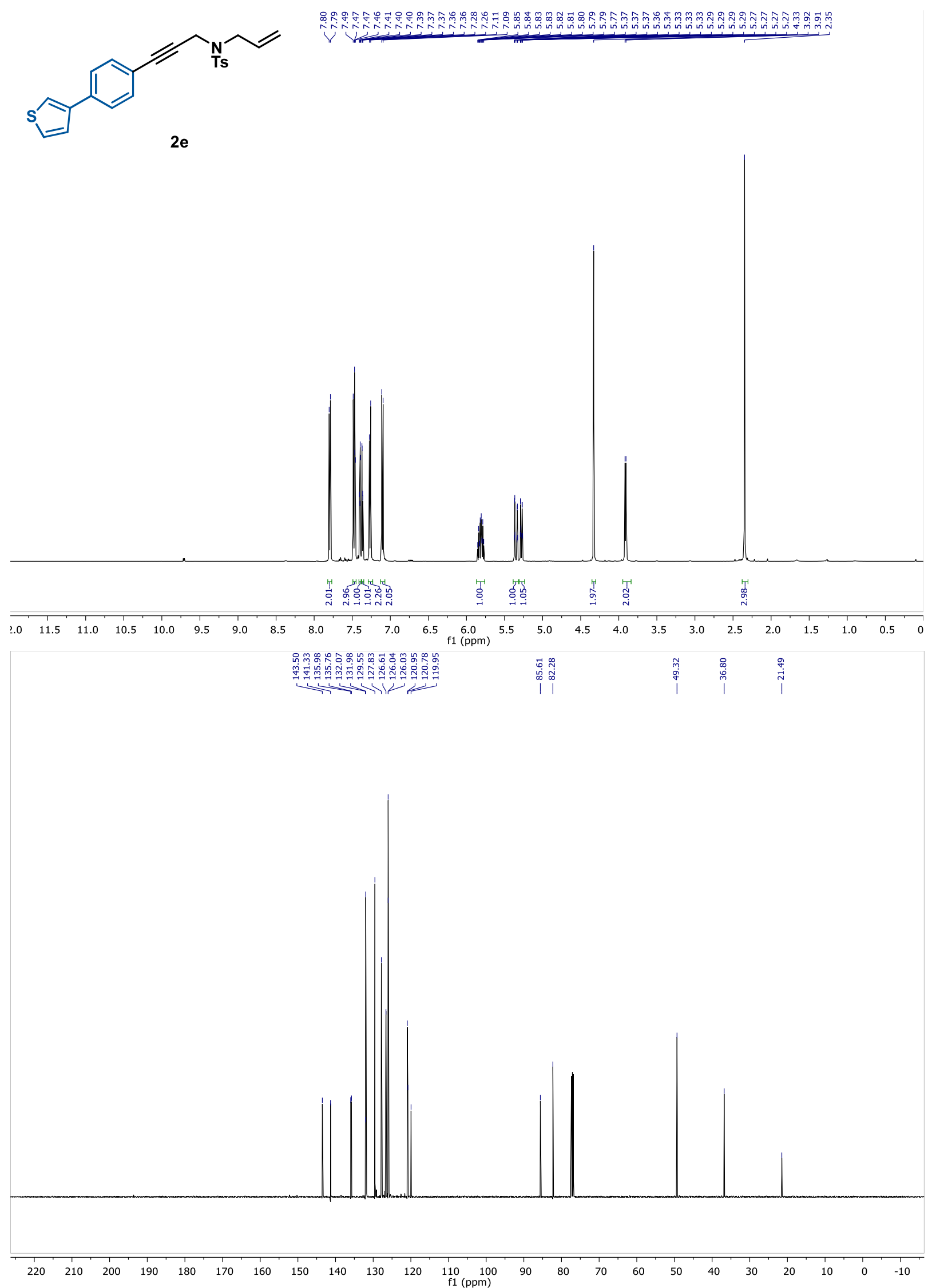

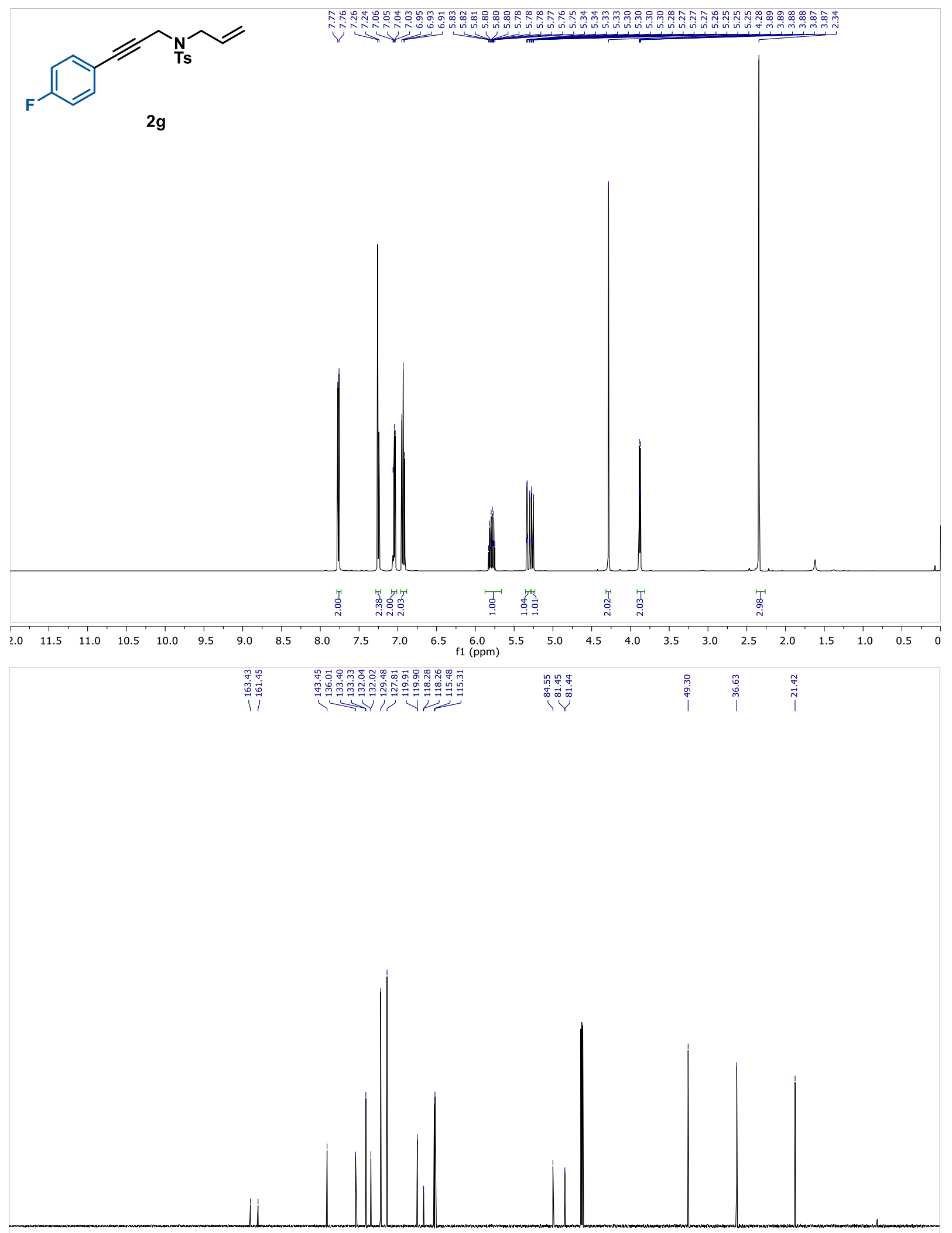

$\begin{array}{lllllllllllllllllllllllll} & 220 & 210 & 200 & 190 & 180 & 170 & 160 & 150 & 140 & 130 & 120 & 110 & 100 & 90 & 80 & 70 & 60 & 50 & 40 & 30 & 20 & 10 & 0 & -10\end{array}$ 


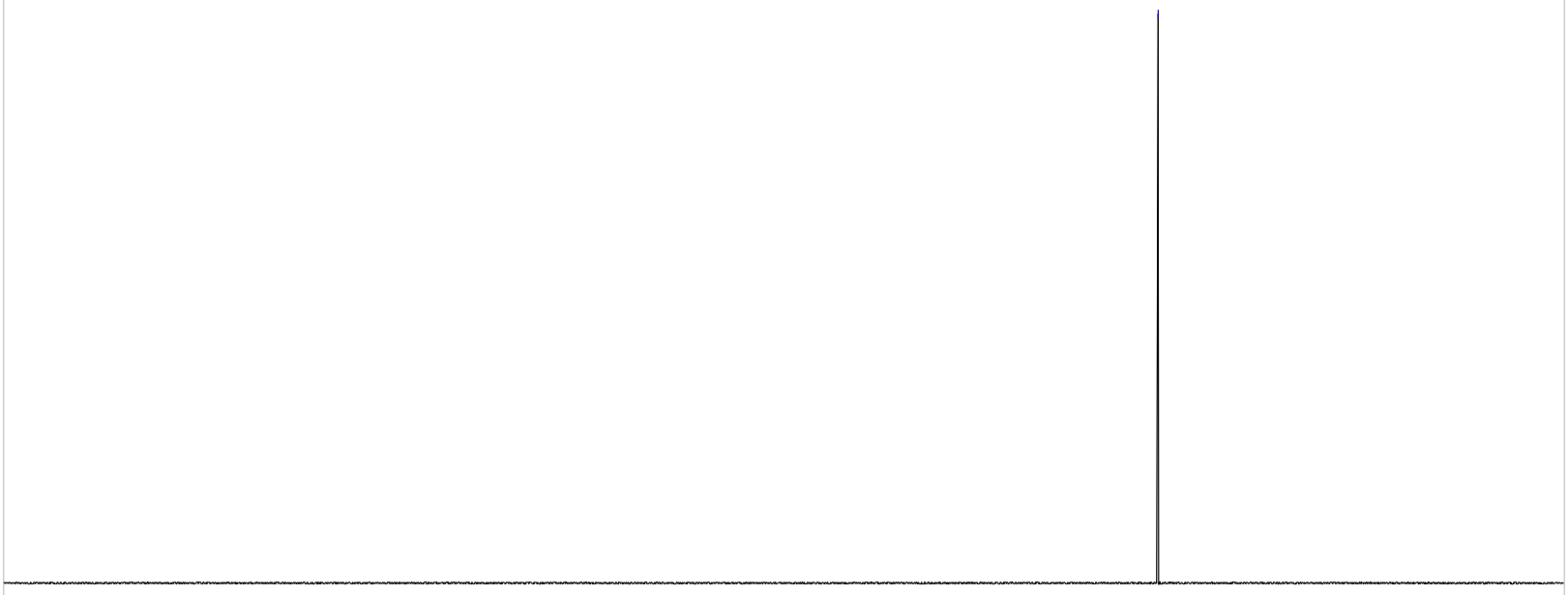

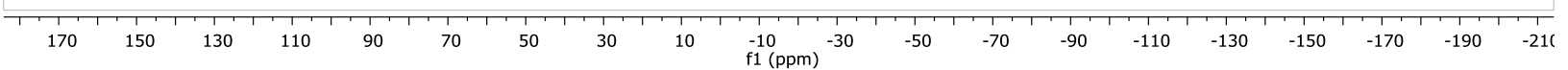



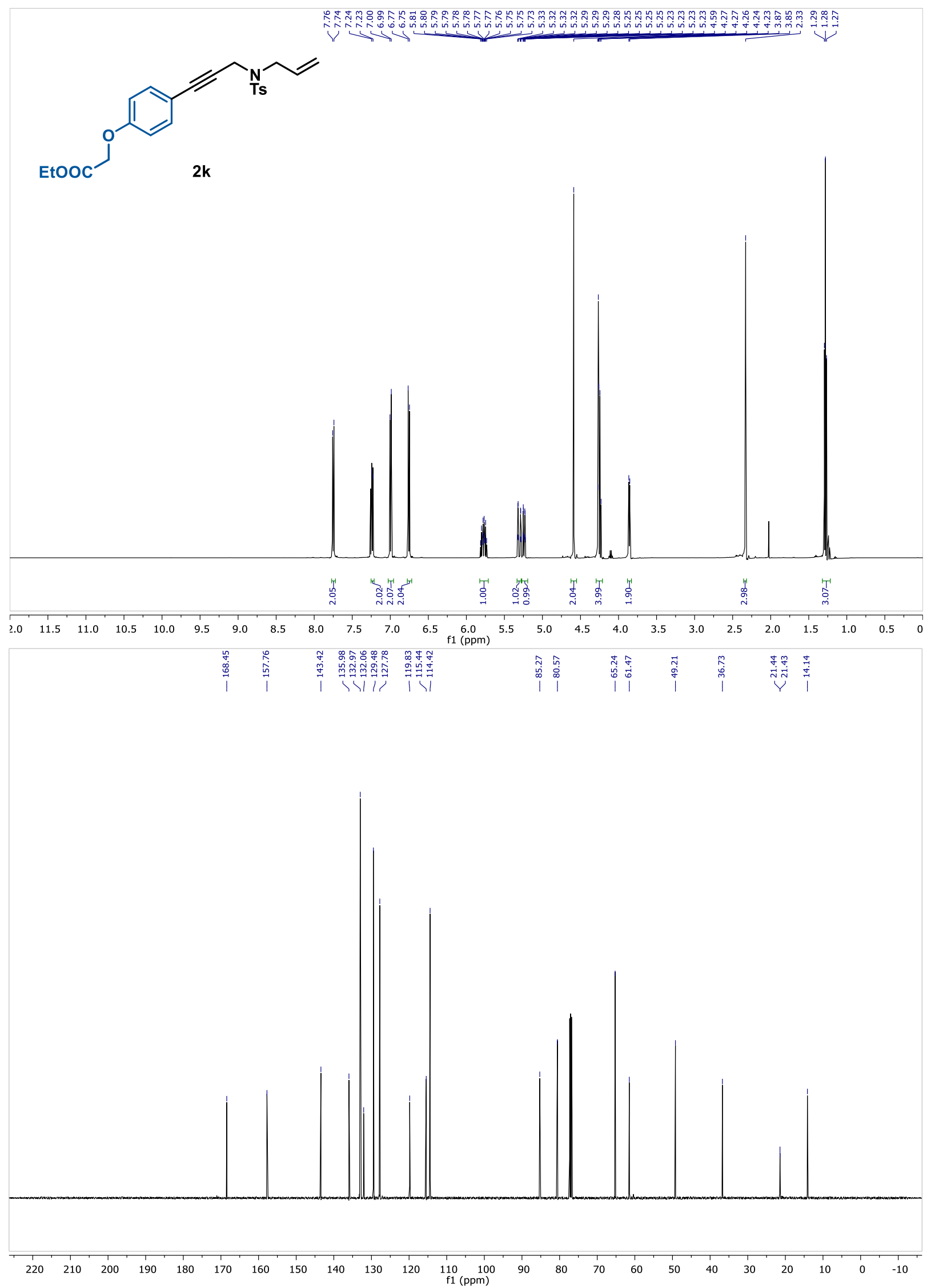

S121 

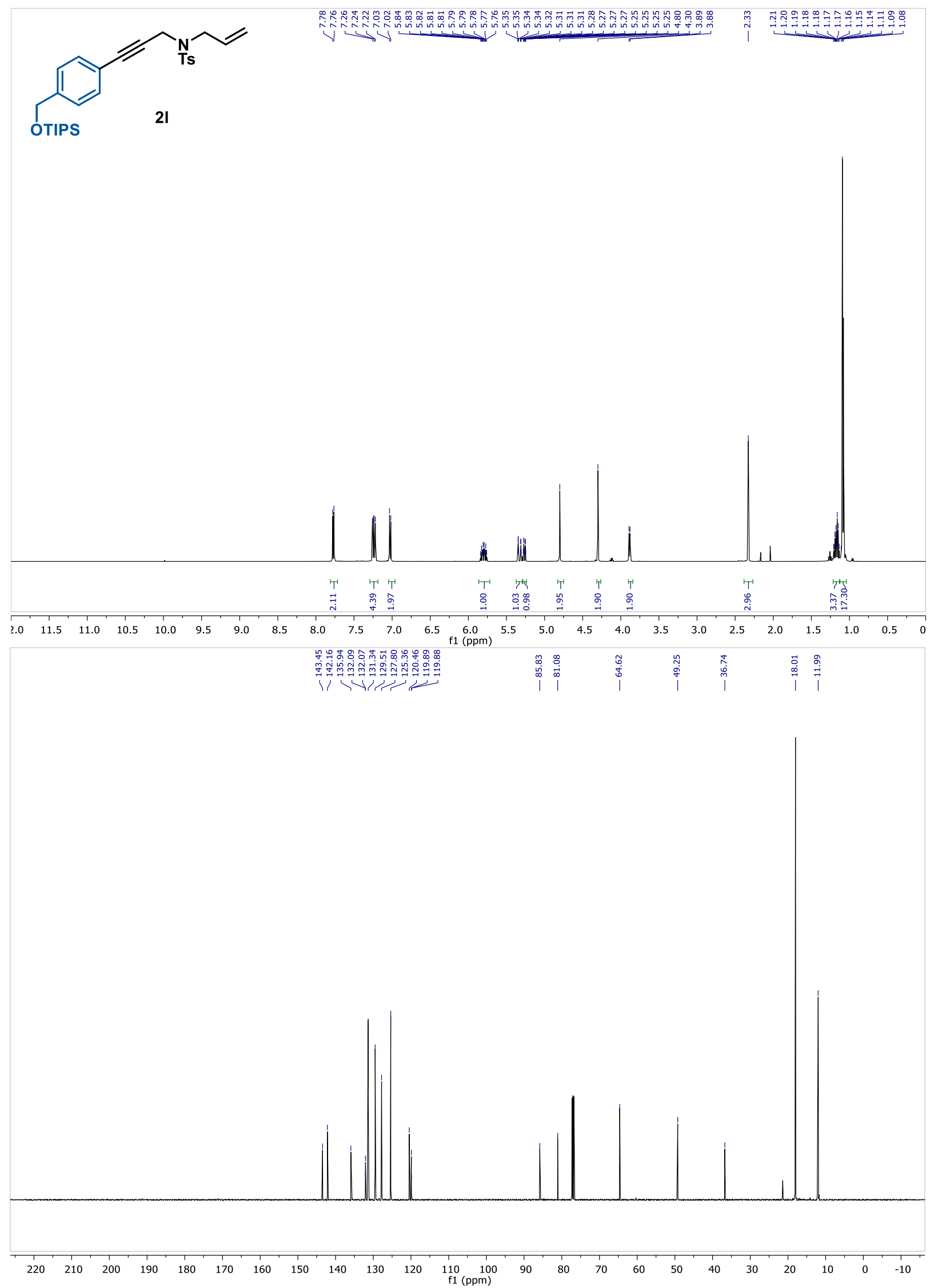


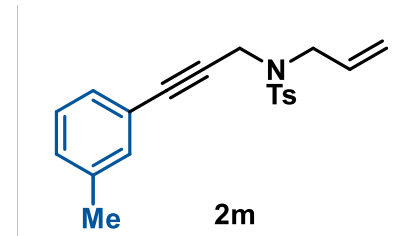

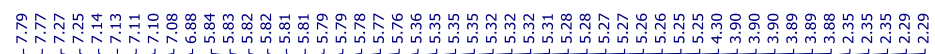
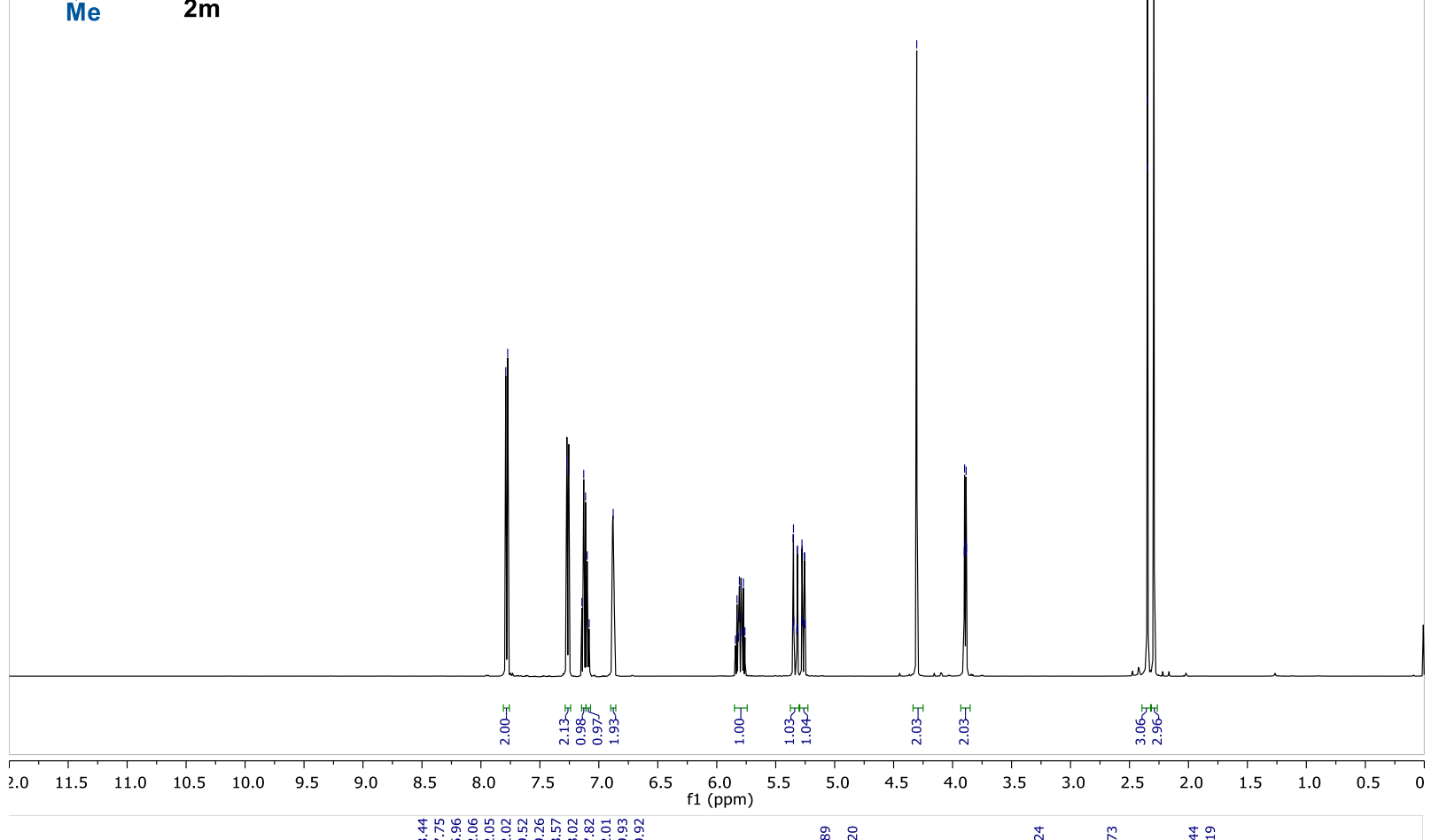

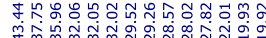

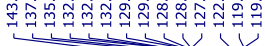

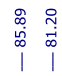

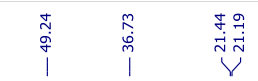

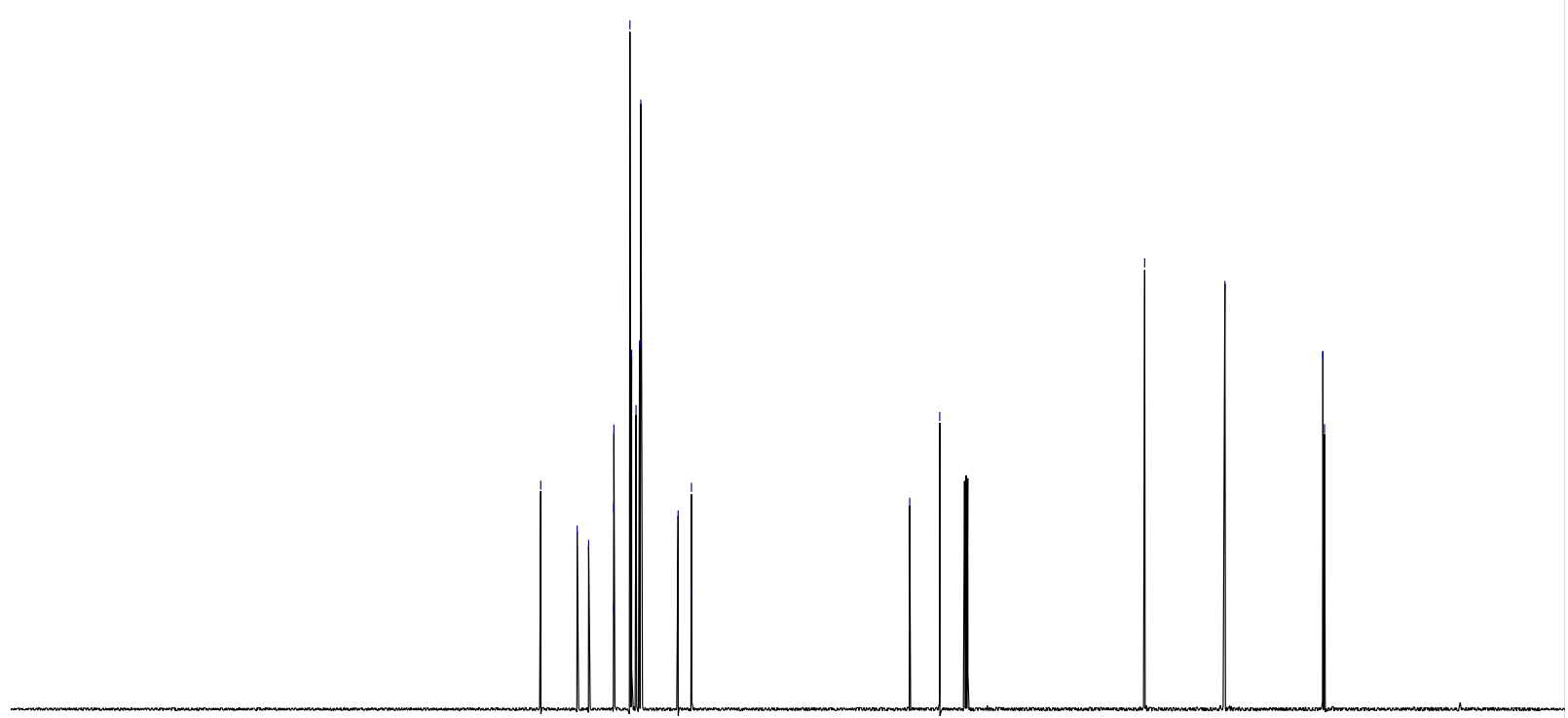

$\begin{array}{llllllllllll}220 & 210 & 200 & 190 & 180 & 170 & 160 & 150 & 140 & 130 & 120 & \begin{array}{l}110 \\ \mathrm{f} 1(\mathrm{ppm})\end{array}\end{array}$ 

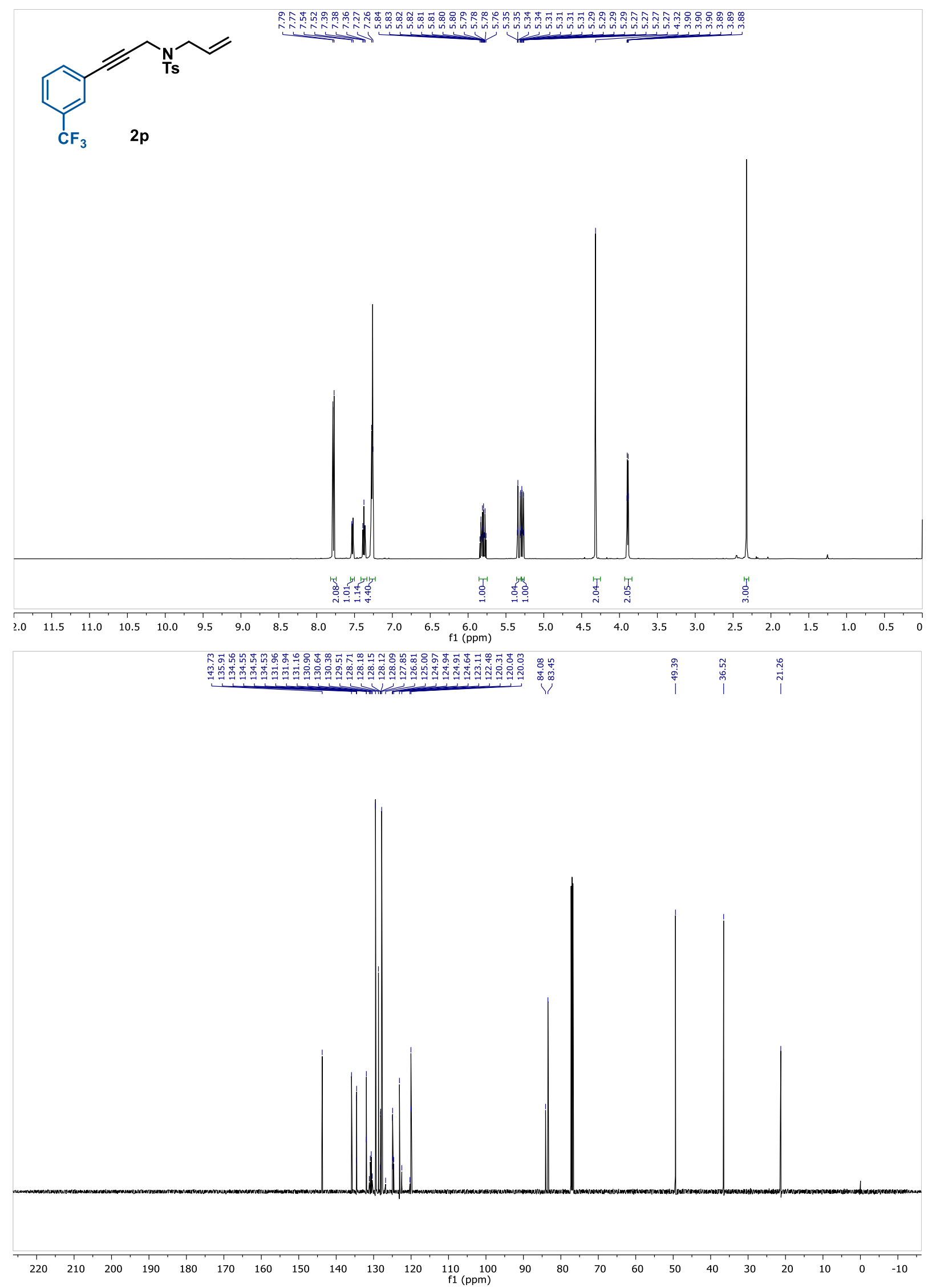


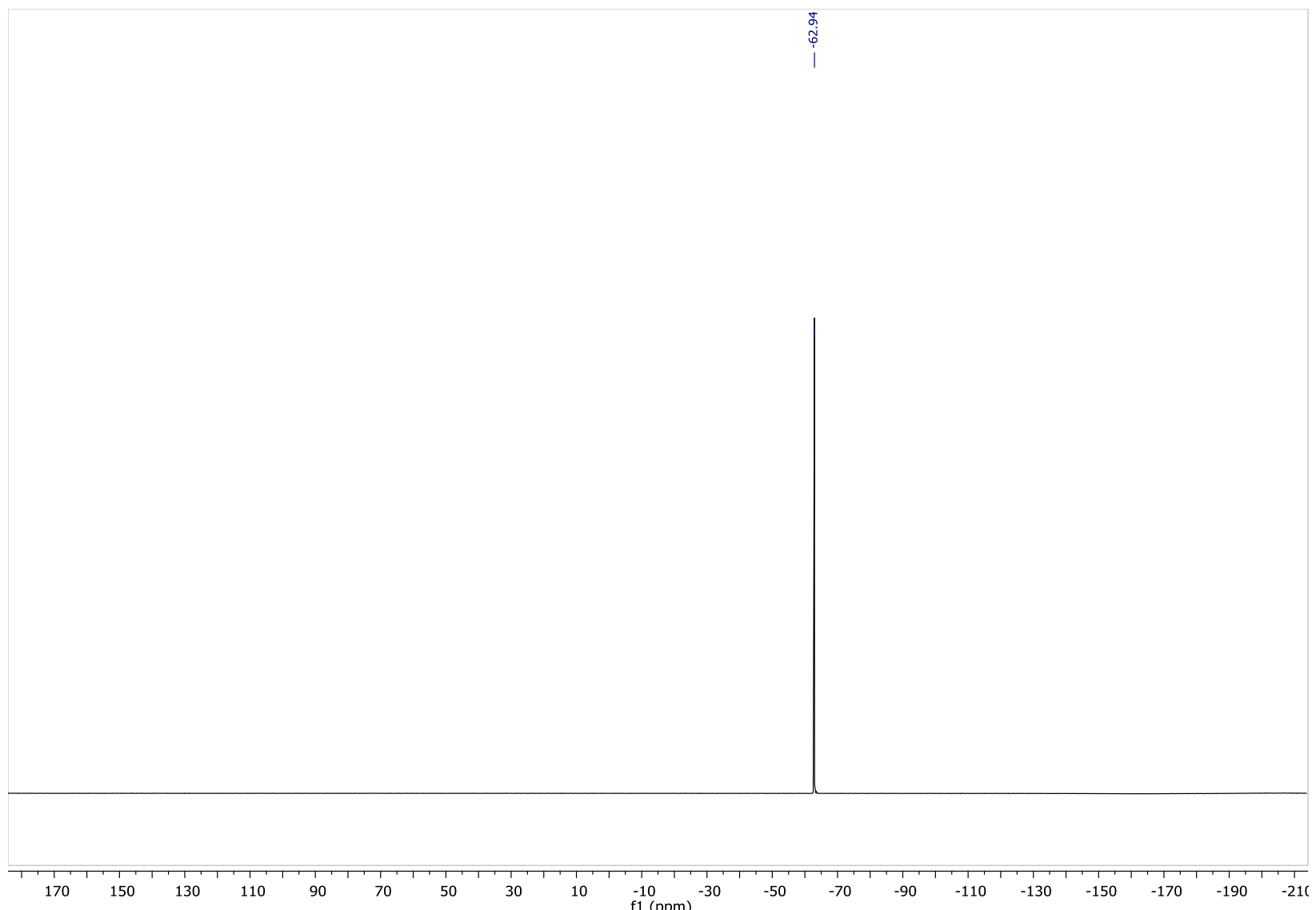



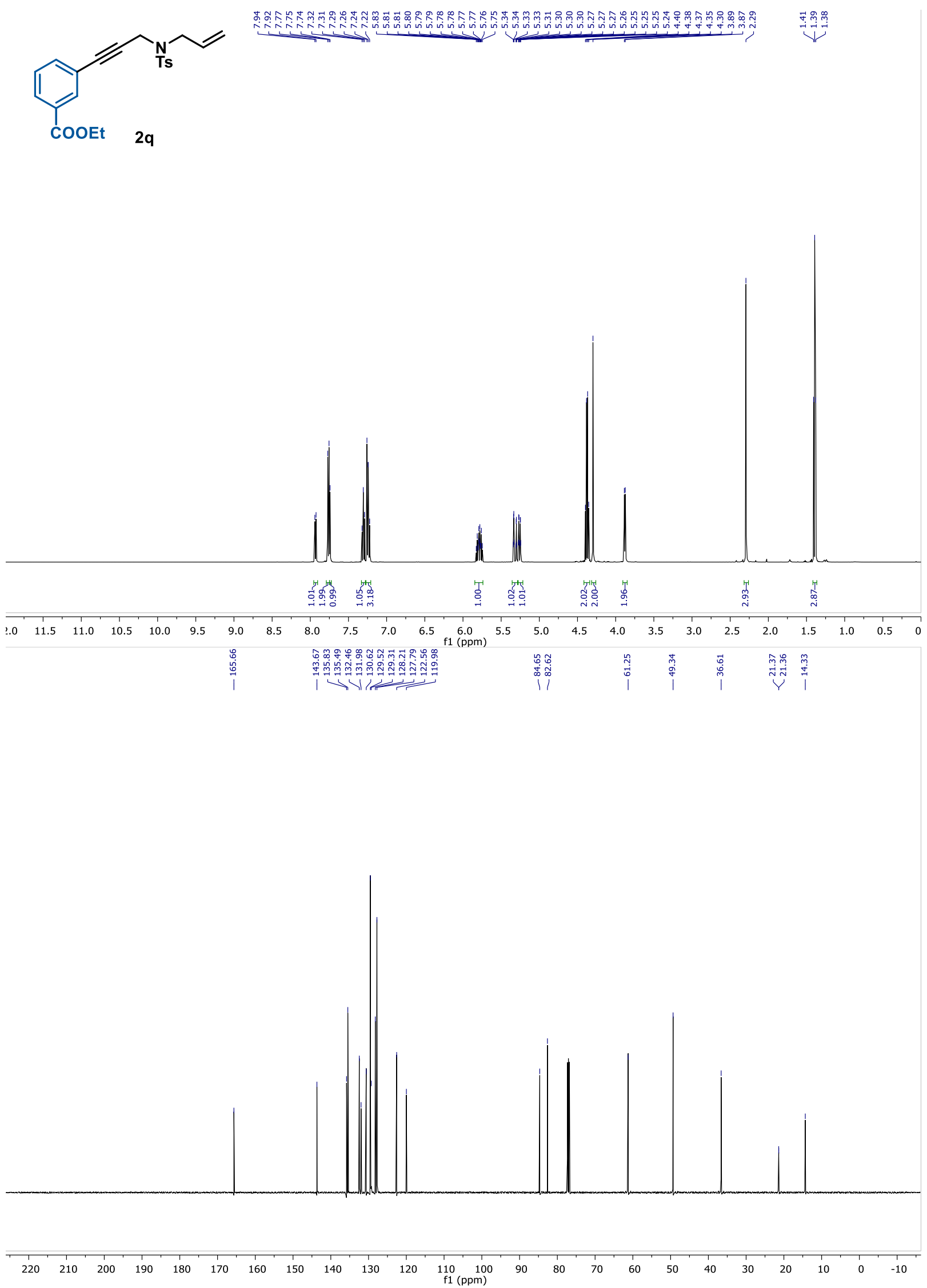

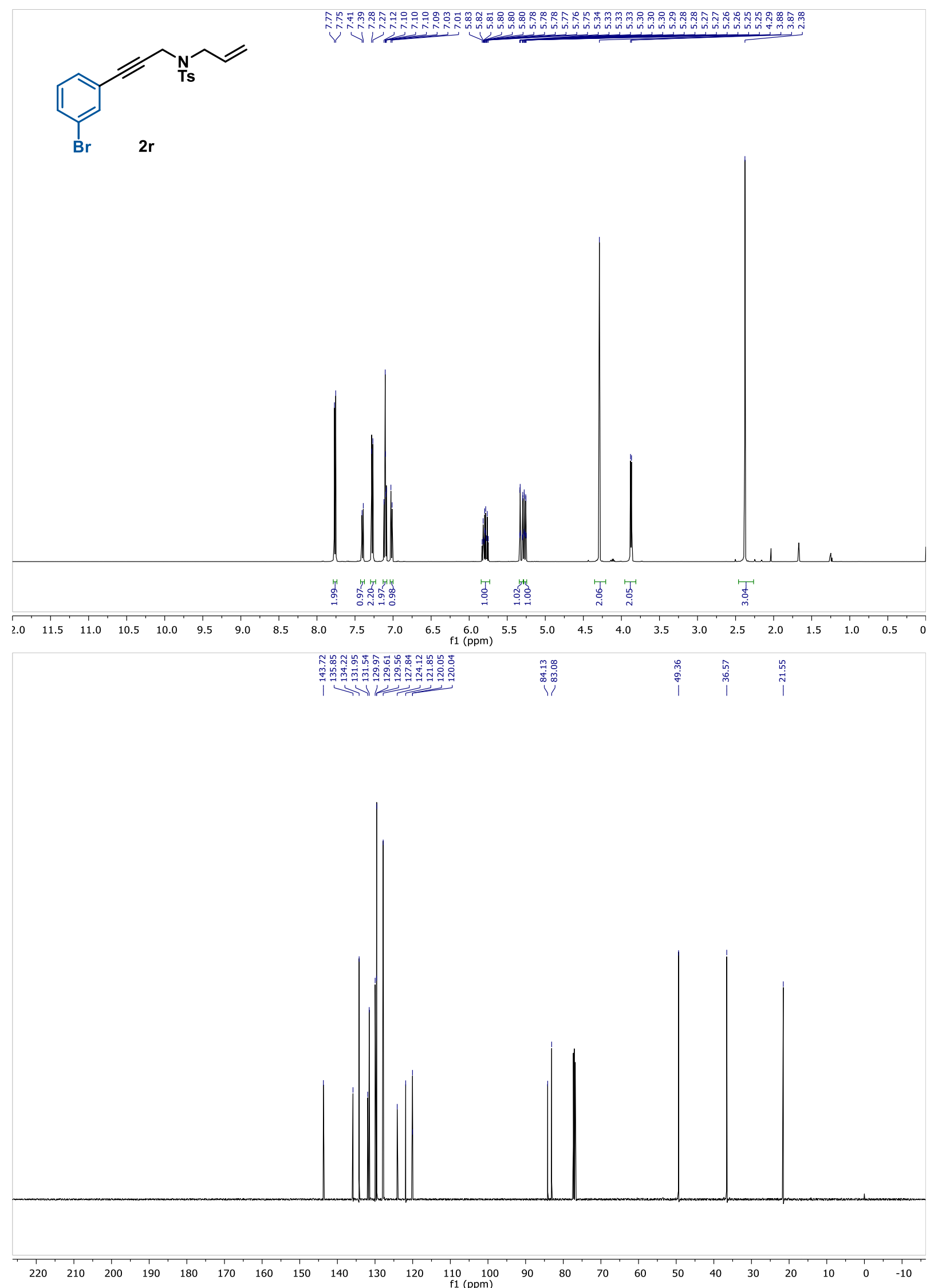

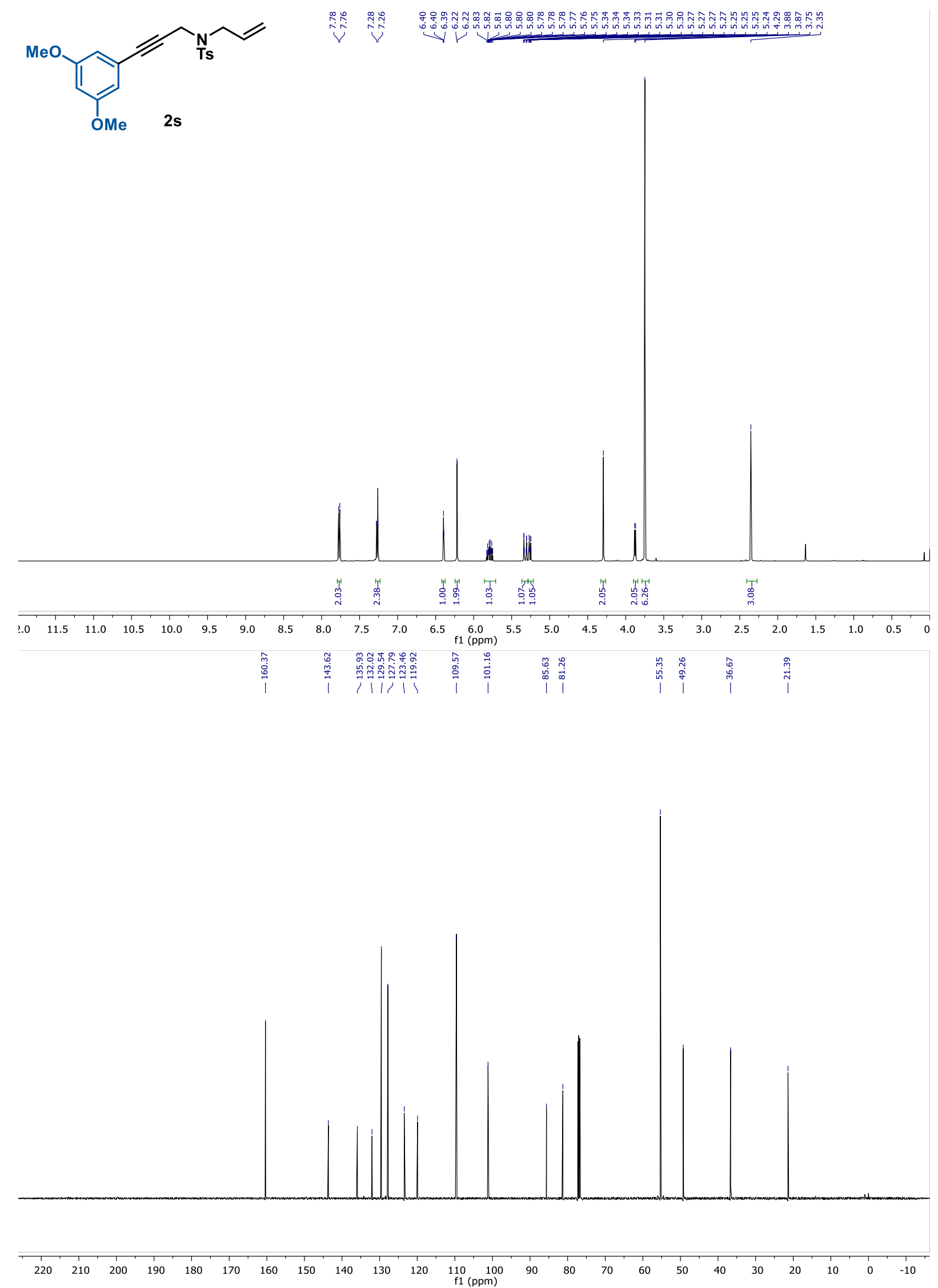

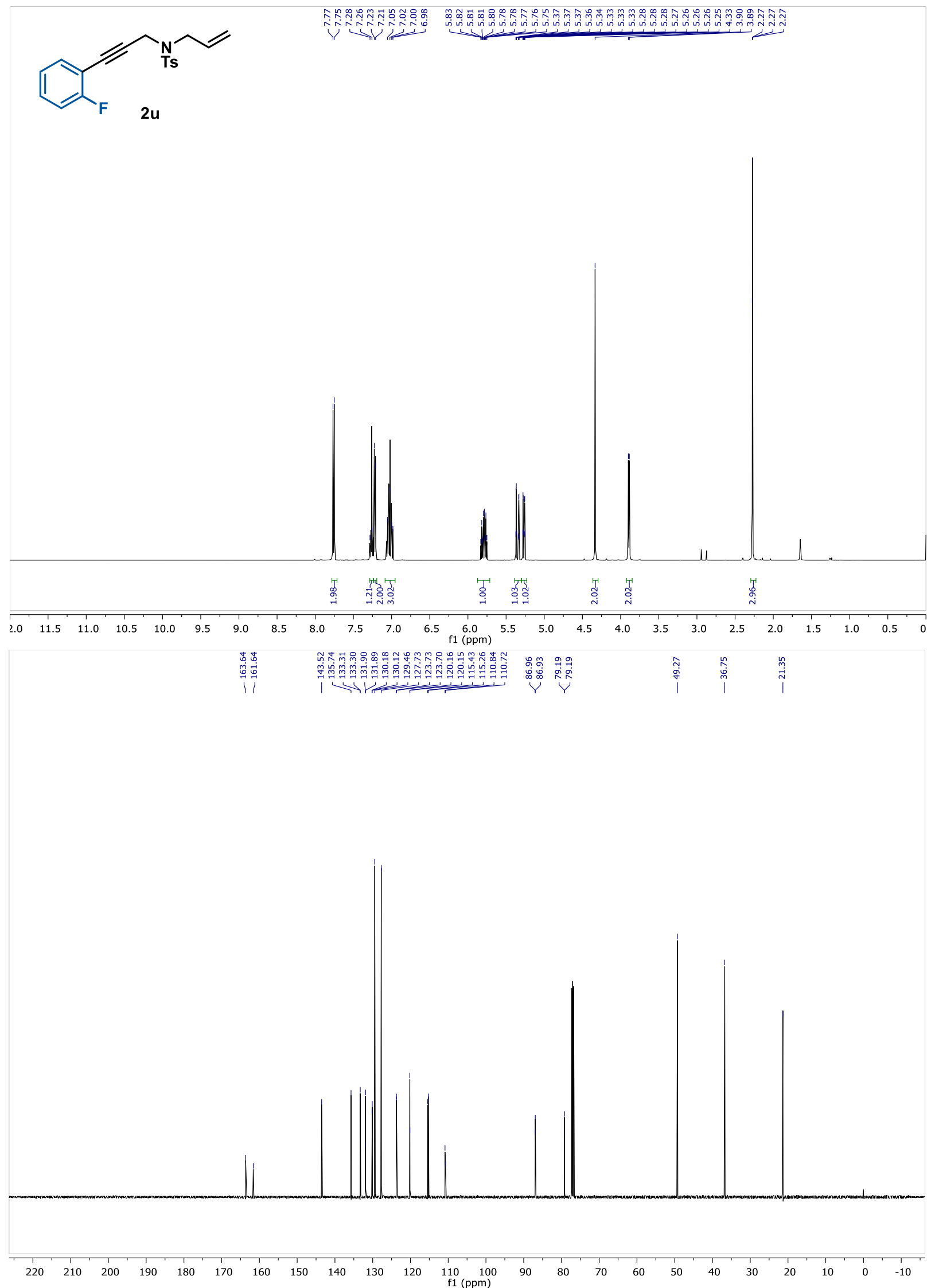


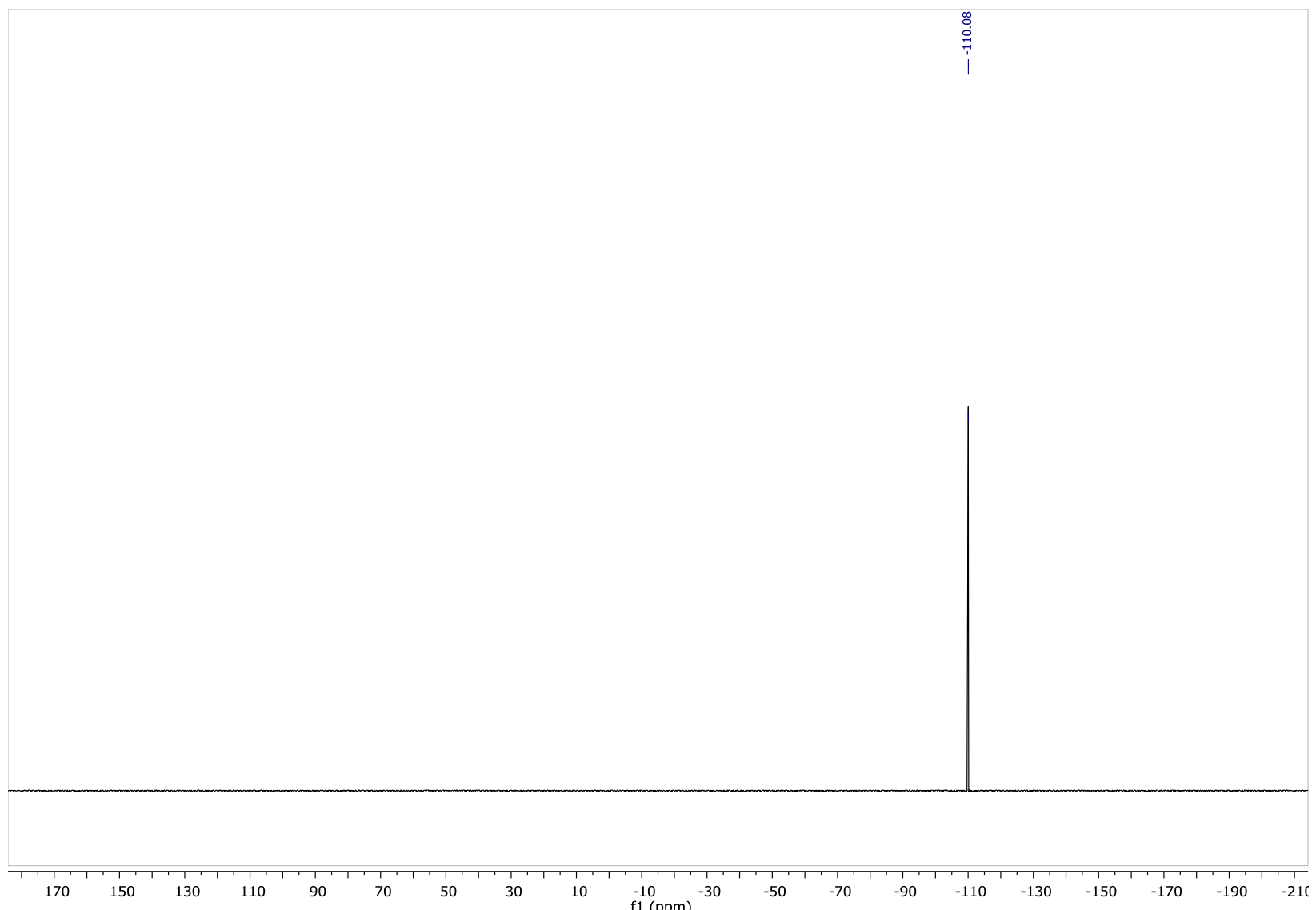



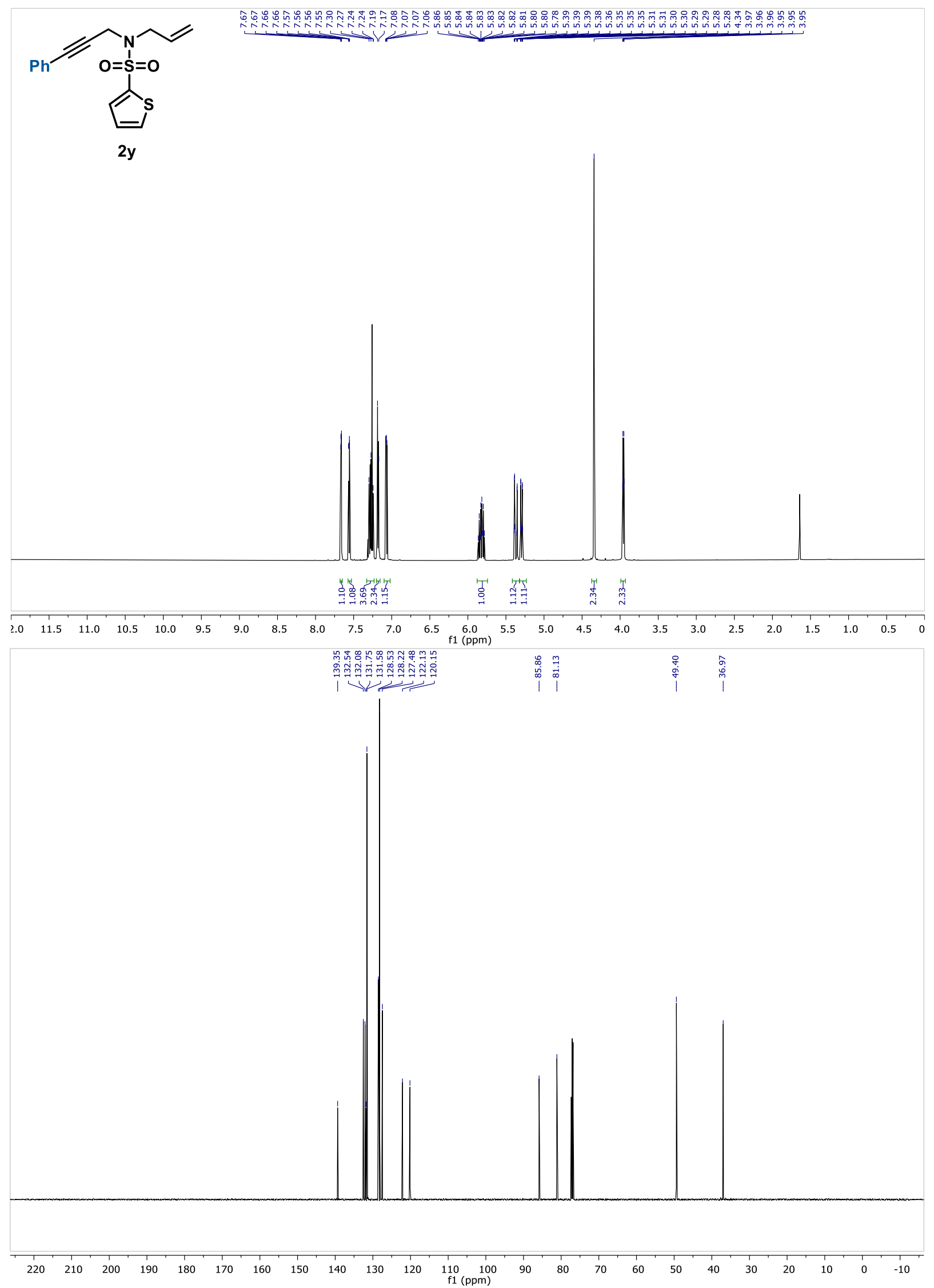

S131 

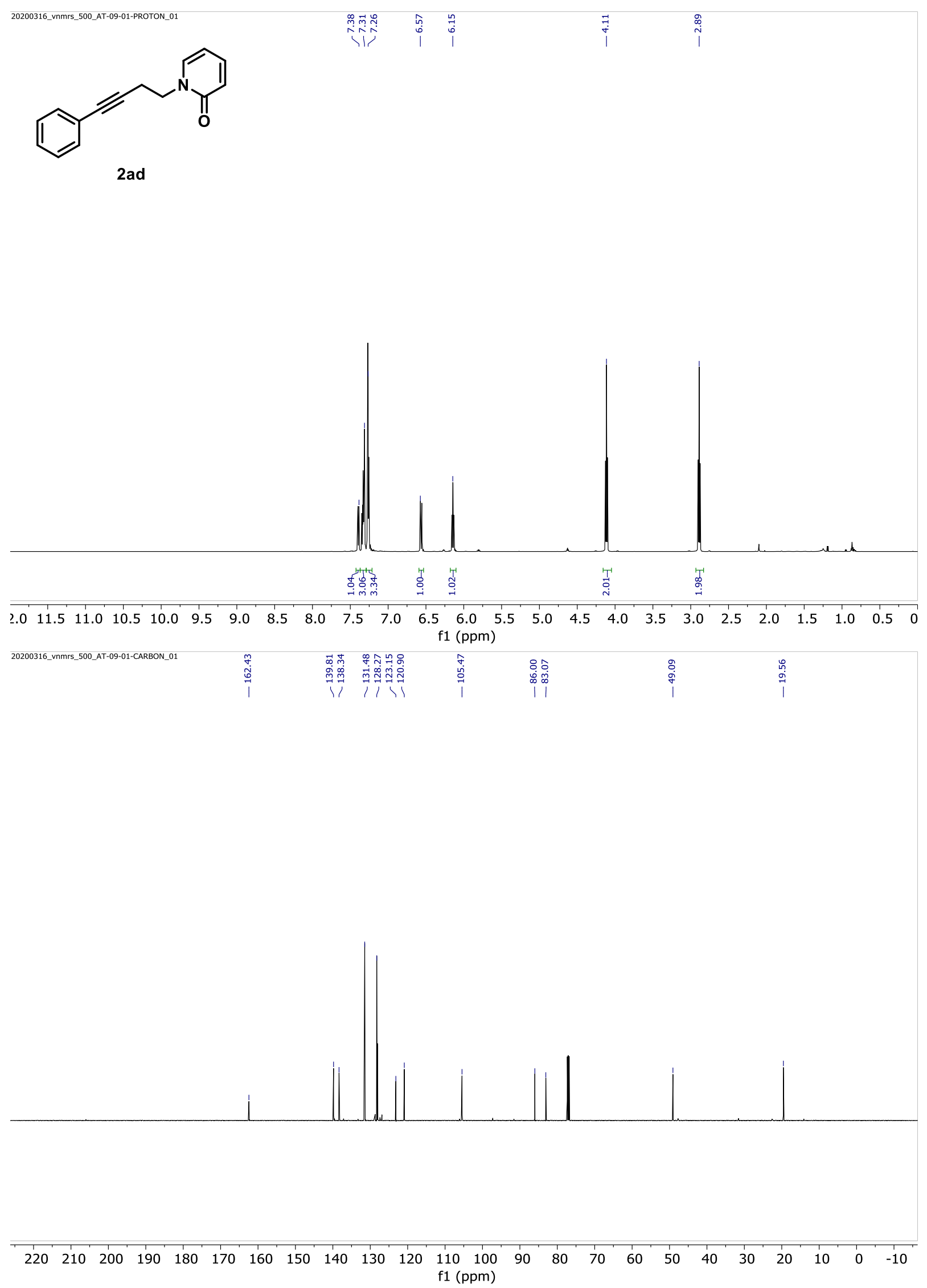

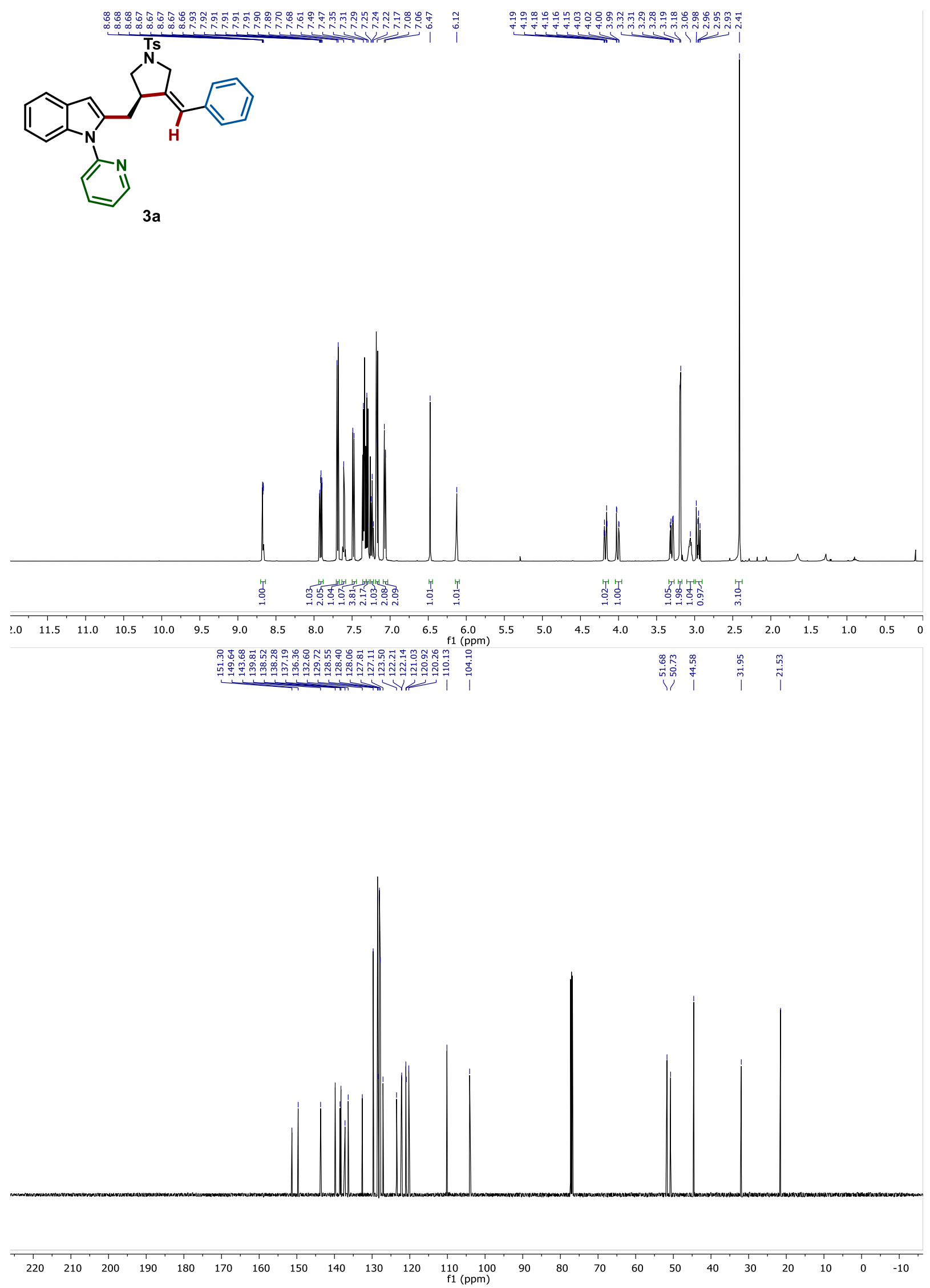

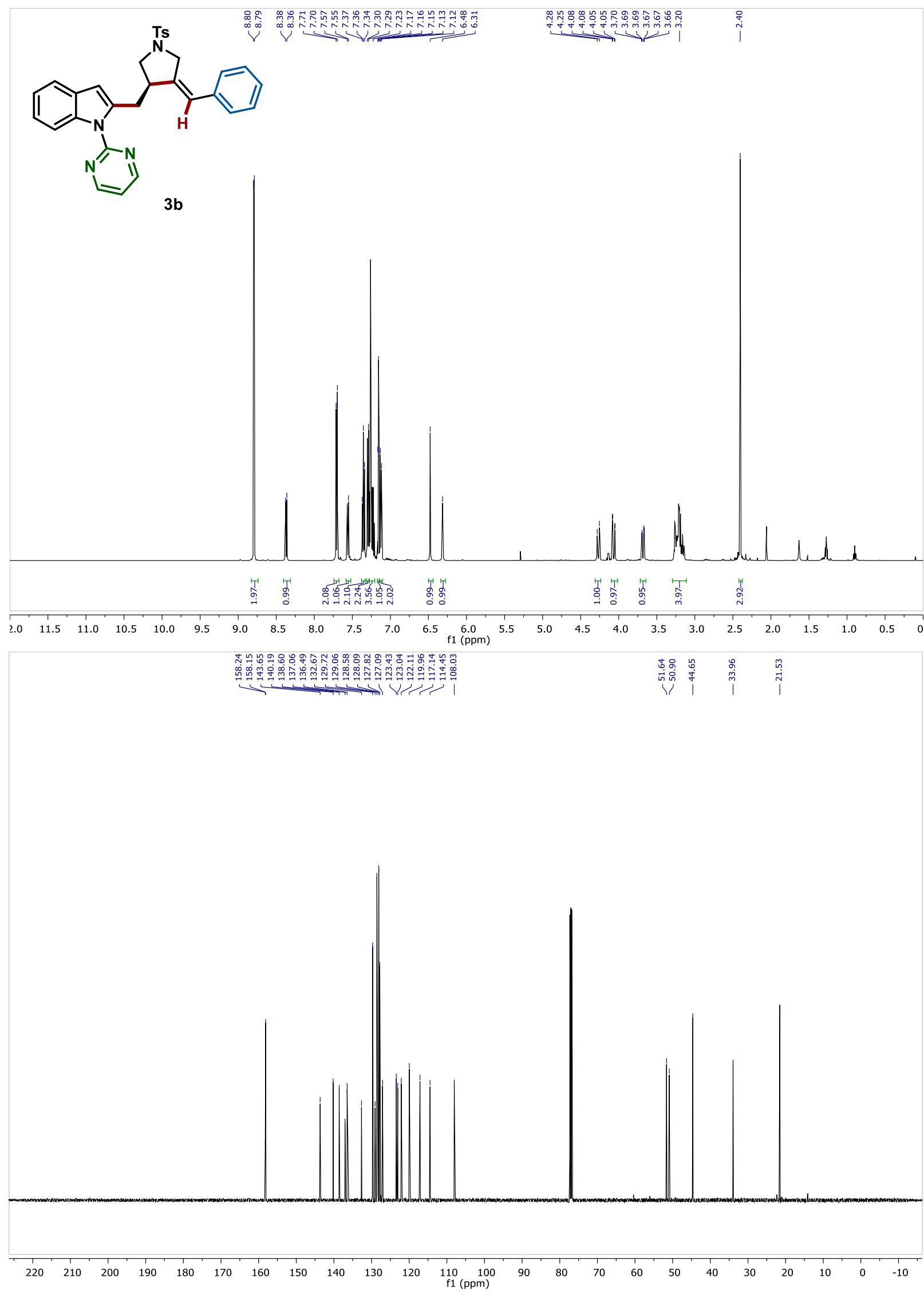


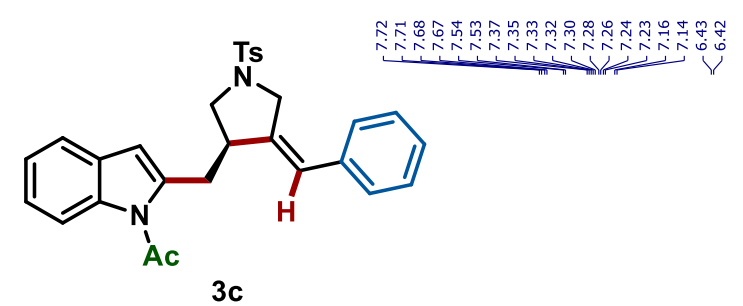

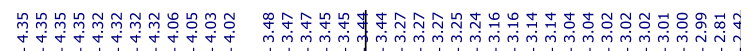
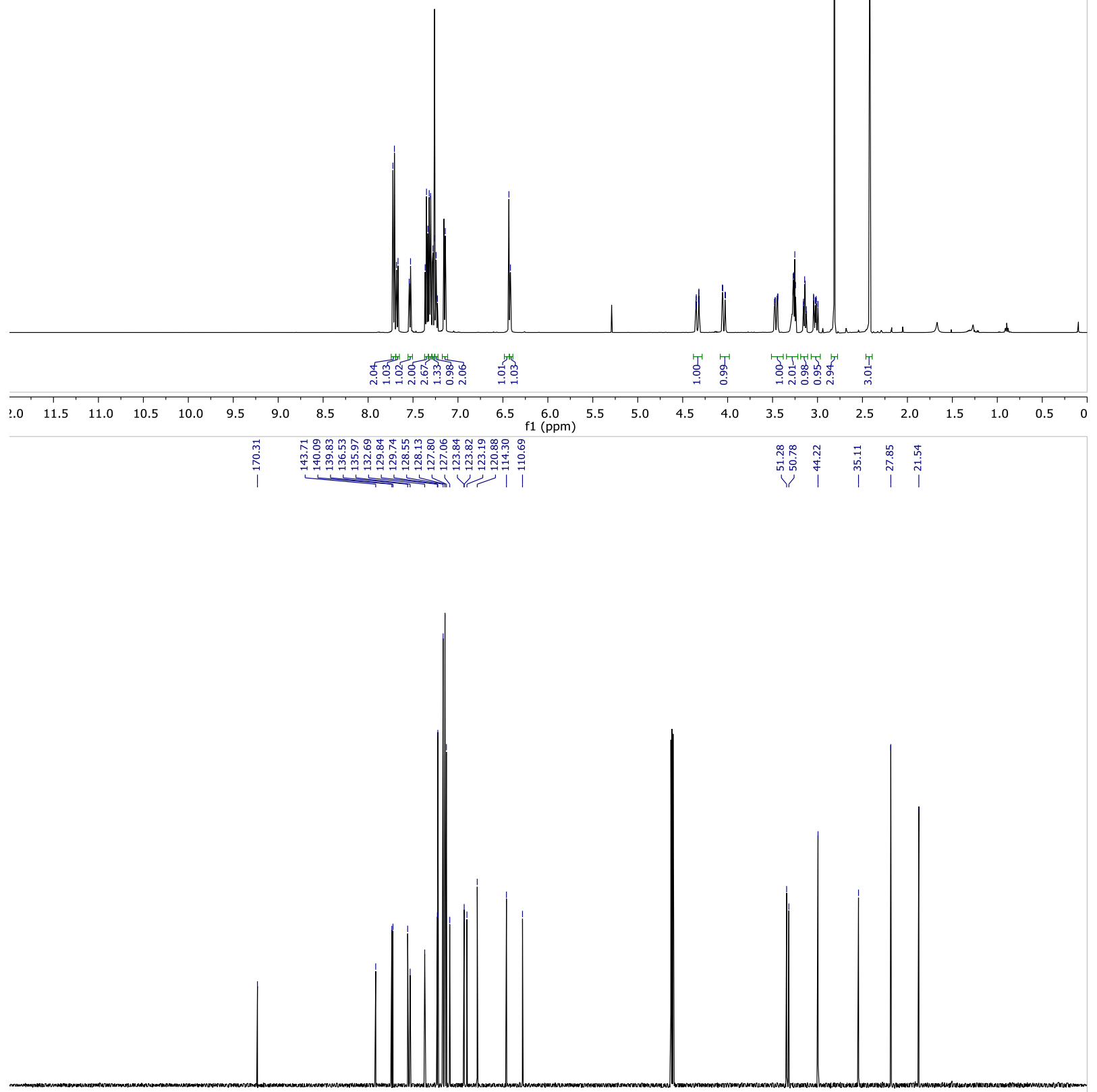

$\begin{array}{lllllllllllll}220 & 210 & 200 & 190 & 180 & 170 & 160 & 150 & 140 & 130 & 120 & 110 & 100 \\ \mathrm{f} 1(\mathrm{ppm})\end{array}$ 

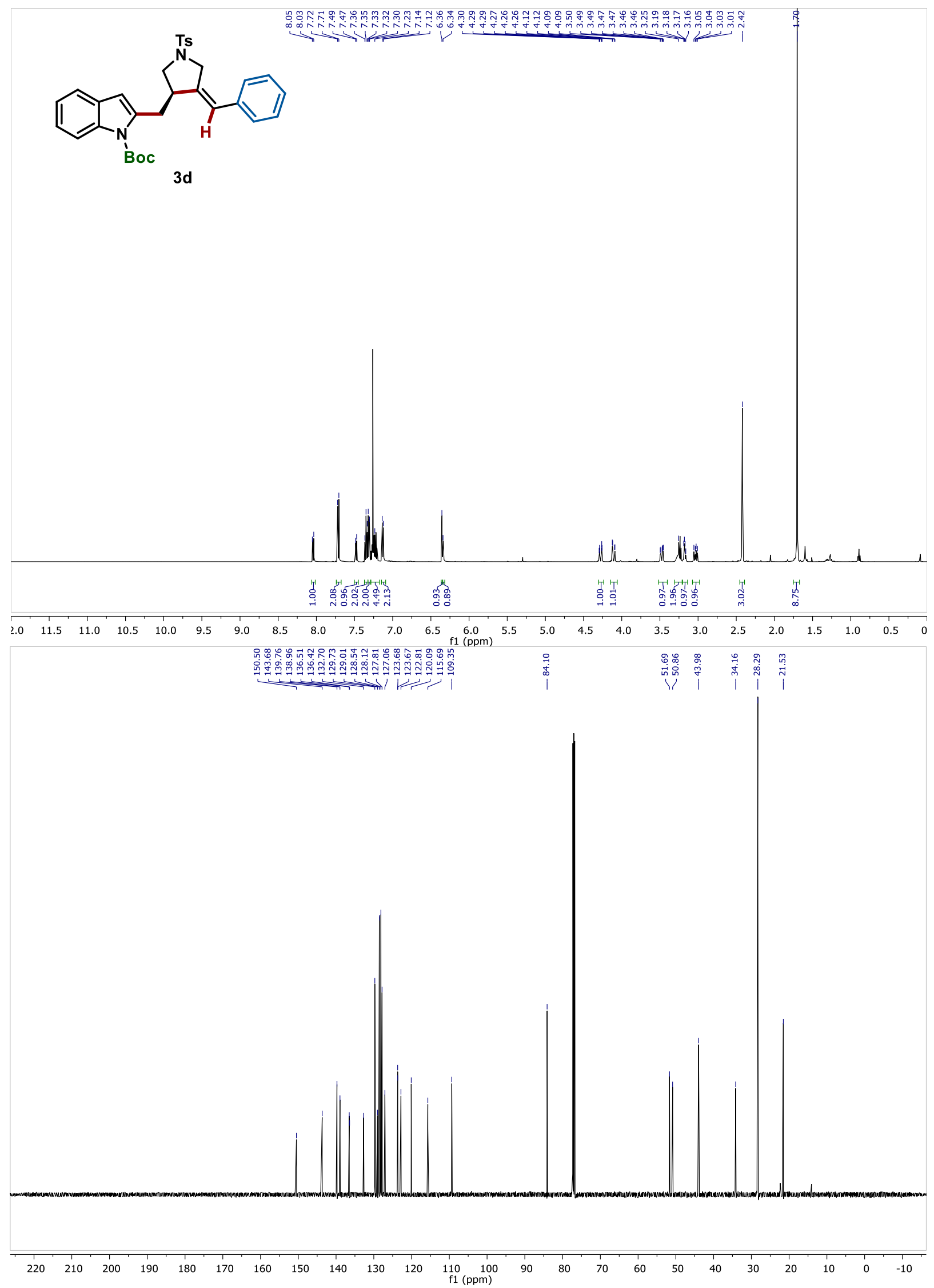

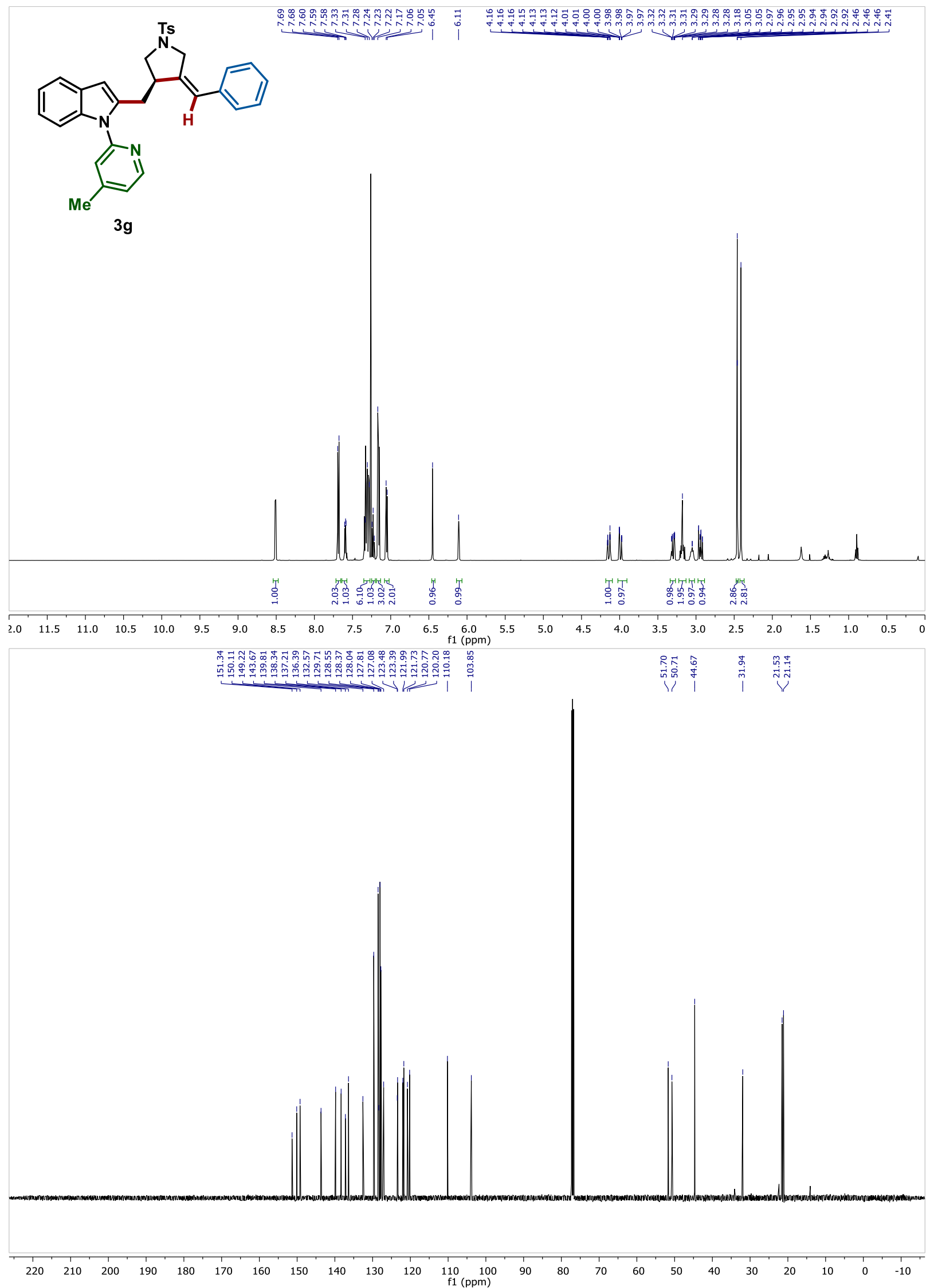

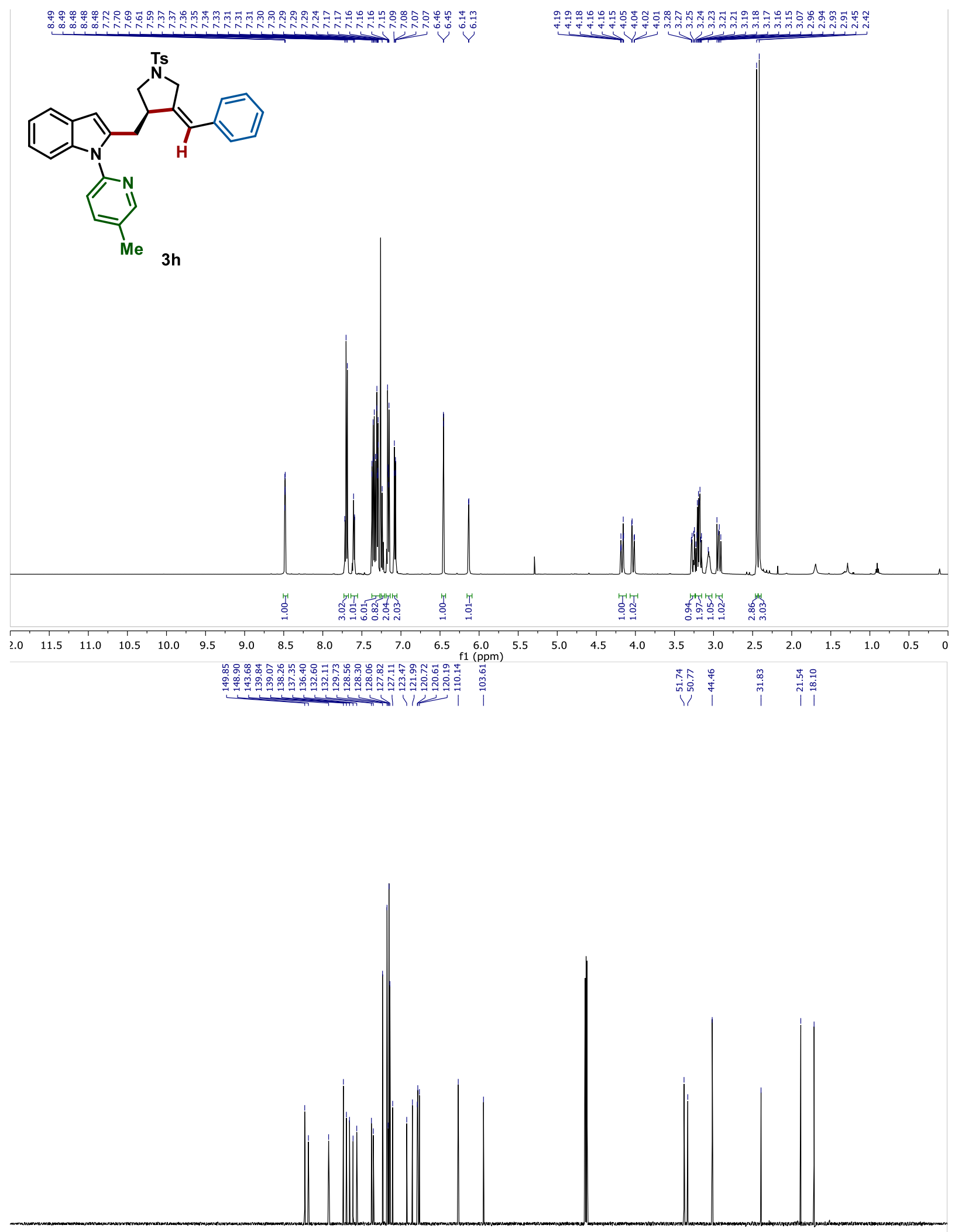

$\begin{array}{llllllllllllllllllllllllll}220 & 210 & 200 & 190 & 180 & 170 & 160 & 150 & 140 & 130 & 120 & \begin{array}{c}110 \\ \mathrm{f} 1(\mathrm{ppm})\end{array} & 90 & 80 & 70 & 60 & 50 & 40 & 30 & 20 & 10 & 0 & -10\end{array}$ 

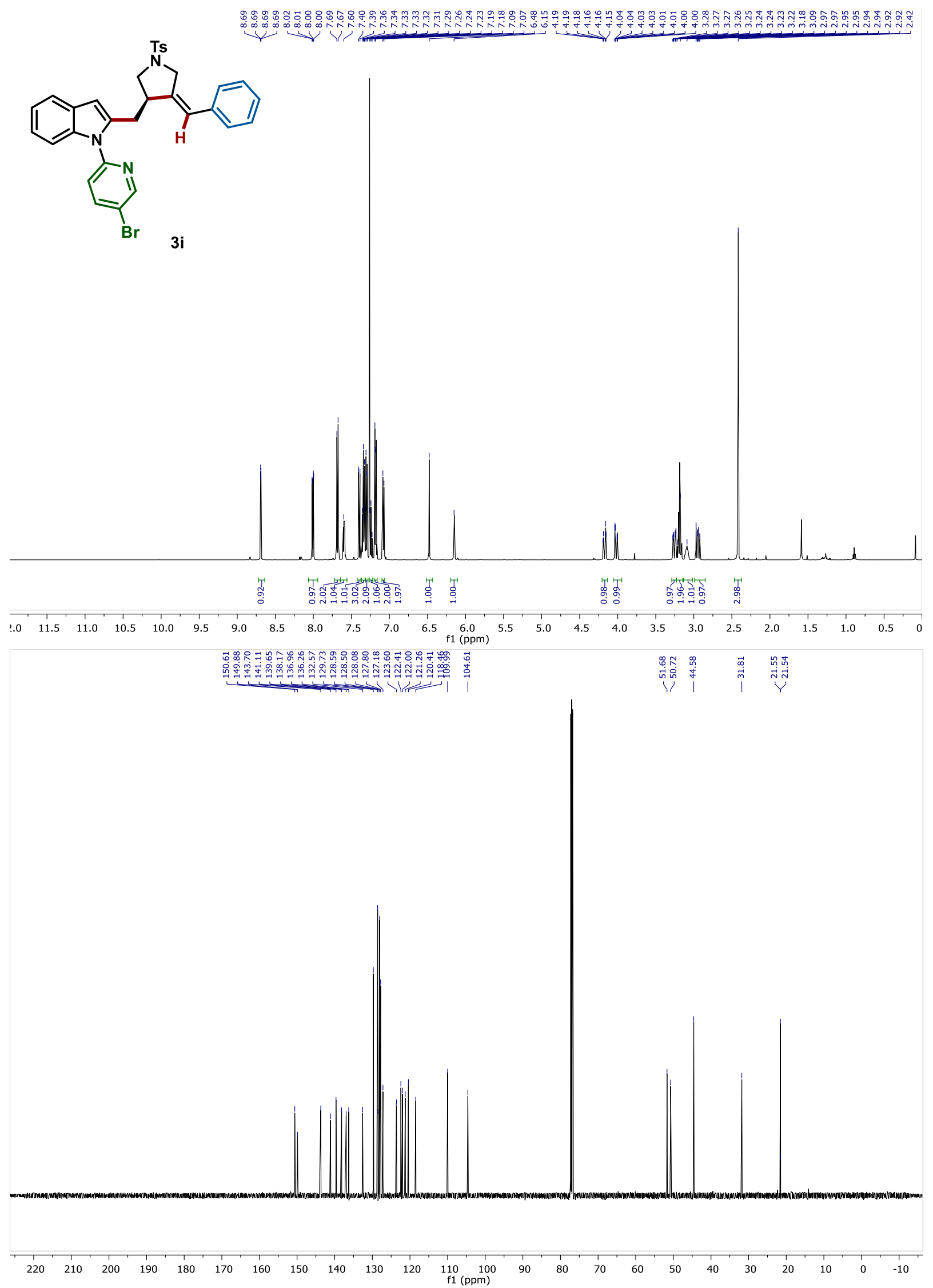

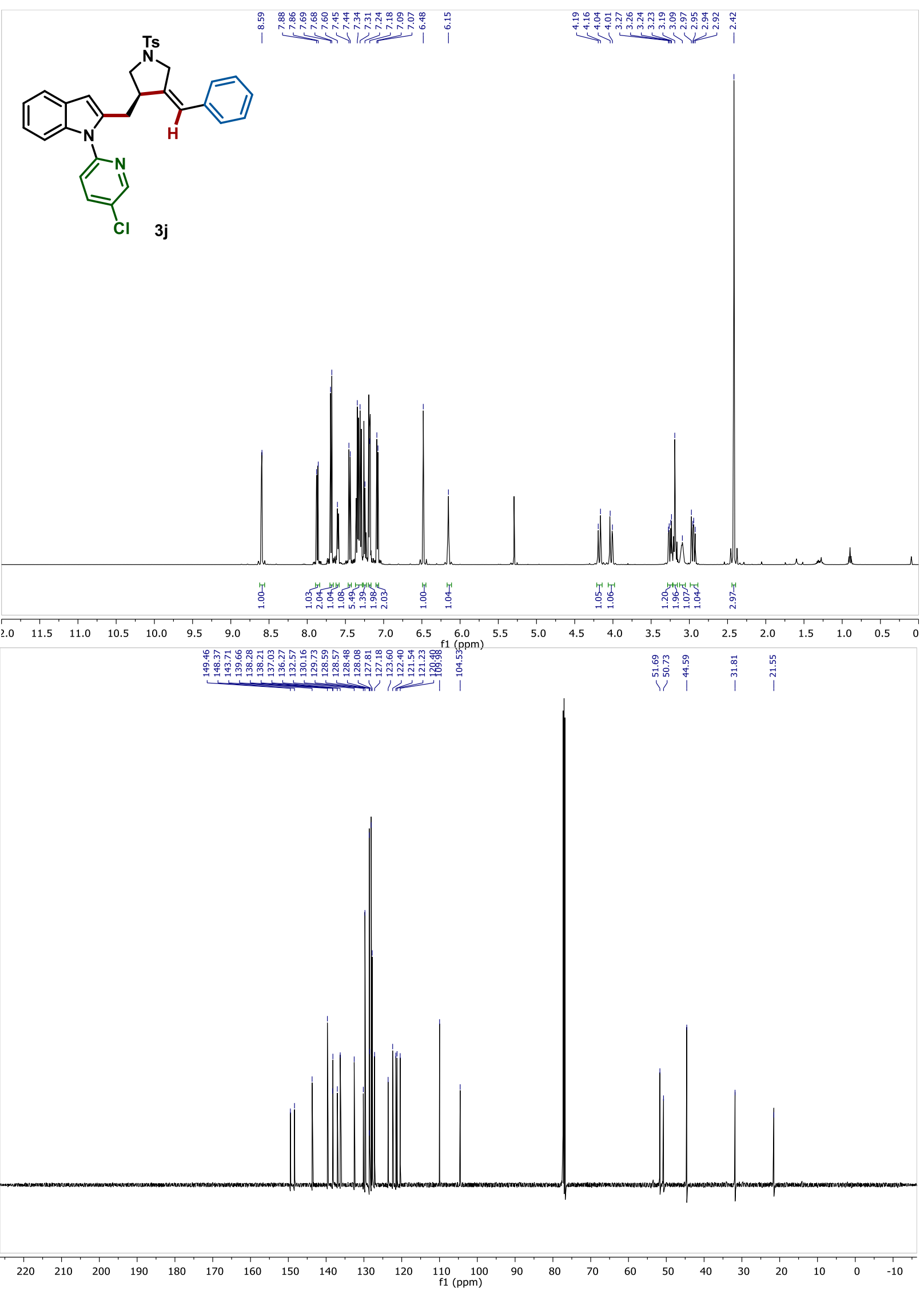

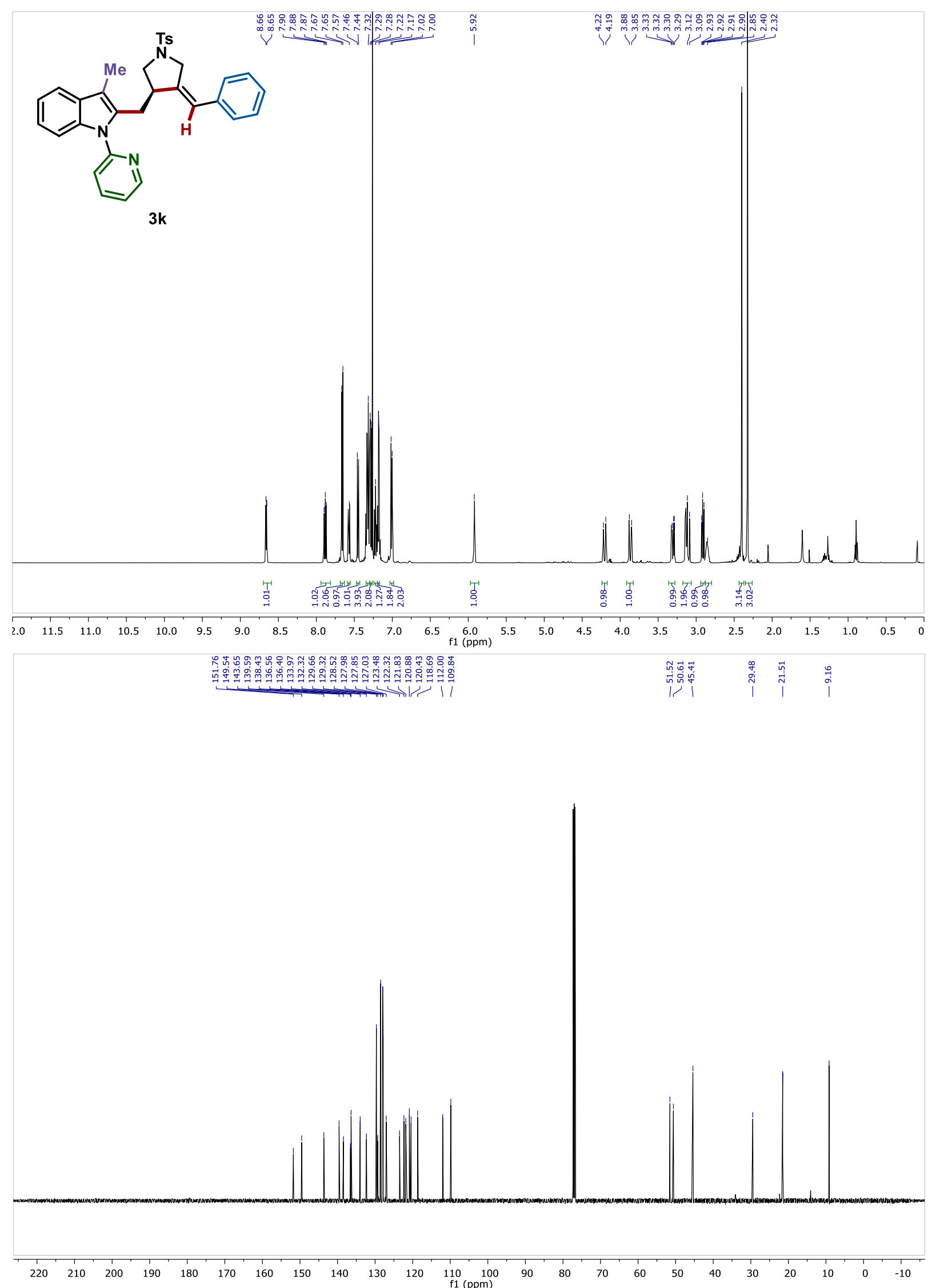

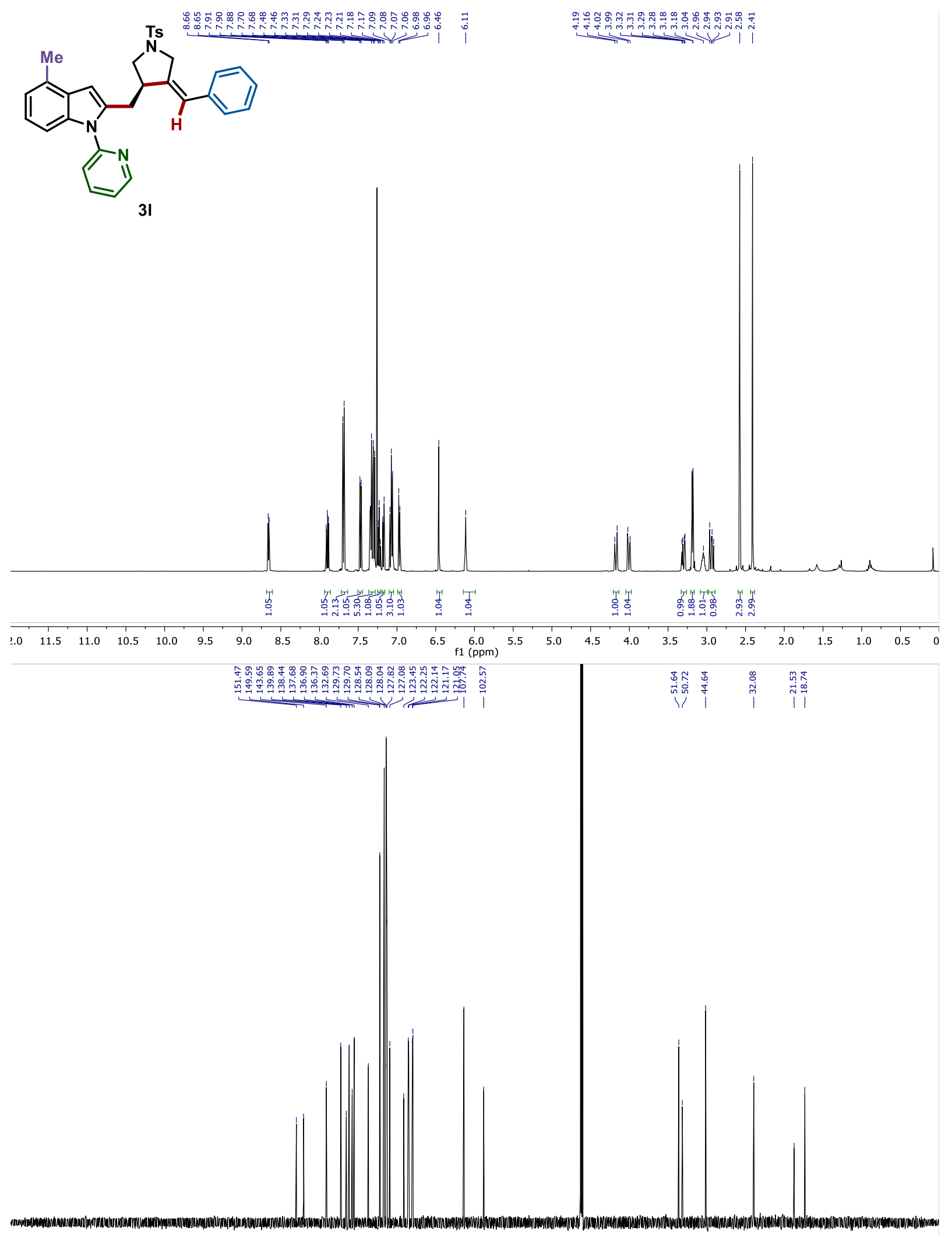

$\begin{array}{lllllllllllllllllllllll}220 & 210 & 200 & 190 & 180 & 170 & 160 & 150 & 140 & 130 & 120 & \begin{array}{c}110 \\ \mathrm{f} 1(\mathrm{ppm})\end{array} & 90 & 80 & 70 & 60 & 50 & 40 & 30 & 20 & 10 & 0 & -10\end{array}$ 

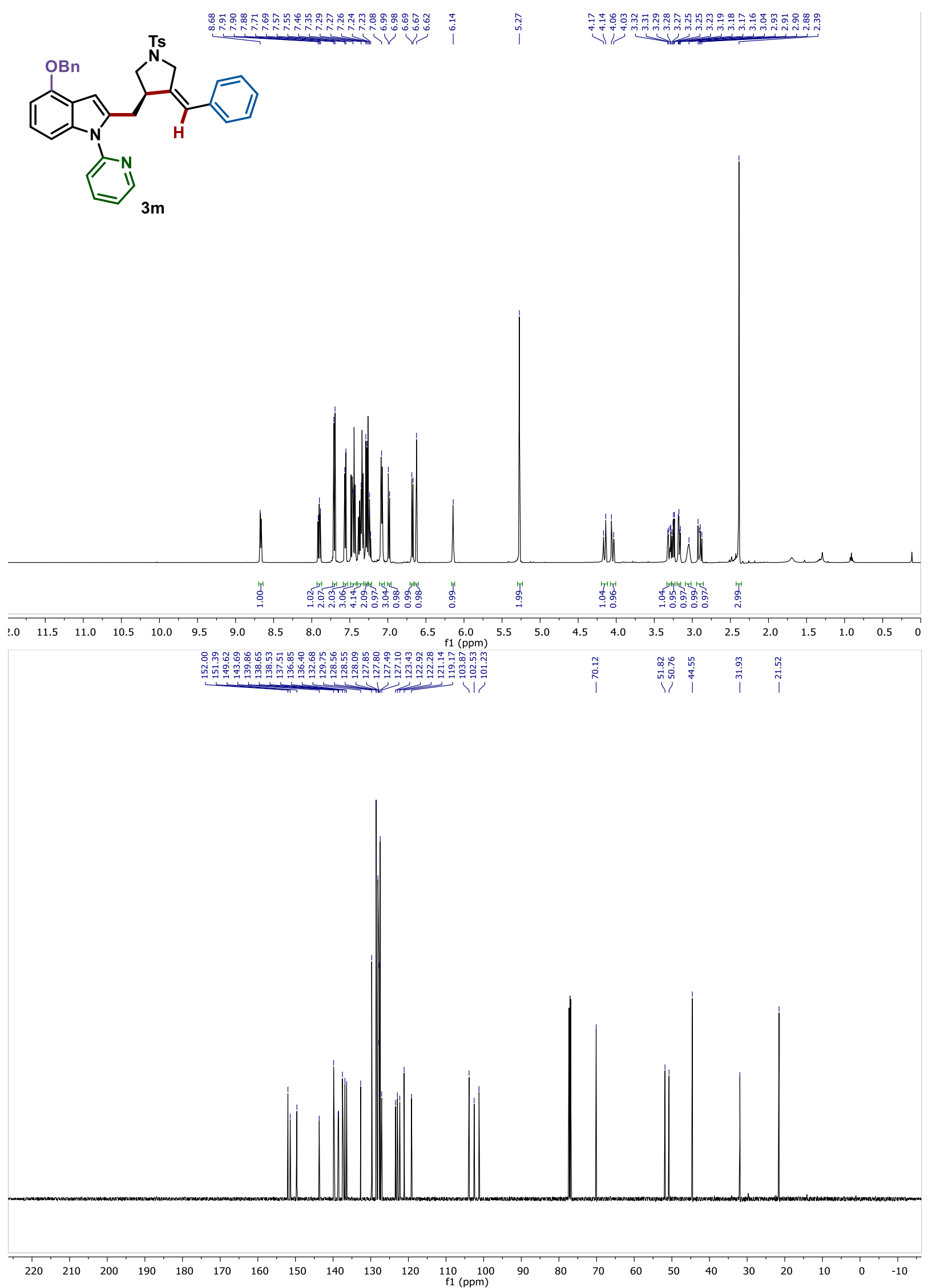

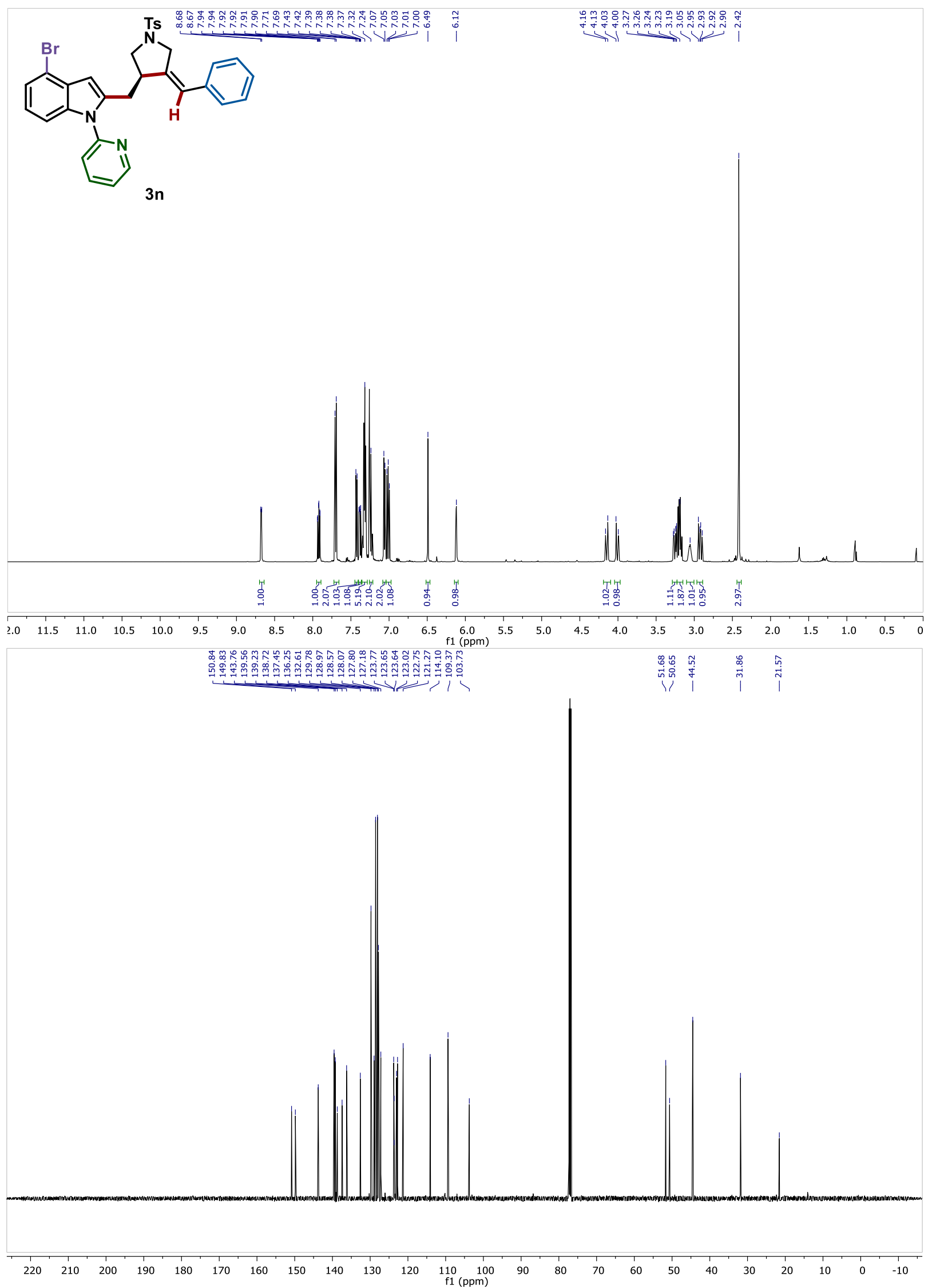

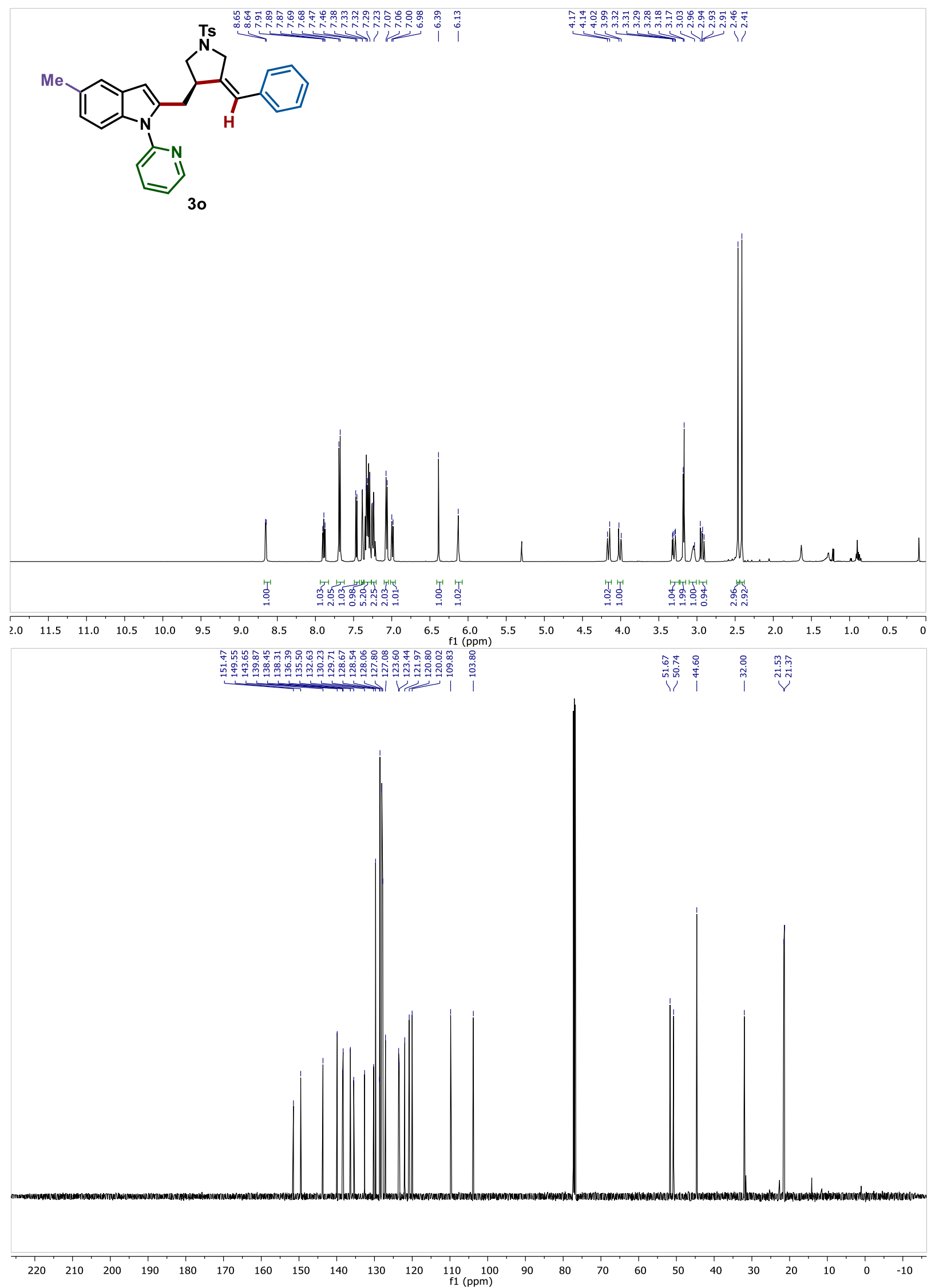

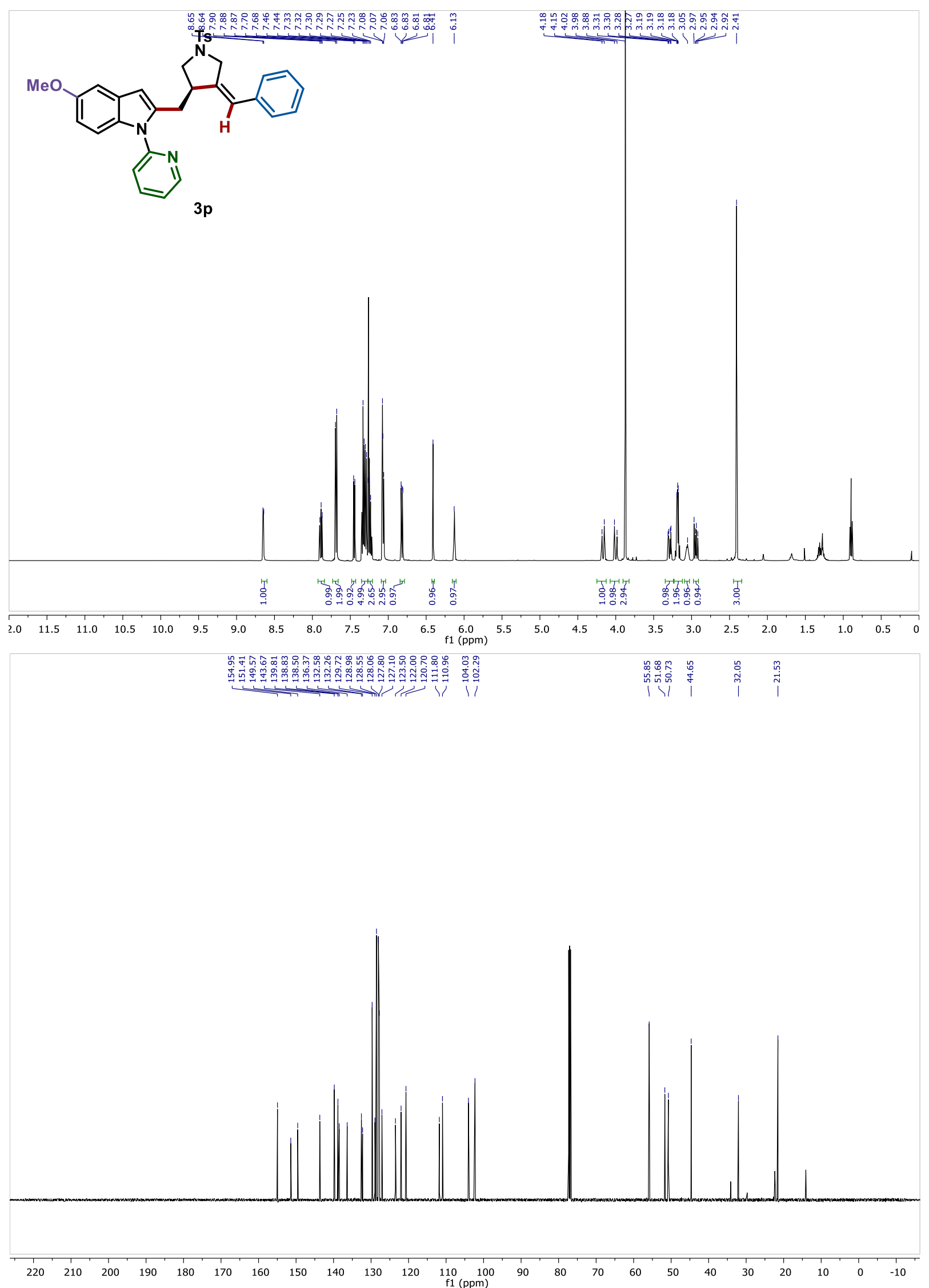

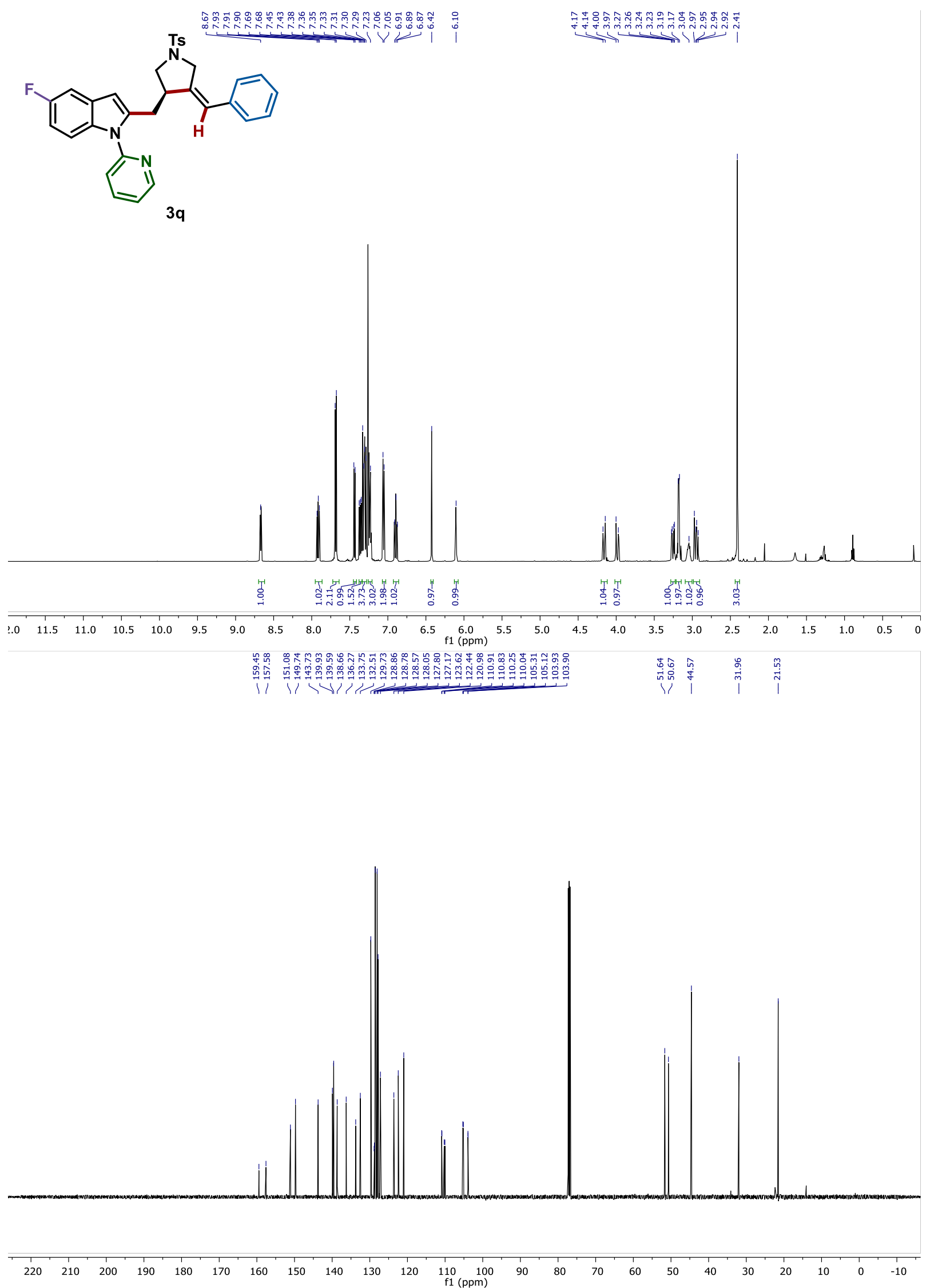


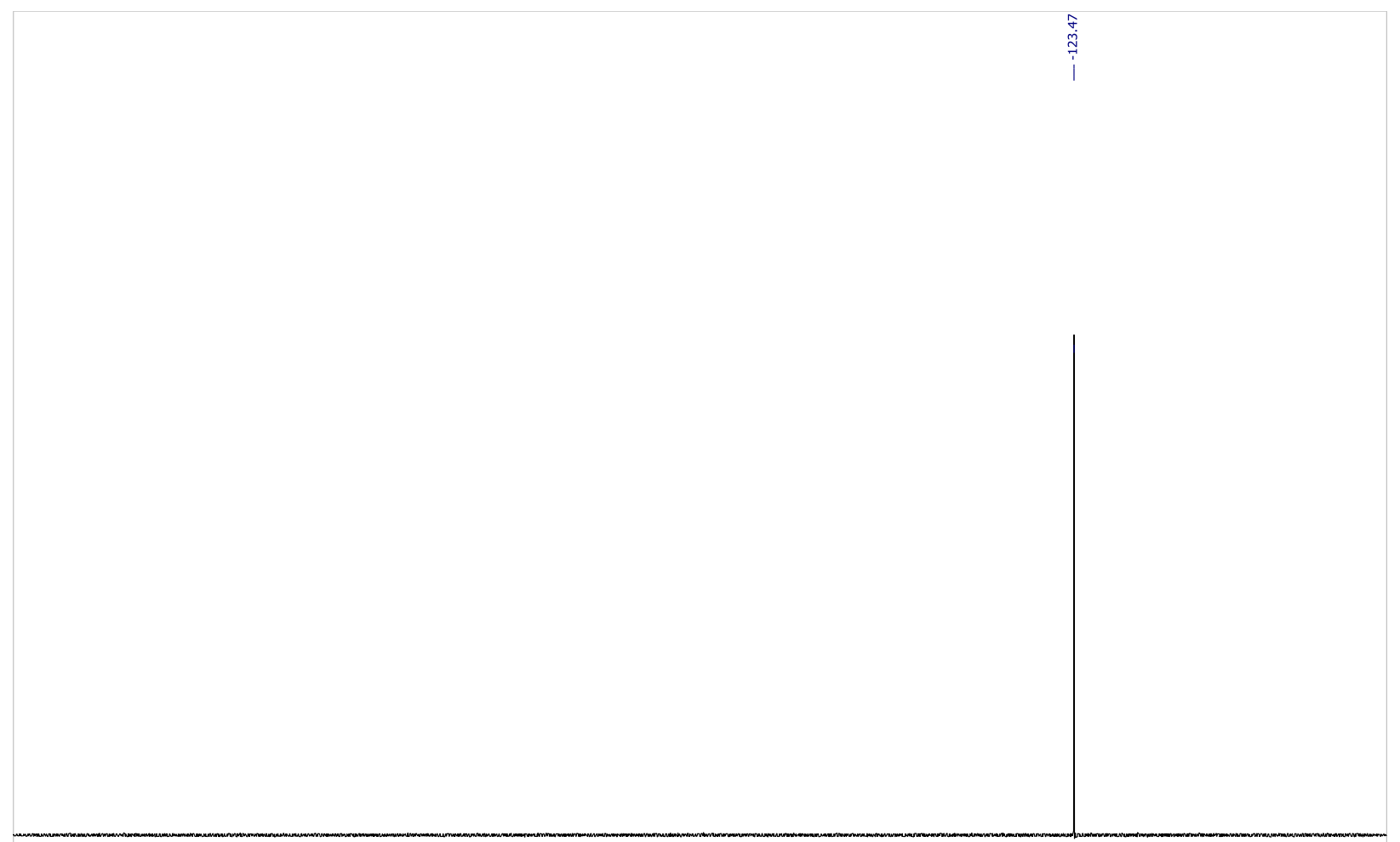

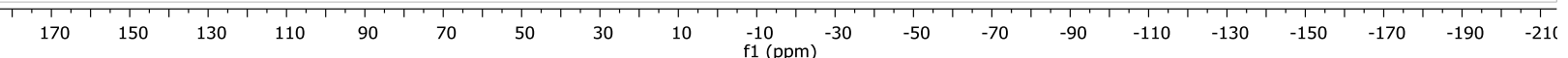



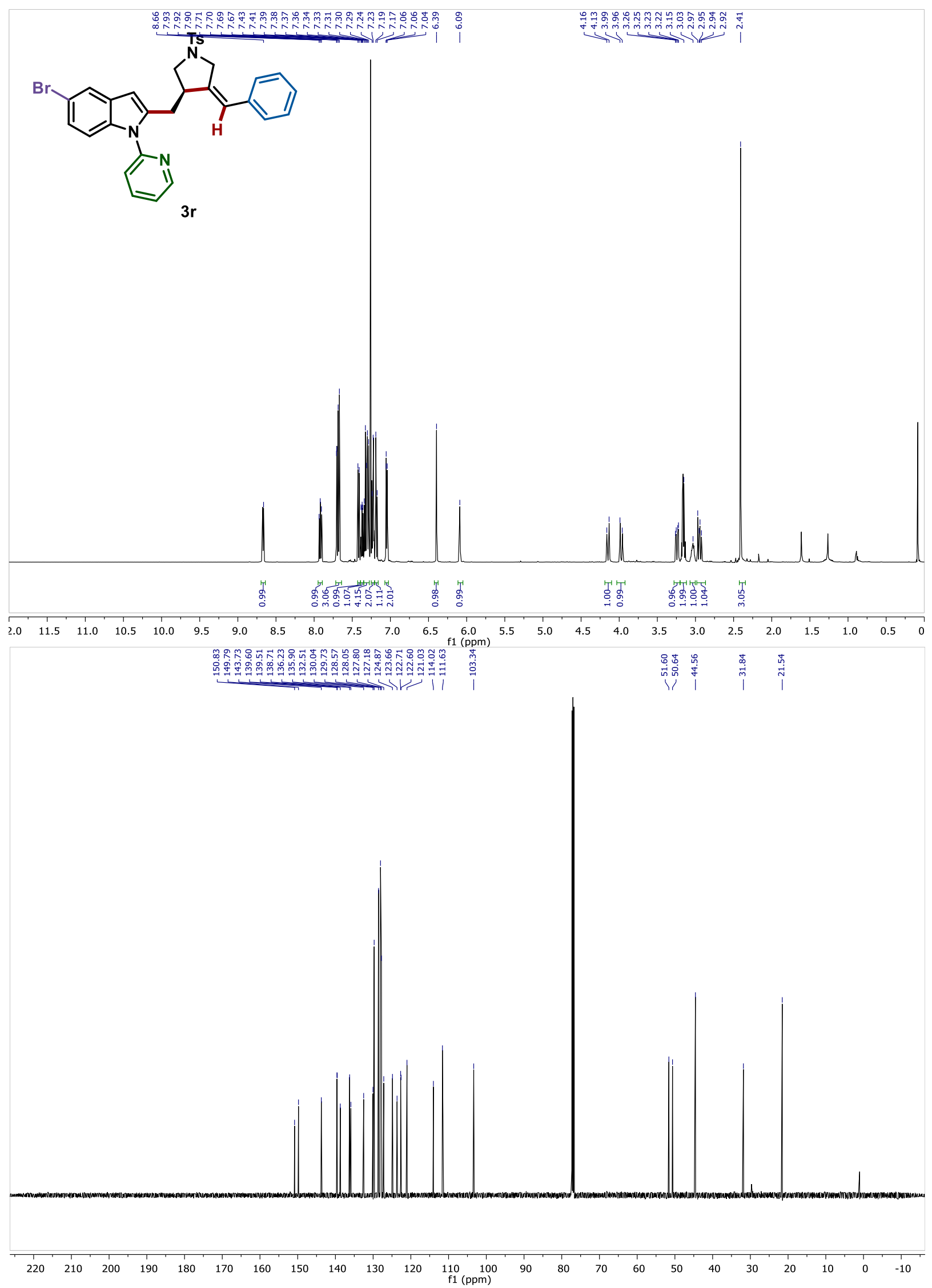

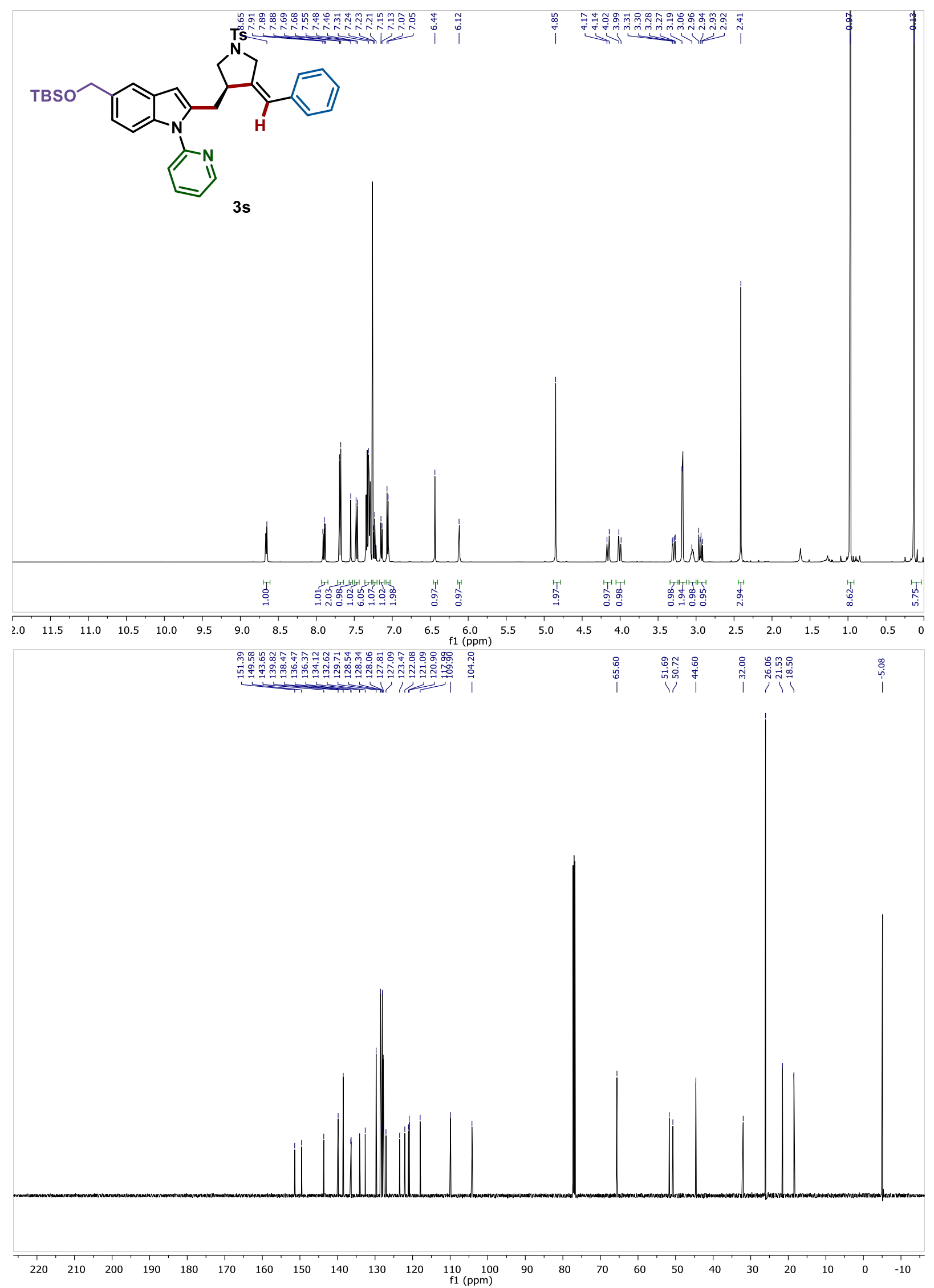


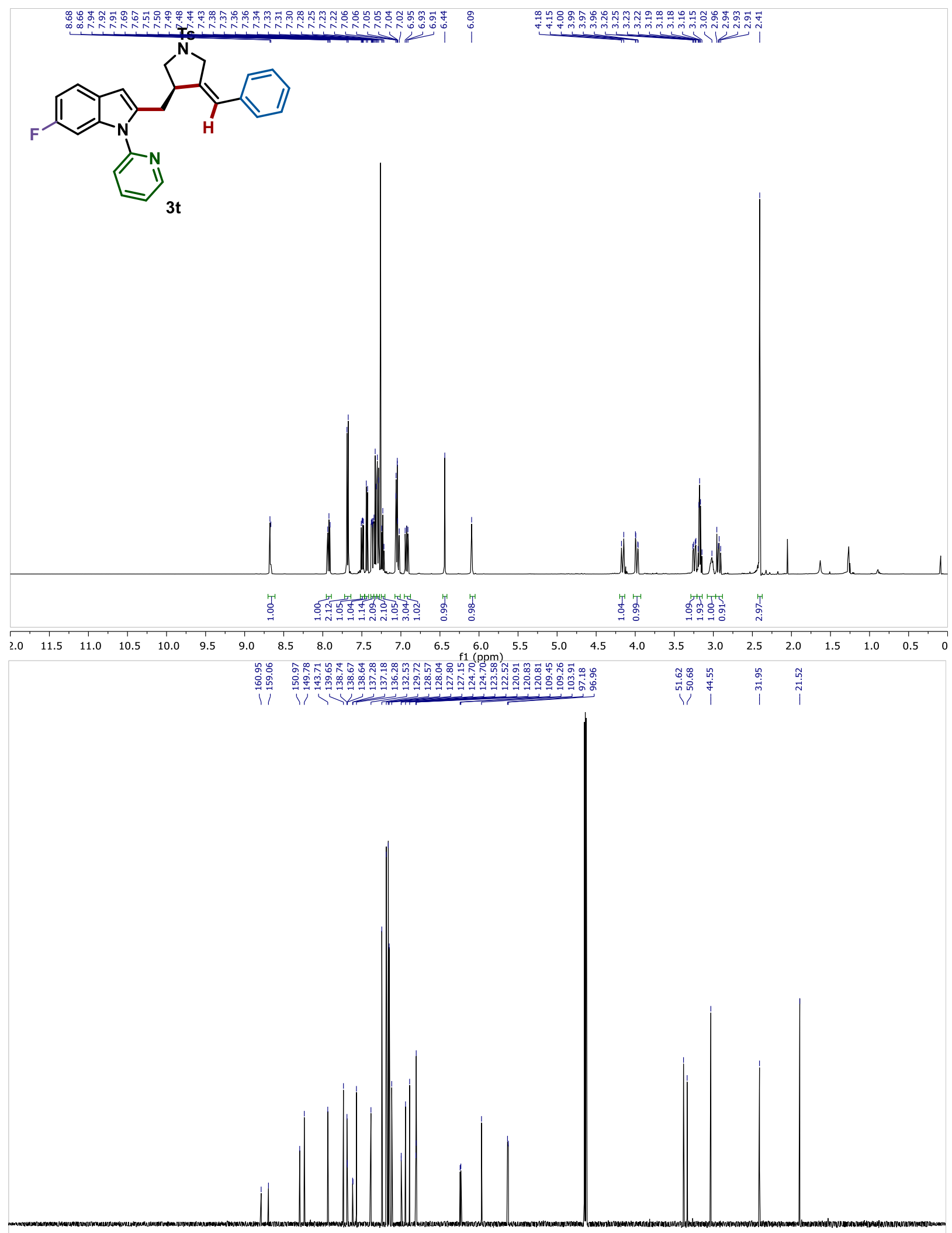

\begin{tabular}{|llllllllllllllllllllllllllll}
$\mid$ & 220 & 210 & 200 & 190 & 180 & 170 & 160 & 150 & 140 & 130 & 120 & $\begin{array}{c}110 \\
\mathrm{f} 1(\mathrm{ppm})\end{array}$ & 90 & 80 & 70 & 60 & 50 & 40 & 30 & 20 & 10 & 0 & -10
\end{tabular} 


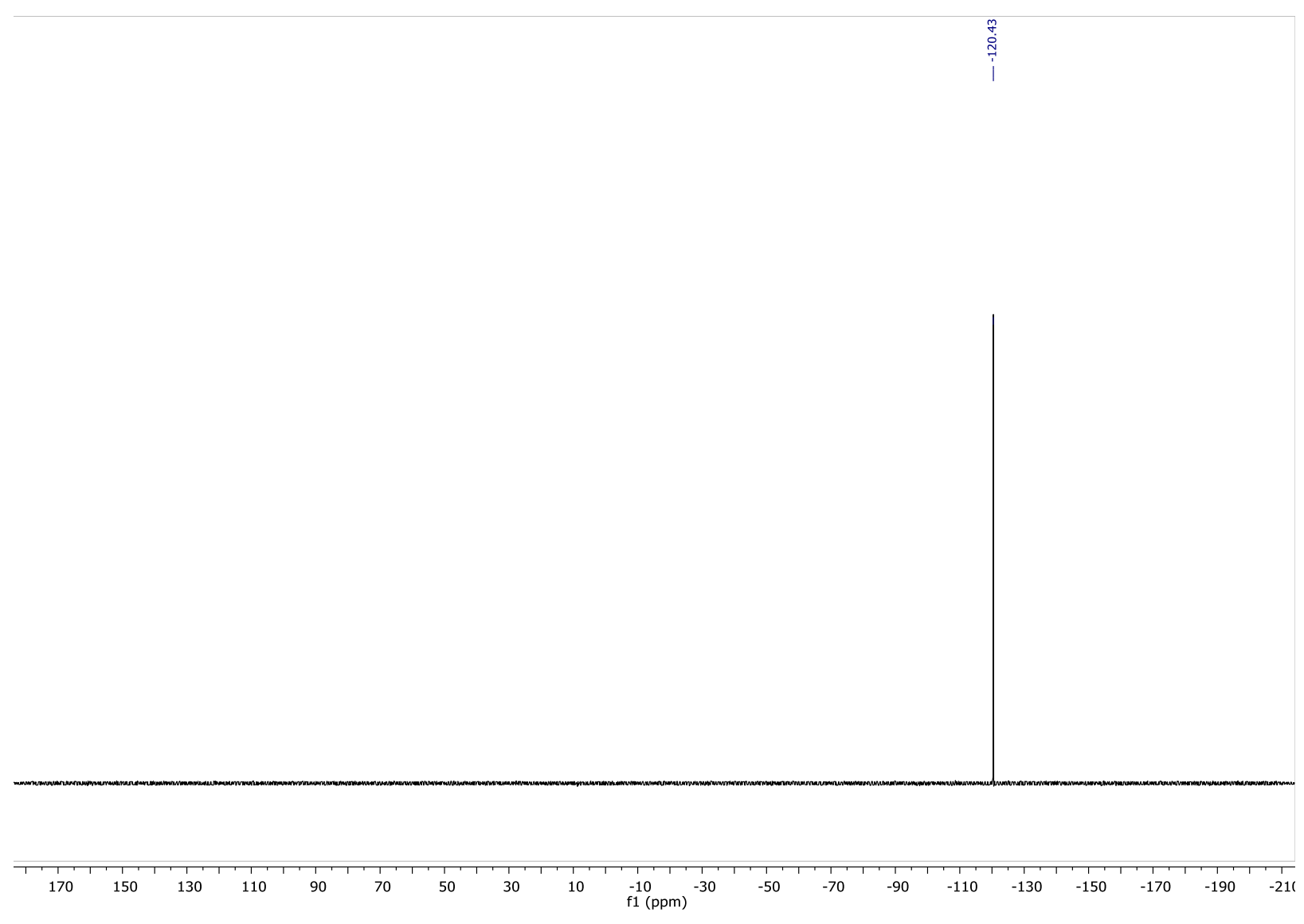




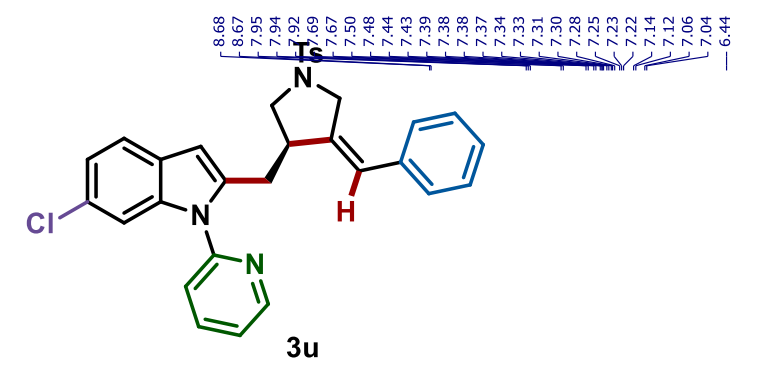

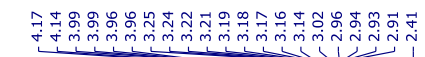

$3 u$

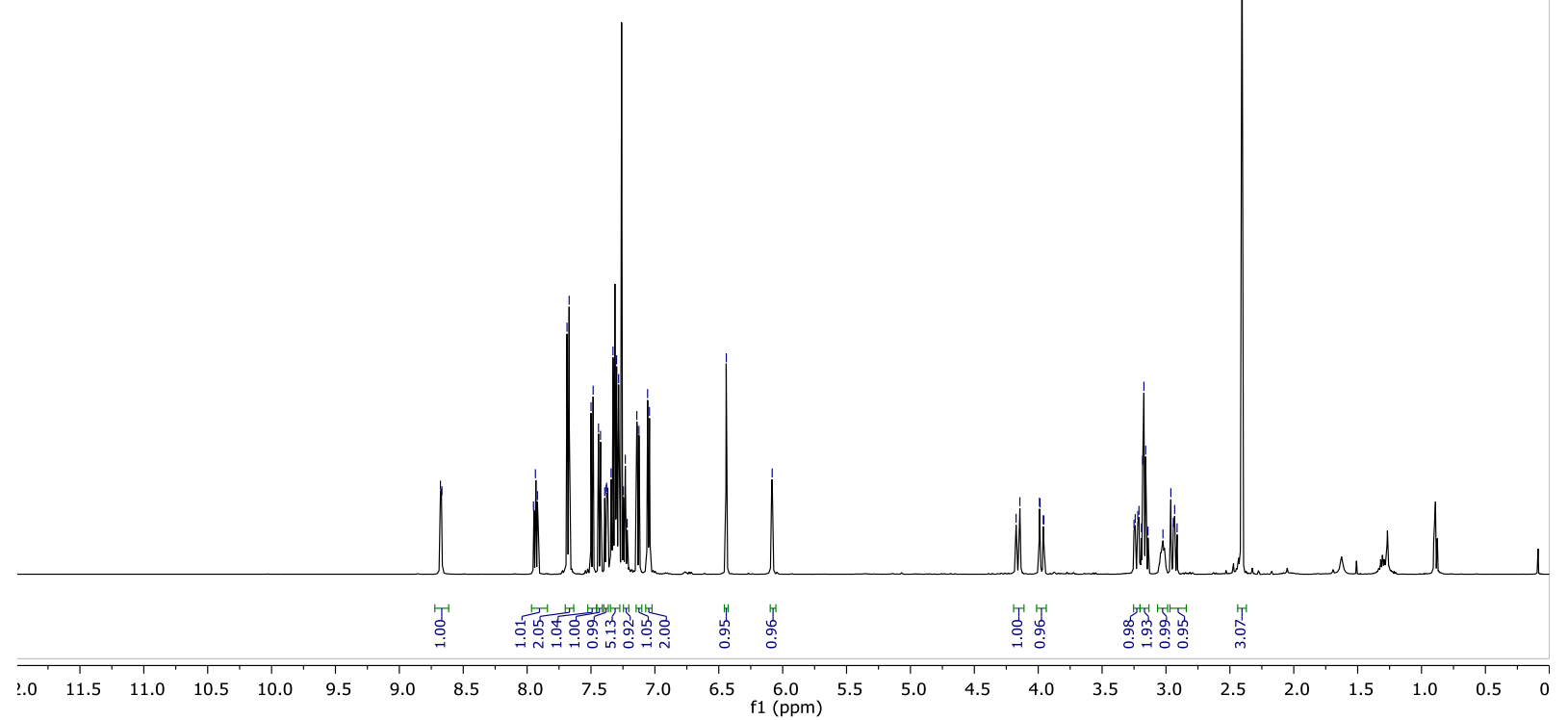

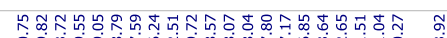

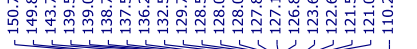
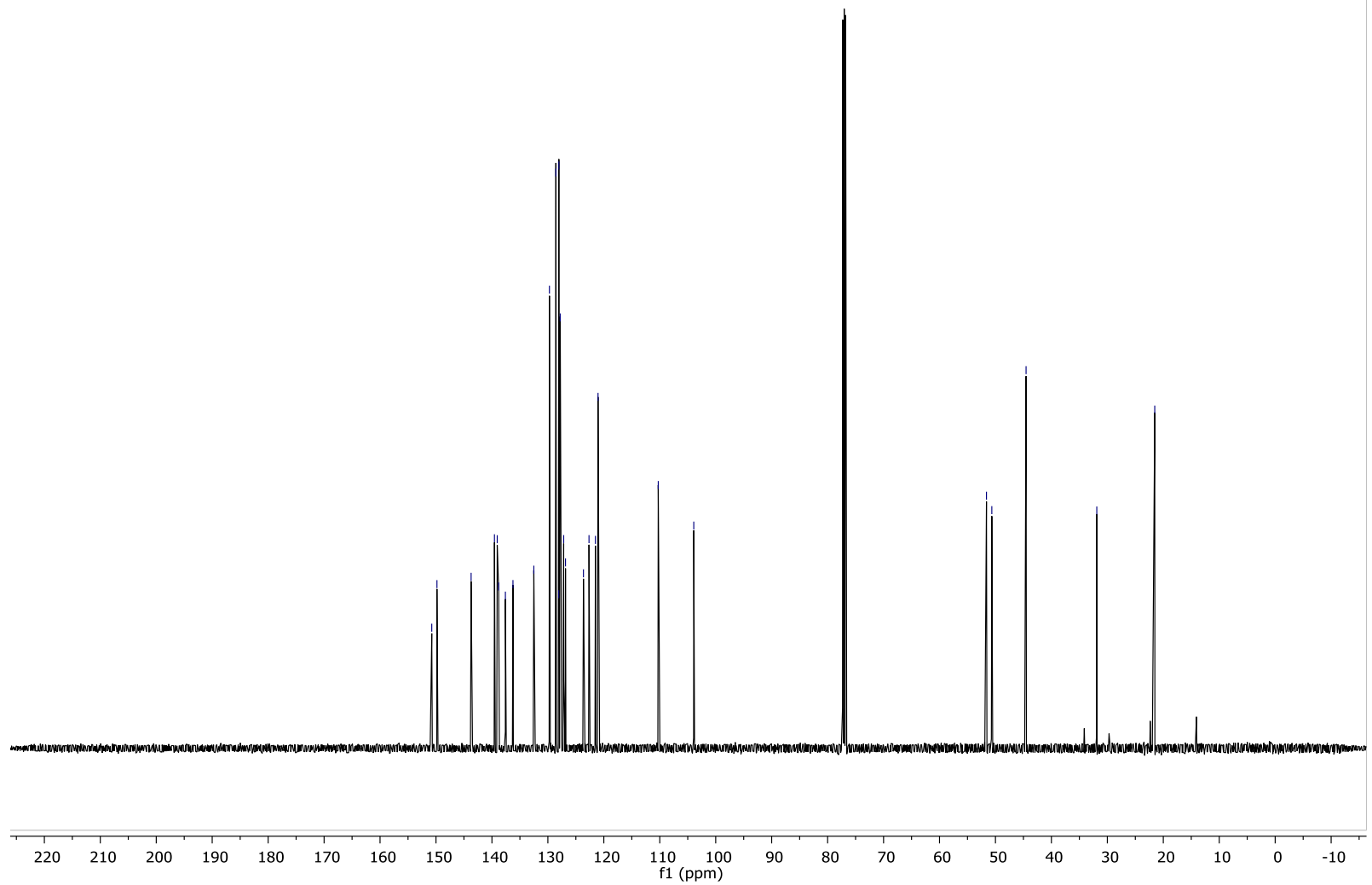

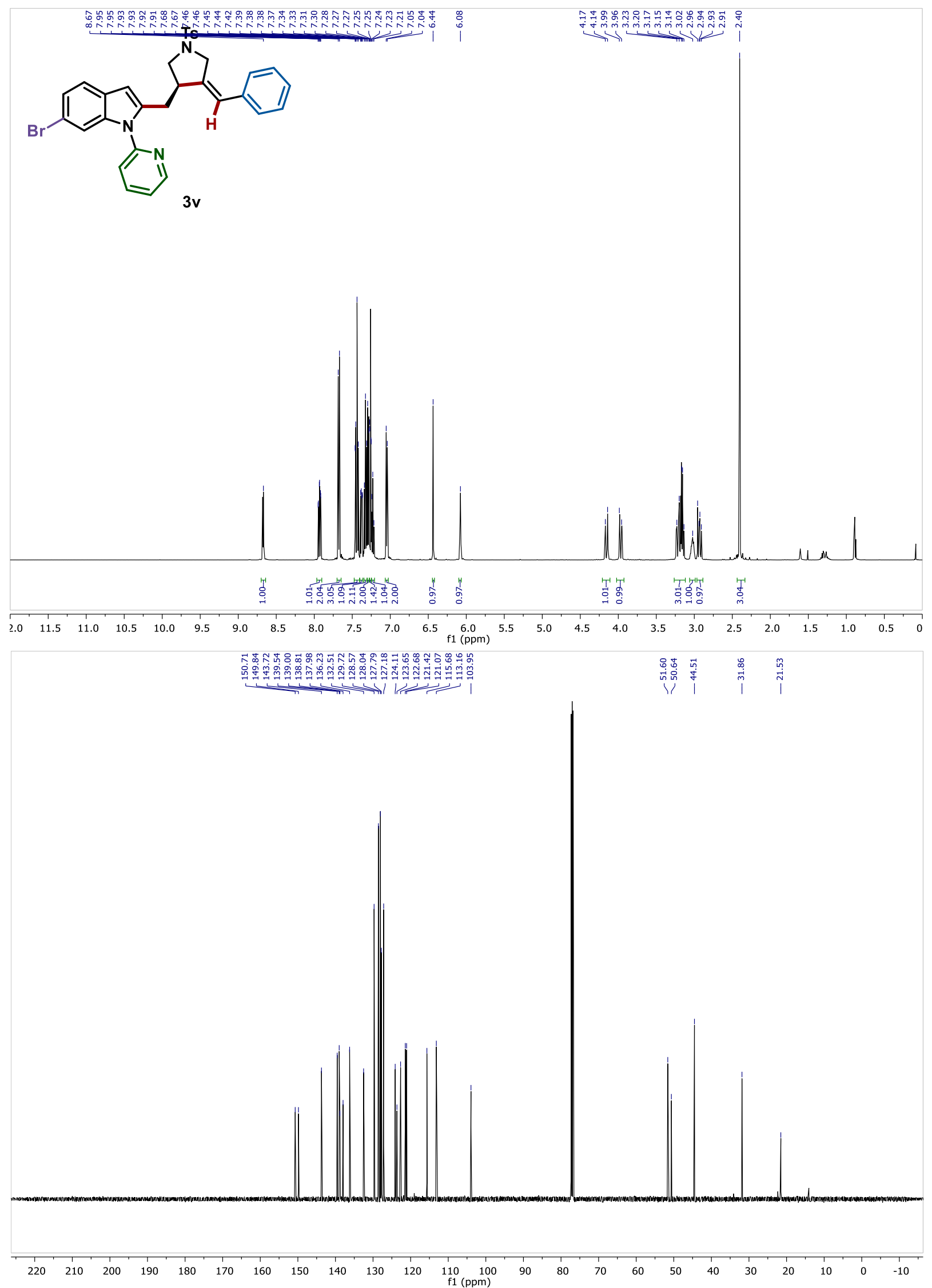

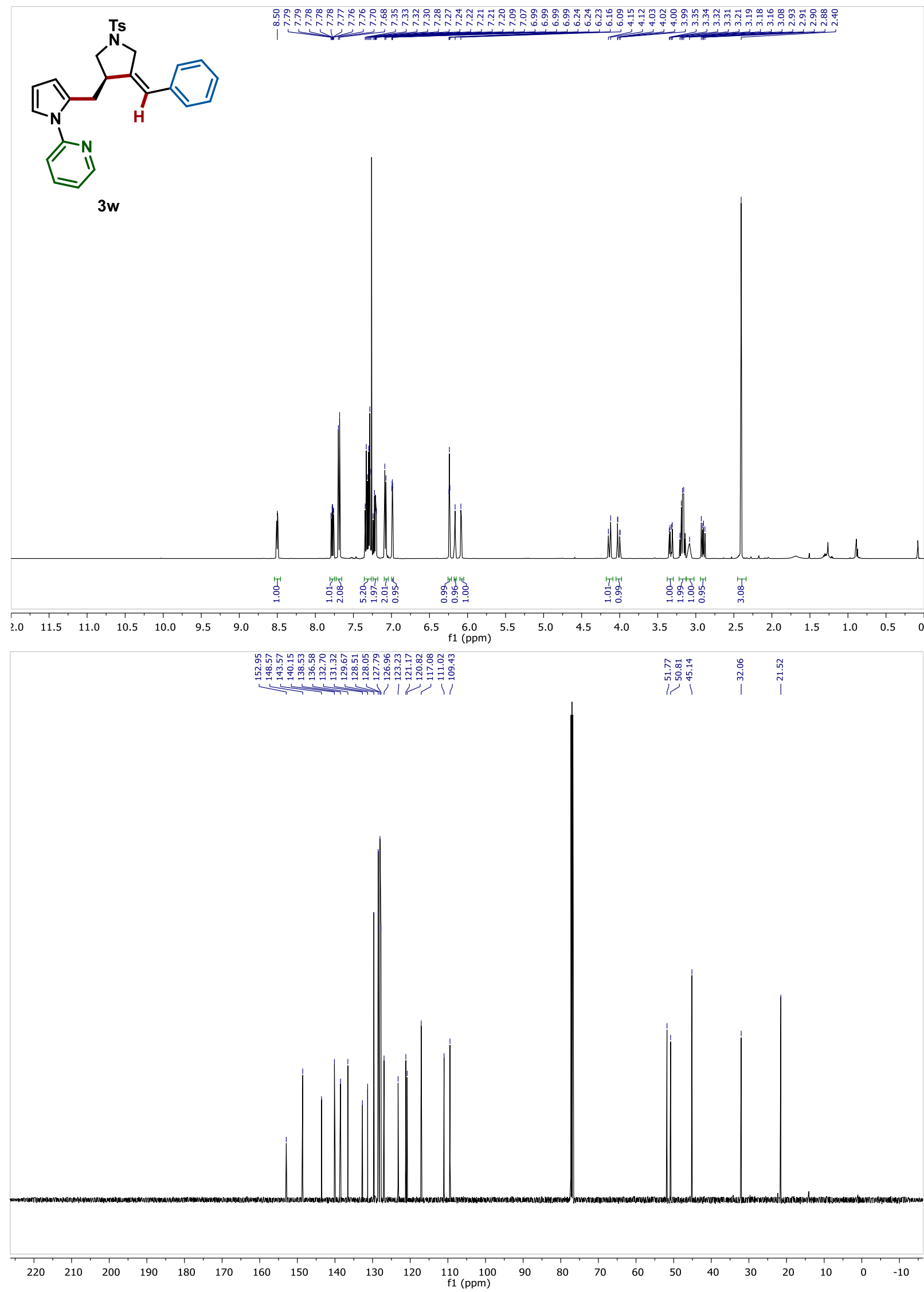

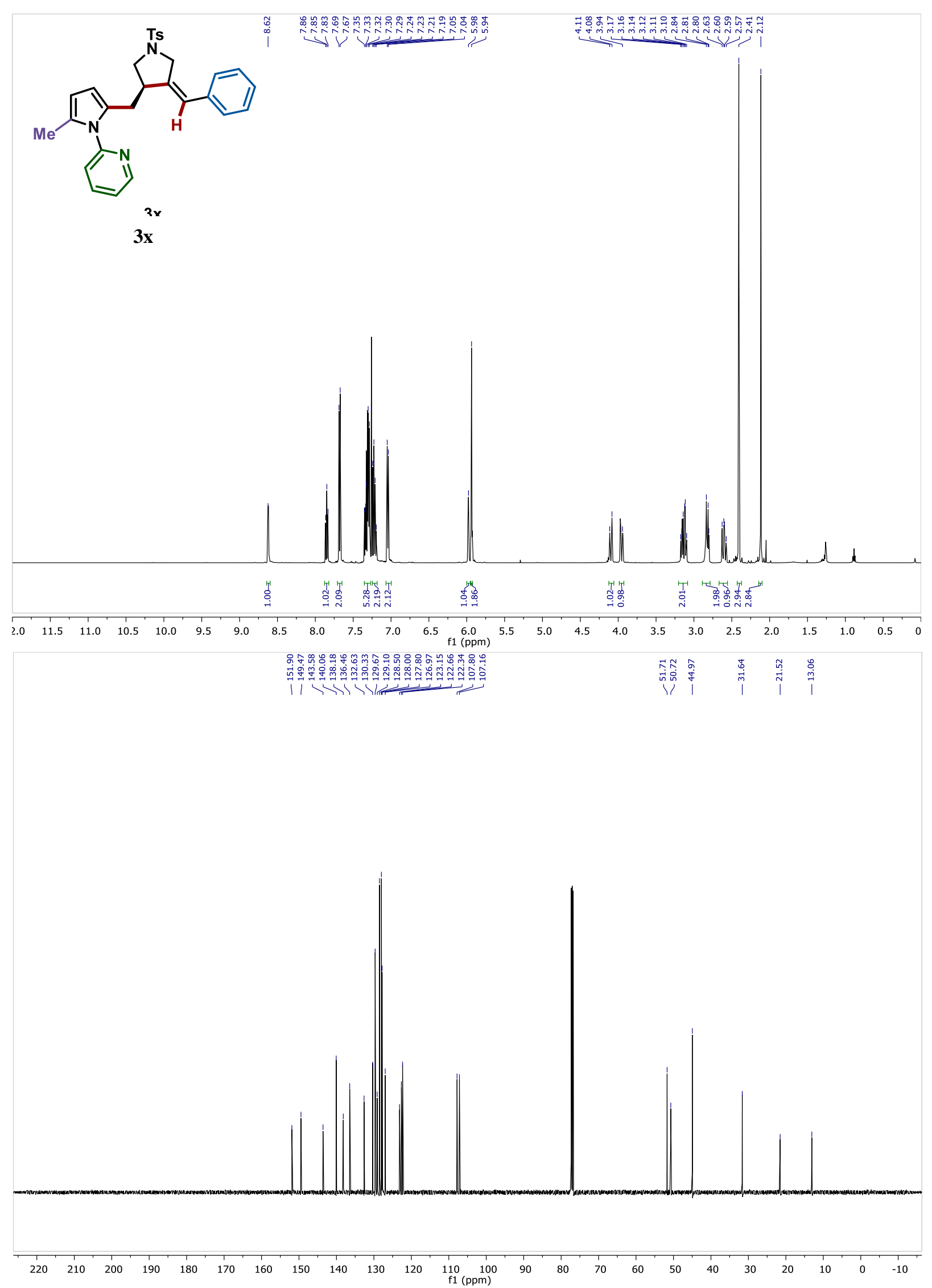


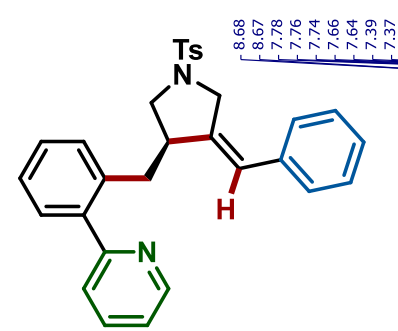

$3 y$
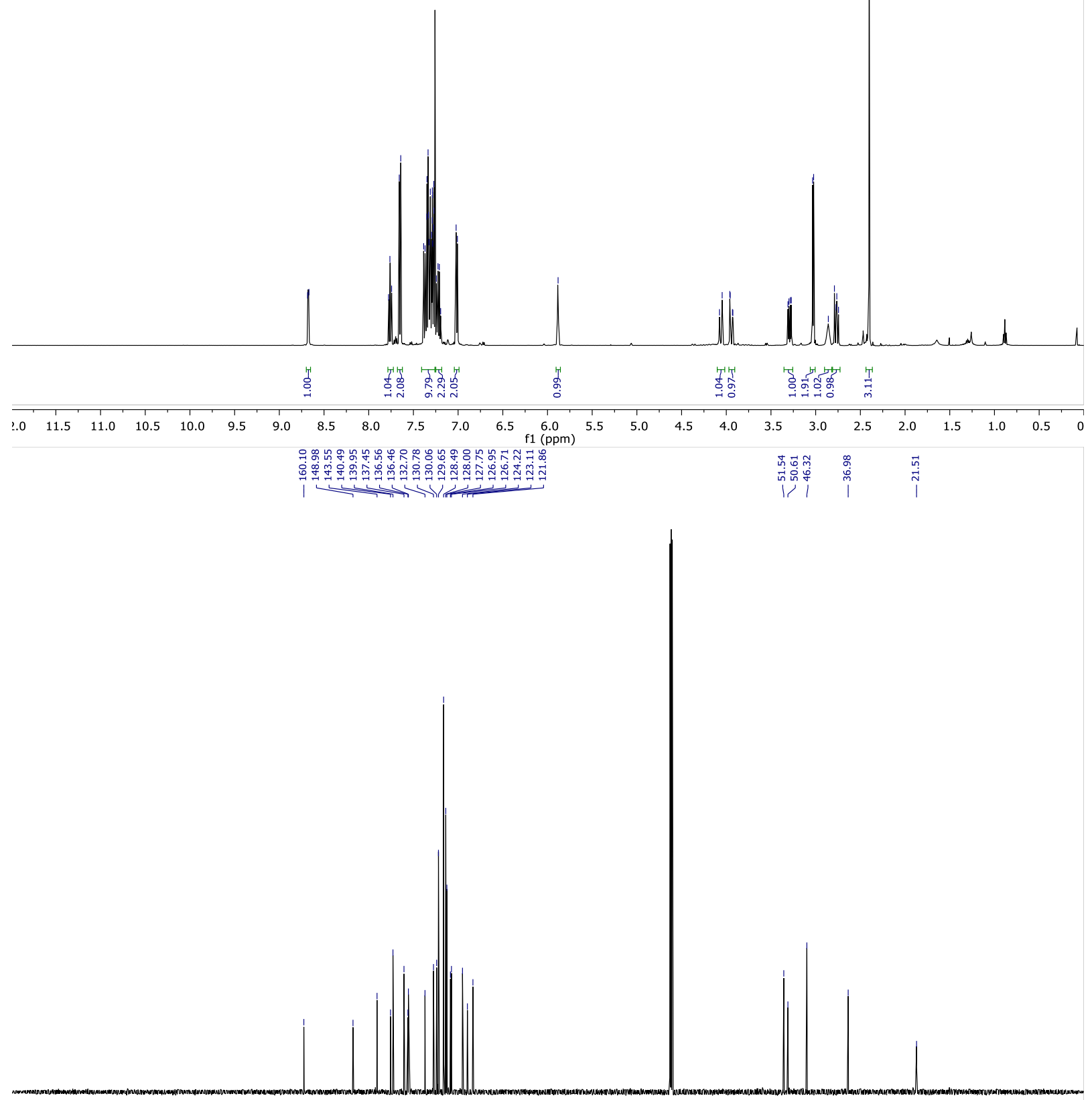

$\begin{array}{lllllllllllll}220 & 210 & 200 & 190 & 180 & 170 & 160 & 150 & 140 & 130 & 120 & 110 & 100 \\ \mathrm{f} 1(\mathrm{ppm})\end{array}$ 

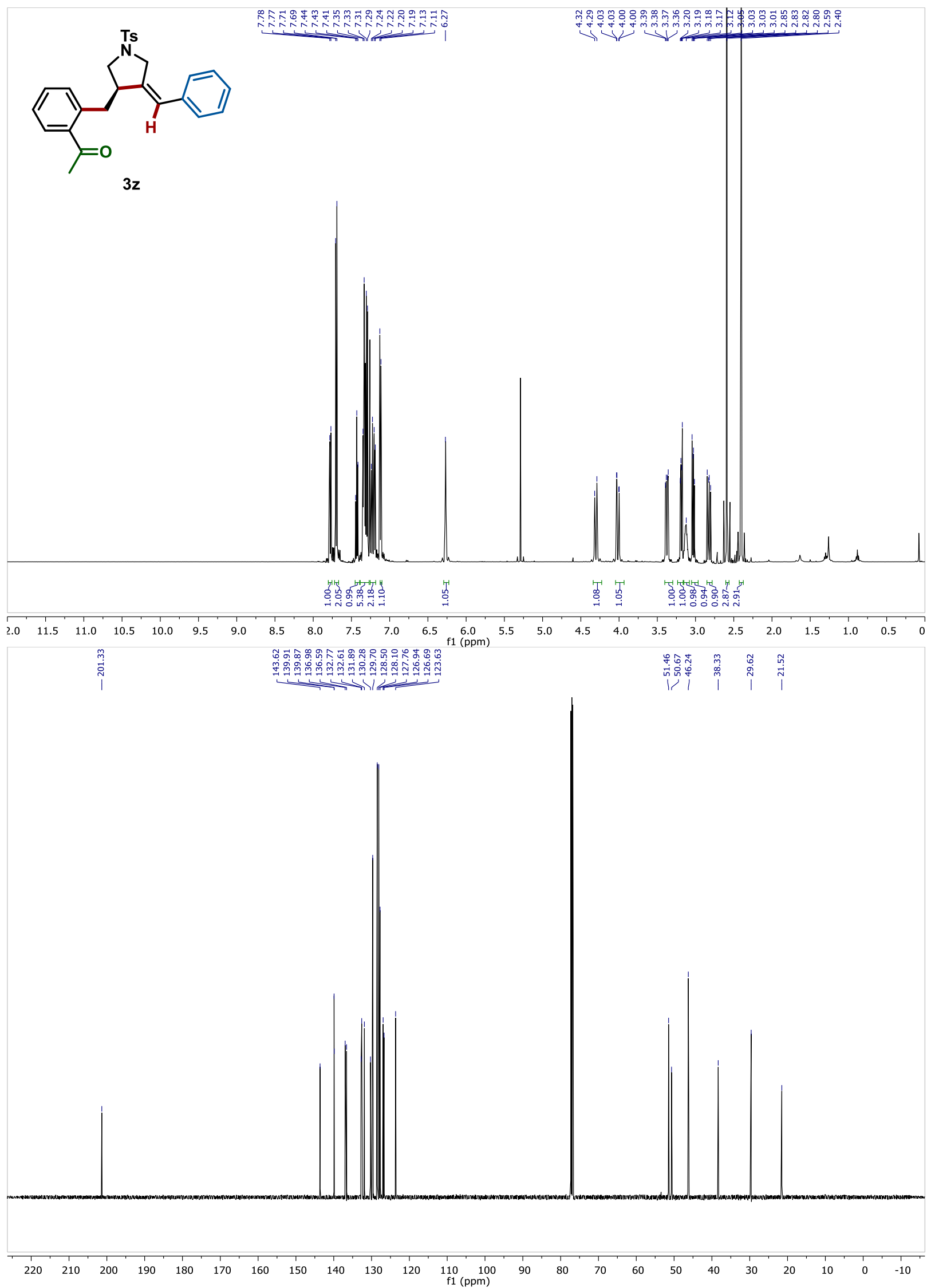

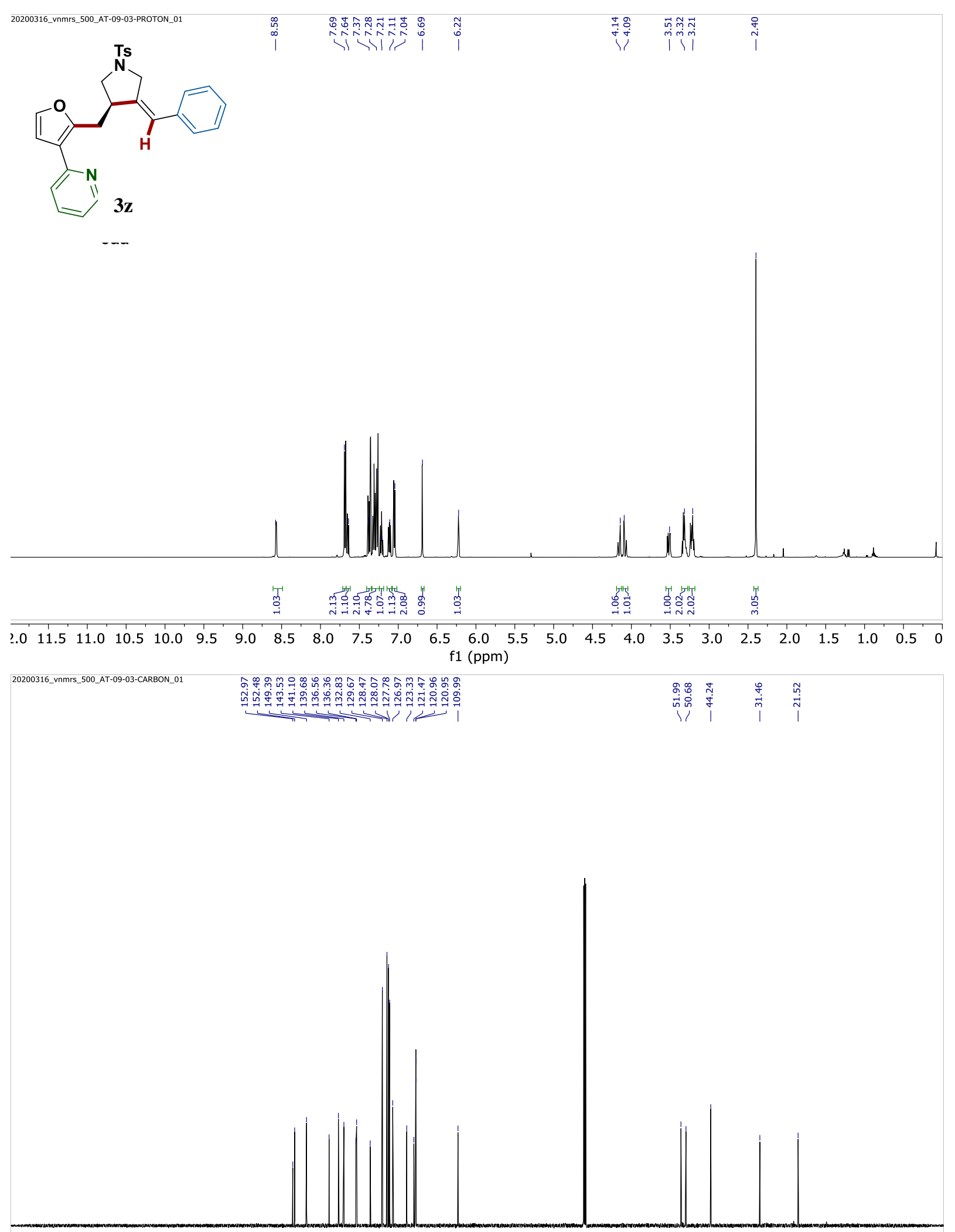

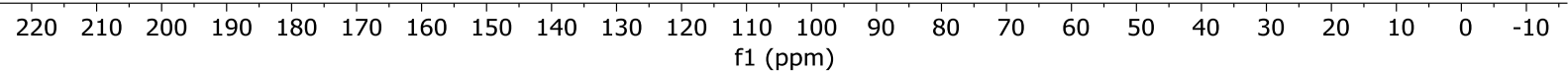



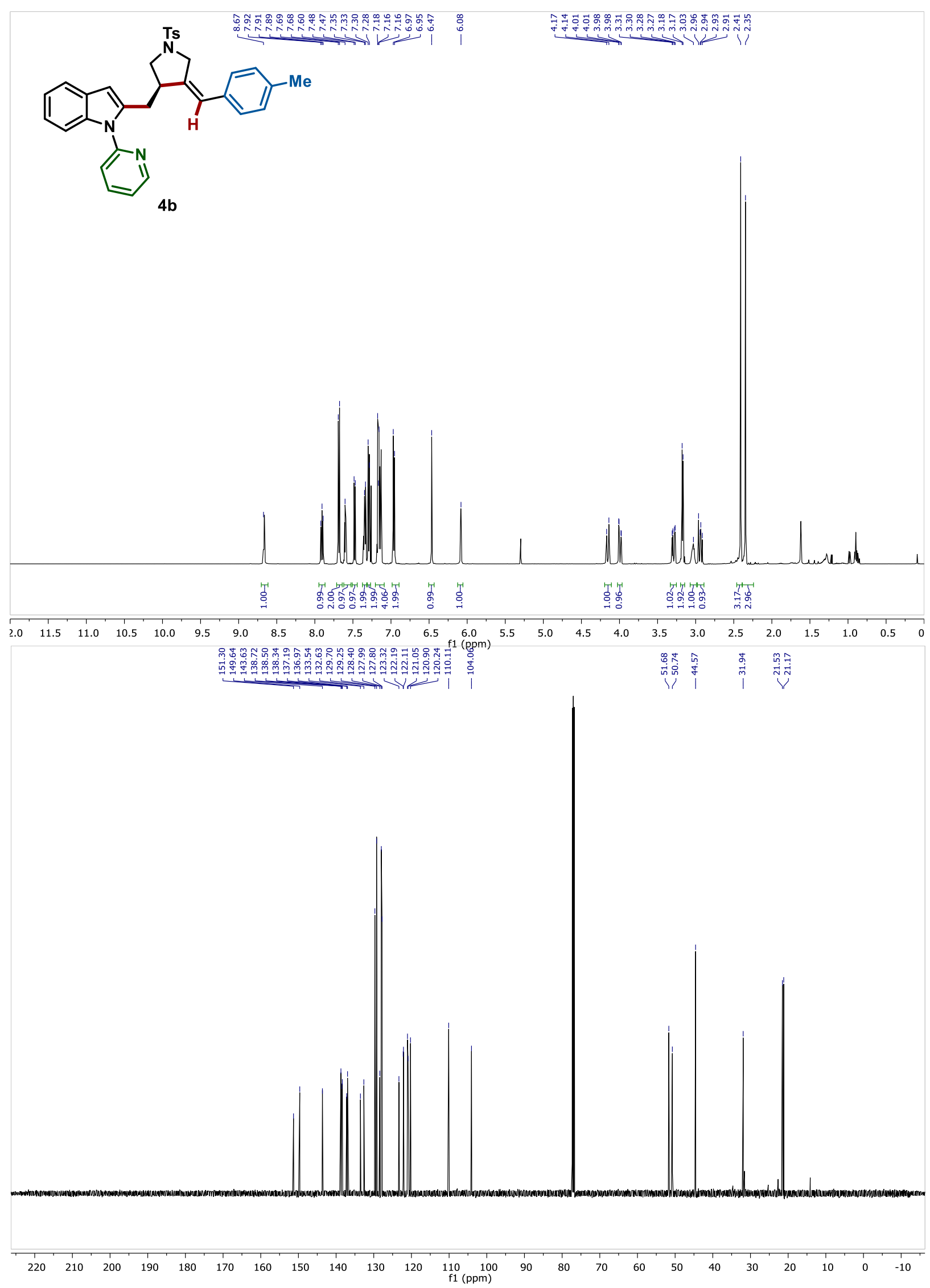

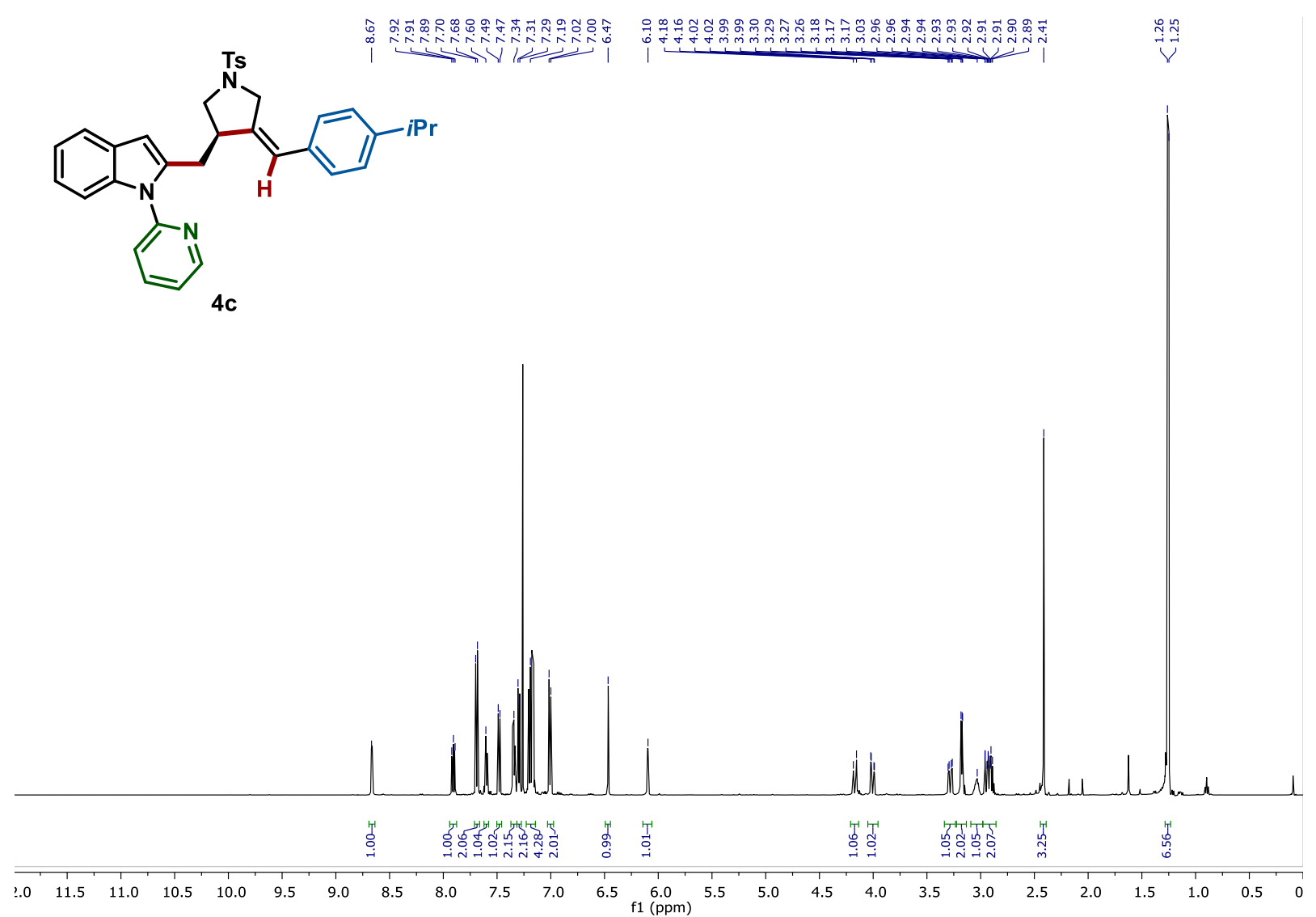

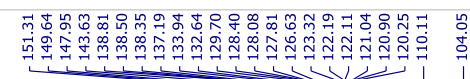
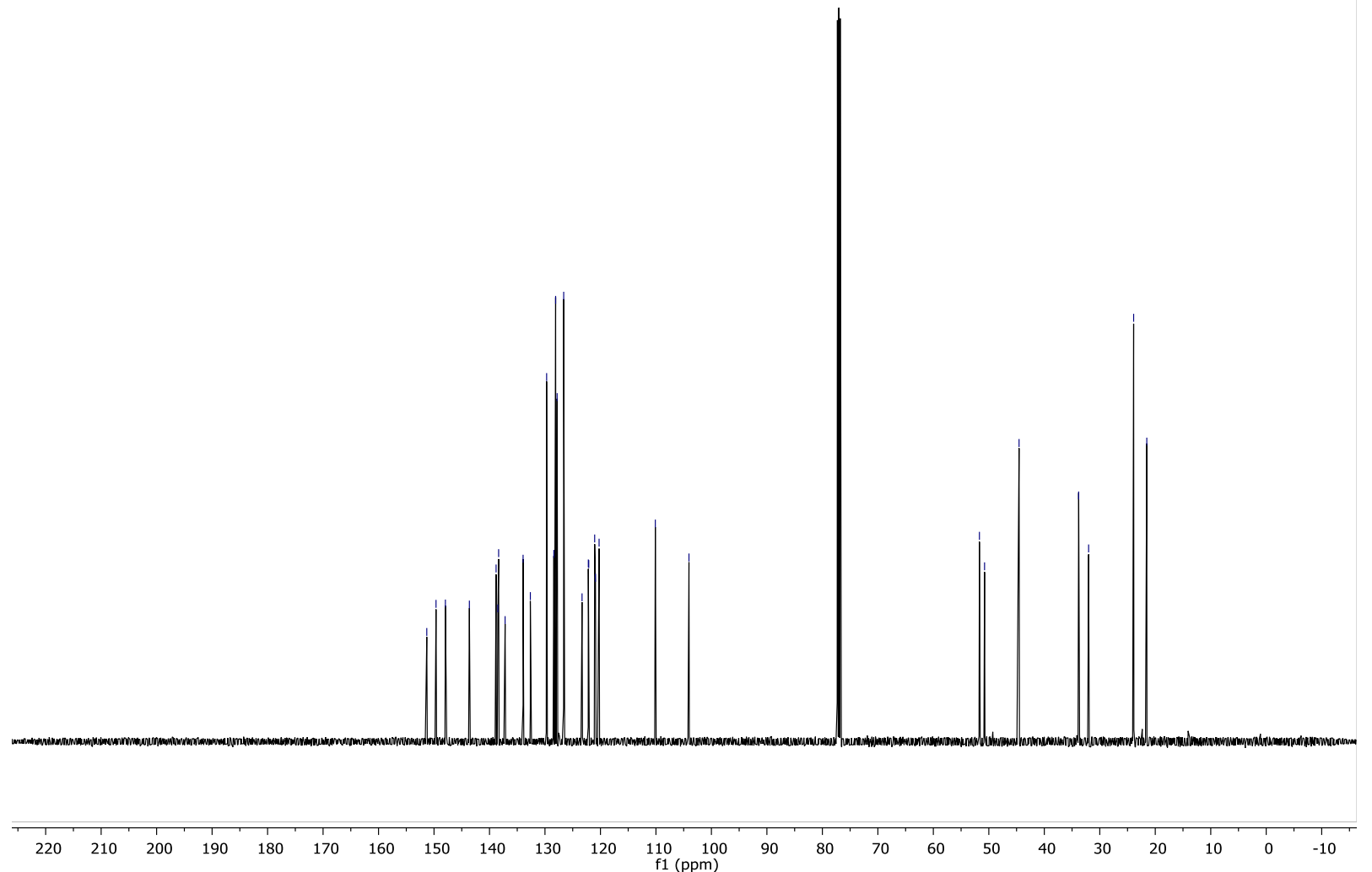

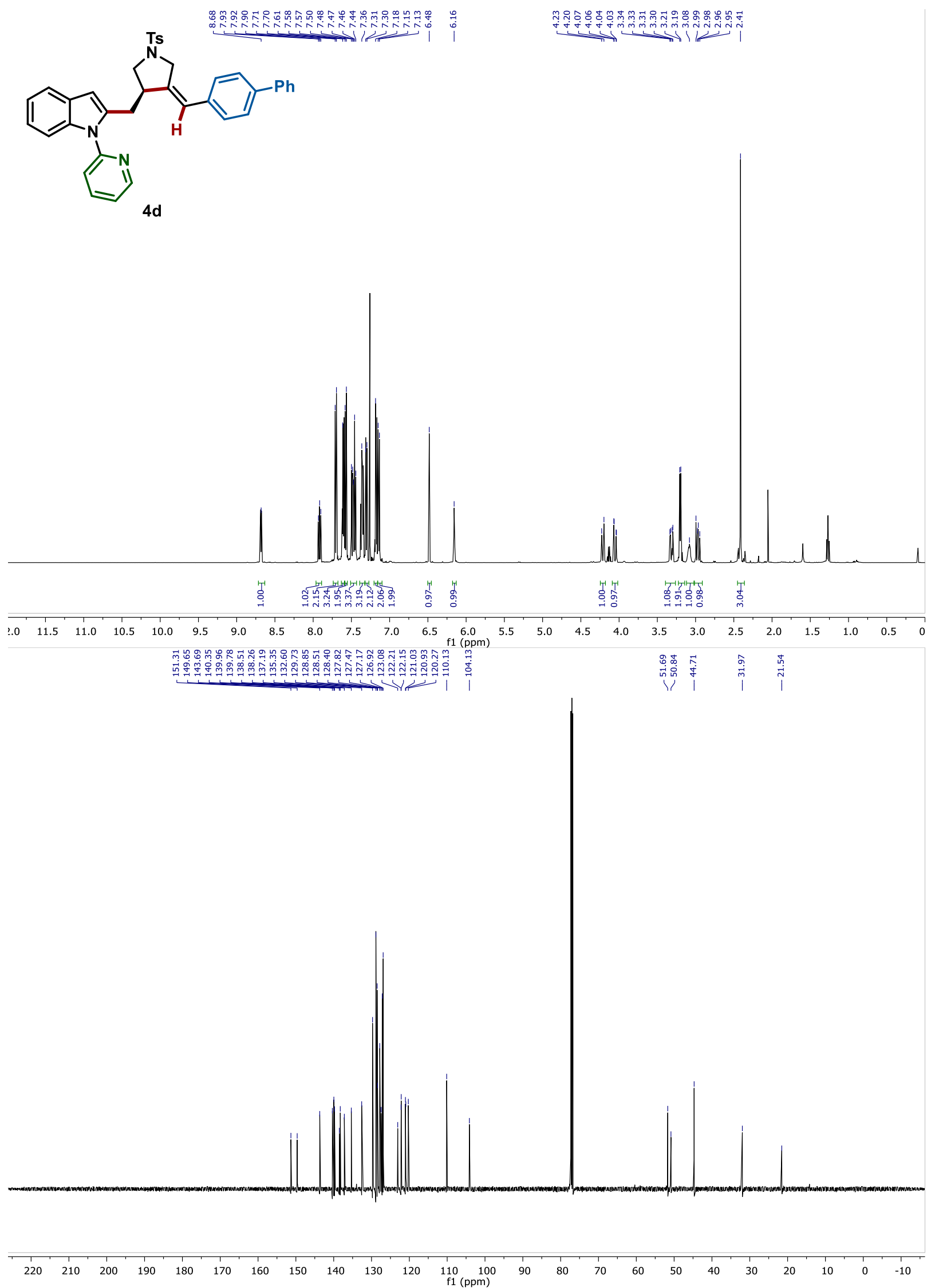

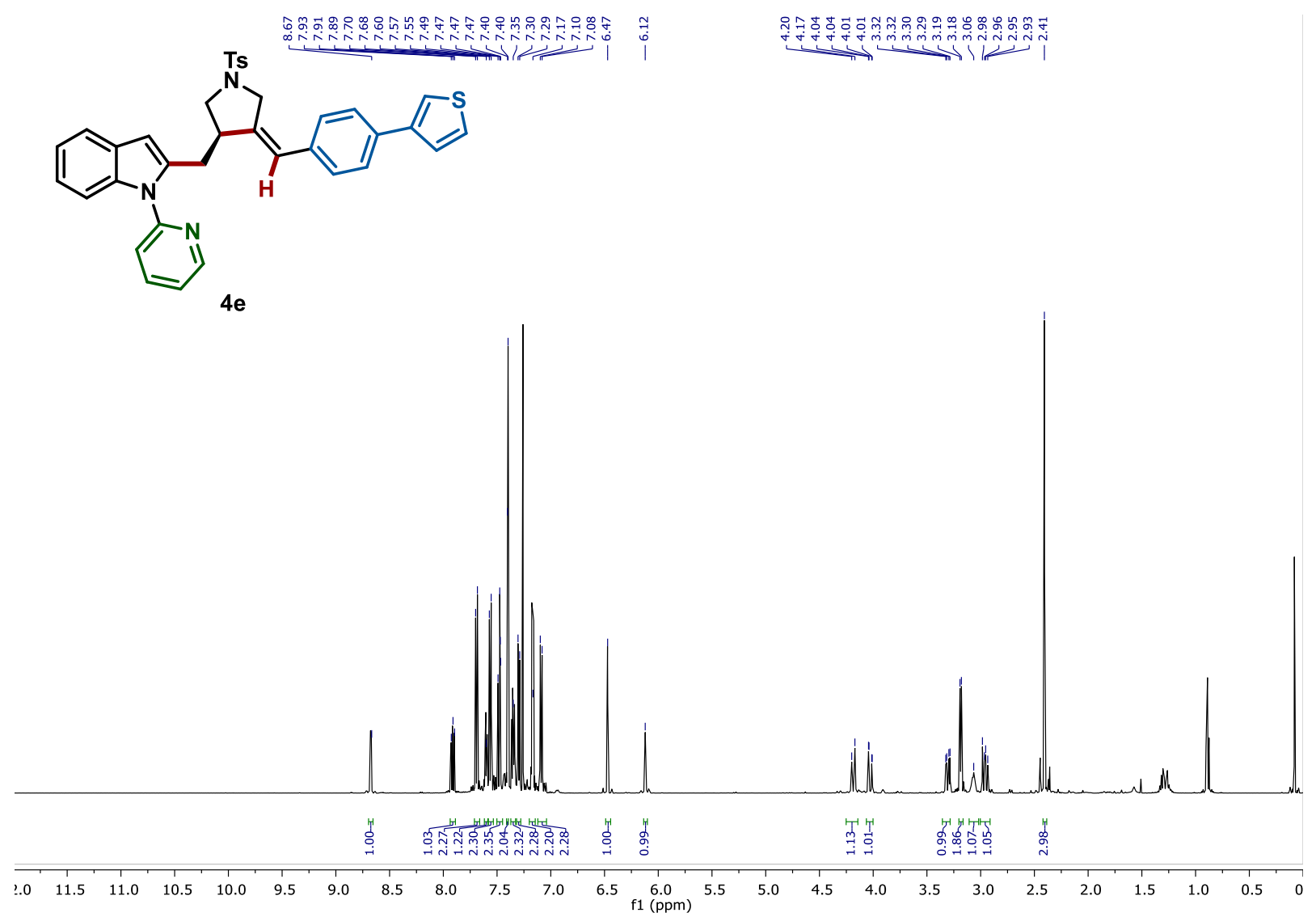

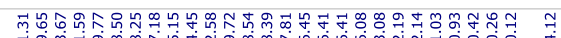

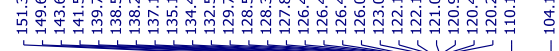

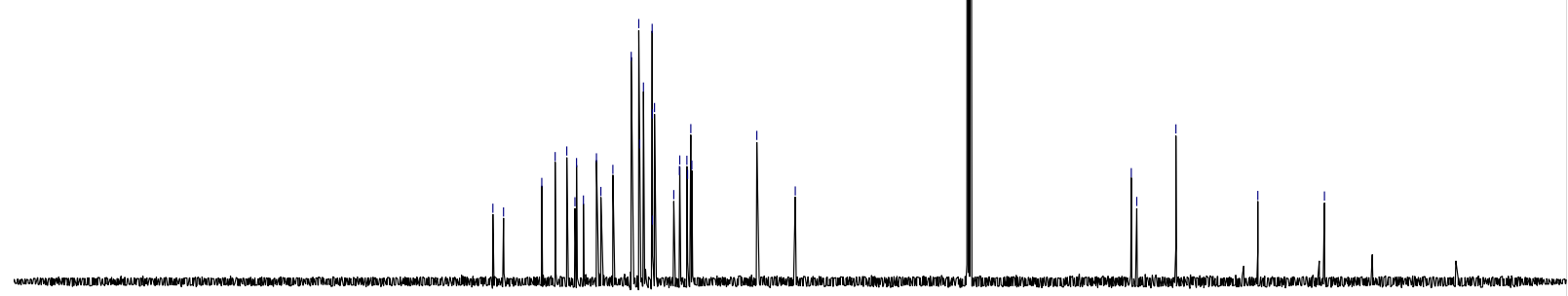

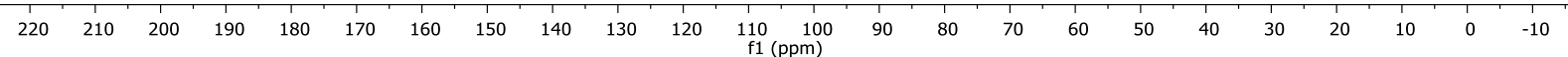



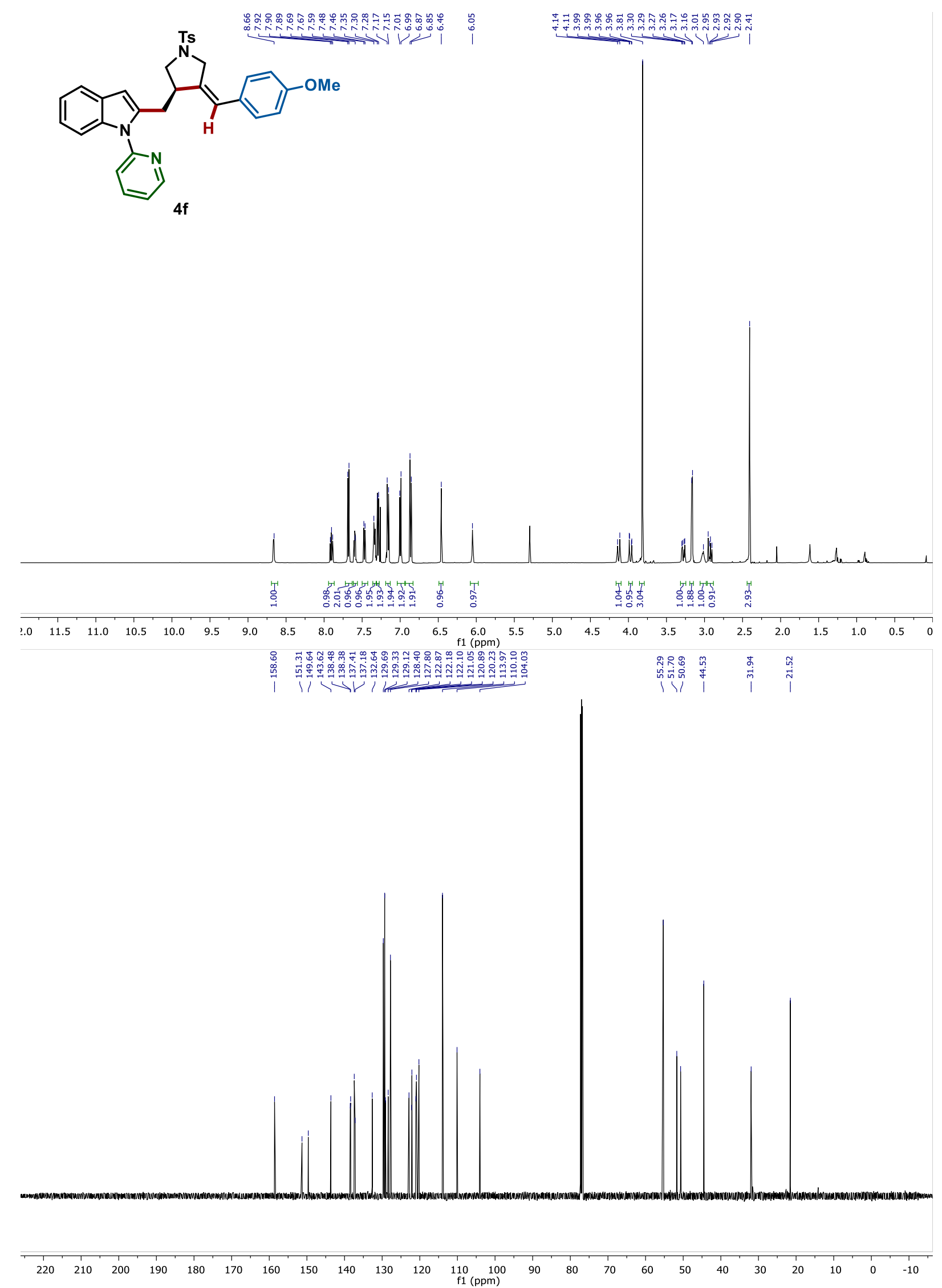

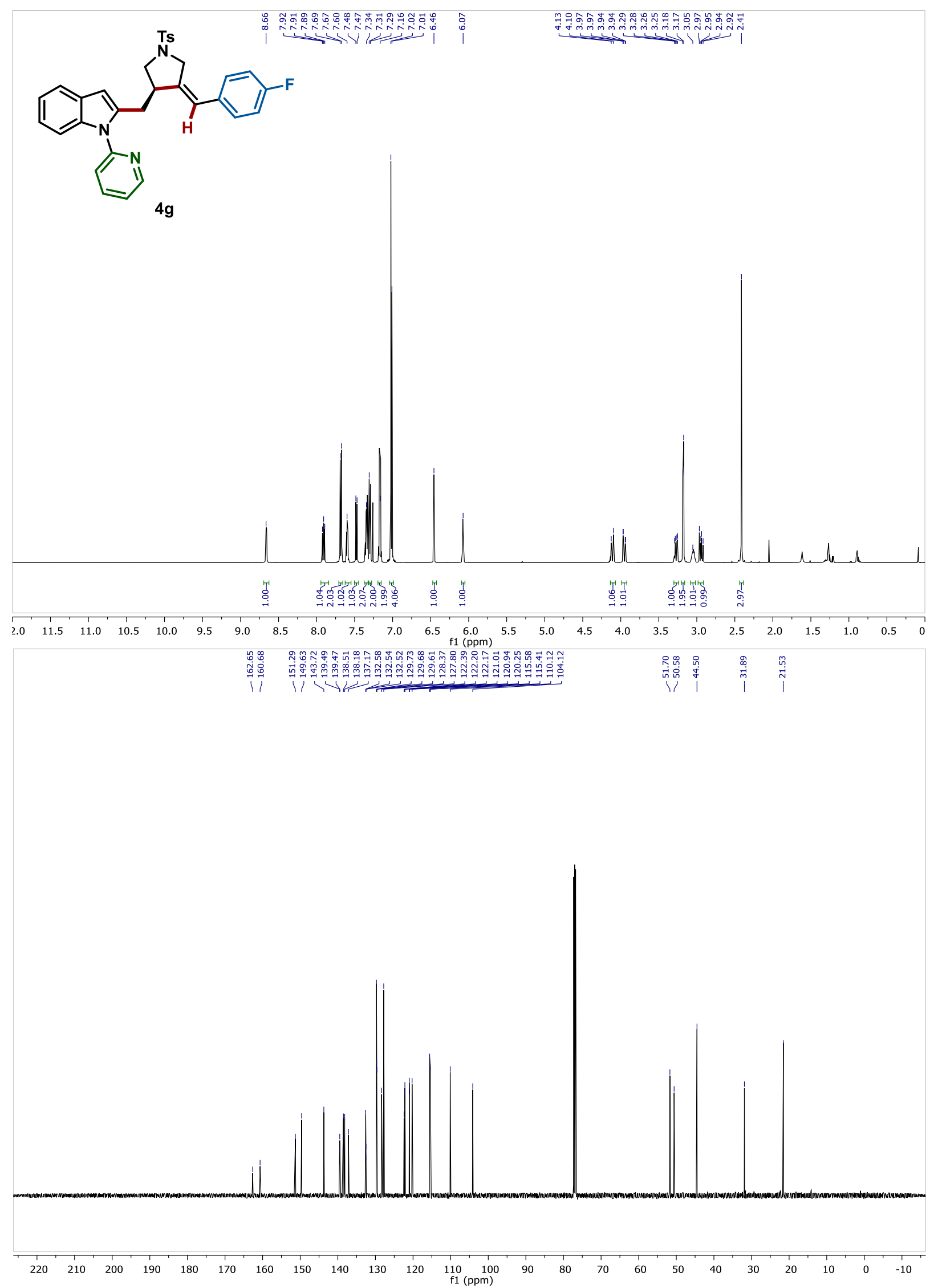


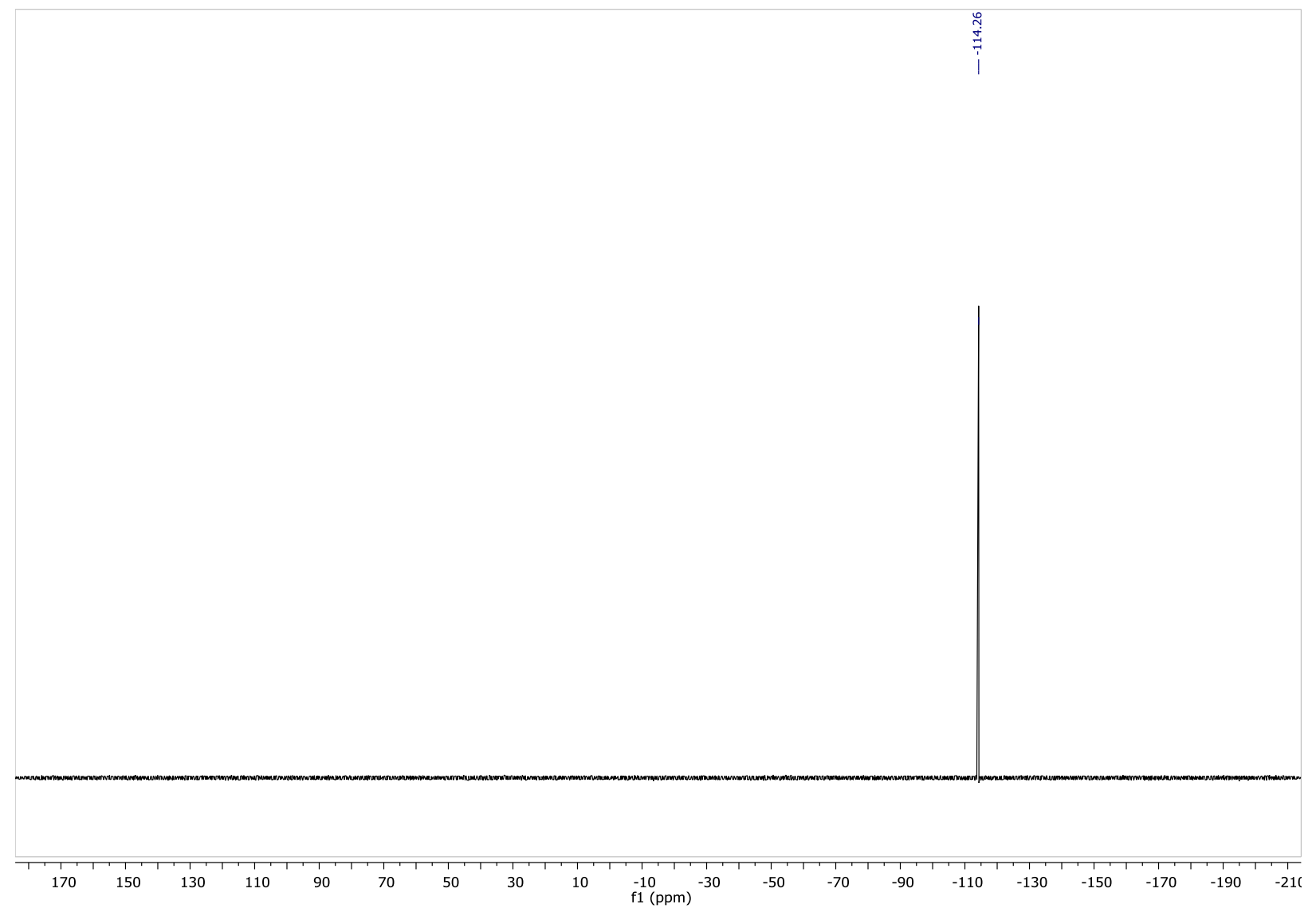



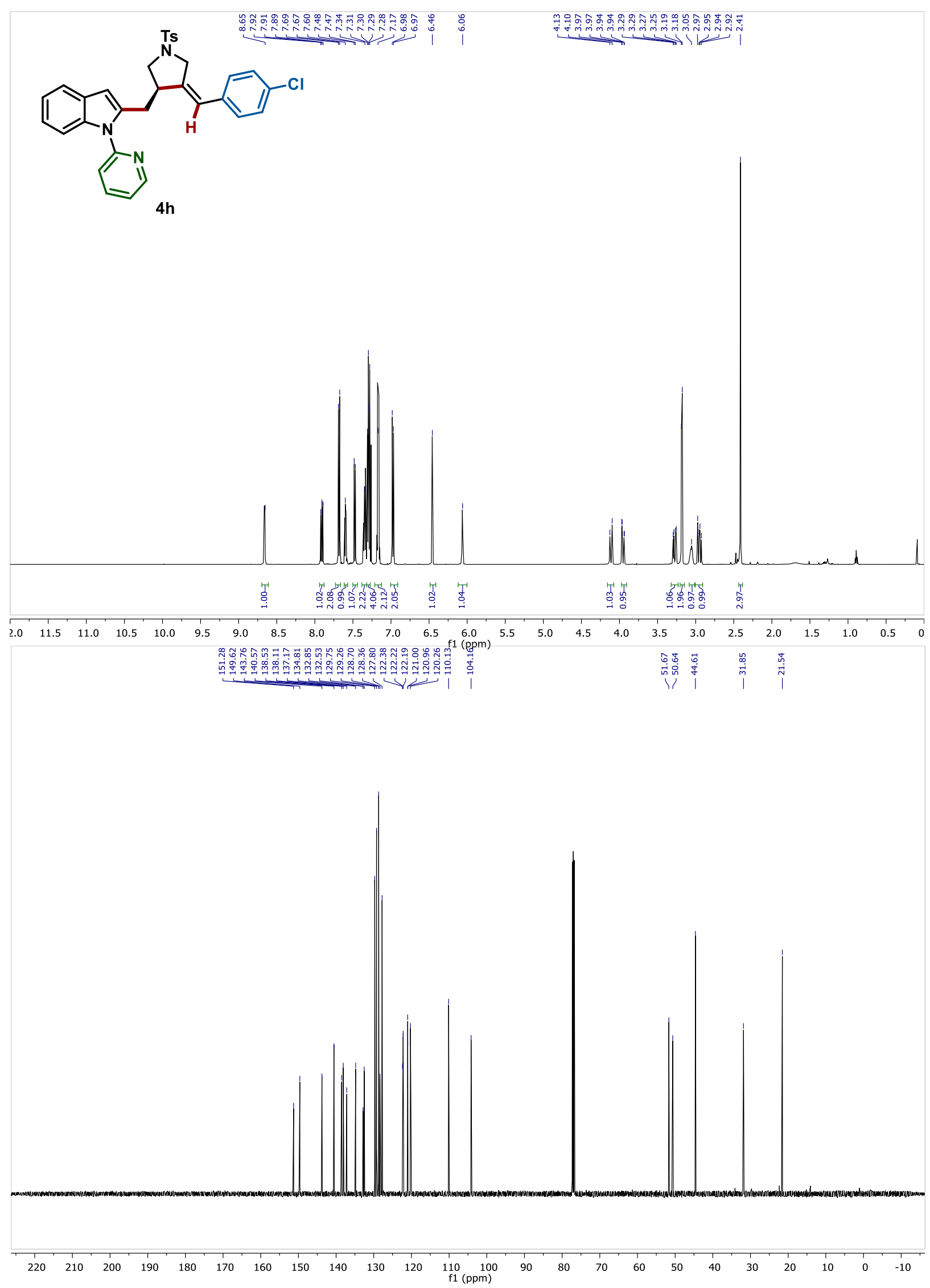

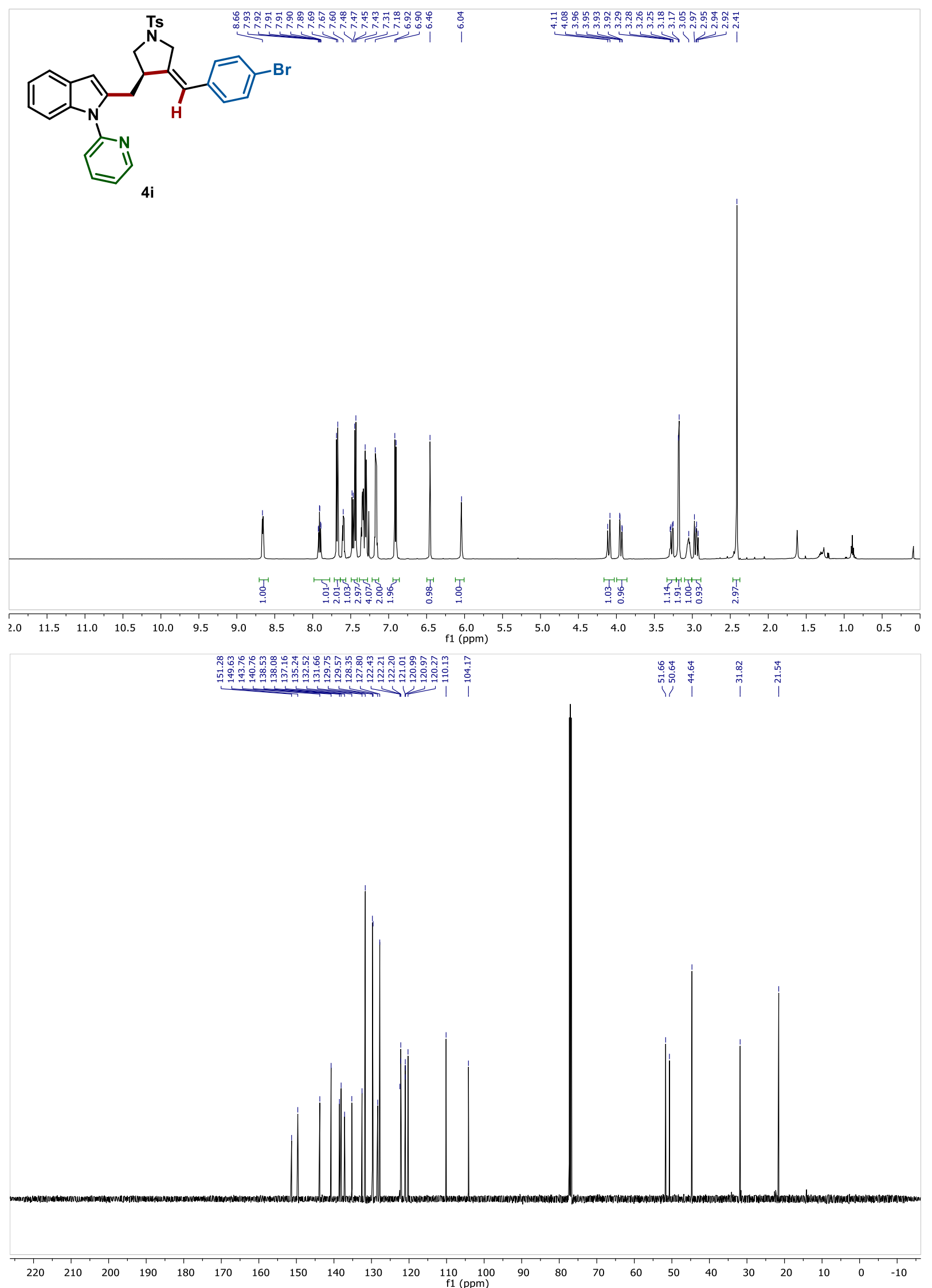


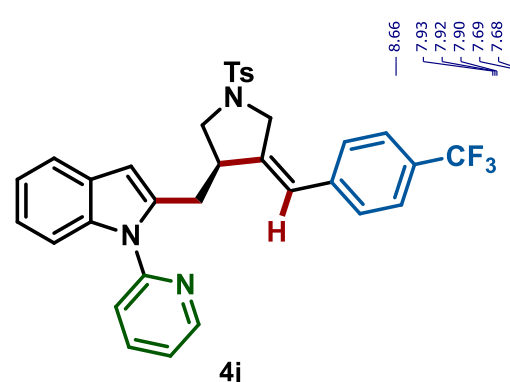

4j
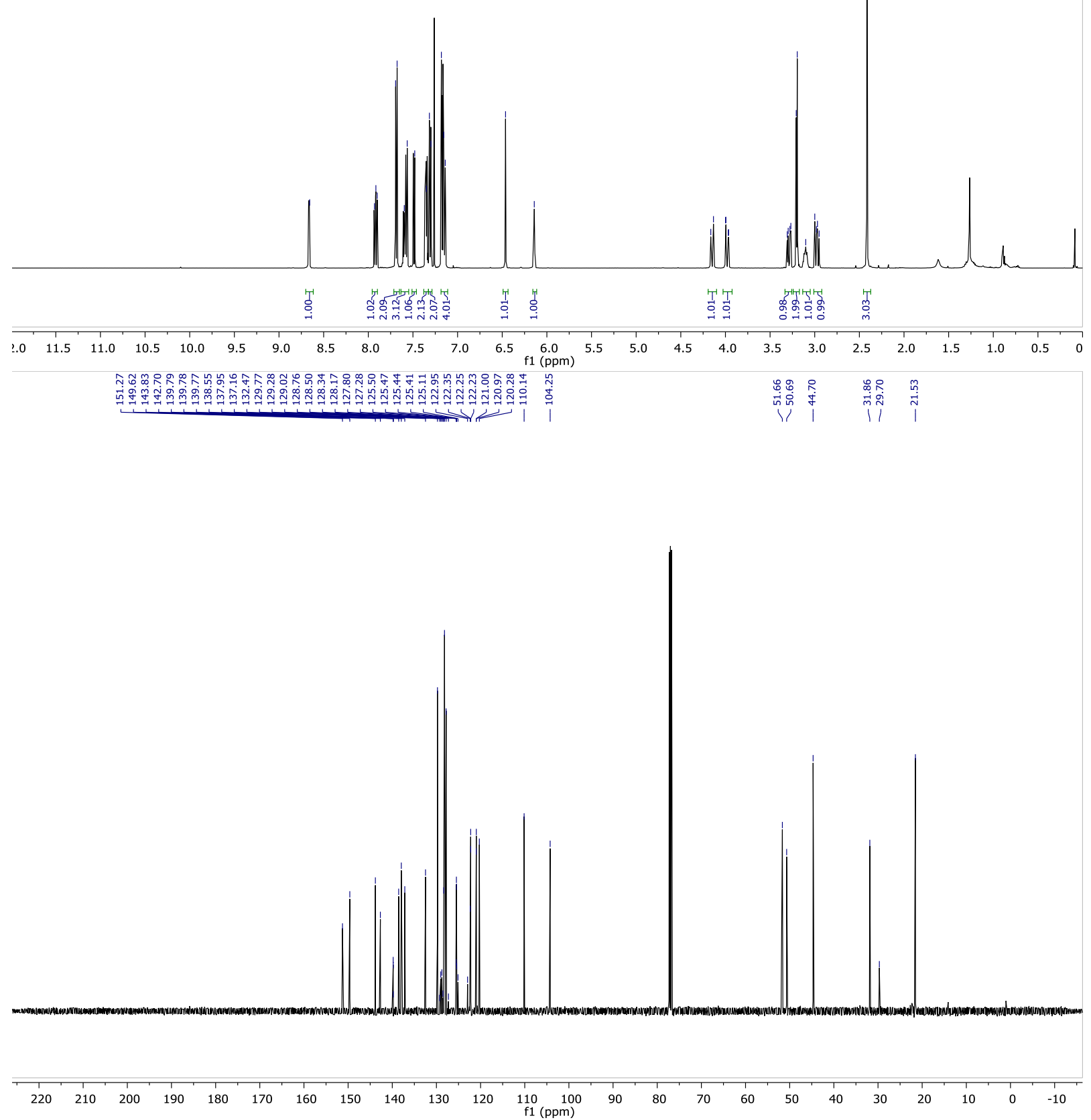


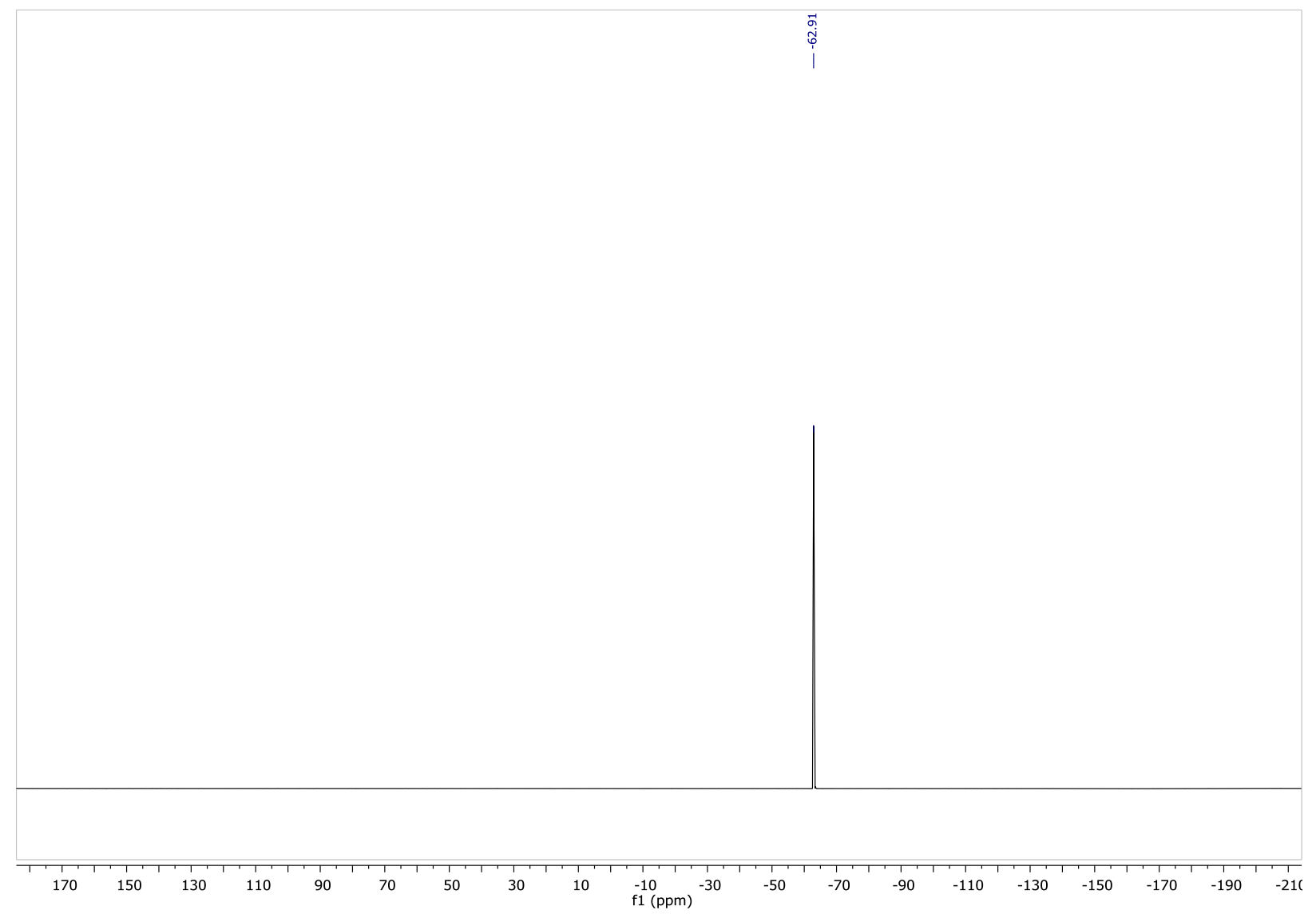



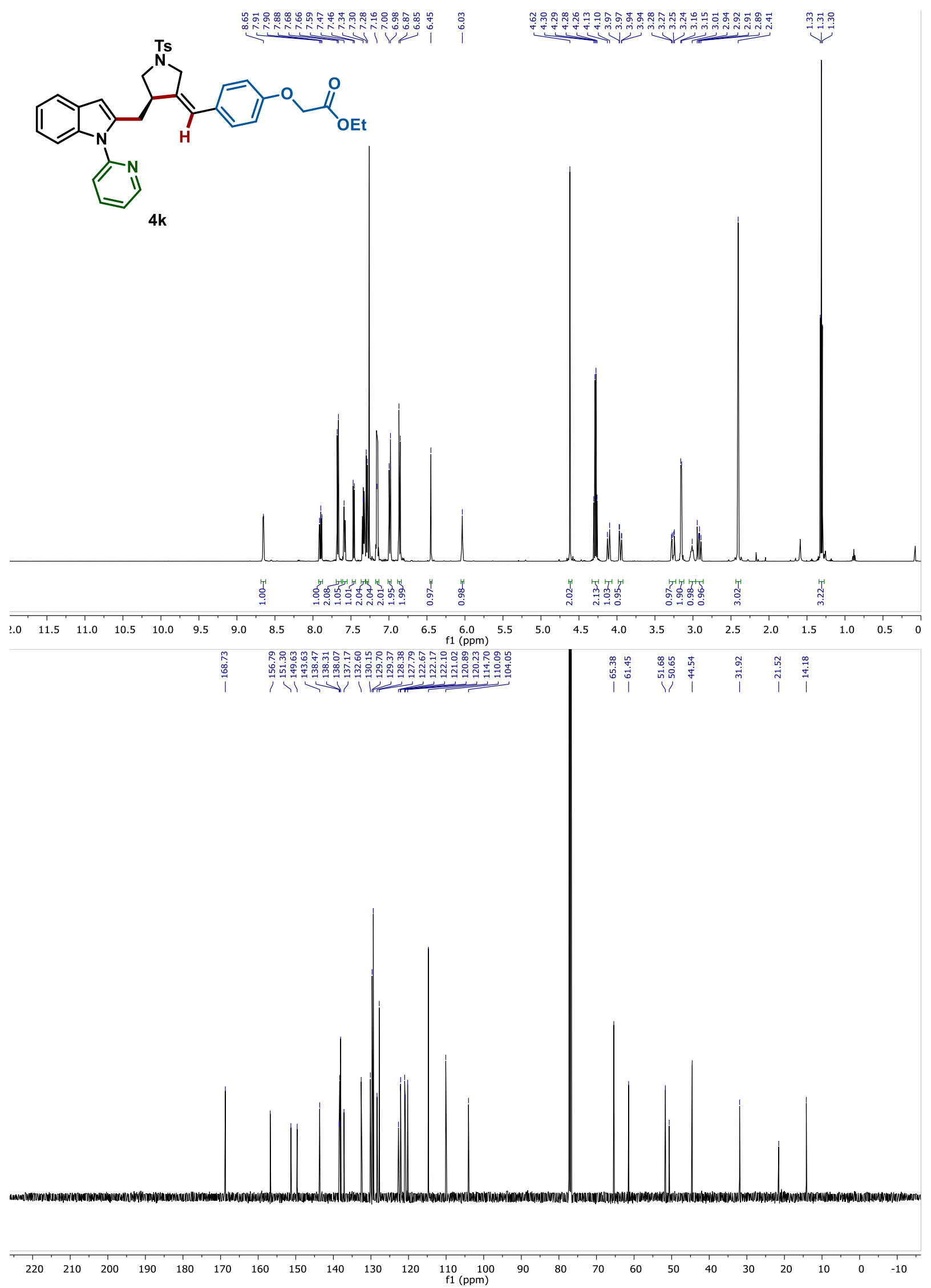

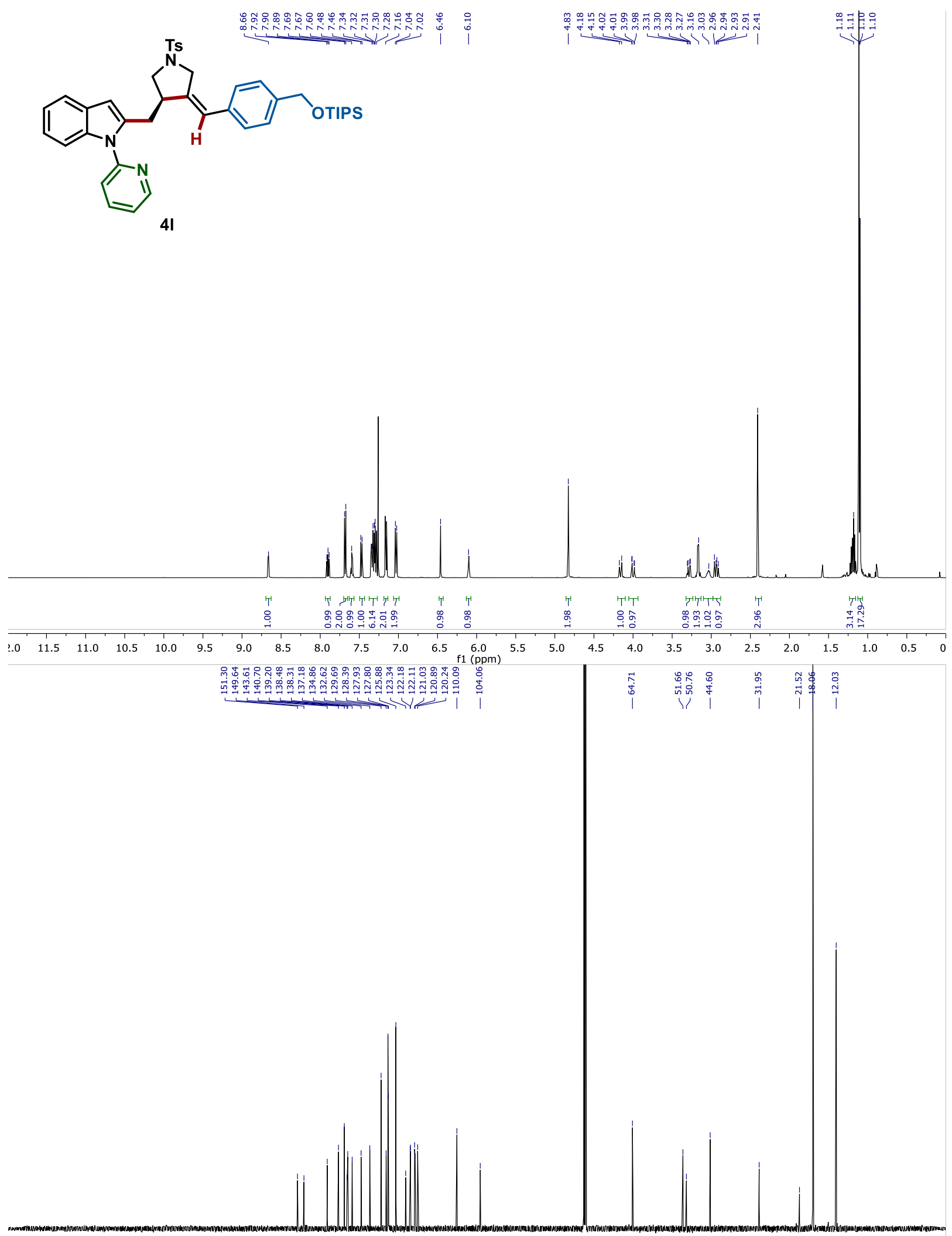

$\begin{array}{llllllllllll}220 & 210 & 200 & 190 & 180 & 170 & 160 & 150 & 140 & 130 & 120 & 110 \\ \mathrm{f} 1(\mathrm{ppm}) & 100\end{array}$ 

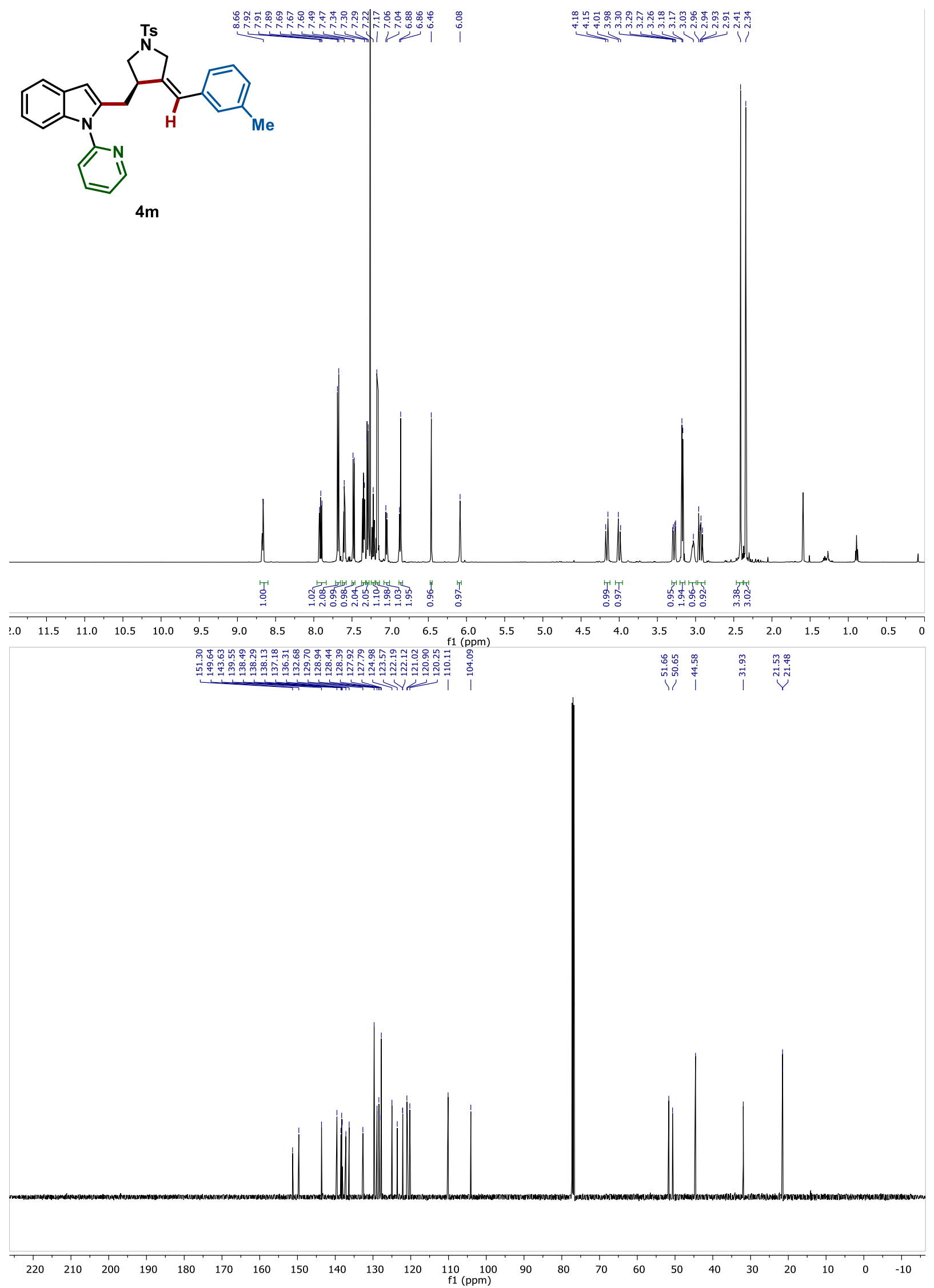

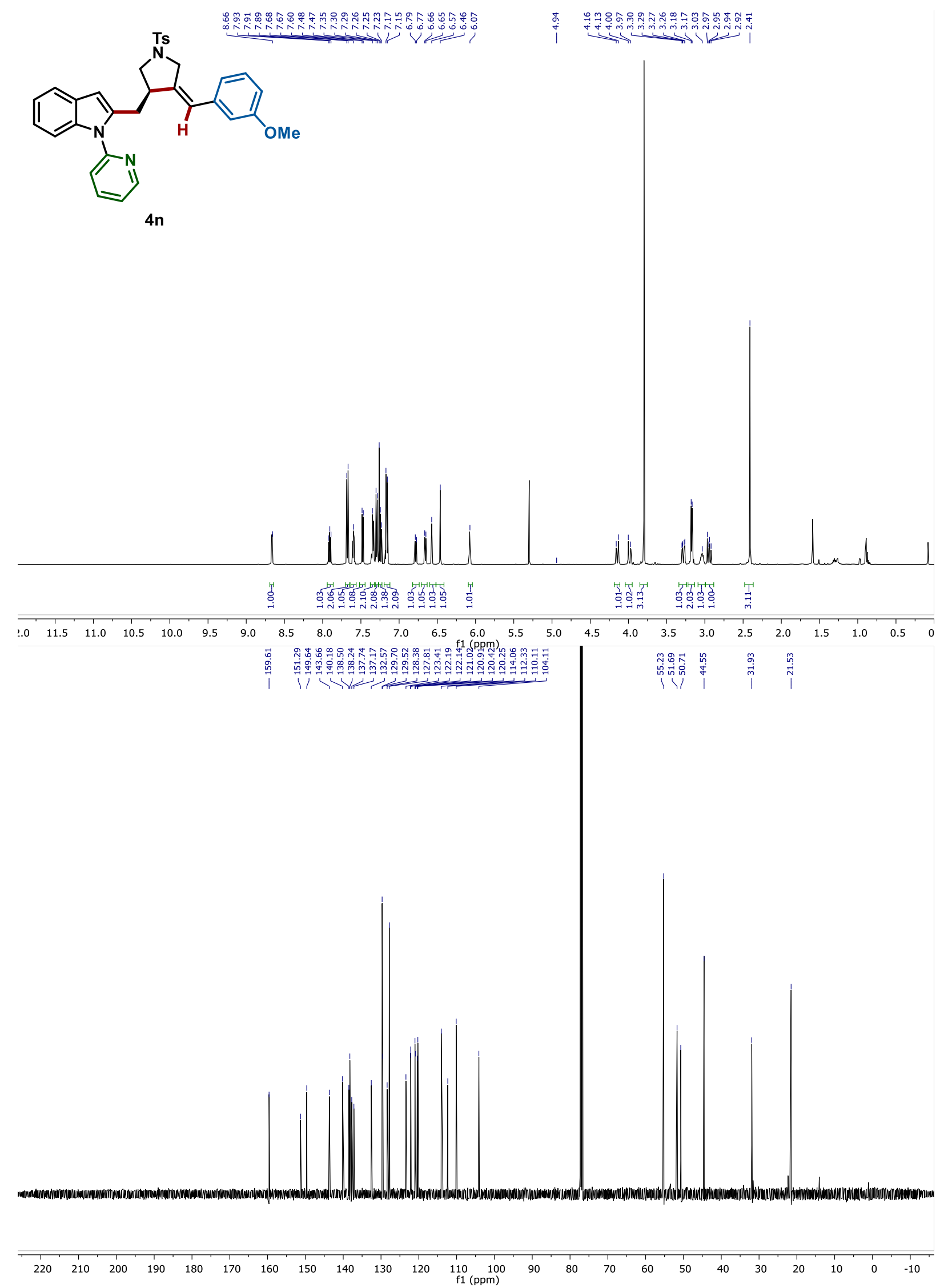

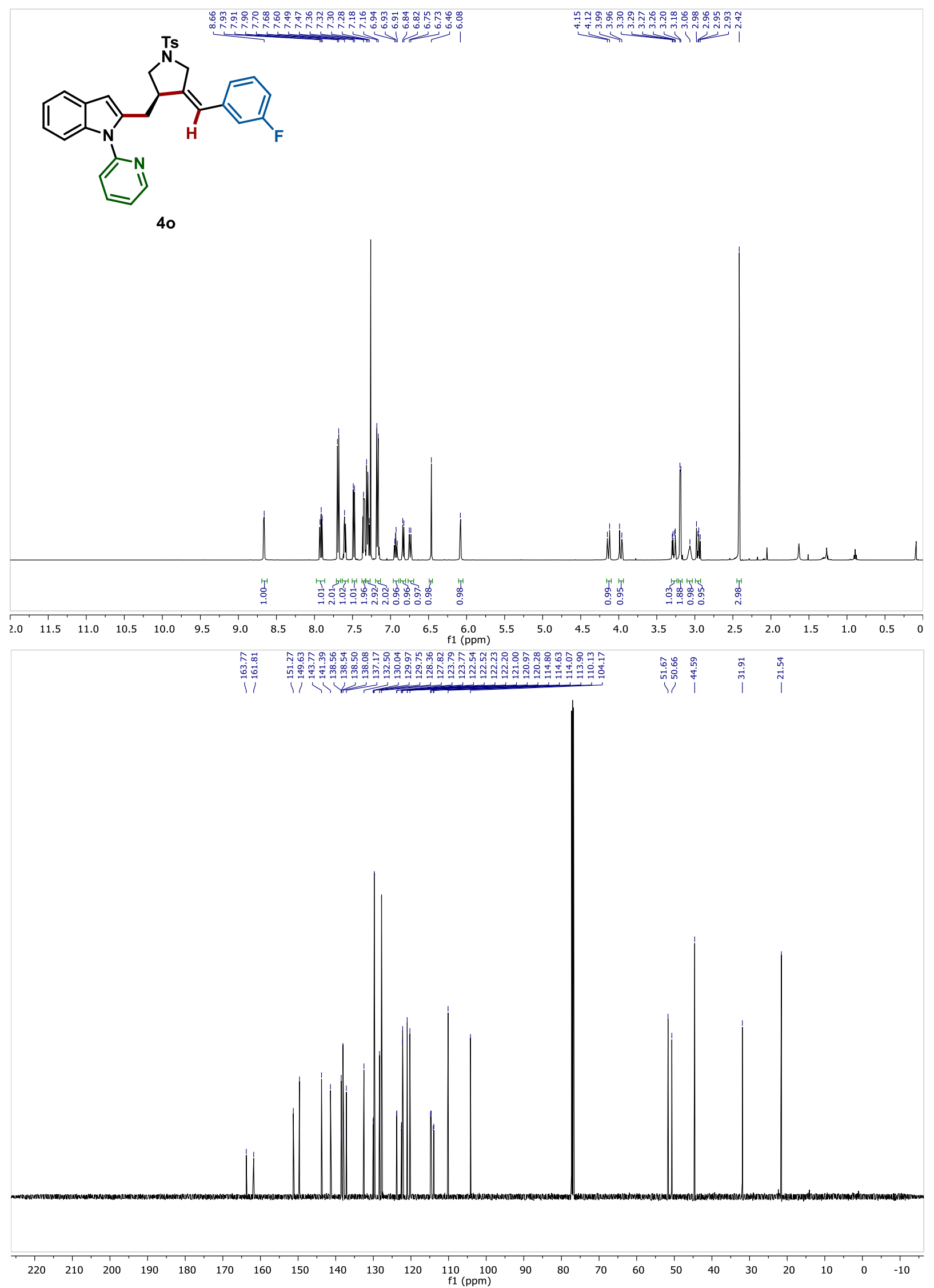


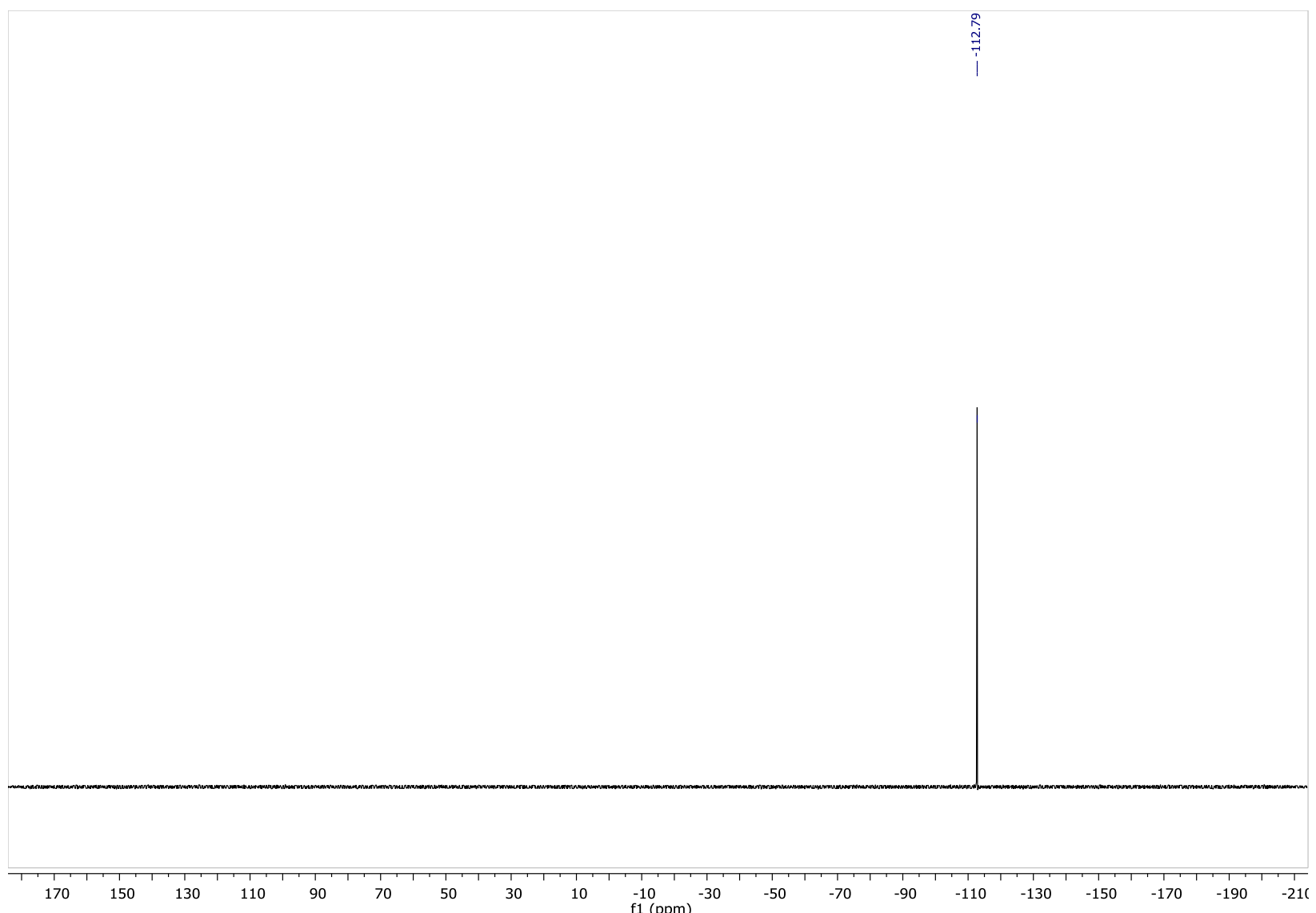




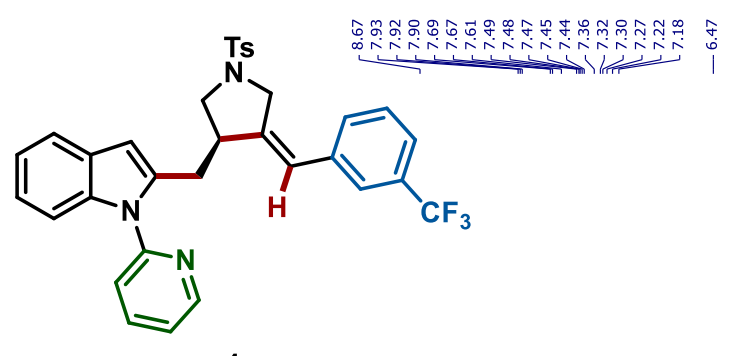

$4 p$
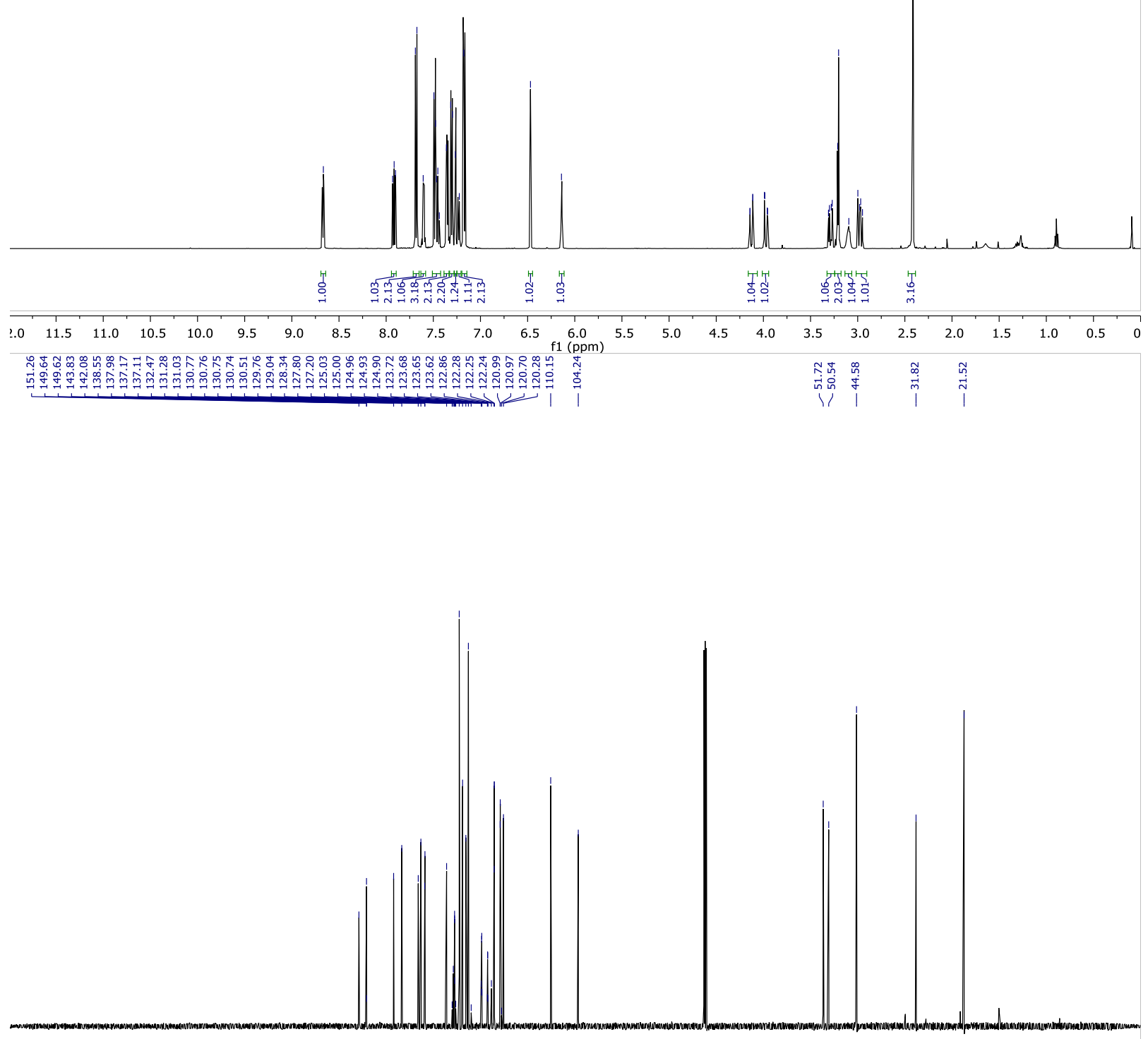

$\begin{array}{lllllllllllll}220 & 210 & 200 & 190 & 180 & 170 & 160 & 150 & 140 & 130 & 120 & 110 & 100\end{array}$ 


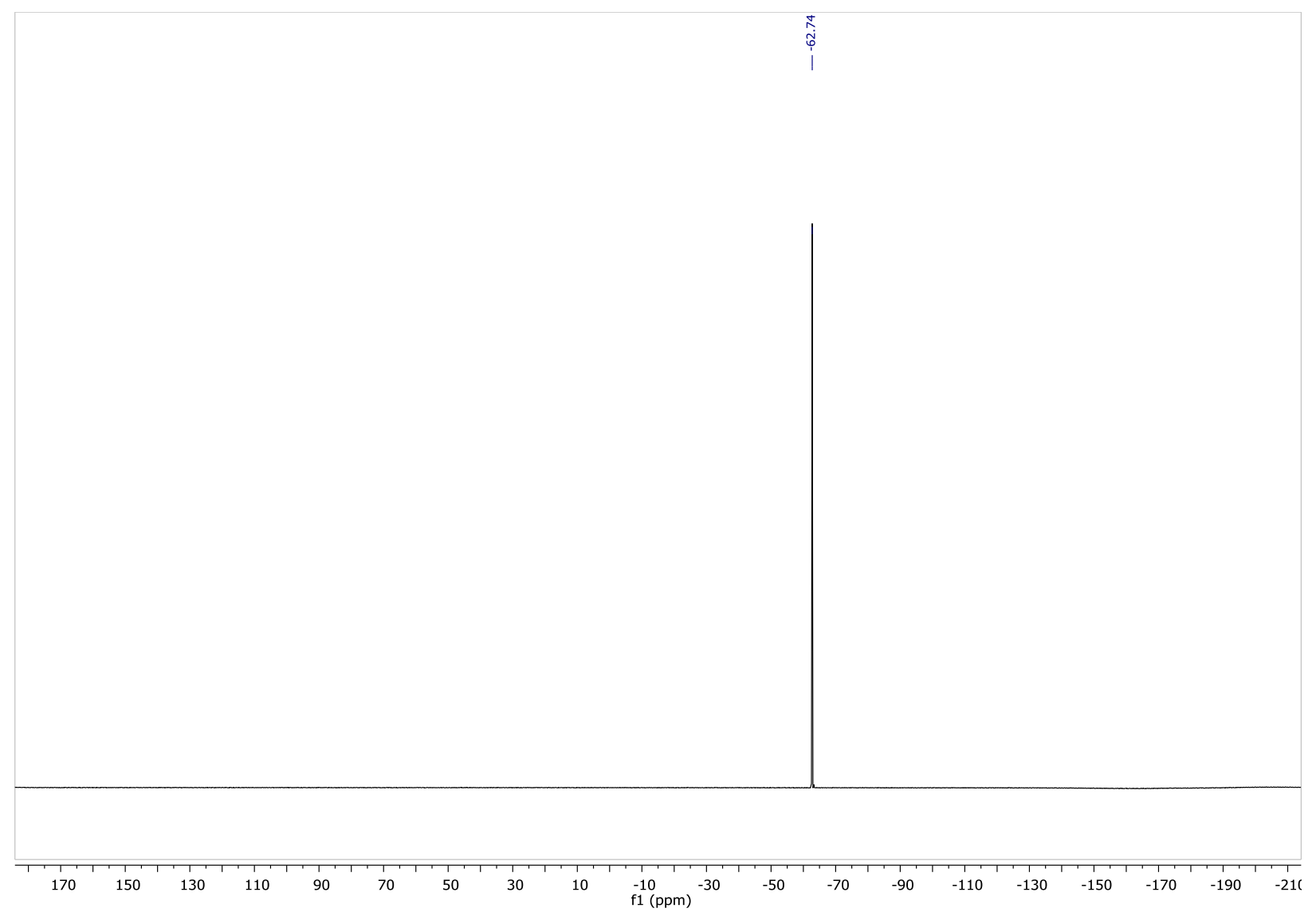



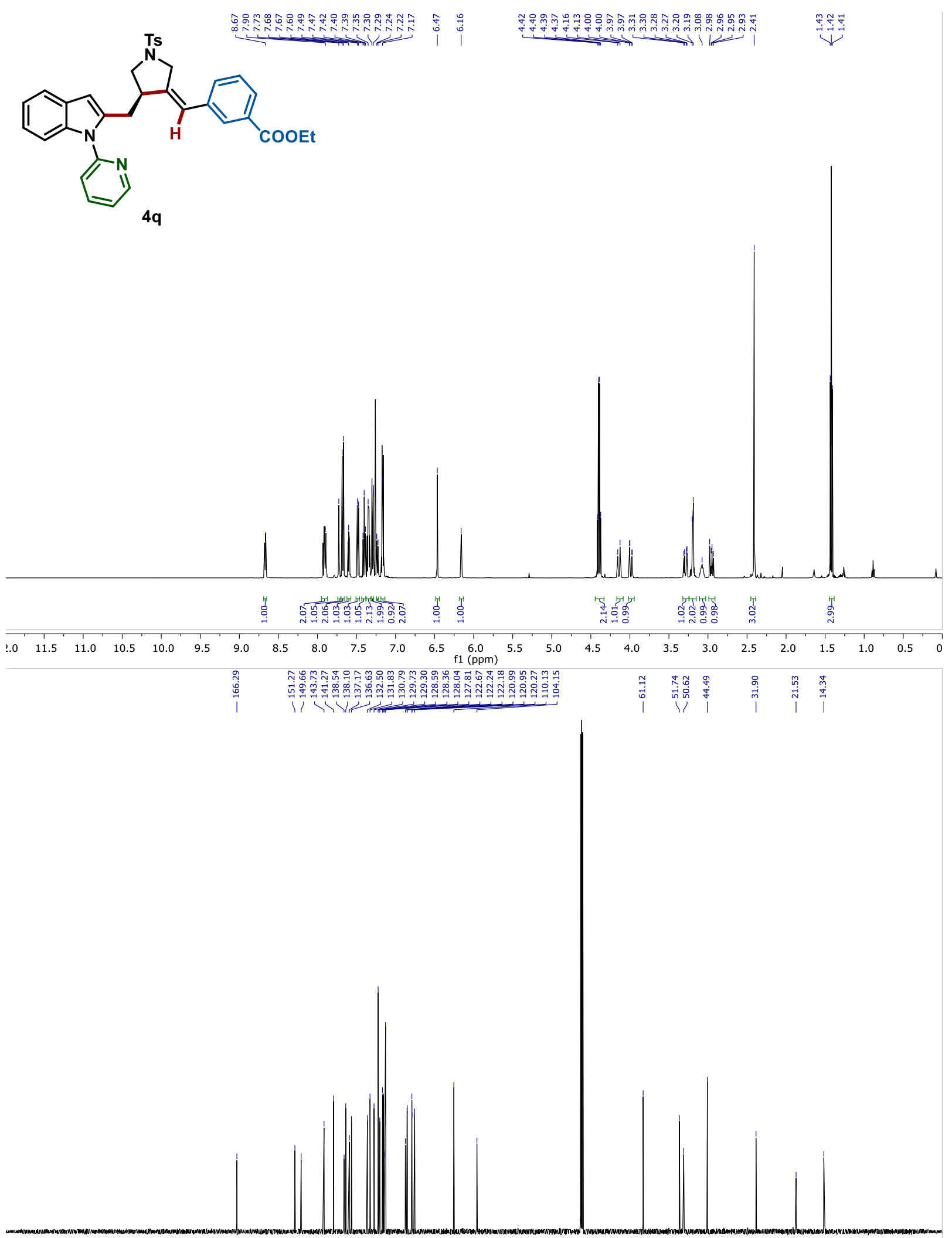

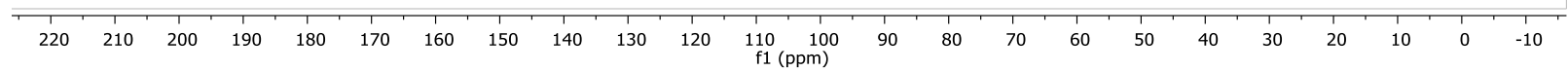



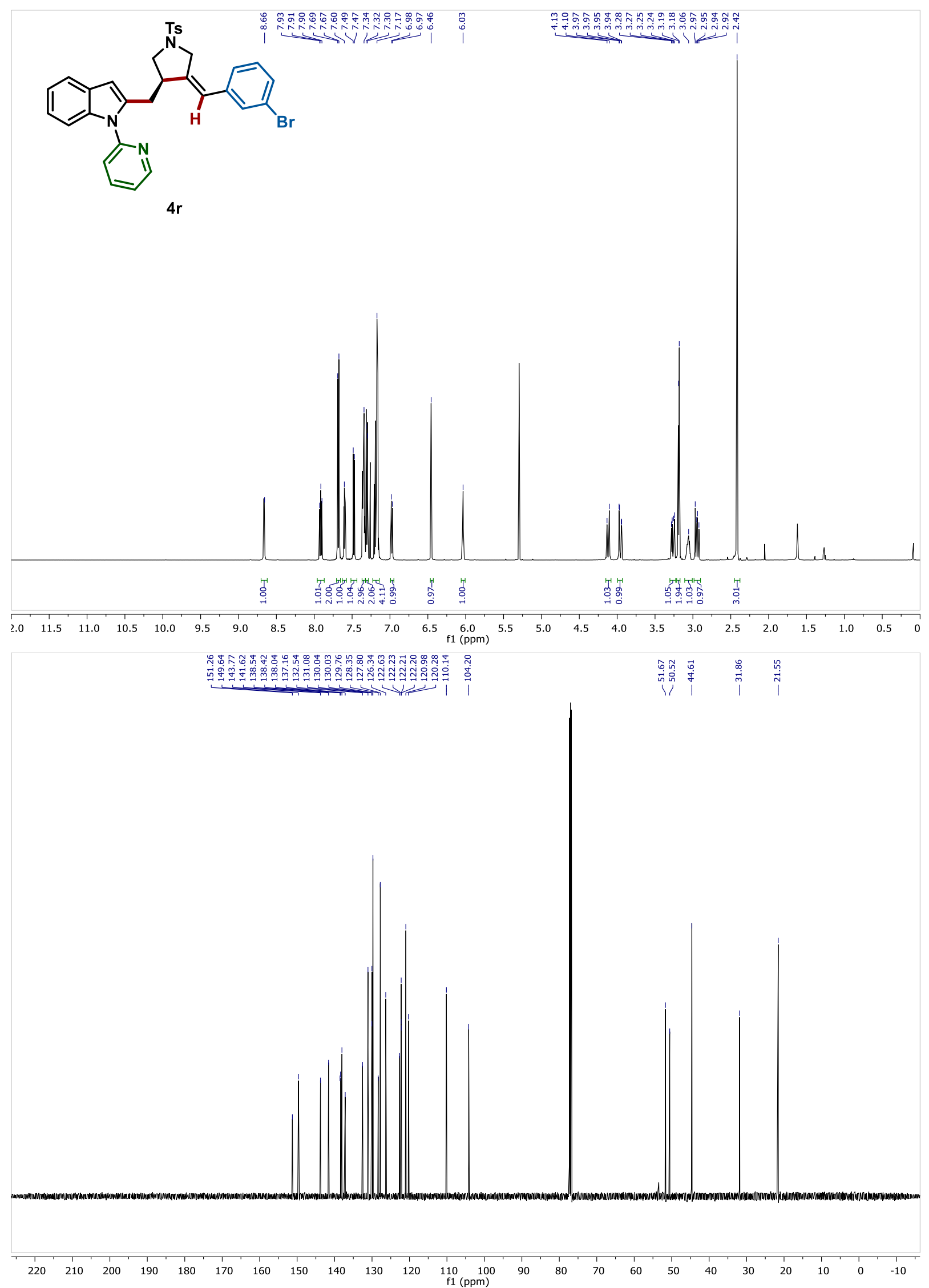

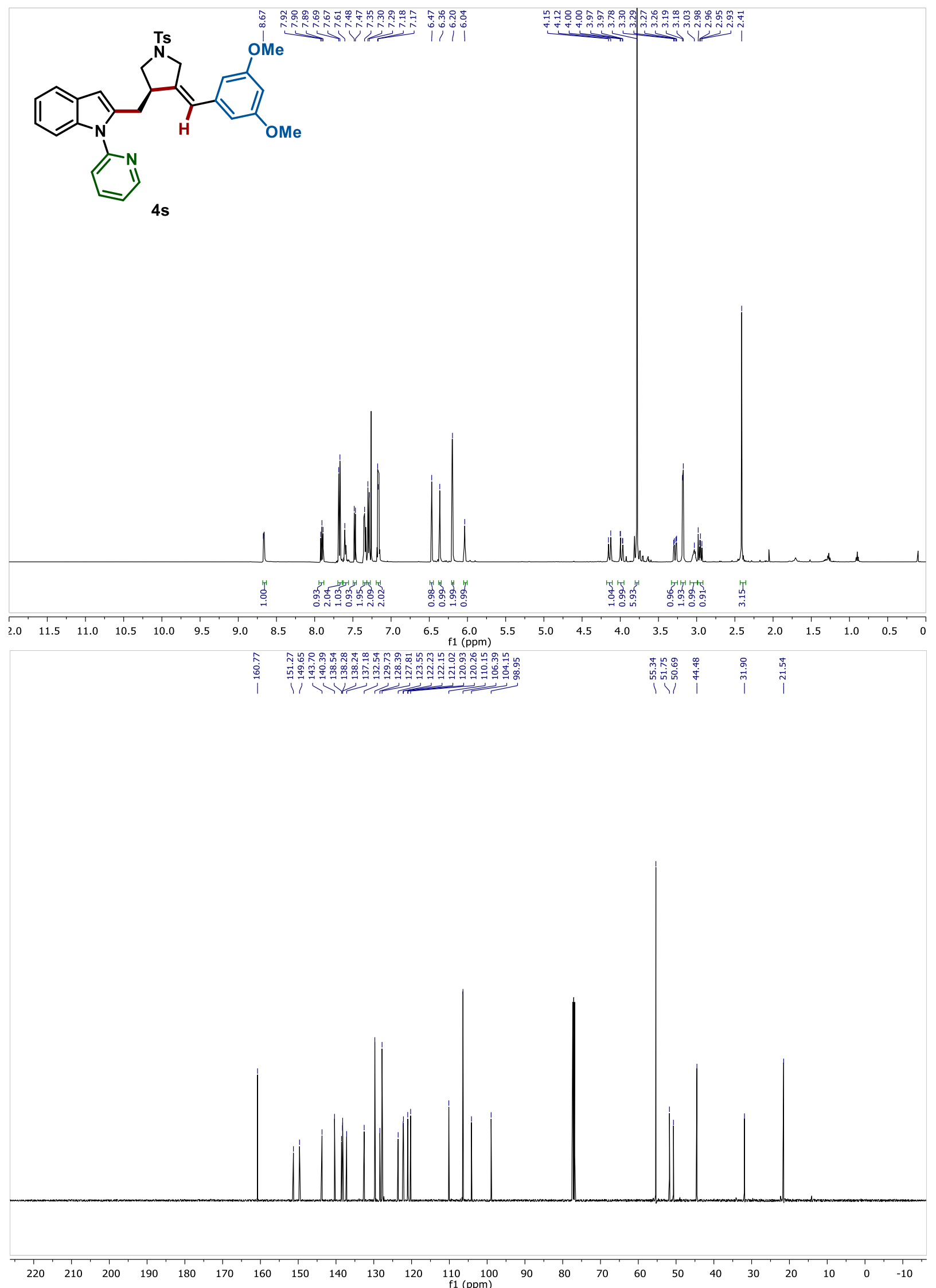

S181 

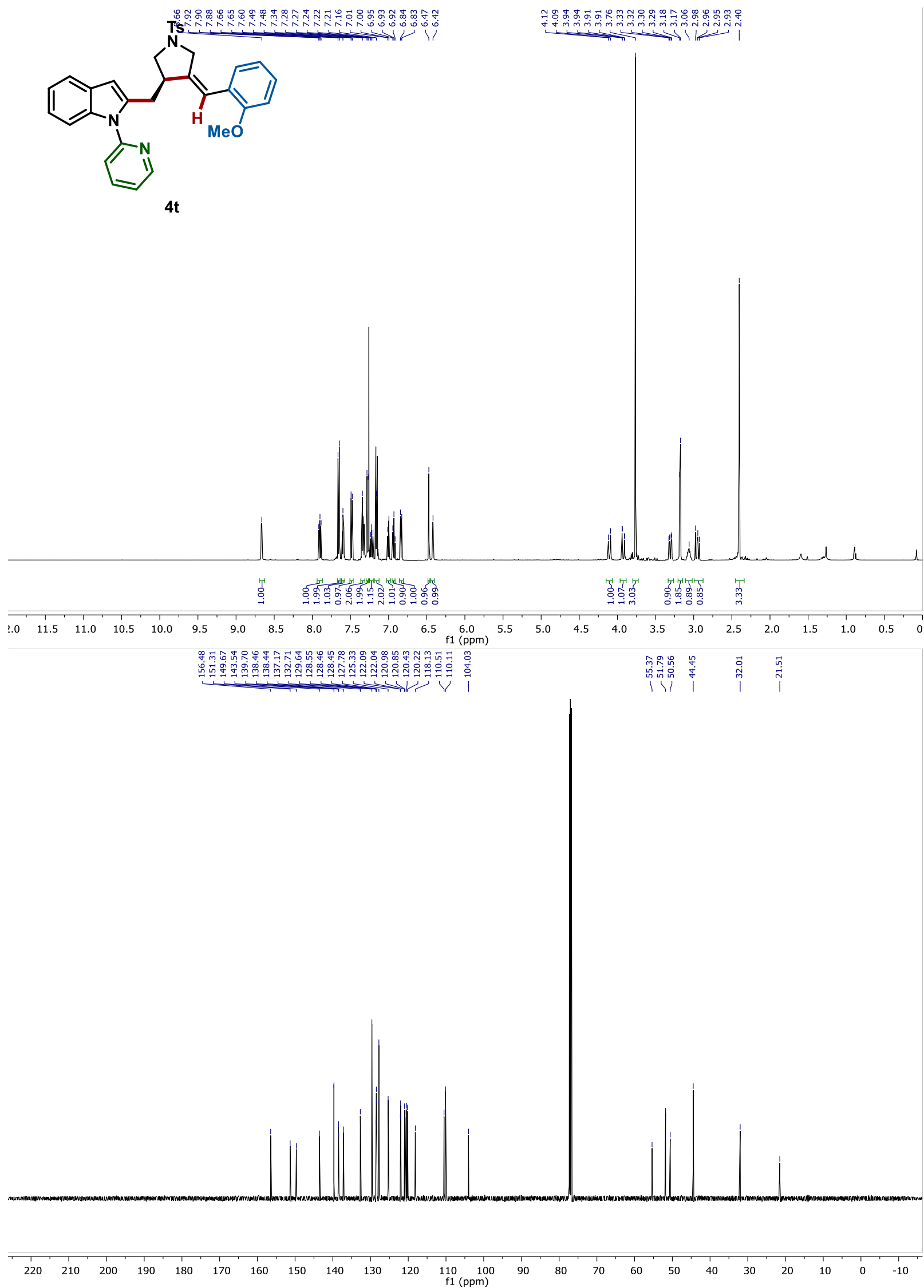

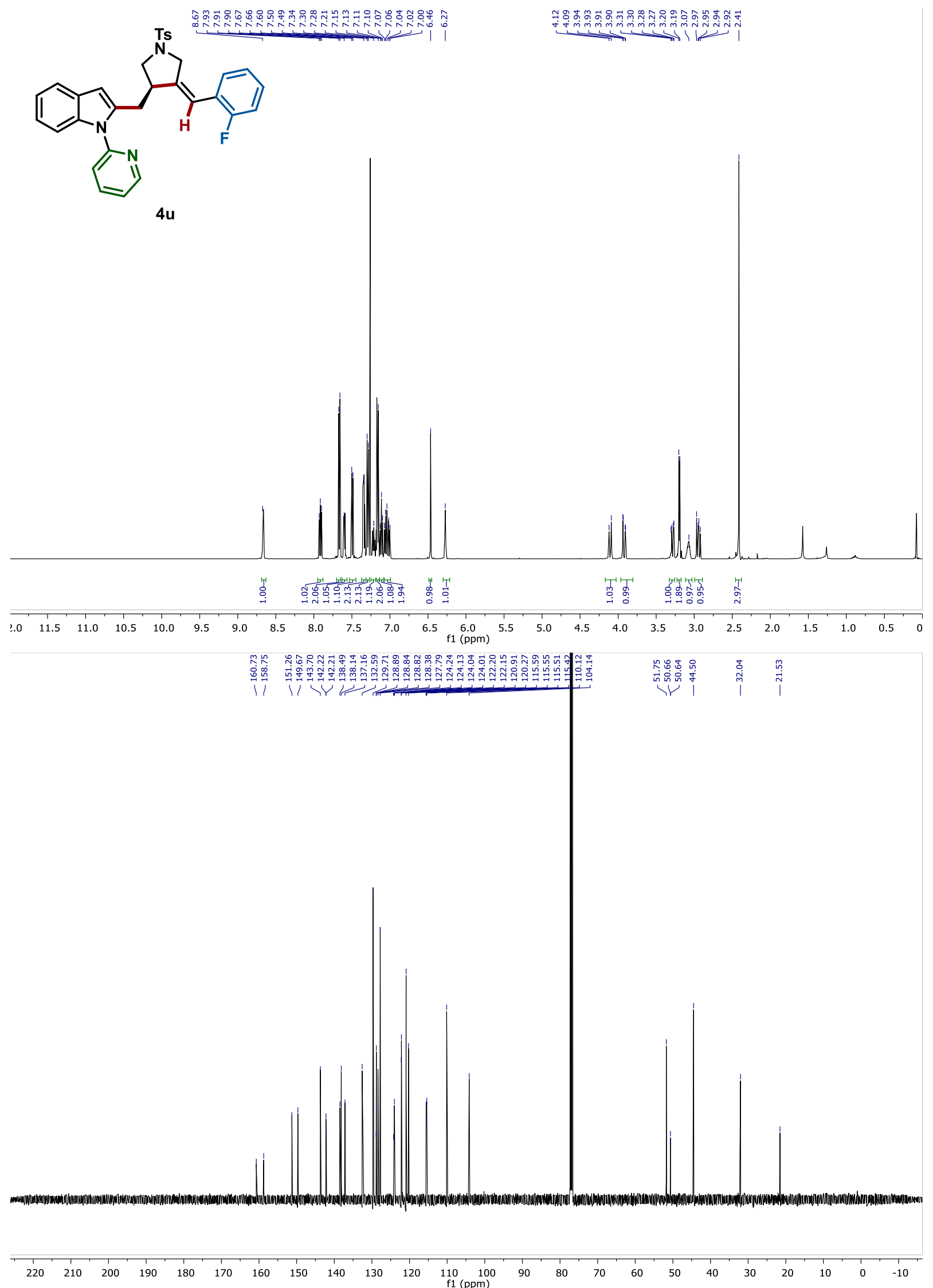


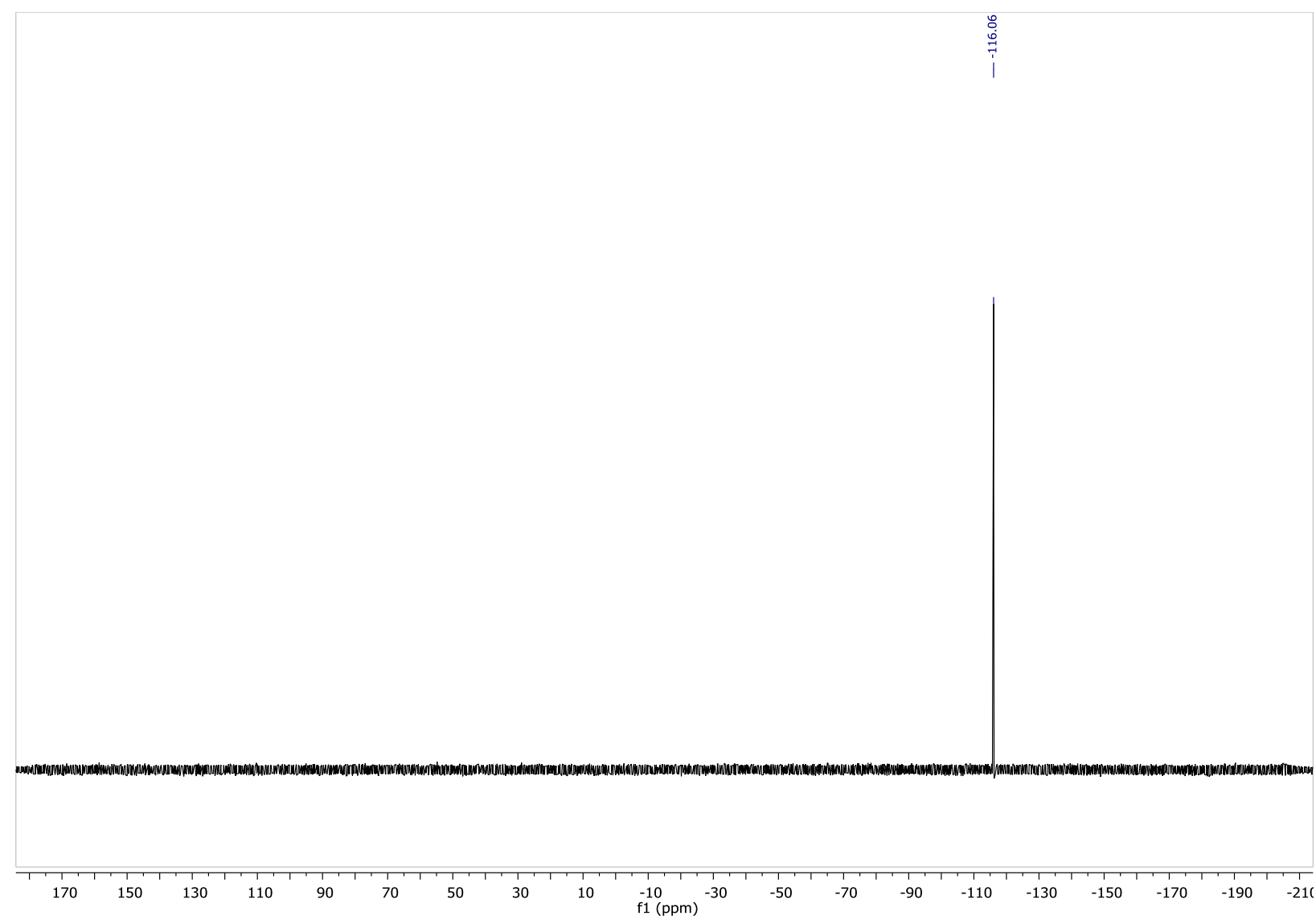



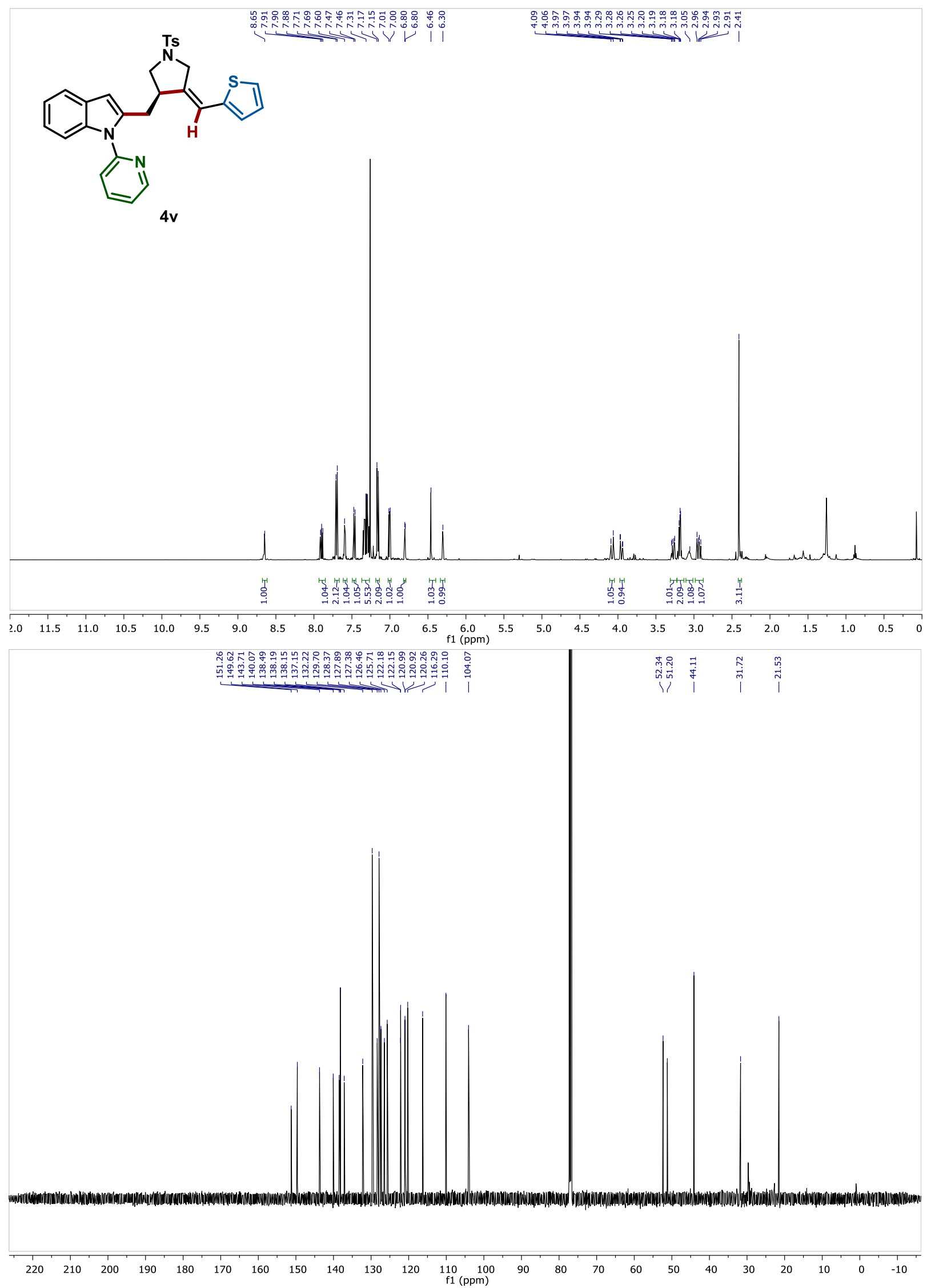

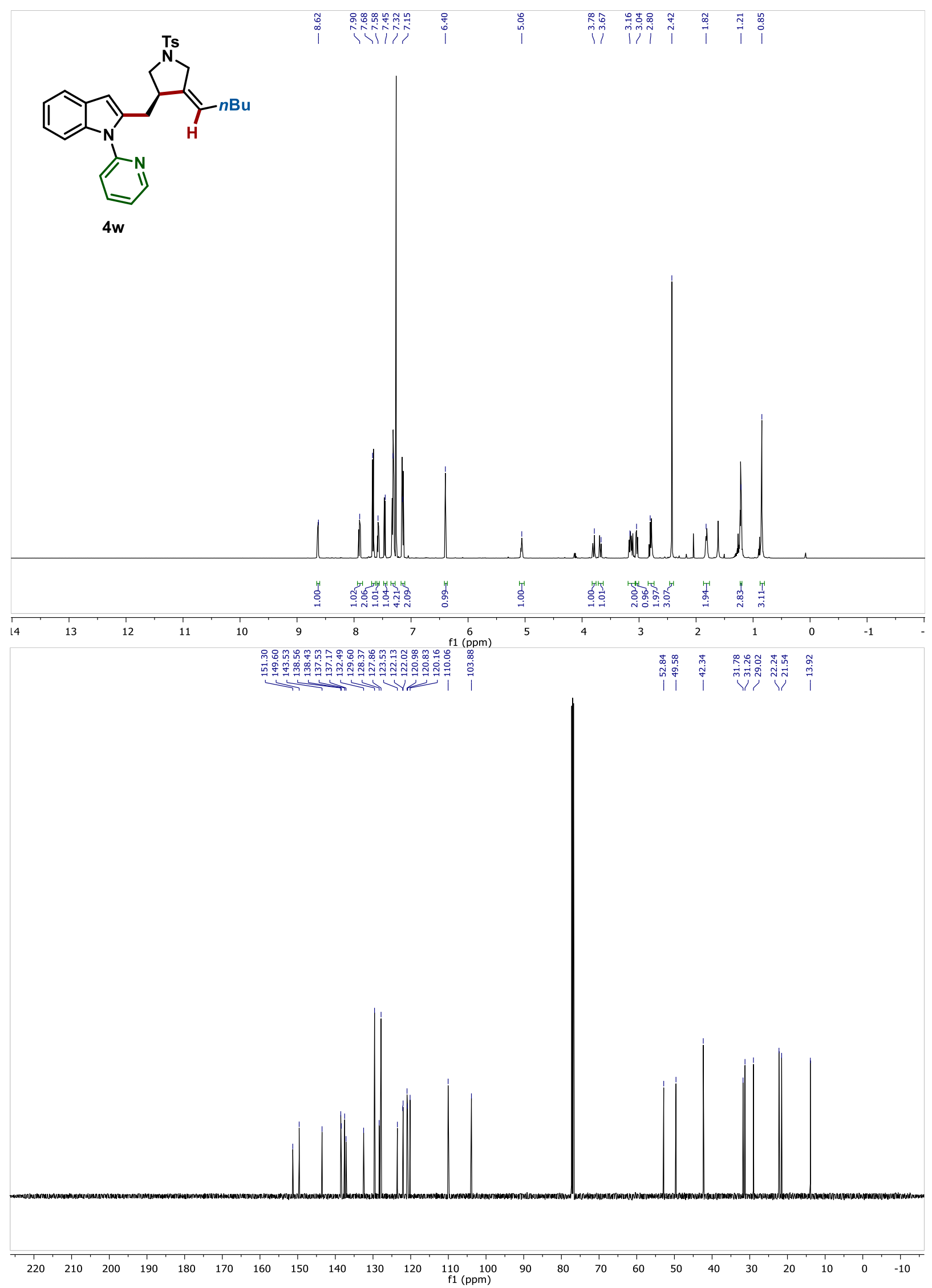

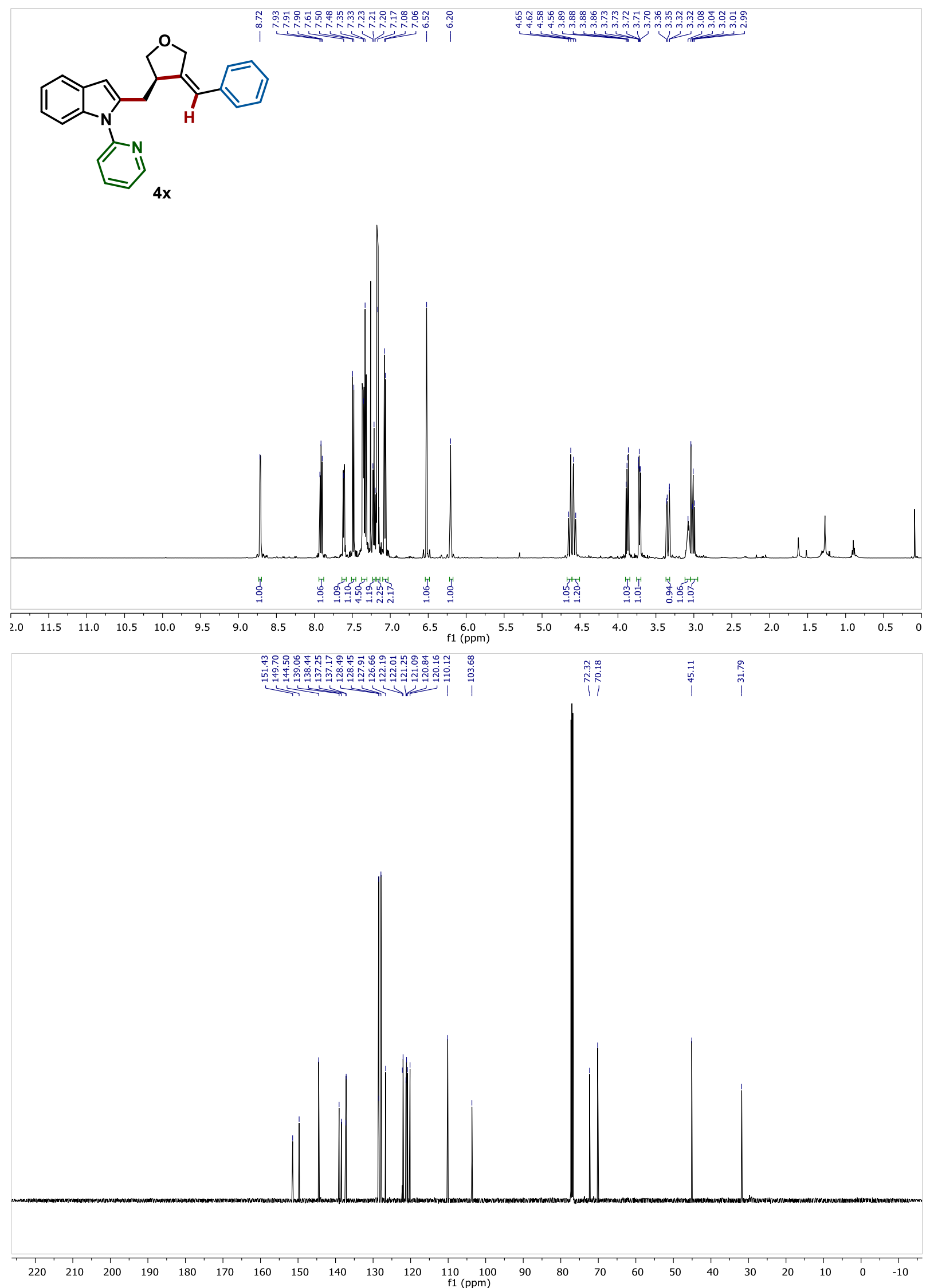

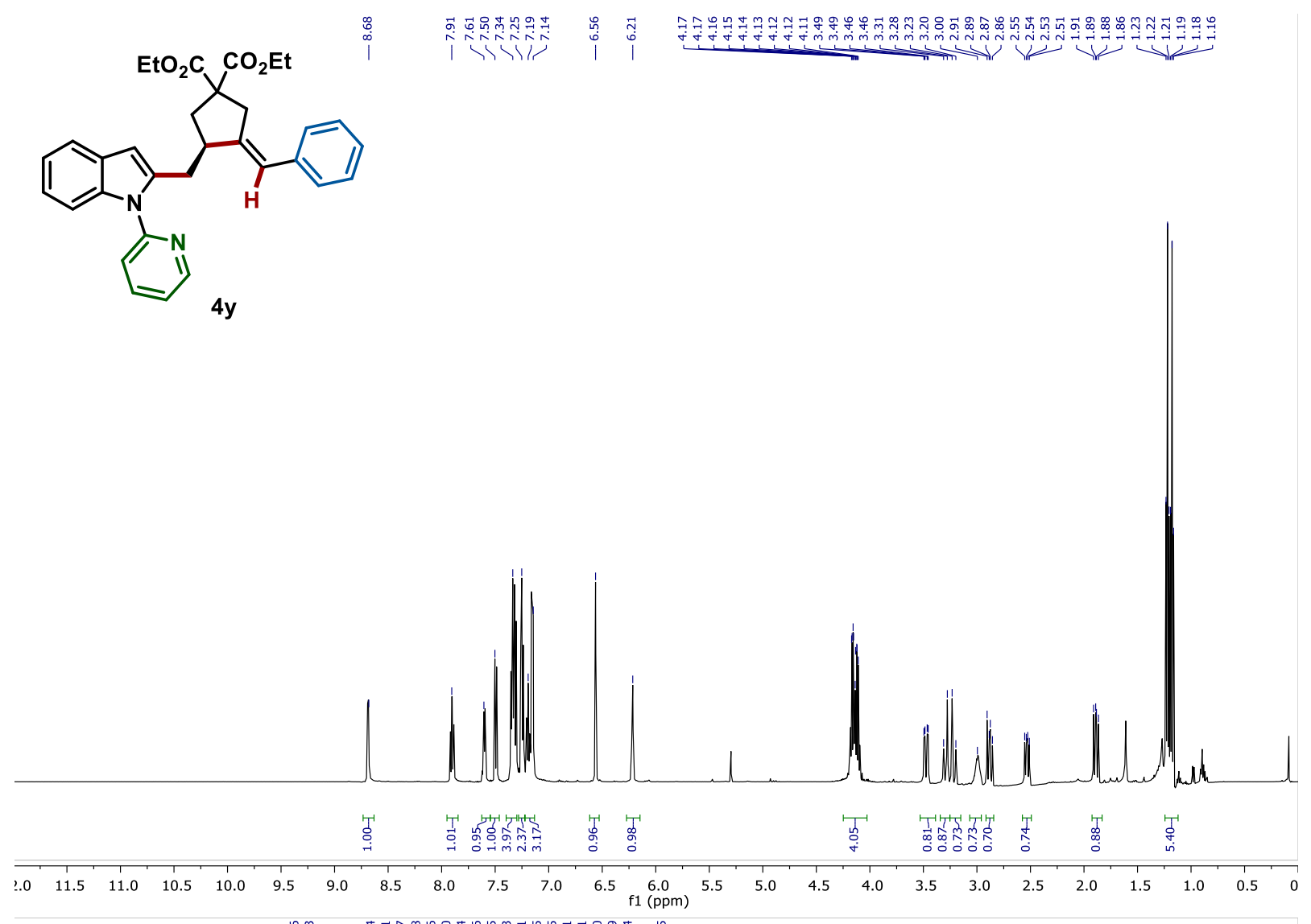
V
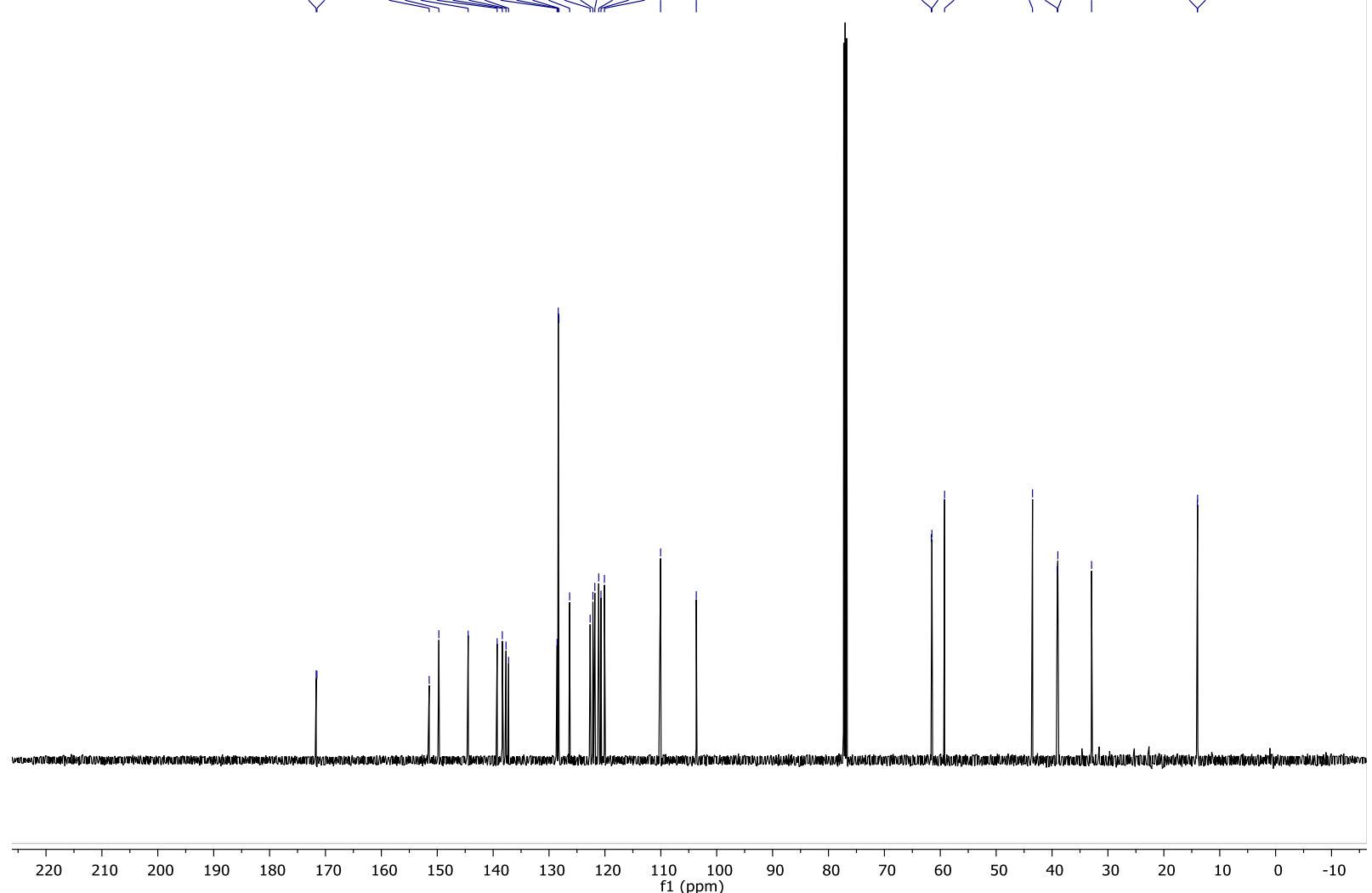

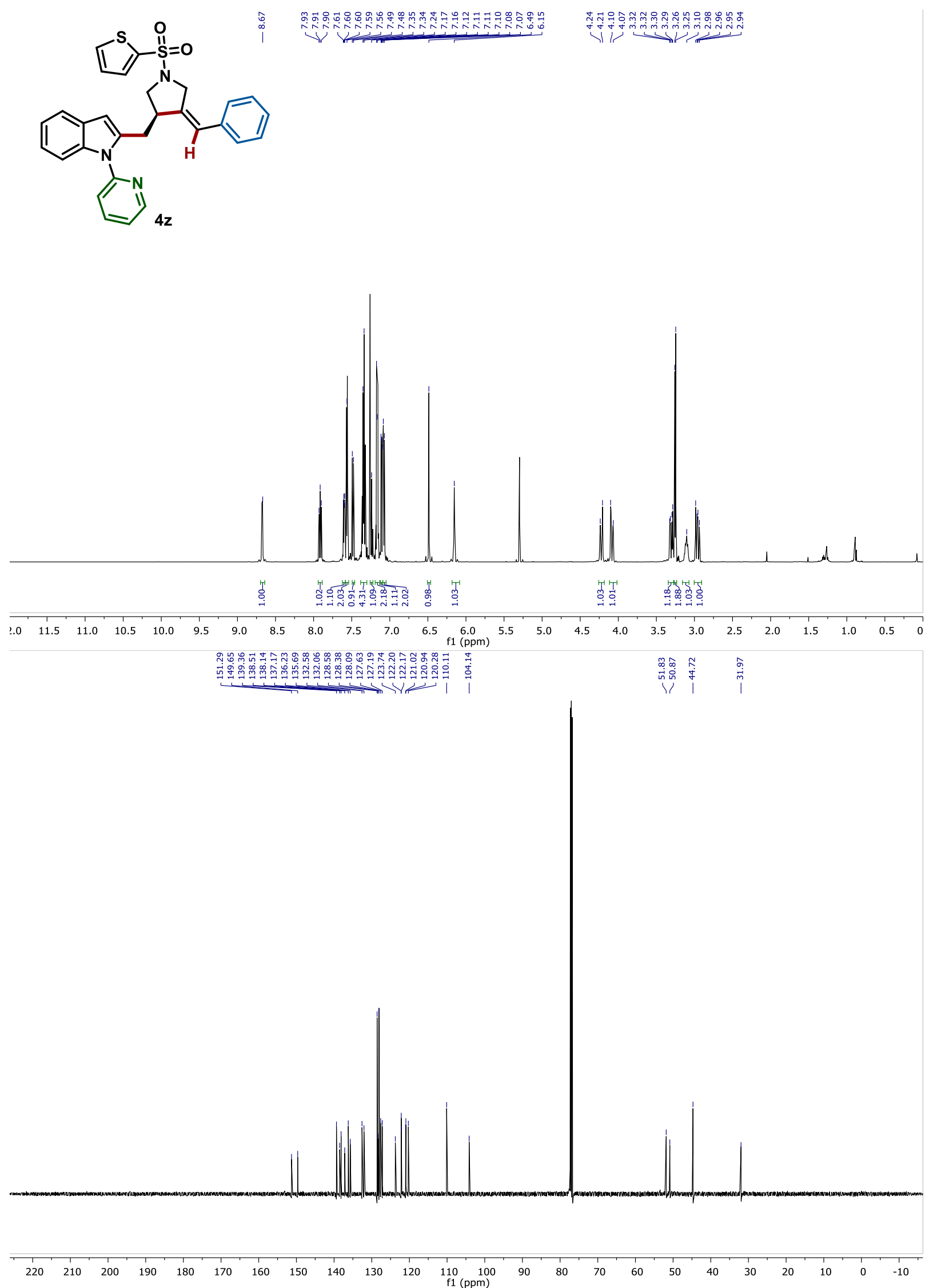


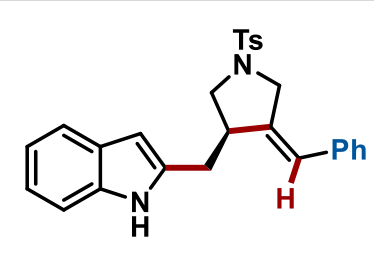

$5 a$

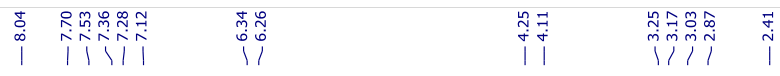
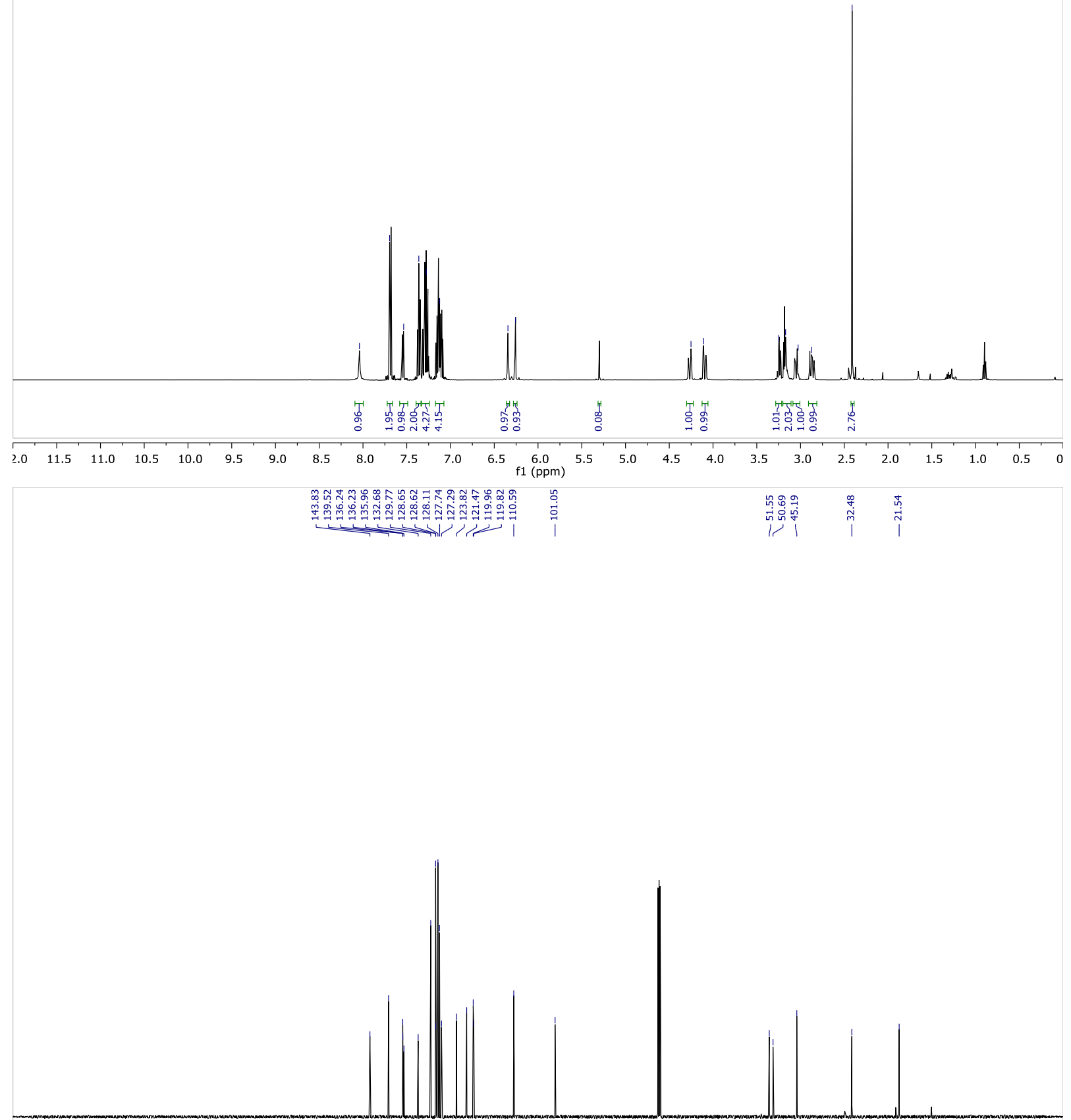

$\begin{array}{llllllllllllllllllllllllllllll} & 220 & 210 & 200 & 190 & 180 & 170 & 160 & 150 & 140 & 130 & 120 & 110 & 100 & 90 & 80 & 70 & 60 & 50 & 40 & 30 & 20 & 10 & 0 & -10\end{array}$ 